\title{
Towards an Architecture for Teaching Virtues, Values and Ethics
}

\section{Berise Thérèse Heasly}

BEd (MCAE), BEdSt (Mel), MEd (Mel), GradDipTheol (MCD)

\author{
School of Education \\ Faculty of Arts, Education and Human Development \\ Victoria University, Melbourne, Australia
}

Submitted in fulfilment of the requirements of the degree of Doctor of Philosophy

November, 2012 


\section{Abstract}

In this thesis, I look towards a new architecture for teaching an eventual curriculum about virtues, values and ethics. I address three sources of research: the voices of teachers who are teaching thinking and thinking skills; the authors who address the various themes and sub-themes emerging from the thesis questions; and an auto-ethnographical retrospective, which marries my experiential learning, formal qualifications and classroom experience.

The focus is on what has happened in education, what is best for teachers; what can be gleaned from the experts' voices, what consequently is helpful in the curriculum, and what can be delivered by teachers to enrich students' learning. Three leading twentieth -century theorists contribute to what counts as virtues, values and ethics. Research findings, personal experiential learning, formal qualifications and my own classroom instruments provide a foundation from which to reach incrementally towards the architecture for the teaching about virtues, values and ethics.

My theory of edu-tensegrity is derived from the architectural concept of tensegrity, which uses tension and compression as a building strategy (e.g., London's geodesic millennium dome; Melbourne’s soccer stadium); models inductive thinking rather than deductive thinking; and employs dialectic, trilectic and quadrilectic logics. Educationally, this applies to push-pull factors in decision-making and moral agentry - for both teachers and students. My conclusions show that such factors, labelled Categories of Influence, model trilectic logic, necessarily present in the detailed responses, response-abilities and responsibilities, that inform teachers' planning, execution and facilitation of the teaching of applied thinking skills: to promote excellence and efficiency that builds architecture that will reach towards a virtues, values and ethics curriculum for the twenty-first century and beyond. 


\section{Student Declaration}

I, Berise Thérèse Heasly, declare that the PhD thesis entitled Towards an Architecture for Teaching Virtues, Values and Ethics is no more than 100,000 words in length including quotes and exclusive of tables, figures, appendices, bibliography, references and footnotes. This thesis contains no material that has been submitted previously, in whole or in part, for the award of any other academic degree or diploma. Except where otherwise indicated, this thesis is my own work.

8 November 2012

Signature

Date 


\section{Acknowledgements}

I wish to acknowledge the following people, who have assisted me in this journey of discovery:

- Professor Brenda Cherednichenko, Pro-Vice Chancellor (Arts and Education) Deakin University, for her support, understanding and early mentoring in my candidature;

- Dr. Anne Davies, Victoria University, for her patient and precise supervision and technical support;

- Professor Constant Mews, Monash University, for his academic support at crucial points of my academic journey;

- Roger Sutcliffe, SAPERE, UK, for his timely mentoring and direction in my progress over a long career of study in understanding the breadth of thinking skills and the teaching skills to accomplish this aim;

- The late John Wilson, University of Oxford and Director of the Farmington Trust, whose mentoring and academic insights directed my learning in the Oxford University Department of Educational Studies in 1990-1991 and beyond;

- The late Professor Matthew Lipman, USA, (and his Australian associates), for personal direction, and whose Philosophy for Children movement informed so much of my subsequent studies;

- Dr. Peter Vardy late of Heythrop College, London, (and his UK and Australian associates) for the provision of so much for the teaching of senior classes via the DialogueAustraliaNetwork (DAN) and for the prosecution of this thesis; 
- Dr Rosalie Holian, then at RMIT Victoria, for academic consultation in the 1990s about direction in the compilation of the Heasly Behaviour Uncertainty Grid;

- Scott Larritt- Evans for technical and ICT support for the geodesic dome;

- Dr. Adrian Hubbard, for timely academic intervention in the construction of my theory of Edu-Tensegrity;

- Adjunct Professor Ian Ling, Victoria University, for his erudite and knowledgeable interventions and generous academic support towards the close of my academic journey;

- Rev. Fr. Michael Elligate, University of Melbourne, for his lifelong friendship and insightful support in all theological, psychological, literary and philosophical matters, over the span of this academic journey and beyond;

- My son, Denis Heasly, for lengthy technical and academic support during this long journey;

- My husband Bernard and my three daughters, Jane, Clare and Sara, whose patience and forbearance I can never adequately compensate, over such a long academic journey. 


\section{Table of Contents}

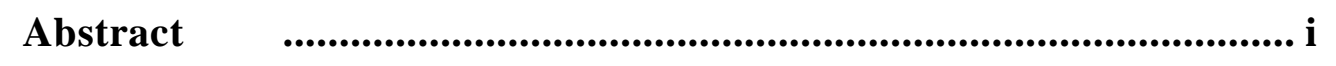

Student Declaration ................................................................................ ii

Acknowledgements..................................................................................... iii

Table of Contents ............................................................................v

List of Figures .................................................................................. i

List of Tables .............................................................................. ii

Chapter 1 Finding the Questions ......................................................1

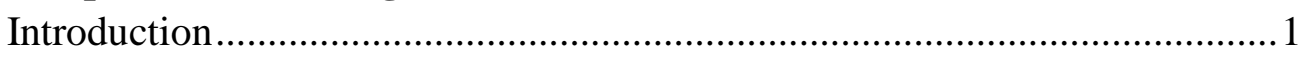

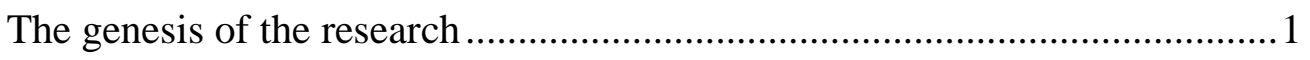

Learning with Wilson, Lipman and Vardy ................................................2

John Wilson 2

Matthew Lipman 3

Peter Vardy 4

The teaching of virtues, values and ethics ................................................... 4

Ontological considerations, epistemological concerns and

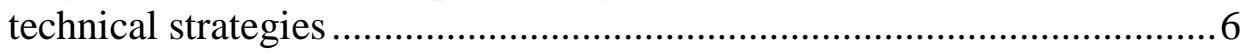

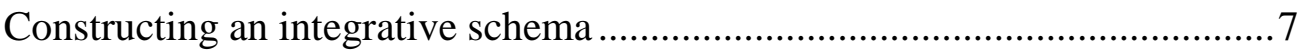

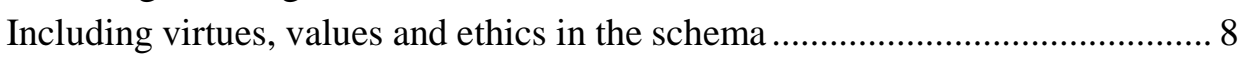

Including the ontological, epistemological and technical aspects in the schema 8

Conducting research in a multi-faceted context............................................

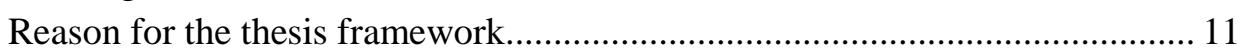

Relevance to teachers of this research ................................................................ 11

New understandings from brain research ....................................................... 14

Students 14

Needs 16

Beginning my research ........................................................................... 18

Learning the broad spectrum of philosophy …................................................. 20

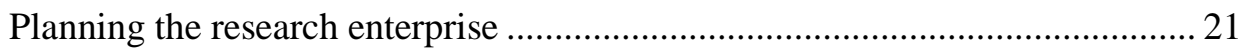

Aims of the research for teacher practice ........................................................... 21

Significance of the research and its contribution to new knowledge.............22

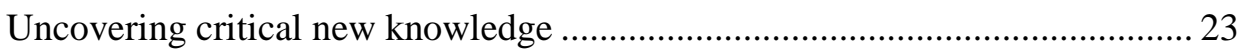

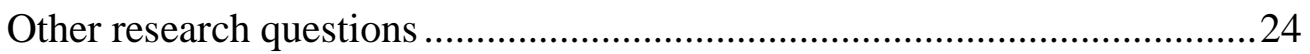


Chapter 2 Answering the Questions: Listening to Many Voices...29

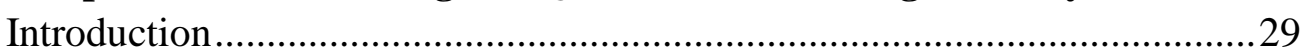

Taking a qualitative and quantitative approach ..........................................31



Teachers' voices 33

My practitioner voice $\quad 34$

Voices from the literature 35

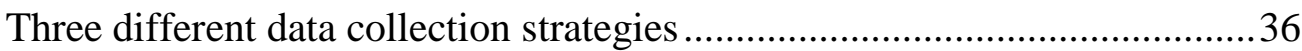

Questionnaire 36

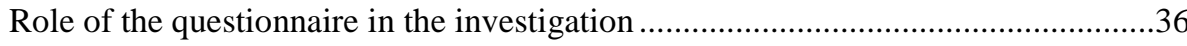

The thinking behind the development of a Questionnaire........................................38

The development of the Questionnaire ......................................................................40



Auto-ethnography $\quad 42$

Literature review $\quad 44$

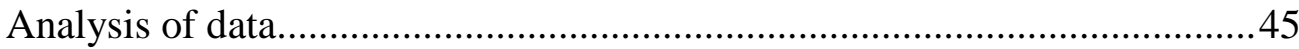

Hesitations, confusions, concerns and silences................................................ 47

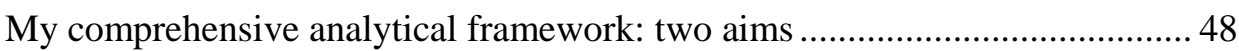

Evaluation of all relevant criteria for this enterprise........................................... 49

Looking forward... 50

Chapter 3

Voices in the Literature: A Multi-Disciplinary Search .......................52

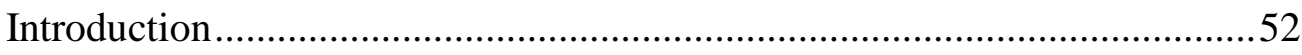

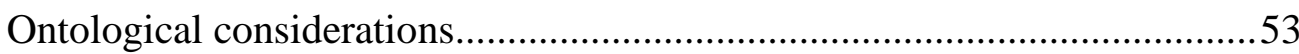

Virtues 53

Values 55

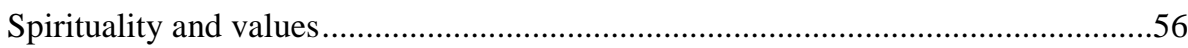

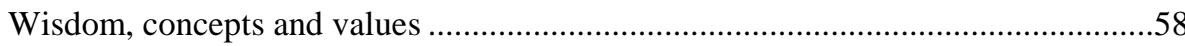

Neuroscience and its implications for education in the teaching-learning process ...60

Ethics

63

Epistemological concerns and consequences.....

Virtues 66

Values 69

The phenomenon of indoctrination

The links between secular and religious values and beliefs ....................................72

Moral argument, the good life and values through reflection and resilience .............76

The links to the building of meaning and of attaining wisdom ..................................78

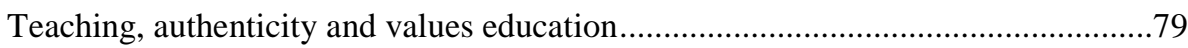

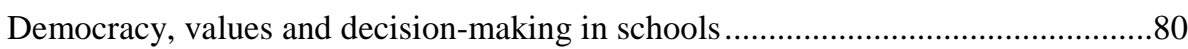

The entry of emotional and other intelligences .......................................................... 
Shining the light on possible reshaped pedagogies .............................................83

Links between educational trail-blazers and the application of thinking skills .........84



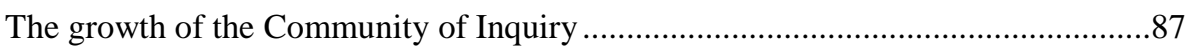

Linking from values and virtues to ethical considerations ......................................8

Ethics 88

Links: Zigler, Dewey and education .............................................................. 91

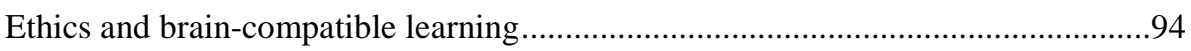

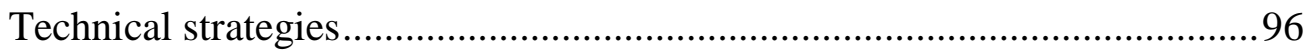

Virtues 96

Links between leadership, educational theory, pedagogy and thinking skills...........97

Understanding how the brain learns.....................................................................98

Values 100

The role of pedagogy: links between theorists, practitioners and experts ................102

Teachers' reflective practices..........................................................................106

Ethics 107

Inserting the pedagogy of the Community of Inquiry ..........................................108

Ethnographical Connections -broadening depth of My Voice .............................110

Looking forward... ....................................................................... 111

\section{$\begin{array}{ll}\text { Chapter } 4 & \begin{array}{l}\text { Practitioner Voices: My Voice, Teachers' Voices, } \\ \text { and Experts' Voices .........................................................112 }\end{array}\end{array}$}

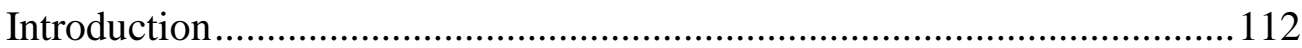

Questions and questioning ................................................................ 114

My practitioner voice $\quad$............................................................... 114

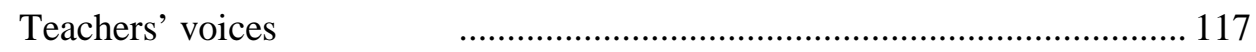



Timing and frequency of teachers' questions......................................................117

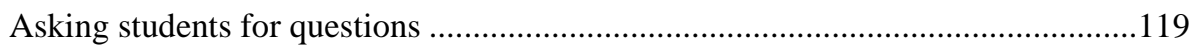

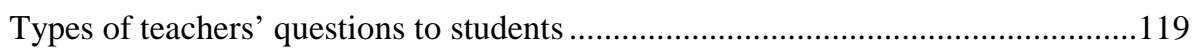

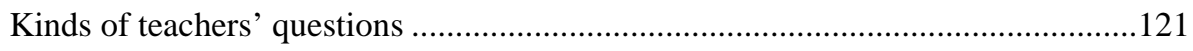

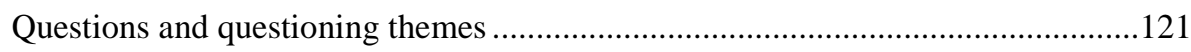

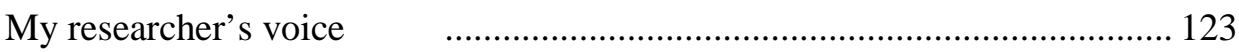

Curriculum frameworks ................................................................ 123

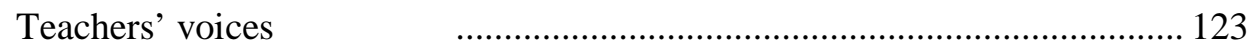

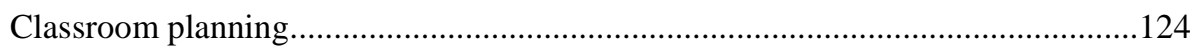

Teachers' range of strategies for documentation used ...........................................125

Variety in participants' chosen strategic frameworks .........................................127

Experts' voices $\quad$................................................................. 130

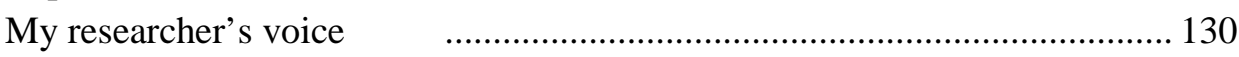

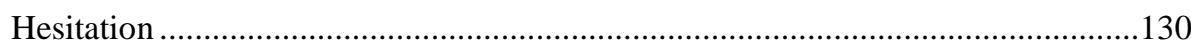

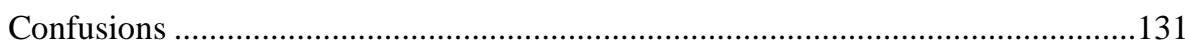






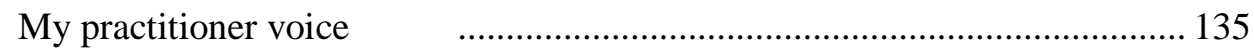

Teachers' voices

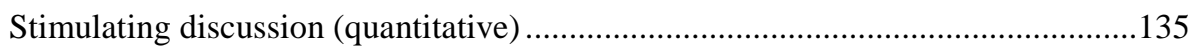

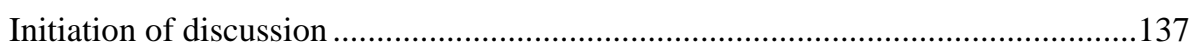

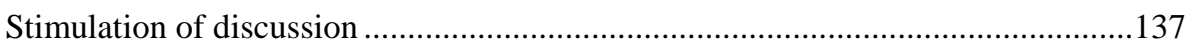

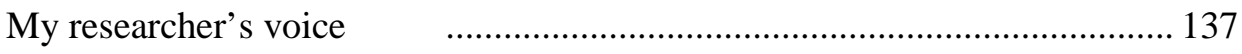

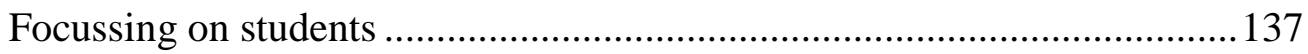



Teachers' voices

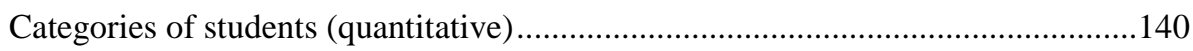



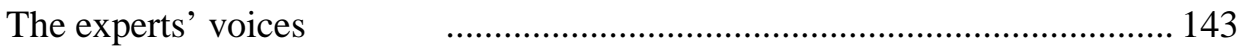

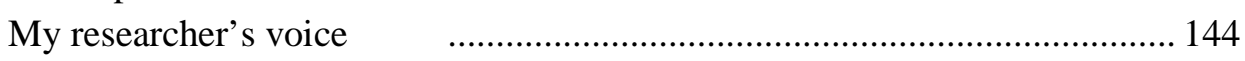

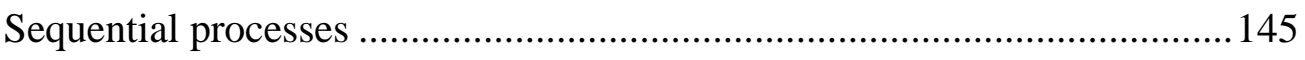

Teachers' voices

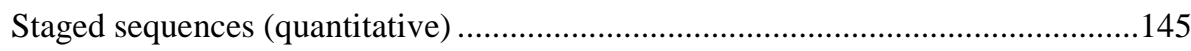

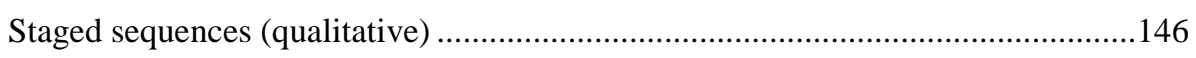

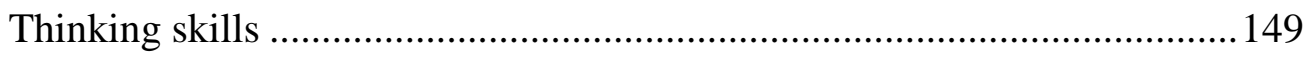

Teachers' voices

Categories, reasons and levels for teaching of thinking skills................................149

Rewards for student use of thinking skills ...........................................................151

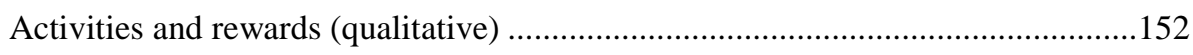

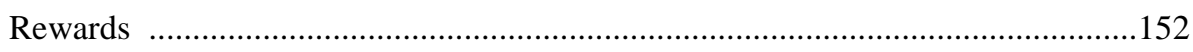

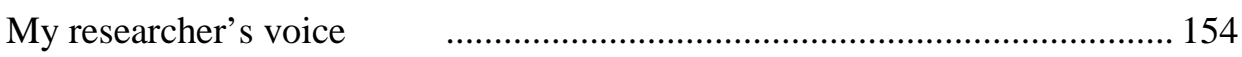

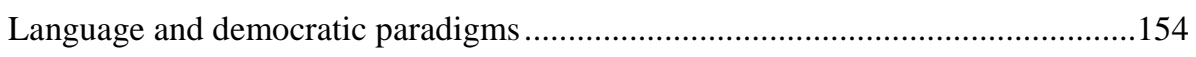

Reasons for, and perceptions of, thinking skills.....................................................157

Teaching the process of decision-making ................................................159

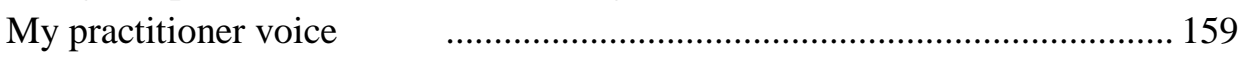

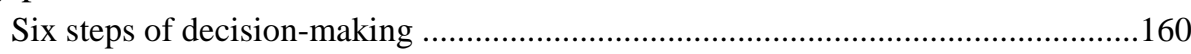

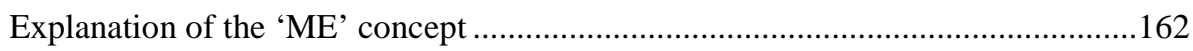

Teachers' voices

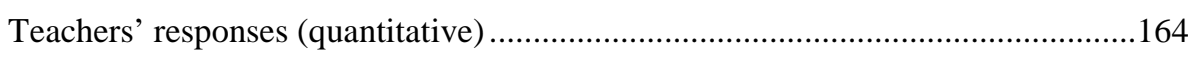

Teachers' responses (qualitative) ...........................................................................165

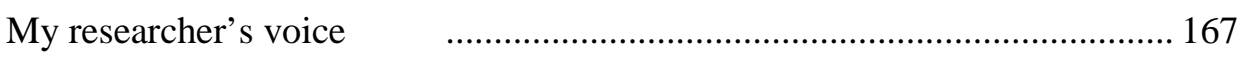

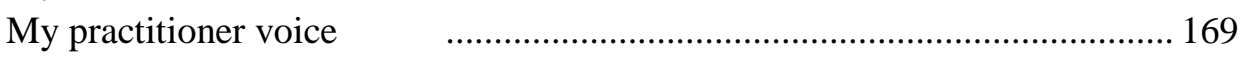

Practices that promote consensus and democracy .....................................170

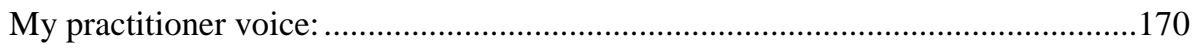

An example of ontological, epistemological and technical (techné) schema ...........170

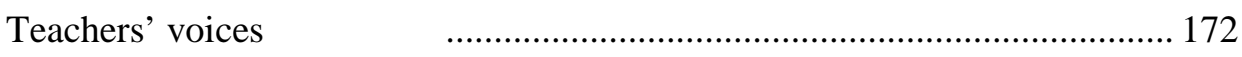

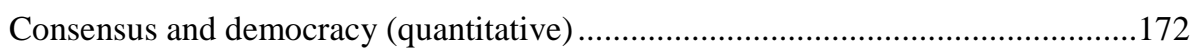

Consensus and democracy (qualitative) ............................................................172

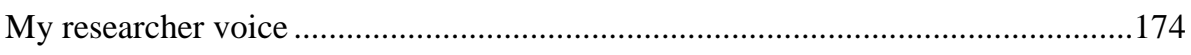

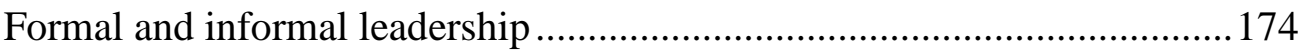




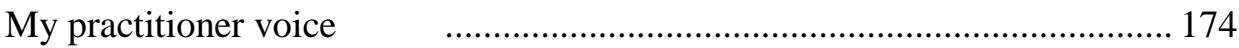

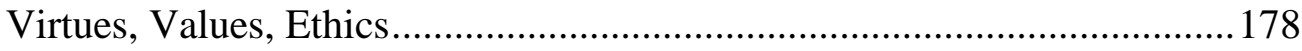

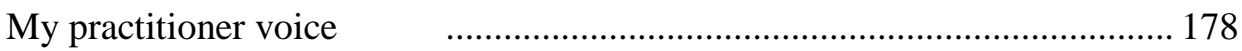

Teachers' voices

My researcher's voice $\quad$.............................................................................. 183

A profile of current teachers' responses: Compilation of the hesitations,

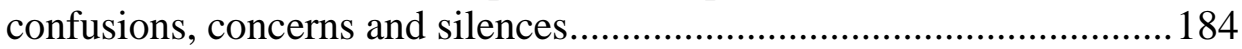

Hesitations

Confusions

Concerns

Silences

Conclusions and comments on Questionnaire content ..............................190

Teachers' voices

New ideas emerging: My researcher's voice .............................................192

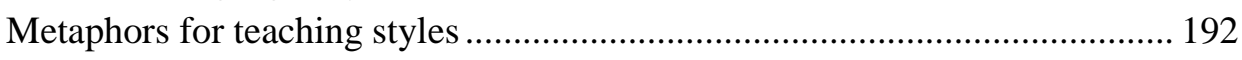

Connecting virtues, values and ethics (VVE) to the purpose of making decisions

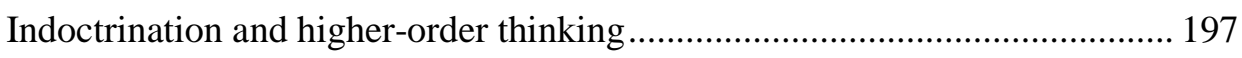

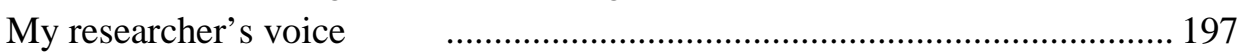

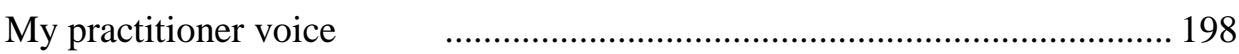

Concept of indoctrination vs. concept of higher-order thinking ...............................199

Control of delivered content vs. exploration of content .............................................199

Other-centred approach vs. self or independent approach ......................................200

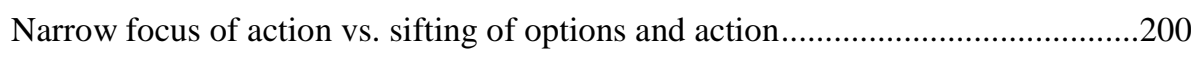

Assumption of power vs. personal autonomy ..........................................................200

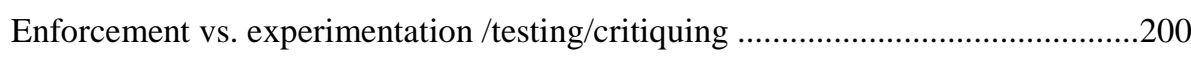

Defiance or dismissal of broad evidence vs. response-ability and responsibility ...201

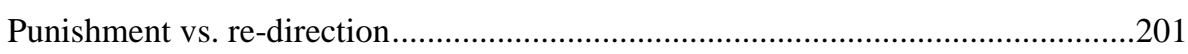

Attribution of guilt vs. acceptance of consequence or recompense...........................201

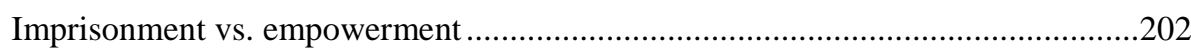

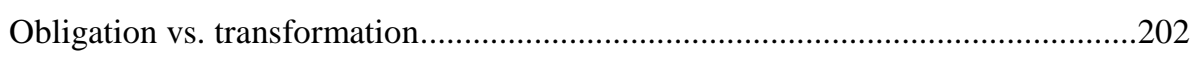

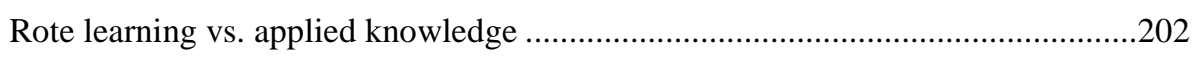

Required obedience vs. gathering of wisdom in action.............................................203

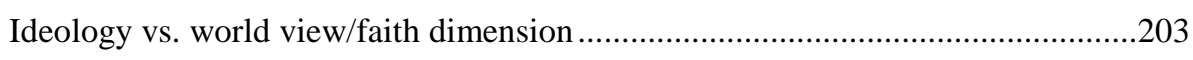

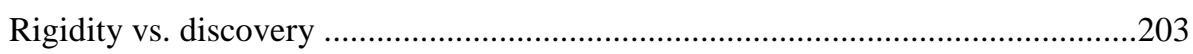

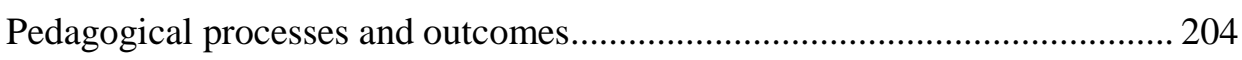

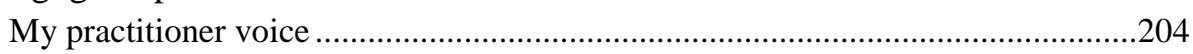

Need for a new architecture .................................................................206

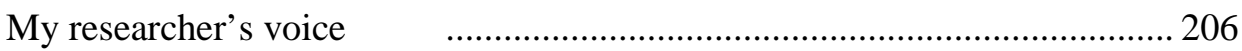




Chapter 5 Auto-Ethnographical Retrospective............................207

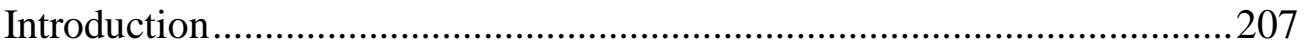

Stages of development: An auto-ethnographic account............................207

Forming 208

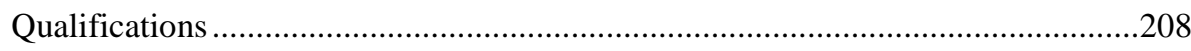

Formal and informal learning........................................................................210

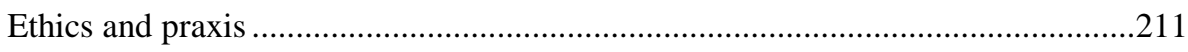

Norming

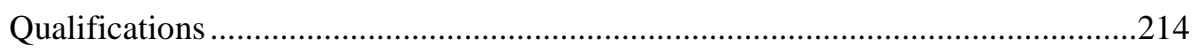

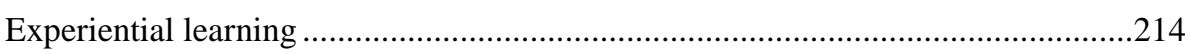

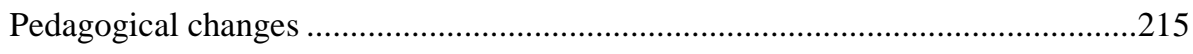

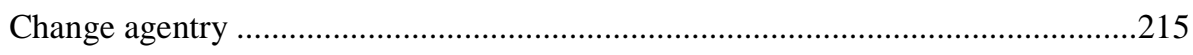

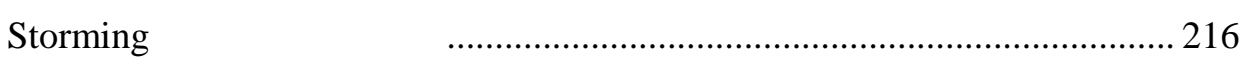

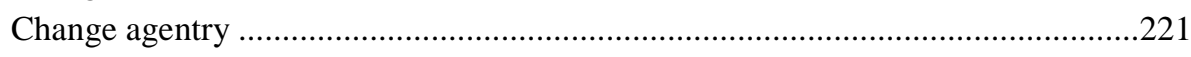

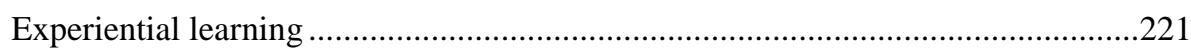

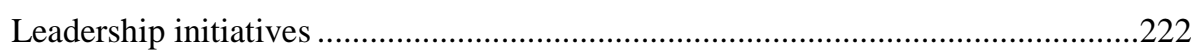

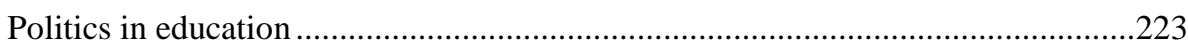

Research and qualification in Philosophy for Children...........................................223

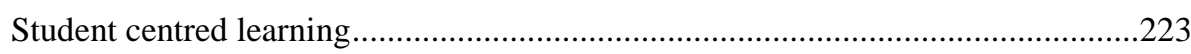

Performing

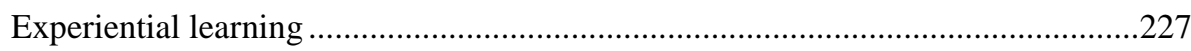

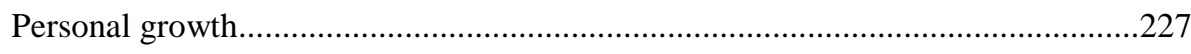

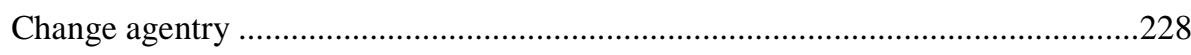

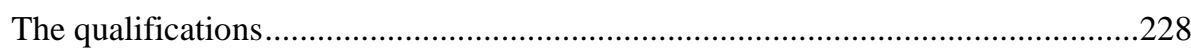

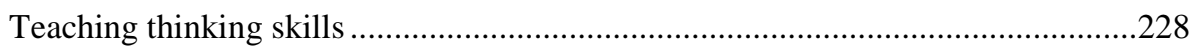

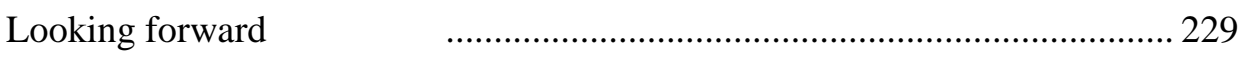

Chapter 6 From Twelve Categories of Influence to the Concept of Edu-tensegrity ............................................2230

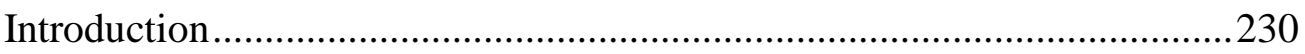

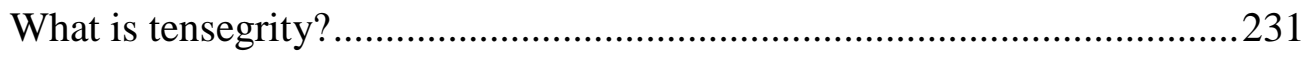

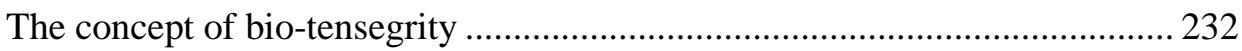

Tensegrity as a concept for education.........................................................234

A new concept of education: Edu-tensegrity ................................................ 236

Three dimensions for each Category of Influence .......................................... 238

Explaining the twelve Categories of Influence ..........................................239

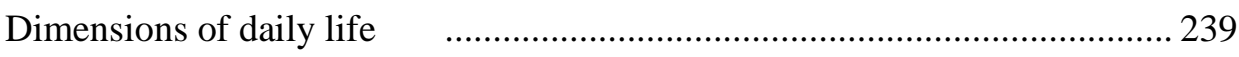

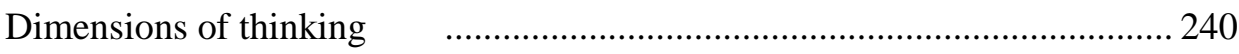

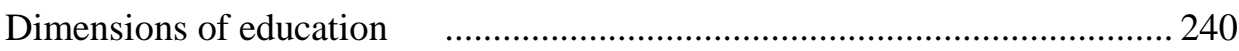

Curriculum theorists

Constructs about knowledge and the thinking curriculum............................... 241

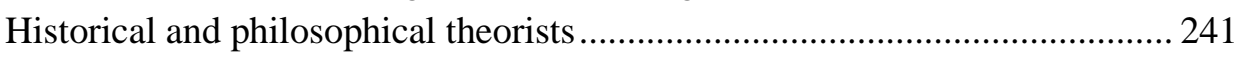

Historical and philosophical constructs ......................................................... 242 
Teachers and teaching $\quad 242$

Instrumental contributions 243

Outcomes and issues of dependence and rich learning.................................... 243

Construction of a belief system, the good life and a career ............................. 244

Integration of student, teacher and curriculum .................................................. 244

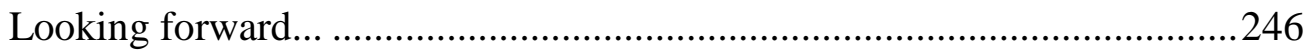

Chapter 7 A new architecture for teaching virtues and values...247

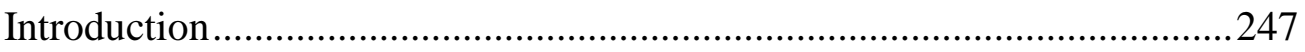

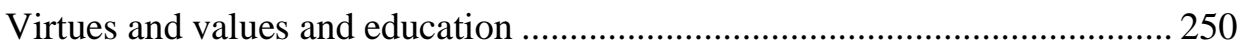

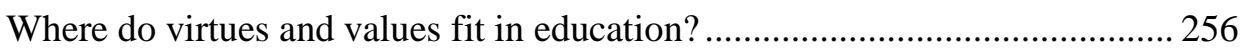

Category of Influence 1: Dimensions of daily life.....................................256

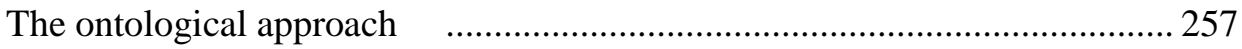

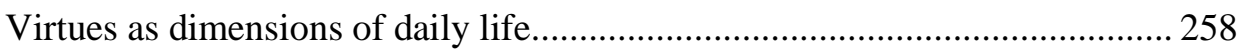

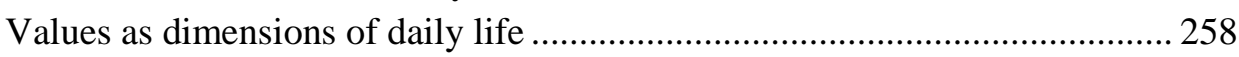

Category of Influence 2: Dimensions of thinking...................................259

A culture of reflection and a culture of resilience............................................ 260

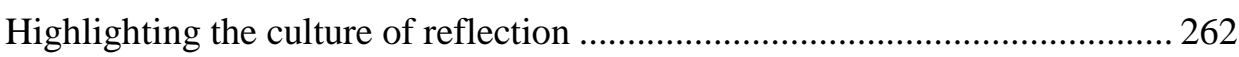

Linking the culture of reflection and the culture of resilience .......................... 263

Highlighting the culture of resilience ............................................................. 264

Connecting through and significance of the process of maieutic thinking ...... 265

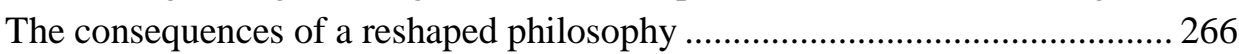

Separating moral matters from religious belief................................................. 268

Levels of moralisation: A reshaped concept of moral sensitivity..................... 269

Establishing open-mindedness, close-mindedness, bias, prejudice,



Teachers and moralisation 271

Category of Influence 3: Dimensions of education ...................................2272

Uncovering detail in dimensions of education................................................ 273

Category of Influence 4: Curriculum theorists and shaping the curriculum 274

Wilson

Lipman

Vardy

Category of Influence 5: Constructs about knowledge and the thinking

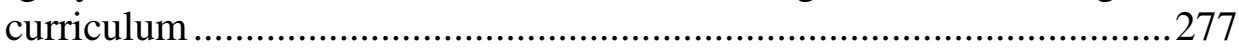

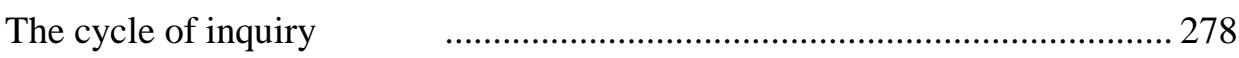

Things that get in the way of teaching thinking............................................... 279

Useful approaches: the role and function of questions ................................... 280

Role and function of questions: teachers' perceptions..................................... 281

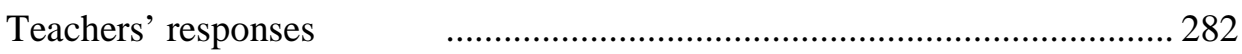

Category of Influence 6: Historical and philosophical theorists..................283

Category of Influence 7: Meaning, reasoning and choice blending and synthesising reflection and resilience............................285

Linking perceptions of theorists and experts in curricula terms ...................... 287 
Some historical links between values-based schools and meaning, reasoning and choice

Extending the links between thinking skills and decision-making ................. 290

Category of Influence 8: Teachers and teaching.......................................291

The unique contribution of Fisher................................................................... 294

Evidence of teacher-oriented languages used ................................................. 297

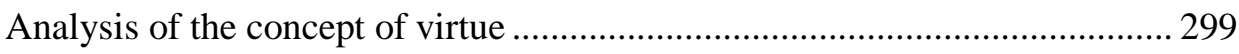

Teachers' perceptions, assumptions and expectations...................................... 301

Emerging relevance of brain research for teaching thinking ............................ 302

Category of Influence 9: My contributions.................................................305

Category of Influence 10: Outcomes, issues of dependency,

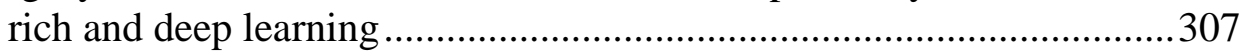

Category of Influence 11: The construction of a personal belief system, a good life and a career ......................................................................308

Category of Influence 12: The integration of student, teacher and the curriculum expert to form the nexus of pedagogical delivery ...............310



\section{Chapter 8 Reshaping Curriculum: Linking Applied Thinking} Skills to Ethics..............................................................312

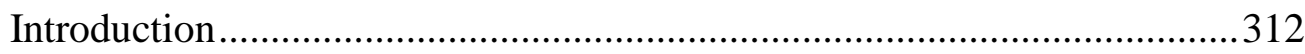

Ethics, ethical leadership, edu-tensegrity and education ............................314

Category of Influence 1: Dimensions of daily life........................................318

Categories of Influence 2: Dimensions of thinking......................................319

Categories of Influence 3: Dimensions of education ....................................320

Categories of Influence 4: Input from research theorists and the

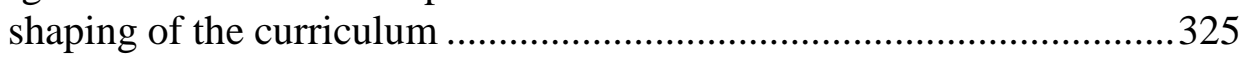

Specific linking of brain research, neuro-plasticity and decision-making ....... 329

Categories of Influence 5: Constructs and knowledge about the thinking curriculum.............................................................................331

Categories of Influence 6: Medical or biological and philosophical theorists lead us to Edu-Tensegrity.........................................................332

Categories of Influence 7: Constructs about meaning, reasoning

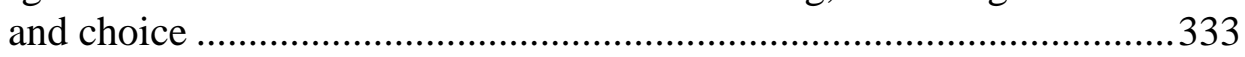

Categories of Influence 8: Teachers and teaching ......................................338

Categories of Influence 9: My curriculum................................................346

Categories of Influence 10: Students' new knowledge, understanding and expanded skills for learning .........................................................349

Categories of Influence 11: Emerging connections of neuroscience to learning

Categories of Influence 12: Collaboration between teachers, student and



Connections of the various Categories of Influence to each other and the classroom. 
Teachers' recognition of inquiry-based learning in classes ................................ 366

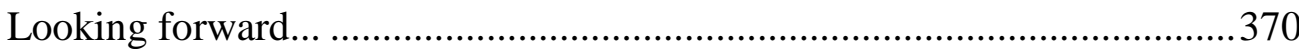

Chapter 9 Reshaping the Values, Virtues, and Ethics (V.V.E.) Future: Responses, response-abilities and responsibilities..............................................................372

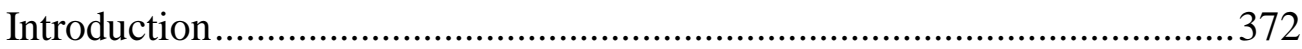



Response-abilities: Improving teaching about V.V.E................................377

Integration of all Categories of Influence into the dome of education ............. 378

Practical implications for the role and function of teacher ................................ 380

Responsibilities: Creating a future for V.V.E.............................................381

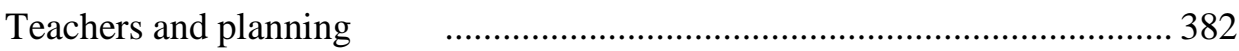

Categories of Influence as responsibilities for education ................................. 383

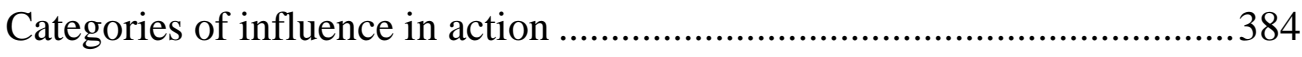

Consequences

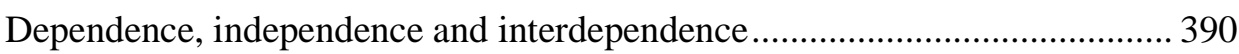

Questions raised by this investigation: Results and answers .......................392

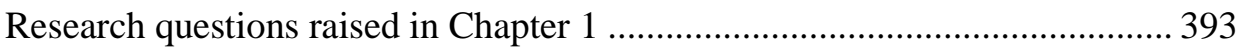

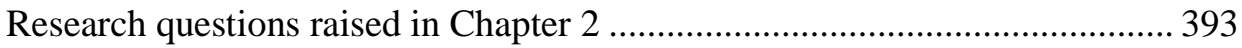

Emerging elements for twenty-first century learning conditions...................... 396

My Final Reflections on the experience of being a researcher Error! Bookmark not defined.

References ….......................................................................................400

Appendix A: Questionnaire ...........................................................422

Appendix B: Splitter \& Sharp......................................................440

Appendix C: John Wilson...............................................................441

Appendix D: Lipman Obituary by Roger Sutcliffe ...........................443

Appendix E: Strategies for teaching (Moore).................................446 


\section{List of Figures}

Figure 1.1

Figure 2.1

Figure 2.2

Figure 3.1

Figure 4.1

Figure 4.2

Figure 4.3

Figure 4.4

Figure 4.5

Figure 6.1

Figure 6.2

Figure 6.3

Figure 6.4

Figure 7.1

Figure 7.2

Figure 7.3

Figure 7.4

Figure 7.5

Figure 7.6

Figure 7.7

Figure 7.8

Figure 7.9

Figure 8.1

Figure 8.2

Figure 9.1

Figure 9.2
An integrative schema

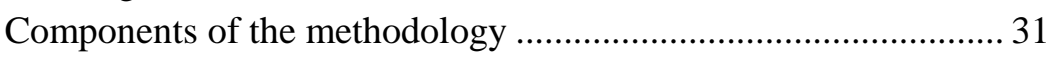

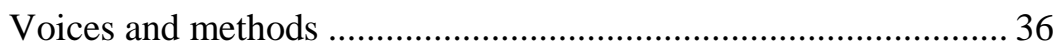

Applying the integrative schema to the literature review................ 53

The Construction of students' questions for enhanced learning .. 115

Decision-making: Considered process v.impulse approach.......... 138

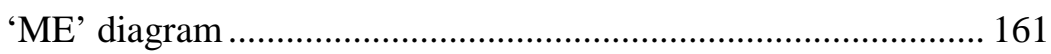

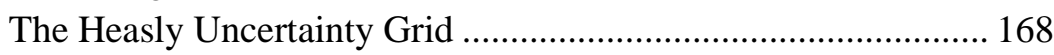

Elements of indoctrination and higher-order thinking ................. 199

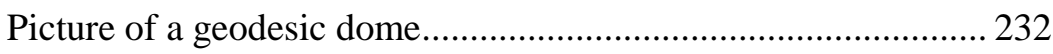

Picture of a giraffe's neck ........................................................ 233

Model: giraffe's neck or cable-and-beam sculpture..................... 233

Edu-tensegrity and the 12 Categories of Influence ....................... 236

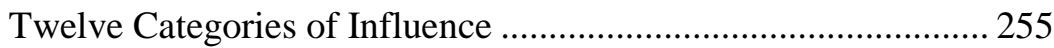

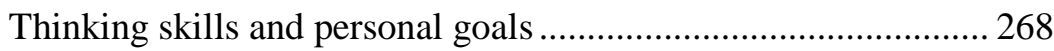

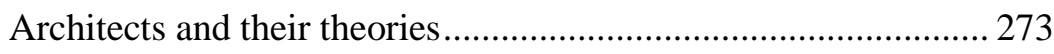

Heasly cycle of inquiry ............................................................. 279

Wassermann's Five Es model ...................................................... 280

A picture of teacher - scaffolding of questions............................ 281

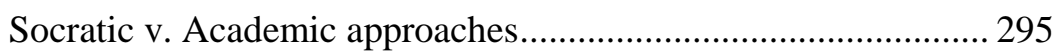

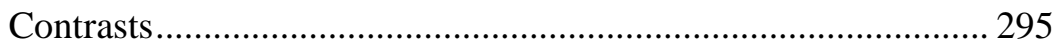

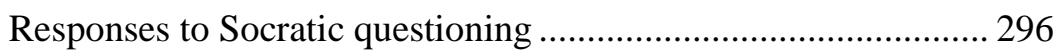

Teachers use of stimuli in classroom .......................................... 340

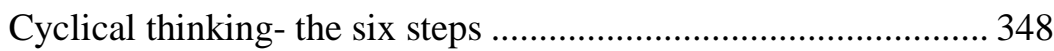

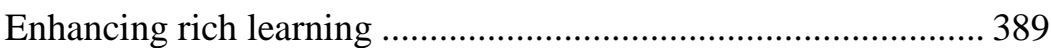

Teachers' role in the democratic inquiry classroom....................... 391 


\section{List of Tables}

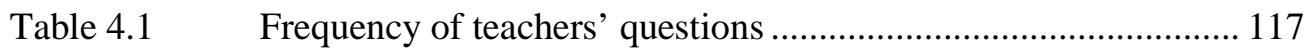

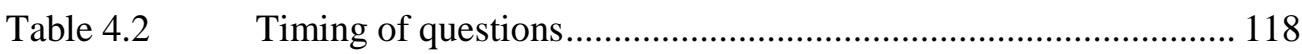

Table 4.3 Timing of questions.................................................................... 119

Table 4.4 Frequency of student questions …….......................................... 120

Table 4.5 Teachers' perceptions of their questions ....................................... 120

Table 4.6 Varieties in types of teachers' questions ...................................... 121

Table 4.7 Questions and questioning themes .............................................. 122

Table 4.8 Teachers' documentation for classroom planning......................... 124

Table 4.9 Government documents employed ................................................ 125

Table 4.10 Classroom planning and suggested schemas................................ 125

Table 4.11 Classroom planning and student-centred thinking and learning .. 126

Table 4.12 Strategic frameworks and examples.......................................... 128

Table 4.13 Student-centred thinking ............................................................. 130

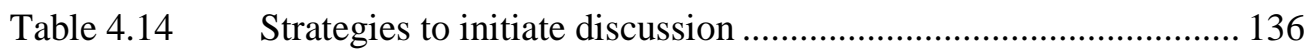

Table 4.15 Strategies to stimulate discussion............................................ 136

Table 4.16 Finding accurate focus through questions ..................................... 140

Table 4.17 Ensuring full student participation in questioning ....................... 141

Table 4.18 Staged sequences.................................................................... 146

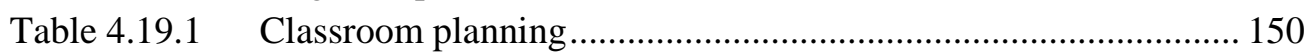

Table 4.19.2 How thinking skills are currently addressed ............................... 150

Table 4.20 Variety of reasoning behind affirmations used ............................ 151

Table 4.21 Activities based on curriculum areas........................................ 152

Table 4.22 Reinforcement strategies and approaches .................................... 153

Table 4.23 Democratic and paradigmatic language ...................................... 155

Table 4.24 Reasons and perceptions of thinking skills ................................. 157

Table 4.25 Teaching of decision-making skills............................................... 165

Table 4.26 Teaching the processes of decision-making ................................. 166

Table 4.27 Teaching the concepts of consensus and democracy ................... 171

Table 4.28 Teaching consensus and democracy............................................. 173

Table 4.29 A listing of formal and informal leadership elements .................. 175

Table 4.30 Responses: teaching virtues, values and ethics ............................ 180

Table 4.31 Teaching values, virtues and ethics.............................................. 181

Table 4.32 Teachers' conclusions and comments .......................................... 190

Table 7.1 Schwab’s commonplaces and Categories of Influence ................. 248 


\section{CHAPTER 1}

\section{Finding the Questions}

\section{Introduction}

I began my teaching career with minimum qualifications and was determined to become expert and efficient in knowledge, skills and, if necessary, leadership. To accomplish this broad aim, I needed to build knowledge through educational studies, supported by classroom experience; to build skills through reflective practice; to deepen understanding through attendance and integration of seminar and conference content; to build a consultative broad base of current educational issues being promoted by Australian and other educational leaders.

This thesis is an inductive narrative in which I research widely to determine the initial and essential questions that might inform and shape my exploratory efforts to accommodate educational change for twenty-first century education.

\section{The genesis of the research}

Over a long career of thirty-six years as a secondary teacher in twelve schools (girls' schools, boys' schools, co-educational schools), including leadership for several faculties (Music, English, Religious Studies, Religion and Values Education), I had become aware that some colleagues focussed their efforts on teaching the subject-content, and the consequent processes, with reason and logic, according to their discipline area. Others, like me, 
focussed their attention on the student, and upon the development of student thinking skills so as to enable an understanding of the subject through the means of the rich learning activities contained in the subject-content. Both approaches seemed legitimate, but with questions in my mind about them I decided to find out what was happening in other places. The search began in the UK and led to the discovery of the work of John Wilson and, almost at the same time, the work of Matthew Lipman; much later, the work of Peter Vardy became valuable.

To accomplish such a wide arc of knowledge, I needed to undertake a comprehensive literature review, so that I was building on what was already known or as Newton said to Hooke 'If I have seen further it is by standing on ye sholdres (sic) of Giants' (Newton, 1676, p. 39).

\section{Learning with Wilson, Lipman and Vardy}

John Wilson, Matthew Lipman and Peter Vardy advocate introducing the concepts of virtues, values and ethics through the medium of teaching thinking skills. The three following sections describe each of their approaches.

\section{John Wilson}

During 1990 and 1991, John Wilson personally encouraged me in three ways. First, he extended an invitation for me to participate in his Master of Education program, but unfortunately because of school leave requirements in Victoria, I could not complete the relevant local Oxford University examinations before it was time to return to teaching in Australia. Second, he invited me to participate in his D.Phil. classes, which I gladly joined, and, through this experience of working with Wilson, I developed a new confidence in my own experience of, and expertise in, teaching. Finally, as Director of the Farmington Trust, he directed my own subsequent research at the Library of the Department of Educational Studies in Norham Gardens, Oxford, so that I could fashion a foundation for further studies and research 
into education in my own milieu. I brought home to Australia extensive notes and books, formal materials and publications, as a foundation for my subsequent studies.

As a result of that year's work I developed an understanding of Wilson's concepts of Moral Components (Wilson, 1971) and his indications of the need to teach students in what he called moral matters.

\section{Matthew Lipman}

Concurrent with this development of personal and professional knowledge and expertise, back in Victoria, Australia, I was introduced to the work of Matthew Lipman, the originator of Philosophy for Children. By attending professional development courses before and after my time spent with John Wilson, I came to realise that for the purposes of my studies, there appeared to be reasons for incorporating Wilson's Moral Components into Lipman's Philosophy for Children.

The structure of Lipman's Community of Inquiry formed a basis from which the concept of teaching children to wonder, to question and to investigate, could proceed. The significance of incorporating an understanding about virtues, values and ethics into the promotion of new knowledge for children was most important if one were to accept Lipman's insistence that children, even in early primary school, could reason right from wrong and use this logic to shape their own behaviour. In my mind this incorporation became coupled with Wilson's efforts to insert moral education into the primary and secondary school curricula by allowing for all participants to apply their own thinking to their own situations.

My hope, despite resistance from some colleagues, was that students in secondary schools would benefit academically, for the sake of their studies, and emotionally, for their personal well-being. 


\section{Peter Vardy}

The work of Peter Vardy $(1998,1999)$ was initially applied to the teaching of concepts and the study of topics for Years 11 and 12 educational purposes. It included accompanying teaching resources and seminar support for teachers of such subject areas as Ethics, Philosophy, and Religion and Values Education (RAVE).

The blending and augmenting of the Wilson Moral Components List, the Lipman Community of Inquiry and the Vardy RAVE curriculum, provided the starting point for this research. Importantly, and following Vardy's lead, the thinking skills I applied ended in my deeper understanding of how synthesis worked in thinking through particular challenging situations prior to personal decision-making.

It is generally agreed that the ability to develop and teach thinking skills (Lipman, 1993a); (Wilson, 1963; 1987) is essential for effective and efficient teaching in the changed and changing culture of the twenty-first century (Cherednichenko, 2000). Lipman’s Community of Inquiry (Lipman, 1993a) also contributes valuably to the aim of teaching the student through the medium of the curriculum (Vardy, 1998).

\section{The teaching of virtues, values and ethics}

The knowledge and strategies required of the teacher (phronesis; colloquially, know-how) as first proposed by Aristotle (Beckett, 1994), are echoed in the teaching-learning strategies constructed by Lipman. The accuracy of the conceptual frameworks constructed by Wilson also appears to align with the chief requirement of Aristotle's phronesis: that the student is of central significance (Shulman, 1987). The strategies are the teachers' classroom tools; the content is the opportunity and the process; the outcomes signify the moral and professional purposes of the teacher's planning and practice (Heasly, 1995). Full recognition of the ethical and epistemological bases of practice (Heasly, 1995) in a classroom marked by a diversity of 
efficient and effective technical (techné) strategies (Aristotle, 1962), results in the enhancement of effective teaching practice.

The combination of these learnings, led me to an understanding of the significance of the teaching about virtues, values and ethics. The sense of virtues and values as shapers of the individual's understanding about what constitutes a good life (Aristotle, 1962) began to emerge, and ethics became comprehendible as the visible behavioural form of personal virtues and values (Singer, 1979; Lipman \& Sharp, 1985).

As I worked with these ideas I realised that I needed to think about the ontological, epistemological and pedagogical dimensions of, and their connections to, the teaching about virtues, values and ethics (Kincheloe, McLaren \& Steinberg, 2011p. 163-177; Lincoln, Lynham \& Guba, 2011, p. 97-128).

I deemed it important to shape an understanding of the various philosophical concepts (drawn from Aristotle, John Dewey and Joseph Schwab) which would emerge for me as applicable to education, connecting into recognisable theories (those of Hedley Beare and Andy Hargreaves). This would provide a framework for a new architecture for the teaching of virtues, values and ethics (as promoted by Wilson, Lipman and Vardy); would provide a new paradigm for the needs of twenty-first century education: one which measured and answered at least in part Frank Crowther's Fourth of Six Dynamics (micro-curriculum deepening) (2010a; 2010b; 2011). This fourth dynamic proposes capacity-building and leadership innovations through micro-curriculum deepening in pursuit of sustainable improvements in education. The new emphasis and new knowledge gained would provide the content, and would in turn reshape the educational processes into a unifying theme (Crowther 2010a, 2010b; Heasly, 1995), based on the parallel architectural principles of 'tensegrity' (Ingber, 1997; Levin, 2002), into a structure for the pedagogical development of existing, but prioritised content - emphasising the connection between the 
student, the teacher, the curriculum (what is taught) and the milieu (Schwab, 1983) in which education took place.

\section{Ontological considerations, epistemological concerns and technical strategies}

Cherednichenko and Kruger (2002) provided me with an early understanding of the ontological considerations, epistemological concerns and consequences, and the technical dimensions of teaching. And Davies (2005, p. 25- 26) indicates, Habermas concentrates on the states of rationality and communicative action, including the relationship between the state and the public sphere, precisely where, I believe, the visible evidence of ethics in action are to be seen.

It became apparent that the technical dimension emerging as strategies and techniques from the Aristotelian idea of techné (Aristotle, 1962), meaning 'art' or 'applied science' or 'skill', is, of course, pedagogical in essence. Aristotle's term refers to skills, and their acquisition from and application to: inductive and deductive thinking patterns; to knowledge itself; to decisions based on ontological considerations, epistemological concerns and their consequences; and to the strategies needed for teaching about virtues, values and ethics. All cultures and communities rely on such patterns to achieve harmony and happiness (Seligman, 1990; Rowland, 1993; Seligman, 2002; Seligman, 2011).

All three dimensions of practice must to be considered, in order to assist the development of ideas about the teaching of virtues, values and ethics. The big ideas and philosophical patterns upon which experience, understanding and commitment rest, translate into ontological considerations. The breadth of knowledge, the variety of skills and the effectiveness of decision-making processes, become merged into epistemological concerns and consequences. The effective strategies and techniques constructed as teachers and curriculum specialists shape the classroom experience of learning for the student cohort, become pedagogical expertise. They uncover, in my 
estimation, the precise and focussed technical strategies which emerge and evolve into rich learning, including eschatological speculations about the meaning of life today.

It is important to me to establish that a number of almost synonymous terms appearing in this study for what takes place in the classroom can be identified:

1. Aristotle's techné, as translated by Ostwald (1962), originally referred to a similar, but not exactly correlative concept of working with the learner to harness understanding.

2. Technical strategies (Anih, 1992; Gardner, 1993b; Lipman, 1993a; Anih, 1995; Lipman, 2003) that a teacher uses to help guide students whose learning styles sometimes differ markedly as they wrestle with new knowledge.

3. Pedagogical content and subject planning (Schwab, 1983) refer to much the same area because of the skill required in making the plans 'fit' the complexities of the learning pattern of a particular class.

\section{Constructing an integrative schema}

- These three considerations, together with the content knowledge associated with the teaching about virtues, values and ethics, became central to this research endeavour, and the integrative schema shown below in Figure 1.1 has shaped its conduct and analysis. The schema functions according to two objectives: one indicates the importance of analysing and understanding about virtues, values and ethics, and a second represents the different aspects of teaching practice. 


\section{Including virtues, values and ethics in the schema}

In using this schema, particular attention was given to the chosen three philosopher-educationalists central to the investigation - Wilson (1963 and beyond), Lipman (1993a, 2003) and Vardy (1998) - and to their key ideas on the teaching about virtues, values and ethics. Their seminal work in the

FIGURE 1.1 AN INTEGRATIVE SCHEMA

\begin{tabular}{|c|c|c|c|}
\hline Virtues & $\begin{array}{c}\text { ONTOLOGICAL } \\
\text { CONSIDERATIONS }\end{array}$ & $\begin{array}{c}\text { EPISTEMOLOGICAL } \\
\text { CONCERNS AND } \\
\text { CONSEQUENCES }\end{array}$ & $\begin{array}{c}\text { TECHNICAL } \\
\text { STRATEGIES }\end{array}$ \\
\hline Values & $\begin{array}{c}\text { Virtues and } \\
\text { ontological } \\
\text { considerations }\end{array}$ & $\begin{array}{c}\text { Virtues and } \\
\text { epistemological concerns } \\
\text { and consequences }\end{array}$ & $\begin{array}{c}\text { Virtues and technical } \\
\text { strategies }\end{array}$ \\
\hline Ethics & $\begin{array}{c}\text { Values and } \\
\text { Ethics and ontologionical } \\
\text { considerations }\end{array}$ & $\begin{array}{c}\text { Ethics and epistemological } \\
\text { and consequences } \\
\text { concerns and } \\
\text { consequences }\end{array}$ & $\begin{array}{c}\text { Values and technical } \\
\text { strategies }\end{array}$ \\
\hline
\end{tabular}

development of the teaching of thinking skills includes discussion of what is to count as virtues, values and ethics in education, and is linked to the efforts of a variety of other authors who have investigated and developed parts of the connections mentioned.

\section{Including the ontological, epistemological and technical aspects in the schema}

Since a number of conceptual layers interlink the theoretical connections of the project, I deemed it crucial that the first strands be established immediately: those relating to ontological, epistemological and technical (techné) strategies. To facilitate this aim, conceptual links are indicated whether consonant with or contrary to virtues, values and ethics - in relation to various authors' contributions to content and new knowledge. The schema diagram shows three sections inspired by Cherednichenko and Kruger (2002): 
- Ontological considerations: which relate to the big ideas and constructs, philosophies and understandings underpinning the teaching of thinking and thinking skills.

- Epistemological concerns and consequences: which relate to the teachers' knowledge of these big ideas and constructs (Crowther, 2010a, 2010b, 2011); it is on this basis that teachers facilitate the development of students' skills.

- Technical strategies: which relate to the further development of how teachers proceed to attain these goals; constructing and implementing effective strategies and techniques as they proceed in an increasingly expert use of pedagogical skills (Costa, 2001).

\section{Conducting research in a multi-faceted context}

During the blithe stampede of the 1990s in which the Australian Federal Government, with typical political zeal, pushed Civics and Citizenship education into the school systems, consequences arose that had never been considered by the politicians of the time, including demands on, and requirements of, teachers that they teach and use their understanding of all underlying concepts (Mackay, 1993) so as to promote such behaviours as foresight, prudence, commitment, as part of the curriculum (Cam, 2002b). Initial reaction by government figures has implied an expectation of mere passivity and quiet acceptance of the recent political landscape's economicrationalist framework on the part of teachers (Splitter \& Sharp, 1995; Gardner, Susan, 1999).

Competition is the central tenet of the harsh world of economicrationalism, but Civics and Citizenship education requires much more. It requires, on a daily basis, the promotion of competition and collaboration, the insights to facilitate personal choice pertinent to a given situation, and the provision of assessment vehicles to monitor one's own progress (Hopkins, 1978). I have found that students currently prefer to spend time tussling daily 
only with those tasks that are to be assessed. They do not want to give credence to any special topic unless, after a combination of teacher input and consequent investigation, they are to be assessed on their work. There, according to Hugh Mackay (1999), Civics and Citizenship education ends!

Principles of equity, quality, flexibility and choice (Pascoe, 1996), are crucial to the broadening of horizons sufficient to allow for the assessment of educators as more than mere mute implementers of government policy. And ways must be found to ensure that their voices are heard. In the contemporary world of pervasive media and advertising influence, there appears to be a very real narrowing in the perspectives of students; and also (I suspect) of teachers. This is experienced as a disallowing of any real choice. Naomi Klein's (2007) work highlights this in a severe way. There is real reason for considering the possibility of an insidious form of subliminal indoctrination. This matter is taken up in Chapter 4.

In their efforts to construct their own future, students come to see that they are a generation which is societally dependent - financially and intellectually - until at least the age of 25 years (Mackay, 1993). As I have observed during my years of teaching, this experience may rob them of their blossoming initiative in their early teens; an outcome with serious concerns for the teachers who face them in their secondary schooling. It can and does, also, lead so often to serious student personal risk-taking that can be breathtakingly life-threatening.

In today's highly technological world, the postmodern phenomenon of blurring the edges of all boundaries is a legacy these students accept from a previous generation who dignify this situation with ideological labels such as 'atomism', 'human rights', 'individual choice' and 'independence'. It is no wonder that we see the emergence of cynicism, nihilism and the consequentialism/anti-consequentialism linear-thinking bind, as we move from classroom to classroom (Connell, Ashenden, Kessler \& Dowsett, 1982). If the new social media has become the current place for lasting socialisation, rather than the school or, as in previous generations, the family and the home, 
then the content and format of teaching needs refurbishment at the very least (Gardner, 1993a). Claims that Lipman's Philosophy for Children addresses these phenomena will be examined (Splitter \& Sharp, 1995). Other existing courses for teacher qualification may also need examination in the context of teachers' contributions. My Heasly 'ME' diagram provided in Chapter 4 addresses and begins the analysis of the socialisation process.

The first and overarching question then, is: how do teachers respond to the implied needs of the student cohort when including such considerations as the teaching of thinking skills at secondary school levels and, as I subsequently postulated, would a proposed virtues, values and ethics curriculum provide a way forward?

\section{Reason for the thesis framework}

Change for change's sake is not answer enough to any of my questions. The importance of change in the student experience in the twenty-first century manifests itself in the broader spectrum of knowledge, facts and information available through the proper use of information technology sources.

Therefore, the existence and promotion of technology in Australian culture, and its consequences for the teaching-learning experience of the whole student cohort, must become part of the studies and skills offered to students on a regulated curriculum basis for their effective and efficient use of research sources.

\section{Relevance to teachers of this research}

It is most relevant here to inspect and analyse the consequences for student teachers whose own experience of education and schooling took place in the climate of the values-neutral classroom pedagogy. Classroom culture is subject to subtle changes, as Schwab (1983) has indicated. Given the huge range of options identified for students to make their own choices, it becomes a matter of concern that many students are confused by such breadth, and because decision-making is left almost entirely to them, or fashioned in the 
opposite manner This phenomenon sometimes emerges where no explorations of options are fully researched, so students are choosing to opt out rather than in. This decision-making is relevant to personal, professional and academic areas, and can on occasions cover the identification of relevant virtues, values and ethics. Thus, the use of advisement, as distinct from advice and from counselling, becomes a skill relevant to the new and continuing teacher, where available options are offered by the teacher to the student for their consideration within a problem-solving context. These ideas are explored further in Chapters 4-9.

Relevant consequences emerge for existing and continuing teachers, who also had to negotiate: the divide between their own experience of the valuesneutral classroom and the existence of gender-based classes; classes of fifty or more students; or the opposite, when student classes were for a variety of reasons only ten to twelve people. My experience of until-now covert agenda of imparting knowledge about virtues, values and ethics relevant to classroom culture, needs to be honestly identified and analysed, so that democracy and consensus can be provided with a balanced foundation from which understanding and harmonious relationships relevant to the progress of study can be established. The reasoning and considerations relevant to the understanding of democracy and consensus in the classroom as espoused within this thesis are discussed in Chapters 7 and 8.

There is also my emerging discovery of the significance of the multicultural classroom, which depends on the presence of migrant populations from across the world, and the possible attendance of refugees whose experiences and reasons for being present are very different from each other and from the everyday experiences of Australian students. The content of class discussion could change depending on the variety and cultural difference of those in each class group. An example of this profitable educational aim is reflected on in my auto-ethnographical introspective (Chapter 5). 
Allied to these reflections are new developments in research into how the brain learns (Beyer, Costa \& Presseisen, 2001; Fogarty, 2005; Dispenza, 2007; Doidge, 2007; Greenfield, 2007; Lipton, 2008). The emerging concept of neuro-plasticity points towards the possibility of new knowledge, especially regarding the role of memory, and needs to be inserted and integrated into the practice of education. This content, I judge as being beyond the scope of this thesis, but it will change the way that classroom pedagogies are constructed, so as to take account of the various conclusions about how best to facilitate and teach the rich knowledge and effective skills necessary for successful twenty-first century living.

The significance of research into how the brain works and learns, and of the evolution of brain-dendrite accommodation in technological/information and communications technology (ICT) experiences, especially during early childhood, is a central part of the considerations of my proposed twenty-first century reshaped theory of education. Changing what is taught will have consequences: consequences for student teachers; consequences along similar lines for continuing teachers; and consequences relevant to the tendency to delegate political and societal changes to the education curriculum. All of these considerations will need subtle but careful development, based on clarity of reasoning and a teaching-learning process that is understood as the teaching of the student through the medium of the subjects taught.

There is a need to promote a continuing and growing educational policy that monitors how much of the existing curriculum is removed in favour of new curriculum initiatives. Stringent monitoring is needed to avoid the problem of the removal of content too quickly or for short-term gain. By connecting this area of concern with Susan Greenfield's $(2004,2007)$ brain research and with Stephen Kagan's (1994) curriculum research and their relevant conclusions, new emphases to the emerging reshaped theory of education for twenty-first century education unfold. 


\section{New understandings from brain research}

There is a need to incorporate some of the very significant new knowledge coming from the study of the mind and its emergent potential. Research by Greenfield (2004, 2007) is now being supported by an array of specialisations in neuroscience, one of which relies on the concept of neuroplasticity - the ability of the brain to alter its structure and function in response to input from the environment - in order to explain learning. A connection with Ingber's (1997) 'architecture of life' becomes significant in relation to Greenfield's unfolding brain-function research.

The research process indicates a need to promote the consequences of these investigated matters into the planning and prosecution of curriculum change to the administrative arm of the Australian education system. If reorganised time use and the promotion of thinking skills in classrooms occurs in a significant and useful manner, then there are consequences for the manipulation of semester time, for the testing and assessment of content, for the monitoring of what constitutes a successful teacher, and even for the successful transformational schools indicated by Caldwell and Spinks' (1992),and Crowther’s (2010a, 2010b) frameworks.

\section{Students}

Students sometimes think that the teaching of applied thinking skills is secondary to the content of the Key Learning Areas described in the Victorian Standards Frameworks II (Victorian Board of Studies, 2000), yet the practise of the science of learning, and indeed of the art of teaching as discussed by Gagné \& Briggs (Gagné \& Briggs, 1974) within the contexts of decision-making and the broad variety of reasoning applicable to thinking, is to support deeper knowledge and understanding in all learning areas as well as in social competence (Dewey, 1944, p.80). It has been my experience that the structure of today's curriculum can lead to a narrowing focus away from thinking skills. Therefore this research contributes to the examination of ways to educate students for their future, by focussing on, amongst other 
things, the provision of thinking skills. As Ann Margaret Sharp notes in Lipman (1993a), there are understandings and practices that explicitly focus on the teaching of thinking, and particularly of self-correction. The study also investigates the possible relationships between current learning, personal decision-making, behaviours, attitudes and preferences, through 'the construct of continuous reconstruction of experience' (Dewey, 1944, p.80); Wilson talks about the same concept (Wilson, 1963, pp. 126 -141). It follows that this whole project is couched in a $21^{\text {st }}$ century reality, and can't be confused with some utopian dream based in unreality.

It has been my experience that Australian students in the twenty-first century expect to find accessible education married to the strategies they need to construct a career, develop a philosophy for life and find the means to investigate eschatological speculations about metaphysical questions on the meaning of life, as they wish. Without the inclusion of the basic and deep thinking strategies that can be incorporated into each topic within each subject area, students are at times subjected to a form of discrimination which I have found can result in a lifetime of negative consequences. I have found that their entry into the career path of their choice is affected by their sometimes tenuous hold on the less obvious, but significant skills that emerge from the various methods of teaching applied thinking skills. This results in the need to insert a balanced curriculum, one which attends to twenty-first century knowledge and education without the unnecessary intrusion of meaningless and self-perpetuating paperwork that substitutes tests and assessments for the opportunity to engage with deep and rich learning in the manner advocated by David Beckett (1994) and noted in Heasly (1995, p.1316):

Beckett is clear that facilitation as a main function of the teacher in the classroom is anything but good teaching practice. ... For Beckett, the shaping of adult learning is based on creativity, reflection and rationality. ... Beckett points out that democracy, autonomy and inclusiveness are 
central to the classroom practices. ... [He implies that] wisdom is part of the aim of education.

Accordingly, this investigation asks the teachers who participated in completing a Questionnaire for their considered opinions of approaches to student development.

\section{Needs}

Twenty-first century Australian students' needs, I believe, encompass the following:

- The integration of, not deletion of, diversity of cultures.

- Balance in the use of ICT.

- Recognition of the management of cultural change.

- The impact of frameworks addressing globalisation.

- The necessity to access educational processes well beyond subject-based curriculum content.

- The need to link tradition and innovation, religious and secular matters, and multicultural and intercultural currents in twenty-first century Australia. This means that students rely on their education to provide opportunities for personal decision-making for career choice, for construction of the good life, and eventually for speculations of an eschatological nature.

Consequently, relevant education experiences need to include:

- Opportunities to learn the construction of relevant questions.

- Learning the preparatory steps to research using appropriate safe ICT facilities.

- Finding appropriate independent thinking, analysis and selfcorrecting skills. 
- Facilitation and development of personal decision-making about career, about constructing the good life, and about choosing a secular or religious journey through life.

This is the multifaceted challenge that educators face in facilitating the flourishing of their students; yet, it extends beyond positive psychology (Seligman, 2011). Chapters 7 and 8 begin to address such relevant education practices.

Teachers' needs, I believe, include:

- Learning their teaching area effectively and efficiently, for deep knowledge.

- Recognising the connections and links between the teaching process and the learning process.

- Having the expertise, for classroom efficiency, to lead and foster thinking skills strategies.

- Knowing and practising the methodology of teaching applied thinking in all subject areas.

- Having broad knowledge about virtues, values and ethics to serve as the foundation of a democratic educational experience for their students.

The emerging recognition and implementation of such educational aims are already with us in various frameworks via disciplinary specialisation. Democratic support rather than prescriptive curricula is warranted for decisions associated with careers education, in the construction of a personal philosophy of life, and in their participation in community domains: I have observed students' expectations of these outcomes of their education increase over time. The teachers with whose practices I am familiar are all already aware of the complexity of these matters, and this investigation is an effort to address their concerns. My Questionnaire was constructed with these particular considerations in mind. 


\section{Beginning my research}

Between 2001 and 2009, as Head of Faculty in English and subsequently of Religion and Values Education at the last college at which I taught, I took up the opportunities available for professional development, sought out curriculum and teaching experts, and decided to focus my research on teachers rather than students.

Before I had begun the physical work on my thesis ten years ago, I started to think through exactly what the focus of my questions were; what the aim of the project was; why it was important to pursue these questions; how it would affect educational practice; and what suggestions for improvements I could offer for twenty-first century education.

To find a respectful theory for twenty-first century education I needed to discover how teachers perceived the teaching of thinking skills in secondary schools. I identified three questions to guide my exploration:

- How could I harness the knowledge, experience and expertise of my own search of over 20 years, to form a foundation for academic research in this area?

- How could I hear precisely what other teachers were saying and, how could I respectfully find accurate evidence from the coalface - the classroom itself?

- How could I find out what educational theorists and expert practitioners were thinking and doing?

I asked myself: 'What are the crucial ontological, epistemological and technical elements that could be incorporated into the development of an effective philosophy and format for the teaching of thinking skills to enable secondary students to learn about, and engage with the concepts of virtues, values and ethics?'

The specific research questions emerged as follows: 
- Ontological Questions: What significance can be assigned to the development of personal perspectives surrounding a teacher's attitude to the teaching-learning process? How do teachers maintain the emphases needed to teach students for rich learning, rather than only the subject content?

- Epistemological Questions: How do teachers ensure that students learn all of the thinking skills relevant to educational knowledge and skills, so that applied thinking skills are recognised, identified, modelled, assessed and demonstrated, in all learning outcomes in all subject areas?

- Technical Questions: How do teachers understand and acquire a comprehensive list of thinking skills with which to model, teach and require of students that they use them in every educational sense: in career choice; in life decisions; and in both secular and religious dimensions and approaches to life's eschatological speculations?

I wanted to develop a respectful theory constructed from a consciously historical perspective, with the reshaped emphases emerging inductively from the literature and from my own and other teachers' experiences. This investigation relied on inductive reasoning for the construction of a picture of what twenty-first century education would include.

It was with relief that I found the latest work of Lincoln, Lynham and Guba, (2011) because chapter 6 sets out the boundaries, the overlaps, the breadth of important paradigms and the need to identify when writing in a research paradigm. As I developed my thesis, I found that learning about the boundaries and concepts underpinned different qualitative research disciplines. I tested early processes like Case Studies, Grounded Theory, and found that inductive paradigms often overlapped. This thesis therefore details the moves I made as I clarified the particular research methodologies and identified why I moved to the concept of a respectful new theory as it 
emerged. The whole is founded on the teaching of thinking skills, and the application to as broad an arc of democratic practice as philosophy and logic would allow.

\section{Learning the broad spectrum of philosophy}

While studying with Wilson, I was introduced to the work of a wide range of philosophers; scholars from the analytical tradition as well as a number of other philosophical schools. Consequently, the history of education in Victoria, its changes and developmental steps, became central to my own questions. Having begun to learn through my studies to identify and understand philosophical perspectives, I began to make comparisons and to find parallels in the decades of change I observed and the educational philosophy behind each shift I discerned. It must be understood, though, that I was a teacher delving into philosophy, rather than a philosopher reaching into educational fields and so, many of the nuanced utterances for which philosophy is renowned may have inevitably escaped my untrained though eager mind. This earlier experiential learning provides the basis for the Literature Review associated with this study.

These considerations are central to the development of my theory for a reshaped twenty-first century Australian classroom, based on clear and historical educational concepts. General histories of philosophical thought traced by Richard Tarnas, Jaroslav Pelikan and Bertrand Russell over the last century appear to inform the values within Australian education. The historical sweep of these educational values is identified in the large bodies of work of significant authors (such as Beare, Hargreaves, Dewey and Bruner) informing the underlying construction of this investigation. The study's conclusions feed into other more precise frameworks (such as Crowther, Hawkes, Ingber, Costa, Greenfield, H. Gardner, and Freakley \& Burgh). Engagement with the broad spectrum of content by Wilson, Lipman and Vardy, and their large bodies of specialised work, allows depth and authenticity to develop as the investigation takes shape, and reliable 
conclusions can then be made. In particular, the work of Laurance Splitter and of I. A. Snook rounds out the broad perspectives underlying my conclusions, which are supported by the works of the authors detailed in the Literature Review in Chapter 3.

\section{Planning the research enterprise}

I wanted to find out how teachers, especially teachers in secondary schools, addressed the concept of the teaching of thinking skills in their classroom practice. And I specifically wanted to know if the 'science' and 'art' of consciously using applied thinking skills from any or all subject areas and disciplines would advance levels of student understanding, thereby achieving for them a richer learning and a more enthusiastic engagement. If, by investigating which authors, educational experts and researchers had proposed innovations to twenty-first century pedagogical and professional learning, I could establish a foundation for my questions by administering my rigorously-designed Questionnaire. It would then be possible to develop richer and more beneficial learning experiences for twenty-first century Australian education scenarios. These two sources would be supported and synthesised through my own experiential and formal learnings and presented in an auto-ethnographical introspective. Inevitably though, I chose to take Newton's approach and stand on the shoulders of giants (Newton, 1676).

\section{Aims of the research for teacher practice}

This project seeks knowledge that will enliven teachers' practice with the aim of providing improved student outcomes (Heasly, 1995) that has been central to my work for over two decades. Students have deeply internalised the tenets and practices of individualism (Singer, 1993) and, simultaneously, the development of capitalism has resulted in the phenomenon of globalisation (Klein, 2007); (Aspin, 2003). Teachers must now contend with the possible student responses to these conflicts - students whose judgement is, as has been observed, focussed very narrowly - and may leave unattended 
consciousness of the connections to considerations of national and international pedagogical significance. A central question has emerged concerning how students should be taught the skills necessary for them to interpret their world, and thus how they can perceive and exercise both their freedoms and responsibilities as formulated (Lipman, 1993a; Splitter \& Sharp, 1995) in Philosophy for Children terms; by Wilson (1963; 1987), in early moral education terms; (Singer, 1979) in terms of ethical choice. Students and their teachers will find ways to think carefully together about the students' environment (Seligman, 1990; Anih, 1992; 1995). To inform such an educational aim, my research seeks to uncover the underlying thinking and practices which teachers employ during the teaching of thinking skills, in order to support the development of student skills and outcomes; a particularly important example of which is the gaining of self-knowledge (Pritchard, 1985). The analytical framework for this research is drawn from teaching and learning theory from a number of sources. (Bloom, 1956; Gagné \& Briggs, 1974; Brown, 1980; Knowles, 1984; Cherednichenko, 2000).

\section{Significance of the research and its contribution to new knowledge}

I decided to investigate how some teachers perceive and understand the teaching of thinking skills in their secondary education practice. This project considers the conflicts which occur when an essentially literary society, media and technology, provide students with a particular world view (Postman, 1986). The research investigates secondary teachers' declared reasons for teaching thinking skills as an extension of previous research with primary teachers conducted by Brenda Cherednichenko (2000), who investigated the reasons given by teachers for their inclusion of a thinking curriculum such as Philosophy for Children in their primary curriculum planning. Her research explores the connections between primary teachers' thinking concerning primary students' backgrounds and social classes, and 
curricula containing primary skills and socio-cultural background. Only a few researchers (Gardner, S, 1999; Cam, 2002b; Leckey, 2009) have investigated secondary curriculum planning on the scale that Cherednichenko achieved in primary settings.

It seemed to me that it was possible to promote thinking skills in the middle years of schooling (Years 5 to 9) without looking towards sociocultural background to provide the outcome of richer student engagement in learning; and anecdotal evidence suggests that many schools implement a thinking-focussed curriculum in the middle years. It may be necessary to align and link the observations and conclusions gleaned from this research with other, perhaps as yet undiscovered, or yet-to-be-written research that emerges in this research field. Eric Jensen (2006) develops a cogent argument for enrichment for successful learning outcomes.

It is hoped that my research makes possible the further development of the theoretical foundations proposed, and of teachers' personal practice of education. We may then say that, like Freire (1972), who when speaking of thinking skills in a Brazilian context, we find that our understanding of the basis of education may become even clearer.

\section{Uncovering critical new knowledge}

My research thesis provides teachers and school administrators with critical new knowledge to enhance educational theory and policy development for secondary classroom practice by placing slightly adjusted emphases on knowledge that is already available. Further, I contribute new knowledge about the pedagogy of the teaching of thinking across the secondary curriculum. This new curriculum connects existing knowledge from my three authorities - Lipman, Wilson, Vardy - to enhance the value of recognisable and parallel processes in the teaching of thinking skills, especially processes which could relate to moral thinking skills (Wilson, Williams \& Sugarman, 1967; Wilson \& Straughan, 1983; Wilson, 1986; Wilson, 1987; Wilson, 1990; Lipman, 1993a; Singer, 1993), for daily use, and begins with the 
teaching of thinking skills already in the curriculum (Griffiths, 1993). My research develops distinctive, as well as collective perspectives on teachers' perceptions, opinions and attitudes towards their teaching of thinking (which is supported by my extensive Literature Review and my auto-ethnographical introspective). It provides significant new knowledge about current curricula and informs new developments in education in Australia, United Kingdom and New Zealand.

\section{Other research questions}

General questions that would shape my research arose from the foregoing discussion, and provided a focus for my planning:

- What have been my own experiences and perceptions of the teaching of thinking and of virtues, values and ethics?

- How do secondary teachers describe their experiences and perceptions of the teaching of thinking and of virtues, values and ethics?

- What do expert practitioners think about the teaching of thinking and of virtues, values and ethics?

- What are the relationships between these experiences and perceptions of the teaching of thinking and the three different curriculum perspectives discussed - Lipman's Philosophy for Children, Wilson's Moral Component List and Vardy's Five Strands for Values Education?

- Given the connections, distinctions and assumptions uncovered in the research, what might comprise my new architecture for the teaching of, and teaching about, virtues, values and ethics?

The thesis is structured in nine chapters, as follows:

- Chapter 1 is my Introduction. In this chapter, I am concerned with discovering what the questions and considerations are - so that I 
may investigate what teachers actually think of their teaching of thinking skills to secondary students - preparatory to assembling the required philosophical and other authorities as secondary sources, and before constructing the auto-ethnographical inductive data reduction chart as my third research source.

- Chapter 2 is a careful effort to 'voice' the questions by 'listening to many voices'. I discuss the use of qualitative research, the methods of analysis, the bundling of similar responses, looking for implied information and seeking to fill in gaps left by the respondents especially to my questionnaire, including my own contributions to the research. Three sets of 'voices' mirror my three sources of research. These voices use an inductive narrative form of text which can detail the various creative and conceptual leaps as new knowledge emerges.

- Chapter 3 identifies further voices: I develop a Literature Review that, because of the issues emerging from the respondents, is necessarily broad. Some authors appear in more than one category in my effort to faithfully include all elements of teachers' concerns (which are ventilated) and the arc of recent educational history (which is respected). The breadth of, and distinction between, these voices, is a result of the many themes and subthemes emerging from the three sources of research. Reference to particular authors is the result of shaping my thesis' content, but the accompanying bibliographical reference list identifies the breadth of the research needed to understand these themes and sub-themes. The importance for me of recognizing that the authors I chose, and on whose work I rely, also stood on the shoulders of giants whose work they relied on, is crucial to the inductive thinking that this thesis exemplifies.

- Chapter 4 records all of the findings obtained by my inductive data reduction. I include diagrams that identify and contrast 
considerations deemed acute enough to need individual analysis. Tables are provided that detail strategies and techniques described by individual respondents to my Questionnaire. My voice is heard through my professional experience in the field, as well as through my own professional development. The result is a diffuse picture of skilled teachers and their ways of teaching thinking skills to secondary students, and is accompanied by the voices of experts who provide the theory informing the practice.

- Chapter 5 details, through the use of inductive thinking grids, an auto-ethnographical retrospective showing the breadth of my experiential learning gained over the breadth of my teaching career. Married to the effort I made to integrate, blend and synthesise this huge field with the formal qualifications I have gained is a resultant modelling of inductive thinking. This is accomplished by allowing the educational construct to emerge from a graphical structure of the schema. This is followed by identification of the emergent concept which then becomes the basis of my synthesis of thinking, planning and study, based on the construct that has emerged. Finally, the relevant theme emerges from the discussion, so that an overview of my learning becomes manageable and can be applied legitimately and accurately as the third source of research.

Chapter 6 begins my synthesis of the investigation. I respond to the data I have documented and differentiate between the teaching of a topic or subject, and teaching the students through the medium of the topic or subject. I introduce, as a unifying theme, the search for a new concept. This concept pays careful attention to the history of education and to the changes that have taken place over 100 years in Australia; and some similar attention is paid to two other countries: New Zealand and England. I introduce what I term my 'Categories of Influence' to give shape to the new concept. Each Category of Influence is a model of trilectic logic, in that three inter-related concepts must be identified and synthesised, before new knowledge emerges and action takes 
place. These Categories of Influence are detailed so that the delivery of teaching instruments and new knowledge will be constructed with relevant results in an overall balanced form of education suitable for twenty-first century conditions; the instruments and knowledge are not themselves subject to interference from outside influences and are single-issue in focus. This autonomy and focus extends beyond the investigation of the perceptions of teachers of their own teaching of thinking skills and encourages a new architecture for the teaching of virtues, values and ethics.

- Chapter 7 is the first application of the Categories of Influence to a reshaping of the curriculum. The Categories lead to a move away from the old educational paradigm to a new educational paradigm emerging as concerned with teaching the student rather than the teaching of discrete topics. A link is established between applied thinking skills, new knowledge gained from neuroscience, and the construction of, and understanding about, meaning attached to various virtues and values.

- Chapter 8 continues my reshaping of the curriculum by recognising the need to further extend teaching about virtues and values into teaching about ethics. This extension is firmly situated within a new educational paradigm, enabling the: construction of a career, the construction of a personal version of the Aristotelian concept of 'the good life' and, thirdly, a capability of constructing exploratory eschatological speculations, whether spiritual or secular.

- Chapter 9 addresses the need to respond to the details of the investigation as a whole. I foreshadow responses, responseabilities and responsibilities that require identification, testing, refinement, restructuring and subjection to rigorous attention, as the pivotal point between the old educational paradigm and my new educational paradigm, and in so doing, answer the questions raised in Chapter 1. 


\section{Looking forward}

This chapter identifies a set of questions investigating the concept of the building of the architecture for the teaching of and about virtues, values and ethics. It recounts the early days of my research journey and my growing familiarity with the authorities and theoretical constructs in use through a self-imposed course of study. It details the formulation of a number of questions central to the thesis, as well as the construction of the actual research question.

Attention is paid in this chapter to the experiences of both students and teachers, but I decided that the latter should form the focus of the research. Considerations of ontological, epistemological and technical matters are undertaken and the presence of new and emerging knowledge in the form of research into the brain is introduced.

Finally a chapter-by-chapter summary of the thesis itself is provided, outlining the discussions and arguments that occur, in their proper place. In the next chapter, I begin to construct the methods and consequent details of the questions raised here, and to listen to the many relevant voices. 


\section{CHAPTER 2}

\section{Answering the Questions: Listening to Many Voices}

\section{Introduction}

For this chapter, I assemble a variety of voices of colleagues - mostly in one school - plus a few other teachers from Australia, UK and NZ. This broadened my recognition of their various concerns and considerations. I did this by means of a Questionnaire and proceeded to develop an understanding of their responses by first undertaking a frequency count, and then by qualitative means that emerged. I evaluated and developed a comprehensive framework that included all their criteria and critiques.

This chapter describes how my research was conceptualised and conducted. The methodology and methods employed were selected to ensure that the research questions were answered by listening to many voices. I had three goals in mind:

1. To investigate the ontological questions that might underpin the teaching about thinking about virtues, values and ethics;

2. To explore epistemological concerns in order to discover which skills and which applications of knowledge and strategies (phronesis) were evident in teachers' delivery. Specific indications were needed regarding which knowledge, which skills and which applications of strategies for education would be relevant for teaching in virtues, values and ethics; 
3. To examine the data in order to develop a systematic approach for effective technical (techné) strategies and techniques pertinent to the understanding of virtues, values and ethics.

In order to achieve these three goals I wanted to learn from a broad spectrum of teacher-colleagues. This desire informed the preparation, process and prosecution of the ensuing investigation, with the Questionnaire, the wide-ranging literature review and the ethnographical introspective serving as support for the themes and sub-themes emerging from the initial response to the teachers' responses.

My research was designed to provide a basis for the development of a reshaped theory about virtues, values and ethics education. To do this I used an evidence-based research approach (Thomas, 2003); (Sutcliffe, 2009). The orientation of my methodology is multidirectional; it involves the integration of six distinct, but related elements:

1. Hearing the voices of three historical leaders in the field of virtues, values and ethics education.

2. Comparing and contrasting each of the theories proposed by the three educational authorities.

3. Describing the practice associated with the three leading theories.

4. Collecting the opinions of local and international teachers, as well as responses from international experts; listening to my own voice.

5. Developing a new theory relating to the thinking skills associated with virtues, values and ethics.

6. Developing a set of proposals for a new approach to the development of thinking skills.

A diagrammatic representation of these components is shown below in Figure 2.1. 
FIGURE 2.1 COMPONENTS OF THE METHODOLOGY

\begin{tabular}{|c|c|c|c|c|c|c|c|c|}
\hline \multirow{2}{*}{ LEADER } & \multirow{2}{*}{$\begin{array}{c}\text { THEORY } \\
\text { PROPOSED }\end{array}$} & \multirow{2}{*}{$\begin{array}{c}\text { PRACTICE } \\
\text { DESCRIBED }\end{array}$} & \multicolumn{3}{|c|}{$\begin{array}{c}\text { DATA } \\
\text { COLLECTION }\end{array}$} & \multirow{2}{*}{\multicolumn{2}{|c|}{$\begin{array}{l}\text { NEW THEORY } \\
\text { DEVELOPED }\end{array}$}} & \multirow{2}{*}{$\begin{array}{l}\text { PROPOSALS } \\
\text { FOR NEW } \\
\text { PRACTICE }\end{array}$} \\
\hline & & & $\begin{array}{l}\text { Lo- } \\
\text { cal }\end{array}$ & $\begin{array}{c}\text { Int' } \\
\mathrm{nl}\end{array}$ & $\begin{array}{l}\text { Ex- } \\
\text { pert }\end{array}$ & & & \\
\hline $\begin{array}{l}\text { John } \\
\text { Wilson }\end{array}$ & $\begin{array}{c}\text { Moral } \\
\text { Components } \\
\text { List }\end{array}$ & Discussions & & & & \multirow{3}{*}{$\begin{array}{l}\text { Thinking } \\
\text { skills for } \\
\text { interpret- } \\
\text { ation: } \\
\text { A New } \\
\text { Theory }\end{array}$} & \multirow{3}{*}{$\begin{array}{l}\text { Virtues, } \\
\text { Values } \\
\text { and } \\
\text { Ethics }\end{array}$} & \multirow{3}{*}{$\begin{array}{l}\text { Theory, Praxis } \\
\text { and Practice: } \\
\text { Thinking Skills }\end{array}$} \\
\hline $\begin{array}{c}\text { Matthew } \\
\text { Lipman }\end{array}$ & $\begin{array}{l}\text { Philosophy } \\
\text { for Children }\end{array}$ & $\begin{array}{l}\text { Community } \\
\text { of Inquiry }\end{array}$ & & & & & & \\
\hline $\begin{array}{l}\text { Peter } \\
\text { Vardy }\end{array}$ & $\begin{array}{l}\text { Religion and } \\
\text { Values } \\
\text { Education } \\
\text { (RAVE) }\end{array}$ & $\begin{array}{c}\text { Dialogue } \\
\text { Australia } \\
\text { Network } \\
\text { Journal }\end{array}$ & & & & & & \\
\hline
\end{tabular}

\section{Taking a qualitative and quantitative approach}

The initial research approach, expressed in a formal proposal, was to employ the methods of Grounded Theory (Glaser, 2002); (Strauss \& Corbin, 1990) and report in a set of three Cases Studies. As the analysis of the data commenced, it became apparent that there were so many themes and subthemes emerging that a revised approach was required: an approach that involved hearing the variety of 'voices' that were emerging, and their analysis, using an inductive method of data reduction. Eventually, these voices came to be considered as part of a 'bricolage', with the researcher's role being that of a 'bricoleur' (Ling, 2012). The essential elements of this bricolage are considered in Chapter 5. The end-point of the research approach was quite different from that which had initially been envisaged: Investigating Teachers' Perceptions of their Teaching of Thinking; this had involved a reshaping of my philosophy of education based on an emergent concept, leading to the eventual thesis title: Towards an Architecture for Teaching of Virtues, Values and Ethics.

The approach taken here is based on an understanding of the initial definitions of the differences and overlaps between and among qualitative and quantitative methodologies and I have drawn on the ideas of Thomas (2003) as well as Lincoln, Lynham \& Guba (2011) who summarise these well. Thomas makes it clear that qualitative methods involve describing all 
kinds of characteristics of people and events. He points out that this form of research employs multi-methods, interpretive approaches, naturalistic expectations, sense-making, and the meaning of relevant research phenomena; however, I knew I must also take account of the differentiations in postmodern qualitative research channels (Constas, 1998) to ensure accuracy.

Significantly, Thomas emphasises that qualitative inquiry includes ethnography, case study, phenomenology, even critiquing education itself. Thomas also refers to grounded theory (Glaser, 2002), and I believe it is important to understand Thomas's comments on the downgrading of the conflict between qualitative and quantitative research methods and also to recognise the differences in each approach. He notes that the skills required to use grounded theory in research include the ability to analyse situations, to recognise bias and to find accurate data. I have endeavoured to meet all of these requirements and have consciously and consistently modelled the stages of inductive thinking. The various considerations, hesitations, confusions and silences of teachers have been addressed as they emerge.

For the purposes of my research, I use the process of ethnography outlined by Thomas (2003) in which he discusses how ethnography might operate within groups -including the need to experience the life of the target group to ensure validation of data through triangulation; or as in my investigation, through trilectic logic. Thus, elements of my personal story are interwoven to incorporate the above considerations into an authentic picture, as follows:

- The voices of teachers in the classroom (in a Questionnaire);

- Further voices from a wide horizon of educational authorities (in a literature review);

- My personal account, detailing the integration of my professional learning with my formal qualifications, over a period of 36 years (in an auto-ethnographical data reduction chart). 
The conscious blending of all three elements - voices of teachers, voices of educational experts, and my own voice (developed as both a practitioner and as a researcher over a 36-year period) - resulted in the construction, within classrooms, of relevant pedagogical instrumental concepts. Emerging data was synthesised into spread-sheets and grids and, during piloting over almost 15 years in secondary classrooms, was augmented by professional learning in Australia and the UK and integrated with my gaining of formal qualifications. Increasingly, through the use of inductive thinking, rather than deductive thinking or lineal reasoning, a set of emerging considerations resulted in my reshaped emphasis for a philosophy of education theory. In retrospect, I realise that the development of this philosophy of education theory has been based entirely on qualitative research methods and methodologies.

Essentially, this is a phenomenological study, in that it examines conscious experience and the phenomena that emerge from acts of consciousness. It has been possible for teachers from the UK, New Zealand and Australia, to be respondents to an open-ended Questionnaire, as well as to provide written anecdotal evidence that allowed comparisons, highlighted the emergence of insightful data and uncovered contrasting responses.

\section{The different voices}

\section{Teachers' voices}

Teachers' own perceptions of the teaching of thinking skills in the classroom were crucial to any new knowledge that may emerge from this investigation. I expected that teachers would be able to comment valuably and so widen my understanding of what worked and what more was possible.

It was necessary, having begun the business of finding the questions highlighted in Chapter 1, to find teachers who would voice, from their current experience at the coalface of contemporary education, those questions 
seen as paramount for consideration of what precisely happens during the teaching of thinking skills.

I chose participants, some from a particular school, from which I would draw the data for collection. I exposed my Questionnaire with its requirements for anecdotal written responses, to five teacher participants from the UK, four teacher participants from NZ, and three random teacher participants from Australia. These were augmented by the responses from nineteen participants from one P-12 college in Melbourne, Australia.

I chose the teachers' profile for participating in this project as follows:

- The teaching of a variety of subject areas.

- Career experience spanning about five years or more continuous service.

- $\quad$ Teaching responsibilities at secondary level of Year 7 to Year 12.

- Willingness to examine a variety of personal teaching-learning practices in the classroom.

It seemed important, as a first goal, to give teachers their own voice, upon which I could set a valid and accurate foundation for the gathering of data about the teaching of thinking skills in secondary schools. I aimed to discover whether the teaching of identifiable thinking skills was a given in their classroom strategies, and whether there were specific skills that particular teachers used that could be universalised for the benefit of rich learning for all twenty-first century students.

\section{My practitioner voice}

I piloted the study and experimented with its operation in my own classes for nearly 15 years, advising students how the learning processes could work, and evaluating each by me-as-teacher viewing the results. This was followed by students performing a self-evaluation and curriculum critique according to previously established criteria. The strategies and tools I used as a teacher in the classroom are recorded in Chapter 4. 
Concurrent with this, I engaged in a deep, personal reflection on each of the themes to emerge from the Questionnaire so that my voice could eventually be mingled with the multiple voices from other participants in the study. This is reported in Chapters 4 and 5.

\section{Voices from the literature}

My personal experience and studies prompted me to connect Wilson and his Moral Components List (Wilson, 1971) to the broad spectrum of classroom strategies in the Philosophy for Children outlined by Lipman (1988). Wilson's List (appendix C) includes reference to the teaching of concepts, the tools for analysis and meaning-making, and the understanding of virtues while Lipman's work focuses on the teaching of concepts, on the use of thinking tools for analysis and meaning-making, and on the understanding of virtues and values. I linked these approaches by testing how Vardy's (1998) Religions and Values Education (RAVE) also connected with the teaching of concepts, with the tools for analysis and meaning-making, and with understanding about virtues, values and ethics.

I chose to analyse the work of all three of these theorist-practitioners Wilson, Lipman and Vardy - in order to find whether applied thinking skills were common to their work, and whether it was appropriate to apply their approaches to the teaching of today's students to think and learn about virtues, values and ethics. I followed this with the Questionnaire so that teachers at the coalface were able to voice evidence of their general practices of the teaching of applied thinking skills in secondary classrooms. I aimed to place particular emphasis on whether teachers believed and practised the teaching of applied thinking skills in relation to what I was to come to consider as virtues, values, and ethics. 


\section{Three different data collection strategies}

The correspondences between the three different voices that heard, and the application of the three different methods employed, are shown in Figure 2.2.

FIGURE 2.2 VOICES AND METHODS

\begin{tabular}{|l|l|}
\hline \multicolumn{1}{|c|}{ THREE VOICES: } & \multicolumn{1}{c|}{ THREE METHODS: } \\
\hline Teachers' voices & Questionnaire \\
\hline My practitioner voice & Auto-ethnography \\
\hline Voices from the literature & Multi-disciplinary literature search \\
\hline
\end{tabular}

\section{Questionnaire}

Role of the questionnaire in the investigation

I began by constructing my Questionnaire to reflect the span of the investigation so far: it needed to marry with the history of philosophical ideas, and to include inputs from experts in the educational field; it needed to integrate with my focus on concrete and experiential learning; it needed to access the voices of teachers at the coalface. The Questionnaire (Appendix A) was delivered to teachers at one outer suburban multi-faith Melbourne College, and then forwarded to teachers in the three countries: Australia, United Kingdom and New Zealand.

The questions, or items, addressed by the Questionnaire focussed on drawing out data concerning teachers' perceptions, considered opinions and their practices, their teaching of thinking skills and their evaluation of what they believed to be success or failure. To avoid any legal issues regarding information the teachers may divulge I prepared and distributed legallybinding confidentiality agreements for their signing and undertook the same myself. Permission was sought from the individual schools or colleges where the participants taught, so that the individual teachers and their schools were confident that no mistakes had been made. 
A legal document to this end was prepared, applied and strictly maintained over the course of the process. Documentation remained within the schools in all three countries.

By posing judicious questions related to what teachers do, this research surveys curriculum experts and classroom teachers and asks them to inquire about such a relational link. In attempting to understand secondary teachers' practice, my research surveys secondary teachers themselves so as to reveal their perceptions, opinions and attitudes about their curriculum decisionmaking and approaches to the teaching of thinking. These perceptions, opinions and attitudes are critical to an understanding of how curriculum decisions are made; they are critical also to the foundations of any theory that strives towards this architecture for teaching about a virtues, values and ethics curriculum.

It was necessary to choose a group of participants who would provide experiential data, based on my Questionnaire. As personal experience had alerted me to certain apparent parallels between the United Kingdom (UK), New Zealand (NZ) and Australian approaches to education, I decided that participants from three countries would constitute one layer of data. Colleagues from a particular school asked to complete the Questionnaire, thus providing the second layer of data, because their experience in that particular community was deemed to hold the potential to show whether identifiable differences between themselves and their UK and NZ colleagues would emerge from their responses. Three acknowledged experts in the teaching of thinking skills, one from each country, the UK, NZ and Australia, were requested to comment on the different sections of the Questionnaire in order to provide a theoretical perspective as a third layer of data. Such triangulation was expected to reveal parallels and contrasts in current practice and current attitudes to the teaching of thinking skills across country and teacher experience (Flick, 2002). These responses are analysed and contrasted in Chapter 4. 
No ethical risks were apparent at this stage, but a constant watch was kept in order to monitor any unexpected concerns of an ethical nature that may have arisen. Initial documentation was considered to have sufficient preparatory text addressing the likely concerns of the participants so as to allay any misgivings they may have had before the project was under way. The relevant proper administrative and ethical channels at Victoria University, Melbourne, Australia, would be the first point of consultation; and of action, if needed.

As the central goal at this point in the process was to identify how teachers do know that they are teaching thinking skills, it was expected that the consultation process via the Questionnaire alone would be quite comfortable for participants.

The Questionnaire was designed by me alone to draw data with an emphasis on co-operation and consultation through data collection, and to direct attention to teachers' responses in qualitative research. The Questionnaire was broad, open-ended, and with transitional, directed, key and evaluative questions all posed with careful intent. The questions also aimed to address the needs of teachers who were charged with teaching Religion and Society units, through Ethics, Philosophy, and Texts \& Traditions, within the Victorian Certificate of Education, to a multi-ethnic, multi-faith and multi-philosophical student cohort.

\section{The thinking behind the development of a Questionnaire}

The Questionnaire asked participating teachers for descriptions and comments related to the following questions:

- What does the term thinking skills mean in your classroom context?

- What teaching content seems to evoke strong thinking skills in students' responses?

- How does the teacher ensure that student response will not focus on the mere parroting of content? 
- How and when is it possible in a specific subject area to enhance regular applied thinking so that higher order comprehension and sophisticated content can be addressed?

- How often is the terminology of thinking skills (e.g., hypothesis, assumption, clarification, false logic, tautology etc.) used by teachers and students in classroom discussions?

- In what ways are discussions promoted regularly and can students be taught to construct relevant questions?

- What proportion of discussions based on thinking skills, is matched only, or most often, to the tasks to be assessed? Is this significant in some way?

- How are the various particular thinking skills taught? As needed? Sequentially? Thematically? Any other way? Is the Philosophy for Children inquiry format familiar and used?

- Is the 'jigsaw pattern’ of inquiry used with any regularity?

- What assignments are used to promote this style of inquiry? How many and what kind of assignments are used to actively promote the variety of thinking skills? Are there certain forms of thinking skills which are particularly helpful in a specific subject area?

Following very early informal discussions and consultations with teachers within schools where I was employed, and the application of my own understanding and experience of the field (using my 'intuitive hunches' - which I now recognise as an early application of inductive data reduction), I clustered these questions, or items as I term them, into eight sections, as follows:

1. Obtaining an accurate profile of the respondents (Items 1-7).

2. Focusing on respondents' professional knowledge and practice (Items 8-11).

3. Understanding the respondents' classroom strategies and management of student learning process (Items 11-15). 
4. Determining the processes used to teach specific tasks, and the student responses to them (Items 16-23).

5. Determining the respondents' knowledge of significant frameworks (Items 24-30).

6. Determining the respondents' processes for the teaching of thinking skills (Items 31-54).

7. Identifying where the teaching of thinking skills fits in the daily teaching-learning process (Items 55-62).

My expectation - based on my practical and theoretical knowledge - was that the responses to these questions would lead to the emergence of a set of higher-order questions, such as:

- Is it possible to further broaden the range of thinking skills listed in Sharp \& Splitter (1995) within a given subject area?

- How do teachers evaluate the success and efficiency of efforts to enhance the use of thinking skills in student work?

- Is evidence of enhanced thinking skills used by students better identified and assessed by oral, written or project-based assignments?

- How does a beginning teacher establish the classroom climate most likely to produce student accessibility to thinking skills?

- Is it better to use thinking skills to enhance understanding of subject content, or to use subject content to enhance the efficient acquisition of thinking skills?

- Are there recommendations possible to provide for continuing teachers in each subject area?

\section{The development of the Questionnaire}

The study involved the collection of directed and open-ended written comment via a number of questions that I constructed after the theoretical framework had become more developed. At this early stage, the thesis 
question and the subsidiary questions became the basis for the whole inquiry and, as a consequence, the Questionnaire.

Following Wilson's insistence that the Philosophy, Sociology and Psychology of Education, are all central to the provision of moral education, I carefully designed questions to address elements of each of these fields.

The Questionnaire was based on elements of pedagogical practice, and on the role and function of person-particular procedures that bring success, pertinent either to a given subject or discipline, or to an underlying philosophy and psychology of education. Ontological questions about the big ideas, fundamental precepts and understandings that underpin the teaching of thinking skills were embedded in each section. Epistemological questions about the level of knowledge and skills necessarily applied to the teaching of the variety of thinking skills for decision-making processes, were focussed in Sections D, E, and F. Effective strategies and techniques (techné) regarding the asking of questions, the use of formats like Philosophy for Children with its Community of Inquiry were framed to discover how teachers go about the teaching-learning process.

\section{Administering the questionnaire}

I deemed it important at this stage to construct the process under which exact and valid information could be requested:

- Arranging for the Director of Studies at the chosen P-12 College to invite willing participants (from a possible total of 70 secondary teachers) to be involved; to collect any completed Questionnaires that were to be handed to me at a later date. There were 19 respondents from the school.

- Organising for possible respondents in the UK, NZ and other Victorian secondary schools, through third persons; obtaining, completing and returning the Questionnaires. There were 11 respondents from these sources. 
- Adapting to a major blow that surfaced when the targeted experts, who were members of the boards of relevant professional journals specialising in Moral Education (Wilson), Philosophy for Children (Lipman) and Religious and Values Education (Vardy), were unfortunately unavailable to address my Questionnaire. Finally, arrangements were made for three practitioner-experts in applied thinking skills to generously act as substitutes. These three people chose to comment, in general terms, on each section of the Questionnaire, rather than on every item.

In total, 33 respondents participated and it took three months to finalise the return of the completed Questionnaires. Despite this being a frustrating process, I considered this to be a random sample: there was no influence, on my part, to impact on the process of obtaining responses.

The findings of the Questionnaire are reported in detail in Chapter 4.

\section{Auto-ethnography}

I realised that in addition to collecting evidence from other teachers, I could also analyse my own experiences, my own expertise and my own knowledge, in parallel with the Questionnaire. I saw that by documenting the variety of teaching instruments that I had myself developed, and by explaining the underpinning concepts, that I could inform some of the investigation's conclusions. But harnessing my knowledge was possible only after my compilation of the completed findings from the Questionnaire. It was important that I address the teachers' voices before and not after I marshalled my own responses to the Questionnaire, because subconscious influences informed by my own thinking could have prejudiced my understanding of individual teachers' voices.

The work of Jean Clandinin and Michael Connelly (1994, pp. 413-427) and their concept of Narrative Inquiry, shaped my reflections on the framework I was to employ for the blending of my classroom experience in 12 schools over 36 years, with the extensive use, for professional 
development purposes, of seminars, conferences, tertiary level qualifications and extensive reflection-in-action and reflection-on-action (Schon, 1983, 1987), cited in (Heasly, 1995). I used these sources to develop a cohesive professional perspective. Without access to such internal and external applied thinking skills and relevant considerations, this thesis would not have been possible.

A further development for me arose as a result of my realising that it was no longer the responses to the Questionnaires or the identifying themes from the literature that provided a satisfactory basis for understanding any conceptual links between virtues, values and ethics; there was a need for a further extension of sources. In particular, I realised that these links were both embedded in, and emergent from, the means by which I had gained my personal perspective with respect to these ideas.

I decided to embark on a reflective process that resulted in an autoethnography of my lifetime in this field, an integration of: the professional experiential learnings over 36 years, workplace learnings, and emergent new learnings obtained through the pursuit of a range of post-graduate qualifications; the teaching skills that underpin the teaching about virtues, values and ethics - a 'bricolage' (Ling, 2012 in press) of my professional life. I had realised there was a need to construct an 'architecture' for the teaching about virtues, values and ethics, to explain these diverse theoretical and practical inputs of contemporary education, and this meant that my autoethnographical account (Chapter 5) would become an essential part of this new construction.

I used the work of Wolff-Michael Roth (2005b) to develop a sufficiently inclusive exposition of the depth and potential of the auto-ethnographical retrospective to allow me to construct this investigation with confidence. I could then appreciate the cyclical nature of my experiential learning and integrate that with my formal studies. Roth (2005a) provides an array of authors versed in auto-biography and auto-ethnography as praxis of research method who support my integration of professional and experiential learning. 
Roth also provides an introduction to the use of auto-biography and autoethnography as tools for learning about learning; Chaim (as cited in Roth, 2005a) highlights the writing of a research journey; Joe Kincheloe's (2005) work, which instructs the reader in how to become a reflective person, encouraged me to critique my learning accurately. Chapters 4, 5 and 6 are the direct outcome.

I recognised the crucial importance of accuracy when listening to my own 'voice', and of recognising that the presence of ideological content could invade both my own self-critique and the voices of the teachers and educationalists I had marshalled. Accuracy, therefore, must be accompanied by transparency in my development of this study.

Clandinin \& Connelly (1994) carefully describe the essence of personal experience methods, drawing on the work of Dewey (1944) and especially emphasis on the primacy of experience to research. For the purposes of this investigation, it was crucial for me to remember their emphasis on the significant inwards/outwards and the backwards/forward elements, examining the autobiographical details designed to elucidate parts of the considerations behind the genesis and prosecution of this thesis. Ethical considerations have been addressed; voice and signature is central to the development of my methods here (Clandinin \& Connelly, 1994).

\section{Literature review}

Having situated both the initial thesis question, and the subsequent questions, for analysis within the Questionnaire, and having established the various methods for valid qualitative research that I would employ, I surveyed a wide range of voices from the literature of various inter-disciplinary authorities. These are attached to a schema that shapes the Literature Review in Chapter 3. These authors are separated into two categories: the first comprises those whose works clarify and define much about the themes and sub-themes which emerge from of my research data; the second details those authors whose works support and clarify many of the surrounding and inter-related 
concepts that contribute to the questions and considerations which emerge from the data in the form of trilectic logic.

\section{Analysis of data}

As has been noted, it may be necessary in the future to inform and link the observations and conclusions gleaned from this research with other, perhaps as yet undiscovered or yet-to-be-written research that emerges in the field of teacher practice as it is framed by these research questions. It is hoped that it also becomes possible to develop further understanding of the theory and practice of education.

Over a long but fruitful period of data analysis of the teachers' responses, I used a range of approaches. I took an inductive approach to determine the key threads, emerging patterns, trends, and a deductive approach to identify inconsistencies, gaps, possible contradictions, confusions, and emphases, in order to discover new considerations that might need to be addressed and collated. I bundled the responses in different ways, looking for implied meanings, and carefully attempting to discern the full meaning of responses. I analysed content by means of collaborative analysis and validation based on the teachers' responses, carefully clarifying coherent pictures of classroom practices; I included some comparisons of classroom practice - all from the content of the Questionnaire. Careful, deep analysis occurred as I strove to fully understand the implications of these observations and my conclusions regarding them. Evidence of certain categories of educational practice began to emerge.

To address the Questionnaire responses thoroughly, one question at a time, I read, understood and reflected on each response, until I was certain that I had discerned (to the best of my ability) the intent and full details of the participant's answer - as many times, the participant answered briefly or in note form. This was followed by comparing the various responses to each question and bundling them under themed headings, until I was confident 
that I had accurately detailed where agreement and disagreement existed. This was followed by another bundling process in which sections of the Questionnaire were aggregated, thus providing a manageable understanding of responses, differences, contradictions, gaps, confusions, concerns and, sometimes, where no information had been entered.

By quantifying the data at this stage, I was able to construct graphs which gave a visual representation of the teachers' experience and practice. This was the only time that the quantitative process emerged within the investigation and was followed by extensive comparison, analysis, and discernment towards the generation of qualitative conclusions. By dint of the completion of these elements, it became possible to identify hesitations, confusions, concerns, and silences. These results are shown in detail in Chapter 4.

I requested responses from three practitioner-experts in the teaching of thinking skills, (one in the UK, one in NZ and one in Australia) and these were examined in detail. All three had been sent a copy of the Questionnaire, and each chose to respond to the different sections with a general comment, rather than to the individual questions appearing under each of the sections. This triangulation of data sources - my personal practices and learnings, the details provided by the teacher-participants, and the opinions and elucidations of the practitioner-experts - provided content that, on analysis, was expected to yield observable national similarities and differences, leading to a space for critical conclusions and observations. It was expected that the specific comments and insights of the teachers would elucidate current practices relating to the teaching of thinking skills. The results yielded more than had been anticipated; these elements are explored in Chapters 6, 7 and 8.

I chose to analyse personally the data provided by the respondents. It became immediately apparent that the colleagues in all three countries had assumed that another teacher (me) would understand their responses, whether summarised or elaborated upon. In the interests of accuracy, understanding 
and transparency, then, I paid careful attention to the varying levels of detail provided by the respondents. Many gave brief answers, apparently expecting that their notes would be sufficient for the purposes of the research project. I took time over the analysis and inspection of the responses - regardless of their length - to be certain that my interpretations were reliable and valid. Because the bundling of comment and references given by participants was undertaken personally and not by an ICT package, I was able to consider the possible implications contained in the brief, succinct responses. Each and every response was taken into account; the detailed responses and outcomes are reported in Chapter 4. Where there was perceived ambiguity, consultation with my thesis supervisors resulted in agreement as to the ultimate interpretation. Each section of the Questionnaire was given individual attention; conceptual cross-links between sections were established and verified. A graphical representation of the frequency of different responses to each section of the Questionnaire is provided in Chapter 4.

\section{Hesitations, confusions, concerns and silences}

Careful inspection and interrogation of the responses was then undertaken to establish a teachers' perception profile that included their hesitations, concerns, confusions, and silences. In this way links and connections between the sets and sub-sets of research questions were located; contradictions and assumptions were detected; various terminologies were checked for consistent application by respondents from the three countries; and thus the findings were validated by internal triangulation. I became confident that conceptual connections and links were present between the list of hesitations, confusions, concerns and silences, and my research questions. Those connections and links emerged, in various ways, in the synthesis of understanding. The list could also be used as a stepping-off point for the wider study program I now set myself, and from which the Literature Review and the accompanying Bibliography emerged. 
I found I could delve into my own experiential learning and formal qualifications at will and at length to make connections; to find links; to overlay elements from each of the research sources in order to identify any contradictions and assumptions, if they existed; and to identify what my 'researcher voice' would provide as the theory unfolded, as the various instrumental tools were applied, and the recommendations that followed were clarified.

I soon recognised that, despite respondents having had an opportunity to register their views in detail in each section of the Questionnaire, these hesitations, signs of confusion, underlying concerns, and silences were concerned with contemporary issues in education in Australia, New Zealand and the UK. My own experience of the writings of leading educationalists and philosophers had focussed my thinking on an understanding of virtues, values and ethics. I had already begun the process of the analysis and synthesis of my studies, integrating relevant contexts and compiling an understanding of the work of Wilson, Lipman and Vardy as preparation for my own practice of teaching thinking skills to secondary students where I taught different subjects and different topics. I became aware that, surprisingly, this was not the case for the majority of the teachers who had completed the Questionnaire. This was particularly unexpected in the case of teachers from my school, as I had initiated a focus on Wilson, Lipman and Vardy in a number of professional development activities over the previous five years.

It became evident that teachers were not prioritising the development of thinking skills for the purposes of teaching about virtues, values and ethics. I realised that I needed to support teachers as their understanding of these elements emerged. These steps are discussed in Chapter 4.

\section{My comprehensive analytical framework: two aims}

My extensive Questionnaire produced a considerable variety of interesting strategies and approaches. I chose as my first aim to list, compare and 
contrast these - for better understanding of the practice of the teaching of thinking skills - by developing an analytical framework that integrated my three chosen educational formats:

Wilson's Moral Components List - a compendium of moral concepts which encompass virtues education (Wilson, 1987) and a focus on moral thinking skills (Wilson, 1987).

Lipman's Philosophy for Children - focus on Community of Inquiry discussion skills like questions, hypotheses, assumptions (Lipman, 1993a; Splitter \& Sharp, 1995).

Vardy's Five-Strand Approach to Religious Studies and Values Education - a complete concept (Vardy, 1998) that integrates rigorous studies regarding Christianity, studies regarding Comparative Religions, studies regarding Values, Virtues and Ethics, with particular relevance only to Year 11 and 12 curricular studies in secondary school.

My second aim was to either confirm or deny the legitimacy of my early understandings of virtues, values and ethics, based on the responses of the participating teachers and experts.

\section{Evaluation of all relevant criteria for this enterprise}

Careful use of criteria for reliability and validity (Flick, 2002), as well as trustworthiness, credibility and dependability, provides authentic data for inspection, interpretation and evaluation. Taking account of the distinctive elements that Mark A. Constas (1998, pp. 36-42) outlines regarding the requirements of postmodern qualitative research, means recognising that the relevant criteria are nuanced very subtly. Reference to important distinctions made by both Constas (1998) and Thomas (2003) allowed me to understand how a synthesis of thinking skills would take place for me; to make sure that I understood the various methods required to address all the imperatives of my thesis research. As my conclusions and recommendations emerged, it became obvious that much that is practised today may need reshaping according to different emphases. 
The evolution of liberal education in the twentieth century has resulted in conflicting outcomes (Wilson, 1987), and educating children for good citizenship has resulted in the emergence of contradictions between what are regarded as rights and what are deemed responsibilities. Value-free and neutral teaching in classrooms has served Australian education well, but has left constraints upon teachers and students alike (Snook, 1975). While this kind of education brought with it knowledge of individual rights, it began an enterprise that was to eventually evolve into the beginnings of the 'stakeholder society’ (Giddens, 1998; Howe, 2007). Significant trends based on a Third Way (Giddens, 1998); for citizenship, question how education prepares students for their uncertain futures. Splitter \& Sharp (1995) argue that students will need broad thinking skills, to understand choices and engage in decision-making (Giddens, 1998). This development supports and advances my effort to refresh social frameworks and personal life situations. This aim is parallel to the reshaped theory of education displayed in Chapters 6, 7 and 8.

The reshaped theory of education rests particularly too on 'paradigmatic controversies, contradictions, and emerging confluences' delineated in chapter 6 by Lincoln, Lynham and Guba (2011). They clarify the path of qualitative research that is emerging in my inductive writing. Also important is an over-arching 'critical pedagogy 'in qualitative research of the work of Kincheloe, McLaren and Steinberg (2011). The importance is the recognition of the clarification of experience as a legitimate source for learning, called bricolage (p. 167), defined as 'an emancipatory research construct' which includes the use of ethnographic methodology as a means of 'selfunderstanding and self-direction’ (p. 171).

\section{Looking forward...}

The following three chapters report the results of my three data collection strategies: 
- Chapter 3 reports my search for theory and voices in my Literature Review.

- Chapter 4 reports the findings of the Questionnaire and my personally designed pedagogical practices.

- Chapter 5 reports my auto-ethnographical retrospective.

By constructing the evidence gained from the Questionnaire, and then deconstructing the details of evidence given by respondents, I could reflect at a deep level on the concepts, the hesitations, the concerns, the possible confusions and even the silences emanating from this rich source. Reconstruction, through the modelling of inductive thinking patterns, has been carefully built into the reporting of all areas of the findings, the Literature Review, the logical emergence of conclusions and my recommendations, and the deliberate construction of a new theory (Chapter 6). Supporting these measures is the comprehensive auto-ethnographical introspective recounting my teaching and learning, which has unearthed new perspectives and emphases which, I contend, contribute towards planning for twenty-first century education.

In the first instance, the title of my thesis was Investigating Teachers' Perceptions of their Teaching of Thinking. The investigation, which began with the same title, was conducted by the use of the Questionnaire as the instrument which would, when secondary school teachers' voices were requested, be listened to, and be reported carefully and accurately, yield precise responses and indicate new knowledge from the coalface. Eventually, this process would lead to a new focus with an accurate title: Towards an Architecture for Teaching of Virtues, Values and Ethics. 


\section{CHAPTER 3}

\section{Voices in the Literature: A Multi-Disciplinary Search}

\section{Introduction}

Upon discerning the breadth of their responses to the Questionnaire, it was vital, in Chapter 3, that I develop a multi-disciplinary search by instituting a wide-ranging effort to listen to significant voices in relevant educational and other literature sources to help me understand the diversity of teachers' concerns and considerations. I aimed to learn, therefore, what questions emerged about teachers' current efforts in a changing learning environment. I sought to discern whatever was to count as systematic teaching that, in a democratic classroom, connected thinking skills with virtues, values and ethics.

This chapter records the results of the multi-disciplinary search for voices in the literature that impact upon this study into the teaching of virtues, values and ethics. I decided to address the various multidisciplinary considerations as many voices, arranged under three headings: the logics of virtues, of values and of ethics; in so doing, I was standing on the shoulders of giants (Newton, 1676) in my field of inquiry. I employed the integrative schema outlined in Chapter 1 to shape this Literature Review. This schema is depicted in Figure 3.1. 
FIGURE 3.1 APPLYING THE INTEGRATIVE SCHEMA TO THE LITERATURE REVIEW

\begin{tabular}{|c|c|c|c|}
\hline & $\begin{array}{c}\text { ONTOLOGICAL } \\
\text { CONSIDERATIONS }\end{array}$ & $\begin{array}{c}\text { EPISTEMOLOGICAL } \\
\text { CONCERNS AND } \\
\text { CONSEQUENCES }\end{array}$ & $\begin{array}{c}\text { TECHNICAL } \\
\text { STRATEGIES }\end{array}$ \\
\hline Virtues & On. $1(1)$ & Ep. $1(4)$ & Te. $1(7)$ \\
\hline Values & On. 2(2) & Ep. 2 (5) & Te. $2(8)$ \\
\hline Ethics & On. $3(3)$ & Ep. $3(6)$ & Te. $3(9)$ \\
\hline
\end{tabular}

The discussion that follows is divided along three main lines ontological considerations, epistemological concerns and consequences, and technical strategies - within each of which I examine the literature relating to virtues, to values and to ethics. Lincoln, Lynham and Guba (2011, p.97-128) give a global perspective to the connections between the various qualitative research paradigms so that it is possible to learn and appreciate all 'paradigmatic controversies, contradictions, and emerging confluences.' This means that the review is divided into nine sections as detailed below.

\section{Ontological considerations}

\section{Virtues}

In this investigation, I define a virtue as follows: 'the accumulation of assimilated personal, disciplined attitudes and dispositions for behaviours seen as the best expression of what an individual can be'. Previously, Wilson (1987) has explored the concept of what is to be regarded as virtue, and its meaning, in a manner that allows for the analysis of sociological, theological, philosophical, psychological, emotional and educational matters relevant to the accumulation of deeper understanding, in the form of, for example, tolerance, fairness, justice and prudence.

Aristotle's deliberative philosophical exploration in Nichomachean Ethics (Aristotle, 1962) provides a foundation from which I have been able to establish, dissect and analyse the definitions, meanings and understandings 
required to imbue a teacher with the skills necessary to present and promote rich learning about virtues, and their connections to values and ethics.

In their considerations of the research into many voices, research that connects the ontological approach to our understanding of what are to be regarded as virtues today, Costa and Garmston (2001) highlight human passions: efficacy, flexibility, craftsmanship, consciousness and interdependence, as generators of effective thought and action. Relevant to investigations into the objective or subjective nature of reality are C. S. Lewis' brief philosophical narratives The Great Divorce and The Problem of Pain (Lewis, 1940, 1946). Lewis follows Plato (Freeman \& Appel, 1956), whose conceptual link -with the notion of unaddressed, unstated pain and the failure to survive or to thrive- is vital to students' understanding prior to decision-making, and an important foundation for teachers' focus and classroom strategies.

In the matter of establishing the acquisition of virtue, Wilson (1987) first defines the word moral before making connections between morality and virtues, exploring what constitutes a moral virtue that promotes human flourishing. The concepts of good, bad, right, wrong and ought, are addressed by John Skorupski (1993) Griffiths (1993), and Martin Seligman (2011). Each considers the meaning of such issues as justice and punishment, as well as what constitutes a moral rule. They consider, too, the distinction between the moral and the ethical, explore the relevance of guilt and blame and the meaning of being sorry, examine the parameters for exclusion and social control, and state their assumption that all of these concepts are underpinned by the framework of practical reason. Sydelle Seiger-Ehrenberg (in Griffiths, (1993) makes distinctions between concept, fact, principle, attitude, skill, or even disposition. Golding (2006) asks teachers to model what they are teaching; Splitter \& Sharp (1995) list a comprehensive set of possibly relevant thinking skills.

A significant number of authors broaden the concept of virtue. Joel Kramer and Diana Alstad (2009), for example, address a range of concepts: 
authority, belief, freedom, and fear. Les Burwood and Rose Wyeth (1998) insist on the need for expertise and efficiency in higher-order thinking skills. Ross Fitzgerald (1993) assembles eleven writers who have written with satire and irony, bitingly truthful short pieces on Eleven Deadly Sins. James McNamara (1983) has written a sensitive volume about compassion, connecting it to powerlessness and meaninglessness; such thoughts have been echoed by Alain de Botton (2004), Seligman (1990, 2002; 2011), Jonathon Sacks (2000b) and Peter Singer (1979). Don Richard Riso (1992) describes the 'Enneagram', a measurement of personality-type connected to the understanding and acquisition of virtues. Natan Sharansky and Ron Dermer (2004) highlight evil in their world, connecting unexpectedly to Anthony Giddens’ Third Way (1998).

\section{Values}

For the purposes of this investigation, I define a value as: the accumulated behaviours that a culture, society, nation, or tribe has mastered, understood, taught, revered and demanded as expression of the best in an individual who belongs to that culture, society, nation, or tribe.

Such values, Singer (1979) suggests, are learned from childhood, practised by most people in each culture, society, nation, or tribe, as a preferred expression of all that is seen to be good for their generation. A link is possible with Adam Hopkins (1978) and his examination of the great debate regarding schools and education. Philip Kaldor, Peter Hughes \& Alan Black (2010) provide research results from an Australian context about Australians making sense about values in life. In a previous work, (Heasly, 1995) I have explored the effort to give balance and reflection to secular and theological life experience, which seems to lead towards an apparent need to counter today's reliance on technology rather than to use the time that such advances save, for thinking. Another conceptual link has been provided by Susan Greenfield's (2011) reflections in an address to our National Press Club on brain function and the expression of the inner personality. 
John Horgan (1999) bravely critiques philosophy, psychology and allied sciences, where efforts to formulate a comprehensive theory of how the brain works were to be found, highlighting the failures, the unfinished efforts and the problems in the field. His term: the 'undiscovered mind', sits at variance with Greenfield’s conception of consciousness (2004).

Leading the educational conversation, Susan Pascoe (2002, pp. 173-183) has assembled a number of experts for the Australian College of Educators Yearbook. The Yearbook feeds directly into the area of teaching and learning about virtues, values and ethics, placing special emphases on values education. Previously, (Pascoe, 1996) had explored the various theories of education and concentrated on broadening the horizons of the educational vista by examining the various trends and factors that lead to effective schools and schooling.

Values also matter to Lane Clark (2004), who, when preparing learners 'for their future NOT our past', uses her 'think box' and her 'think tower' as stable, initial stepping stones towards quality in thinking. Jean-Jacques Rousseau (1974) wrote Emile: or, On Education as a means of exploring what was meant by education of the young. Isaac Bashevis Singer (1983) provides insight into values and concepts that feeds into his well-established cultural perspective and philosophy of a unique Jewish milieu in his novel, The Penitent.

In my search I found that so many examinations of what I was to propose as the understanding of virtues, values and ethics, were already established. Yet, so much more had to be explored- in order that teachers could provide for the emergence and possible practice of more virtues, values and ethics that are chosen as relevant for contemporary student living and learning.

\section{Spirituality and values}

To aid in my search for a rewarding or reshaped twenty-first century philosophy of education that addressed my concerns, I found experts who had analysed this connection already. Eugene Stockton (1995) offers a 
unique picture of the spirituality of the Aboriginal people of Australia by asking what mainstream Australian society would be like if it were to seriously adopt this ancient wisdom. Sacks (2003) asks a similar question. David Tacey (1995, 2003), like Stockton, gives a detailed insight to the spiritual change and political development needed to understand Aboriginal Australians. Charles Taylor (2007) connects thinking, logic and values, and asks what it means to live in a secular age. Paul H. Hirst \& Richard S. Peters (1970) address the logic of education in the methods, history and philosophy of education; their considerations preceded similar examinations by Schwab (1983) who details the effectiveness of the four commonplaces of the curriculum: the student, the teacher, the curriculum content(what is taught) and the milieu in which it is taught. The result is the emergence of many connections between spiritualities, secular belief systems, philosophies and values systems.

In attempting to clarify the prevailing ideologies that influence organisations, William Kay (1975) takes a different route towards values: moral values are considered as problems. More recently, Irwin Edman (2009) has overviewed emerging curriculum concepts underpinning the growing development of a balanced Religion and Values Education subject, for multiculturalism and interculturalism in Australian schools. Kramer and Alstad (2009) develop a realistic values goal by expanding personal and social awareness.

Pushing this line of interrogation further, Wilson (1987), Robert Fisher (2003), and Peter Vardy (1999), provide exemplars of values, be they virtuesor values-based, or religious, political or philosophical in texture: honesty, fair play, social inclusion, social justice, active care for all forms of disablement, reason-based, impulse, previous experience, risk taking, needed outcomes, possibilities, or obedience to previously internalised concepts. Furthermore, when clarifying the notion of civilian values, they draw a distinction between duty and virtue. In so doing, they show that virtues and values are first and foremost the concern of the individual; whereas, the 
concept of ethics becomes the domain of standards accepted by a particular population, and eventually, a universal concept for living the 'good life'.

\section{Wisdom, concepts and values}

In expanding considerations that include wisdom as well as concepts and values, Griffiths (1993, pp. 31-35) highlights practical wisdom and ethical virtue, asking also for consideration of civilian virtues - civic pity, civic compassion - and military virtue. In the same volume, Onora O’Neill (1993) sets out a clear and concise outline of the distinctions between duty and virtue. Writing at the same time, Howard Gardner (1993a) points out that effective relationships and self-knowledge have strong links to ethical behaviour, as follows:

[T]he interpersonal nature of an individual's sense of self in secular systems of thought, ethical behaviours and the underpinning logic and reasoning is predicated on thinking skills that provide the individual with conclusions of what and why some issue is seen as right or wrong.

Addressing social policy in a secular context, Brian Howe (2007) details changes in Australia, and describes attitudes towards risk that include concerns similar to my own regarding the spectre of social exclusion:

\footnotetext{
The core capacity here is the ability to notice and make distinctions among other individuals, and in particular, among their moods, temperaments, motivations, and intentions.
}

Michael Domeyko Rowland (1993), also speaking from a secular context, develops definitions of what he deems can constitute happiness. Right and wrong behaviours are supported by laws of life, including response-ability, taking control, moral mindfulness and decision-making. Mackay (2004) sees 'moral mindfulness' as a pathway to moral clarity, underscoring the earlier issue (Mackay, 1993) of taking control of one’s self and one’s life. 
Greenfield (2008) sees the twentieth century as a battleground between a blatantly decadent consumerism and tougher, purer, simpler lifestyles dictated by the blueprints of ideologies, be they political, religious or some cultish cocktail of the two. Whether the determinants of identity lie in the genes, as the Nazis would have it, or the environment, as Marxism prefers, it seems that the individual has in both cases been cast in a predictable mould: identity shifts away from the unique person with their idiosyncratic personality, in favour of the collective persona; the collective narrative.

Creativity is included when Khaled Hosseinee (2008) investigates the impact of a creativity-teaching program on teachers' knowledge, attitude and skills. Elements pertaining to thinking skills and reasoning link her research to that of J. G. McAdam (2011). K. Helmut Reich (1995) distinguishes between pure logic and relational contextual reasoning. C. C. Verharen (1997) states:

The dialogue takes place, only against a supporting framework of assumptions common to both parties in the dialogue. Kuhn assumes that all theories must face a battery of tests: accuracy, consistency, utility, meaningfulness, simplicity, scope, and fertility.

Phillip Cam (2002b, p. 26), a leading Australian specialist in Philosophy for Children, insists:

I began with Dewey's claim that philosophy may take on the role of integrating our empirical beliefs and our values. In an educational setting, it is really only philosophy that can take on this role. Only philosophy combines the breadth of subject matter with the development of a holistic understanding in which both facts and values have their place. And only philosophy has the tools that are appropriate for such a reflective inquiry.

As a leading English specialist in Philosophy for Children and teaching thinking, Fisher (2003) adds:

People believe in many things, but their beliefs are often egocentric and unconsidered habits of mind. Some of these beliefs are spontaneously 
formed; some are derived from the beliefs of others such as parents and teachers. What these beliefs have in common is that they are rarely raised to what Piaget called conscious realisation.

and he continues, (p.165):

The goal of Socratic discussion is to be self-correcting and to teach the constructive resolution of disagreement ... Through Socratic education, with its focus on questioning and on explanation, every learner also becomes a teacher, and plays a part in the continuing quest for understanding and for ways of life that are worth living

\section{Neuroscience and its implications for education in the teaching-learning process}

To achieve the self-correction, the art of questioning, the promotion of rich learning indicated by the various authors like Fisher, Wilson, Lipman, Vardy, and provide a channel for such achievement, I have also turned to neuroscience and the attention needed to identify the old educational paradigm, and my new educational paradigm.

Spencer Kagan (1994), Arthur L. Costa (1992, 2000, 2001, 2001a, 2001b), Robin Fogarty (1991, 1993, 2001, 2005), and Susan Gardner (1996, 1997, 1998; 1999) have dedicated long careers and extensive educational study to medical-educational research towards the construction of personal and classroom thinking skills. They deploy the extant coherence and thinking patterns of the brain, to promote teaching and learning. Costa (2001) accomplished this aim in his comprehensive scenario of Habits of Mind.

V. H. Menec (2003), Greenfield (2004), Eric Jensen (2006), Hosseinee (2008), and McAdam (2011), are all concerned to allow creativity to surface as the emerging brain faculties improve. Jensen (2006), Norman Doidge (2007), Joe Dispenza (2007), and Bruce Lipton (2008), are involved in brain research towards a better understanding of the brain's potential in a range of human activities, including creativity and learning.

Greenfield (2004) shows that our real self - our imagination, individuality, memory, love, free will - might otherwise soon be lost forever. 
She presented this dramatic claim as keynote speaker at the Norrköping (Sweden) Conference in 2007, alerting educators to the necessity of including the new knowledge from neuroscience for learning and following these matters in subsequent years with further research and addresses (Greenfield, 2008, 2011).

Ingber (1997, pp. 48-57) clearly delineates a universal set of building rules that seems to guide the design of organic structures, and provides the informing concepts and philosophy connected to brain research. Interesting paradigms incorporating the media and values emerge as Neil Postman (1996) gives a challenging cameo on the values and expertise of the media, whose role in the skewing of thinking skills, he says, requires exploration by relevant experts.

Costa (2000) brings values in action to the fore, introducing new perspectives on intelligences, assembling authors who give emphasis and clarity to flexibility and to the capacity to shift perspectives; to the capacity to express prior knowledge and apply it to new situations; to meta-cognition and to our ability to reflect on our own thinking; to questioning and the capacity for healthy scepticism; to the need for accuracy, precision and elegance in learning and teaching; and to the human capacity for selfrenewal. Links with Matthew Lipman, Ann Margaret Sharp \& Frederick S. Oscanyon (1977), with Lipman \& Sharp (1978), with Wilson (1987), and with Vardy (1998) consequently apply. Fogarty (1991, p. 67) has assembled a number of relevant articles by Costa in his development of the possibility of infusing thinking into the curriculum in a conscious and efficient manner. He details thinking as neither an add-on nor a quick-fix, and sets out the steps for the teaching of a thinking skill efficiently.

Questions about how we understand the source of emotions like hate and compassion are examined in the literature, too. Paul Ekman (2001, 2008), whose research into the science of emotions is strikingly akin to that of Doidge (2007) and Daniel Goleman (2007), stretches both East and West in an effort to understand the nature of our emotional lives. Links to Wilson 
(1963), and to Vardy when paying attention to emotional intelligence, from Ekman, Doidge and Goleman are clear.

The recent feature article of an Australian Council for Educational Leaders (ACEL) journal, Leading \& Managing, provides a precise description of the author's (Crowther, 2011) eponymous COSMIC C-B model; a model for sustainable capacity-building in schools. He explains in detail how the parallel pathway operates. A. J. Ayer (1973) addresses the relevant value connections and claims of metaphysics, meaning and common sense, philosophical analysis, problems of perception, the construction of the physical world, the examination of body and mind connections, facts and explanations, order and chance, logic and existence and, finally, the claims of theology. Michael Rowland (1993) produces a secular concept called Absolute Happiness. Connections to and contrasts with John Wilson, Norman Williams \& Barry Sugarman (1967) and Wilson's Moral Components list, and to Goleman’s (1998b) emotional awareness, apply, though this would be a study in its own right, when approaches to curriculum design based on my outcomes are developed.

The historical element of thinking skills is addressed when Bertrand Russell (2004) traces the history of ideas. Richard Tarnas had done so previously (1991). Gary Watson (1982), as editor, identifies aspects of free will and delineates how to recognise the inner workings of the individual, reasoning towards an ability to live the good and ethical life. Concepts such as freedom, responsibility, necessity, and moral luck are also included. This links to Wilson, Williams \& Sugarman (1967) and the Moral Components List, as well as to Vardy's (1998) Five-Strand Approach to Religion and Values Education. Alan John Watt (1976) provides an accessible investigation and exploration to establish what rational moral education really is. Wittgenstein (1972) writes extremely densely about ethical propositions in philosophy; these propositions have been translated by Pears and McGuinness (in Pears, (1971). I am indebted to the work of Russell (2004) and of P. F. Strawson (1963), for drawing my attention to the value of 
rational, relevant content, to meaning and to logic; and for their conclusions which enabled me to make connections that had not previously been apparent. These connections are further developed in the approach advocated in Lipman's (1969) Community of Inquiry; the outcome enabled students to engage in and understand rational moral education. A very lucid profile of Wittgenstein, highlighting the changes in his stance, appears in Seligman (2011). This helped me to understand and appreciate the relevance to my enquiry of Wittgenstein's work

The Catechism of the Catholic Church (JohnPaul, 1994), the most recent compendium of Catholic doctrine, tries to explain in a question-and-answer format, the central elements of what it is that the Catholic Church offers. Similar compendia from other religions and belief systems could be substituted as and when appropriate. Significantly, Grayling (2011) has compiled a secular bible, mirroring in part the approach of the JudeoChristian scriptures by offering a channel for rational but secular moral education, allowing a scenario for a secular approach to the investigation of knowledge, often consigned to the studies in religion, about virtues, values and ethics. The approach implied in this thesis is that knowledge about virtues, values and ethics belong in the realm of general knowledge in Lifelong Learning.

\section{Ethics}

The third set of ontological reflections addresses what are to be regarded as ethics, an ethical life and moral development. For the purposes of this investigation, I define an ethic as the considered result or outcome in action by the individual in daily contribution to the owned culture, society, nation, or tribe. These outcomes have been used to solve most dilemmas, questions, paradoxical situations and vexed problem in life.

Singer (1979, pp. 1-4) highlights for teachers and students the connections between ethics, an ethical life and moral development:

- $\quad$ Morality is not really out of date. 
- Traditional moralists tend to defend one moral code only

- Ethics is not about a set of prohibitions particularly concerned with sex.

- Ethics is not a construct system which is all very noble in theory but no good in practice.

- People sometimes believe that ethics is inapplicable to the real world because they believe that ethics is a system of short and simple rules like do not lie ...

- Ethics is broader than the framework of one or more religions.

- Ethics is a framework beyond relativism or subjectivism. It is not just personal opinion.

Schwab (1983, pp. 239-265) further delineates the critical value and ethical dimensions of the four commonplaces of the curriculum - the student, the teacher, the subjects taught and the context (the 'milieu', according to Schwab) - by highlighting the need to be balanced and coherent so that decision-making about teaching and learning is aimed directly at student knowledge and understanding.

Extending the notion of an educational ethic further, Alan Walker's (2011, p. 28) monograph examines school leadership as a specific connective activity, re-emphasising Robert J. Starratt's view that educational leaders are expected to be moral in enacting the foundational virtues of responsibility, authenticity and presence. This is cultural values in action, and feeds into eight perspectives on the leading of learning as set out by Michael Bezzina (2009), and provides another glimpse into the importance of affective and academic leadership in the life of a school.

Recent research about how the brain learns and our understanding of moral development, is examined by R. L. Zigler (1999), whose insights suggest that we must think more seriously about the formation of the moral impulse as the basis for moral development and education, rather than simply for moral reasoning. Far from providing entirely novel insights about the growth of morality, however, this research relies on insights that Zigler attributes to the medieval Jewish philosopher Maimonides:

It is already apparent to those who study character development that as a culture and a community we have been involved, directly or indirectly, in 
the perpetuation and support of an agenda of values, virtues and vices which are being transmitted to the young: directly when we choose a direction; indirectly when, principally by abdication, we allow the messages and values of the media, peer group and culture around us to occupy the time of our young.

Moral agency has been explored by Robert Boostrom (1998, pp. 179190) who unpacks an instructional episode from Plato's Meno, and then draws out three necessary secular elements of moral agency - choice, vision and an end-in-view:

In a general sense a moral agent is rational and deliberative. A moral agent acts and judges in accordance with her own understanding of the good. A moral agent is a subject, not an object, and thus cannot be a receptacle, but how do these general terms translate into classroom practice?

Demonstrating values and ethics in action, Fairbairn (1999) indicates that the possession of such qualities as competence, expertise, empathy, and intuition as they pertain to the teaching-learning process, is what makes an expert teacher. The modelling of values in truth and ethics has been undertaken by Susan T. Gardner (1998, p.55):

The seduction of relativity...If there is no truth in ethics, there can be no value education...If there is no truth in ethics, there can be no ethical philosophical inquiry. If there is no truth, there is no expertise, no apparent value to education, and no intelligence....An undeniable practical belief in truth...Soft relativism: truth in the empirical realm...Misconceptions about truth and the truth-seeking process...Scientists do not assert that claims are true...Scientists assert that claims are true...Establishing truth through the process of falsification is not possible: establishing truth is...The transcendental deduction of truth...The product is not separate from the process, in evaluating a truth claim; therefore, one can only evaluate the process...Morality requires that one be as objective as possible...We must teach the process (not the product)... We must teach our children that in ethics, as in science, it is important to think, not merely feel and emote; 
and show reason why thinking is important is because, if done objectively, rigorously and creatively, it will lead the thinker closer to the truth.

Finally, in relation to the ontology of ethics, Susan J. Blackmore (2005) probes our understanding of consciousness, of how that understanding explains the working of the brain, as well as how neuro-science and the phenomenon of neuro-plasticity can be harnessed accurately and authentically for effective learning.

\section{Epistemological concerns and consequences}

Having delved into meaning in ontological terms about the links and connections with virtues, values and ethics, I now apply an epistemological lens to focus on all three sets of concepts to accumulate knowledge, to define new concerns and to deepen meaning.

\section{Virtues}

In breaking down, in analytical form, the concept of each virtue, Wilson's work provides a deductive channel through which to accomplish Aristotle's phronesis, and to pinpoint the consequences of the deeper understanding and knowledge that I now consider as constitutive of meaning. Connections of meaning include: virtue and morality; values and morality; morality and ethics. Wilson, Williams \& Sugarman (1967) link and analyse these concepts from both philosophical and educational viewpoints; these links are revisited by Costa \& Garmston (2001); Wilson (1971) identifies the connections between the emotions and religion and applies his Moral Components List (see Appendix C). He explores each concept as a tool for self-knowledge and for the consideration of ethical matters; he calls the outcome of this exploration moral education. Earlier, when probing the elements of higherorder thinking skills, Wilson (1963, p. vii), when undertaking analysis of each concept, had identified a single, coherent technique of thought. In this work, he examines the concepts of morality and freedom; morality and 
reason; problems in moral education; moral dimensions; and youth culture. He concludes by integrating his analysis into knowledge and action which can then serve his concept of moral education. As a result, it is possible to make distinctions between and connections with social conditions, democracy and morality - or, according to my experience, in today's terminology: values.

In a consideration of the social class determinants of morality, Kay (1975) examines morality from the perspective of different outcomes conditions in the home; the requirement of existing relationships for morality to flourish; the rigidity or alternative flexibility that ensues from these differences - and points towards consequentialism. This knowledge, which is a central focus of his analysis, is clearly outlined in his Introduction and Overview, and leads to the work of Vardy (1998), who takes what he terms a Five-Strand Approach to Religion and Values Education to cover knowledge, experience, the exposure to concepts of values and of ethics, and to concepts concerning what constitutes religious belief.

I now turn to cumulative approaches to cultivating knowledge about virtues, values and ethics in the classroom. Lipman (1988) sought to develop young children's skills of wondering and discovering. Mark Freakley \& Gilbert Burgh (2000, p. x) record in their acknowledgements how Lipman's Philosophy for Children program grew out of the tradition of Dewey. Lipman's (1993a) aim is in line with that of Dewey, namely to help all students to find what they will eventually call virtues or values by the method of deeper understanding and knowledge, and to do this within the Community of Inquiry - in which students question the text or resource first and then participate in discussion with others in a congenial classroom atmosphere. This approach broadened the array of thinking skills listed by Splitter and Sharp (1995). Lipman advocates continual use of the Community of Inquiry in primary and secondary education, and, by extension, tertiary education. I do too! 
Susan R. Easterbrooks and Nanci A.Scheetz (2004) claim that it is especially important for students who are deaf or hard of hearing to learn to think critically, and point out that:

Character education (CE) refers to the effort to teach basic values and moral reasoning (Doyle \& Ponder, 1977). Values clarification (VC) is the process of examining one's basic values and moral reasoning (Rokeach, 1973).

Their article provides a worthy summary of the links and connections between three interlinking concepts: critical thinking, character education and values clarification. Linked to this approach is the Values Education Resources for Primary and Secondary Teachers, entailing 32 units of work with research findings and teacher professional development (PD), and provided after a seminar conducted at University of Melbourne by Neil Hawkes (2010). It is my belief, on the basis of my teaching experience, that these links apply not only to the hearing impaired, but to all learners.

For accurate observations, Steven Schwartz (2000) on The Seven Deadly Sins of the Christian Tradition is outstanding. Using humour, satire and sly wit, he supports the readers' understanding of his subject matter, and explores concepts of what counts as pride, envy, anger, covetousness, gluttony, sloth and lust. These considerations, for me, provide a worthy opportunity to develop what connects with and constitutes Lipman \& Sharp's (1985) concept of 'self-correction' and what Stan Anih (1992) has quaintly called 'fallibilistic flexibility'.

Recently, in the developmental content for Christianity, David Martin (2011) provides connections between violence, democracy, religion, and secularisation. And Karl W. Giberson \& Francis S. Collins (2011) provide further discussion of the language and understanding of the links between science and faith. 


\section{Values}

Fearlessly for his era, Wilson (1965) sought ethical and moral insights into four areas: sex and logic, sex and education, sex and society, and sex and the individual. He was an early education specialist in the investigation of what constitutes a serious understanding of teaching for the good life, of the place of religion and philosophy in daily life (Wilson, 1987, pp. 22-34), and of the importance of a modern understanding of what should be regarded as moral education or perhaps in values education.

In further developing Lipman's thinking skills approach, Splitter \& Sharp (1995) effectively highlight a long list of thinking skills which inform the normal development of those pedagogical strategies chosen as values. It is very clear to me that these values include respect, honesty, sensitivity, reasonableness, and the strengthening of judgement.

Significantly, the link between Wilson's Moral Components List has eventually been explored in values education by educators such as Hawkes (2010, Chapter 1). A similar approach led to pedagogical experts, including Basil Moore (1986), being able to build pedagogical strategies and techniques in classroom milieux. Similarly, Bruce R. Joyce \& Marsha Weil (1986, pp. 1-22) delineate the many recognised models of teaching, and distinguish between a model of teaching and a model of learning.

I have drawn on valuable input from the work of Andy Hargreaves (2009) and of Hargreaves \& Dennis Shirley (2009) who address the sweep of historical reforms since the middle of last century. Hargreaves (2009, p. 29) is particularly concerned with a twenty-first century education based on renewed professionalism and active democracy. Similarly, Hedley Beare and the ACEL (2010) provide a monograph that details the sweep of historical development starting a decade or so earlier than Hargreaves and concentrating exclusively on Australian conditions. In his conclusion, Beare stresses the sense of mission for teachers and colleagues: to make improvements vital for twenty-first century schooling and learning. 
Twenty-first century student needs are recognised by Crowther (2010), who provides a twenty-first century model for building and sustaining capacity in a school: COSMIC C-B. He also reshapes some of the current educational emphases by using a list of six dynamics to be developed within a phenomenon he calls parallel leadership. The fourth dynamic in relation to this thesis is the most significant to this investigation - micro-pedagogical deepening.

Working within the same secular paradigm as Rowland (1993), Seligman (2002) provides a profile of Learned Helplessness - a phenomenon which teachers have found in classrooms containing students who have never learned the use of resilience, or whose levels of pain and suffering have overwhelmed their innate ability to focus and learn. Seligman (1990) outlines a conceptual process to help the reader, the teacher, the parent, and perhaps the student, to understand what is happening in their experience, and provides psychological steps that lead the individual from pessimism to optimism. Finally, Seligman (2011) follows the same process as Howard Gardner (2008) by providing applied thinking skills and learned judgement to constructing a theory that shows how his work can be internalised by students and teachers alike. The concept of well-being is firmly established in order to allow individuals to flourish, and his definition of what it means for humans to flourish is the central focus of this thesis. The connections are pronounced: with the work of Schwab (1983, p. 245) and his four commonplaces; with that of Costa (2000) and the Habits of Mind scenario; with the multi-intelligences theory of H. Gardner (1993a); and with content in Chapters 4, 5 and 6 of this thesis. All are interlinked and provide new knowledge for inclusion in a specific new architecture of virtues, values and ethics for the classroom.

Epistemological considerations about the connections between science, spirituality, psychology and organisational functioning can be profitably analysed in conjunction with the work of Peter Senge (2004). Senge integrates knowledge from science, spiritual values, psychology, 
management thought, and experience with learning organisations - in particular with systems thinking - into what he calls 'the fifth discipline'. He details core disciplines central to the building of organisations: personal mastery, mental models, building shared vision, team learning, and systems thinking. In the accompanying resource book Senge and his colleagues (2000) directly target schools and undertake a careful and disciplined indeed, an ethical - planning process to address all facets of school renewal. Particularly important is Senge's conversation with Howard Gardner (2000, pp. 555-566). Subsequently, ACEL writers (2010 newsletter), in constructing a best- practice methodology for teaching and learning, align with Senge (2011), who provided a keynote address via Skype at a recent ACEL conference in Adelaide.

What follows are twelve sub-themes derived from the literature concerning the epistemological dimension of values.

\section{The phenomenon of indoctrination}

At this point in the examination it is prudent to include within the acquired knowledge and discernment, the phenomenon of indoctrination. Snook (1972) assembles experts who relate and discuss various concepts of indoctrination. From the evolution of these considerations as an independent concept, to its connection with rationality, religious and other beliefs, respect for the individual, moral and values education, the existence of doctrine, democratic method, freedom, personal responsibility and the possibility of what Snook calls mis-education, in any form, the study is a careful exploration of exactly what indoctrination is and what it is not. In this sense, it has strong links to the work of Wilson, Williams \& Sugarman (1967), James Gribble (1969), Vardy (1999), Lipman (2003) and Fisher (2003).

To counter the possibility of such undesirable impositions as indoctrination in a classroom setting, it is necessary for teachers to understand the process by which narratives can highlight right and wrong behaviours in all sorts of ways and for all sorts of reasons. This is familiar 
territory for teachers of English and English Literature: as the protagonists and characters of texts are studied, their motivations examined and themes are discovered. The potential for such texts to elucidate behavioural motivations and reasoning lies behind Matthew Lipman's authoring of a number of novelettes. A more current example is the Bolognan morality tale, The Devil's Trousers - by Maurice Saxby \& Julie Gross (1999).

\section{The links between secular and religious values and beliefs}

The parallels in the use of thinking skills in order to attain knowledge, as demonstrated in the work of Wilson (1987), Gardner (1993b), Mackay (1993) and Fisher (2003) are apparent; however, where some of these authors use the term 'moral' in their determinations, others retain 'values' as a synonym. Singer (2009) expands the notions of values and beliefs to include fitting into society, and contributing to the wellbeing of others as well as self.

Increasingly, secular interests have emerged about the use of thinking skills for the acquisition of knowledge. Denham Grierson (2009) provides his reflection on the question of the degree to which secularity that has existed historically in Australian society. Daniel J. Harrington (2005) profiles how Catholics read the Bible. These are useful contributions, in which significant distinctions between religious and secular thinking are made. Such contributions at least relate directly to the decision-making process I contribute and outline in Chapter 4 of this thesis. The process of reasoning, contrasting and choosing reliable conclusions leads to student success rather than failure in decision-making. A. C. Grayling’s (2011) discussion parallels and addresses some of these distinctions. By connecting Grierson, Harrington and Grayling approaches, I have incorporated into this decision-making process a teaching tool which bridges the religious- secular divide.

Richard M. Gula (1989) offers a comprehensive volume from a Christian's perspective that defines the nature of moral theology, and delineates the task of the moralist. As an antidote to the challenging content of Daniel Jonah Goldhagen (2002), Gula’s volume pinpoints a framework 
regarding foundations for a Catholic morality, giving broad contrasting content for higher-order thinking skills. Similarly, Gula and Goldhagen provide vital content leading me to an appropriate methodology for all classes to investigate.

The work of a particular group of authors has added to my understanding of secular and religious values and beliefs: I can thank the work of Martin (2011), for providing a contemporary analysis of the paradoxes and apparent insolubility of many of today's ethical and political dilemmas, so that we as teachers are armed with the knowledge, distinctions and pitfalls attending to the existence of violence, of prioritized secularisation and religious diversity in democracy in twenty-first century change and philosophical subtlety. Haring (1978) addresses Christian biblical questions: the concepts of freedom and morality; liberty and responsibility; belief and fidelity; the fundamental option to believe or not; life-decisions; conscience; traditions and law; sin and conversion; and the relationship with the Christian concept of the Creator. The concepts are constructed with clarity and integrity, supporting Wilson's Moral Components List, connecting to Community of Inquiry content, and feeding Vardy's approach. Barbara Stead (1996) provides a careful study of the use of the Bible as a curriculum resource in Victoria, Australia, giving recommendations about its place in the curriculum for primary schools. Supporting the need for careful attention to the link between the Bible and values, Anthony John Hicks (1997) demonstrates the innate abilities of students to recognise justice, both formally and informally, so that teachers must take account of the positive nature of existing knowledge brought by students to their class studies. Authors Haring, Stead and Hicks are speaking from a particular and religious point of view but can't quite be ignored.

Richard Leonard's (2009) particular approach to education examines how film scholars and others can identify the spiritual dimension of films and movies. The paradigm that underpins Leonard's work is able to be linked to Vardy (1999), Wilson (1971), Goleman (1998), Lipman \& Sharp (1978), 
Rowland (1993) and Fisher (2003). Greenfield's (2007) research highlights that imagination, empathy and response-ability (ability to respond) can have contradictory impacts on Leonard's proposals.

Examination of concepts such as inclusivity and exclusivity give rise to an appreciation of cultural and moral outcomes in pluralist societies such as Australia, New Zealand and the United Kingdom. A. J. Watt (1976) outlines the need in a pluralist society to be inclusivist in the teaching of concepts of moral education, making it clear that divisive behaviours do not achieve desired outcomes; there are links between his conclusions to those of Wilson (1987).

Christopher Hodgkinson (1991) demonstrates that to achieve desired outcomes, leadership needs to be versed in an understanding of what is involved for education and leadership to become inclusive during the teaching of values; taking account of both value theory and value praxis.

The Values Education movement in the UK, led by Neil Hawkes (2010), among others, appears to have a close relationship with the work of Wilson (1987; 1990). Hawkes, a contemporary of Wilson's in Educational Studies at Oxford University, was a practising primary teacher (Wilson worked at tertiary level at the same time). The congenial conditions and the essential promotion of those attributes that were central to Hawkes' community, show a direct link to Wilson's (1987) broader analyses. Hawkes delineates a precise scenario within a single school. The educational aim was the application of thinking skills to life, incorporating 'educare and educere' (education knowledge and training, in Socratic and Aristotelian terms).

The movement founded by Lipman, Sharp \& Oscanyon (1977) in the USA had a similar beginning to that of Values Education. Lipman established the Community of Inquiry as a formal concept for pedagogical purposes in the hope of leading the way in enhancing community values and maximising potential changes of personal behaviour in children (including self-correction); these elements show distinct links to the work of Costa (2001a) and Anih (1992). 
Insights from social research uncover parallels in the knowledge applicable to the more complete attendance to students' needs: Mackay (1993) was suspicious that Australia was becoming more violent, less fair and less comfortable for families; at that time, he discerned developments in political thinking and philosophical conversation that were concerned with the issue of justice for the 'lower' strata of society. Race Mathews (1999) outlines the doctrine of Distributism, linking it to the social reform movements of his time. The development of contemporary ethical thinking was linked to earlier value paradigms and is evident in Crowther's (2010) concept of parallel leadership. Des Storer (1985) assembles a variety of experts who provide a profile of specific values across nine ethnic family groups in Australia. Storer's work has echoes in the multiculturalintercultural perspectives, subsequently highlighted by Constant Mews (2009), all pointing to a need for teaching thinking skills for twenty-first century education purposes.

Importantly, since Existentialism often remains unexamined today in the accumulation of the various links being highlighted here, Robert G. Solomon, (1974, p. ix-xix) has amassed a remarkable bibliography on Existentialism (from Soren Kierkegaard to David Miller): his philosophical statements are a form of conceptual sculpture; his Introduction gives a valuable ontological overview of what this theory is, and is not. William Barrett (1964) endeavours to define 'Existentialism' and identifies two forms of the concept. His volume centres on the question of implied meaning, from the position taken by Martin Heidegger, one of the founders of Existentialism. Mary Warnock (1970, p. 140), by echoing many of the leading thinkers in Existentialism (which appears to inform much Educational thinking today), sets a seal on its exploration as a philosophical theory. David Aspin (2002) identifies and develops what he calls 'an ontology of values'.

Perhaps anticipating the historical sweep of Russell (2004), Tarnas (1991),and Pelikan (1990), T. S. Eliot (1962) models ways in which 
terminology can be dissected from different perspectives in order to derive understanding and meaning; his work Notes Towards a Definition of Culture is superbly crafted and would be useful in any tertiary level Community of Inquiry.

Students need the opportunity to explore links to the 'good life' through their participation in their Community. In drawing attention to the emphasis placed on individual choice and the status of ethics that relate to what counts as religious belief, Patrick Gardiner (1988) adds clarity and insight about the thinking of Kierkegaard. Jürgen Habermas (1987) provides a profound study on knowledge and human interests, in which he uses many philosophical theories to uncover how the processes of inquiry inform our thinking and speech as we determine what we mean and what we want to communicate. His statement that beliefs are crystallised in concepts, feeds directly into the ontological and epistemological considerations of this project and relates directly to my inductive data reduction chart in Chapter 5. Charles Handy (2001) talks of the signs of the times; he exemplifies the effort to construct, in Wilson et al (1967) terms, the good life, paying due respect to the old and the new, the past and the future. Gardiner, Kierkegaard, Habermas and Handy address critical concepts which are foundational to my claim that teaching thinking skills can be a general starting point for the teaching about virtues, values and ethics for twenty-first century education.

\section{Moral argument, the good life and values through reflection and resilience}

When addressing the concept of moral thinking as an analytical philosopher, R. M. Hare (1981, p. 25) provides an understanding of what a moral argument is, concluding with a discussion about what is to be regarded as equality in education. He distinguishes two forms of moral thinking intuitive moral thinking and critical moral thinking - thus linking into elements of the works of Wilson, Lipman and Vardy. Hare's (1992) concepts of relativism and rationality, and his exploration of why a moral language is useful, provide links to Wilson's body of work. I have endeavoured to 
highlight here that the historical connections of Hare, Wilson, Lipman and Vardy all feed into my construction of a refreshed philosophy of education which is characterised by a culture seemingly more intent of instant thinking rather than reflective thinking. I have trialled the various instruments that appear in this thesis based on such considerations.

John Hick (1973, pp. 1-3) begins with an exploratory section on the Philosophy of Religion. His writing provides an overview of the foundations of philosophy in an arc which stretches from ultimately religious perspectives, through the spectra of faith and belief, to secularism today, providing relevant epistemological insights that might guide the teaching of decision-making processes.

Barack Obama's (2008) autobiography is an instructive example of how to think carefully about career, the good life and the possibility of a religious paradigm within which to live.

Peter O’Connor (2001), Stockton (1995) and Tacey (1995) provide valuable insights in relation to elements of thinking skills underpinning response-ability and responsibility. Unusually, O’Connor (2001) takes his own family roots, and the ancient mythology of Ireland, as a foundational concept from which to attempt to find some of the meanings and truths of life: love, hate, rage, despair and death. I found crucial connections with the work of Stockton (1995) and Tacey (1995) and also with Wilson's (1963) emphases on emotional content in determining meaning in life. These crucial connections underpin my considerations of a theory for philosophy of education.

I now turn to links between Classical and Modern Philosophy as a model for the building of meaning. Eugene Freeman and David Appel's (1956) translation of Plato's The Wisdom and Constructs of Plato, by dint of its analysis and integration of the Classical Greek Philosopher's writings, makes Plato's work accessible both ontologically and epistemologically. Chapters of interest and value to my study include: The Unexamined Life is Not Worth Living; Not Even God Can Alter the Good; What is the Source of 
Evil?; A Construct is Like a Bird in a Cage; the Nature of Being or Is-ness; The Search for Justice; and The Parable of the Cave. Building of meaning underpins the teaching of thinking skills, preparatory to applying those thinking skills in life, as personal choices made regarding virtues, values and ethics.

\section{The links to the building of meaning and of attaining wisdom}

To commence the building of meaning and the attaining of wisdom, Copthorne Macdonald (1996) provides an example of how to recognise, analyse and understand the concept of wisdom, and then to find legitimate ways of inserting wisdom into our experience of the good life. The contrasting study, completed by Remke Klapwijk \& Els Rommes (2009), regarding education in the Netherlands, however, shows evidence of certain career-specific values only, rather than a whole of life philosophy.

I found that De Botton (2004) follows the secular channels of Rowland (1993) and Seligman (1990, 2002; 2011) in order to identify anxiety and its effects. Additionally, he uses philosophy to recognise the values that are placed on the good life, addressing the various difficulties he posits as causes of conflict in the search for the good life, and pointing towards the building of meaning and the attaining of wisdom.

As a means of clarifying the many faces of what is called democracy, Anthony Giddens (1998) writes of The Third Way, in which he analyses the two main ideological forces in Western politics, Left and Right, and the various titles under which they appear depending upon which country is inspected. He names six dilemmas facing seekers of the good life: globalisation, individualism, left and right in political ideology, political agency, ecological issues and third way politics, as ethically important.

While, of course, Catholics appear to live faithfully and to the best of their ability and belief, the values and ethics espoused in the Catechism, converse behaviour has been observed in the Church's hierarchy by Goldhagen (2002), who constructs a philosophical and moral critique of the 
Jewish Holocaust which can not be ignored. His assembling of evidence and exploration of the visible values and ethical behaviours exhibited during that time, provides a challenging format for the building of meaning and gaining of wisdom. Applied thinking skills would provide relevant foundations for rigorous and logical outcomes, if decision-making is allowed to be democratic, and respect for difference is preserved.

As a leader in the development of academic understanding in the connections between neuroscience, biology and psychology, Marc Hauser (2006, p. 279) develops a perspective on the way in which humans have developed their values and their sense of right and wrong: 'She is born knowing that killing is wrong, helping is good, breaking promises is bad, and gratuitously harming someone is evil'.

\section{Teaching, authenticity and values education}

Building up the list of various authors who highlight aspects of thinking skills leading to the building of meaning and the gaining of wisdom culminates in teaching thinking skills for personal authenticity, through a broad palette of virtues, values and ethics as well as the knowledge pertaining to specific learning domains.

A number of writers have addressed the issue of authenticity in the teaching of values in schools. The concept of achieving authenticity is the focus of a study undertaken by Suzanne Macqueen (2009). Obama (2006) provides an autobiographical storyline which authenticates his personal values about himself, his family, his society and his future. Robert Theobald (1997), focussing on communities, writes about the contrasting potential for, and the possibilities of, reshaping the world around us by knowledge about, and perhaps the inculcation of, virtues, values and ethics. He suggests this through criteria that include ecological integrity and respect for the whole of the planet; and it is justice that underpins his aims.

Beare (2010, p. 16) provides a concise history of education in Australia. He details five discernible historical periods during which pivotal 
transformations in policy served to clarify community educational values. He also observes a 'globalised schooling in a borderless world in which patterns of schooling, curricula, assessment methods, learning programs [and] student achievement data are in the process of becoming international and interchangeable too, at least in the developed world'.

An alternative view is given by $\mathrm{H}$. Douglas Brown (1980) who ranges over a number of interlinking considerations, including human learning and cognitive variations, and personality and socio-cultural variables in language development. He leaves the reader to contemplate issues that are central to the teaching of thinking skills and language acquisition, and which still form part of the dilemma of finding world's best practice today. This is especially transparent in the figures he provides showing the diversity and profiles of channels for first and second language acquisition.

John Biggs \& Ross Telfer (1981) provide an exploration of the process of learning. Considerations of educational aims, models of learning, the role and function of memory, much about motivation and stages of development in understanding and language development, are examined alongside moral and social development, individual differences and the role of evaluation in learning - all necessarily values-related. Thankfully, we have progressed in our understanding of the various ways in which the brain learns to manage the understanding of levels of intelligence; thankfully, too, we have also discarded the use of the term 'retardation' (p. 415).

\section{Democracy, values and decision-making in schools}

An outline of what constitutes leadership under the newly-established concept of a self-managing school is provided by Brian Caldwell \& Jim Spinks (1992). Their concept involves changing the context of education through the development of cultural, strategic, educational and responsive leadership; the link to Beare, Caldwell \& Ross H. Millikan (1989) is precise. Smyth (1993) provides a critique and balance of the self-managing school concept, detailing dilemmas, and conflicts of meaning and values: the 
confusion of leadership with site-management; paradigmatic shifts in educational perspective; the role of devolution; and the existence of serious stress seen to be hiding within the concept of the self-managing school.

A significant examination of ethical leadership in the context of structural and organisational change in pastoral care, is provided by A. H. R. Chittenden (2004). This has links back to the work of Donald Cave (1976) who details four ideas about change highlighting the links between existentialism and education; self-actualisation; democracy and equality; and the role of religion - representing what I have called the old educational paradigm. The presence and continuity of existing values, ethics and leadership patterns suggested by both Chittenden and Cave, are logical predecessors to Beare (2010), his historical critique of these issues as a bellwether leading towards the architecture for a virtues, values and ethics curriculum.

An emphasis on the role of the social scientist in the so-called 'great school debate' was initiated by Adam Hopkins (1978). This pattern was followed by Raewyn Connell, Dean Ashenden, Sandra Kessler \& Gary Dowsett (1982), who analyse the impact of educational changes, based on social status and division, developing in Australia in the 1980s. The parallels were similar: the connections with school and family proximity are important; the social division behind the developments inside schools connect with the findings of C. Wright Mills (1978). Clear echoes and links back to notions of democracy, values and decision-making, initiated by Lipman, Sharp \& Oscanyon (1977), resonate two and three decades later in the writings of Giddens (1998) and Beare (2010).

John Carroll (2004) has taken an alternate view to the recognised historical substance of the Western culture: an exercise in values contrast, implying ethical educational leadership, and pertinent to my purpose of canvassing a very broad set of considerations upon which I can balance my refreshed philosophy of education explorations. 
Coming almost from left field for the 1990s, Colin W. Evers \& Gabriele Lakomski (1991; 1996) explore the concept of coherentism in school administration, addressing the same questions for improvement of school success rate undertaken by Caldwell \& Spinks (1986) - that of encouraging higher-order thinking to flourish. It is disappointing to note that the concept of coherentism was not extended and expanded to include coherent planning between the needs of student, teacher, the subject and the milieu, as conceptualised by Schwab (1983).

\section{The entry of emotional and other intelligences}

By proposing the inclusion into educational processes, recognition of the various intelligences that he had identified, Howard Gardner (1993a) contributes to a better understanding of multiple intelligences. H. Gardner, Mihaly Csikszentmihalyi \& William Damon, (2001) have provide a detailed reflection on what counts as good work. The subtitle of their volume provides a hint as to their recommendations: 'When excellence and ethics meet'. As a natural consequence of his earlier work, H. Gardner (1991) bases the foundations of his posited multiple intelligences on the quest of humankind for meaning in life. His work appears to provide established links about virtues, values and ethics with Wilson (1963; 1967), Lipman \& Sharp (1978) and Vardy (1998, 1999).

A comprehensive, detailed profile of those adverse effects on children with suspect social and educative conditions, that pose as hurdles in their attempts at undertaking successful education, is provided by William Glasser (1969). His thesis implies that children use their reflection time positively, both intellectually and emotionally; this might not be so if Greenfield's (2004) research into the brains of children today, prevails. The nurture groups’ pedagogy begun as a pilot project by Boxall but described, published and continued by Sylvia Lucas (2010) is a crucial link, as is the values education in a primary school addressed by Hawkes (2010). 
Goleman (1998a, 2007) develops some significant insights into emotional intelligence, closely aligned, in part, with $H$. Gardner's multiple intelligences. Goleman (1996) uses the concept 'schooling the emotions' in a rough parallel to Wilson's (1971) concept of 'educating the emotions', drawing information from internal areas of the self about how the brain functions so as to produce the tendency to bury painful personal episodes in life. This has links with the recent writings of Jensen (2006), Doidge (2007), Greenfield (2008) and Lipton (2008). Relevant understanding in emotional response is detailed by Dirk Wellham (2010) who provides details of a school project in which emotional intelligence (in its four Gardnerian categories) helps facilitate the development and optimisation of academic performance. In exploring how the use of technology changes the patterns of how the neurons connect in the young brain, and the way this informs understanding, thinking and feeling, Greenfield (2004) becomes a connecting link to Robin Fogarty, David Perkins \& John Barell (1992); and to Costa (1991; 2000; 2001a).

\section{Shining the light on possible reshaped pedagogies}

By providing information of the various issues and concerns that considered the educational debates of his time, Gribble (1969) establishes obvious connecting links between the skills of teaching: elements of how we know; the connections between morality and education; the social aspects of education addressed by Wilson (1963), by Wilson, Williams \& Sugarman (1967); and subsequently by Snook (1972), Singer (1979) and Mackay (2004).

Three stages of educational history (and a proposal for a fourth) have been constructed by Hargreaves (2009), in a manner which has parallels with a planetary system; they are echoed in Beare (2010); previously, Hargreaves (2003) had detailed the educational concept of the knowledge society itself. In a historical reconstruction of how knowledge has been and is capable of reinvention, Ian F. McNeely \& Lisa Wolverton (2009) trace the intellectual 
traditions of the Chinese, Islamic and Indian civilisations - and of the West until they observe all four traditions converge in the twentieth century to produce a truly global civilisation. These considerations have connections to the work of Wilson (1987), Russell (2004), Tarnas (1991) and Beare (2010) and show relevant channels useful for broadening the teaching of thinking skills in multicultural and intercultural milieux.

\section{Links between educational trail-blazers and the application of thinking skills}

Donald A. Norman (1969) makes an early attempt in psychology to sketch out the elements of memory and attention as an introduction to human information processing. The significance and links between Norman's examination of memory, attention, and acquisition of information, resonates with the recent writings of Greenfield (2004), Jensen (2006), Doidge (2007), and Lipton (2008). Martha C. Nussbaum (2001) presents me with a compelling account of emotional intelligence - to be recognised, understood, analysed, and accurately articulated. Nussbaum's work, I believe, builds unconsciously on Wilson's (1971) deliberations, highlighting the connections to the understanding of the function of emotions; as well, Nussbaum points towards Goleman (1996), particularly in her dissection of everyday life.

Though the content offered by D. D. Raphael (1976) refers to the problems of political philosophy, there are sections that overlap with the work of Giddens (1998), and which are of significance: the problem of distinguishing between power and authority; the limits of political obligation; and the boundaries of meaning implied in concepts of liberty, democracy and freedom. Gidden's exploration of the deepest meaning of democracy (that does not devolve into anarchy), is crucial for student understanding. Martin Rich (1972), gathers a group of experts who provide a broad spectrum of contributions that explain the aims and values of education which, I suggest, may be integrated with the values schools of Hawkes (2010).

By accentuating logical theory involving careful and honest attention to the meaning of words and terms, addressing examples of inconsistency, 
reasoning, formal logic, inductive reasoning and probability, Strawson (1963) provides me with a base for the subsequent work of Lipman, Wilson, and Vardy. In particular, the link with Philosophy for Children and the Community of Inquiry is obvious as cultural meaning morphs into new approaches to communication. In a later volume, Strawson (1985) explores the assumptions and distinctions derived from theories of scepticism and naturalism also applied, and how they resonate today. More recently, in what is obviously an ethical enterprise, Robert J. Swartz, Arthur L. Costa, Barry K. Beyer, Rebecca Regan \& Bena Kallick (2008, p. xiii and pp.11-32) provide an integrated learning process: researching, promoting, training, conceptualising and translating into classroom practice for the teaching of skilful thinking at all levels of education.

A summary statement that establishes a firm foundation for a better understanding of ethics and morality is provided by John Hospers (1982, p. 1, original italics):

\footnotetext{
Ethics is a branch of the study of human conduct. ....The aspect of human conduct that concerns ethics is morality. ... Ethics ...is a philosophical study of morality. ... What reasons can be given for or against a position someone holds...?
}

\section{Religious and secular pathways}

As a logical means of respecting the needs of people preferring the secular channel for life’s journey, Seligman (1990, 2002; 2011) suggests a learnedoptimism as a paradigm for positive emotions, chosen virtues and a variety of strengths. His subsequent analysis, application and signposts, provide subtle depth for rich learning for both student and teacher, and point towards a reshaped architecture for the teaching of virtues, values and ethics. This paradigm links to and contrasts with Lipman (1993a), Joe Dispenza (2007) and Lipton (2008), as well as identifying with Wilson's (1987) Moral Components List. 
Tarnas (1991) provides a summary of the History and Philosophy (and the History of Philosophy) that form the knowledge foundation of the Western world view: the Ancient Greek world view; the transformation of the Classical era; the clarity and confusion of the Christian view; the transformation of the Medieval era; modern world view; the transformation of the Modern era. He also provides an overview that integrates and synthesises all these influences on contemporary Western society.

In assembling a number of philosophers and educators, expert in related fields of philosophy, philosophy of moral education, and education itself, Monica Jean Taylor (Taylor, 1975) points out that there is at present, in the pluralistic society in which we live, much general interest in morality. She deals with progress and the problems that exist, and asks eminent practitioner-philosophers (including John Wilson) to address ethical matters ranging from where moral education fits in the curriculum, through the phenomenon of the neutrality of the teacher, to the goal of providing religious education in a pluralist society.

An exploration of the writings and philosophical deliberations of the early twentieth century French philosopher, Simone Weil, including a careful analysis of her religious, social and political thought, is provided by Peter Winch (1989). He relates Weil's development of a controversial conception of the connection between humankind and the natural world, which shaped further her religious appreciations of metaphysical dimensions -or what I have termed: eschatological speculations. Winch struggles to integrate some of Weil's concepts - in particular, just what thinking is - and in so doing acknowledges a specific connection with Wittgenstein's (1972) conception of thinking; a contrast to the profile drawn by Seligman (2011). In this regard, the work of Barrett (1966), Pears (1971), Anthony Kenny (1973) and Freeman and Appel (1956) provides some depth of understanding that helps to link thinking and applied thinking skills to Baruch de Spinoza (1955), and Plato. The missing link in our understanding of thinking - a consideration of 
the way in which the brain works - is provided by Jensen (2006), Doidge (2007), Greenfield (2008) and Lipton (2008).

In order to further improve our understanding of Wittgenstein's (1972) writings on the philosophy of language and philosophy of mind, Kenny (1973) attends to an apparent contradiction. Kenny’s explorations extracted Wittgenstein's meaning in its early and its more mature depths, although there are no connections made to Wittgenstein's particular personal history as made by Seligman (2011). In the teaching of thinking skills, such apparent contradictions can creep into discussions and conclusions, and Splitter \& Sharp (1995) gave pointers to help in monitoring in Community of Inquiry format in an educational setting; similarly, Anih (1992; 1995) provided pointers in a political setting.

\section{The growth of the Community of Inquiry}

Michael S. Pritchard (1985) was one of the early band of practitioners in the Philosophy for Children movement, with Lipman, (1977). He emphasises the relevance of concepts such as fairness, evidence, proof, controlling thoughts, reciprocity and self-knowledge. Ronald F. Reed (1992), another of the earliest practitioners (Lipman \& Sharp, 1978), provides details of the construction and facilitation of this format. Revered members of the movement - such as Sandra MacColl and Richard Morehouse - appeared and were joined by experienced philosophers, psychologists, teachers and artists to make recommendations about structuring and how to produce the desired student conversations in truth and meaning.

It is useful to examine eschatological, theological and spiritual perspectives and their links to educational knowledge. Anih (1992; 1995) provides a large body of work (coining the phrase: 'fallibilistic flexibility' to highlight self-correction for Nigerians), with affiliation to the Philosophy for Children headquarters and to Lipman (1969) and his principles. Sacks (2000b; 2003) examines Jewish philosophical and religious writings and, like Henry L. M. Nouwen (1983) before him describes how personal faith and 
values inform daily experience and provide a wealth of wisdom which is relevant to Values. Echoing Isaac Bashevis Singer (1983), Sacks (2003) also provides a view of matters of faith from a Jewish perspective which he claims considers Judaism to have the strongest ethical conception of freedom and dignity of the individual.

\section{Linking from values and virtues to ethical considerations}

Possible ethical responses to the concept of a preventive war and to the concept of globalisation - knowledge of each of which is the right of all students - are addressed, by Singer (2002) and by Francis Fukuyama (2007), respectively.

In the tradition of Tarnas (1991), Carroll (2004), Russell (2004) and Taylor (2007), -who assemble the thoughts and words of philosophers, theologians, and modern scholars,- Pelikan (1990) provides a comprehensive compendium of modern religious thought with clarity, detail and excellence. Such streams of ethical thinking clarify for teachers the previously unaddressed elements in their personal thinking as part of a shared intellectual heritage, and contribute to insights into knowledge about virtues, values and ethics of our civilisation. Conversely, Douglas Pratt (2003), under the banner of rethinking religion investigates, what is and what is not relevant to the teaching of thinking skills. His Foreword (p. vii) and Introduction (pp. ix, xii) gave valuable definitions and distinctions about how to study what religion is about: highlights of teaching about, and teaching in religious matters. As an integral part of differentiating between secular and theological concepts, this content is significant, connecting in secular terms to Seligman, Rowland and Aristotle; and in religious terms to Wilson, Lipman and Vardy.

\section{Ethics}

Like Wittgenstein, Spinoza (1955) explores how we improve our understanding and recognise the depth of meaning in ethics; because they 
came from different backgrounds, a link is required between the epistemological starting point of Wittgenstein and Spinoza, and today's teachers so that they might understand the depth of meaning in ethics.

A. C. Davies (2005) addresses many themes that emerge regularly in this study. She provides a link between earlier writers and the emerging new knowledge of the flexibility of brain function, led by researchers such as Greenfield and Doidge. Davies' examination of the needs of teachers for a New Democracy for Professional Development and Research provides a conclusion from sources of impeccable educational expertise when she alludes to the voices that are central to the activities which will emerge in the new educational paradigm. She looks to many voices in the literature sourcing Anthony Giddens, Eva Cox, Anna Yeatman, Amartya Sen and Zygmunt Bauman and Jon Bird. She refers to a program of rethinking, responding, reshaping, reconceptualising, reconstructing and recreating in educational scenarios.

There is now the possibility that pre-established concepts of brain function will lead to a reshaping and re-creation of what is and what is not ethics. Doidge (2007), with careful research and a number of remarkable stories of people who triumphed over serious injury, provides content about how the brain can change its processes. His area of research and expertise is neuro-plasticity, connecting to Wilson particularly through the development of alertness to the meaning of individual concepts of virtue, dealt with earlier in this chapter. Doidge (2010) includes, in a two-part DVD, a sequel about how it is possible to change the functions of your mind: it is beyond the confines of this investigation to develop this concept further, but its existence for teachers' new knowledge is vital - unless this new knowledge is included in the educational program, what is or what is not ethics might be termed both very old-fashioned and irrelevant.

In parallel with Wittgenstein (1972) and Spinoza (1955), Brink (1989) provides a foundational volume of ethics based on what I have increasingly 
come to regard as the old paradigm. He delves into meta-ethics, moral epistemology, moral psychology, value theory, and moral theory:

I argue that a realist meta-ethics, a coherentist moral epistemology, an externalist moral psychology, a non-reductive form of ethical naturalism, an objective theory of value, and an objective form of utilitarianism are individually plausible and mutually supporting.

The foundations of ethics have been shaken by the emerging research into brain structure and function by Greenfield (2004, 2008) and others, and by the positive psychology of Seligman (1990, 2002); so the old paradigm based on logic and free will - may no longer apply! Meanwhile, it is appropriate to recognise Davies' (2005) research as a pivotal point between the old and the new paradigms. Meta-cognition - knowing about knowing is pertinent to my investigation, and has links to Brink (1989). Ten years later, Francis J. Schweigert (1999), when writing about education and justice in the context of the old paradigm, states:

\footnotetext{
This study investigates the educative process in restorative justice reforms, revealing three characteristics effective in facilitating moral learning for the common good. These three characteristics can be formulated as principles to guide the theory and practice of community-based moral education.
}

In the new paradigm, the educative process that Schweigert proposes figures in all three of virtues education, values education and ethics education, as discussed in this study; so, empathy is manifested in virtues, compassion equates to values, and restorative justice takes the form of ethics. Being concepts not necessarily familiar to students, they must be taught in ways that include discussion and exploration. Such forms of empathy and compassion are implied in Wilson, Lipman, and Goleman. Brenda Richardson (1997) refers in her paper to Lipman's two kinds of care: 
Lipman distinguishes between two KINDS of care. One care protects, preserves, and keeps. Another care intervenes to make a difference for the sake of what is cared for. Both kinds of care are active.

The article from Pedro O. Ruiz \& Ramon L. Vallejos (1999), in concert with James McNamara (1983), affirm the concepts of compassion and empathy:

We propose compassion as a new model for moral education. The insufficiency of Kohlberg's cognitive model for such education is shown, as in the absence of compassion in dialogical ethics. We review briefly some authors who have treated the theme of compassion and propose the development of empathy as a foundation for educating for compassion. Specifically, we propose emotional guidance and observation-based tasks. Education for compassion, as it is understood here, does not only lead to Knowing the reasons of other people, to understanding them (empathy), but also to taking responsibility for the real situation of the other (compassion).

\section{Links: Zigler, Dewey and education}

Ronald L. Zigler (1998) inspects a neglected dimension of Dewey's work: an examination of the influence that Dewey had on the formalisation of four domains of moral education. Zigler reveals that Dewey clearly anticipated the research and constructs which Goleman (1996, 1998a, 1998b, 2007) recently sought to popularise about emotional intelligence. Zigler highlights that among Goleman's conclusions was the recommendation that the education of moral character address the development of emotional habits and the fostering of emotional literacy. From my perspective, the concept of moral character fits into the pedagogical support that Lipman's Community of Inquiry provides. Further, it categorises Wilson's Moral Components list and inserts itself into Vardy's Five-Strand approach to education in religion and values. Zigler claims that democratic classroom pedagogy, e.g., the Community of Inquiry, is linked directly with ethics. I agree with this conclusion and my thesis provides an extensive response to support this. 
Connected to these considerations are further insights from Wilson (1998) who highlights in his article the difference between actual morality and what he bemoans as 'ideology and unreason':

Two aspects or aims of morality are distinguished: (1) the need to avoid trouble and ensure appropriate behaviour, and (2) forms of life which invoke sharing, and hence require certain basic dispositions as well as behaviour-patterns. Analytical philosophy hence has an important part to play: it is no good just trying to run up some appealing form of life and hope that it will be persuasive - that leads directly to ideology and unreason. We badly need some picture or pictures of life which, although no doubt fitting some individuals more than others, will give some kind of contextual substance and solidity for the deployment of moral equipment and moral virtue.

Nicholas Michelli (1999, p. 10) comments on the importance of critical enculturation:

We must remember that the critical enculturation of students into an emerging political and social democracy must be an integral part not only of primary and secondary education, but of a university education as well. This does not mean blind acceptance of the status quo, indoctrination, simple study of the key documents or laws of a democracy, or learning how to cast a vote. It involves learning to apply critical thinking to making good judgments as a citizen.

The higher-order thinking skills required to achieve such matters without confusion have been developed carefully in the Community of Inquiry approach; connections to Flew (1972a, 1972b), which warn against false reasoning and possible indoctrination, predominate.

Overall, links to the works of Snook, Wilson, Lipman (especially for the Community of Inquiry) and to those of Vardy, Fisher, Sutcliffe, Wills and Golding (especially for the teaching of values and ethics) emerged as significant to my thesis question; Kristjannsson (2000, p. 13) points out the importance of the inclusion of emotion education in teacher training: 
A good teacher must be his [sic] pupils' keeper: act (as we have already seen) as a moral exemplar himself, shepherd to the best of his abilities their moral and emotional development, and make reflective use of the didactics of emotion education. Unfortunately, without the appreciation and implementation of these truths in teacher-training programs, any suggestions to the effect that teachers should help students promote morally valuable emotions will continue to fall on deaf ears and be of little avail.

At a philosophical level, Susan T. Gardner (1997, p. 55-62) concludes:

There are a lot of critical thinking programs that focus on skills and many focus on attitude. My contention is, however, that philosophy has a unique contribution to make to the recent contemporary effort to enhance good thinking because it promotes good thinking by focusing on philosophical topics. Of course, if we are unconcerned about ends, if we do not care whether or not our youngsters ever ask themselves what kind of people they want to be and how their immediate actions and goals bare on that end, if we do not believe that it matters whether anyone wonders what kind of community best nurtures human well-being, if we ourselves think it is unimportant to ask what counts as a good or worthwhile life, then any reputable thinking program is probably as good as any other.

In my attempts to support the professional development of teachers, links to Lipman and Vardy, are crucial. According to Susan Wilks (1996, p. 4546), whose terse summary was central for teachers' perceptions of the new techniques and strategies for teaching thinking skills, the underlying beliefs behind the Community of Inquiry are as follows:

The members' beliefs remain their own, but it is easy to seek reasons for their Constructs and submit them to public inquiry. In a pluralistic society the community should be composed of a number of diverse participants capable of public inquiry in which they can think well for themselves. Each member of a community of inquiry is someone who lives in the present, remembers the past and anticipates the future, each in a unique way. 
Thus, in its development of new teacher training programs, the new paradigm needs to take careful account of the contribution of Kristjannsson (2000), Susan Gardner (1997), and Susan Wilks (1996).

\section{Ethics and brain-compatible learning}

My understanding of the deeper elements and connections of their theories of good and evil, moral beliefs, the ought/is debate, the various interpretations of moral philosophy, what it is we call rules, and forms of utilitarianism: all are explored for the distinctions, the links, the sometimes false assumptions that may slip into our thinking. Phillipa Foot (1967) assembles the research of twelve practitioner-experts to ensure that such links are pertinent to teachers' efforts to instil a culture of reflection in the classroom.

The Journal of Moral Education (Volume 36 No. 1) contains much pertaining to neuro-scientific research into Neuro-imaging with children: ethical issues and case scenarios pinpointed in this edition by Coch (2007) and the accompanying Tancredi (2007) book review - 'Hardwired behaviour: what neuroscience reveals about morality' - within the same issue. These two contributions link with the work of Greenfield (2004) and Doidge (2007) and, by extension, the work of Costa (1992) and Fogarty (1991).

Lynn Revell \& James Arthur (2007) propose that character education is akin to the teaching of values education in schools; their assessment of the situation in the UK resonates with a developing curriculum need in Australia

Mackay (1993, 1997) introduces a need for the authentic profiling of present-day Australian society; he explores the attitudes, values and outlook of three generations of Australians. In his subsequent study of Australians, conducted as the nation faced the new millennium, Mackay (1999) provides a summary of what today are called Values. His overview (Mackay, 1999, 1999, p. viii) includes what he calls 'crosscurrents of confusion [...] undercurrents of hope' and feeds into teacher response-ability (i.e., the ability: possession of knowledge of the background ontological considerations, the epistemological concerns and consequences and the 
technical (techne) strategies - the pedagogical detail) to teach about virtues, values and ethics. Later, in a sociological survey, Mackay (2004) focuses on how Australians decide what is right and what is wrong.

J. L. Mackie (1977) provides a comprehensive discussion of what is to count as ethics. I recognise his point that scepticism is the inner response, intellectually and emotionally, to a challenging new knowledge that does not yet fit with our previous experience. This position connects with Wilson's Moral Component List (1971), Anih's self-correction (1992), and Hawkes’ values education (2010).

In a personal exploration, from a logical viewpoint, of what ethics means, Singer (1979, 1993, 2009) applies ethics to practical situational issues and problems. In contrast, A Phillips Griffiths (1993), in a meta-analysis, assembles practitioners' writing on aspects of ethics, covering objective prescriptions, integrity and self-identity, ethical absolutism and education, morals and politics, duties and virtues, a definition of morality, justice and ethical knowledge, and institutional ethics. These issues highlight the overlap between the earlier paradigm and the new paradigm arising from my investigations. As a representative of the former paradigm, and consistent with Wilson’s Moral Components List, Bernard Williams (1972) discusses connections between what constitutes morality and its partner, ethics; at that time, there was no established link with brain research - the latter had not yet emerged.

In subsequent research into Australian conditions, Garth Boomer (1982) focuses on which, and how many, of the 200 Australian cultures that constitute the nation's multicultural identity were central and significant for human rights, values education, justice and bullying phenomena, ESL practices, and teacher expertise; as he emphasises the rich diversity brought to schools by the children from these cultures. These links between new cultures and cultures of previous decades connect with, and make logical reference to the contributions of H. Gardner (1993b) and Goleman (2007); 
and with the emerging brain research of Greenfield (2004), Doidge (2007) and Lipton (2008).

\section{Technical strategies}

I consider that specific approaches are vital when applying the strategies and techniques to be used in the classroom for the teaching of what each virtue and value means. These considerations entail elements of the pedagogical skills as detailed above (technical strategies and techniques; techne). These approaches are discussed in this section.

\section{Virtues}

Costa and Garmston's (2001, p. 18- 22) 'passions', which underpin the acquisition of virtues, are the basis for habituation in the mind. They provide, therefore, a foundation for Costa's (1992) Habits of Mind, in which he recommends using questions as diagnostic tools, as constructs with which we can assess the cognitive development of ourselves, other individuals, and groups. This is akin to considerations that led Wilson (1987) to compose his Moral Components List; however, Wilson did not take the next step of setting the contents into a formal curriculum in modern pedagogical terms. Earlier, Wilson (1963) had placed emphasis on teaching higher-order thinking skills - in particular, the construction, deconstruction, and reconstruction of concepts; this was taken up by Costa.

In the meantime, Lipman (1988) developed the Community of Inquiry to provide a support system for the acquisition of accurate and comprehensive varieties of thinking skills for students. This process developed the steps of social interaction and mutual inquiry where students prompt each other and learn together. May Leckey (2009) provides a study in an Australian school that highlights the various processes, difficulties and mistaken expectations that may accompany the setting up of the Community of Inquiry. Leckey's conclusions support Lipman's expectation of continuous use of the 
Community of Inquiry, and strengthen my own belief in such continuous use in the classroom.

Separately, Vardy (1998) began the Five-Strand Approach to the study of virtues, values and ethics, setting each topic into a curriculum design. The two approaches of Lipman and Wilson discussed above, linked with the approach of Vardy, provide in my experience, a strong technical basis for a higher-order thinking program in virtues, values and ethics at senior secondary levels.

\section{Links between leadership, educational theory, pedagogy and thinking skills}

I have found there to be a need to link leadership, educational theory, pedagogy, and thinking skills in order to embed rich learning about virtues, values and ethics into the culture of a school. This position is supported by Dorothy Andrews (2010, p. ii) who writes, succinctly, that the 'current focus on leadership, be it distributed, instructional, educative, transformational or sustainable, is on affecting the quality of teaching and hence the learning outcomes of students'.

A new concept, that of parallel leadership as the key to successful school capacity-building, is encountered in the work of Crowther (2010, p. 27) who outlines the CONSTRUCTS project, and the six dynamics leading to his COSMIC $C-B$ model of school leadership. In a separate monograph of the ACEL series, Crowther, in collaboration with Lindy Abawi, Dorothy Andrews, Joan Conway, Mark Dawson, Marian Lewis, Allan Robert Morgan \& Shauna Petersen (Crowther et al., 2010) details how the concept of sustainable school capacity-building came to fruition through the research of the Constructs team. He provides a comprehensive description of the underpinnings and concepts behind his COSMIC C-B model. The applied thinking skills approach feeds in to his dynamic 4: micro-pedagogical deepening. This is set out in detail in his subsequent publication (Crowther, 2011). 
In a consideration of coherentism, John P. Keeves \& Gabriele Lakomski (1999) include the following: philosophical issues; feminist perspectives; the impact of positivism, anti-positivism and empiricism; the nature of phenomenology and its connection to existentialism; and the link to the development of the theory. I have learned that these issues deepen support for Lipman's (2003) Community of Inquiry in that they centrally situate the recognition of an educational theory that underpins classroom pedagogy.

\section{Understanding how the brain learns}

In moving from the old paradigm to the new proposed paradigm, information based on brain research describes the cellular changes affecting the brain's functioning and how the cells of the body can be affected by the individual's thoughts. Lipton (2008) argues that the cellular changes unleash the power of consciousness. He goes on to explain the new science of the brain, indicating opportunities for understanding the link between mind and matter. Doidge (2007) provides new information which, I believe, connects to Lipman's Community of Inquiry. Increasingly, the connections become clearer that the power of the brain expands learning; therefore, this is an area that needs to be integrated into pedagogical processes. Dispenza (2007) extensively expands on how the brain has evolved in response to the requirement that it process incoming and escalating data flows. Relevant connections can be made to Wilson (1963), Lipman (1993a), Vardy (1999), and Costa - with distinct links to Lucas (Lucas, 2010) regarding nurture groups.

H. Gardner (2008) also provides an insightful profile of what he calls 'five minds for the future' taking account of the changed conditions in his first chapter, that are emerging within education for the needs of today's student cohort. His concept of disciplined, synthesising, creating, respectful and ethical 'minds', provides a parallel and possible contrast, to the conclusions and the hesitations, concerns, confusions and silences of teachers responding to the Questionnaire underpinning this investigation that will be encountered in subsequent chapters. 
Significantly, Dispenza (2007) provides some startling information about how the brain can be used for learning in lifestyle and health considerations; these considerations are implied in Greenfield's (2011) address to the Australian National Press Club, especially in her examples of the London taxi-drivers' brains and research into piano-playing research: this research indicated that certain parts of the brain expand to accommodate challenging requirements like remembering an enormous mapping of a city like London; and similar, but different parts of the brain that also expand and re-pattern to accommodate challenging requirements like memorising large classical symphonies complete. These have central consequences for and impact upon the development of learning and new knowledge for educational purposes as well.

What was promoted as a life-changing approach to learning for parents, is set out by Dawna Markova (1992); this approach shows patterns that fit well with H. Gardner's multi-intelligences. Applying the old paradigm, Markova refers to natural persuaders, storytellers and empathetic children. I believe that if Greenfield's thesis holds in the face of further research, children will need first to be taught empathy to achieve Markova’s old paradigm indicators to become successful kinaesthetic, as well as visual and auditory learners. In the sub-categories of pedagogy, there are familiar strategies pertaining to the delivery of a subject area, and strategies of learning that encourage the student to engage with the patterns of applied thinking skills through the medium of the subject content.

In his considerations of freedom of choice, and emerging from the old paradigm, Mackay (1999, 2004) explores guilt, shame and forgiveness as moral factors in deciding what is right and what is wrong. These explorations, in turn, meet ontological and epistemological requirements in teaching about virtues, values and ethics - a requirement that goes beyond the teaching of virtues, values and ethics; it takes account also of the democratic subtlety in which students learn about these concepts with no 
evidence that given concepts of certain virtues will take precedence or be promoted as of prior significance to students.

Respect for the diversity in historical Christendom and the cultures of belief and unbelief, are addressed when C. Taylor (2007) makes connections between historical Christendom in Western Culture, the emergence of Modernity, and the quirks and eddies of the historical use of power in religious, spiritual, cultural and secular milieux. He also makes connections and distinctions that are useful in understanding the modern concepts of virtues, values and ethics. I consider that his work shows elements of both ontological and epistemological relevance to this investigation. Taylor's content connects with Wilson, Lipman and Vardy; Aristotle, Dewey and Schwab.

\section{Values}

On first recognising the central relevance of questioning, Lipman (1991) realised that in response to their early schooling, young children lose the art of questioning, and the ability to wonder and speculate on the daily experience of life; this was part of the old paradigm. In the emerging new paradigm, Lipman's insights are materialised in the work of Costa, Fogarty, Doidge, and Dispenza. A fresh starting point, that reflects the acceptance of the new paradigm, emerges in a promotional statement made to me by Roger Sutcliffe $(2009,2010)$ prior to a SuperSeminar that he conducted in London in 2010:

Inquiry-based Learning could be said to have its roots in the very beginnings of formal education in the West: in the Greek schools inspired by Socrates and his questioning approach. Sadly, as education became institutionalised, teaching and learning tended increasingly towards a banking model - with knowledge being transmitted from teacher to learner, largely through lecturing. Despite overwhelming evidence and reason to suppose that such learning is shallow and hard to sustain, many lessons are still designed on the basis that the more information that can be presented by the teacher, the better the students will learn. Inquiry-based 
learning, which has survived in many forms but with few champions in the mainstream, emphasises two aspects of learning that are fundamental, but often under-valued by teachers: firstly, the importance of nurturing students own curiosity (their will to inquiry); and, secondly, the importance of developing their imagination and reasoning (their skills to inquire).

The banking-transmission approach (Freire, 1972) to institutionalised teaching and progressively being employed in inquiry-based learning led to the latter being under-valued, thus diminishing students' will and skill to engage in inquiry. Startling, too, is the deficit approach to teaching; as I have reported previously (Heasly, 1995, p. 71), this approach views students as being short of knowledge and understanding in certain areas within a topic or language in content, in cultural mores, and in ability to respond to new topic content. The student is seen as in deficit before any teaching is delivered when, in fact, they bring a broad palette of ideas, knowledge and experience to the classroom. I believe that teachers now need to recognise teaching about each or as many of the concepts of virtues, values and ethics. This, in Wilson's terms, is central to student learning.

Yvette Jackson (2001) addresses this issue, investigating how underachievement, particularly in urban students, might be reversed with a pedagogy of confidence, using Lipman's effective and efficient thinking skills as the central component. Jackson defines pedagogy specifically:

\footnotetext{
Pedagogy is an art or method of teaching, developed and refined when teachers are confident in their ability to successfully affect their students. This sense of confidence results from knowing what to do and believing you have the skills and abilities to meet those expectations.
}

The old paradigm represents what I call a deficit model of pedagogy; the new paradigm represents a new 'pedagogy of confidence'. Noteworthy in its address of the concept of this new 'pedagogy of confidence' in learning, Lucas (2010) updates a research project that was initiated by the late Boxall and her teams in UK. Together, Lucas and Boxall emphasise the usefulness 
of Nurture Groups that began when Boxall researched, very successfully, how teachers used new skills to educate traumatised and emotionallydeprived students.

\section{The role of pedagogy: links between theorists, practitioners and experts}

For the purposes of this investigation, I define pedagogy as the established practices, outlined frameworks and systematised formats, through which teachers, supported by Curriculum Corporations, Education departments and other specializations and experts initiate and sustain the teaching-learning process daily; this is the old paradigm. Support for the work of Greenfield (2007) in the understanding of how neuroscience would increasingly be a factor in improved teaching and learning processes: this provides the foundation of the new paradigm. Martha Burns (2010) provides a vivid outline of the new paradigm in action; my exploration of this new paradigm is outlined, described and analysed in the following chapters of this thesis.

Lipman (1993a) gives voice to all elements of the theory and practice of the Community of Inquiry. Likewise, Wilson, Williams \& Sugarman (1967) propose a specific list of Moral Components as the foundation of a pedagogy for the purposes of moral education. Vardy (1998) responds in parallel fashion to the needs of students with a pedagogy based on his Five -Strand approach to religious and values education in Australia.

An understanding of thinking and thinking skills that includes applied thinking skills as a measure of learning is provided by Fisher (2003). He gave impetus to the actual teaching of thinking skills enriching and improving every area of the curriculum. In this approach, Fisher (2003) concentrates on philosophical inquiry in the classroom. He begins with the question: Why philosophy? He highlights the importance of the skill to think about one's thinking. He then leads teachers to the point of deciding what is to count as the best practice for teaching children to think.

With experience in New Zealand and Australian education, Clinton Golding (2006), one of the practitioner-experts who responded to my 
Questionnaire, has explored the meaning of fourteen of what are now familiarly called the rich concepts: belonging, change, identity, natural, ownership, life, choice, thinking, education, values, happiness, boredom, progress and proof. He shows how Lipman's Community of Inquiry may be applied in all values education.

Costa, addresses the connections and links between the five underlying passions: efficacy, flexibility, craftsmanship, consciousness and interdependence that drive effective thinking. Costa (2001) provides a new channel for the teaching of conceptual development, creative learning and problem-solving strategies. In the US at that time, much emphasis was being placed on forms of these that teachers could use.

Mews (2009) explores the possibilities of reason and fruitful communication for religions from a different perspective from that outlined above, by analysing 'Catholicism, Nostra Aetate and interreligious dialogue'. He provides groundwork for the exploration of the concepts of multiculturalism and interculturalism, without losing, in values education, the depth and content of any of the participating major religions of the world. Indications within his article attend to the considerations and epistemological concerns in my thesis.

In further analysis and attending to the emerging new paradigm I am exploring, Sarah Redshaw (1995) with her long history in Philosophy for Children (Heasly, 1995), provides an analysis designed to ask whether enough has been done to promote the various forms of thinking processes in tertiary education. The use of modern technology can be enhanced in the lecture theatre by the immediate deployment and teaching of applied thinking skills. Siva Krishnan (Krishnan, 2009) has also focussed on what I am calling the new paradigm for education and has proved this through his whole investigation!

Another leading exponent of Philosophy for Children, Splitter (1997) provides an analysis of exactly what is, in practice, the Community of Inquiry. Splitter (1997) gives a descriptive list for students of what counts as 
Community then identifies exactly what is meant by Inquiry: with a philosophical foundation underlying their pedagogical processes, teachers can assist students in their quest for knowledge by teaching them how to make progress in good judgments and to think efficiently

Connection between the Community of Inquiry and Educational Reform are set out by Pavel Lushyn \& David Kennedy (2000); they give points relevant to: study of a proposed text; the construction of an agenda; the solidifying of the learning community; the use of exercises and discussion plans; the encouragement of further responses. They connect their analysis directly to the theory and practice of Philosophy for Children (1984; 1991). By taking account of the focus, complexities and subtleties of a philosophical discussion, Tock Keng Lim (1998), analyses the format and effectiveness of the Philosophy for Children program. Linking this to Lipman (1993a), he pays specific attention to how teachers' understanding and assessment work, in order to identify when and how students go from ordinary thinking to critical thinking and philosophical reasoning. A connection to Redshaw (1995), Golding (2006), Fisher (2003) and Krishnan (2009) is implied. Like Redshaw, Krishnan has given fresh impetus and emphasis to the value and significance of collaborative styles of learning in a tertiary setting. Had the students in his investigation been more familiar with the format of the Community of Inquiry shaped through Lipman's paradigm, Krishnan concludes that they may have had deeper learning outcomes.

Barry Dwyer \& Graham English (1988b, 1988a) provide a teachers' manual and matching students' books for study by Australian Catholics in junior secondary classes. In the later spirit of Mackay $(1997,1999)$ these two companion volumes were an effort to provide for an emerging multicultural society. Ray H. Elliott (1986, 1988) provides a foundational pedagogy appropriate for religious education in Australia; it was followed, among many others, by Vardy (1998) and had echoes of Wilson's (1967) Moral Components List. Elliott addresses four aspects of the curriculum: the 
explicit, the implicit, the ritual and the classroom, connecting, I believe, with Schwab (1983) and his four commonplaces of the curriculum.

Similarly to Harrington (2005) and Moore (1986), Terence J. Lovat (1989) takes the microscope to 'this thing called Religious Education'. Lovat highlights some models of the teaching process meant to distinguish between two teaching models for religious education: a faith-forming model and an inter-faith model. The consequence of this process is the delivery of a further model Lovat calls the critical model of teaching. The following are implied in his later model: H. Gardner's (1993a) multiple-intelligences; Mews (2009) and his link between multiculturalism and interculturalism; and to Moore (Moore, 1986) and the six-strategy process of studying one's own cultural or religious foundation before addressing that of other cultures. It is only possible, now, to appreciate Lovat's integrated and critical pedagogy. To me, this resembles a version of Philosophy for Children - although neither Lovat nor Lipman, working quite separately and probably unknown to each other at the time, would have recognised such a correspondence. The teacherfacilitator in Lipman's Community of Inquiry has six separate management techniques which provide similar outcomes to those objectives stated by Lovat, in a similarly democratic classroom format, and both experts would have accepted these aspects of teaching efficiently.

Separately from the foregoing, Howard Gardner (1993a) identifies what he views as separate intelligences; de Bono (1996) constructs his thinking hats program; Costa (1992) focuses on developing a series of what he calls habits of mind; and Fogarty (1993, 2001a) provides strategies and techniques that successfully enhance how the brain works during learning.

Now, in 2011, we have five new sets of findings out of the knowledge of various parts of neuroscience: Greenfield, Lipton, Doidge, Jensen and Dispenza, all of which address new knowledge that, rapidly, has focused on the aim of improvement of learning for students. The implications for possible changes in teachers' pedagogical strategies and techniques uncovered are quite remarkable. The teaching of thinking in education now 
means refocussing on pedagogy: technical strategies and techniques (or, using Aristotle’s term: techné).

\section{Teachers' reflective practices}

Whereas Splitter addresses the disconnection between philosophers and teachers for Lipman's Philosophy for Children, Vardy addresses the disconnection between Theology and Philosophy for Years 11 and 12 study designs in Australia and New Zealand. In the latter case, Vardy and his team thoroughly explain the curriculum content in professional development seminars and published materials for participating teachers linked through the DialogueAustraliaNetwork (DAN).

Robert J. Marzano (2001) provides a model of how human thought can be facilitated towards efficient and higher order thinking: 'the decision the student makes and her subsequent actions will be determined by the interaction of herself, meta-cognitive and cognitive systems, as well as her knowledge'.

Co-operative learning is the basis of de Bono's Thinking Hats extending inspection in thinking, connecting to Lozano (2001, pp. 192-196) towards efficient learning. Lozano highlights the development of thinking styles: monarchic, hierarchic, oligarchic and anarchic. Edward de Bono (1996) provides a comprehensive overview of the thinking systems that he has devised and claims to have perfected. His short profile (pp. 9-15) of those he terms the 'gang of three': classical philosophers - Socrates, Plato and Aristotle - is central to these systems. Richard W. Paul (2001) outlines the specific styles and special aims of dialogical and dialectic thinking. The clear understanding to emerge was that many logic patterns exist, and that students need to recognise and use them efficiently for learning.

John Ralston Saul (2009) provides analysis of the observable eddies and currents including the reassessment of financial values and ethics, surrounding globalisation and globalism. These eddies have connection to Splitter \& Sharp (1995), to Fukuyama (1999), to Naomi Klein (2007) and 
these all have fed into the conceptual thinking of Mackay (1993), Giddens (1998), and Howe (2007). The Community of Inquiry is central to clarifying these eddies and currents for students and teachers alike.

Costa (2000, p. vii-xii) also provides staged classroom strategies teaching for efficient development of all the intelligences for a wide variety of subjects. Brenda Cherednichenko \& Susan Wilks (1997) provide analysis of the role of teacher as facilitator, provoker, modulator, monitor, and supporter. As Fisher (1990, 2003) indicates, the teacher is to be clear about how the strategies, built from Aristotle and Plato and by implication, from Socrates, enhance the use of specific pedagogical perspectives (Heasly, 1995).

Schwab (1983) has given a timely pause to educational planning by insisting that we must never forget that there are four commonplaces in the curriculum: student, teacher, subjects taught, and milieu; these must be given equal emphases in all planning and practice. I see a link between Schwab (1983) and Jenny Hill (2011) who provides an overall scenario which lists tools and programs for leadership in schools. Further implications emerge with Paul Thornton, Renata Phelps \& Anne Graham (2011), who detail the emerging new knowledge of teacher leadership with a view to improving educational practice.

\section{Ethics}

Kristjannsson (1998) believes that self-respect is widely and rightly considered an important value in moral education. He suggests Aristotle's analysis of his much-neglected crown of the virtues, megalopsychia, should be examined:

I recommend, in line with Lipman's (1991) Constructs, that for the purpose of raising or consolidating students self-respect, the classroom be converted into a community of inquiry in which students learn to listen to one another with respect, build on one another's Constructs, challenge one another to supply reasons for otherwise unsupported opinions, assist each 
other in drawing inferences from what has been said, and seek to identify one another's assumptions.

These are, in essence, ethical behaviours.

\section{Inserting the pedagogy of the Community of Inquiry}

In the tradition of Cherednichenko (2000), and later, of Wilks (2005) and Murris (2000), I found that Macqueen (2009), concentrates on the teachers' role.

In completing the analysis of the basic premises of the Philosophy for Children program, it is necessary to stop and discuss the role of the teacher. The role of the teacher in the Philosophy for Children classroom is unusual and quite complicated. As opposed to other subjects, the teacher here is neither an expert in philosophy nor a judge. The teacher is, before anything else, an organiser of philosophical discussions, which are not directed to the transfer of total knowledge from the field of philosophy.

In the tradition of Cherednichenko \& Wilks (1997) Bohm (1998) highlights one of the significant elements of Lipman's Community of Inquiry in the classroom. He draws our attention to a central feature of the Community of Inquiry and other similar strategies.

Another leading exponent in Philosophy for Children, Murris (2000) examines the sometimes controversial role of the facilitator in communities of philosophical inquiry. It had significant ethical consequences for how we evaluate Philosophy with Children. She argues that the philosophical dimension of an inquiry depended squarely on the facilitating skills and attitude of the teacher. There will always be a tension between the role of the teacher and the facilitator in Philosophy for Children. Today's practitioners must integrate both considerations and sets of skills into their pedagogy.

Susan Gardner (1996, p. 41) makes a very salient point, alerting new practitioners to Philosophy for Children: 
If a community of inquiry is to be successful both in its main goal of moving toward truth and its side goals of enhancing good thinking and developing good character, it will require the firm guidance of an ever vigilant facilitator who maintains direction and forces depth with respect to the philosophical truth toward which the inquiry points. We will do the novice no small favour by letting them know at the outset that inquiry is not mere conversation and that facilitation of inquiry is hard work.

This is a clear and ethical educational aim.

Separately, Jim Burdett (1999, p. 6), working in a sensitive area, has focused on two significant factors: the malignant environment factors (MEF) that exacerbate vulnerable personal factors (VPF) to push unprecedented numbers of youth to the desperate act of suicide. Burdett maintains that Philosophy for Children might also offer a solution to this problem of MEF:

If we take another look at Splitter \& Sharp's list of thinking skills we discover processes that are particularly appropriate for looking critically at social and political issues.

He explicitly links his whole analysis to Splitter \& Sharp (1995).

As an example of the Community of Inquiry, Freakley \& Burgh (2000) have voiced an area of curriculum often lost in the over-crowdedness and expectations of State and regional curricula content. Using the ethical pedagogical methods laid down originally by Lipman (1969), the authors have supported teacher-training in ethics for teachers, current ethical dilemmas, values and the curriculum, education and democracy, difference and pluralism, and reward and punishment. These considerations provide significant direction for teachers’ professional development.

Hawkes (2010) has written of his experience on whether teaching values in education within primary schools does in fact provide a deeper learning experience and quality of education for primary pupils. His work has, in my estimation, connections into the extensive research of Lovat (1989) from Newcastle University, Australia. Hawkes (2010, p. 271) established an 
emphasis and focus on Values, and the implementation within the school in which he was Head Teacher. Hawkes concludes that:

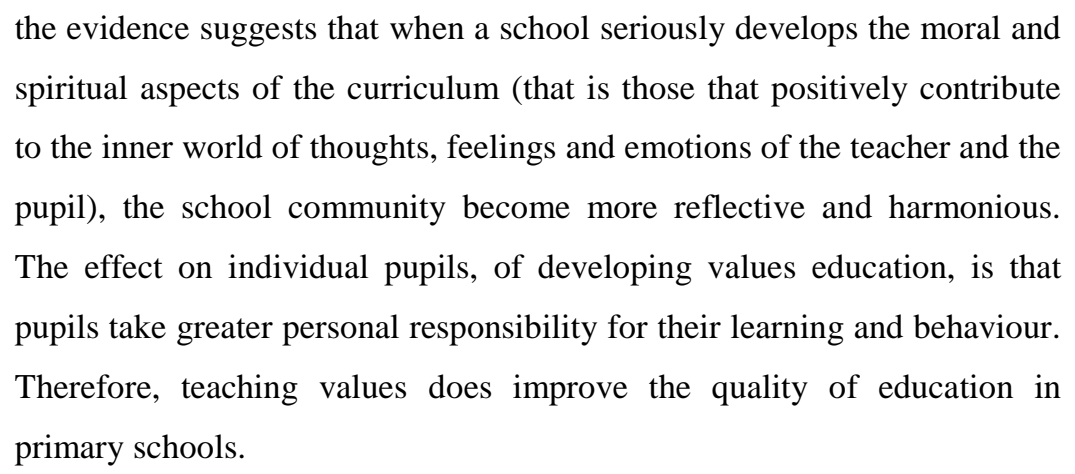

Crowther (2010a) describe the theory of distributed leadership as a firm foundation for the aim of sustainable and capacity-building for a school, detailing along the way one form of leadership, which he calls 'parallel leadership'. He provides steps towards achieving this form of school reform, by the use of milestones in the journey of change. Marion Lewis (2010) extends these considerations in her editorial of the same journal.

Providing a study which signals the need for open communication, consultation, top-down and bottom-up decision-making processes, Chittenden (2004) advocates for effective ethical leadership.

\section{Ethnographical Connections -broadening depth of My Voice}

Having realised the value of an ethnographical research approach to this investigation quite late in the development of the thesis content, I have now recognised the value of personal narrative in the teaching of thinking skills in my career. This recognition led me to the work of Wolff-Michael Roth (2005) with its wide-ranging development of what is to count for autoethnographical studies within qualitative research. The blending of my experiential learning over 36 years of continuous involvement in seminars, staff days, local conferences and overseas conferences, and my participation in movements such as the Association of Moral Education and the Philosophy for Children, both in Australia and overseas, led me to find the 
connections, links, gaps and questions for my personal learning. Integrating these matters from this rich source of learning with formal qualifications has meant deeper learning. Application of Roth's concepts, constructs, thematic developments and new knowledge, has led me to Mary Beattie (2009) and the further development of how central is the quest for meaning in the learning arc of each individual student. The link to the development of thinking skills for students' careers, their construction of the good life, and their quest for meaning from all sources, is inherent in Beattie’s work.

Finally, the extra-ordinary breadth of the fourth compendium of Denzin \& Lincoln (2011) gives overviews of the kinds and processes of each form of qualitative research, and provides a sustainable understanding of the significance of understanding of the concepts and overlaps inherent in instituting the processes needed for successful and balanced qualitative research.

\section{Looking forward...}

Having established the questions, the methods of analysis (including the Questionnaire for teachers), the informing literature and the autoethnographical retrospective, it is now time to analyse the rich content of teachers' voices, in conjunction with the other 'voices'. In Chapter 4, the auto-ethnographical narrative that introduces my voice is included. As a consequence the teachers' voices, the experts' voices and both my researcher and my practitioner voices are heard. 


\section{CHAPTER 4}

\section{Practitioner Voices: My Voice, Teachers' Voices, and Experts' Voices}

\section{Introduction}

In this chapter, I document in auto-ethnographical format, an inductive data reduction chart of my own learning resulting from my classroom experience, from integration of content of seminars, conferences and short courses, and from my formal tertiary studies.

Having completed my examination of the 'voices' in the Literature, I now turn to examine the practitioner 'voices'. In this chapter, I systematically examine the connections possible from three sources:

- my own experience over 36 years of teaching;

- results and understandings from teachers' responses from the Questionnaire;

- integration of relevant content from the expanse of the Literature Search.

The findings from my Questionnaire as completed by thirty teachers and three practitioner experts, together with my reflections, both as a practitioner and as researcher, are reported here. The findings are manifested in the four 'voices' that emerge as a consequence of analysis.

The 33 respondents, which include the three 'experts', to the Questionnaire came from three countries: Australia, New Zealand and the United Kingdom. My analysis and evaluation of their responses has uncovered ten teacher approaches. I used inductive processes to analyse and 
evaluate my own profile as a practitioner from ontological and epistemological, and from the technical perspectives and deductive processes, in order to identify unanswered questions.

Nine independent themes emerged from the Questionnaire. As a consequence, this Chapter is divided into nine sections:

1. Questions and questioning.

2. Curriculum frameworks.

3. Discussion.

4. Focussing on students.

5. Sequential processes.

6. Thinking skills.

7. Teaching the process of decision-making.

8. Consensus and democracy

9. Teaching of and about virtues, values and ethics

Each of these nine sections is treated in the following way: first, there is a consideration of my practitioner voice; second, there is a consideration of the teachers' voices - derived from an inductive data analysis of the Questionnaire responses; third comes a consideration of the experts' voices derived from Questionnaire responses, if a response was received; fourth, there is consideration of my researcher voice - consisting of two elements (where identified): one derived from my analysis of the literature and the previous four findings, and the other derived from my judgement of hesitations, confusions, concerns and silences on the part of the respondents.

I have also used a triad of terms which are central to understanding the founding of this investigation. The terms are:

\section{Idea (Comment) $\longrightarrow$ Concept $\longrightarrow$ Construct}

These terms mirror the ontological, epistemological and pedagogical schema that appears in Chapter 3. The individual discovers a new idea about something inspirational (ontological thinking) and pursues the idea which 
will translate into a concept (epistemological thinking), which thus allows for new knowledge, and proceeds to test, improve and validate a new construct in their daily milieu (pedagogical thinking) in order to teach the new knowledge, or at least to announce, broadcast and promote the new knowledge and its application. It is important then to note that as each idea becomes more substantial, new themes emerge and blossom into new knowledge, so a new construct has been born. This is creative inductive thinking at its best.

\section{Questions and questioning}

Three voices only are heard within this theme: my practitioner voice, the teachers' voices, and my researcher voice.

\section{My practitioner voice}

Many of the classroom strategies I practised were based on my approach to the use of, and the role of questions, on the sharing of questioning, on the format of the Community of Inquiry and on the inevitable discussions (which often did not finish at the end of the teaching period). On occasion, if the subject matter was particularly contentious, students could be heard by others present, deep in further conversation in the Library or the school recreation areas and I found this a further step in the process of growing confidence in their use of thinking skills. Valuable understandings for students came from the early part of the Community of Inquiry format, in which students composed and constructed questions before research and discussion could take place. This experience taught me to make more efficient use of targeted questions to aid deeper learning.

Reflecting on my practice I realised that the better the art of questions and questioning exercised, the better the responses from students. This was a significant realisation. As a kid I knew there was a simple list for asking 
FIGURE 4.1 THE CONSTRUCTION OF STUDENTS' QUESTIONS FOR ENHANCED LEARNING

\begin{tabular}{|l|c|c|}
\hline \multicolumn{1}{|c|}{$\begin{array}{l}\text { QUESTION } \\
\text { STARTERS }\end{array}$} & CLOSED & OPEN \\
\hline How? & & $\checkmark$ \\
\hline Where? & $\checkmark$ & \\
\hline When? & $\checkmark$ & \\
\hline What? & $\checkmark$ & \\
\hline Which? & $\checkmark$ & \\
\hline Who? & $\checkmark$ & $\checkmark$ \\
\hline Why? & & $\checkmark$ \\
\hline What if? & & \\
\hline
\end{tabular}

questions: How? Where? When? What? Which? Who? Why? Without the support of the list that was familiar to me from childhood, I knew that teaching how to construct questions would enjoy limited success (see Figure 4.1). It was important to walk the students through each step, acknowledging closed and open questions, and dismissing no question till it was tested because often we found that what seemed to be a silly question would lead to something more significant. Important too was the additional conditional question: what if?

We chose which question starters were factual (closed) and which encouraged further exploration (open). This made sense when the students and I decided to link these questions to the concept of analysis. Students accepted that there was a reason for the exercise and began to discover its impact on their information retrieval, their knowledge levels, and, of course, on their improved assessment. The students' evaluation sessions always seemed to indicate that the system I used helped produce the final result.

I could see that the art of questioning was an intricate skill, and the variety of questions that could be asked and investigated were numerous. Its exercise gave me the opportunity to teach the difference between deductive and inductive reasoning. It also resulted in a teacher-driven classroom 
activity that students anticipated with enthusiasm: to build questions about the current subject as a classroom activity, from which the students could select those questions they deemed to be worth pursuing. What started as my burgeoning efforts to be a good teacher emerged in the Lipman mould became the catalyst for a serious development of how to teach the art of questioning by systematic use of question starters. The immediate value of teaching and modelling evaluative and clear thinking processes was startling, in that Year 12 students returned to my class to consult and to check the processes because they wanted to use this activity in other classes and other milieux.

The initial ontological approaches that shaped the teaching-learning process were accompanied by emerging teacher-knowledge of an epistemological nature leading to the development of skills which were technically accurate in aim, to provide for classroom success (technical techné), essentially changing perceptions of pedagogical purpose and delivery.

In items 11, 12 and 13 of my Questionnaire, teachers were asked about:

- How frequently they raise questions in their classrooms

- How frequently they ask students for their questions

- The timing of questions

- The different kinds of questions they then asked.

Extending this construct, they were asked whether these questions happened at the beginning of the lesson, end of the lesson, regularly or randomly during the lesson.

In the Questionnaire, participants were asked a number of questions relating to how and when they used questions in their teaching process. I used as many of questions listed and explicated by Splitter \& Sharp (1995) to focus the students on thinking skills, as I taught the various curricula. 


\section{Teachers' voices}

Teachers use questions for a variety of reasons, and in a variety of ways in order to facilitate the process of learning and understanding.

\section{Teachers' questions}

All respondents surveyed responded to the item regarding the relative frequency with which they asked questions of students. Over half (18/33) indicated that they often asked questions and just under half of the teachers $(14 / 33)$ indicated that they asked questions rarely.

\section{Timing and frequency of teachers' questions}

More than half the teachers used questions continuously at various times during the topic or the class. Two respondents indicated that they used questions irregularly throughout a lesson - at the beginning, at the end or randomly. Just under half of the teachers (15/33) reported asking questions throughout the topic or including them at the beginning class, at the end or randomly, given that a topic can range over a number of classes and that they take place in different venues. These data are reported in Table 4.1.

\section{TABLE 4.1 FREQUENCY OF TEACHERS' QUESTIONS}

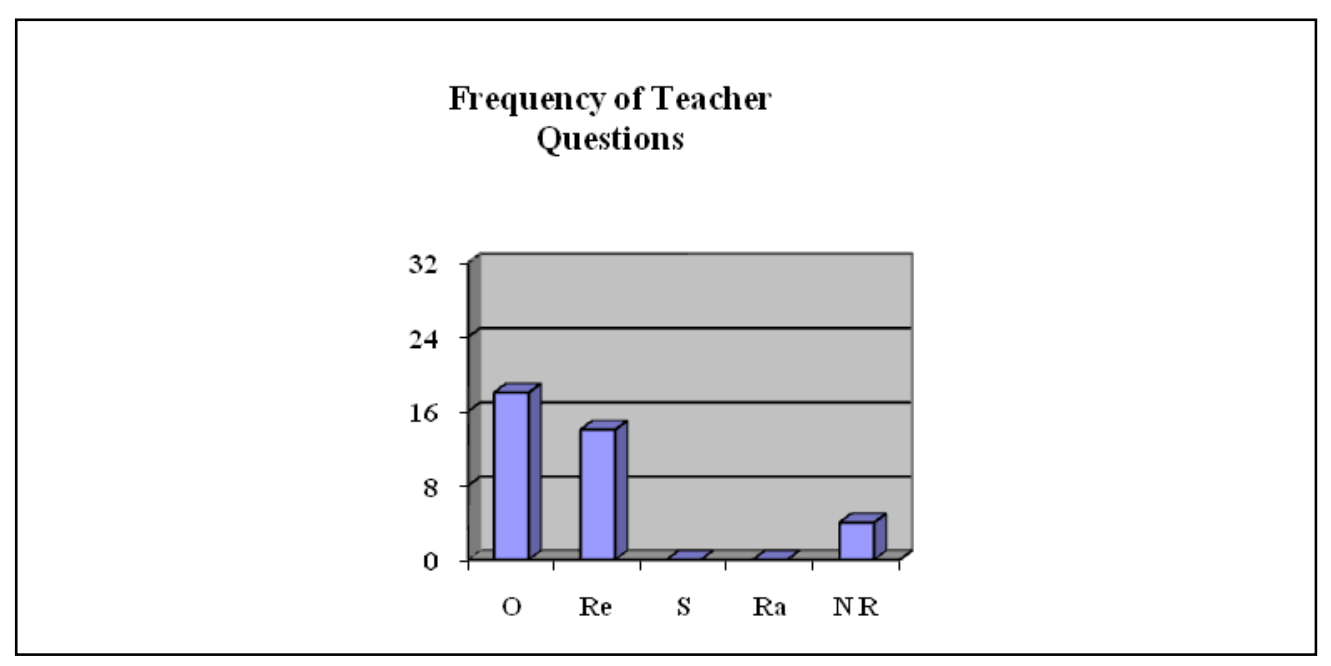

$(\mathrm{O}=$ Often; $\mathrm{Re}=$ Regularly; $\mathrm{S}=$ Start of lesson; Ra = Rarely; $\mathrm{NR}=$ No response $)$ 


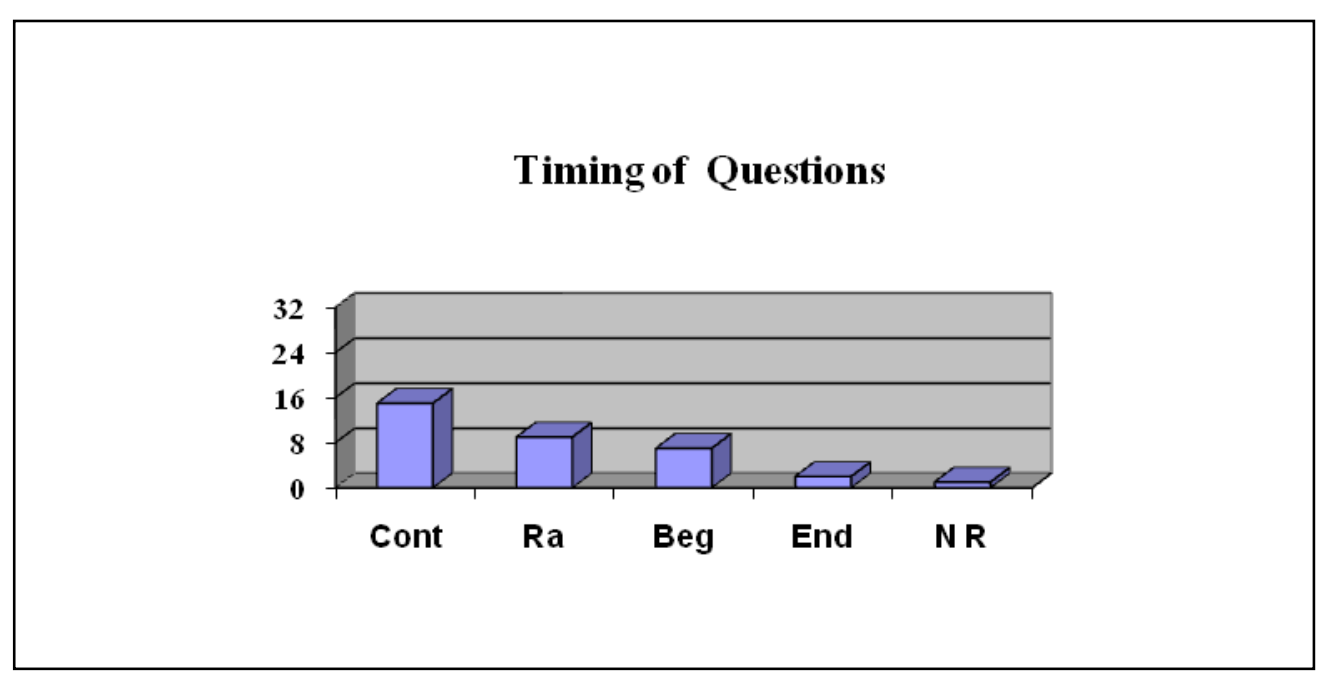

(Cont = continually; Ra = rarely; Beg = beginning of class; End = End of class; $\mathrm{N}$ R = no response $)$

Significantly, just over half of all of the teachers (18/33) indicated that questions were used at a particular time of the session or topic. Half (9/18) of these indicated that they raised questions randomly; just under a half (7/18) have specified that they asked questions at the beginning of the topic or class, while the remaining two teachers indicated that they used questions solely at the end of the class. These data are reported in Table 4.2.

In their responses, the teachers indicated that they used questions for a variety of reasons. While teachers had different perceptions about asking students for their own particular questions, in all of the extended responses the theme that emerged was that teachers were concerned with the studentcentred learning. The majority favoured a timing of questions that stimulated student thinking. Their comments were exact; the ontological issue was present; the concept was adhered to; and the pedagogical approach that was indicated was student-centred. Teachers took an approach to learning, which stimulated retention and recall. These responses are reported in Table 4.3; all names are pseudonyms; all comments are direct quotes. 
TABLE 4.3 TIMING OF QUESTIONS

\begin{tabular}{|c|c|c|c|}
\hline COMMENT & CONSTRUCT & CONCEPT & THEME \\
\hline $\begin{array}{l}\text { Connie and Georgia used questions randomly } \\
\text { during class for retention and recall so that } \\
\text { students constructed their knowledge during the } \\
\text { discussions. }\end{array}$ & $\begin{array}{l}\text { Stimulate retention and } \\
\text { recall in a Constructivist } \\
\text { setting. }\end{array}$ & $\begin{array}{l}\text { Constructivist } \\
\text { approach }\end{array}$ & \multirow{8}{*}{$\begin{array}{l}\text { Student- } \\
\text { centred } \\
\text { learning }\end{array}$} \\
\hline $\begin{array}{l}\text { Ben chose to empower students with a beginning } \\
\text { question like "What do you think?" }\end{array}$ & $\begin{array}{l}\text { A focus question that } \\
\text { empowers students }\end{array}$ & \multirow{2}{*}{$\begin{array}{l}\text { Focus on } \\
\text { students } \\
\text { interests }\end{array}$} & \\
\hline Dennis asked questions for focus. & $\begin{array}{l}\text { Questions that provide a } \\
\text { lesson focus. }\end{array}$ & & \\
\hline $\begin{array}{l}\text { Questions for specific reasons were used to } \\
\text { stimulate thinking and focus on retention and } \\
\text { recall. Magda, for example, would put a question } \\
\text { on the board to begin the class. }\end{array}$ & $\begin{array}{l}\text { Focus question to } \\
\text { stimulate thinking; } \\
\text { focus on retention and } \\
\text { recall. }\end{array}$ & $\begin{array}{l}\text { Question as } \\
\text { advance } \\
\text { organiser }\end{array}$ & \\
\hline $\begin{array}{l}\text { Olag, a Music teacher, asked students a broad } \\
\text { question at the start of a lesson - How would you } \\
\text { organise a band? - and then kept asking questions } \\
\text { about areas they needed to think through during } \\
\text { the class. }\end{array}$ & $\begin{array}{l}\text { A broad question at the } \\
\text { start with follow-up } \\
\text { questions requiring } \\
\text { student input }\end{array}$ & \multirow{4}{*}{$\begin{array}{l}\text { Stimulating } \\
\text { student } \\
\text { thinking }\end{array}$} & \\
\hline $\begin{array}{l}\text { Portia said that she used questions to arouse } \\
\text { curiosity and then aimed for a discussion which } \\
\text { was student-directed. }\end{array}$ & $\begin{array}{l}\text { Arousing curiosity } \\
\text { leading to a student- } \\
\text { directed discussion. }\end{array}$ & & \\
\hline $\begin{array}{l}\text { Franco also kept asking questions while he was } \\
\text { looking for students' responses and a flow of } \\
\text { Constructs. }\end{array}$ & $\begin{array}{l}\text { Asking questions that } \\
\text { stimulate and encourage } \\
\text { Constructs. }\end{array}$ & & \\
\hline $\begin{array}{l}\text { Participants indicated that questions were random } \\
\text { at times to stimulate new thinking, like Michael's } \\
\text { indication of his leading questions. }\end{array}$ & $\begin{array}{l}\text { Random questions used } \\
\text { to stimulate thinking. }\end{array}$ & & \\
\hline
\end{tabular}

\section{Asking students for questions}

The following graph shows that of the 28 teachers who responded to the above Item, all reported asking for questions frequently. Just under half (13/28) indicated that they asked questions often, while just over half (15/28) indicated that they ask students for questions regularly. It was not possible to conclude from their responses what were practices of the remaining four teachers in regard to asking students for questions. These details are presented in Table 4.4.

\section{Types of teachers' questions to students}

Teachers had different perceptions about asking students for questions. Questions by teachers of the students are one type of question: asking students for their questions completes the cycle of teachers' questions. Each 
TABLE 4.4 FREQUENCY OF STUDENT QUESTIONS

\section{Frequency of Student Questions}

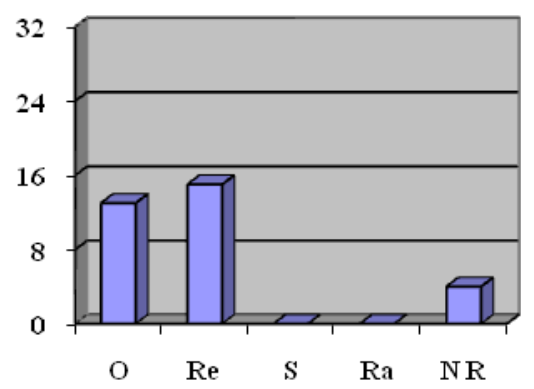

(O = Often; Re = regularly; $\mathrm{S}$ = Start of lesson; Ra = Rarely; $\mathrm{N}$ R = No response)

is important for facilitating inquiry. Four representative responses are contained in Table 4.5. These responses suggest that there is a dichotomy between questions that are student-centred and those that are teacherdirected. For the former, the teachers actively seek student support; for the latter, teachers use questions to stimulate discussion.

TABLE 4.5 TEACHERS' PERCEPTIONS OF THEIR QUESTIONS

\begin{tabular}{|c|c|c|c|}
\hline COMMENT & CONSTRUCT & CONCEPT & THEME \\
\hline $\begin{array}{l}\text { Terence: I always fish for more with open } \\
\text { questions. }\end{array}$ & $\begin{array}{l}\text { Using open-ended } \\
\text { questions }\end{array}$ & \multirow[b]{2}{*}{$\begin{array}{l}\text { Seeks student } \\
\text { input }\end{array}$} & \multirow[b]{2}{*}{$\begin{array}{l}\text { Student- } \\
\text { centred }\end{array}$} \\
\hline $\begin{array}{l}\text { Jane: found her place in amongst the exchanges } \\
\text { between students: When a student raises an } \\
\text { issue/question I let other students } \\
\text { answer/contribute and then go from there. }\end{array}$ & $\begin{array}{l}\text { Focus on exchanges } \\
\text { between students }\end{array}$ & & \\
\hline $\begin{array}{l}\text { Giovanni: Uses a cyclical questioning strategy } \\
\text { where he raises an issue/ theme/ topic and then it } \\
\text { is discussed ...I ask questions, they ask questions } \\
\text { throughout the discussion. }\end{array}$ & $\begin{array}{l}\text { Raises issue, themes } \\
\text { and topics for } \\
\text { discussion }\end{array}$ & \multirow{2}{*}{$\begin{array}{l}\text { Uses teachers } \\
\text { questions to } \\
\text { stimulate } \\
\text { discussion }\end{array}$} & \multirow{2}{*}{$\begin{array}{l}\text { Teacher- } \\
\text { directed }\end{array}$} \\
\hline $\begin{array}{l}\text { Jane: [I] raise a quote, a fact, an object, an } \\
\text { example from the text or experiment (science- } \\
\text { based) and sees how students respond. }\end{array}$ & $\begin{array}{l}\text { Raises a quote, fact, } \\
\text { object or example } \\
\text { and explores } \\
\text { students responses }\end{array}$ & & \\
\hline
\end{tabular}


TABLE 4.6

VARIETIES IN TYPES OF TEACHERS' QUESTIONS

\begin{tabular}{|c|c|}
\hline EXAMPLE & TYPE OF QUESTION \\
\hline $\begin{array}{l}\text { Paul: I try to find a provocative question - one to which there is no } \\
\text { obvious answer. }\end{array}$ & Provocative \\
\hline $\begin{array}{l}\text { Giovanni: I raise an issue/theme/topic and we discuss it. I ask } \\
\text { questions/ they ask questions throughout the discussion. }\end{array}$ & Basic \\
\hline $\begin{array}{l}\text { Jane: In Science, thinking skills are to a limited extent taken into } \\
\text { account in the brainstorming for an experiment. Students are actively } \\
\text { encouraged to ask how something might happen and why. Some } \\
\text { problem-solving is generally involved. In English, thinking skills are } \\
\text { used in student analysis of media articles and in criticism and } \\
\text { discussion of text. I encourage thinking skills by asking students to } \\
\text { generate questions they might like to ask a character/journalist/author } \\
\text { and why they would like to ask that question. I then encourage them to } \\
\text { think about how they believe such people would respond. }\end{array}$ & Open \\
\hline $\begin{array}{l}\text { Susan: Often by saying we all have different solutions and some may } \\
\text { work better than others - depends on the situation; e.g.: should a clock } \\
\text { face have numbers on it? Is logging in native forests ethical or not? } \\
\text { Can be seen from an environmental, economic or social point of view } \\
\text { - different people, different solutions or Constructs - does not mean } \\
\text { any are wrong or right. }\end{array}$ & Construct-based \\
\hline $\begin{array}{l}\text { Samantha: Pre-unit testing e.g., in Legal Studies; Human Rights; Post } \\
\text { self-evaluation and Course Evaluation. Use empathy and de Bono's } \\
\text { Thinking Hats to help students move perhaps from seeing no problem } \\
\text { to showing compassion and value for others. This approach is } \\
\text { especially useful in English, Legal Studies, Religious Studies and even } \\
\text { some Commerce units such as Civics and Citizenship. }\end{array}$ & Philosophical \\
\hline $\begin{array}{l}\text { George: I challenge all students to think - I don't select a group! I ask } \\
\text { questions/give information/tell stories/give counter arguments/I } \\
\text { monitor interest - then modify my approach. }\end{array}$ & Planning \\
\hline
\end{tabular}

\section{Kinds of teachers' questions}

The responses in the Questionnaire indicated that teachers used different kinds of questions. Table 4.6 contains some of the different types of questions.

\section{Questions and questioning themes}

From the responses that teachers gave to the specific item regarding the use they made of questioning, four themes dealing with questions and questioning emerged, three of which were student-centred - student-centred thinking, student-centred issues, and stimulating student thought; the fourth related to a constructivist approach by the teacher to student learning. These responses are summarised in Table 4.7. 
TABLE 4.7 QUESTIONS AND QUESTIONING THEMES

\begin{tabular}{|c|c|c|c|}
\hline COMMENT & CONSTRUCT & CONCEPT & THEME \\
\hline $\begin{array}{l}\text { Terence is always fishing for more with open } \\
\text { questions. }\end{array}$ & Fishing for more & $\begin{array}{c}\text { Open } \\
\text { questions }\end{array}$ & \multirow{4}{*}{$\begin{array}{l}\text { Student- } \\
\text { centred } \\
\text { thinking }\end{array}$} \\
\hline $\begin{array}{l}\text { Olag, a music teacher, asked students a broad } \\
\text { question at the start of a lesson: How would you } \\
\text { organise a band? And then kept asking questions } \\
\text { about areas they need to think through. }\end{array}$ & Broad question & \multirow{3}{*}{$\begin{array}{l}\text { Stimulating } \\
\text { interest }\end{array}$} & \\
\hline $\begin{array}{l}\text { Olag kept asking questions about areas they need } \\
\text { to think through. }\end{array}$ & $\begin{array}{l}\text { Areas to think } \\
\text { through }\end{array}$ & & \\
\hline $\begin{array}{l}\text { Franco also kept asking questions while he was } \\
\text { looking for children's response and a flow of } \\
\text { Constructs. }\end{array}$ & Flow of Constructs & & \\
\hline $\begin{array}{l}\text { Franco also kept asking questions while he was } \\
\text { looking for children's response and a flow of } \\
\text { ideas }\end{array}$ & $\begin{array}{l}\text { Initiating } \\
\text { children's } \\
\text { responses }\end{array}$ & \multirow{2}{*}{$\begin{array}{l}\text { Student- } \\
\text { directed } \\
\text { discussion }\end{array}$} & \multirow{2}{*}{$\begin{array}{l}\text { Student- } \\
\text { centred issues }\end{array}$} \\
\hline $\begin{array}{l}\text { Portia said that she used questions to arouse } \\
\text { curiosity and then aimed for a discussion that was } \\
\text { student directed. }\end{array}$ & To arouse curiosity & & \\
\hline $\begin{array}{l}\text { Magda: questions for specific reasons were used } \\
\text { to stimulate thinking and focus on retention and } \\
\text { recall. }\end{array}$ & $\begin{array}{l}\text { Focus on retention } \\
\text { and recall }\end{array}$ & \multirow{3}{*}{$\begin{array}{l}\text { Teacher } \\
\text { directing }\end{array}$} & \multirow{4}{*}{$\begin{array}{l}\text { Stimulating } \\
\text { student } \\
\text { thought }\end{array}$} \\
\hline $\begin{array}{l}\text { Jane: raises a quote, a fact, an object, an example } \\
\text { from the text or experiment (science-based) and } \\
\text { see how students respond. }\end{array}$ & $\begin{array}{l}\text { I use [content] and } \\
\text { see how students } \\
\text { respond }\end{array}$ & & \\
\hline $\begin{array}{l}\text { Magda would put a question on the board to } \\
\text { begin the class. }\end{array}$ & Stimulates thinking & & \\
\hline $\begin{array}{l}\text { Michael indicated that questions were random at } \\
\text { times to stimulate new thinking, like Michael's } \\
\text { indication of his leading questions. }\end{array}$ & $\begin{array}{l}\text { Stimulates new } \\
\text { thinking }\end{array}$ & $\begin{array}{l}\text { Teacher- } \\
\text { directed } \\
\text { leading } \\
\text { questions }\end{array}$ & \\
\hline $\begin{array}{l}\text { Giovanni used a cyclical questioning strategy } \\
\text { where he raised an issue/ theme/ topic and then we } \\
\text { discuss it ...I ask questions, they ask questions } \\
\text { throughout the discussion. }\end{array}$ & $\begin{array}{l}\text { I ask questions, } \\
\text { they ask questions }\end{array}$ & $\begin{array}{l}\text { Cyclical } \\
\text { questioning } \\
\text { strategy }\end{array}$ & \multirow{3}{*}{$\begin{array}{l}\text { Constructivist } \\
\text { approach by } \\
\text { teacher }\end{array}$} \\
\hline $\begin{array}{l}\text { Connie and Georgia used questions randomly } \\
\text { during class for retention and recall so that } \\
\text { students constructed their knowledge during the } \\
\text { discussions. }\end{array}$ & $\begin{array}{l}\text { Used questions } \\
\text { randomly }\end{array}$ & $\begin{array}{l}\text { Stimulating } \\
\text { student } \\
\text { learning }\end{array}$ & \\
\hline $\begin{array}{l}\text { Ben chose to empower students with a beginning } \\
\text { question like what do you think? }\end{array}$ & $\begin{array}{l}\text { What do you } \\
\text { think? }\end{array}$ & $\begin{array}{c}\text { Student } \\
\text { empowerment }\end{array}$ & \\
\hline $\begin{array}{l}\text { Jane found her place in amongst the exchanges } \\
\text { between students: When a student raises an issue/ } \\
\text { question I let other students answer/ contribute } \\
\text { and then go from there }\end{array}$ & $\begin{array}{l}\text { Uses exchanges } \\
\text { between students }\end{array}$ & $\begin{array}{l}\text { Student } \\
\text { empowerment }\end{array}$ & \multirow{2}{*}{$\begin{array}{l}\text { Constructivist } \\
\text { approach by } \\
\text { teacher }\end{array}$} \\
\hline Dennis asked questions for focus, & Focussing issues & $\begin{array}{l}\text { Teacher } \\
\text { initiating } \\
\text { questions }\end{array}$ & \\
\hline
\end{tabular}

It was clear to me that the teachers took their questioning technique very seriously; there was a mix of student and teacher focus within the four themes. Open questions were used to stimulate interest and focus student 
learning, to orient student-directed discussion, and to empower students. To achieve this, teachers focussed on providing direction to discussions by means of leading questions that stimulated thinking, focussed on retention and recall, aroused student curiosity and interest, and encouraged a flow of Constructs. These findings are supported by the teachers' perceptions of their questions reported in Table 4.5 .

\section{My researcher's voice}

Splitter \& Sharp (1995, pp. 117, 128- 129) delineate three sets of cognitive actions to raise awareness of how thinking skills may broaden the discussion during classroom reflections. The questions used in the techniques I have described above aligned with the first set: Reasoning and Inquiry; the instruments that I used in classroom discussions and which were allied to Wilson and Lipman, answered their second set: Concept Formation; and the instrumental list is applied to the third heading: Meaning Making. Under the heading Reasoning and Inquiry, there is a formidable list of thinking skills that beginning and continuing teachers could safely use as a testing instrument to enhance meaning making.

\section{Curriculum frameworks}

Within this theme, two voices only are heard: the teachers' voices, and my researcher voice.

\section{Teachers' voices}

In answer to the eighth Item of the Questionnaire, which focuses on professional knowledge and practice, teachers were asked: Are there particular curriculum frameworks that you use for the teaching of thinking skills? If so, please indicate what these are. 


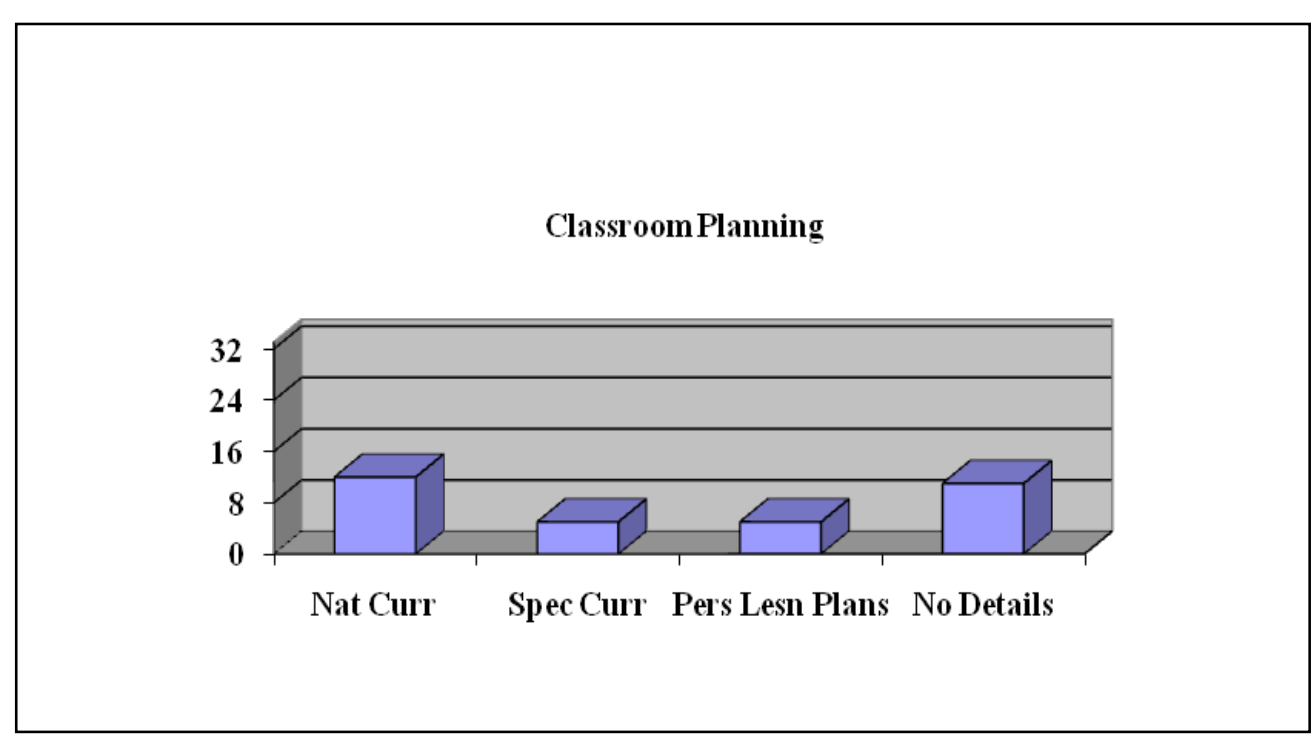

(Nat Curr = National Curriculum documents; Spec Curr - Special curriculum documents; Pers Lesn Plans = Personal Lesson Plans; No Details = no response)

The responses underlined the different ways of employing the term curriculum frameworks. Some teachers planned, using the frameworks provided in relevant Government documents: others used specific curriculum frameworks; some used a personal framework for lesson planning, while a fourth group did not give any detail about specific frameworks, but indicated that they used a topic planning approach.

\section{Classroom planning}

The respondents indicated variations in their use of curriculum documentation for their classroom planning. These variations are shown in Table 4.8.

Twelve teachers (12/33) stated that they used formal national or regional curriculum documents to plan their lessons. The government documents mentioned by teachers, by country, are summarised in Table 4.9. 
TABLE 4.9 GOVERNMENT DOCUMENTS EMPLOYED

\begin{tabular}{|c|c|}
\hline COUNTRY & EXAMPLES \\
\hline Australia (Victoria) & $\begin{array}{l}\text { In Australia (Victoria), teachers mentioned: } \\
\text { - Curriculum Standards Frameworks (CSF) } 2 \\
\text { - Victorian Certificate of Education Study Designs } \\
\text { - Language other than English LOTE documents } \\
\text { - Victorian Essential Learning Standards. }\end{array}$ \\
\hline England & $\begin{array}{l}\text { In England, teachers mentioned: } \\
\text { • The England and Wales National Curriculum. }\end{array}$ \\
\hline New Zealand & $\begin{array}{l}\text { In New Zealand, teachers mentioned: } \\
\text { - English Curriculum document } \\
\text { - NZ Performing Arts document } \\
\text { - } \quad \text { NCEA Levels } 1,2 \text { and } 3 \\
\text { - International Baccalaureate Theory of Knowledge in } \\
\quad \text { NZ. }\end{array}$ \\
\hline
\end{tabular}

\section{Teachers' range of strategies for documentation used}

Teachers were not specific about the strategies that they associated with each of these documents. Five (5/33) stated that they used specific published curriculum frameworks from which they constructed sequences to incorporate in their lessons. Four of these five teachers indicated a familiarity with Lipman's Philosophy for Children framework and one teacher used both Lipman's framework and Vardy's 5-Strand (RAVE) curriculum framework.

Two major themes emerged from the responses of the teachers and experts, who all completed the questionnaire. The first theme relates to the use of a variety of schemas (see Table 4.10).

TABLE 4.10 CLASSROOM PLANNING AND SUGGESTED SCHEMAS

\begin{tabular}{|c|c|c|c|}
\hline COMMENT & CONSTRUCT & CONCEPT & THEME \\
\hline $\begin{array}{l}\text { Magda, UK: I follow the QCA Scheme of work } \\
\text { guidelines and use the Badger Music as my main } \\
\text { resource. I don't use any particular thinking } \\
\text { framework. }\end{array}$ & $\begin{array}{l}\text { No particular } \\
\text { thinking framework }\end{array}$ & $\begin{array}{l}\text { Badger } \\
\text { Music main } \\
\text { resource }\end{array}$ & $\begin{array}{l}\text { QCA } \\
\text { scheme of } \\
\text { work } \\
\text { guidelines }\end{array}$ \\
\hline $\begin{array}{l}\text { Susan, in Art, Craft and Design: } \\
\text { Some think after research-reading, others after } \\
\text { seeing pictures, others after drawing, some after } \\
\text { discussions and others by actually making and } \\
\text { modifying. }\end{array}$ & $\begin{array}{l}\text { Different } \\
\text { approaches to } \\
\text { reflection }\end{array}$ & \multirow{2}{*}{$\begin{array}{l}\text { Accommo- } \\
\text { dating } \\
\text { difference }\end{array}$} & \multirow{2}{*}{$\begin{array}{c}\text { Applying } \\
\text { Gardner's } \\
\text { Multiple } \\
\text { Intelligences }\end{array}$} \\
\hline $\begin{array}{l}\text { Pietr, PE: Pre and post-testing; questions; VAK } \\
\text { (Visual/ Auditory/ Kinaesthetic) dominance testing } \\
\text { and CONSTRUCTS kite }\end{array}$ & $\begin{array}{l}\text { Measuring growth } \\
\text { in learning and } \\
\text { thinking }\end{array}$ & & \\
\hline
\end{tabular}




\begin{tabular}{|c|c|c|c|}
\hline COMMENT & CONSTRUCT & CONCEPT & THEME \\
\hline $\begin{array}{l}\text { Giovanni and Rose, English and Social Studies: } \\
\text { Giovanni and Rose used role play and drama. }\end{array}$ & $\begin{array}{l}\text { Use of role play and } \\
\text { drama }\end{array}$ & & \\
\hline $\begin{array}{l}\text { Samantha, Humanities/Legal Studies: } \\
\text { Use empathy. }\end{array}$ & Empathy & Attitude & \multirow[b]{2}{*}{$\begin{array}{l}\text { Bloom's } \\
\text { Domains }\end{array}$} \\
\hline $\begin{array}{l}\text { Paul, English and Social Studies: } \\
\text { Paul commented: Dispassionate and intellectual } \\
\text { skills are essential, but students must be able to } \\
\text { identify other responses e.g., emotional, subjective. }\end{array}$ & $\begin{array}{l}\text { Objective and } \\
\text { subjective skills }\end{array}$ & $\begin{array}{l}\text { Knowledge } \\
\text { and attitudes }\end{array}$ & \\
\hline $\begin{array}{l}\text { Susan, in Art, Craft and Design: } \\
\text { I try to tap into different learning and thinking styles } \\
\text { so kids who think in different ways are stimulated to } \\
\text { think it through. }\end{array}$ & $\begin{array}{l}\text { Different learning } \\
\text { and thinking styles }\end{array}$ & $\begin{array}{l}\text { Accommo- } \\
\text { dating } \\
\text { difference }\end{array}$ & $\begin{array}{l}\text { Construct- } \\
\text { ivism }\end{array}$ \\
\hline $\begin{array}{l}\text { Samantha, Humanities/Legal Studies: } \\
\text { De Bono's Thinking Hats to help students move } \\
\text { perhaps from seeing no problem to showing } \\
\text { compassion and value for others. }\end{array}$ & Problem finding & $\begin{array}{l}\text { Compassion } \\
\text { and value }\end{array}$ & $\begin{array}{c}\text { De Bono's } \\
\text { Hats }\end{array}$ \\
\hline $\begin{array}{l}\text { Vaughan, PE: } \\
\text { Vaughan listed De Bono's Hats; question starters; } \\
\text { visual and case study stimulus; problem-solving and } \\
\text { guided discovery }\end{array}$ & $\begin{array}{l}\text { Different } \\
\text { approaches to } \\
\text { learning }\end{array}$ & $\begin{array}{l}\text { Using } \\
\text { different } \\
\text { stimuli }\end{array}$ & $\begin{array}{c}\text { De Bono's } \\
\text { Hats }\end{array}$ \\
\hline $\begin{array}{l}\text { Matthew, AUST: I have been using the 5-Strand } \\
\text { Approach as a basic model for my Religious and } \\
\text { Values Education programs in each of the last three } \\
\text { schools I have worked in over } 17 \text { years. }\end{array}$ & $\begin{array}{l}\text { Three schools, } 17 \\
\text { years }\end{array}$ & $\begin{array}{l}\text { 5-strand } \\
\text { approach a } \\
\text { basic model }\end{array}$ & \multirow{3}{*}{$\begin{array}{l}\text { Vardy's 5- } \\
\text { Strands } \\
\text { RAVE }\end{array}$} \\
\hline $\begin{array}{l}\text { Matthew, AUST: Encouraging young people to } \\
\text { think for themselves is fundamental to this } \\
\text { approach. }\end{array}$ & $\begin{array}{l}\text { Thinking for } \\
\text { themselves }\end{array}$ & $\begin{array}{l}\text { Fundamental } \\
\text { to this } \\
\text { approach }\end{array}$ & \\
\hline $\begin{array}{l}\text { Matthew, AUS: I am very familiar with the Vardy } \\
\text { Five-Strands for Teaching World Religions and } \\
\text { Values as I have been instrumental in the } \\
\text { establishment of the Dialogue Australasian Network } \\
\text { in Australia and New Zealand. }\end{array}$ & $\begin{array}{l}\text { Dialogue } \\
\text { Australasian } \\
\text { Network, Australia } \\
\text { and New Zealand }\end{array}$ & $\begin{array}{l}\text { Teaching } \\
\text { world } \\
\text { religions and } \\
\text { values }\end{array}$ & \\
\hline
\end{tabular}

The second theme is a focus on student-centred thinking and learning (see Table 4.11).

\section{TABLE 4.11 CLASSROOM PLANNING AND STUDENT-CENTRED THINKING AND LEARNING}

\begin{tabular}{|l|l|l|c|}
\hline \multicolumn{1}{|c|}{ COMMENT } & CONSTRUCT & CONCEPT & THEME \\
\hline $\begin{array}{l}\text { Dennis (English, NZ ): Most areas of the } \\
\text { curriculum especially reading, writing, } \\
\text { speaking; written and question and answer; } \\
\text { group discussion; }\end{array}$ & $\begin{array}{l}\text { Reading, writing, } \\
\text { speaking }\end{array}$ & $\begin{array}{l}\text { Question and } \\
\text { answer; } \\
\text { group } \\
\text { discussion }\end{array}$ & $\begin{array}{c}\text { Student- } \\
\text { centred } \\
\text { learning }\end{array}$ \\
\cline { 1 - 2 } $\begin{array}{l}\text { Magda (UK): My scheme of work encourages } \\
\text { questioning and reflection, especially degrees of } \\
\text { success, why and how to improve. }\end{array}$ & $\begin{array}{l}\text { Encourages } \\
\text { questioning and } \\
\text { reflection }\end{array}$ & $\begin{array}{l}\text { Success and } \\
\text { improvement }\end{array}$ & \\
\hline
\end{tabular}




\begin{tabular}{|l|l|c|c|}
\hline \multicolumn{1}{|c|}{ COMMENT } & CONSTRUCT & CONCEPT & THEME \\
\hline $\begin{array}{l}\text { Samantha (Aust Humanities/Legal Studies): } \\
\text { highlighted the following: pre-unit } \\
\text { Questionnaires e.g., Legal Studies: Human } \\
\text { Rights - post self and course evaluation. }\end{array}$ & $\begin{array}{l}\text { Measuring growth in } \\
\text { thinking and } \\
\text { learning }\end{array}$ & $\begin{array}{c}\text { Self and } \\
\text { course- } \\
\text { evaluation }\end{array}$ & \\
\hline $\begin{array}{l}\text { Lavenda (English and Social Studies): Pre post } \\
\text { testing; check knowledge (who, what, why, } \\
\text { where ...); Comprehension (describe and } \\
\text { explain); application (solve/ use); Analysis } \\
\text { (what do you think? How do you think?); }\end{array}$ & $\begin{array}{l}\text { Measuring growth in } \\
\text { thinking and } \\
\text { learning } \\
\text { pynthesis (imagine/ predict/ draw/ write/ } \\
\text { application or transferral to real life situations. }\end{array}$ & $\begin{array}{c}\text { Bloom's } \\
\text { Domains }\end{array}$ & \\
\hline $\begin{array}{l}\text { Paula (Health-Human Development, and } \\
\text { Physical Education): Give a bright student a } \\
\text { question or statement that is the opposite of } \\
\text { what they know and require them to formulate a } \\
\text { response against their right answer. }\end{array}$ & $\begin{array}{l}\text { Special strategy for } \\
\text { senior students }\end{array}$ & $\begin{array}{l}\text { Working } \\
\text { outside } \\
\text { comfort zone }\end{array}$ & $\begin{array}{c}\text { Student- } \\
\text { centred } \\
\text { learning }\end{array}$ \\
\hline
\end{tabular}

I found that the focus on student-centred thinking and learning amplified a constructivist approach to both skills, involving the following: developing language arts (reading, writing, speaking, listening through question-andanswer sessions and group discussions); encouraging questioning and reflection; measuring growth in thinking and learning; extending students beyond their comfort zone; using Philosophy for Children as a core for education; being a critical thinker; and developing problem-solving frameworks.

\section{Variety in participants' chosen strategic frameworks}

In their answers to this Item, participants detailed many strategies they used in classrooms, whether planned as part of the lesson or as a response to an unexpected student query. They named strategic frameworks including, research-based; sequential; non-sequential; visual; auditory; written; and kinaesthetic. Examples of techniques related to each framework are contained in Table 4.12. 
TABLE 4.12 STRATEGIC FRAMEWORKS AND EXAMPLES

\begin{tabular}{|c|c|}
\hline STRATEGIC FRAMEWORKS & EXAMPLES \\
\hline Research-based & $\begin{array}{l}\text { - CONSTRUCTS kite } \\
\text { - de Bono's Hats } \\
\text { - pre and post testing } \\
\text { - inquiry-based research } \\
\text { - internet research } \\
\text { - case studies } \\
\text { - Thinking Keys } \\
\text { - data charts } \\
\text { - Bloom's Taxonomy }\end{array}$ \\
\hline Sequential & $\begin{array}{l}\text { - } \text { CONSTRUCTS kite } \\
\text { - VAK dominance testing } \\
\text { - de Bono's Hats } \\
\text { - PMI sequences } \\
\text { - literature circles } \\
\text { - pre and post testing } \\
\text { - } \text { plenary sessions with student questions } \\
\text { - Socratic approach, with more formal approach to logic } \\
\text { - } \text { the reasoning method } \\
\text { - } \text { brain-storming } \\
\text { - critical analysis } \\
\text { - comparative analysis }\end{array}$ \\
\hline Non-sequential & $\begin{array}{l}\text { - question starters: How? When? Where? Why? What? } \\
\text { - } \text { visual aids and stimulus material } \\
\text { - feedback techniques } \\
\text { - evaluation of self and curriculum } \\
\text { - written analysis } \\
\text { - LOTE memory strategies } \\
\text { - Venn diagrams } \\
\text { - logic and thinking games } \\
\text { - } 5 \text { W H (New Zealand) } \\
\text { - Gardiners intelligences } \\
\text { - empathetic teaching and embedded-ness } \\
\text { - values assessment } \\
\text { - lateral thinking/ creative thinking }\end{array}$ \\
\hline Visual & $\begin{array}{l}\text { - CONSTRUCTS kite } \\
\text { - VAK dominance testing } \\
\text { - de Bono's Hats } \\
\text { - literature circles } \\
\text { - visual aids and stimulus material } \\
\text { - internet research } \\
\text { - LOTE memory strategies } \\
\text { - data charts } \\
\text { - Venn diagrams }\end{array}$ \\
\hline Auditory & $\begin{array}{l}\text { - discussion } \\
\text { - Philosophy for Children's Community of Inquiry } \\
\text { - inquiry-based classroom } \\
\text { - de Bono's Hats } \\
\text { - PMI sequences } \\
\text { - literature circles } \\
\text { - question starters } \\
\text { - visual aids and stimulus discussions } \\
\text { - feedback techniques }\end{array}$ \\
\hline
\end{tabular}




\begin{tabular}{|c|c|}
\hline STRATEGIC FRAMEWORKS & EXAMPLES \\
\hline & $\begin{array}{l}\text { - } \text { evaluation of self and curriculum discussions } \\
\text { - } \text { LOTE memory strategies for class work } \\
\text { - } \text { Thinking Keys } \\
\text { - logic and thinking games } \\
\text { - } \text { Sonary sessions with student questions } \\
\text { - } \text { and argument } \\
\text { - the reasoning method } \\
\text { - empathetic teaching and embedded-ness } \\
\text { - } \text { lainstoral thinking/ creative thinking. } \\
\end{array}$ \\
\hline Written & $\begin{array}{l}\text { - literature circles } \\
\text { - } \text { pre and post testing } \\
\text { - feedback techniques } \\
\text { - } \text { gvaluation of self and curriculum } \\
\text { strategy. } \\
\text { - } \text { written analysis } \\
\text { - Thinking Keys } \\
\text { - } \text { Blooms Taxonomy of cognitive skills } \\
\text { - crical analysis especially in essay form } \\
\text { - } \text { values assesseanentysis }\end{array}$ \\
\hline Kinaesthetic & $\begin{array}{l}\text { - } \text { CONSTRUCTS kite } \\
\text { - Game Sense } \\
\text { - } \text { VAK dominance testing } \\
\text { - } \text { case studies investigation }\end{array}$ \\
\hline
\end{tabular}

Teachers across a range of disciplines indicated that particular frameworks were central to their lesson planning. These frameworks, all of which were directed towards student-centred thinking, are summarised in Table 4.13. Six concepts emerged, as follows: building learners, developing values and creating thinkers; using Philosophy for Children to encourage students to think for themselves; using guided reading to introduce thinking skills; developing critical thinking and reflective skills; developing concept formation and problem solving frameworks in Mathematics and Science; and developing what Michael (teacher) attributed directly to Bloom's taxonomy for higher order thinking skills. 
TABLE 4.13 STUDENT-CENTRED THINKING

\begin{tabular}{|c|c|c|c|}
\hline COMMENT & CONSTRUCT & CONCEPT & THEME \\
\hline $\begin{array}{l}\text { Clint, NZ: I see it as the core of a proper } \\
\text { education because through the structure of the } \\
\text { Community of Inquiry it builds learners, values } \\
\text { and thinkers. }\end{array}$ & $\begin{array}{l}\text { Philosophy for } \\
\text { Children core of a } \\
\text { proper education }\end{array}$ & $\begin{array}{l}\text { Building } \\
\text { learners, values } \\
\text { and thinkers }\end{array}$ & \multirow{4}{*}{$\begin{array}{l}\text { Student- } \\
\text { centred } \\
\text { thinking }\end{array}$} \\
\hline $\begin{array}{l}\text { Clint, NZ: This [Philosophy for Children] is my } \\
\text { specialist area. }\end{array}$ & $\begin{array}{l}\text { Students thinking } \\
\text { for themselves }\end{array}$ & $\begin{array}{l}\text { Encourage- } \\
\text { ment }\end{array}$ & \\
\hline $\begin{array}{l}\text { Dennis, Eng: Guided reading [introducing } \\
\text { thinking skills] as early as possible after the } \\
\text { introductory overview of the topic. }\end{array}$ & Guided reading & $\begin{array}{l}\text { Introductory } \\
\text { thinking skills }\end{array}$ & \\
\hline $\begin{array}{l}\text { Magda UK: Development of skills requires a } \\
\text { good self critic. }\end{array}$ & Skill development & Self-critic & \\
\hline $\begin{array}{l}\text { Michael (Maths): Most problems in Maths and } \\
\text { Science require thinking skills. The framework } \\
\text { involves distilling the key information that is } \\
\text { given. Selecting the appropriate tool to solve, then } \\
\text { I obtain feedback. }\end{array}$ & $\begin{array}{l}\text { Distilling the key } \\
\text { information in maths } \\
\text { and science }\end{array}$ & $\begin{array}{l}\text { Problem } \\
\text { solving } \\
\text { framework }\end{array}$ & \multirow{2}{*}{$\begin{array}{l}\text { Student- } \\
\text { centred } \\
\text { thinking }\end{array}$} \\
\hline $\begin{array}{l}\text { Michael (Maths): Bloom's Taxonomy is quite } \\
\text { effective because it enables the teacher to vary the } \\
\text { depth to which thinking skills are used. }\end{array}$ & $\begin{array}{l}\text { Vary depth of } \\
\text { thinking skills }\end{array}$ & $\begin{array}{l}\text { Use Blooms } \\
\text { Taxonomy [of } \\
\text { Cognitive } \\
\text { skills] }\end{array}$ & \\
\hline
\end{tabular}

\section{Experts' voices}

There were no hesitations, confusions, concerns or silences within the comments given by experts in their completion of the Questionnaire. Rather, they commented with accuracy on pedagogical matters. They were not prey to indecision and provided valuable clarity to each section of the Questionnaire in their answers.

\section{My researcher's voice}

In this sub-section I reflect on one point of hesitation and six individual points of confusion encountered in the responses.

\section{Hesitation}

The hesitation arose when teachers were asked if they would actually teach the concepts of virtues, of values, and of forms of ethical thinking. There was some evidence to suggest that teachers may be aware of this as a contentious issue, preferring to abide by the official policy of a previous generation regarding the desirability of a values-neutral classroom (in Australia). One of 
the respondents, Giovanni, an Australian teacher of English and Social Studies, highlights a particular teaching approach:

Ethics seems to mean Virtues and Values. I DONT believe we should teach any particular ritual to be ethical e.g.: going to church is good OR dying for your country is admirable [emphasis in original].

It has now been established that in many schools under the benign influence of a teacher in the classroom, students explore many virtues and values when studying narrative, but where unfocussed pedagogy can leave some unfair situations unaddressed. Life Skills work, which comes into play often in Administration time, is the likely useful time for this, especially in secondary school. It is the responsibility of the classroom or homeroom teacher. Life Skills topics can be experienced as full of unaddressed or underaddressed values that form part overt and covert agenda of the particular class, teacher or school.

\section{Confusions}

The first confusion showed in the responses of several respondents who admitted that to them, virtues and values were versions of the same thing. Consequently, it made an appreciable difference to the texture of some of the answers given. Teacher-participant Pietr defined the two concepts as follows:

\footnotetext{
Virtues are characteristics that a person outwardly shows as a result of their inner personality. Values are beliefs/abilities that a person uses/has in their interaction with others.
}

A second confusion rested on the teachers' familiarity with and commitment to the 'free and secular' educational policy of their respective countries (but especially in Australia); the striving for a values-neutral classroom that had been their priority. Apparently, values creep in by stealth or by accident, and the presence of covert agenda may not really be addressed. Respondents did not always distinguish between a values-neutral 
classroom and a possibility of unaddressed and mistaken concepts of tolerance, justice and respect. Where they tried to do so, there was noticeable confusion as to how this might be done. It also emerged that in a school such as the one from which more than half the respondents taught, values could be taught in Religion classes, or some values perhaps could be discussed briefly in Life Skills classes, accompanying Drug Education and Sex Education. Teacher Jane delineated her boundary lines clearly:

I don't use the term virtue. I think it is a crucial part of teaching that manifests in our expectations of ourselves and of our students. In Life Skills, these characteristics are explored more actively and at times in English. In managing a homeroom, these are encouraged/ modelled/praised/acknowledged all the time. ... I consider virtues to be favourable, desirable qualities that show care and respect for self and others. Virtues education is encouraging students to develop skills/ qualities that allow them to practise such things in their interactions with others.

The third confusion appeared to rest in the teachers' education (once again, especially in Australia), which in the main would have taken place in that same values-neutral classroom which had operated for years and which was consequently the pedagogical paradigm most advocated by these teachers. I have found that, though the word is rarely used in contemporary academic conversations, there was an apparent confusion in the responses as to what does and does not constitute indoctrination. Having assumed it to be chiefly the preserve of Religion classrooms (and it cannot be denied that indoctrination did take place in many confessional schools as a direct and defiant response by enthusiastic staff to the imposition of a values-neutral education upon their faith-based pedagogy), today's teachers may not have opportunities to fully investigate the extent to which indoctrination may be present today. The confusion further is exacerbated by the unexamined belief that indoctrination is only found in religious spheres. It would seem that today's teachers do not impinge on matters where there is an expectation of 
choice in the lives of today's students - whether it is career-oriented, or under the auspices of philosophical frameworks or religious belief systems.

Teacher Paul answered the Questionnaire (item 44) regarding a definition of Virtues Education thus: 'I take it to mean character education and I believe this has no place in the curriculum as a subject in its own right'.

Bernard saw Virtues Education as ‘values, standards a belief in one’s self and purposes'. Giovanni said:

Virtues are possibly the Big Picture Headings e.g., Virtues is underlined with constructs, ethics, morals, values surrounding it. Ethics seems to mean virtues and values.

Henry asked: 'What sort of virtues?' when asked about the thinking curriculum he used in his classroom, yet his responses to other questions showed clearly the value he assigned to respect, harmony and critical thinking in relation to his Art and Design curricula.

The fourth confusion concerned the documentation detailing the specific technical (techné) strategies used in classrooms. Some participants relied clearly, and only, on those governmental documents which give content guidance and outlines. No other process of information was given as to how this content was translated into step-by-step strategies to allow students space to achieve understanding, and for the formation of relevant questions so that actual learning takes place. It was not possible to extract more about what the teachers' strategies used.

Other participants were clear in their recognition of how they designed their step-by-step strategies so that students' questions were clarified and rich learning took place. These respondents used a variety of work processes devised under Lipman's Community of Inquiry and Fogarty's BrainCompatible Classrooms and others.

The fifth confusion emerged when participants answered questions about the difference between teaching the student, each student, and teaching just the curriculum. Dennis stated clearly that virtues are not taught directly, but 
rather only in the context of the themes/characters in situ during a specific topic; however, teacher-participant Lavenda related the organisation of her curriculum topics with teaching strategies in detail:

\footnotetext{
When planning a unit, I anticipate what I think the students will already know - prior learning; what the students will be able to work out; what they will need to be taught. Then I elicit their prior learning/knowledge, affirming or correcting that, and extend it with the presentation of new information, in a variety of ways. To process new information, students may work individually, in pairs or selected small groups.
}

Here she is detailing those strategies and the different techniques employed to ensure that rich learning takes place in a variety of settings. This is something that is not accounted for by the implementation of tests. It was not possible to conclude from the responses whether the design of assignments was seen as sufficient for rich learning to take place. Getting through the curriculum according to the requirement of their internal documentation was seen by some respondents as the only aim of delivery of curricular content.

A sixth confusion emerged for participants who found it difficult to answer some of the specific questions because of the precise attention they paid to the requirements of their respective National Curricula. Ben, who baldly stated that the only curriculum framework he used was the National Curriculum replied, in relation to the question (item 11) relating to how he organised curriculum topics and teaching strategies, only that: 'It is a key part of Geography and Citizenship therefore it is introduced from the beginning. Many lessons start with Q \& A'.

This same disinterested approach was particularly noticeable in participants from New Zealand and the United Kingdom. Little evidence of a negotiated curriculum was shown in most of the participants' responses, although several teachers related how they helped students to compose questions relevant to the strategies used in Lipman's Community of Inquiry. 
It was not possible to discern just how constricted or otherwise, teachers seemed to view the delivery of a National Curriculum.

\section{The use of discussion}

Within this theme, two voices are heard: my practitioner voice and the teachers' voices; because my concerns emerge from the analysis of my practitioner voice, my researcher voice is not present.

\section{My practitioner voice}

Development of the process of the Community of Inquiry required me to teach to each person in the class the value of listening, to use the various question starters to test and to accurately assess and apply logic and reason to each part of the discussion about the deeper meanings involved. I found that students grew in confidence, in their trust in each other, and in the role of the teacher (which often became that of a facilitator), over the course of the learning process until the exploration of contentious, awkward or complicated concepts was successfully navigated.

\section{Teachers' voices}

Teachers were asked to provide information relating to their understanding of their personal classroom strategies and management skills to spark and advance classroom discussion. The use of questions generated by the teacher and by the student, engendered by the study text or through discussion, was apparent; teachers used a variety of strategies to broaden discussion on a relevant topic. These are discussed in the followed sub-sections.

\section{Stimulating discussion (quantitative)}

Two categories were identified by the teachers: the initiation of discussion and the stimulation of discussion. These data are reported in Tables 4.14 and 4.15 . 


\section{TABLE 4.14 STRATEGIES TO INITIATE DISCUSSION}

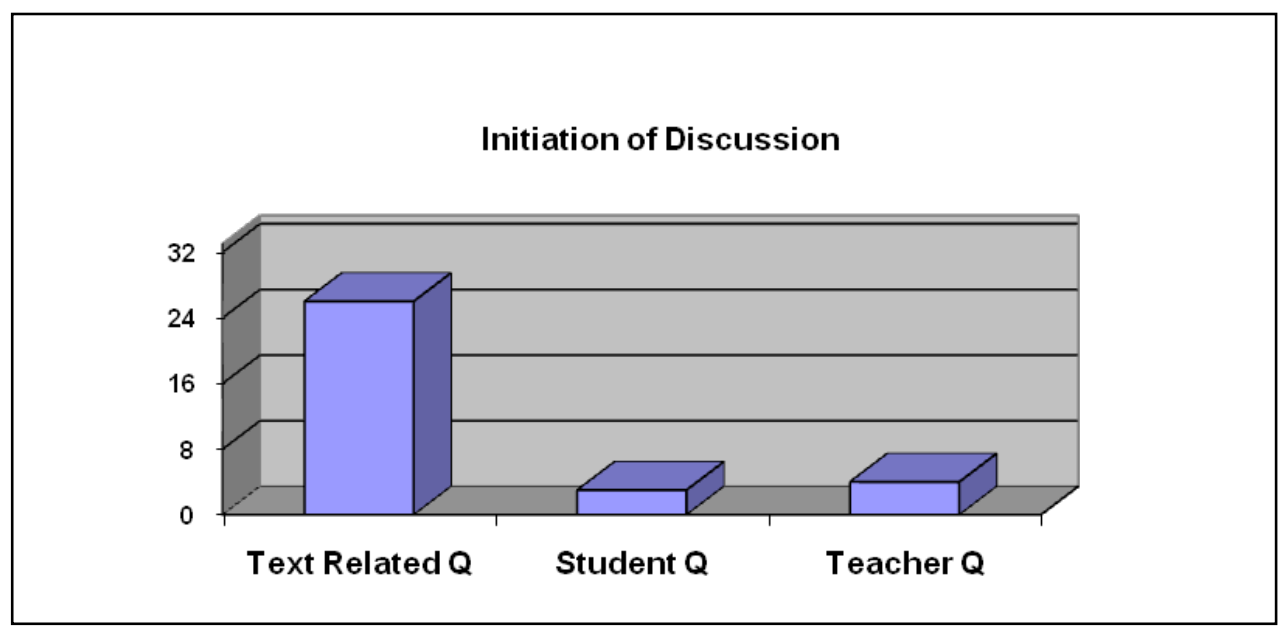

(Text Related Q = Teacher-generated questions from texts; Student Q = Questions generated directly from Students; Teacher Q = Questions generated directly by Teachers.)

\section{TABLE 4.15 STRATEGIES TO STIMULATE DISCUSSION}

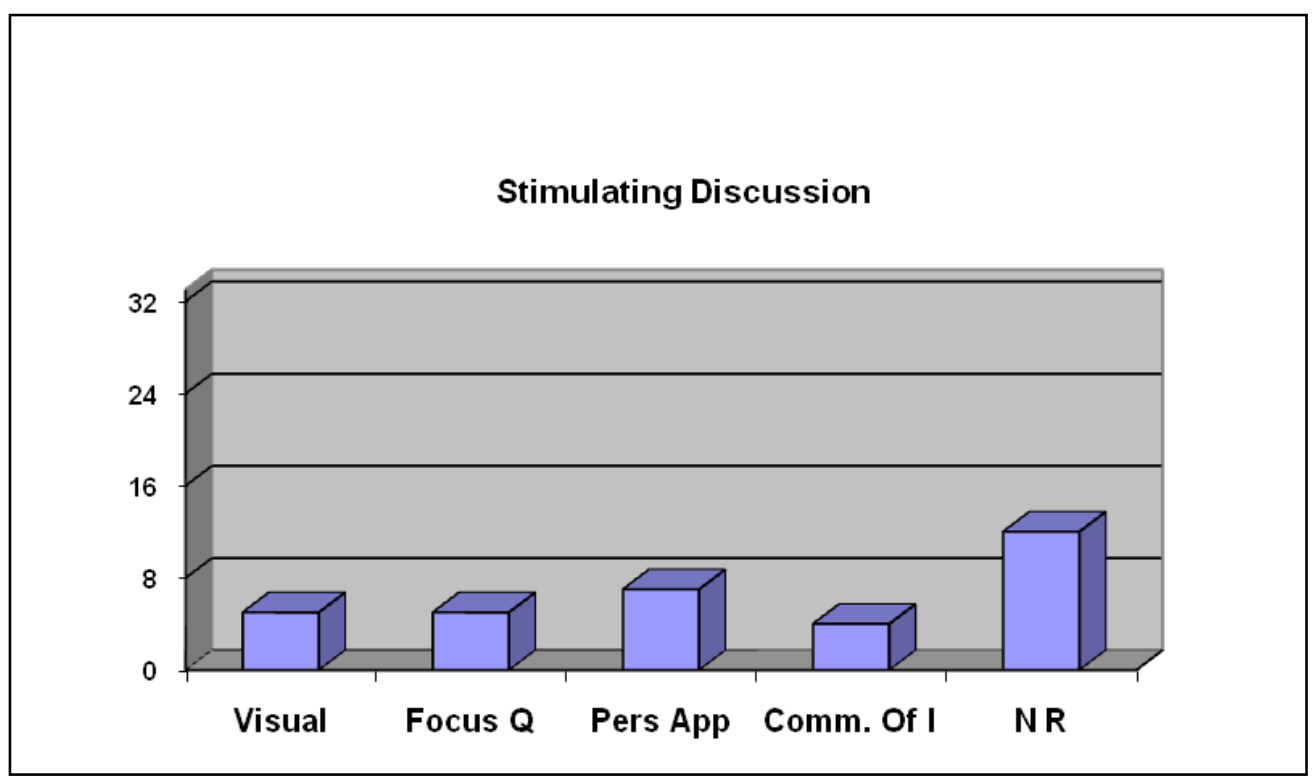

(Visual = using visual materials to stimulate thinking and questions; Focus $\mathrm{Q}=$ using focus questions to stimulate discussion; Pers App = Teachers using personal approaches and application of materials to engender discussion; Comm. Of I = teachers using the precise format of Community of Inquiry relating to the Philosophy for Children class; $\mathrm{N} \mathrm{R}=$ no response)

Most teachers (26/33) indicated that text-related questions led to discussion. Very few indicated discussion results from students' questions (3/33) or teachers questions (4/33). 


\section{Initiation of discussion}

Most teachers (26/33) indicated that text-related questions led to discussion. Very few indicated discussion results from students' questions (3/33) or teachers' questions (4/33).

\section{Stimulation of discussion}

Five teachers (5/33) used visual stimuli to generate questions for discussion while five others (5/33) used focus questions about the text. Seven teachers (7/33) gave no indication of regular classroom processes; instead, they used very personal approaches to student questions. The three experts: Roger, Clint and Matthew - made it clear that questions should form an expected part of a Community of Inquiry classroom. Twelve participants (12/33) did not specify a response.

\section{My researcher's voice}

Because my concerns emerge in my practitioner's voice, my researcher voice here is not in evidence.

\section{Focussing on students}

Within this next theme, however, four voices are heard: my practitioner voice, the teachers' voices, experts' voices and my researcher voice.

\section{My practitioner voice}

Apart from the actual feat of teaching students to focus on the relevant questions for a given topic, it became increasingly obvious to me during my teaching that students at all levels began to apply the newly acquired system of questions and testing to their own lives, especially in English, Social Studies, Religion Studies, Business studies and Ethics: my subjects. Teaching a process of decision-making was a next vital step, and through my focussing on students and their needs I constructed two processes to this end. 
These processes resulted in students making a distinction between urgent and impulse (not impulsive) decisions and what I referred to as reflective and impulse decision-making, as shown in Figure 4.2.

FIGURE 4.2 DECISION-MAKING: CONSIDERED PROCESS V.IMPULSE APPROACH
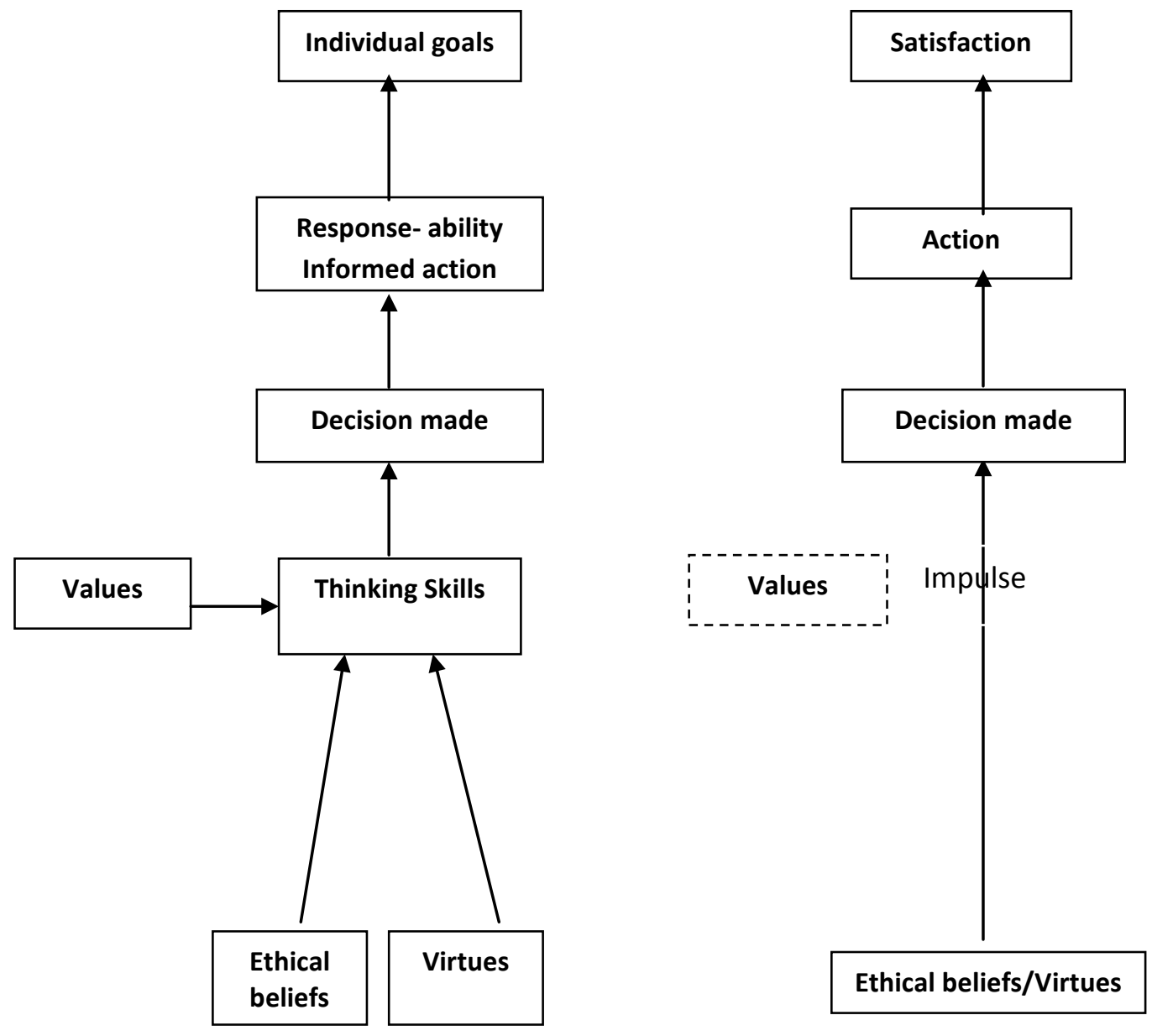

The following process evolved from discussions and consultations with the educational experts who took part in the study, and who tested their advisements (but not their advice). It is important to recognise here that advice is the offering of personal experience similar to the problem being experienced by another person, whereas advisement is my term for the process of seeking by the problem-solver of persons who are expert in the problem area and can offer educated options to the problem-solver. This meant that calculated risks could be taken, their accuracy could be tested and 
mistakes could be discarded. It is my experience the students exhibit both considered (reflective) and impulse (urgent) processes in their decisionmaking. The left shows a step-by-step reflective process (the result of careful thinking) while the right side of the diagram shows an impulse process (made as an instantaneous response to a stimulus) in two different types of decisionmaking.

Students were fascinated by the self-discovery that attended the discussions about the parallels and contrasts implied by the application of two thinking patterns as they appear above. Without fail, they responded immediately to the role of Values as the left diagram showed a stronger presence. Further investigation and consultation with others, including parents, brought together a wide variety of people who began to appreciate that each style of thinking was important in differing circumstances; students were able to recognise the significance of each style and to use both with dawning discernment. I noted that the ability to differentiate between advice (detailed personal experience of another person who offered to help) and advisement (detailed expert information regarding the question showing options available for consideration), which emerged as they sought information from their chosen experts, aided their thinking and planning, since it was an experience that underscored the existence of dependent/independent/interdependent elements when decision-making was needed.

It is worth highlighting that the reflective process for a considered outcome rests on a conscious understanding of personal ethical beliefs and relevant virtues (whether of secular, philosophical or religious origin). By contrast, the impulse approach rests on the sudden need as foundation for action, where ethical beliefs and relevant virtues may not always be clearly identified as part of the process, but are invisible in the response of the individual. 


\section{Teachers' voices}

The Questionnaire required teachers to identify (item 16) whether student behaviour influenced their efforts to ask questions of particular students. The Item focussed particularly on whether a teacher was able to stimulate certain individuals or certain categories of students to deeper reflection and analysis. The categories of student selection for questioning listed for response in a multiple choice item on the Questionnaire were as follows: leaders, quiet ones, noisy ones, boys, girls, alternating boy and girl, random choice, student choice, all students.

\section{Categories of students (quantitative)}

Teachers either ticked the last box as a way of covering "All the above" categories, but sometimes they highlighted some categories in particular. These data are displayed in Table 4.16. Seventeen teachers (17/33) showed that they stimulated all students towards thinking skills. Five teachers (5/33) highlighted that they stimulated students in a random fashion, while eleven $(11 / 33)$ gave insufficient details for assessment.

\section{TABLE 4.16 FINDING ACCURATE FOCUS THROUGH QUESTIONS}

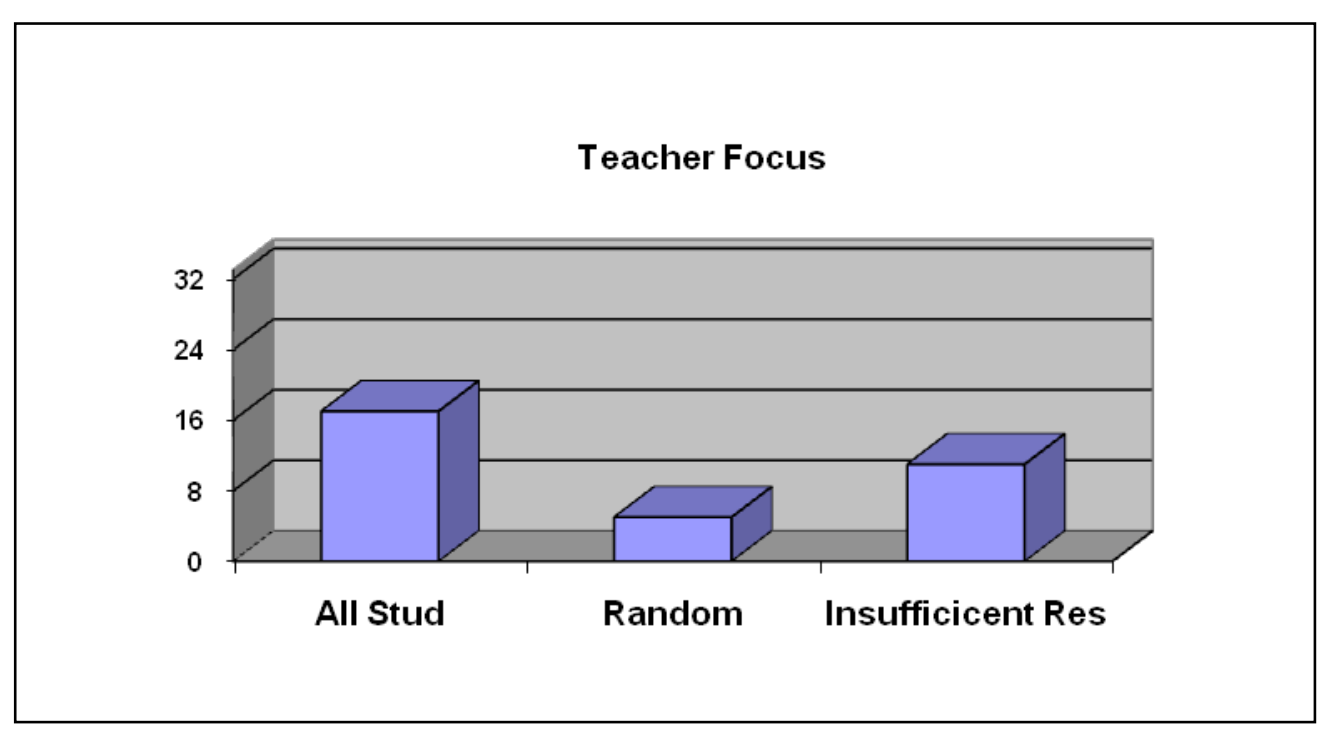

(All Stud = teachers used questions for all students; Random = teachers used questions randomly; Insufficient Res = there was insufficient response in the questionnaire) 


\section{Inclusivity in questioning}

I found that teachers highlighted their intentions to be inclusive and to give all class members equal opportunity to benefit from questions promoting thinking skills. It was clear that all these teachers tried to be inclusive; however, they did distinguish between quiet and noisy students, leaders and followers, and boys and girls, when this was deemed necessary. Some teachers referred to questions directed to all students; some emphasised that their practice was of a random nature to allow for maximum input from a variety of student questions.

Several responses indicated that by using questions to students in rotation, teachers promoted equal benefit for all class members. Teachers stated that they deliberately did not select individual students in an effort to give freedom to question as students have need. All teachers indicated also, that, to the best of their ability, they attempted to give equal opportunity to all students' questioning. The constructs, concepts and themes that emerged from this item are contained in Table 4.17.

TABLE 4.17 ENSURING FULL STUDENT PARTICIPATION IN QUESTIONING

\begin{tabular}{|c|c|c|c|}
\hline COMMENT & CONSTRUCT & CONCEPT & THEME \\
\hline $\begin{array}{l}\text { Giovanni, in English and Drama, ticked all boxes } \\
\text { in an effort to show his clarity about consciously } \\
\text { directing all categories of students towards } \\
\text { thinking skills. }\end{array}$ & $\begin{array}{l}\text { Focus on all } \\
\text { students }\end{array}$ & $\begin{array}{l}\text { Directing all } \\
\text { categories of } \\
\text { students }\end{array}$ & \multirow{5}{*}{$\begin{array}{l}\text { Full student } \\
\text { participation }\end{array}$} \\
\hline $\begin{array}{l}\text { Terence, in Humanities, English and Life skills, } \\
\text { targeted leaders, quiet ones, noisy ones, boys, } \\
\text { girls. }\end{array}$ & & & \\
\hline $\begin{array}{l}\text { Harriette targeted all students: I would cover all } \\
\text { of these (categories) not necessarily in one lesson. } \\
\text { The quiet one's would need to be coaxed to } \\
\text { comment only after personal previous discussion } \\
\text { with them to help confidence. If possible, in } \\
\text { rotation, but if not completed, try to continue next } \\
\text { lesson if topic lends itself. }\end{array}$ & $\begin{array}{l}\text { Targeting all } \\
\text { students }\end{array}$ & $\begin{array}{l}\text { Rotation of } \\
\text { questions }\end{array}$ & \\
\hline $\begin{array}{l}\text { Magda (language classes and in Music studies) } \\
\text { indicated that while she did stimulate all students } \\
\text { towards thinking skills, it does depend upon the } \\
\text { task. It is handy to refocus particular pupils. }\end{array}$ & $\begin{array}{l}\text { Thinking depends } \\
\text { on task }\end{array}$ & $\begin{array}{l}\text { Refocus } \\
\text { particular } \\
\text { students }\end{array}$ & \\
\hline $\begin{array}{l}\text { Commenting about random use of stimulating } \\
\text { students, Frank said: Quiet one's, random choice. }\end{array}$ & Random choice & $\begin{array}{l}\text { Stimulating } \\
\text { all students }\end{array}$ & \\
\hline
\end{tabular}




\begin{tabular}{|c|c|c|c|}
\hline COMMENT & CONSTRUCT & CONCEPT & THEME \\
\hline Pietr said: Leaders, random choice, all students. & \multirow[b]{2}{*}{$\begin{array}{l}\text { Encourage quiet } \\
\text { students }\end{array}$} & \multirow{8}{*}{$\begin{array}{l}\text { Targeting } \\
\text { all students }\end{array}$} & \\
\hline $\begin{array}{l}\text { Maryanne, in History, commented: Quiet ones. I } \\
\text { do try to encourage here; otherwise random } \\
\text { choice; all students. }\end{array}$ & & & \\
\hline $\begin{array}{l}\text { June, in Art and Visual Communications, added: } \\
\text { Watch for students who are not connected to the } \\
\text { task, try to get their interest. }\end{array}$ & \multirow{2}{*}{$\begin{array}{l}\text { Encourage quiet } \\
\text { students }\end{array}$} & & \\
\hline $\begin{array}{l}\text { Portia, in both Maths and Humanities, paid } \\
\text { particular attention to the quiet one's }\end{array}$ & & & \\
\hline $\begin{array}{l}\text { Connie, in Gymnasium and Physical Education, } \\
\text { highlighted five categories: leaders, quiet ones, } \\
\text { noisy ones, boys, girls, all students. I ask all pupils } \\
\text { to participate. }\end{array}$ & $\begin{array}{l}\text { Five categories of } \\
\text { students }\end{array}$ & & \\
\hline $\begin{array}{l}\text { George, in Maths or Religious Studies, also } \\
\text { challenges all students to think. I don't select a } \\
\text { group. }\end{array}$ & $\begin{array}{l}\text { Challenging all } \\
\text { students }\end{array}$ & & \\
\hline $\begin{array}{l}\text { Sandy, in relation to English, History and } \\
\text { International Baccalaureate studies replied: All } \\
\text { students. Try to! - showing commendable } \\
\text { identification that one’s intention may not always } \\
\text { surface fully in practice. }\end{array}$ & $\begin{array}{l}\text { [Involve] all } \\
\text { students }\end{array}$ & & \\
\hline $\begin{array}{l}\text { Dennis, also in English, stated similarly: All } \\
\text { students - ideally. }\end{array}$ & Ideally, all students & & \\
\hline
\end{tabular}

The overwhelming response from teachers to inclusivity in questioning was to ensure full student participation. Five teaching concepts emerged: directing all categories of students (a focus on all students); rotation of questions (so that all students were regularly targeted); refocussing particular students (to ensure that the task is clear); stimulating all students to respond (by randomly choosing them to respond); and, generally, targeting all students (by recognising at least five student types, challenging and involving all students and ensuring that the quiet students were encouraged to respond), Clearly, for the effective teaching of Philosophy for Children, questioning skills need to be developed to a very high level, and the individual behavioural types in a class need to be identified with a view to including them all in active responding.

There were indications that teachers had differing interpretations as to what constituted inclusivity. They stated that questioning could be random. If quiet students needed to be coaxed to enter the conversation, they might be needy because of shyness, hesitancy over accuracy, or even because they choose not to expose themselves to the possibility of derision so frequently 
practiced among students. Teachers did not indicate within their responses whether they believed that the specific use of random questioning techniques helped them to maintain the student-centred classroom pedagogy. It was consequently quite legitimate to expect that students who exhibited disengagement might become more focussed by means of strategic questioning about the topic under discussion.

\section{The experts' voices}

The three experts highlighted, without ticking individual boxes, some valuable general considerations regarding particular methods of stimulating the students towards thinking skills.

Roger, the UK educational expert, commented within the Questionnaire:

I would not over-complicate the business by trying to identify and target different skills for different individuals. I am much taken with the efficiency and naturalness of the Community of Inquiry approach, in which students can - and I believe do - learn skills and strategies for better thinking from each other (and also, of course, from skilled facilitation of teachers). I think there does need to be an element of challenge from teachers or facilitators to students, but it is better if the challenges are to the community as a whole, and/or if they actually come spontaneously from within the community.

Clint, the New Zealand expert, identified some of the categories of thinking in a particular process:

Best practice is not to teach skills at all but to consciously model the thinking in the different disciplines. Also asking the students to engage in the same thinking will help. For example, science requires predictive thinking, so the teacher models making predictions and checking their accuracy and modifying the predictions if they are not accurate ... Then they ask the students to predict (what do you think will happen next? Why? How can we test if our predictions are correct...) 
Australian expert, Matthew, expected all students to be involved fully in the Community of Inquiry. His explanation was detailed:

\begin{abstract}
We are currently engaged in the process of setting up exemplars of best practice in teaching Philosophy and Ethics, and foundational to this course is the teaching of Critical thinking skills. Creating communities of inquiry is an essential aspect to both the Philosothon and the course itself. The Philosothon has been a great way to get together Tertiary and Secondary teachers with a view towards encouraging and identifying best practice in critical thinking skills as it relates to senior secondary students.
\end{abstract}

Matthew, the Australian expert, whose efforts had resulted in the establishment of a lunchtime activity given the name of Philosothon, also made it clear that he expected the role of teacher in the Community of Inquiry to include not just teaching, but also facilitating at appropriate times during the course of the period in which the Community of Inquiry operated. He insisted that the usefulness of the Community of Inquiry experience includes the consistent building of a variety and diversity of critical thinking skills.

\title{
My researcher's voice
}

In this sub-section I reflect on one apparent hesitation about an understanding of what are to be regarded as virtues. In this instance, teacher-participant Jane delineated her boundary lines clearly:

I don't use the term virtue. I think it is a crucial part of teaching that manifests in our expectations of ourselves and of our students. In Life Skills, these characteristics are explored more actively and at times in English. In managing a homeroom, these are encouraged/ modelled/praised/acknowledged all the time. [...] I consider virtues to be favourable, desirable qualities that show care and respect for self and others. Virtues education is encouraging students to develop skills/ qualities that allow them to practise such things in their interactions with others. 
It is interesting to note how this teacher responds so transparently, and with relevance, to homeroom practices. This is particularly pertinent to my underlying requirement that teachers teach the student through the various pedagogical and other practices.

\section{Sequential processes}

It is far more important that the voices of the teachers are heard here than that my practitioner voice emerges. Thus it is my researcher voice that is presented here. The teachers' sequential processes are central to this investigation. Teachers were asked at what stage they preferred to introduce thinking skills (item 10) and to describe some indications of any process they used to achieve this in classroom discussion.

\section{Teachers' voices}

The participants indicated that they most often used specific strategic sequences as they set up questions to facilitate class progress. They indicated links between their own questions and the ensuing discussions and tried to distinguish between a teacher-directed classroom and a student-directed classroom from their individual perspectives.

\section{Staged sequences (quantitative)}

An overlap in answers given by teachers trying to indicate which students they targeted for questions about promoting learning, came about because teachers ticked in several ways the list of possible categories of students whom they might consciously wish to target for questioning. Seventeen teachers (17/33) indicated that their questions were initiated as teacher. Seventeen others (17/33) indicated that their process was two-pronged with discussion following a question. Five respondents (5/33) indicated that they actively used groups as a means of handling questions, and to explore topics through the use of questions. 
Another five teachers (5/33) indicated the use of the jigsaw strategy in group work. Two others stood out (2/33) by highlighting a variety of stimuli as bases for eliciting questions, implying the strategies most suitable for each of these stimuli.

\section{Staged sequences (qualitative)}

I found that the respondents emphasised four main concepts: student-centred learning, philosophical questioning, group learning, and using a variety of strategies. To a lesser extent, they were concerned with higher-order thinking, employing a joint approach, and taking account of the range of multiple intelligences existing in their classes. Their comments are summarised in Table 4.18.

\section{TABLE 4.18 STAGED SEQUENCES}

\begin{tabular}{|c|c|c|c|}
\hline COMMENT & CONSTRUCT & CONCEPT & THEME \\
\hline $\begin{array}{l}\text { Kerri: [I use] brain storming techniques and } \\
\text { mind-mapping on white board or as group tasks. }\end{array}$ & $\begin{array}{l}\text { Brain storming \& } \\
\text { mind-mapping }\end{array}$ & \multirow{4}{*}{$\begin{array}{c}\text { Group } \\
\text { learning }\end{array}$} & \multirow{8}{*}{$\begin{array}{c}\text { Staged } \\
\text { sequences }\end{array}$} \\
\hline $\begin{array}{l}\text { Sandy: Jigsaw strategy to facilitate the focus on } \\
\text { significant questions for the groups. }\end{array}$ & Jigsaw strategy & & \\
\hline $\begin{array}{l}\text { Paula: students placed in groups for working then } \\
\text { has question time }\end{array}$ & Question time & & \\
\hline $\begin{array}{l}\text { Vaughan: I may throw out a question for } \\
\text { discussion and see how it goes. Similarly, a } \\
\text { student may do the same. }\end{array}$ & $\begin{array}{l}\text { Student and teacher } \\
\text { questions }\end{array}$ & & \\
\hline $\begin{array}{l}\text { Matthew, Australian Expert, commented: The } \\
\text { model that we use allows students to engage in } \\
\text { higher-order thinking and this seems to naturally } \\
\text { progress from the processes which are in place for } \\
\text { a Community of Inquiry. }\end{array}$ & $\begin{array}{l}\text { Community of } \\
\text { Inquiry }\end{array}$ & $\begin{array}{l}\text { Higher-order } \\
\text { thinking }\end{array}$ & \\
\hline $\begin{array}{l}\text { Paul, in English, German Studies or History: } \\
\text { reached for a provocative question; }\end{array}$ & $\begin{array}{l}\text { Provocative } \\
\text { questioning }\end{array}$ & $\begin{array}{c}\text { Joint } \\
\text { approach }\end{array}$ & \\
\hline $\begin{array}{l}\text { Lavenda, in English: ensured there was always a } \\
\text { visual, aural and kinaesthetic component to each } \\
\text { activity or lesson; }\end{array}$ & $\begin{array}{l}\text { Variety of learning } \\
\text { components }\end{array}$ & $\begin{array}{l}\text { Multiple } \\
\text { Intelligence } \\
\text { components }\end{array}$ & \\
\hline $\begin{array}{l}\text { Clint, the NZ Expert: Questions are important to } \\
\text { coach students in their thinking - e.g., why do you } \\
\text { think this? Ask them to give reasons. However, } \\
\text { the questions can be used badly to lead the } \\
\text { students to the answer the teacher thinks is best. }\end{array}$ & $\begin{array}{l}\text { Coaching students in } \\
\text { thinking }\end{array}$ & $\begin{array}{c}\text { Philosophical } \\
\text { questioning }\end{array}$ & \\
\hline
\end{tabular}




\begin{tabular}{|c|c|c|c|}
\hline COMMENT & CONSTRUCT & CONCEPT & THEME \\
\hline $\begin{array}{l}\text { Matthew, Australian Expert: Addressing and } \\
\text { asking questions is an essential part of any course. } \\
\text { In our Communities of Inquiry we get students to } \\
\text { each ask a question about the source material and } \\
\text { then write these on a white board together with the } \\
\text { students' names. We then address the questions to } \\
\text { the group and ask students to abide by the rules } \\
\text { associated with the Community of Inquiry. }\end{array}$ & $\begin{array}{l}\text { Community of } \\
\text { inquiry }\end{array}$ & & \\
\hline $\begin{array}{l}\text { Pietr: Post a question relating to the topic and ask } \\
\text { students to stand in a certain place depending on } \\
\text { their point of view and discuss; break up and } \\
\text { rediscuss. }\end{array}$ & Defending a position & & \\
\hline $\begin{array}{l}\text { Connie: Questions providing feedback and for } \\
\text { reviewing the previous topic. }\end{array}$ & $\begin{array}{l}\text { Feedback and } \\
\text { review }\end{array}$ & & \\
\hline $\begin{array}{l}\text { Sandy: Jigsaw strategy to facilitate the focus on } \\
\text { significant questions for the groups. }\end{array}$ & Jigsaw strategy & & \\
\hline $\begin{array}{l}\text { Roger, UK Expert: The basic approach to } \\
\text { teaching questioning is to open students' minds to } \\
\text { the nature and importance of philosophical } \\
\text { questioning for meaning, reasoning, and value. }\end{array}$ & $\begin{array}{l}\text { Making meaning, } \\
\text { reasoning, valuing }\end{array}$ & & \\
\hline $\begin{array}{l}\text { Pietr: Post a question on the board and select } \\
\text { students in small groups to answer and report } \\
\text { back. }\end{array}$ & Reporting back & & \\
\hline $\begin{array}{l}\text { Roger, UK expert, the only respondent to pinpoint } \\
\text { the actual business of teaching questioning: There } \\
\text { is also need to be an element of creative/ radical } \\
\text { alternatives, best captured in the overall strategy } \\
\text { of asking: Could this thinking/ acting/ product be } \\
\text { better? }\end{array}$ & $\begin{array}{l}\text { Teaching } \\
\text { questioning }\end{array}$ & & \\
\hline $\begin{array}{l}\text { Magda, in Class Music, commented that the } \\
\text { development of thinking skills requires a good } \\
\text { self-critic; }\end{array}$ & Being a self-critic & \multirow{8}{*}{$\begin{array}{l}\text { Student- } \\
\text { centred } \\
\text { learning }\end{array}$} & \\
\hline $\begin{array}{l}\text { Clint, the NZ expert: [The] recommended } \\
\text { process is the community of inquiry -start with a } \\
\text { problematic situation and students learn to think in } \\
\text { various disciplines by engaging with and resolving } \\
\text { this problem. }\end{array}$ & $\begin{array}{l}\text { Community of } \\
\text { inquiry }\end{array}$ & & \\
\hline $\begin{array}{l}\text { Franco: [I am] looking for children's response } \\
\text { and flow of Constructs. }\end{array}$ & Flow of Constructs & & \\
\hline $\begin{array}{l}\text { George does not use regular process or structure: I } \\
\text { can go either way depending on level of interest. }\end{array}$ & $\begin{array}{l}\text { No regular process } \\
\text { or structure }\end{array}$ & & \\
\hline $\begin{array}{l}\text { June, in Art Theory, relied on question-starters } \\
\text { supported by sequenced instructions; }\end{array}$ & $\begin{array}{l}\text { Question starters by } \\
\text { sequenced } \\
\text { instructions }\end{array}$ & & \\
\hline $\begin{array}{l}\text { Jane, in Psychology, Biology and English, also } \\
\text { relied on question starters; }\end{array}$ & $\begin{array}{l}\text { Question starters by } \\
\text { sequenced } \\
\text { instructions }\end{array}$ & & \\
\hline $\begin{array}{l}\text { Susan, in Art, Craft Design and Technology, } \\
\text { concentrated on students being encouraged to } \\
\text { think divergently. }\end{array}$ & $\begin{array}{l}\text { Thinking } \\
\text { divergently }\end{array}$ & & \\
\hline $\begin{array}{l}\text { Susan, a Design and Technology teacher: always } \\
\text { presents the work as a problem so students are } \\
\text { encouraged to think divergently and discuss the } \\
\text { suggestions. }\end{array}$ & $\begin{array}{l}\text { Thinking } \\
\text { divergently }\end{array}$ & & \\
\hline
\end{tabular}




\begin{tabular}{|c|c|c|c|}
\hline COMMENT & CONSTRUCT & CONCEPT & THEME \\
\hline $\begin{array}{l}\text { Clint, the NZ expert: thought encouraging } \\
\text { questions should be used with an open-attitude } \\
\text { where teachers are consciously focusing on the } \\
\text { thinking of students, not on whether they have the } \\
\text { right answer or not . }\end{array}$ & $\begin{array}{l}\text { Thought- } \\
\text { encouraging } \\
\text { questions }\end{array}$ & & \\
\hline Frank: Visual stimulus to elicit student response. & Visual stimuli & & \\
\hline $\begin{array}{l}\text { Hyacinth used a variety of strategies in a mixture } \\
\text { of teacher-directed, and student-directed but } \\
\text { teacher-guided process: think/pair/share or other } \\
\text { brainstorming strategies; written materials; visual } \\
\text { stimuli; a strategy like corners; anecdotes; student } \\
\text { questions/teacher questions; thinking keys } \\
\text { activity. }\end{array}$ & $\begin{array}{l}\text { Teacher, student and } \\
\text { teacher-student } \\
\text { processes }\end{array}$ & \multirow{3}{*}{$\begin{array}{l}\text { Variety of } \\
\text { strategies }\end{array}$} & \\
\hline $\begin{array}{l}\text { Lavenda uses stimulus prompts like: Can you tell } \\
\text { us...? How do you feel about...? What do you } \\
\text { think about...? }\end{array}$ & $\begin{array}{l}\text { Teacher, student and } \\
\text { teacher-student } \\
\text { processes }\end{array}$ & & \\
\hline $\begin{array}{l}\text { Samantha, who took a similar approach, listed the } \\
\text { following sequences: teacher guidance and an } \\
\text { emphasis on student inquiry; case studies; chalk } \\
\text { and talk; videos; texts; comprehension tasks; } \\
\text { inquiry-based research; internet. }\end{array}$ & $\begin{array}{l}\text { Teacher, student and } \\
\text { teacher-student } \\
\text { processes }\end{array}$ & & \\
\hline
\end{tabular}

When summarising, in my researcher's voice, the overall effect of the details set out in table 4.18, it is important to note that this was the first time that I appreciated in full the divergence between my expectations and the actual documented responses from the teachers. Where there was important and careful stimuli centred on asking questions in different ways, the aim was to focus students' attention upon their own learning, the philosophical questioning in a perhaps limited form, with team-building for group learning. Teachers managed this by inclusion within their classroom activities and strategies as much variety as they could insert.

As a consequence, I found that in their responses there seemed to be distinctly less emphasis by teachers on the conscious promotion of higherorder thinking skills, and evidence of little inclination to identify the various multiple intelligences (H. Gardner, 1993a) presenting in the way students operated their own learning. 


\section{Thinking skills}

Within this theme, two voices are heard: the teachers’ voices and my own researcher voice.

\section{Teachers' voices}

Teachers were asked to describe features of their current teaching practice that they applied in their daily teaching of thinking skills. They indicated that they sought successful teaching-learning processes and classroom strategies based on what was seen to be good for the student, was helpful for the class, and which contributed to the effective delivery of the curriculum content.

\section{Categories, reasons and levels for teaching of thinking skills}

I found that the teachers used a variety of pedagogical language to talk about the Questionnaire items. They also used different kinds of activities to achieve their teaching-learning goals; to stimulate questions and questioning; and to lead to discussion. It seems that within this concept of the use of questions and questioning, there is embedded a democratic paradigm, that allows students to explore in every direction. Efforts in their responses to distinguish between teaching the subject and teaching the student were productive, as teachers gave information about the thinking skills they employed.

The first set of responses, contained in Table 4.19.1, referred to classroom planning. Fifteen respondents (15/33) explained their philosophy of education, with general statements of a policy nature given. Four teachers (4/33) concentrated their efforts on the successful delivery of the curriculum content. The remaining five (5/33) did not name their exact philosophy in the classroom but comments elsewhere indicate that they operated under similar classroom strategies and objectives as noted herein. Two participants did not respond to this question and the three experts: Roger, Clint and Matthew gave generalised responses, only. 
TABLE 4.19.1 CLASSROOM PLANNING

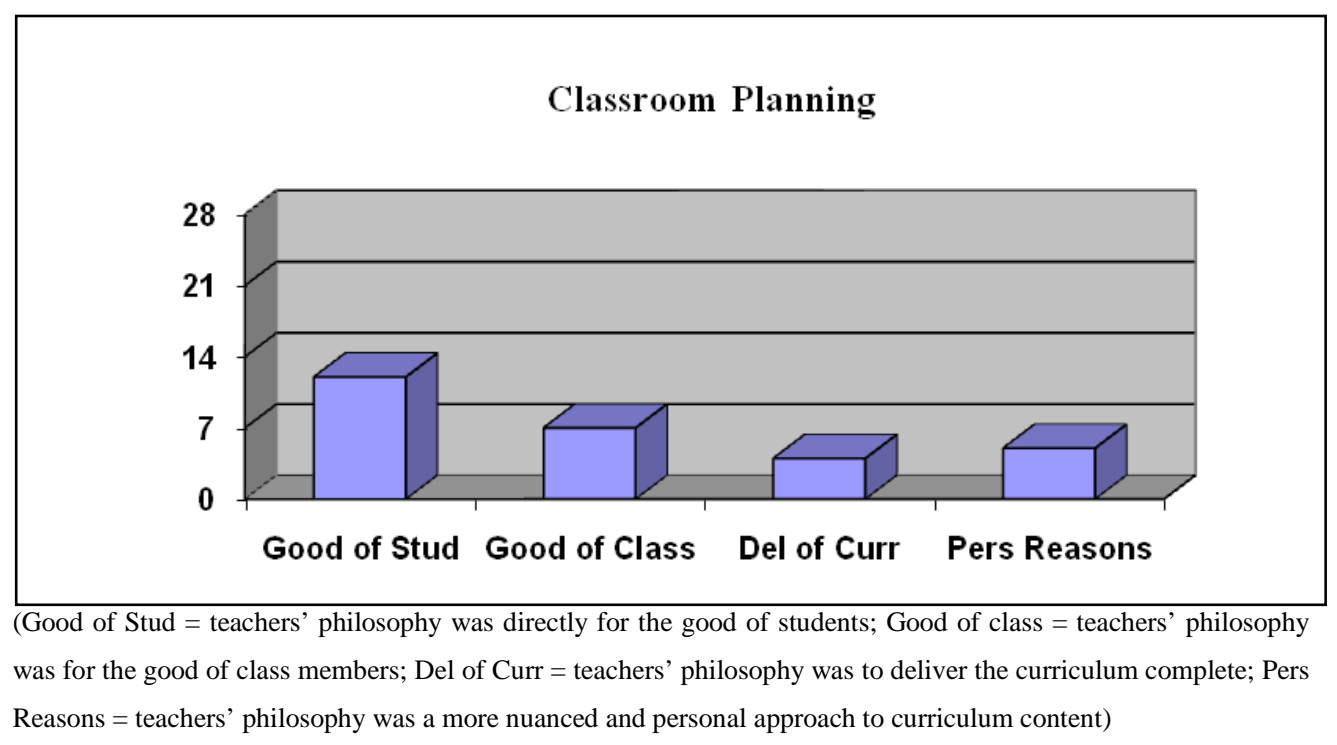

The data contained in Table 4.19.2 refer to thinking skills used by teachers. Fourteen teachers gave a list of thinking skills used most often and which were therefore most familiar to their own students. Eight had reasons

TABLE 4.19.2 HOW THINKING SKILLS ARE CURRENTLY ADDRESSED

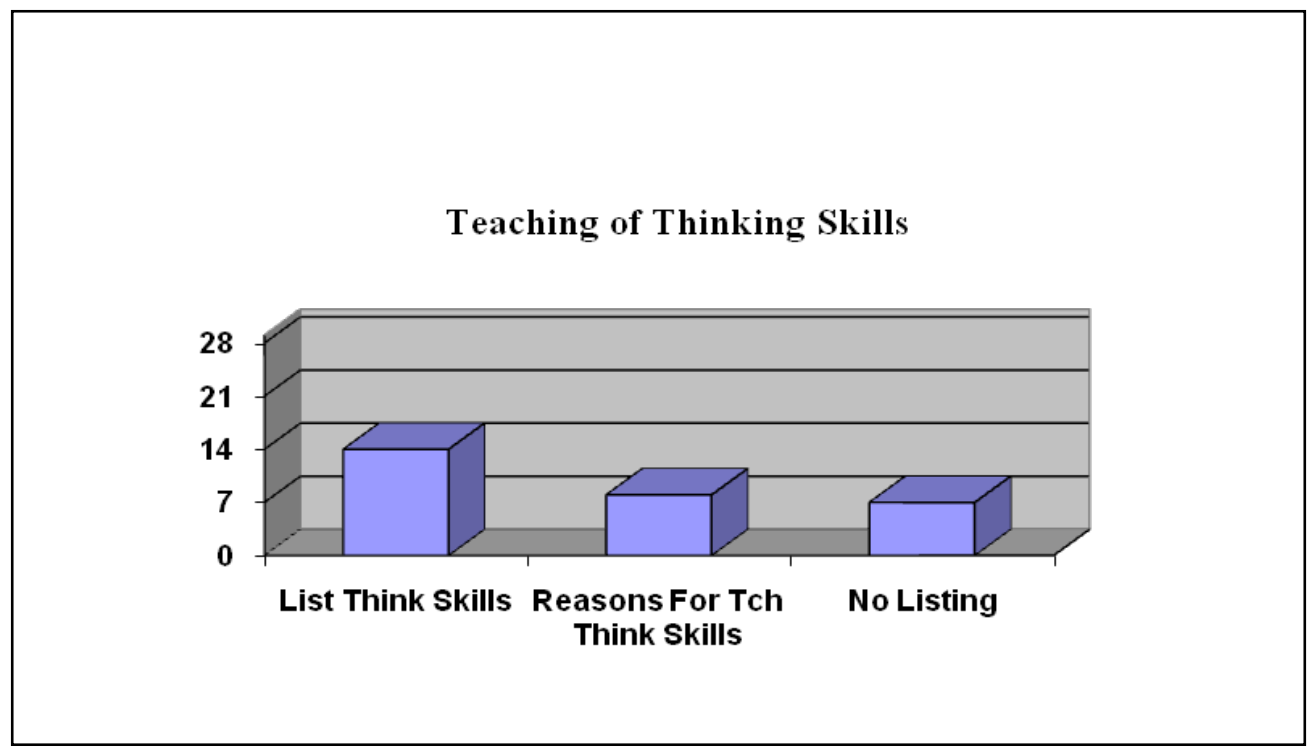

(List Think Skills = Teachers listed thinking skills as needed; Reasons For Tch Think Skills = teachers had specific reasons for teaching relevant thinking skills; No Listing = teachers showed no listing of specific thinking skills taught) 
for teaching thinking skills as they were applied to the particular and relevant content of a topic for student learning, and seven did not list the thinking skills most often used in their discipline. Twenty-six teachers gave clear reasons for how their teaching of thinking skills operated. A small number did not respond to this question.

\section{Rewards for student use of thinking skills}

Teachers used a variety of rewards, from verbal affirmation to lollies (confectionery), when students displayed applied thinking skills within the classroom experience. These data are reported in Table 4.20. Two teachers (2/33) gave lollies as reward for displaying the use of applied thinking skills; however, twenty teachers (20/33) preferred verbal rewards as a means of showing approval for successful use of thinking skills. Nine others (9/33) concentrated on what they termed visible and lasting rewards for the display of successful thinking skills in class. Three teachers (3/33) did not specify the particular rewards, if any, that they used.

TABLE 4.20 VARIETY OF REASONING BEHIND AFFIRMATIONS USED

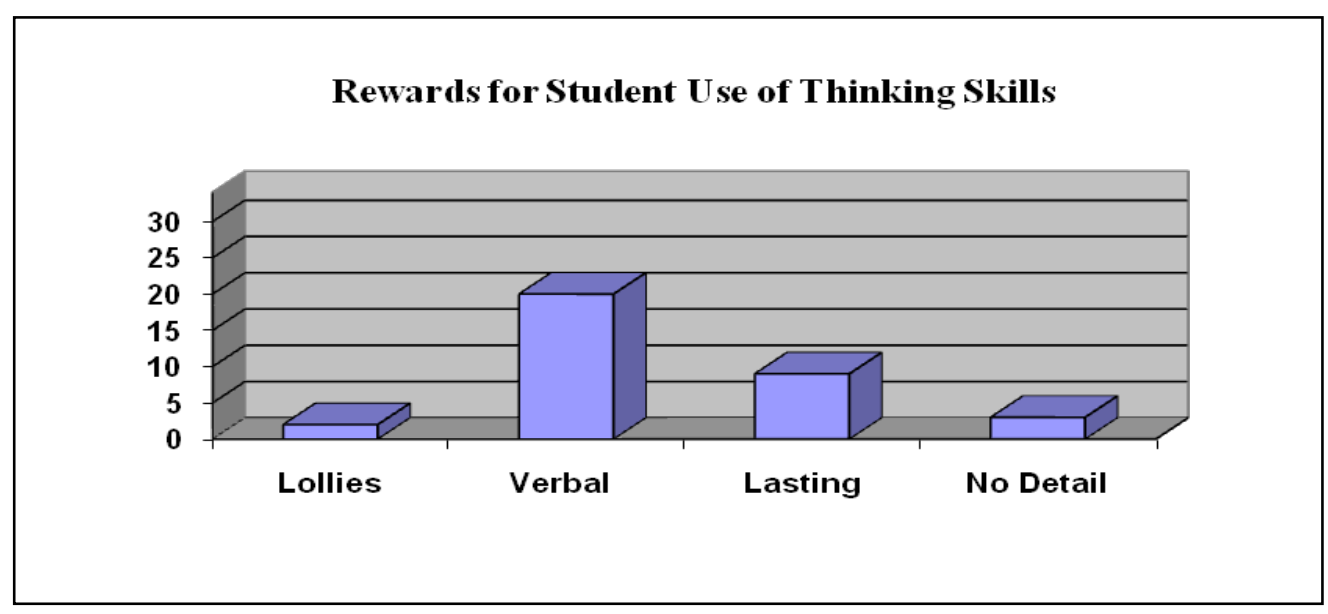

(Lollies = teachers used sweets as a reward for student thinking skills; Verbal = teachers used verbal affirmations for student thinking skills; Lasting = teachers used other affirmation channels like display of Star or other certified affirmations; no detail = teachers did not respond to this question) 
TABLE 4.21 ACTIVITIES BASED ON CURRICULUM AREAS

\begin{tabular}{|c|c|c|c|}
\hline COMMENT & CONSTRUCT & CONCEPT & THEME \\
\hline $\begin{array}{l}\text { English or Religious Studies: Teachers often } \\
\text { started with a question on the board, after which } \\
\text { they invited written responses before questions } \\
\text { could be formulated. Harriette: Dictate and } \\
\text { expect pupils to give negative or positive } \\
\text { comment - give suggestions only, collect } \\
\text { statements and discuss views. }\end{array}$ & Provide a question & $\begin{array}{l}\text { Invite a } \\
\text { written } \\
\text { response }\end{array}$ & $\begin{array}{l}\text { Student- } \\
\text { centred } \\
\text { responses }\end{array}$ \\
\hline $\begin{array}{l}\text { Science: Teachers provided precise sources like a } \\
\text { fact or an example to stimulate student response. } \\
\text { Jane: I will provide a quote, a fact, object, } \\
\text { example and see how students respond. }\end{array}$ & $\begin{array}{l}\text { Provide a fact or an } \\
\text { example }\end{array}$ & $\begin{array}{l}\text { See how } \\
\text { students } \\
\text { respond }\end{array}$ & $\begin{array}{l}\text { Student- } \\
\text { centred } \\
\text { responses }\end{array}$ \\
\hline $\begin{array}{l}\text { Physical Education: Teachers were more direct } \\
\text { and perhaps physical in their strategies. Pietr: } \\
\text { Pose a question relating to the topic and ask } \\
\text { students to stand in particular spots depending on } \\
\text { their point of view they were expected to discuss } \\
\text { their reasons. }\end{array}$ & $\begin{array}{l}\text { Teachers employ } \\
\text { direct, physical } \\
\text { stimuli }\end{array}$ & $\begin{array}{l}\text { Students } \\
\text { move to a } \\
\text { location; } \\
\text { explain their } \\
\text { reasons for so } \\
\text { doing }\end{array}$ & $\begin{array}{l}\text { Student- } \\
\text { centred } \\
\text { responses }\end{array}$ \\
\hline $\begin{array}{l}\text { Art, Design and Technology: Visual stimuli } \\
\text { were used to begin the project or study. Susan: I } \\
\text { always present the work as a problem so students } \\
\text { are encouraged to think. }\end{array}$ & Visual stimuli used & $\begin{array}{l}\text { Present the } \\
\text { work as a } \\
\text { problem }\end{array}$ & $\begin{array}{l}\text { Student- } \\
\text { centred } \\
\text { thinking }\end{array}$ \\
\hline
\end{tabular}

\section{Activities and rewards (qualitative)}

Four curriculum areas were represented in the individual responses: Art, Design and Technology; English; Physical Education; and Religious Studies. Once again, the themes emphasised a student-centred orientation. The details are contained in Table 4.21.

\section{Rewards}

Teachers displayed their recognition of the use of thinking skills within the classroom in various ways. I found that five themes emerged: collaborative reinforcement; reinforcement of student-centred thinking; use of rewards systems; the closely-related teacher-directed reinforcement; and a single recognition that teacher-directed reinforcement needed to be included in the individual teacher’s repertoire. These details are contained in Table 4.22. 
TABLE 4.22 REINFORCEMENT STRATEGIES AND APPROACHES

\begin{tabular}{|c|c|c|c|}
\hline COMMENT & CONSTRUCT & CONCEPT & THEME \\
\hline $\begin{array}{l}\text { Samantha: Always use positive } \\
\text { reinforcement both inside the classroom and } \\
\text { beyond; communicate collaboratively at all } \\
\text { times with staff and students. }\end{array}$ & $\begin{array}{l}\text { Positive } \\
\text { reinforcement }\end{array}$ & $\begin{array}{l}\text { Collaborative } \\
\text { learning }\end{array}$ & \multirow{2}{*}{$\begin{array}{l}\text { Collaborative } \\
\text { reinforcement }\end{array}$} \\
\hline $\begin{array}{l}\text { Giovanni: Engages with their Constructs - } \\
\text { set hypotheticals, mark work and provide } \\
\text { comments and challenges. }\end{array}$ & $\begin{array}{l}\text { Engages with } \\
\text { students Constructs }\end{array}$ & $\begin{array}{l}\text { Recognition of } \\
\text { students } \\
\text { thinking skills }\end{array}$ & \\
\hline $\begin{array}{l}\text { George: I don't think I reward, except for } \\
\text { simple praise like Well done! Good } \\
\text { question. Great answer. }\end{array}$ & Simple praise used & $\begin{array}{l}\text { Recognition of } \\
\text { students } \\
\text { thinking skills }\end{array}$ & \multirow{3}{*}{$\begin{array}{l}\text { Student- } \\
\text { centred } \\
\text { thinking } \\
\text { reinforced }\end{array}$} \\
\hline $\begin{array}{l}\text { Paul: You raise an interesting point. That } \\
\text { opens up another area to explore. Yes, there } \\
\text { are scholars/researchers who have a similar } \\
\text { view. }\end{array}$ & $\begin{array}{l}\text { Using language as } \\
\text { reinforcement }\end{array}$ & $\begin{array}{l}\text { Recognition of } \\
\text { students } \\
\text { thinking skills }\end{array}$ & \\
\hline $\begin{array}{l}\text { Bernard: Make the student aware of how } \\
\text { valuable applied thinking is. }\end{array}$ & $\begin{array}{l}\text { Valuing applied } \\
\text { thinking }\end{array}$ & $\begin{array}{l}\text { Recognition of } \\
\text { students } \\
\text { thinking skills }\end{array}$ & \\
\hline $\begin{array}{l}\text { Portia: Encourages; say positive comments; } \\
\text { give stickers to the quiet ones. }\end{array}$ & Teacher approval & $\begin{array}{l}\text { Encourage } \\
\text { quieter students }\end{array}$ & \multirow{6}{*}{$\begin{array}{l}\text { Rewards } \\
\text { systems }\end{array}$} \\
\hline $\begin{array}{l}\text { Kerri: Uses positive reinforcement because } \\
\text { it works to encourage the quieter students to } \\
\text { participate. }\end{array}$ & $\begin{array}{l}\text { Positive } \\
\text { reinforcement }\end{array}$ & $\begin{array}{l}\text { Encourage } \\
\text { quieter students }\end{array}$ & \\
\hline $\begin{array}{l}\text { Michael: This year I have used a merit page } \\
\text { for students who make good use of thinking } \\
\text { skills ... [I] had a system, whereby students } \\
\text { were rewarded with snakes [i.e.. lollies] for } \\
\text { displaying thinking skills. }\end{array}$ & $\begin{array}{l}\text { Merit page for good } \\
\text { thinking skills }\end{array}$ & Rewards system & \\
\hline Franco: A smile; a reward; class accolade. & Simple approach & $\begin{array}{l}\text { Smile, reward, } \\
\text { class accolade }\end{array}$ & \\
\hline $\begin{array}{l}\text { Four other teachers identified teacher } \\
\text { approval: Hyacinth, Sandy, Lavenda, and } \\
\text { Maryanne. }\end{array}$ & Teacher approval & $\begin{array}{l}\text { Teacher } \\
\text { approval }\end{array}$ & \\
\hline $\begin{array}{l}\text { Five teachers used teacher praise, namely, } \\
\text { Dennis, Frank, Connie, Magda, and } \\
\text { Harriette. }\end{array}$ & Teacher praise & Teacher praise & \\
\hline $\begin{array}{l}\text { Olag: There is a sequence. The teacher } \\
\text { plans, predicts, co-opts, and affirms; then } \\
\text { students are influenced, taught, and become } \\
\text { efficient learners. }\end{array}$ & Learning sequence & $\begin{array}{l}\text { Collaborative } \\
\text { learning }\end{array}$ & \multirow{4}{*}{$\begin{array}{l}\text { Teacher- } \\
\text { directed } \\
\text { reinforcement }\end{array}$} \\
\hline Paula: Uses excited teacher approval & Teacher approval & Excited response & \\
\hline $\begin{array}{l}\text { Samantha: }[\mathrm{I}] \text { avoid rejection; promoting } \\
\text { resilience. We want to inspire students to be } \\
\text { the best they can possibly be. }\end{array}$ & Promote resilience & $\begin{array}{l}\text { Inspiring } \\
\text { student } \\
\text { behaviour }\end{array}$ & \\
\hline $\begin{array}{l}\text { June: Acknowledges students in class and } \\
\text { asks them to read great answers; photocopy } \\
\text { their responses for other class members. }\end{array}$ & Teacher approval & $\begin{array}{c}\text { Positive } \\
\text { acknowledge- } \\
\text { ment }\end{array}$ & \\
\hline $\begin{array}{l}\text { Susan: [I] always display the work at the } \\
\text { end; hold it up to talk about; [I] read out the } \\
\text { good research or provide copies for others } \\
\text { (with permission). }\end{array}$ & $\begin{array}{l}\text { Displays and } \\
\text { discusses } \\
\text { stimulating, and } \\
\text { challenging tasks }\end{array}$ & $\begin{array}{c}\text { Positive } \\
\text { acknowledgeme } \\
\text { nt }\end{array}$ & \multirow{2}{*}{$\begin{array}{l}\text { Teacher- } \\
\text { directed } \\
\text { reinforcement }\end{array}$} \\
\hline $\begin{array}{l}\text { Vaughan: Teacher approval and further use } \\
\text { of the strategies used. }\end{array}$ & Teacher approval & $\begin{array}{l}\text { Positive } \\
\text { reinforcement }\end{array}$ & \\
\hline
\end{tabular}




\begin{tabular}{|c|c|c|c|}
\hline COMMENT & CONSTRUCT & CONCEPT & THEME \\
\hline $\begin{array}{l}\text { Terence: A three-point approach of positive } \\
\text { affirmation of opinions given; avoidance of } \\
\text { putdowns and always looking to build; } \\
\text { occasional note in diary, though rare. }\end{array}$ & $\begin{array}{l}\text { Three-point } \\
\text { approach }\end{array}$ & $\begin{array}{c}\text { Positive } \\
\text { reinforcement }\end{array}$ & \\
\hline $\begin{array}{l}\text { Pietr: The inclusion of more demanding and } \\
\text { stimulating tasks that bring extra } \\
\text { responsibility and challenge. }\end{array}$ & $\begin{array}{l}\text { Demanding, } \\
\text { stimulating, and } \\
\text { challenging tasks }\end{array}$ & $\begin{array}{l}\text { Responsibility } \\
\text { and challenge }\end{array}$ & \\
\hline $\begin{array}{l}\text { Samantha: Big-picture framework } \\
\text { Teamwork, risk taking in terms of } \\
\text { contribution, individualism becoming } \\
\text { evident, etc. }\end{array}$ & $\begin{array}{l}\text { Big-picture thinking } \\
\text { rewarded }\end{array}$ & $\begin{array}{l}\text { Teaming, risk } \\
\text { taking, } \\
\text { contributing, } \\
\text { personal views }\end{array}$ & \\
\hline $\begin{array}{l}\text { Rose: Something I perhaps need to work on } \\
\text { - I expect my students to think and thus } \\
\text { probably do not adequately reward students } \\
\text { display of thinking skills. }\end{array}$ & Lacking in rewards & $\begin{array}{l}\text { Change in } \\
\text { teacher } \\
\text { behaviour } \\
\text { required }\end{array}$ & $\begin{array}{c}\text { Teacher- } \\
\text { directed } \\
\text { reinforcement } \\
\text { needed }\end{array}$ \\
\hline
\end{tabular}

\section{My researcher's voice}

To view the teachers responses overall, it seems that immediate and positive responses are the intention of the teacher, but it is not clear to me that the students would always view the above responses within the table as enough. Teacher-directed reinforcement and promotion of individual work needs to be coupled with student understanding of the value of student-demonstrated new learning.

\section{Language and democratic paradigms}

My analysis of the various responses showed that the language pertaining to each given subject is democratic in nature as well as paradigmatic in content. The themes that emerged, contained in Table 4.23, were as follows: democracy in the classroom; flexible thinking environment; guiding student learning paradigm; personal empowerment; philosophical paradigm; writing as a thinking tool. 
TABLE 4.23 DEMOCRATIC AND PARADIGMATIC LANGUAGE

\begin{tabular}{|c|c|c|c|}
\hline COMMENT & CONSTRUCT & CONCEPT & THEME \\
\hline $\begin{array}{l}\text { George: specific about expecting his students to } \\
\text { keep an open mind; use different perspectives; } \\
\text { allow for shades of grey. }\end{array}$ & $\begin{array}{l}\text { Maintaining open } \\
\text { minds, taking } \\
\text { different } \\
\text { perspectives. }\end{array}$ & $\begin{array}{l}\text { Allow for } \\
\text { shades of grey }\end{array}$ & \multirow{3}{*}{$\begin{array}{l}\text { Democracy in } \\
\text { the classroom }\end{array}$} \\
\hline $\begin{array}{l}\text { Sandy: uses lots of questioning - open-ended and } \\
\text { problem-solving ... [I] provide analytical } \\
\text { strategies ... [we do] ... a lot of group work with } \\
\text { focus questions. }\end{array}$ & $\begin{array}{l}\text { Group work with } \\
\text { focus questions }\end{array}$ & $\begin{array}{l}\text { Analytical } \\
\text { strategies } \\
\text { applied }\end{array}$ & \\
\hline $\begin{array}{l}\text { Henry: I challenge individual perceptions. } \\
\text { Education must be about more than knowledge! }\end{array}$ & $\begin{array}{l}\text { Challenges } \\
\text { individual } \\
\text { perceptions }\end{array}$ & $\begin{array}{c}\text { Broadens } \\
\text { expectations }\end{array}$ & \\
\hline $\begin{array}{l}\text { Pietr (Physical Education classes): talked about } \\
\text { self-knowledge and creativity, problem-solving } \\
\text { and guided discovery. }\end{array}$ & $\begin{array}{l}\text { Higher order } \\
\text { psycho-motor } \\
\text { focus }\end{array}$ & $\begin{array}{c}\text { Broadens } \\
\text { expectations }\end{array}$ & \multirow{6}{*}{$\begin{array}{l}\text { Democracy in } \\
\text { the classroom }\end{array}$} \\
\hline $\begin{array}{l}\text { Olag (Music classes): lists and expects leadership } \\
\text { skills, predicting skills, synthesising skills, } \\
\text { analytical skills, cooperation skills for his } \\
\text { students. }\end{array}$ & $\begin{array}{l}\text { Stresses high } \\
\text { higher order } \\
\text { cognitive and } \\
\text { affective skills }\end{array}$ & $\begin{array}{l}\text { Broadens } \\
\text { viewpoints }\end{array}$ & \\
\hline $\begin{array}{l}\text { Samantha: uses thematic approaches, linear } \\
\text { development of the topic and inquiry-based } \\
\text { learning to enhance the opportunities for learning. }\end{array}$ & $\begin{array}{l}\text { Broadening } \\
\text { approaches }\end{array}$ & $\begin{array}{l}\text { Enhancing } \\
\text { classroom } \\
\text { opportunities }\end{array}$ & \\
\hline $\begin{array}{l}\text { George: I ask questions/give information/tell } \\
\text { stories/give counter arguments. I monitor interest } \\
\ldots \text { then modify my approach. }\end{array}$ & Variety of stimuli & $\begin{array}{l}\text { Flexible in } \\
\text { approach }\end{array}$ & \\
\hline $\begin{array}{l}\text { Susan (Art, Craft and Design Technology): talked } \\
\text { about expecting students to investigate/design/ } \\
\text { produce/ evaluate their work. }\end{array}$ & $\begin{array}{l}\text { Investigating, } \\
\text { designing, } \\
\text { producing and } \\
\text { evaluating work. }\end{array}$ & $\begin{array}{c}\text { Lifting } \\
\text { expectations } \\
\text { in the } \\
\text { classroom }\end{array}$ & \\
\hline $\begin{array}{l}\text { Bernard: provided a careful slant on democracy } \\
\text { as he talked about the freedom to fail; freedom to } \\
\text { create; put things in perspective. }\end{array}$ & $\begin{array}{l}\text { Freedom to fail; } \\
\text { freedom to create }\end{array}$ & $\begin{array}{l}\text { Maintaining a } \\
\text { democratic } \\
\text { perspective }\end{array}$ & \\
\hline $\begin{array}{l}\text { Terence: talked about questions, flexibility and } \\
\text { causing a positive environment for thinking. }\end{array}$ & $\begin{array}{l}\text { Questions, } \\
\text { flexibility, positive } \\
\text { environments }\end{array}$ & $\begin{array}{l}\text { A positive } \\
\text { thinking } \\
\text { environment }\end{array}$ & \multirow{3}{*}{$\begin{array}{c}\text { Flexible } \\
\text { thinking } \\
\text { environment }\end{array}$} \\
\hline $\begin{array}{l}\text { George: I can go either way depending on the } \\
\text { level of interest. }\end{array}$ & Level of interest & $\begin{array}{l}\text { Democratic } \\
\text { or } \\
\text { paradigmatic }\end{array}$ & \\
\hline $\begin{array}{l}\text { Vaughan: looks at use of lateral thinking, } \\
\text { embedding thinking skills within the unit for } \\
\text { deeper meaning }\end{array}$ & $\begin{array}{l}\text { Encouraging and } \\
\text { embedding lateral } \\
\text { thinking }\end{array}$ & $\begin{array}{l}\text { Looking for } \\
\text { deeper } \\
\text { meanings }\end{array}$ & \\
\hline $\begin{array}{l}\text { Dennis (senior English): uses questions and } \\
\text { answer, group discussion, guided reading. }\end{array}$ & $\begin{array}{l}\text { Question and } \\
\text { answer as } \\
\text { methodology }\end{array}$ & $\begin{array}{l}\text { Pedagogical } \\
\text { method }\end{array}$ & $\begin{array}{c}\text { Guiding } \\
\text { student } \\
\text { learning } \\
\text { paradigm }\end{array}$ \\
\hline $\begin{array}{l}\text { Hyacinth: The Community of Inquiry provides a } \\
\text { framework for teaching any topic, but is } \\
\text { especially helpful in teaching a literary text. It } \\
\text { takes students out of their traditional roles and } \\
\text { develops their thinking in a different environment } \\
\text { and in a different way. They take control of their } \\
\text { thinking and generate their learning. It removes } \\
\text { dependence on teacher as a fountain of knowledge }\end{array}$ & $\begin{array}{l}\text { Liberating students } \\
\text { to learn and think. }\end{array}$ & $\begin{array}{l}\text { Encourages } \\
\text { personal } \\
\text { responsibility } \\
\text { for students } \\
\text { learning. }\end{array}$ & $\begin{array}{c}\text { Personal } \\
\text { empowerment }\end{array}$ \\
\hline
\end{tabular}




\begin{tabular}{|c|c|c|c|}
\hline COMMENT & CONSTRUCT & CONCEPT & THEME \\
\hline $\begin{array}{l}\text { Giovanni (English and Drama): used topic and } \\
\text { issue questions for continual response to written } \\
\text { material - read/ say/ write, what they think. }\end{array}$ & $\begin{array}{l}\text { Read, say, write } \\
\text { what they think }\end{array}$ & $\begin{array}{l}\text { Continual } \\
\text { response to } \\
\text { written } \\
\text { material }\end{array}$ & \multirow{3}{*}{$\begin{array}{c}\text { Philosophical } \\
\text { paradigm }\end{array}$} \\
\hline $\begin{array}{l}\text { Paul (English, German and History): talked about } \\
\text { questions to which there is no obvious answer. He } \\
\text { expected students to: evaluate, make abstractions, } \\
\text { and use generalisations and to associate. }\end{array}$ & $\begin{array}{l}\text { Questions with no } \\
\text { obvious answers }\end{array}$ & $\begin{array}{c}\text { Problem } \\
\text { solving }\end{array}$ & \\
\hline $\begin{array}{l}\text { Clint (NZ expert): highlights that the teacher } \\
\text { needs to be focused on student thinking processes, } \\
\text { not the correct answer (his underlining. }\end{array}$ & $\begin{array}{l}\text { Focus on student } \\
\text { thinking processes }\end{array}$ & $\begin{array}{c}\text { Process over } \\
\text { product }\end{array}$ & \\
\hline $\begin{array}{l}\text { Giovanni (English and Drama), Always respect } \\
\text { others right to have an opinion. Always support } \\
\text { your opinion with a rational argument. }\end{array}$ & $\begin{array}{l}\text { Respect opinions } \\
\text { of others }\end{array}$ & $\begin{array}{l}\text { Support } \\
\text { opinion with } \\
\text { rational } \\
\text { argument }\end{array}$ & \multirow{3}{*}{$\begin{array}{c}\text { Philosophical } \\
\text { paradigm }\end{array}$} \\
\hline $\begin{array}{l}\text { Roger (UK expert), highlights the breadth and } \\
\text { range of the philosophical nature of thinking and } \\
\text { the value of questions, creating radical thinking. }\end{array}$ & Value of questions & $\begin{array}{l}\text { The } \\
\text { philosophical } \\
\text { nature of } \\
\text { thinking }\end{array}$ & \\
\hline $\begin{array}{l}\text { Rose (Biology and Science): talked about } \\
\text { questions that involved Venn diagrams, concept } \\
\text { mapping, debates, fish bowl group work, } \\
\text { continuums, open-ended questions, argumentative } \\
\text { essays, POE (predict, observe, explain); word } \\
\text { posters, deep thinking challenges. }\end{array}$ & $\begin{array}{l}\text { Deep thinking } \\
\text { challenges }\end{array}$ & $\begin{array}{l}\text { Variety of } \\
\text { processes }\end{array}$ & \\
\hline $\begin{array}{l}\text { Jane (Psychology, Biology, English or Life } \\
\text { Skills): I encourage students to stop and write } \\
\text { what they mean/ want to say. Sometimes students } \\
\text { speak before thinking. Writing makes them pause } \\
\text { longer to think. }\end{array}$ & $\begin{array}{l}\text { Writing what they } \\
\text { mean or want to } \\
\text { say }\end{array}$ & $\begin{array}{l}\text { Pausing } \\
\text { before } \\
\text { responding }\end{array}$ & $\begin{array}{l}\text { Writing as a } \\
\text { thinking tool }\end{array}$ \\
\hline
\end{tabular}

Comments from various teachers featuring in this table are ethical in tone and involve self-reflection which is to be promoted and admired. In varying degrees, I was impressed by the teachers who exhibit an intention towards real democracy as experienced by students within the classroom; there is effort aimed at promoting a flexible thinking environment; some effort is expended in a democratic strategy to guide student learning as a daily practice so that personal empowerment is a real part of the student experience; some attention is paid in varying degrees also to the construct if not the full practice of a philosophical paradigm underpinning pedagogical planning and delivery; more could be done to promote the activity of writing as a thinking tool.

I found the evidence of diversity in approach to be exciting; it is a possibility of understanding each other's methods and pedagogical practices 
that provide development of technical strategies and techniques worthy of a democratic, thinking classroom atmosphere conducive to rich learning.

\section{Reasons for, and perceptions of, thinking skills}

There are various approaches to the concept of the teaching of thinking skills. Some teachers perceive the whole construct as embedded in their particular subject or domain area, and can see no reason for instituting a discrete category of teaching that is thinking skills. Others can see some reasons for a pronounced set of strategies for the teaching of thinking skills, seemingly happy to make this aim part of their philosophical approach, but not necessarily an explicit goal within their teaching pattern for their own area of expertise in education.

In my analysis of the extensive responses made to the question relating to thinking skills, eight themes emerged: corollary to creativity; developing a personal style; facilitating better learning; independence; life skills; moral and ethical behaviour; relevant thinking skills; required core skills. The details relating to these themes are contained in Table 4.24.

TABLE 4.24 REASONS AND PERCEPTIONS OF THINKING SKILLS

\begin{tabular}{|c|c|c|c|}
\hline COMMENT & CONSTRUCT & CONCEPT & THEME \\
\hline $\begin{array}{l}\text { Paul (Language and History): Questioning is } \\
\text { fundamental to understanding, but also to } \\
\text { extension of learning. I believe it is a corollary } \\
\text { of creativity. }\end{array}$ & $\begin{array}{l}\text { Fundamental to } \\
\text { understanding }\end{array}$ & $\begin{array}{l}\text { Essential in } \\
\text { learning }\end{array}$ & \multirow{3}{*}{$\begin{array}{c}\text { Corollary to } \\
\text { Creativity }\end{array}$} \\
\hline $\begin{array}{l}\text { Portia (Maths and Humanities): Thinking skills } \\
\text { encourages active participation, builds and } \\
\text { expands on Constructs. It also encourages } \\
\text { creative thinking. }\end{array}$ & Active participation & \multirow[b]{2}{*}{$\begin{array}{c}\text { Expanding on } \\
\text { Constructs }\end{array}$} & \\
\hline $\begin{array}{l}\text { Paula (Physical Education and Health/Human } \\
\text { Development), in reference to Year } 12 \text { students: } \\
\text { It is often easy to respond as expected - if you } \\
\text { can respond to a question you weren't expecting } \\
\text { then you are really thinking and also going over } \\
\text { the work you know. }\end{array}$ & $\begin{array}{l}\text { Unexpected } \\
\text { responses significant }\end{array}$ & & \\
\hline $\begin{array}{l}\text { June: clarity of approach for her Art students is } \\
\text { obvious: Theory - the analysis of art works and } \\
\text { visual communication is a major component in } \\
\text { the VCE subjects, so I teach these skills. Prac. } \\
\text { Work - the development of personal styles } \\
\text { through art must be student driven - again for } \\
\text { interest as well as to meet criteria at Year } 12 \text {. }\end{array}$ & $\begin{array}{l}\text { Combining theory } \\
\text { and practice }\end{array}$ & $\begin{array}{l}\text { Developing } \\
\text { analytical } \\
\text { skills }\end{array}$ & $\begin{array}{c}\text { Developing } \\
\text { a personal } \\
\text { style }\end{array}$ \\
\hline
\end{tabular}




\begin{tabular}{|c|c|c|c|}
\hline COMMENT & CONSTRUCT & CONCEPT & THEME \\
\hline $\begin{array}{l}\text { Rose (Biology): [It] engages students in their } \\
\text { own learning and is thus more effective. Enables } \\
\text { deconstructing and clarifying of previously } \\
\text { constructed concepts and facilitates better } \\
\text { learning as concepts (are) processed into long } \\
\text { term memory. }\end{array}$ & $\begin{array}{l}\text { Engages students in } \\
\text { their own learning }\end{array}$ & $\begin{array}{l}\text { Highlighting } \\
\text { the theoretical } \\
\text { perspective: }\end{array}$ & $\begin{array}{c}\text { Facilitating } \\
\text { better } \\
\text { learning }\end{array}$ \\
\hline $\begin{array}{l}\text { Susan (Design and Technology): It gives } \\
\text { students confidence to believe in themselves and } \\
\text { their ability to come up with Constructs. It } \\
\text { provides a great variety of solutions that keep all } \\
\text { students enthused and excited about thinking } \\
\text { and learning. }\end{array}$ & $\begin{array}{l}\text { Developing self- } \\
\text { belief and } \\
\text { confidence }\end{array}$ & $\begin{array}{l}\text { Independence } \\
\text { in learning } \\
\text { and thinking }\end{array}$ & $\begin{array}{c}\text { Independenc } \\
\text { e }\end{array}$ \\
\hline $\begin{array}{l}\text { Pietr makes clear that students gain a deeper } \\
\text { understanding as to the processes required to } \\
\text { develop non-physical as well as physical skills. } \\
\text { They can then apply this knowledge to other } \\
\text { topics and improve their performance. }\end{array}$ & $\begin{array}{l}\text { Deeper } \\
\text { understanding of } \\
\text { physical and non- } \\
\text { physical processes }\end{array}$ & $\begin{array}{c}\text { Application of } \\
\text { knowledge }\end{array}$ & \multirow{5}{*}{ Life skills } \\
\hline $\begin{array}{l}\text { Terence (Humanities): shows a realistic } \\
\text { approach to a long-term goal: Students in } \\
\text { middle school view their educational } \\
\text { experiences from a particular exponential } \\
\text { position that can have, sometimes, created a } \\
\text { dependence on being informed and a reliance on } \\
\text { special outcomes dictated by schools. Whilst } \\
\text { such are important, I hope to rekindle the } \\
\text { interest in finding out for one's-self as much as } \\
\text { possible. }\end{array}$ & $\begin{array}{l}\text { Finding out for } \\
\text { one's-self }\end{array}$ & \multirow[t]{2}{*}{$\begin{array}{c}\text { Becoming } \\
\text { independent } \\
\text { learners }\end{array}$} & \\
\hline $\begin{array}{l}\text { Magda (Languages, SOSE, Religion studies, } \\
\text { and Music in Australia and England): [I want] to } \\
\text { help pupils realise that they are responsible for } \\
\text { their own learning, that they can work out where } \\
\text { they fall short, and find a way to fix it. Life } \\
\text { Skills! }\end{array}$ & $\begin{array}{l}\text { Engages students in } \\
\text { their own learning }\end{array}$ & & \\
\hline $\begin{array}{l}\text { Giovanni (English): English is about getting } \\
\text { students to develop their Constructs and to be } \\
\text { able to communicate them in a variety of styles } \\
\text { - you need to be able to do this to cope with life. }\end{array}$ & $\begin{array}{l}\text { Developing and } \\
\text { communicating } \\
\text { Constructs }\end{array}$ & $\begin{array}{l}\text { Coping with } \\
\text { life }\end{array}$ & \\
\hline $\begin{array}{l}\text { Hyacinth (English, administrative leadership, } \\
\text { experience in Welfare and Social Work): } \\
\text { Thinking skills not only make students perform } \\
\text { better academically but equips them for life. It } \\
\text { takes away the high emphasis we place on } \\
\text { emotional thinking. It helps to develop in } \\
\text { students a thinking framework in which they can } \\
\text { operate. }\end{array}$ & $\begin{array}{l}\text { Provides a thinking } \\
\text { framework }\end{array}$ & $\begin{array}{c}\text { Enhances } \\
\text { academic } \\
\text { performance }\end{array}$ & \\
\hline $\begin{array}{l}\text { Samantha: (Legal Studies and English): [It is] } \\
\text { to help me and the students evaluate what we } \\
\text { do, teach values and not only talk the talk, but } \\
\text { walk the walk also. }\end{array}$ & $\begin{array}{l}\text { Talk the talk and } \\
\text { walk the walk }\end{array}$ & $\begin{array}{l}\text { Developing } \\
\text { values }\end{array}$ & $\begin{array}{l}\text { Moral and } \\
\text { ethical } \\
\text { behaviour }\end{array}$ \\
\hline $\begin{array}{l}\text { Michael (Maths and Sciences) teaches relevant } \\
\text { thinking skills because they are an effective tool } \\
\text { for successfully negotiating a pathway to } \\
\text { solving problems as well as developing more } \\
\text { robust thinking strategies. }\end{array}$ & $\begin{array}{l}\text { Tools for solving } \\
\text { problems }\end{array}$ & $\begin{array}{l}\text { Providing } \\
\text { pathways to } \\
\text { learning }\end{array}$ & $\begin{array}{c}\text { Relevant } \\
\text { thinking } \\
\text { skills }\end{array}$ \\
\hline
\end{tabular}




\begin{tabular}{|l|l|l|l|}
\hline COMMENT & CONSTRUCT & CONCEPT & THEME \\
\hline A sample of six teachers: & $\begin{array}{l}\text { Required in teaching } \\
\text { of English; relate to } \\
\text { texts studied; } \\
\text { identifies the work } \\
\text { of a historian; used } \\
\text { to provide feedback; } \\
\text { underpin } \\
\text { knowledge; supports } \\
\text { sharing of opinions; } \\
\text { central to final year } \\
\text { assessment. }\end{array}$ & Core skills & $\begin{array}{l}\text { Required } \\
\text { core skills }\end{array}$ \\
\hline
\end{tabular}

It became clear to me that the comments reported in Table 4.24, above, from the six teachers mentioned last, are straightforward in intent; they are reasoned, reflective and educational in tone; they imply conscious delivery of focussed pedagogical approaches; and they are consonant with the overall responses in the Questionnaire and its questions. Teachers' perceptions are crucial to this investigation and are central to my understanding of the initial thesis title as well as being central to the focus of the present title. The constructs are familiar; concepts derived from them are practical and focused on student learning; themes are philosophically connected and consistent with a thrust towards a democratic classroom. How this happens is the underpinning theme of the following chapters.

\section{Teaching the process of decision-making}

Within this theme, three voices are heard: my practitioner voice, the teachers' voices, and the experts’ voices.

\section{My practitioner voice}

In this sub-section, two teaching instruments are explained: my Six Steps of Decision-making and my 'ME' diagram. 


\section{Six steps of decision-making}

The Six Steps of Decision-Making that I have taught are as follows:

1. Define the problem

2. List possible options, no matter how unusual

3. Investigate each possible option carefully

4. Invite expert opinion, advisement and research internet.

5. Choose the best likely option, listing other options in order of preference

6. Carry out the action, then evaluate.

If successful, the problem is solved; if unsuccessful, I always found it necessary to take the students back to whichever stage was needed in the light of new information, and follow the process through once more.

I developed a precise scenario based on the use of the 'ME' diagram which is displayed in Figure 4.3. This crystallised most of the students' responses as they could see that in discussion, much rested on the experience of each class member. Through discussion, it became obvious that students could disagree for their own reasons at times with a general expected consensus. This surprised many students, but became the reason why I first used a brain-storming session to construct the 'ME' diagram as I showed them how to integrate their similarities and their differences and how to appreciate and learn from each other. The eventually complete concept also taught them - and me - just how much potential there was for composing their futures in a positive way. This led to the connections between decisions made for career; for quality of life (their term for philosophical world view); and eventually to the possibility of a secular or religious approach to the meta-questions about the meaning of life generally. Distinctions between the need for impulse or lightning decisions where needed were identified and the need for reflective decisions making use of the Six Steps outlined above became part of our deliberations from Year 8 to Year 12.

Teachers, already, were aware of the content of this diagram. It is in the specific application within the classroom, that I found the impact of the 


\section{FIGURE 4.3 'ME' DIAGRAM}

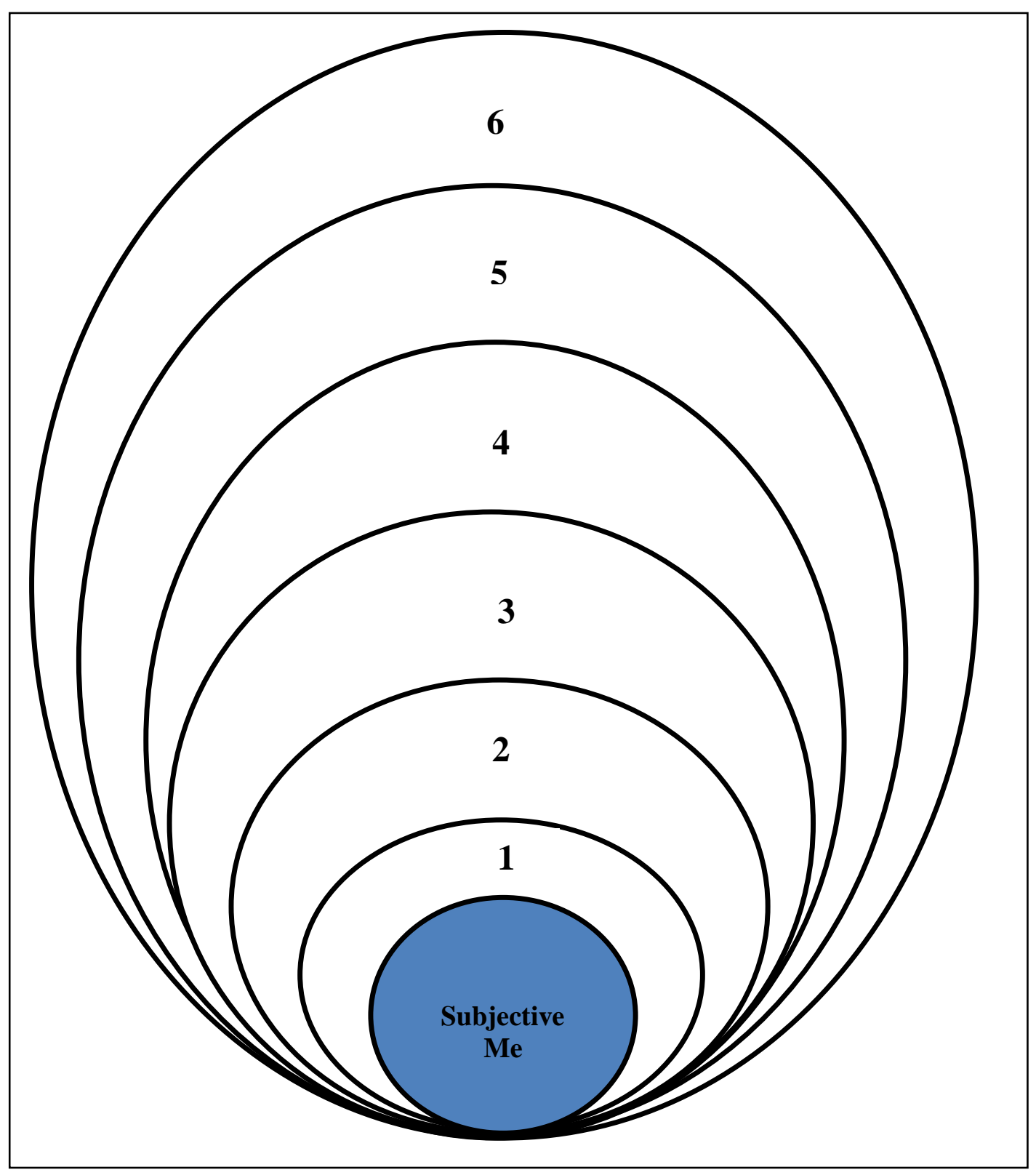

knowledge contained begins to register in the students' mind. The concept of 'ME' appeals to the students and can be inserted in many discussions and attached to many questions about characters in novels, historical figures, geoscience, and historico-political considerations even in business studies. It certainly underpinned the variety and diversity of decision-making in both a personal and community sense. The use of relevant and linking concepts 
were ontological in content; the particular choice of the terms and concepts in each area were epistemological as they conveyed the relevant knowledge and skills required for the successful applied thinking skills and relevant classroom strategies (technical: techné) to provide educational outcomes of value to students through discussion of the Community of Inquiry format and the consequential use of written and oral reflections.

\section{Explanation of the 'ME' concept}

Beginning at the centre point, the teacher, by use of handouts, power point or brainstorming on the board highlights the starting block: the ME in the diagram is each individual student. The democratic element was established immediately by teacher comment that what followed applied in the unique experience of each person listening.

Each ME experiences the first influences, both positive and negative, of the world from the family with its own culture of religious, secular and cultural mores. The tiny baby is situated within its first influences and absorbs and adapts to the small world within which life is lived. The child is totally dependent for succour and survival.

When judged appropriate by parent and carers, the child of today's parents experiences a second set of broader influences in day-care, or with a nanny and eventually in a kindergarten-like setting. Learning about the world becomes a bigger picture, absorbing and adapting to the surrounding influences. The child is assuming gradually the skills to decide and choose in myriad ways as an individual. The long-term result will be the gradual forming of a personal world view, general opinion, or personal perspective.

The third set of influences which impact on the growing child include primary, secondary school influences. These come thick and fast, as the child again absorbs and adapts to new experiences, new possibilities, more friends and a dawning understanding of the complexities that exist in this much broader world where interdependence can be of value. Influences of parental settings are integrated with the much larger world of learning in school. The 
response-ability and responsibility of parents, carers, teachers, and significant others aid in the growing adaptability and new living skills required to absorb and flourish in the expanded world around the individual child. Concurrently, but more randomly, the influences of suburb, town, city or shire and state governmental sources have their impact. The realisation by the individual, that there are more complex circumstances than personal preferences, brings the need to find reasonable responses. The experience of the individual is tempered by the needs and wishes of others, and interdependence and community become part of this experience.

The fourth set of influences that the child discovers sometimes begins to surface as hobbies, friendship clubs, sporting teams and training, school excursions and camps that broaden the child's view of the bigness of the world around them. Learning accelerates, and discernment of real knowledge, distinct from cultural complexity, enters the world of the child's experience. Integrating those experiences and that burgeoning knowledge, including the impact of the broad spectrum of IT allows for the child to begin the process of making distinctions about what to believe, what to recognise as truth, what responses make sense in the world.

The fifth set of influences are often forgotten as the child comes to terms with whatever physical, emotional, social, intellectual and spiritual needs are identified and developed, so that maturity can begin. Whether the child lives in a suburb, a city, a town, or a shire has a specific impact on how life is lived. The district, the State, and even the National arena will have influences at particular times, during which the child needs to integrate political and other influences, absorbing and adapting to the explosions of knowledge. At some distance: international or whole world or global impacts intrude. The student must choose whether or how to respond. Quality of life is constructed throughout this process. This perhaps is globalisation if viewed as a bigpicture diagram. At the opposite end of the spectrum, this would be viewed as the personal picture of the individual facing the future. 
Whichever perspective is assumed, the first choice would be whether to engage in constructing an owned individual future: the options including either to follow a demanding religious paradigm or to allow Fate or the Universal Energy to steer a personal secular future based on impulse, whimsy, optimism, and happiness, commitment, satisfaction. The level of personal responsibility for the student-self will rest on the quality of decision-making and the accompanying skill to self-correct where needed.

Beyond that, as a sixth influence, increasingly there are the influences of a globalised world whose impact at times can be catastrophic. Natural disasters and man-made suffering can impact on the life of that child in a way that is often unseen. Teachers can help with such integration within the various educational disciplines by giving students relevant opportunities in discussion to articulate their concerns, to integrate their responses and to make sense of a very complex world. National, international, universal considerations like climate change, while political in nature, have varying impacts on the child, and discernment of what can be dealt with, and what needs to be left to the experts, is needed. In this way, education is timely, accurate in delivery, allowing for democratic understanding and is a central feature of teachers' array of classroom skills.

\section{Teachers' voices}

In the description of how the teachers do actually teach decision-making in their classroom pedagogies, respondents have given further detail of the use of questions and questioning. This is the applied science of teaching thinking skills, embedded in the classroom strategies and management especially of contentious topics.

\section{Teachers' responses (quantitative)}

The responses of 33 teachers are reported in Table 4.25. Twenty-four teachers stated that they do NOT teach decision-making skills - although some seemed to relate this approach to their subject area, rather than 
TABLE 4.25 TEACHING OF DECISION-MAKING SKILLS



(Teach Decision Making = teachers do specifically teach decision-making skills; Do Not Teach Decision Making = teachers have not specifically taught decision-making as part of the curriculum; No Details = teachers have given no details about whether they do teach decision making or otherwise)

generalise the skills. Three were the experts whose knowledge and experience highlight how they teach questioning and how they extend this to finding answers and conclusions. Four teachers stated that they do not teach decision-making skills as a discrete educational aim. Five teachers did not specifically state either way.

\section{Teachers' responses (qualitative)}

I found that the strongest theme to emerge from the responses related to achieving personal mastery, a lesser theme related to algorithmic thinking; and there was a single reference to values clarification. These data are summarised in Table 4.26. 
TABLE 4.26 TEACHING THE PROCESSES OF DECISION-MAKING

\begin{tabular}{|c|c|c|c|}
\hline COMMENT & CONSTRUCT & CONCEPT & THEME \\
\hline $\begin{array}{l}\text { Terence: uses a similar approach: Decision- } \\
\text { making can take many forms. I would } \\
\text { endeavour to develop decision-making skills } \\
\text { through the avoiding direct answers to questions } \\
\text { by reflecting the question back to the student to } \\
\text { formulate and develop his/her own responses } \\
\text { and become accountable for their own } \\
\text { Constructs and indeed actions. }\end{array}$ & $\begin{array}{l}\text { Reflect the question } \\
\text { back ... formulate their } \\
\text { own responses ... } \\
\text { become accountable } \\
\text { for their own } \\
\text { Constructs and actions }\end{array}$ & $\begin{array}{l}\text { Avoid direct } \\
\text { answers }\end{array}$ & \multirow{7}{*}{$\begin{array}{l}\text { Achieving } \\
\text { personal } \\
\text { autonomy }\end{array}$} \\
\hline $\begin{array}{l}\text { Jane (Psychology, Biology, English and Life } \\
\text { Skills): I like to encourage students to make } \\
\text { decisions as to how they might manage their } \\
\text { time, a task or a situation with their peers. If a } \\
\text { student approaches me to say they could not } \\
\text { hand in their homework because it isn't finished, } \\
\text { I ask them firstly to think about what decisions I } \\
\text { might then have to make my assessment and } \\
\text { why. I then ask them to think about what } \\
\text { decisions they made which lead them to end up } \\
\text { having to tell me the work was incomplete. } \\
\text { Finally we then generate a list of possible } \\
\text { alternative strategies/decisions for them which } \\
\text { may then influence how/if I will assess the work } \\
\text { when they submit it. }\end{array}$ & $\begin{array}{l}\text { Encouraging students } \\
\text { to manage their time, } \\
\text { task or situation with } \\
\text { their peers. }\end{array}$ & $\begin{array}{c}\text { Considering } \\
\text { the } \\
\text { consequences }\end{array}$ & \\
\hline $\begin{array}{l}\text { Samantha (Legal studies): The discussion } \\
\text { might be about Refugees. Perhaps a student has } \\
\text { a racist or insular response. Control the } \\
\text { discussion by ensuring that everyone takes their } \\
\text { turn and does not become heated. Intervene } \\
\text { when necessary with Legal and Human Rights } \\
\text { information - provide a structured debate with } \\
\text { evidence. Don't allow bullying. Counsel and } \\
\text { teach students to breathe, wait and cope. }\end{array}$ & $\begin{array}{l}\text { Control the discussion } \\
\ldots . . \text { structure the debate } \\
\text { with evidence ... } \\
\text { breathe, wait and } \\
\text { cope. }\end{array}$ & $\begin{array}{c}\text { Control the } \\
\text { discussion } \\
\text { and intervene } \\
\text { when } \\
\text { necessary }\end{array}$ & \\
\hline $\begin{array}{l}\text { Giovanni: Writing an essay involves decisions } \\
\text { i.e.: what will I put in each paragraph? Their } \\
\text { written work doesn't involve RIGHT } \\
\text { ANSWERS; it involves them CHOOSING what } \\
\text { to include (his emphasis). }\end{array}$ & $\begin{array}{l}\ldots \text { it involves them } \\
\text { choosing what to } \\
\text { include. }\end{array}$ & \multirow{4}{*}{$\begin{array}{c}\text { Intuitive } \\
\text { decision- } \\
\text { making } \\
\text { encouraged }\end{array}$} & \\
\hline $\begin{array}{l}\text { Dennis: Of those teachers who stated they did } \\
\text { not teach decision-making, for instance, says: I } \\
\text { expect a lot of decision-making to be made. I } \\
\text { don't specifically teach it. }\end{array}$ & $\begin{array}{l}\text { I don't specifically } \\
\text { teach [decision } \\
\text { making]. }\end{array}$ & & \\
\hline $\begin{array}{l}\text { Hyacinth (English teacher): description of a } \\
\text { decision-making process is clear: In Literature } \\
\text { circles, students need to decide the various roles } \\
\text { they will undertake, and then determine the } \\
\text { process they will use to complete the task, } \\
\text { including homework. They need to decide on } \\
\text { how the group will function, in terms of the day } \\
\text { to day tasks, who will lead the group and how } \\
\text { they will convey learning to other class } \\
\text { members. As in all decision-making, they have } \\
\text { to live with the consequences. This develops } \\
\text { effective decision-making. Students also decide } \\
\text { which text they will study and they need to } \\
\text { validate their reason. }\end{array}$ & $\begin{array}{l}\text { In all decision-making } \\
\text { they have to live with } \\
\text { the consequences. }\end{array}$ & & \\
\hline $\begin{array}{l}\text { Bernard: Make up your own mind - a clear } \\
\text { direction; }\end{array}$ & $\begin{array}{l}\text { Make up your own } \\
\text { mind! }\end{array}$ & & \\
\hline
\end{tabular}




\begin{tabular}{|c|c|c|c|}
\hline COMMENT & CONSTRUCT & CONCEPT & THEME \\
\hline $\begin{array}{l}\text { Olag (Music teacher): When working in } \\
\text { ensembles, students have to make decisions, } \\
\text { about: roles in the ensemble; arrangements; } \\
\text { rehearsal techniques. I take a back seat if I can } \\
\text { but encourage them to: Substantiate all } \\
\text { discussion with good arguments; Listen to each } \\
\text { other; Find a compromise. }\end{array}$ & $\begin{array}{l}\text { Substantiate, listen, } \\
\text { compromise }\end{array}$ & \multirow[t]{2}{*}{$\begin{array}{l}\text { Intuitive } \\
\text { decision- } \\
\text { making } \\
\text { encouraged }\end{array}$} & \multirow{3}{*}{$\begin{array}{c}\text { Achieving } \\
\text { personal } \\
\text { autonomy }\end{array}$} \\
\hline $\begin{array}{l}\text { Rose (Biology teacher): Said she does not } \\
\text { currently teach thinking skills in her subject but } \\
\text { has done so when teaching Sex education and } \\
\text { Drug education units. }\end{array}$ & $\begin{array}{l}\text { Thinking skills } \\
\text { encouraged in sex and } \\
\text { drug education. }\end{array}$ & & \\
\hline $\begin{array}{l}\text { Henry (Design and Technology): Students } \\
\text { practise in manufacturing: which process/which } \\
\text { tool/safety /physical sensibility/control/ } \\
\text { precision? ... What is important is to identify } \\
\text { their own values/ positions. }\end{array}$ & $\begin{array}{l}\text { Important [for } \\
\text { students] to identify } \\
\text { their own values and } \\
\text { positions. }\end{array}$ & $\begin{array}{l}\text { Practise the } \\
\text { process }\end{array}$ & \\
\hline $\begin{array}{l}\text { Paul: expands a little: Students must give a } \\
\text { reason. It is also part of developing a discourse } \\
\text { style, which I teach discretely. Students have to } \\
\text { learn formulae, such as: I can see the point you } \\
\text { are making, but I have a problem with XXX, a } \\
\text { matter you rely on in your argument. }\end{array}$ & $\begin{array}{l}\text { Students must give } \\
\text { reasons [for their } \\
\text { decisions]. }\end{array}$ & $\begin{array}{l}\text { Developing a } \\
\text { discourse } \\
\text { style }\end{array}$ & \multirow{3}{*}{$\begin{array}{l}\text { Algorithmi } \\
\text { c thinking }\end{array}$} \\
\hline $\begin{array}{l}\text { Pietr (Physical Education teacher): Most often } \\
\text { whether teaching using Game Sense or through } \\
\text { general/direct teaching, I use the FREEZE and } \\
\text { replay of a particular teaching moment. Students } \\
\text { observe then comment on what they observed. } \\
\text { They can ask the performers questions to further } \\
\text { understand what happened. }\end{array}$ & $\begin{array}{l}\text { Replays a particular } \\
\text { teaching moment: } \\
\text { observe, comment, } \\
\text { question. }\end{array}$ & $\begin{array}{l}\text { Freeze and } \\
\text { replay to } \\
\text { improve } \\
\text { understandin } \\
\quad \mathrm{g}\end{array}$ & \\
\hline $\begin{array}{l}\text { Michael (Science teacher): When solving a } \\
\text { scientific problem the following decisions need } \\
\text { to be made: What is the relevant information } \\
\text { that has been given?; Are there any assumptions } \\
\text { that can/need to be made? Decide on which } \\
\text { tools/procedure to use to solve the problem. }\end{array}$ & $\begin{array}{l}\text { Relevant information, } \\
\text { assumptions, } \\
\text { decisions on tools or } \\
\text { procedures to solve a } \\
\text { problem. }\end{array}$ & $\begin{array}{c}\text { Rational } \\
\text { problem } \\
\text { solving }\end{array}$ & \\
\hline $\begin{array}{l}\text { Franco: Is careful to use decision-making as } \\
\text { part of the normal clarification of values and } \\
\text { morals which are embedded in themes and } \\
\text { issues. }\end{array}$ & $\begin{array}{l}\text { Clarification of values } \\
\text { and morals embedded } \\
\text { in themes and issues. }\end{array}$ & $\begin{array}{l}\text { Normalising } \\
\text { decision- } \\
\text { making }\end{array}$ & $\begin{array}{c}\text { Values } \\
\text { clarificatio } \\
\mathbf{n}\end{array}$ \\
\hline
\end{tabular}

\section{My researcher's voice}

I concluded that teachers use a variety of instrumental strategies to engage students in decision-making within their own subject areas. No one seems to give evidence that this decision-making can be shaped into a set of steps that can be used for almost all kinds of decision-making. Teachers' comments are pragmatic and focussed; some reflect secular or religious bases within their constructs; most seem to use strategies that they understand well and see this as a useful tool for teaching; most see the concepts allied to this topic as 
FIGURE 4.4 THE HEASLY UNCERTAINTY GRID

\begin{tabular}{|c|c|c|c|c|c|c|}
\hline OUTCOME & & & & & & OUTCOME \\
\hline Behaviour & $\begin{array}{c}\text { Consequenc- } \\
\text { es }\end{array}$ & Features & ELEMENTS & Features & $\begin{array}{c}\text { Consequenc } \\
\text {-es }\end{array}$ & Behaviour \\
\hline Aggression & $\begin{array}{l}\text { Stultificat- } \\
\text { ion }\end{array}$ & Laziness & $\begin{array}{l}\text { PHYSICAL } \\
\text { RESPONSE }\end{array}$ & $\begin{array}{c}\text { Skills } \\
\text { Appetites }\end{array}$ & Strength & $\begin{array}{l}\text { Persever- } \\
\text { ance } \\
\text { Courage }\end{array}$ \\
\hline $\begin{array}{c}\text { Tyranny } \\
\text { Deceit }\end{array}$ & $\begin{array}{l}\text { Unpredict- } \\
\text { ability } \\
\text { Hopeless- } \\
\text { ness }\end{array}$ & $\begin{array}{c}\text { Hysteria } \\
\text { Moodi- } \\
\text { ness }\end{array}$ & $\begin{array}{l}\text { EMOTIONAL } \\
\text { RESPONSE }\end{array}$ & $\begin{array}{l}\text { Wishes } \\
\text { Dreams }\end{array}$ & $\begin{array}{l}\text { Response- } \\
\text { ability }\end{array}$ & Honesty \\
\hline $\begin{array}{c}\text { Greed } \\
\text { Avarice }\end{array}$ & Narcissism & $\begin{array}{l}\text { Selfish- } \\
\text { ness }\end{array}$ & $\begin{array}{c}\text { SOCIAL } \\
\text { RESPONSE }\end{array}$ & $\begin{array}{c}\text { Communi- } \\
\text { cation } \\
\text { Love }\end{array}$ & Openness & $\begin{array}{c}\text { Patience } \\
\text { Justice } \\
\text { Forgiveness }\end{array}$ \\
\hline $\begin{array}{c}\text { Jealousy } \\
\text { Envy }\end{array}$ & $\begin{array}{c}\text { Prejudice } \\
\text { Inconsist- } \\
\text { ency }\end{array}$ & $\begin{array}{c}\text { Arro- } \\
\text { gance } \\
\text { Presumpt } \\
\text {-ion }\end{array}$ & $\begin{array}{l}\text { INTELLECTUAL } \\
\text { RESPONSE }\end{array}$ & $\begin{array}{c}\text { Inquiry } \\
\text { Logic }\end{array}$ & $\begin{array}{l}\text { Critical } \\
\text { skills }\end{array}$ & $\begin{array}{l}\text { Discern- } \\
\text { ment }\end{array}$ \\
\hline $\begin{array}{c}\text { Abuse - } \\
\text { self or other }\end{array}$ & Despair & Nihilism & $\begin{array}{l}\text { SPIRITUAL } \\
\text { RESPONSE }\end{array}$ & $\begin{array}{c}\text { Quest - } \\
\text { satisfaction } \\
\text { wholeness }\end{array}$ & $\begin{array}{c}\text { Personal } \\
\text { paradigm }\end{array}$ & Integrity \\
\hline
\end{tabular}

imposed by their own subject area; all are intended to promote student independence.

The Heasly Uncertainty Grid/ Behaviour Uncertainty Grid (HUG/BUG), shown in Figure 4.4, is definitely not to be a measuring tool - rather an illustration for students to explore. The central column is the starting point, showing elements of personality (as discussed by Laurence, 1991; Riso, 1992; Rohr \& Ebert, 2009), so diversity is immediately accommodated. By recognising the first column on the left and right of this central column, it can be seen that contrasts or choices of response are listed. By comparing the middle column on the left and right of the central column, it can be seen that certain consequences of choices emerge and these consequences can, by implication, influence our choices and our experience. By checking the outer columns on the left and the right of the central column, contrasting 
behaviours can be identified as suitable or otherwise. This does not mean that a person is one thing or another rather their decisions place them along a continuum.

One other illustration for students is that the names in these columns can be researched, analysed and discussed for deeper meaning and fuller understanding. This will apply to these and many other virtues, values and ethics that might appear in the curriculum under many guises.

It is to be noted also that the Cartesian effect of looking at both ends of the spectrum as contrast only, is mitigated when the teacher takes deliberative action to show the spectrum visually, and identifies all the points of degree between the outer ends and along the path of the continuum. This element is central to student understanding so that chosen stances in each spectrum can be identified as being moderate in nature, rather than the extremes possible. I understand the term 'spectrum' to be a way to identify that the line of inquiry can stretch backwards and forwards infinitely, whereas I understand the term 'continuum' to have a starting point and to move in one direction only.

\section{My practitioner voice}

Once the value and benefit of the decision-making sequences, and the $\mathrm{ME}$ diagram were applied in my classrooms over about 15 years, it became obvious that the Heasly Uncertainty Grid (HUG/BUG) shown in Figure 4.4 had much to offer. The two parts of the title refer to the positive and negative influences inherent in the concept: a hug is beneficial and positive but a bug is distasteful and negative. It was helpful for testing the possible pathways available when certain decisions were made, but it began to make sense for students through the use of curriculum topics that trace behaviours for English studies; understanding why historical figures behaved in certain ways; why political figures made certain decisions about war and other matters; why criminal behaviour was so awkward to understand, was equally possible using this grid. 


\section{Practices that promote consensus and democracy}

Within this section, three voices are heard: my practitioner voice, the teachers' voices and my researcher’s voice.

My practitioner voice:

\section{An example of ontological, epistemological and technical (techné) schema}

An example of how world events can cause trauma came with my Year 9 Special Education class of 21 students, when the Twin Towers were blown up. Students had arrived two minutes before me and were screaming at each other about who to blame for such a calamity. I established immediately that we were each going to take part in a class assignment regarding the questions surrounding the event. We went immediately into Community of Inquiry mode: listing as many questions as we could manage. This was followed by discussions about how to research, taking account of every subject discipline in their school curriculum.

Individual students researched the weight and type of planes used (maths); the amount of fuel carried by each plane (business studies); Art work, such as painting and papier maché; Indian Ink drawings (Art Design and Appreciation); researchers finding out the beliefs of Islam and Christianity particularly where each religion agreed on internal principles (Religion and Values Education); several essays (English and English Literature); a short play; some poetry (Drama); collages on themes like grief, war and its aftermath (Cultural Studies); a CD with a rapper song composed by one student (Music Studies); papier-maché replicas of the type of plane used (Technical Design and Drawing); reports based on collections of newspaper headlines (current affairs and English studies); maps of countries involved because of the terrorists apparent background (Geography); in all, we chose 20 possible assignments and to pass the semester's requirements, we agreed that each person must complete 10 assignments. 
I encouraged students to work together in small teams and to make good use of their subject teachers' knowledge as well as the facilities in the Library and IT laboratories. We did our evaluation by allowing each person to display and present their personal work. Invitations were issued for significant others internally and externally to attend the presentations, and some work ended up on display in the Library, and in various other special locations around the college. Those students of various world faiths, nations and cultures ended up, in their own words, comfortable and more understanding of a lot of people and a lot of things at semester's end; so did their Special Education teacher!

TABLE 4.27 TEACHING THE CONCEPTS OF CONSENSUS AND DEMOCRACY

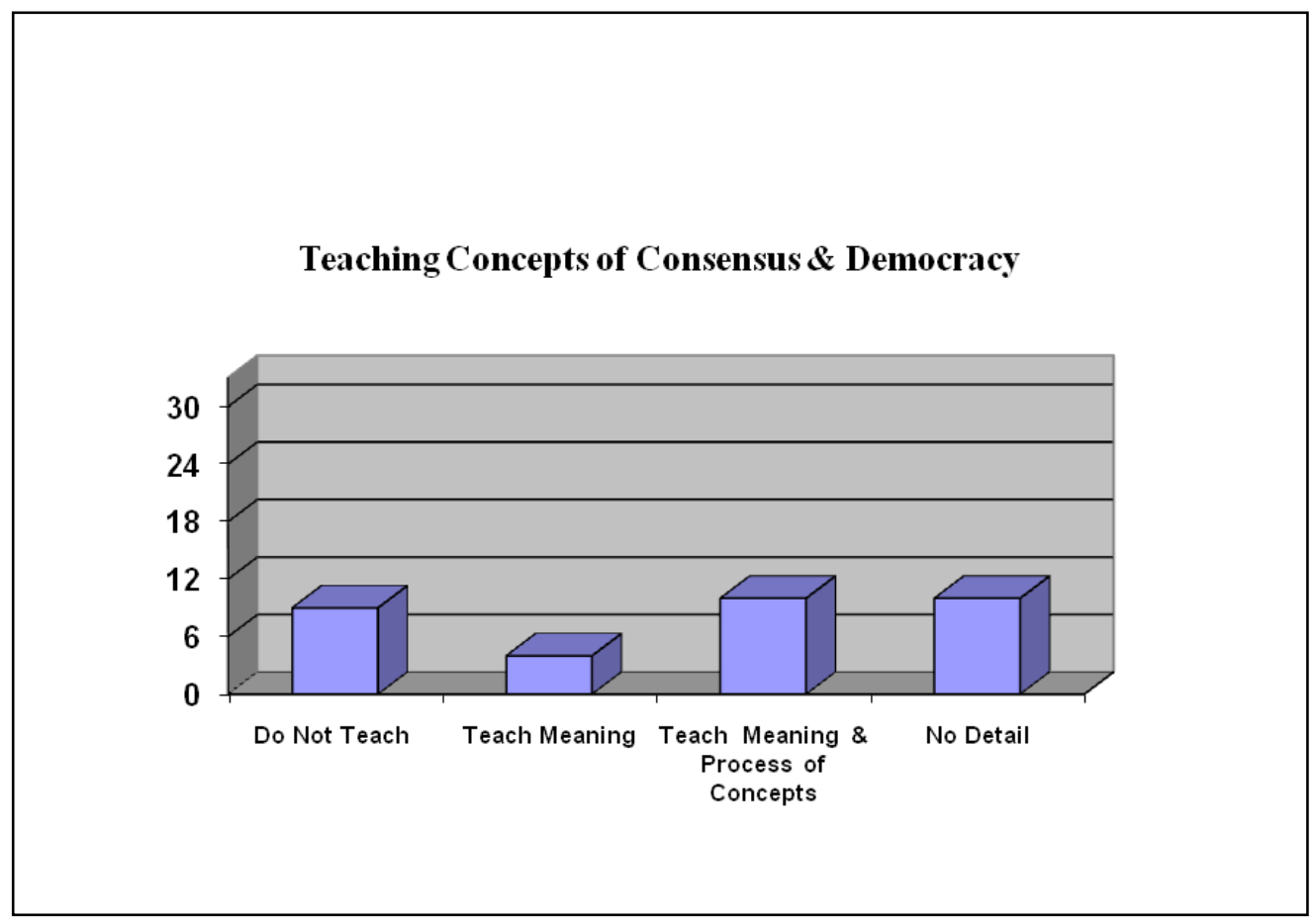

(Do Not Teach = teachers did not specifically teach processes of consensus and democracy; Teach Meaning = teachers did teach the meaning of consensus and democracy; Teach Meaning \& Process of Concepts = teachers teach both the meaning and the active process of the concepts of consensus and democracy; No detail = teachers did not specify any details about this matter.) 


\section{Teachers' voices}

Teachers were requested to explain whether they taught explicitly the concepts of consensus and democracy or perhaps used one or both in classroom strategies as a means of applying thinking skills.

\section{Consensus and democracy (quantitative)}

The data contained in Table 4.27 refer to the responses of 33 teachers. Nine teachers did not teach either of the two concepts at all though the responses to other questions made it clear that they modelled both concepts in differing ways in day-to-day classroom strategies. Four teachers teach the meaning of both concepts but not the process of either concept. Ten teachers teach the meaning and the process of the two concepts of consensus and democracy. Ten teachers gave no details to this question. The suggestion is that twothirds of the teachers in the survey engage in active teaching of consensus and democracy in their classes.

\section{Consensus and democracy (qualitative)}

I identified two themes that emerged from the responses: establishing a Community of Inquiry and applying the principles of Philosophy for Children. These data are summarised in Table 4.28. 


\begin{tabular}{|c|c|c|c|}
\hline COMMENT & CONSTRUCT & CONCEPT & THEME \\
\hline $\begin{array}{l}\text { Paul: I don't expect my own behaviour to be any } \\
\text { different from that of the students. I explain my } \\
\text { reasons and expect questions from students. }\end{array}$ & $\begin{array}{l}\text { I don't expect my } \\
\text { own behaviour to be } \\
\text { any different from } \\
\text { that of the students }\end{array}$ & $\begin{array}{c}\text { Explanations } \\
\text { given; } \\
\text { questions } \\
\text { expected. }\end{array}$ & \multirow{4}{*}{$\begin{array}{c}\text { Community } \\
\text { of Inquiry }\end{array}$} \\
\hline $\begin{array}{l}\text { Hyacinth: I don't teach either overtly, but it is } \\
\text { part of the Community of Inquiry approach. I } \\
\text { teach these principles in all teaching. }\end{array}$ & $\begin{array}{l}\text { I don't teach either } \\
\text { [of these] overtly. }\end{array}$ & $\begin{array}{l}\text { Implicit } \\
\text { principles are } \\
\text { to be applied } \\
\text { in all } \\
\text { teaching. }\end{array}$ & \\
\hline $\begin{array}{l}\text { Jane: I am thinking of a Year } 9 \text { classroom and I } \\
\text { think I perhaps teach the meaning of the words } \\
\text { without being aware that I do it. Sometimes class } \\
\text { decisions require consensus for the activity to go } \\
\text { ahead. At other times I encourage students' right } \\
\text { to be individual in their opinion and to be } \\
\text { respected for this. }\end{array}$ & $\begin{array}{l}\text { Class decisions may } \\
\text { require consensus; } \\
\text { students' right to be } \\
\text { individuals is } \\
\text { preserved. }\end{array}$ & $\begin{array}{l}\text { No dichotomy } \\
\text { between the } \\
\text { two: } \\
\text { consensus and } \\
\text { democracy. }\end{array}$ & \\
\hline $\begin{array}{l}\text { Rose says: I tend not to state my own position, } \\
\text { certainly not at the beginning of any topic. As } \\
\text { stated previously I tend to play devil's advocate } \\
\text { throwing up what ifs and have you thought about } \\
\text {... If asked, I will state my view and reasons for it. } \\
\text { Always adding that there is no right or wrong } \\
\text { regarding many of the issues we tackle in Biology. }\end{array}$ & $\begin{array}{l}\text { There is no right or } \\
\text { wrong regarding } \\
\text { many of the issues } \\
\text { we tackle in } \\
\text { biology. }\end{array}$ & $\begin{array}{l}\text { Views } \\
\text { expressed; } \\
\text { positions } \\
\text { encouraged; } \\
\text { tolerance of } \\
\text { disparate } \\
\text { views. } \\
\end{array}$ & \\
\hline $\begin{array}{l}\text { Matthew (Australian expert): As already } \\
\text { indicated, I am new to teaching thinking skills and } \\
\text { as such I am yet to develop strategies that promote } \\
\text { decision making skills which lead from efficient } \\
\text { thinking skills. Suffice to say that good thinking } \\
\text { skills are foundational to good decision making. }\end{array}$ & $\begin{array}{l}\text { Good thinking skills } \\
\text { are foundational to } \\
\text { good decision } \\
\text { making }\end{array}$ & $\begin{array}{c}\text { Good thinking } \\
\text { requires } \\
\text { efficient } \\
\text { thinking skills }\end{array}$ & \multirow{2}{*}{$\begin{array}{l}\text { Philosophy } \\
\text { for Children }\end{array}$} \\
\hline $\begin{array}{l}\text { Clint (NZ expert): Teaching decision-making is } \\
\text { still missing the point - the aim is to have students } \\
\text { who engage in reflective decision making as part } \\
\text { of everyday life. Creating a culture in the } \\
\text { classroom which encourages and expects } \\
\text { reflective decision making is essential. Philosophy } \\
\text { for Children is the best way I know to do this. }\end{array}$ & $\begin{array}{l}\text { The aim is to have } \\
\text { students who engage } \\
\text { in reflective } \\
\text { decision making as } \\
\text { part of everyday } \\
\text { life. }\end{array}$ & $\begin{array}{l}\text { Reflective } \\
\text { decision } \\
\text { making is } \\
\text { essential }\end{array}$ & \\
\hline $\begin{array}{l}\text { Roger (UK expert): Oh dear! I'm not very happy } \\
\text { about this link at all. Talk of decision-making } \\
\text { skills reminds me too much of the less attractive } \\
\text { side of de Bono's work, in which he supposes that } \\
\text { wise decisions can be made by applying routine, } \\
\text { mechanical techniques. Most of these are at best } \\
\text { applicable to machines and decisions about } \\
\text { objects; they hardly begin to develop the } \\
\text { necessary subtle, comprehensive and intuitive } \\
\text { thoughts and feelings that go towards truly wise } \\
\text { decision-making. In the end, the challenge is to } \\
\text { develop good judgment, and this is a lifetime } \\
\text { challenge best met by cultivating various } \\
\text { dispositions, such as sincerity, openness, curiosity, } \\
\text { reflectiveness, accuracy, tenacity, empathy, sense } \\
\text { of humour/ proportion, etc. In my view, already } \\
\text { articulated, these are best developed through } \\
\text { repeated exposure to the practice of philosophical } \\
\text { inquiry and reflection. }\end{array}$ & $\begin{array}{l}\text { Need to develop the } \\
\text { necessary subtle, } \\
\text { comprehensive and } \\
\text { intuitive thoughts } \\
\text { and feelings that go } \\
\text { towards truly wise } \\
\text { decision-making. }\end{array}$ & $\begin{array}{c}\text { Requires } \\
\text { philosophical } \\
\text { inquiry and } \\
\text { reflection. }\end{array}$ & $\begin{array}{l}\text { Philosophy } \\
\text { for Children }\end{array}$ \\
\hline
\end{tabular}




\section{My researcher voice}

Two major themes emerged: developing a Community of Inquiry and applying a set of key concepts using the Philosophy for Children curriculum. A Community of Inquiry develops the following concepts: the teacher gives explanations and these are followed by questions from students; the implicit principles of consensus and democracy are to be applied in all teaching; there need not be a dichotomy between the two constructs: it is consensus and democracy; and views can be expressed, positions encouraged and disparate views tolerated. The key concepts in Philosophy for Children that specifically encourage consensus and democracy are as follows: good thinking requires efficient thinking skills; reflective decision-making is an essential component; this reflection requires the inquiry and reflection encouraged by the application of philosophy. Thus, Philosophy for Children, which encourages the development of a Community of Inquiry directly, supports seeking consensus and developing democracy as an integrated, holistic outcome.

\section{Formal and informal leadership}

In this section, the only 'voice' heard is my practitioner voice.

\section{My practitioner voice}

In an effort to analyse further the element of leadership, both in my career and in my research, I devised over time a framework consisting of a list of matching pairs, partly stimulated by the Bryman framework, (in the presentation from his doctoral thesis by Gurr at University of Melbourne in my class lectures in 2000 regarding Values in Principal Leadership), and partly by my reflective practice. These pairs, contained in Table 4.29, below, identify the differences in thinking, skills, behaviours, knowledge, and wisdom levels. To understand this construct, it is necessary to read each set 
TABLE 4.29 A LISTING OF FORMAL AND INFORMAL LEADERSHIP ELEMENTS

\begin{tabular}{|c|c|}
\hline $\begin{array}{l}\text { FORMAL LEADERSHIP } \\
\text { The formal leader is using (or may be, or } \\
\text { will use) the following: }\end{array}$ & $\begin{array}{l}\text { INFORMAL LEADERSHIP } \\
\text { The informal leader is using (or may be, } \\
\text { or will use) the following: }\end{array}$ \\
\hline $\begin{array}{l}\text { - visible, with designated title and incumbent } \\
\text { responsibilities. }\end{array}$ & $\begin{array}{l}\text { - less visible or somewhat invisible in } \\
\text { normal daily processes }\end{array}$ \\
\hline $\begin{array}{l}\text { - collaborative or non-collaborative } \\
\text { behaviours }\end{array}$ & - operates out of collaborative modes \\
\hline - sees always the overall picture & - attends to detail with care \\
\hline - may miss significant detail & $\begin{array}{l}\text { - is concerned with reading the particulars in } \\
\text { processes }\end{array}$ \\
\hline - operates in universals & - is concerned with implementation of policy \\
\hline - initiator of ,responsibility for, policy & $\begin{array}{l}\text { - operates almost continually in a mentoring } \\
\text { mode }\end{array}$ \\
\hline - uses consultative processes & $\begin{array}{l}\text { - implements and consults and facilitates } \\
\text { when change is in progress }\end{array}$ \\
\hline - balances existing mores with change & - looks at options and response-ability levels \\
\hline - reaches decisions, uses responsibility & - representing sectional concerns \\
\hline - represents the whole organisation & - is legally designated a follower \\
\hline - is the legal face of the organisation, CEO & $\begin{array}{l}\text { - uses a curriculum rather than an } \\
\text { administrative focus }\end{array}$ \\
\hline - uses an administrative focus & $\begin{array}{l}\text { - operates out of a transformational/ } \\
\text { transactional/ transparent behaviour set }\end{array}$ \\
\hline $\begin{array}{l}\text { - operates out of transactional and } \\
\text { transformational frameworks as } \\
\text { appropriate }\end{array}$ & $\begin{array}{l}\text { - is concerned with focus and meaning when } \\
\text { addressing vision and mission }\end{array}$ \\
\hline $\begin{array}{l}\text { - is taken up with organisational vision and } \\
\text { mission }\end{array}$ & - has a concern for analysis of initiatives \\
\hline $\begin{array}{l}\text { - has a concern for political and strategic } \\
\text { development }\end{array}$ & $\begin{array}{l}\text { - is concerned with implementation of } \\
\text { purpose, rather than defining it }\end{array}$ \\
\hline - has a central role in defining purpose & - uses empowering skills continually \\
\hline $\begin{array}{l}\text { - exhibits some facets of charismatic } \\
\text { behaviours }\end{array}$ & $\begin{array}{l}\text { - translates inspiration into consultative } \\
\text { language and process }\end{array}$ \\
\hline $\begin{array}{l}\text { - is expected to display inspirational } \\
\text { approach }\end{array}$ & $\begin{array}{l}\text { - eases processes for consultation and } \\
\text { collaboration }\end{array}$ \\
\hline $\begin{array}{l}\text { - is expected to display individualised } \\
\text { consideration }\end{array}$ & $\begin{array}{l}\text { - has abilities to counsel and empathise and } \\
\text { so support without endangering balance in } \\
\text { the organisation }\end{array}$ \\
\hline $\begin{array}{l}\text { - is able to identify contrary behaviours } \\
\text { likely to destroy organisational balance }\end{array}$ & $\begin{array}{l}\text { - exhibits a regular pattern of personal } \\
\text { professional development and empathy } \\
\text { with other followers }\end{array}$ \\
\hline $\begin{array}{l}\text { promotes a regular pattern of affirmation } \\
\text { and opportunity for staff personal } \\
\text { development }\end{array}$ & $\begin{array}{l}\text { operates within the organisation, identified } \\
\text { as part of the organisation }\end{array}$ \\
\hline $\begin{array}{l}\text { - is often an import from elsewhere, with } \\
\text { attendant need to learn local organisational } \\
\text { mores }\end{array}$ & $\begin{array}{l}\text { - accepts the challenge of new goals and sets } \\
\text { about methods of analysis and } \\
\text { implementation }\end{array}$ \\
\hline $\begin{array}{l}\text { - needs those who carry out well the } \\
\text { informal leadership role to achieve goals. }\end{array}$ & - shows worthiness of trust regularly \\
\hline - has to earn the trust of all staff & $\begin{array}{l}\text { - uses the myriad yet invisible opportunities } \\
\text { to demonstrate integrity }\end{array}$ \\
\hline $\begin{array}{l}\text { - has to watch for opportunities to } \\
\text { demonstrate empathy and integrity }\end{array}$ & $\begin{array}{l}\text { - regularises gap between local cultural } \\
\text { norms and those introduced by } \\
\text { CEO/formal leader } \\
\end{array}$ \\
\hline
\end{tabular}




\begin{tabular}{|c|c|}
\hline $\begin{array}{l}\text { FORMAL LEADERSHIP } \\
\text { The formal leader is using (or may be, or } \\
\text { will use) the following: }\end{array}$ & $\begin{array}{l}\text { INFORMAL LEADERSHIP } \\
\text { The informal leader is using (or may be, } \\
\text { or will use) the following: }\end{array}$ \\
\hline $\begin{array}{l}\text { - must learn prevailing present } \\
\text { organisational cultural norms }\end{array}$ & $\begin{array}{l}\text { - recognises apparently doomed initiatives } \\
\text { and mediates }\end{array}$ \\
\hline $\begin{array}{l}\text { often, specially at first, has insufficient } \\
\text { channels to identify apparently doomed } \\
\text { initiatives }\end{array}$ & $\begin{array}{l}\text { may, or usually may not be, part of the } \\
\text { existing team leadership }\end{array}$ \\
\hline - may be supported by team leadership & $\begin{array}{l}\text { - identifies and eases difficulties in time } \\
\text { lag before team leadership has become } \\
\text { efficient }\end{array}$ \\
\hline $\begin{array}{l}\text { - } \begin{array}{l}\text { must accept time lag involved with } \\
\text { development of team leadership } \\
\text { complementarity }\end{array} \\
\end{array}$ & $\begin{array}{l}\text { - } \text { facilitates processes for equity/ } \\
\text { excellence/ effectiveness/ efficiency at } \\
\text { many levels }\end{array}$ \\
\hline $\begin{array}{l}\text { - is expected to initiate processes to attain } \\
\text { equity/ excellence/ effectiveness/ } \\
\text { efficiency at all levels }\end{array}$ & $\begin{array}{l}\text { - facilitates networking internally and } \\
\text { externally }\end{array}$ \\
\hline $\begin{array}{l}\text { - institutes organisational networking } \\
\text { structures }\end{array}$ & - has subtle attributed authority \\
\hline $\begin{array}{l}\text { - has prescribed, attributed and } \\
\text { proscribed authority }\end{array}$ & $\begin{array}{l}\text { - eases barriers existing between many } \\
\text { cells of responsibility within } \\
\text { organisation }\end{array}$ \\
\hline $\begin{array}{l}\text { - enhances interconnectivity of the many } \\
\text { cells of responsibility within the } \\
\text { organisation }\end{array}$ & $\begin{array}{l}\text { - explains and enhances historical context } \\
\text { to ensure continuity of organisation }\end{array}$ \\
\hline $\begin{array}{l}\text { - recognises and enhances tradition to } \\
\text { facilitate continuity in the organisation }\end{array}$ & $\begin{array}{l}\text { - facilitates best practice methodologies } \\
\text { and technologies to integrate and } \\
\text { maintain balance }\end{array}$ \\
\hline $\begin{array}{l}\text { - introduces best practice methodologies } \\
\text { with maintenance and innovation } \\
\text { balanced }\end{array}$ & $\begin{array}{l}\text { - provides curricular access for } \\
\text { adaptation within innovation }\end{array}$ \\
\hline - promotes adaptation within innovations & $\begin{array}{l}\text { - translates innovative leadership into } \\
\text { identifiable ethical behaviours for staff }\end{array}$ \\
\hline $\begin{array}{l}\text { - exhibits ethics as an element of } \\
\text { innovative leadership }\end{array}$ & $\begin{array}{l}\text { - exhibits skills for transmitting } \\
\text { framework of existing cultures } \\
\text { accurately }\end{array}$ \\
\hline $\begin{array}{l}\text { - exhibits skills of reading organisational } \\
\text { culture accurately }\end{array}$ & $\begin{array}{l}\text { - encourages diversity as a contribution } \\
\text { to organisational wholeness }\end{array}$ \\
\hline $\begin{array}{l}\text { - enhances unity while respecting } \\
\text { diversity }\end{array}$ & $\begin{array}{l}\text { - } \begin{array}{l}\text { conducts a mediating role in contra- } \\
\text { cultures and sub-cultures within the } \\
\text { organisation }\end{array} \\
\end{array}$ \\
\hline $\begin{array}{l}\text { - is aware of contra-cultures and sub- } \\
\text { cultures existing within the organisation }\end{array}$ & $\begin{array}{l}\text { - eases the processes existing when } \\
\text { consensus causes potential conflict }\end{array}$ \\
\hline - recognises the limits of consensus & - helps handle ambiguity and confusion \\
\hline - identifies ambiguity and confusion & $\begin{array}{l}\text { - recognises dissent and works to ease } \\
\text { consequences for refusers }\end{array}$ \\
\hline $\begin{array}{l}\text { - recognises that organisational members } \\
\text { may actively resist innovation }\end{array}$ & $\begin{array}{l}\text { - uses explanatory processes to aid } \\
\text { innovation and is distant from mind } \\
\text { games }\end{array}$ \\
\hline $\begin{array}{l}\text { - does not play mind games to aid } \\
\text { innovation processes }\end{array}$ & $\begin{array}{l}\text { provides curriculum support and } \\
\text { development when external turbulence } \\
\text { forces organisational change } \\
\end{array}$ \\
\hline $\begin{array}{l}\text { - recognises and communicates when } \\
\text { external turbulence forces innovation in } \\
\text { the organisation }\end{array}$ & $\begin{array}{l}\text { - empathises and supports those coping } \\
\text { with innovative fatigue }\end{array}$ \\
\hline $\begin{array}{l}\text { - recognises and respects innovation } \\
\text { fatigue }\end{array}$ & $\begin{array}{l}\text { - engenders interest in and possible } \\
\text { acceptance of steps needed for potential } \\
\text { change }\end{array}$ \\
\hline
\end{tabular}




\begin{tabular}{|c|c|}
\hline $\begin{array}{c}\text { FORMAL LEADERSHIP } \\
\text { The formal leader is using (or may be, or } \\
\text { will use) the following: }\end{array}$ & $\begin{array}{c}\text { INFORMAL LEADERSHIP } \\
\text { The informal leader is using (or may be, } \\
\text { or will use) the following: }\end{array}$ \\
\hline $\begin{array}{l}\text { recognises the steps of development } \\
\text { needed to effect change }\end{array}$ & $\begin{array}{l}\text { makes the changes necessary to } \\
\text { encourage steps of development. }\end{array}$ \\
\hline $\begin{array}{l}\text { is concerned with the external } \\
\text { considerations of image and } \\
\text { appearance. }\end{array}$ & $\begin{array}{l}\text { is concerned with internal } \\
\text { considerations and consequences } \\
\text { regarding image and appearance. }\end{array}$ \\
\hline
\end{tabular}

of descriptions to develop the whole perspective of each form of leadership formal and informal. The second step is to consider each pair together to allow an easier comparison.

At the end of such a creative process, I believe it is apparent that informal leadership is practised by those with sufficient experience, professional development and current knowledge to recognise the realities, to read the strategic intent needed, as staff came to terms with the detail of changes: modelling thinking skills and consequent behaviours already identified in the prosecution of this enterprise.

Informal Leadership, I realise, is a separate area of organisational responsibility which emerges when existing staff with sufficient understanding and experience within the organisation of school find themselves in roles supporting many initiatives in the life and growth of their educational organisation. In direct comparison to the descriptive list of Formal Leader behaviours and responses, the Informal Leader shows different conceptual responses.

Having compiled these lists in tandem over a long period, I realised that in my pedagogical practice, motives of transparency were central in my personal educational theory as it was evolving. By modelling to both faculty members and class members, I seemed to be developing a deeper understanding of the processes that were embedded in the teaching- learning process. I undertook my M Ed thesis (Heasly, 1995) and other studies concurrently, all of which would contribute to my growing maturity as a practitioner. My learning in the work place was a necessary step in 
contributing to my new knowledge, which I have endeavoured to critique as I developed this study.

No responses pertaining to the informal and formal leadership question was given by teachers as this was not a significant question to investigate within the Questionnaire. Experts did not comment, and my researcher voice is not heard, because the extensive grid on the two forms of leadership was composed over many years in my practice as teacher.

\section{Virtues, Values, Ethics}

Within this section, three voices are heard: my practitioner voice, and the teachers’ voices and my researcher voice.

\section{My practitioner voice}

Given that the teaching of virtues, values and ethics is already present in the political push to include such matters as drug education and driving skills, it becomes important how, when, and why these matters are part of the curriculum. Before addressing the findings, I will clarify what I mean by virtues, values and ethics:

- Virtues

If Virtues are seen as encompassing attitude, preference, sense of right and wrong, it becomes important to recognise whether this happens in the stated agenda of the curriculum, rather than the covert agenda that may otherwise apply. Teachers’ voices give us accurate detail.

\section{- Values}

If Values are part of the promotion of community harmony inside the school and elsewhere, it emerges that overt and covert agenda needs to cohere so that teachers and students have accurate readings of current and significant Value systems. Teachers can provide such information. 


\section{- $\quad$ Ethics}

If Ethics is the culmination of the integration of deep learning about virtues and values, then teachers can promote the kinds of behaviours that students have democratically chosen through developed thinking skills and integrated ethical behaviour.

\section{Teachers' voices}

Nineteen teachers (19/33) were clear that they actively teach virtues, values, and ethics in their classroom practice. Eleven teachers (11/33) were equally clear that they do not actively teach virtues, values or ethics within their classrooms. Three teachers (3/33) gave no details. The data are contained in Table 4.30.

The teachers who did respond couched their comments in careful language that highlights their unstated concerns about what exactly is the meaning of each of these three terms. Teachers provided me with qualitative information about the actual situation in the classroom in the teaching about virtues, values and ethics. Before addressing the findings, I will clarify what they appear to mean by teaching virtues, values and ethics in the dot points below:

\section{- Virtues}

Teachers identified clearly that some do actively teach some virtues. Others have indicated that they do not pursue this avenue.

\section{- Values}

Similarly, teachers have shown that they make efforts to complete curriculum content and do not necessarily include concepts of values.

\section{- $\quad$ Ethics}

Some teachers seemed to be confused by the separation of the three elements: virtues, values and ethics. If concentration on the requirements of assessments is central to teachers' concerns, then ethics was really completion of requirements as expected. 


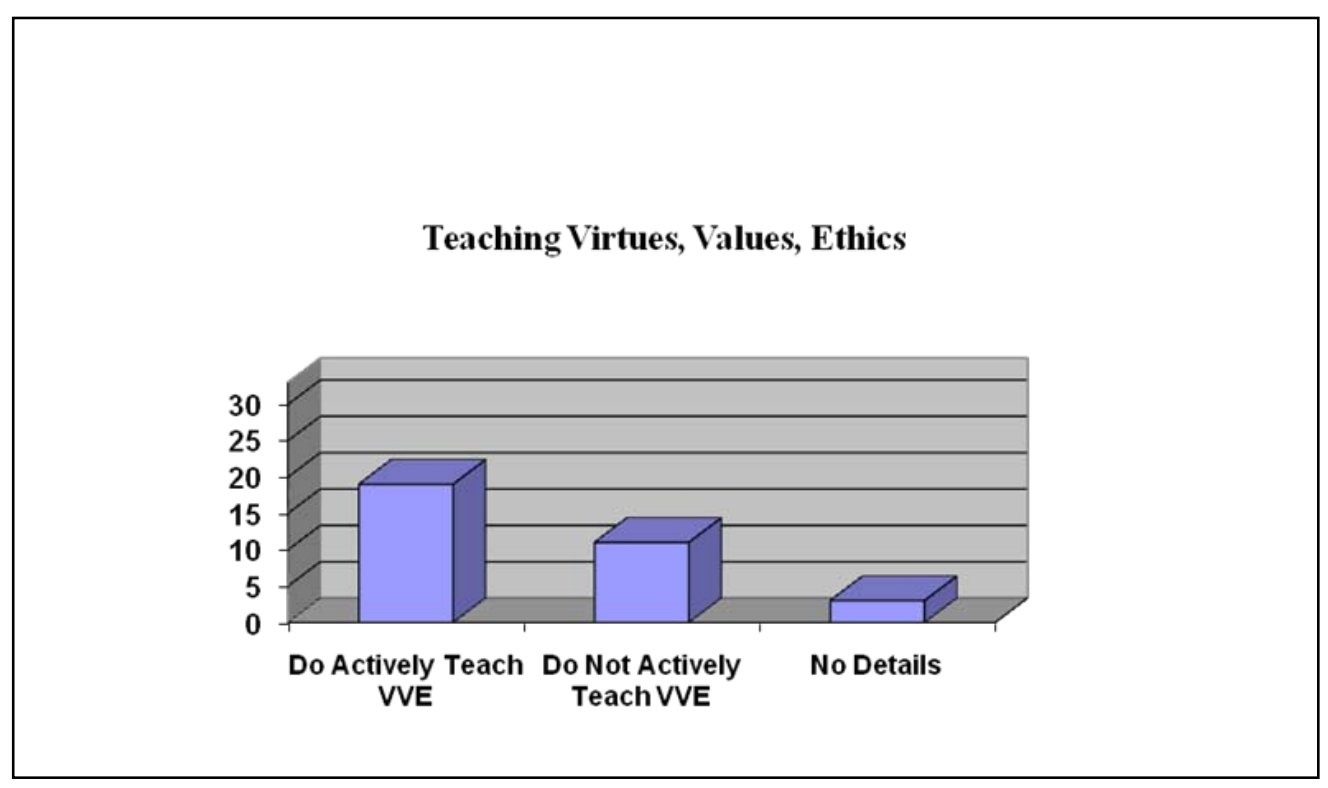

(Do Actively Teach V.V.E. = teachers do include the teaching about Virtues, Values and Ethics in their daily curriculum; Do Not Actively Teach V.V.E. = teachers do not actively include specific content for teaching about Virtues, Values and Ethics.)

As a consequence of the question referring to the teaching of virtues, values and ethics, a connection was expected between the way democracy and consensus emerged in the classroom. These questions were expected to follow the teachers whose teaching of thinking skills focused on democracy, and on harmonious classroom communities. If teachers were aware of the three frameworks named in the Questionnaire, they could comment on other similar frameworks, if it was necessary.

When related with my practitioner voice and the position stated abovethat there is uncertainty in the minds of the teachers as to the meanings of the concepts of virtues, values and ethics - it is scarcely surprising that the teachers' comments were addressed, only, to the theme of concept clarification; however, three respondents were still concerned with moral agency in schools. These data are summarised in Table 4.31. 
TABLE 4.31 TEACHING VALUES, VIRTUES AND ETHICS

\begin{tabular}{|c|c|c|c|}
\hline Comment & Construct & Concept & Theme \\
\hline $\begin{array}{l}\text { Michael: It is virtuous to move forward in a } \\
\text { way that contributes to the common good; that } \\
\text { it is important to teach about Virtues education } \\
\text { as thinking skills; that teaching morals as } \\
\text { difference between good and bad/ right and } \\
\text { wrong is important; that it is necessary to } \\
\text { teach students how to decide what it is that } \\
\text { they value for life decisions; that ethics takes } \\
\text { the idea of virtue further to the principle of } \\
\text { rules and regulations. This notion is central to } \\
\text { the pursuit of scientific thinking (my area). }\end{array}$ & $\begin{array}{l}\text { It is virtuous to move } \\
\text { forward in a way that } \\
\text { contributes to the } \\
\text { common good ... it is } \\
\text { important to teach } \\
\text { about virtues } \\
\text { education as thinking } \\
\text { skills ... ethics takes } \\
\text { the idea of virtue } \\
\text { further to the principle } \\
\text { of rules and } \\
\text { regulations. }\end{array}$ & $\begin{array}{l}\text { Being virtuous } \\
\text { contributes to } \\
\text { the common } \\
\text { good ... virtues } \\
\text { education is } \\
\text { taught as } \\
\text { thinking skills ... } \\
\text { ethics establishes } \\
\text { principles of } \\
\text { rules and } \\
\text { regulations. }\end{array}$ & \multirow{5}{*}{$\begin{array}{c}\text { Concept } \\
\text { clarification }\end{array}$} \\
\hline $\begin{array}{l}\text { Portia: Virtues education is an effective } \\
\text { holistic education which uses a constructive } \\
\text { paradigm and walks the talk of authentic } \\
\text { character education. Values education is } \\
\text { education in values and it is a universal } \\
\text { phenomenon intrinsic to all learning and } \\
\text { education whereas Virtues education is for } \\
\text { character development. Ethics involves } \\
\text { defending and recommending concepts of } \\
\text { right and wrong behaviour so it should be } \\
\text { inculcated in the thinking skills curriculum. It } \\
\text { enables students to think for his/her actions. }\end{array}$ & $\begin{array}{l}\text { Virtues education ... } \\
\text { walks the talk of } \\
\text { authentic character } \\
\text { education ... values } \\
\text { are a universal } \\
\text { phenomenon intrinsic } \\
\text { to all learning and } \\
\text { education ... ethics } \\
\text { involves defending } \\
\text { and recommending } \\
\text { concepts of right and } \\
\text { wrong [and] enables } \\
\text { students to think about } \\
\text { their actions }\end{array}$ & $\begin{array}{l}\text { Character } \\
\text { development } \\
\text { (virtues); } \\
\text { intrinsic to all } \\
\text { learning and } \\
\text { education } \\
\text { (values); } \\
\text { defending and } \\
\text { recommending } \\
\text { concepts of right } \\
\text { and wrong } \\
\text { (ethics) }\end{array}$ & \\
\hline $\begin{array}{l}\text { Georgia: I practise the encouragement of a } \\
\text { democratic, but respectful classroom } \\
\text { environment. }\end{array}$ & $\begin{array}{l}\text { Encouraging a } \\
\text { democratic, but } \\
\text { respectful classroom } \\
\text { environment. }\end{array}$ & $\begin{array}{c}\text { Developing a } \\
\text { democratic, } \\
\text { respectful } \\
\text { classroom } \\
\text { environment }\end{array}$ & \\
\hline $\begin{array}{l}\text { Susan: The classroom is practice for adult life } \\
\text { so that if we can teach students about doing } \\
\text { the right thing for the right reason then we will } \\
\text { develop a society who cares and supports. }\end{array}$ & $\begin{array}{l}\text { The classroom is a } \\
\text { practice for adult life } \\
\text { [during which] we can } \\
\text { teach students about } \\
\text { doing the right thing } \\
\text { for the right reason [in } \\
\text { order to] develop a } \\
\text { society that cares and } \\
\text { supports. }\end{array}$ & $\begin{array}{l}\text { Doing the right } \\
\text { thing for the } \\
\text { right reason in } \\
\text { order to develop } \\
\text { a caring, } \\
\text { supporting } \\
\text { society. }\end{array}$ & \\
\hline $\begin{array}{l}\text { Samantha: Teaching students the right thing } \\
\text { to do, wanting the right thing and putting these } \\
\text { into action in the right time and place. Every } \\
\text { human is capable of this and we can therefore } \\
\text { bring about change in the world. She also } \\
\text { details: Qualities of human spirit, integrity, } \\
\text { kindness, compassion, self-control, respect } \\
\text { and honesty. ... Virtues education teaches and } \\
\text { models standards of good behaviour that } \\
\text { develop a sound school culture and spirit. The } \\
\text { virtues must be taught and modelled from the } \\
\text { leaders down to the staff and all the students. } \\
\text { Inherently linked are needs to think rationally, } \\
\text { understand another's perspective and practise } \\
\text { all the virtues in everyday life. Teach/ allow } \\
\text { students in a moral situation to assess the } \\
\text { conflict, collect all of the relevant facts, } \\
\text { analyse, and make a virtues/values decision } \\
\text { and come up with a just course of action. }\end{array}$ & $\begin{array}{l}\text { Virtues education } \\
\text { teaches and models } \\
\text { standards of good } \\
\text { behaviour that } \\
\text { develop a sound } \\
\text { school culture and } \\
\text { spirit. }\end{array}$ & $\begin{array}{c}\text { Maintaining } \\
\text { standards of } \\
\text { good behaviour } \\
\text { and developing a } \\
\text { sound school } \\
\text { culture. }\end{array}$ & \\
\hline
\end{tabular}




\begin{tabular}{|c|c|c|c|}
\hline Comment & Construct & Concept & Theme \\
\hline $\begin{array}{l}\text { June: Virtues education = } \\
\text { fairness/acceptance/morals ... teaching them } \\
\text { to behave with respect and justice in school } \\
\text { and out of school. Her definition of Values } \\
\text { education was as follows: Values to me means } \\
\text { a similar thing to Virtues but virtues I think } \\
\text { relates to personal behaviour and morals. } \\
\text { Values can refer to possessions and } \\
\text { background of family and culture and religion. } \\
\text { She defined Ethics education this way: Ethics } \\
\text { = moral questions/rules of conduct }\end{array}$ & $\begin{array}{l}\text { Virtues relates to } \\
\text { personal and moral } \\
\text { behaviour ... values } \\
\text { refer to possessions } \\
\text { and background of } \\
\text { family and culture and } \\
\text { religion ... ethics } \\
\text { equates to moral } \\
\text { questions and rules of } \\
\text { conduct. }\end{array}$ & $\begin{array}{c}\text { Personal and } \\
\text { moral behaviour } \\
\text { (virtues); family } \\
\text { and cultural } \\
\text { backgrounds } \\
\text { (values); moral } \\
\text { questions and } \\
\text { rules of conduct } \\
\text { (ethics) }\end{array}$ & \\
\hline $\begin{array}{l}\text { Hyacinth: Being a Christian teacher in a } \\
\text { Christian school, values education is overtly } \\
\text { and covertly taught right through the } \\
\text { curriculum. This includes thinking skills } \\
\text { curriculum. Values taught are those which are } \\
\text { consistent with Christian beliefs, in particular, } \\
\text { the values held by the Church which controls } \\
\text { the school. ... We also want students to } \\
\text { develop an ethical approach to life in general } \\
\text { within a Christian framework and teach } \\
\text { accordingly. }\end{array}$ & $\begin{array}{l}\text {... in a Christian } \\
\text { school, values } \\
\text { education is overtly } \\
\text { and covertly taught } \\
\text { right through the } \\
\text { curriculum.... [the] } \\
\text { values taught are those } \\
\text { which are consistent } \\
\text { with Christian beliefs } \\
\ldots \text { we also want } \\
\text { students to develop an } \\
\text { ethical approach to } \\
\text { life in general within a } \\
\text { Christian framework } \\
\text { and teach accordingly }\end{array}$ & $\begin{array}{l}\text { Values and } \\
\text { ethics result in a } \\
\text { link between the } \\
\text { secular and the } \\
\text { religious in the } \\
\text { curriculum of a } \\
\text { Christian school. }\end{array}$ & \\
\hline $\begin{array}{l}\text { Jane: In Life Skills classes these } \\
\text { characteristics are explored more actively and } \\
\text { at times also in English. In managing a home } \\
\text { room, these are encouraged/modelled/ } \\
\text { praised/ acknowledged all the time. }\end{array}$ & $\begin{array}{l}\text { In Life Skills classes } \\
\text { these characteristics } \\
\text { [values, virtues and } \\
\text { ethics] are explored } \\
\text { more actively and at } \\
\text { times also in English }\end{array}$ & $\begin{array}{l}\text { Values, virtues } \\
\text { and ethics are } \\
\text { explored actively } \\
\text { in life skills and } \\
\text { English classes. }\end{array}$ & \\
\hline $\begin{array}{l}\text { Henry: Does not formally teach such matters } \\
\text { but both Virtues and Values tie into teaching } \\
\text { and modelling integrity, which is often talked } \\
\text { through in class. }\end{array}$ & $\begin{array}{l}\text { Virtues and values tie } \\
\text { into teaching and } \\
\text { modelling integrity, } \\
\text { which is often talked } \\
\text { through in class. }\end{array}$ & $\begin{array}{l}\text { Virtues and } \\
\text { values involve } \\
\text { modelling } \\
\text { integrity. }\end{array}$ & \\
\hline $\begin{array}{l}\text { Paula: I teach virtues in all classroom } \\
\text { practices - it is the basis of how the room } \\
\text { operates - to be allowed to have an opinion/ to } \\
\text { be encouraged to think deeply for excellence/ } \\
\text { to respect others opinions. I feel some } \\
\text { behaviours must be taught, and I honestly feel } \\
\text { our parent base does not give all of the virtues } \\
\text { education needed. I try to role model good } \\
\text { behaviours, treatment, respect, etc. and just } \\
\text { doing the right thing. }\end{array}$ & $\begin{array}{l}\text { Virtues is the basis of } \\
\text { how a classroom } \\
\text { operates - to be } \\
\text { allowed to have an } \\
\text { opinion/to be } \\
\text { encouraged to think } \\
\text { deeply for } \\
\text { excellence/to respect } \\
\text { others opinions. }\end{array}$ & $\begin{array}{l}\text { Virtues } \\
\text { education and } \\
\text { values education } \\
\text { respect personal } \\
\text { opinion, } \\
\text { thinking deeply } \\
\text { for oneself and } \\
\text { respecting the } \\
\text { opinions of } \\
\text { others. }\end{array}$ & \\
\hline $\begin{array}{l}\text { Vaughan mentions that embedded in his } \\
\text { teaching content are the virtues of everyday } \\
\text { life. He lists compassion, justice, self-control, } \\
\text { values that we hold, beliefs also, and he } \\
\text { highlights moral judgements and beliefs about } \\
\text { the right and wrong debate as also central to } \\
\text { his teaching. }\end{array}$ & $\begin{array}{l}\text { Embeds virtues, which } \\
\text { included values, in his } \\
\text { teaching content. }\end{array}$ & \multirow{2}{*}{$\begin{array}{c}\text { Virtues } \\
\text { education equal } \\
\text { values education }\end{array}$} & \\
\hline $\begin{array}{l}\text { George: I am confused by the subtle } \\
\text { differences in definition. My understanding is } \\
\text { that it (virtues education) is related to Values } \\
\text { and so should be foundational to the } \\
\text { application of any knowledge. Ethics is the } \\
\text { application of values. }\end{array}$ & $\begin{array}{l}\text { Virtues education is } \\
\text { related to values ... } \\
\text { Ethics is the } \\
\text { application of values }\end{array}$ & & \\
\hline
\end{tabular}




\begin{tabular}{|c|c|c|c|}
\hline Comment & Construct & Concept & Theme \\
\hline $\begin{array}{l}\text { Paul, in Victoria, equates Virtues education } \\
\text { with character education; he does not see any } \\
\text { real distinction between Virtues education and } \\
\text { Values education: I think the concepts of } \\
\text { ethics are too arcane for secondary students, } \\
\text { whereas applied ethics, or morals, can be } \\
\text { understood. I believe ethics consists in the } \\
\text { study of philosophical works of people such as } \\
\text { Kant and Schopenhauer -all too hard for } \\
\text { young people. }\end{array}$ & $\begin{array}{l}\text { No real distinction } \\
\text { between virtues and } \\
\text { values education. }\end{array}$ & & \\
\hline $\begin{array}{l}\text { Bernard, a Victorian, also sees no difference } \\
\text { between Virtues and Values education. He } \\
\text { uses more intangible rather than tangible } \\
\text { teaching strategies and practices as Virtues } \\
\text { education means Values, Standards - a belief } \\
\text { in one's self and purpose. He believes that } \\
\text { Ethics education is respect for oneself and } \\
\text { others. }\end{array}$ & $\begin{array}{l}\text { No difference between } \\
\text { virtues and values } \\
\text { education ... ethics } \\
\text { involves respect for } \\
\text { oneself and others. }\end{array}$ & $\begin{array}{l}\text { Virtues, values } \\
\text { and ethics are } \\
\text { closely entwined. }\end{array}$ & \\
\hline $\begin{array}{l}\text { Lavenda, from New Zealand: Teaching } \\
\text { values is very important in building an open- } \\
\text { minded and tolerant citizenry: scenarios/ role } \\
\text { plays - examining different peoples world } \\
\text { views/ perspectives; cross cultural } \\
\text { comparisons; pair and small and whole group } \\
\text { discussions/ formal and informal debates } \\
\text {...needed }\end{array}$ & $\begin{array}{l}\text { Values are important } \\
\text { in building an open- } \\
\text { minded and tolerant } \\
\text { citizenry. }\end{array}$ & $\begin{array}{l}\text { Building an } \\
\text { open-minded } \\
\text { and tolerant } \\
\text { society }\end{array}$ & \multirow{3}{*}{$\begin{array}{l}\text { Moral } \\
\text { agency in } \\
\text { schools }\end{array}$} \\
\hline $\begin{array}{l}\text { Dennis, a New Zealander: Basic moral values } \\
\text { relate to right and wrong. }\end{array}$ & $\begin{array}{l}\text { Basic moral values } \\
\text { relate to right and } \\
\text { wrong. }\end{array}$ & $\begin{array}{l}\text { Developing } \\
\text { concepts of right } \\
\text { and wrong }\end{array}$ & \\
\hline $\begin{array}{l}\text { Sandy, with experience in New Zealand and } \\
\text { Victoria, states: Looking at moral basis of } \\
\text { decisions we make. }\end{array}$ & $\begin{array}{l}\text { Looks at the moral } \\
\text { basis of decisions } \\
\text { made. }\end{array}$ & $\begin{array}{l}\text { Good and bad } \\
\text { decision-making }\end{array}$ & \\
\hline
\end{tabular}

\section{My researcher's voice}

An intriguing summary can be constructed here to highlight the widely divergent and sometimes idiosyncratic educational definitions of virtues, values and ethics. Some teachers saw large overlaps between the definitions of what is to count as virtue and what is to count as values; others saw no difference whatsoever. June showed clarity in her definitions about the difference between and the boundaries of virtue, values and ethics. Some teachers define virtues, values and ethics only in relation to their own subject area; others see no reason to open up the topic in a classroom. Some teachers favoured a secular framework, and others favoured a religious framework. No teachers identified differences in relation to other religious traditions beyond Christian. 
All accepted the construct of personal respect as the foundation of this whole area of education. Most teachers were concerned with matters developing concepts of right and wrong in behaviour and in thinking. The underlying theme of concept clarification pervaded within these very divergent views, and thereby highlighted the convergence of educational ethics within their delivery of content for student learning.

\section{A profile of current teachers' responses: Compilation of the hesitations, confusions, concerns and silences}

I believe it is important to recognise that, within the responses of the participants, there surfaced some general hesitations, signs of confusion, underlying concerns, and some silences to do with current issues in education in Australia, New Zealand and England, despite respondents having an opportunity to register their views in detail in each section of the Questionnaire. These have emerged in previous considerations within this chapter, and they need to be detailed together to give an overview of the whole picture of the teachers' voices. Thus here, in reflective mode, my researchers' voice considers these matters prior to addressing a set of overarching principles which might drive a reshaped theory of education.

The Questionnaire was based on elements of pedagogical practice and the role and function of person-particular procedures that bring success either pertinent to a given subject or discipline, or to an underlying philosophy and psychology of education. Ontological questions about the big Constructs and understandings that underpin the teaching of thinking were embedded in each section. Epistemological questions about the level of knowledge and skills necessarily applied to the teaching of the variety of thinking skills for decision-making processes were focused in Sections D, E, and F. Effective strategies (techné or pedagogy) and techniques regarding the asking of questions, the use of formats like Philosophy for Children with its Community of Inquiry were framed to discover how teachers go about the teaching learning process. 


\section{Hesitations}

The first hesitation was within the actual responses to the Questionnaire items. Explanations and examples were either not always outlined when requested, or were non-existent. It is possible that concentration on the actual title of the Questionnaire: 'Investigating Teachers' Perceptions of their Teaching of Thinking Skills' occupied the participants so that further expansion was seen as irrelevant.

The second was about slight differences in the wording of different section questions throughout the whole Questionnaire. Remarks like 'answered this already' and 'see earlier answer' seemed to indicate that the respondent was in a hurry or thought the question was repeated or did not recognise the changed emphasis attached to the later question.

The third was particularly noticeable when questions referred to the secular approach to philosophy of education as distinct from religious approaches to philosophy of education. As many respondents belonged to a college of the Christian mainstream, the hesitation could be for a number of reasons, including privacy and democracy. No evidence existed in other answers to explain.

The fourth centres on the possibility of discrete teaching of thinking skills, as a series of tools of logic, as a contrast to the possibility of teaching content, or as outcomes under the guise of teaching thinking skills. Little evidence of this as a live issue showed in responses. The teachers seemed unaware that discrete teaching of thinking skills rests on the transmission of a series of brain-compatible thinking and interrogating skills, so that students can be confident about democratic thinking and the quality of decisionmaking they need. They were cognisant apparently of the possibility that the opposite is indoctrination and is to be queried in whatever guise it appears.

The fifth is the potential implication when teachers may be asked to actually teach the concepts of some virtues, some values, and some logical forms of ethical thinking. There is some evidence to suggest that teachers may be aware of this as a contentious issue, preferring to keep to what was 
the official policy of a previous generation in Australia about having a values-neutral classroom. It has now been established that whatever the benign influence of a teacher in the classroom, students explore many virtues and values when studying narrative, when unfocused pedagogy can leave some unfair situations unaddressed; Life Skills, which came into play in Administration time, is the likely useful time for this, especially in secondary school. It is the responsibility of the classroom or homeroom teacher. Life Skills topics are full of unaddressed or under-addressed values that are overt and covert agenda of the particular class, teacher or school.

The sixth refers to teaching decision-making processes as part of thinking skills in the classroom. Respondents were at pains to highlight the efforts that they made to ensure the independent decisions of their class members. However, little evidence was noted that showed that teachers could teach how such a process would evolve without any unnecessary influence on outcomes.

\section{Confusions}

The first confusion showed in the responses of several respondents who admitted that to them, virtues and values were versions of the same thing. Consequently, it made an appreciable difference to the quality of some of the answers given.

The second seemed to emerge when respondents were asked about the student-directed classroom as distinct from a teacher-directed classroom. One respondent answered for a teacher-directed classroom, and left the rest of the response.

The third was to do with the documentation about the specific technical (techné) strategies used in classrooms. Some participants relied clearly and only on the governmental documents giving content and outlines. No other process of information was given about how this content was translated into step-by-step strategies giving students space for understanding, and for the formation of relevant questions so that actual learning takes place. It is not 
possible to extract what their strategies were. Other participants were clear in their recognition of how they designed their step-by-step strategies so that students' questions were clarified and rich learning took place. These respondents indicated processes that sometimes used a variety of work devised by Lipman’s Community of Inquiry and Fogarty’s Brain-compatible classrooms and others.

The fourth was the assumption that a Community of Inquiry in Lipman's framework was the same as an Inquiry-based classroom. The details of the Community of Inquiry rest on the careful use of each of the steps in Lipman's process and in which students can ask any questions of themselves, their classmates, and their teacher. Then they begin the prioritising of questions, allocating who does what research to provide for the class. Everyone helps in a cooperative and collaborative effort. An inquiry-based classroom, though, relies on some input from the teacher about the content, followed by individual research prior to completion of individual work assignments. No sound evidence of recognition of the difference was present in most responses.

The fifth emerges when participants answer questions about the difference between teaching the student, each student, and teaching just the curriculum. Getting through the curriculum according to the requirement of their internal documentation was seen by some respondents as the only aim of delivery of curricular content.

The sixth rests on the previous policy, especially in Australia, of striving for a values-neutral classroom. Consistently, values creep in by stealth or by accident, and the covert agenda is not really addressed. Respondents did not always distinguish between a values-neutral classroom and a possibility of unaddressed and mistaken concepts of tolerance, justice and respect. Where they tried to do so, there was noticeable confusion as to how this might be done. It also emerged that in a school such as the one from which more than half the respondents taught, values could be expected to be taught in Religion classes or perhaps some values could be discussed briefly in Life Skills 
classes along with Drug education and Sex education, both scenarios contributing to student learning.

The final confusion rests on the experience of teachers of today's Australia especially, whose education in the main would have been (for many) in that values-neutral classroom that operated for several generations. Though the word is not used in today's academic conversations, there is an apparent confusion about what constitutes indoctrination. Today's teachers may not have opportunities to investigate the full scale of what does constitute indoctrination and what does not. The confusion further is exacerbated by the unexamined belief that indoctrination is only found in religious spheres. The alternative seems to be that today's teachers do not impinge on matters where there is an expectation of choice in the lives of today's students whether career oriented, or within philosophical frameworks or religious belief systems.

\section{Concerns}

My first identification of a concern, and one that seems to be behind some participants' responses, is whether there is a responsibility, especially in secondary classes, for teachers to discipline their students. Some evidence shows that teachers perhaps expect that the respect and cooperation that makes class work operate seamlessly, is the province of the students and perhaps their parents. Not much evidence of how the relevant rules, for a teachers' class would operate, was offered. As there was not a particular question asking this precise matter, it is difficult to conclude more than a generalised concern.

A second concern emerged for participants who found it difficult to answer some of the specific questions, because they kept precisely to the requirements of the National Curriculum. This disinterested approach was particularly noticeable in participants from New Zealand and England. Not much evidence of a negotiated curriculum was shown in any of the participants' responses, except several who helped students to compose the 
questions relevant to the strategies used in the Community of Inquiry. It was not possible to discern just how constricted or otherwise teachers seemed to view the delivery of a National Curriculum.

\section{Silences}

Even though opportunities were given in the prosecution of the Questionnaire, for respondents to highlight problems in delivering teaching of thinking skills in whatever way seemed appropriate to their disciplines, and the exigencies of daily inclusion of extra-curricular activities, no respondent actually explained how it is possible to provide on a regular basis exactly and only what a National Curriculum required; this was the first silence. If this is the result of the experience of teachers in England where it is possible to deliver exactly the same content to four classes of differingabilities in each week of the term, then the silence is deafening. It becomes obvious that there are problems relating to the actual student learning rates in such a program.

Similarly, teaching to the exam, as teachers appear to do in delivering only the set curriculum according to government documentation (especially in Years 11 and 12), is unaddressed; this was the second silence. The same constraints seem to apply to the NAPLAN testing and My School in Australia. No specific question was included in the Questionnaire, but the silence is conspicuous, because to teach thinking skills includes teaching students how to construct relevant questions and then helping them to prioritise the questions list. No time allocation has been given, especially when the curriculum for the Victorian Certificate of Education (VCE) in Victoria is so broad, so choices are made by the teacher and the school curriculum team, and often these choices do not include any reference to the class to be taught. Short-sighted as this may appear, no evidence of teacher concern has emerged in the responses to the Questionnaire. 


\section{Conclusions and comments on Questionnaire content}

Teachers were requested directly as a conclusion to the Questionnaire to advise any other matters that they saw as relevant to the nature of the Questionnaire and the topic being investigated. A number of respondents had significant points to make and these are detailed in Table 4.32.

TABLE 4.32 TEACHERS' CONCLUSIONS AND COMMENTS

\begin{tabular}{|c|c|c|c|}
\hline COMMENT & CONSTRUCT & CONCEPT & THEME \\
\hline $\begin{array}{l}\text { Samantha: Leaders need to be seen to act. } \\
\text { e.g.,: via discipline, justice, harmony, } \\
\text { communication, support, PD etc. Great } \\
\text { principals and staff build a values based } \\
\text { learning community, which in turn leads to } \\
\text { an ethical school culture. Lack of discipline, } \\
\text { justice, proper redemption processes lead to } \\
\text { an attitude of I can do what I like. . }\end{array}$ & $\begin{array}{l}\text { Building a values- } \\
\text { based learning } \\
\text { community, which in } \\
\text { turn leads to an ethical } \\
\text { school culture? }\end{array}$ & $\begin{array}{l}\text { Seeking a } \\
\text { philosophy of } \\
\text { education that is } \\
\text { school-wide. }\end{array}$ & \multirow{4}{*}{$\begin{array}{l}\text { Building a } \\
\text { learning and } \\
\text { thinking } \\
\text { organisation. }\end{array}$} \\
\hline $\begin{array}{l}\text { Rose: Not enough is being done in schools, } \\
\text { particularly at the senior levels to encourage } \\
\text { students to be independent learners. } \\
\text { Learning is a lifelong process and if we want } \\
\text { students to become engaged in society and } \\
\text { the issues that are important to them, then } \\
\text { students must be given the skills to question, } \\
\text { to do further research, to critically analyse } \\
\text { what they read or hear in the popular media } \\
\text { and to acknowledge that there are legitimate } \\
\text { views that may differ from their own. }\end{array}$ & $\begin{array}{l}\text { Seeing learning as a } \\
\text { lifelong process to } \\
\text { ensure that our } \\
\text { students become } \\
\text { engaged in society and } \\
\text { face issues that are } \\
\text { important to them by } \\
\text { means of questioning, } \\
\text { researching, critically } \\
\text { analysing, and } \\
\text { recognising } \\
\text { differences. }\end{array}$ & $\begin{array}{l}\text { Seeing the big } \\
\text { picture: } \\
\text { Learning as a } \\
\text { life-long } \\
\text { experience. }\end{array}$ & \\
\hline $\begin{array}{l}\text { Pietr: Only that it is much more effective if } \\
\text { all staff at the school believe and teach them } \\
\text { as part of their subject for it to have most } \\
\text { impact. }\end{array}$ & $\begin{array}{l}\text { Involving all staff in a } \\
\text { school in the teaching } \\
\text { of thinking skills } \\
\text { within the frame of } \\
\text { values, virtues and } \\
\text { ethics. }\end{array}$ & $\begin{array}{l}\text { Taking a systems } \\
\text { approach to the } \\
\text { development of } \\
\text { thinking skills. }\end{array}$ & \\
\hline $\begin{array}{l}\text { Hyacinth: When you begin using thinking } \\
\text { skills frameworks or activities, it is like } \\
\text { teaching new knowledge. The more you } \\
\text { teach thinking skills, the more integrated } \\
\text { they become - until it becomes part of your } \\
\text { style, you use it all the time and become a } \\
\text { more effective teacher. }\end{array}$ & $\begin{array}{l}\text { To integrate thinking } \\
\text { skills in order to } \\
\text { become a more } \\
\text { effective teacher. }\end{array}$ & $\begin{array}{l}\text { Using thinking } \\
\text { skills to develop } \\
\text { a new style of } \\
\text { teaching. }\end{array}$ & \\
\hline
\end{tabular}




\begin{tabular}{|c|c|c|c|}
\hline COMMENT & CONSTRUCT & CONCEPT & THEME \\
\hline $\begin{array}{l}\text { Henry, who has taught in three states in } \\
\text { Australia as well as India and Canada, } \\
\text { admitted to being a bit confused about the } \\
\text { content of the Questionnaire and how it } \\
\text { applies to his area of Technology and } \\
\text { Design, but his comment is revealing: I think } \\
\text { more could be done to recognise importance } \\
\text { of being human: creativity/ personal } \\
\text { honesty/ accountability/ integrity/ Going } \\
\text { Deeper - How we affect others when we } \\
\text { share this stuff, ... Education must be more } \\
\text { than knowledge. Must have meaning- } \\
\text { personal awareness/ growth/ spirituality - } \\
\text { context of relationship society. The } \\
\text { underlining of emphasised points shown is } \\
\text { Henrys effort to highlight some very } \\
\text { relevant details behind the Questionnaire. }\end{array}$ & $\begin{array}{l}\text { Developing the } \\
\text { following: creativity, } \\
\text { personal honesty, } \\
\text { accountability, } \\
\text { integrity, going } \\
\text { deeper, our impact on } \\
\text { others when we share } \\
\text { this philosophy: } \\
\text { making meaning } \\
\text { through personal } \\
\text { awareness, growth, } \\
\text { spirituality; working } \\
\text { in the context of a } \\
\text { relationship society }\end{array}$ & $\begin{array}{l}\text { Applying } \\
\text { thinking skills } \\
\text { for personal, } \\
\text { cultural and } \\
\text { spiritual } \\
\text { development. }\end{array}$ & $\begin{array}{l}\text { Making } \\
\text { meaning in a } \\
\text { relationship } \\
\text { society. }\end{array}$ \\
\hline $\begin{array}{l}\text { Sandy: I don't find that the VCE requires } \\
\text { me to look at thinking skills to the extent } \\
\text { that the IB did, and I don't find the VCE } \\
\text { students as open to being challenged as the } \\
\text { IB students. TOK (Theory of Knowledge) as } \\
\text { a subject is geared to thinking skills. Easy } \\
\text { for History - be content-driven. IB English } \\
\text { (is) better suited than VCE English. TOK } \\
\text { demands an analysis of how we gain } \\
\text { knowledge across a variety of subject areas }\end{array}$ & $\begin{array}{l}\text { VCE students not as } \\
\text { open to being } \\
\text { challenged to think as } \\
\text { those following the } \\
\text { International } \\
\text { Baccalaureate diploma } \\
\text { program. }\end{array}$ & $\begin{array}{l}\text { IB demands the } \\
\text { application of } \\
\text { thinking skills } \\
\text { through its } \\
\text { compulsory } \\
\text { Theory of } \\
\text { Knowledge } \\
\text { component. }\end{array}$ & $\begin{array}{l}\text { Epistemology/ } \\
\text { Theory of } \\
\text { Knowledge } \\
\text { incorporated } \\
\text { in the } \\
\text { secondary } \\
\text { school } \\
\text { curriculum. }\end{array}$ \\
\hline $\begin{array}{l}\text { George: I find it difficult to break down } \\
\text { these theories into practical differences, or } \\
\text { separate approaches in the classroom. I } \\
\text { focus on the topic and use various } \\
\text { techniques to crack it open for the students. } \\
\ldots \text { challenging them on the journey to be } \\
\text { open to new Constructs and opinions. I } \\
\text { always challenge them to think: how can I } \\
\text { apply my knowledge? George uses an } \\
\text { example of one of the textbooks from } \\
\text { Religion, Values, and Ethics class: } \\
\text { Introduction to Ethics. }\end{array}$ & $\begin{array}{l}\text { Focusing on the topic } \\
\text { and use various } \\
\text { techniques to crack it } \\
\text { open for the students } \\
\ldots \text { challenging them } \\
\text { on the journey to be } \\
\text { open to new } \\
\text { Constructs and } \\
\text { opinions. }\end{array}$ & $\begin{array}{l}\text { Overcoming the } \\
\text { difficulty in } \\
\text { relating theory to } \\
\text { practical } \\
\text { differences or } \\
\text { separate } \\
\text { approaches in } \\
\text { the classroom. }\end{array}$ & $\begin{array}{c}\text { Relating } \\
\text { theory and } \\
\text { practice } \\
\text { within the } \\
\text { classroom. }\end{array}$ \\
\hline
\end{tabular}

\section{Teachers' voices}

Teachers highlighted constructs that focused on a values-based school community; on learning as a life-long experience needing skills in questioning, searching, analysing and recognising difference, all of which are thinking skills; on a need for cohesion in curricular and administrative school frameworks through teaching thinking skills; on a thinking skills integration in effective pedagogical practice; on ensuring development of thinking skills to allow knowledge about virtues, values and ethics to emerge; on needs of senior students to rely on previously acquired thinking skills for success; on being able to crack it open as an attempt to help students find meaning in curriculum. 
The concepts deriving from these considered opinions include a need for philosophy of education school-wide; a big picture approach; a system-wide approach to teaching thinking skills - even to integrating these skills into a new model of teaching, focused on personal, cultural and spiritual development; and preference as a consequence for IB curriculum with its theory of knowledge rather than the VCE curriculum. Themes emerging include building the thinking and learning organisation by:

- making meaning in a relationship-based society;

- incorporating the successful IB approach to teaching thinking rather than VCE ;

- making sure that theory and practice in classroom milieux are integrated and cohesive.

Links and connections to authors and educationalists I have chosen as references which are noted in chapter 3, and parallels to the autoethnographical retrospective (Chapter 5) are graphically illustrated.

\section{New ideas emerging: My researcher's voice}

Subsequent to the 'voices' heard in previous sections, there are no other voices than my researcher voice in what follows:

\section{Metaphors for teaching styles}

As the analysis of teachers' responses emerged, it became obvious to me that it is possible to recognise the differing hues that inform teachers in their daily attitudes and preferences to the technical strategies that were emerging. I have attempted to develop a set of metaphors that represent teacher styles. It is important, however, that in identifying those teachers who fit one or more of the following profiles, individual colleagues are not being judged; nor is one style seen as inferior to any other! What is of significance is that teachers are given opportunities to recognise and understand their similarity to one or more of the profiles. They may decide there is a reason for, or value in, 
varying their personal teaching styles for value and worth in their pedagogical (techné) practices. I have identified ten possible styles:

1. Curriculum slaves - those who take a Curriculum document direct from the relevant Education Department, and put the stated aims of the document directly to students, planning and delivering by instruction and other means that lead the students through moves needed to achieve the final outcome.

2. Administrative ideologues - those who are converted to one curriculum strategic process (e.g., Community of Inquiry or Inquiry Learning framework). These teachers use the individual strategies from that process that they perceive will be effective and that they have learned to use, exclusively.

3. VOK Inter-actors- those aware of the three learning styles emergent decades ago in research about excellence in teaching learning processes: Visual, Oral, and Kinaesthetic learning styles and Visual, Oral and Kinaesthetic teaching styles. Having internalised the relevant strategies when they first encountered this process, they concentrate on completing topic aims. This is different in context from the other two profiles above because the approach allows for three particular styles of strategies designed by the teacher to teach each particular segment. Students sometimes are confused when the teacher appears to be saying the same thing in three differing ways.

4. Gen X Innovators - those whose exposure to current professional development and learning has included knowledge of the connections between successful curriculum outcomes and streamlined processes e.g.: RVE, PMI, de Bono's Thinking Hats, et al. These people are broadening the teaching of thinking skills format.

5. Democratisers- those with personal methodologies relying on facilitation and team work, with other teachers and with students, rather than highlighting the expertise of teachers in relation to the 
incoming students, e.g.,: Inquiry learning, Team teaching, Fish-bowl strategy, Corners, Debating and Drama.

6. IT buffs - those who know and understand various insertions of IT skills as a facilitating factor in student learning, e.g., student directed classrooms, whole-class strategies like the research used for the Fishbowl; where tasks are shared and skills learned through sharing of approaches used. Team learning and collaboration techniques often accompany the use of IT skills.

7. Philosophisers - those who plan careful blending and specific use of Government documents with the role and function of the teacher in the teaching-learning process, and incorporate underlying use of teaching of concepts and the use of questions and questioning as bridges to the acquisition of applied thinking skills.

8. Study Concentrators - those who see the role and function of teacher as exclusively teaching and delivering the content of a particular discipline, unit or topic pitched at a preconceived standard for a given grade level. Pre- and Post- testing often accompanies a Deficit Model of teaching strategies meant to develop student acquisition of knowledge and skills relevant to current tasks.

9. Producers of Excellence -those who use an educational policy at a personal level, to promote deep and rich learning for each individual student, relying on the use of content of the given topic first for knowledge within the topic itself, then extending that knowledge into application through applied thinking skills to the student. A variety of classroom strategies and deliberately focused teaching processes are used separately or concurrently as the class progresses through the introduction, engagement and completion of tasks by students who evaluate what has just been achieved.

10. The converters - those who use strategic questions, or deliberate and planned questioning in class discussions to lead the students to produce a preconceived and preferred outcome. Note is made here 
that preferred outcomes for disciplines like Science and Mathematics would need to use this pedagogy, whereas disciplines like English, Philosophy, Religious Studies are entirely different and this approach may be, on occasions, far more dangerous in nature.

There is no measurement here of teachers who might adhere to one of these profiles. Further reflections in Chapter 6 identify some of the significant considerations that could apply.

\section{Connecting virtues, values and ethics (VVE) to the purpose of making decisions}

In this section, I endeavour to clarify the links, connections, gaps, contradictions and assumptions according to my experience in Philosophy for Children classes in order to influence the construction of my reshaped educational theory. I contend that Understanding begins with the teaching of thinking skills so that the individual meets, learns, understands, and applies the following elements:

- Virtues

- Learning what? Learning how? Learning what if? Learning self-correction.

- Students are exploring risk and contrasting it with a stable response; they are embedding understanding and definition of this set of concepts as an internal attitude and chosen habit of mind, which they plan to usefully inform personal behaviours for positive value.

- The list of test question starters connects here.

- Values

- Learning about others; learning to appreciate; learning best practice; learning tolerance; learning to value and accept what was deemed appropriate; recognising what and why the culture around us is characterised by certain standards of behaviour. 
- Students are embedding understanding and definition of the set of concepts as a recognised chosen set of community behaviours of a given tribe, nation or group.

- The ME concept and the Heasly HUG/BUG connects here by providing channels that they can use to test their knowledge, understanding and choice for quality in their decision-making, independent of others.

\section{- Ethics}

- Behaviours arc; Equality; Rights; Responsibilities; Respect; Reconciliation skills. Students are embedding understanding of this set of concepts, chosen by them as best practice for the general good of most people.

- The use of the HUG/BUG connects here, in order to make decisions about:

\section{- Career Planning:}

- Knowledge; Education; Experience; Advisement (not advice); Evaluation; Self-Correction; Achievement. Students are testing, exploring and shaping their increasing knowledge of themselves, their growing knowledge and their increasing skills level.

- The ME concept connects here.

\section{- Quality of Life:}

- Democratic Engagement; Philosophy of Right and Wrong; Community Contribution; the assumption of Independence; the Practice of Interdependence. Students are self-correcting where necessary, allowing for the mistakes they might make as their testing and mistaken assumptions bring reconstruction of their thinking and expectations of themselves.

- $\quad$ The use of the HUG/BUG applies here. 


\section{- Secular Belief System:}

- Safety; Equality; Life Achievement; Rest and Recreation; Satisfaction; Happiness; Quality of Life. A regular understanding by students of an active democracy emerges as a consequence.

- The Heasly Six Steps in Decision-making, the two forms of Decisions, the Heasly HUG/BUG and the ME concept all connect here, to provide teachers with initial channels for teaching about virtues, values and ethics.

\section{- Metaphysical Meaning:}

- Gradual Understanding of Meta-questions from religious or non-religious personal belief systems; personal philosophy of life achieved through self-correction, personal experience, cultural and religious choices. The best constructs of religious, secular and philosophical thinking underpin this outcome.

\section{Indoctrination and higher-order thinking}

In this section, my researcher's voice is heard first, and then my practitioner voice is heard.

\section{My researcher's voice}

In considering the various teaching styles, and considering the links, connections, gaps, contradictions and assumptions, one particular skill to emerge is the need by teachers in the classroom to understand what constitutes indoctrination and how it differs in concept, understanding and teaching strategy from the delivery of higher-order thinking skills. Neither indoctrination, nor higher-order thinking skills, is better than the other; the important thing is when one, or the other, is needed. They are not congruent with each other.

As a consequence I have found that it was necessary for me to spend time analysing the ontological approaches exemplified in the teachers' responses. 
In the past, I found it important to distinguish indoctrination from religious or political belief; to recognise the differing roles of lineal and lateral thinking; to understand deeply what the difference was between fundamentalist thinking patterns and democratically constructed personal and communal options. These distinctions represent, for me, a need for a major epistemological development in virtues, values and ethics education. It was also vital that teachers actually recognise that while some of these thinking patterns can be dangerous, it is not logical to dismiss or ignore them. These patterns, called sometimes indoctrination and sometimes fundamentalist thinking, have existed from time immemorial, and have had their uses in previous generations. In my experience, stimulating discussions occur when senior students become adept at abstract thinking and can concretise at will, developing the best and most balanced personal understandings through appropriate pedagogical (techné) strategies.

\section{My practitioner voice}

I have begun with a comparison between the individual concepts of indoctrination and higher-order thinking skills, and followed through with a number of distinctions, assumptions, gaps, contradictions and misunderstandings. I have identified 15 separate ways to develop valid delivery of curriculum and strategic practices that connect to the practice of Consensus and Democracy. For me, distinctions between indoctrination and higher-order thinking become important in order to distinguish between problem, difficulty, dilemma and paradox - this helped define the boundaries between secular and religious reasoning. In this endeavour, I was guided by David Martin's (2011) exploration of the multiple ironies and necessary paradoxes that accompany a review of religion, fanaticism and violence in our twenty-first century world. These distinctions function to ensure that through imagination, broad thinking and questions are modelled to facilitate maximum progress in students' rich learning. The 15 elements are contained in Figure 4.5; each element is considered below in two parts: the first, concerned with indoctrination; the second with higher-order thinking: 
FIGURE 4.5 ELEMENTS OF INDOCTRINATION AND HIGHER-ORDER THINKING

\begin{tabular}{|c|l|l|}
\hline SERIAL & \multicolumn{1}{|c|}{ INDOCTRINATION } & \multicolumn{1}{c|}{ HIGHER-ORDER THINKING } \\
\hline $\mathbf{1}$ & Concept of Indoctrination & Concept of Higher-Order thinking \\
\hline $\mathbf{2}$ & Control of delivered content & Exploration of content \\
\hline $\mathbf{3}$ & Other-centred approach & Self or independent approach \\
\hline $\mathbf{4}$ & Narrow focus of action & Sifting of options and action \\
\hline $\mathbf{5}$ & Assumption of power & Personal autonomy \\
\hline $\mathbf{6}$ & Enforcement & Experimentation/ testing/ critiquing \\
\hline $\mathbf{7}$ & $\begin{array}{l}\text { Defiance/ Dismissal of broad } \\
\text { evidence }\end{array}$ & Response-ability and responsibility \\
\hline $\mathbf{8}$ & Punishment & Re-direction \\
\hline $\mathbf{9}$ & Attribution of guilt & $\begin{array}{l}\text { Acceptance of consequence or } \\
\text { recompense }\end{array}$ \\
\hline $\mathbf{1 0}$ & Imprisonment & Empowerment \\
\hline $\mathbf{1 1}$ & Obligation & Transformation \\
\hline $\mathbf{1 2}$ & Rote-learning & Applied knowledge \\
\hline $\mathbf{1 3}$ & Required Obedience & Gathering of wisdom in action \\
\hline $\mathbf{1 4}$ & Ideology & World View/ Faith dimension \\
\hline $\mathbf{1 5}$ & Rigidity & Discovery \\
\hline
\end{tabular}

\section{Concept of indoctrination vs. concept of higher-order thinking}

I believe that it is important to indicate very clearly that indoctrination is the business of imparting one, sometimes simplistic, answer to a complex question or problem, insisting that the one answer was enough. I have found that higher-order thinking, however, provides steps of analysis, looking for options and elements of complexity in order to deepen understanding.

\section{Control of delivered content vs. exploration of content}

If teachers like me controlled the content to be delivered by strategies like instruction alone, by time constraints, by demanding a regurgitated answer, by assessment strategies which dismiss parts of the content, then indoctrination is present. In higher-order thinking, I must ensure that 
inspection of every element of meaning by judicious use of questions, discussion, testing, hypothesising and other thinking skills from the Splitter \& Sharp list, and then deep and rich learning can take place.

\section{Other-centred approach vs. self or independent approach}

If the approach and direction of the teacher was seen to be the only way to learn content in classroom topics, then the student would ingest what was necessary and identify what must be returned as evidence of learning. If I ensure that inquiry, research, questions, discussions whether fish-bowl style or community of inquiry were used to broaden and clarify, then the independence and self-regulation of the student would be preserved as deep learning was promoted.

\section{Narrow focus of action vs. sifting of options and action}

If the teacher were to allow too short a time for the outline and content of a topic or question to be addressed, then the students would not internalise the depth of understanding that was possible, and would become immune to the benefits of sifting for the best options for help, understanding and learning, preferring to grab an expected answer only.

\section{Assumption of power vs. personal autonomy}

If I, through the use of classroom strategies, control every move and stop further investigation, then I, as teacher, maintain the power of the classroom over how learning takes place, consequently student personal autonomy would be diminished, and passivity would take the place of choice for learning and how to proceed with that aim.

\section{Enforcement vs. experimentation /testing/critiquing}

If $\mathrm{I}$, as the presenter, facilitator or teacher push the topic in a certain direction without recourse to the need for questions, for testing, for an array of experimentation, then it would be a teacher-directed classroom with no 
freedom to explore. Students would lose interest and shallow learning and possible indoctrination would take the place of enthusiastic research and questioning.

\section{Defiance or dismissal of broad evidence vs. response-ability and responsibility}

In matters of Values and Ethics, there may be a tendency for me as a teacher to provide a preferred answer to a difficulty, a problem, a dilemma, a paradox without analysing the depth of meaning already indicated, and without time allotted for researching further. This would stop students from their independent exploration of content and diminish their response-ability. Response-ability is different from responsibility, in that it is necessary for the individual student, or anyone else involved, to reflect on attitudes, preferences, contradictions and connections in order to decide responses that deepen the understanding of concepts and topics. Then the responsibility of that individual would be to articulate, clarify, develop and discuss elements of the matter under consideration in order to share, to critique, and to learn.

\section{Punishment vs. re-direction}

The teaching-learning process would be at risk when those in authority, whether the actual teacher like me, or a Principal or Principal's delegate, prefer to punish when a mistake was made. The mistake may be seen as evidence of failure rather than an opportunity to clarify further, recognise confusion, and allow for questions and hypothesis in order that re-direction of the students' attention will provide a channel for learning. Re-direction can even come in the form of a teacher question or another student asking for more information.

\section{Attribution of guilt vs. acceptance of consequence or recompense}

When students' experience of the classroom included being labelled guilty of laziness or worse by a teacher like me, the psychological effect becomes a message of power over the students' progress. If instead, there was an 
acceptance by both teacher and student that the consequences of making a mistake was that more work was needed to repair the understanding of the lesson, that experience of control-over, would be lessened and learning could take place unimpeded.

\section{Imprisonment vs. empowerment}

Of course, this imprisonment was not necessarily incarceration, but the business of severe detention experiences becomes counter-productive and furthers the possibility of the students' feeling that there was control-over, rather than an alternative of empowerment based on positive strategies that focus on good processes enhancing learning potential.

\section{Obligation vs. transformation}

In times past, there was a tendency for some teachers to place students under all sorts of obligations because of the students' extra needs. The student reacted by focusing only on the exact directions of the teacher hoping that learning would be possible. There is an alternative way for a teacher in my position that focuses on teacher strategies to transform the student experience into something pleasant, independent and helpful. The student reacts with an accelerated burst of energy striving towards learning well.

\section{Rote learning vs. applied knowledge}

Rote-learning has never been totally obliterated in the classroom. It was useful in some discipline areas, but must never be the only strategy for learning. Sometimes, a short-cut list can be given by me as teacher, but if the student does not have time to recognise the links and connections, there is little chance to understand and apply the content appropriately. Applied knowledge comes from making sense through inductive and deductive thinking skills, not from a rote-learned piece with little recognition of meaning. Confining a student to rote-learning can be experienced as a serious constraint. 


\section{Required obedience vs. gathering of wisdom in action}

Teachers have always been aware, as I have, of the value of self-reflection in the matter of how they deliver in a classroom. There is a tendency when under pressure to revert to one's earliest experiences of the classroom. Teaching skills do not include those archaic styles of teaching that were based on obedience before learning. Treating students as though they have no initiative was always counter-productive. Redirection by classroom strategy towards the questioning, the testing, the research, and the discussion needed to explore all facets of the topic under consideration means that the students gathered an experience of what it meant to consciously begin gathering knowledge and applying it wisely: A Socratic approach, a Lipman approach, a Vardy approach! And, by extension, a Heasly approach!

\section{Ideology vs. world view/faith dimension}

Ideology is the concept of gathering a universal conclusion about a world matter, a contentious issue, a political conundrum, or a deliberate choice, deciding on a particular way of responding. Ideology is never faith, in any of the various religions of twenty-first century. If an ideology (sometimes today called spin) took the place of the eschatological speculations so familiar in life today, there is no possibility of developing an astute and balanced wisdom or faith, whether religiously-based or secularly-based. Ideology can be about any subject, and can permeate classroom strategies, and there is value for a teacher like me to be clear about the likelihood that at times, students will exhibit various unexamined and alternative views about some preferred contentious topics, especially about ethical behaviour.

\section{Rigidity vs. discovery}

Teaching I have found was always about discovery, about learning, about new fields of importance. If the approach of the teacher is rigid and unyielding, students identify the resistance and give only expected answers without much personal thought and without internalising particular or 
significant elements of classroom content. If instead, teachers like me can feel comfortable with admitting when they don't know enough about a certain topic to provide some re-direction where necessary, the team approach to learning together settles the class into a student-directed classroom experience where learning is positive and wise.

Most recently, van Hooft (2012, p. 13), in a comment relating to religious classes in state schools, strongly affirmed that a liberal society should guard against indoctrination by 'religious adherents':

\begin{abstract}
A liberal society should protect children in public schools from indoctrination by well-meaning religious adherents while also protecting the private right of religious groups to set up their own schools. Schools set up by a liberal state and pursuing public good should not be intruded upon by the private convictions of any groups within society.
\end{abstract}

van Hooft's position echoes mine: that, in a democratic society, we are all entitled to learn enough to be able to choose wisely.

\title{
Pedagogical processes and outcomes
}

In this section, only my practitioner voice is heard.

\section{My practitioner voice}

Had I not listened to Basil Moore at a conference held in Melbourne in 1986, I would not have realised the importance of making the distinction between teaching styles; nor would I have recognised the need for a new educational model that encourages collaborative learning between students and teachers. At this Eleventh Conference of the Australian Association for Religious Education, Moore (1986) provided a booklet entitled Five Strategies for Teaching in which he described, in parallel, five models:

- Concept Attainment Model

- Inquiry Learning Model 
- Communications Model

- Negotiated Model

- Shared Praxis Model

He provided information about the five relevant originators, the distinctive key goals, with the accompanying critique of education, the individual rationale, the key theoretical assumptions and the basic structure for the teaching-learning process. Accompanying each module was a hypothetical example to flesh out the meaning in practice. In particular, this showed the difference between the Inquiry Learning model and the Philosophical Community of Inquiry which was central to Lipman's format. This booklet was as much about the best practice of teaching and the role of teacher as it was an example of the diversity that accompanied the teaching and learning within the theological or spiritual milieux. It was, however, broader than just these two milieux; it also considered the appropriate choice of models to suit particular students, teachers and subjects in all milieux. In this sense, Moore was akin to Schwab's four commonplaces of the curriculum, though I was not aware of Schwab at this stage of my learning curve, and Moore had not specified any connection.

Over 36 years of teaching in secondary schools, for me, these five teaching models became the processes for development of my attitude, elements of my knowledge, and an expansion of my set of skills that focused on efforts to teach the student rather than only the topic content, no matter in which discipline I was teaching. Each of the models, as Moore described them, gave the underlying philosophy (ontological influence), followed by the theoretical assumptions and rationale (epistemological underpinning) and the sequential classroom steps (pedagogy-techné) with which the teacher would improve the teaching learning process, and become a more efficient practitioner. I became more conscious of the three concepts of ontology, epistemology and pedagogy-techné as I tried to improve the use of these five models in my teaching-learning processes. In retrospect, I can now make 
more deliberate analysis and evaluation of what Moore was indicating, and what I was practising.

\section{Need for a new architecture}

In this concluding section, only my researcher's voice is heard.

\section{My researcher's voice}

In integrating the Moral Components of Wilson, the Community of Inquiry of Lipman, and eventually the more advanced curriculum content of Vardy, it became obvious that, as well, I was developing and trialling certain conceptual frameworks of my own. These streams of educational expertise were building on the foundation of the five Teaching Models that Moore delineated.

I was investigating therefore the ontological considerations pertinent to my practice; the epistemological concerns and considerations that resulted from my personal perspectives and personal philosophy; and, in Aristotelian terms, a format of technical strategies fused into pedagogical (techné) practice.

The development and trialling of my own conceptual frameworks demanded that I integrate my thinking, my successes and mistakes into a coherent and practical practice for the areas of values, virtues and ethics. With these areas as content, I have been able to translate this demand ontologically, epistemologically and pedagogically into a new, unifying concept which will be detailed in Chapter 6 .

\section{Looking forward}

In the next chapter, I explore my experiential learning through the channel of an inductive data reduction chart focused on my career of 36 years. This is a necessary step to shape and reshape the integration of the experiential learning and the formal qualifications, before I begin the task of applying the three research sources to the business of constructing a new theory of education. 


\section{CHAPTER 5}

\section{Auto-Ethnographical Retrospective}

\section{Introduction}

In this chapter, using a simplified auto-ethnographic approach and apply an inductive data reduction technique, I present a synthesis of the experiential learning that provides another voice (mine) as a source of the background thinking that contributed to the original design of my Questionnaire. The account is addressed in terms of my 'stages of development' across a period of 36 years in secondary schools and in universities. This permits my voice to emerge and to be heard in the mix of sources contributing to the detail of a reshaped theory for teaching virtues, values and ethics.

\section{Stages of development: An auto-ethnographic account}

To enable my voice to be heard in this thesis, I decided to write a brief autoethnographic account of my professional development across a period of some 40 years. This account is structured according to Tuckman's (1965) categories for the team development of small groups: forming, norming, storming, and performing. A subsequent exposure and development of these categories was provided by Mulford's (1977) application of these concepts in Australian classrooms - with the addition of a fifth category; mourning (Tuckman, later, added a less dramatic category of 'adjourning'). Since I am still actively involved in the development of thinking about virtues, values 
and ethics, and have no intention stopping, the fifth and sixth stages, fortunately, does not apply!

\section{Forming}

In the early formational phase of my teaching-learning career there were four themes that were influential: qualification, formal and informal learning, ethics and praxis; of these, the search for qualifications was the most urgent. These auto-ethnographic themes, and my account of their origins, are summarised in Table 5.1.

In line with Tuckman (1965) and Mulford (1977), I have detailed the entries in the left column with as many of the significant experiential learning processes as I can record. These experiences fall into groups which are highlighted by the change of colour as the reader proceeds through the four major stages of my career: forming, norming, storming and performing.

The construct column translates the personal comment from Column 1 into a theoretical construct from which I could glean concepts about the needs of teachers and students, the possible problem-solving to be done, and the identification of which studies and which professional seminars and certificates of understanding I would need to become a fully expert teacher. The right hand column assigns a thematic title to the directions perceived that I would need to take to attain the expertise required, so that further planning of my studies could be implemented: formal qualifications, ethical considerations, integration of formal and informal learning, and the foundations of teaching praxis (Cherednichenko, 2000).

\section{Qualifications}

Because my earliest qualifications related to individual performance in piano, organ and voice, I lacked the necessary qualification for the teaching of Music in classrooms: this created the need for classroom qualifications. In addition, in the process of balancing work and home activities, I engaged in a 
TABLE 5.1 FORMING

\begin{tabular}{|c|c|c|c|c|}
\hline REF & COMMENT & CONSTRUCT & CONCEPT & THEME \\
\hline F1 & $\begin{array}{l}\text { I began with a tertiary Music } \\
\text { qualification at Melba Memorial } \\
\text { Conservatorium in piano, } \\
\text { performance and teaching. }\end{array}$ & $\begin{array}{l}\text { Began tertiary } \\
\text { teaching } \\
\text { qualification for } \\
\text { music }\end{array}$ & $\begin{array}{l}\text { Need for } \\
\text { classroom } \\
\text { qualifications }\end{array}$ & \multirow{3}{*}{ Qualification } \\
\hline F5 & $\begin{array}{l}\text { This qualification took } 7 \text { years } \\
\text { because of fulltime teaching, four } \\
\text { children and home duties. }\end{array}$ & $\begin{array}{l}7 \text { years } \\
\text { undergraduate } \\
\text { studies part-time }\end{array}$ & $\begin{array}{l}\text { Careful } \\
\text { integration of } \\
\text { formal and } \\
\text { informal } \\
\text { learning }\end{array}$ & \\
\hline F3 & $\begin{array}{l}\text { Because of staff difficulties, at one } \\
\text { school, I was asked to teach Yr } 8 \\
\text { History, English and RE on a fulltime } \\
\text { basis. This magnified the discomfort } \\
\text { regarding teaching practice and praxis } \\
\text { so I needed to decide what to do. }\end{array}$ & $\begin{array}{l}\text { extension into core } \\
\text { subjects an extra } \\
\text { challenge }\end{array}$ & $\begin{array}{l}\text { Implications } \\
\text { for my } \\
\text { qualification } \\
\text { level }\end{array}$ & \\
\hline F6 & $\begin{array}{l}\text { I began to see that experience with } \\
\text { my children helped me understand } \\
\text { students at comparable ages, and vice } \\
\text { versa. Valuable connections were } \\
\text { made regarding the difference } \\
\text { between teaching just the bones of a } \\
\text { subject and the student need to be on } \\
\text { a closer orbit with the teacher to } \\
\text { achieve real understanding of many } \\
\text { areas within topics that I taught. }\end{array}$ & $\begin{array}{l}\text { Constructing and } \\
\text { clarifying various } \\
\text { content that } \\
\text { blended learning } \\
\text { from my children, } \\
\text { my students and } \\
\text { my studies }\end{array}$ & $\begin{array}{c}\text { First glimmers } \\
\text { of the concept } \\
\text { of teaching the } \\
\text { student } \\
\text { through the } \\
\text { medium of the } \\
\text { subject - } \\
\text { necessitating } \\
\text { accurate } \\
\text { planning, } \\
\text { listening and } \\
\text { thinking skills }\end{array}$ & \multirow{2}{*}{$\begin{array}{l}\text { Formal and } \\
\text { informal } \\
\text { learning }\end{array}$} \\
\hline F7 & $\begin{array}{l}\text { For most of my subsequent career, I } \\
\text { was appointed as Home room teacher. } \\
34 \text { of the } 36 \text { years were blends of } \\
\text { curriculum development, pastoral care } \\
\text { initiatives, professional development } \\
\text { seminars and conferences, personal } \\
\text { study and the inevitable } \\
\text { responsibilities of leading in the } \\
\text { homeroom. In this early stage, I was } \\
\text { appointed internally as Head of RE } \\
\text { faculty, a position which I was to } \\
\text { occupy in several schools. }\end{array}$ & $\begin{array}{l}\text { constructing a } \\
\text { personal } \\
\text { perspective of } \\
\text { blending formal } \\
\text { and informal } \\
\text { learning and } \\
\text { training }\end{array}$ & $\begin{array}{l}\text { Shaping and } \\
\text { reshaping of } \\
\text { new knowledge } \\
\text { into a } \\
\text { continuous and } \\
\text { professional } \\
\text { approach to the } \\
\text { teaching } \\
\text { learning } \\
\text { process }\end{array}$ & \\
\hline F2 & $\begin{array}{l}\text { The reason I taught was the result of a } \\
\text { request to teach class music in several } \\
\text { schools on a part-time basis. I was } \\
\text { uncomfortable teaching in class music } \\
\text { when my earlier qualifications were } \\
\text { for single performance in piano, organ } \\
\text { and voice. }\end{array}$ & $\begin{array}{l}\text { Request to teach } \\
\text { class music }\end{array}$ & $\begin{array}{c}\text { Personal ethical } \\
\text { standards }\end{array}$ & Ethics \\
\hline F4 & $\begin{array}{l}\text { I refused to start the music and other } \\
\text { subject teaching till the following } \\
\text { year because then, I could enrol in a } \\
\text { part-time Education qualification at } \\
\text { MCAE, so I then took the position. }\end{array}$ & $\begin{array}{l}\text { refusal to expand } \\
\text { teaching } \\
\text { responsibility } \\
\text { without relevant } \\
\text { qualifications }\end{array}$ & $\begin{array}{l}\text { Inadequacy of } \\
\text { previous } \\
\text { qualifications } \\
\text { for new } \\
\text { responsibilities }\end{array}$ & Praxis \\
\hline
\end{tabular}


careful integration of formal and informal learning. An extension of my role to encompass the teaching of History, English and Religious Education (not Religious Instruction, which was an earlier term for learning about religion in some confessional schools) demanded more than was offered in my preliminary qualification, especially as I became aware, that

because of staff difficulties, at one school, I was asked to teach Yr 8 History, English and RE on a fulltime basis. This magnified the discomfort so I needed to decide what to do (see F3 in Table 5.1).

The urgent, initial need to establish a set of classroom qualifications in order to gain a four-year teacher qualification was superseded by a careful integration of formal and informal learnings to gain, as Shulman (1987) describes in his categories of knowledge: 'content knowledge' and 'curriculum content knowledge; to develop the necessary pedagogical skills, i.e., 'pedagogical content knowledge' which, when combined, lead to 'pedagogical content knowledge'. At the same time, the need to upgrade my formal qualifications became more pressing, as the growing awareness of my own needs for pedagogical content knowledge expanded.

At the same time, I experienced the first glimmers of the concept of 'teaching the student through the medium of the subject' (F6); in this sense, I felt the need to expand on Shulman's (1987) knowledge of learners and their characteristics. Engaging in formal and informal learning enabled me to shape and reshape new knowledge into a continuous and professional approach to the teaching learning process.

\section{Formal and informal learning}

My child-rearing experience, combined with my initial experience of a range of classroom teaching, gave me the first glimmers of the concept of teaching the student through the medium of the subject - necessitating accurate planning, listening and thinking skills. I have observed that 


\begin{abstract}
I began to see that experience with my children helped me understand students at comparable ages, and vice versa. Valuable connections were made regarding the difference between teaching just the bones of a subject and the student need to be on a closer orbit with the teacher to achieve real understanding of many areas within topics that I taught. (F6)
\end{abstract}

I began to construct a personal perspective whereby a blending of formal and informal learning and teaching led to the shaping and reshaping of new knowledge into a continuous and professional approach to the teachinglearning process, i.e., a synthesis of knowledge that emerged as my Philosophy of Education.

\title{
Ethics and praxis
}

Two other significant themes began to emerge: ethics and praxis. The former arose because of my personal ethical standards: I was asked to teach Classroom Music and I wished to do my best for my students. This led to my taking a position that was unusual:

I refused to start the teaching till the following year because then, I could enrol in a part time Education qualification at Melbourne College of Advanced Education (MCAE), and then took the position (F4).

I recognised the inadequacy of my previous qualifications for these new responsibilities and that I needed to combine my teaching and learning practice with my technical/practical and theoretical expertise in Musical Performance: it was a personal awareness of the need for praxis.

At the ethics level, I began to develop a set of personal ethical standards, first in response to my personal discomfort at not being sufficiently prepared by my performance-based Music studies to enable me to teach Classroom Music. Allied to this, was a practical determination to become sufficiently qualified to take on the successful prosecution of the teaching-learning process. 
I didn’t have what I believed I needed - ontologically, epistemologically, and pedagogically. An intention was formed to take immediate action towards becoming suitably qualified as the proper classroom Humanities teacher, regardless of the time and energy that it took.

\section{Norming}

Over the norming phase of my teaching-learning career four themes emerged: the ongoing theme of qualifications, together with the necessity of broadened and continuous experiential learning, pedagogical change and moral change agentry. These themes, which enabled me to meet the expectations of being a professional teacher, are summarised in Table 5.2.

\section{TABLE 5.2 NORMING}

\begin{tabular}{|c|c|c|c|c|}
\hline REF & COMMENT & CONSTRUCT & CONCEPT & THEME \\
\hline N1 & $\begin{array}{l}\text { I completed B.Ed. at University of } \\
\text { Melbourne. I immediately embarked } \\
\text { on a further P/G degree to understand } \\
\text { how Administration, Philosophy of } \\
\text { education, Curriculum Change and } \\
\text { other related areas fitted into the } \\
\text { bigger picture of Victorian education. }\end{array}$ & $\begin{array}{l}\text { Embarked on } \\
\text { further studies to } \\
\text { understand bigger } \\
\text { picture }\end{array}$ & $\begin{array}{l}\text { Importance of } \\
\text { expanding my } \\
\text { knowledge of } \\
\text { Education }\end{array}$ & Qualification \\
\hline N2 & $\begin{array}{l}\text { I joined the Education Union and each } \\
\text { of the professional associations where } \\
\text { I was expected to teach: English, } \\
\text { History, Social Studies and Religious } \\
\text { Education - either in the Catholic } \\
\text { scenario or the wider Victorian } \\
\text { milieux. }\end{array}$ & $\begin{array}{l}\text { Expectation that } \\
\text { these resources } \\
\text { would deepen my } \\
\text { knowledge and } \\
\text { understanding of } \\
\text { Education }\end{array}$ & $\begin{array}{l}\text { Integration of } \\
\text { local and } \\
\text { universal } \\
\text { elements of the } \\
\text { broad } \\
\text { unfolding } \\
\text { picture of } \\
\text { education }\end{array}$ & \multirow{3}{*}{$\begin{array}{c}\text { Experiential } \\
\text { learning }\end{array}$} \\
\hline N3 & $\begin{array}{l}\text { It was necessary to focus and integrate } \\
\text { these new knowledge bases into a } \\
\text { manageable and advanced } \\
\text { methodology that I could feel was } \\
\text { sufficient for the needs of the students } \\
\text { I taught. }\end{array}$ & $\begin{array}{l}\text { Focus on } \\
\text { integrating new } \\
\text { knowledge for } \\
\text { student need }\end{array}$ & $\begin{array}{l}\text { Reshaping of } \\
\text { advanced } \\
\text { methodology } \\
\text { for student } \\
\text { learning }\end{array}$ & \\
\hline N4 & $\begin{array}{l}\text { In the } 70 \text { s, much emphasis was placed } \\
\text { on the development of study skills and } \\
\text { plagiarism problems - students were } \\
\text { inclined when expected to work in } \\
\text { pairs to copy each other's work. }\end{array}$ & $\begin{array}{l}\text { development of } \\
\text { study skills to avoid } \\
\text { student plagiarism } \\
\text { and cheating } \\
\text { problems }\end{array}$ & $\begin{array}{l}\text { Upgrading of } \\
\text { pedagogical } \\
\text { skills for } \\
\text { student richer } \\
\text { learning }\end{array}$ & \\
\hline
\end{tabular}




\begin{tabular}{|c|c|c|c|c|}
\hline REF & COMMENT & CONSTRUCT & CONCEPT & THEME \\
\hline N5 & $\begin{array}{l}\text { I decided that I needed to investigate } \\
\text { on a long-term basis exactly what I } \\
\text { needed to study about teaching } \\
\text { learning styles, where matches and } \\
\text { mismatches could be addressed and } \\
\text { what VAK (visual/ aural and oral/ } \\
\text { kinaesthetic) styles of learning meant } \\
\text { for the teacher to address to facilitate } \\
\text { real learning. }\end{array}$ & $\begin{array}{l}\text { Decision to } \\
\text { incorporate VAK } \\
\text { learning styles into } \\
\text { my teaching styles }\end{array}$ & $\begin{array}{l}\text { Provision to } \\
\text { match teaching } \\
\text { styles and } \\
\text { learning styles } \\
\text { in pedagogical } \\
\text { practice }\end{array}$ & \\
\hline N6 & $\begin{array}{l}\text { Alongside this stream of learning for } \\
\text { me was a new challenge: Pastoral } \\
\text { Care responsibilities especially for } \\
\text { underprivileged kids. This underlined } \\
\text { the already conscious efforts I was } \\
\text { making to become as expert as } \\
\text { possible in teaching the student } \\
\text { through the medium of the subject. } \\
\text { More integration of my thinking made } \\
\text { it possible to have a blended and more } \\
\text { advanced and hopefully more expert } \\
\text { professional approach to teaching- } \\
\text { synthesis of thinking/planning. }\end{array}$ & $\begin{array}{l}\text { Pastoral Care } \\
\text { responsibilities to } \\
\text { be inserted into } \\
\text { pedagogical } \\
\text { practice as well as } \\
\text { above initiatives }\end{array}$ & $\begin{array}{l}\text { Advanced } \\
\text { theory and } \\
\text { practice of } \\
\text { expertise in } \\
\text { classroom }\end{array}$ & \\
\hline N8 & $\begin{array}{l}\text { I developed the perspective of } \\
\text { changing schools regularly, once it } \\
\text { became obvious that it was possible, } \\
\text { and it was beneficial for my expertise } \\
\text { levels in teaching and leading: I } \\
\text { would apply for a boys school, } \\
\text { followed by a girls school, followed } \\
\text { by a coeducational school ... }\end{array}$ & $\begin{array}{l}\text { Changing of } \\
\text { schools to upgrade } \\
\text { pedagogical finesse } \\
\text { for student diversity }\end{array}$ & $\begin{array}{l}\text { Upgrade of } \\
\text { expertise in } \\
\text { teaching and } \\
\text { leading }\end{array}$ & \multirow{4}{*}{$\begin{array}{c}\text { Pedagogical } \\
\text { changes }\end{array}$} \\
\hline N9 & $\begin{array}{l}\text { This had the effect of improving my } \\
\text { understanding of student diversity, } \\
\text { their need for scaffolding in different } \\
\text { styles. }\end{array}$ & $\begin{array}{l}\text { Improvement of } \\
\text { pedagogical } \\
\text { strategies }\end{array}$ & $\begin{array}{l}\text { Improved } \\
\text { understanding } \\
\text { of student } \\
\text { needs, } \\
\text { including } \\
\text { scaffolding } \\
\text { techniques }\end{array}$ & \\
\hline N11 & $\begin{array}{l}\text { PET/ TET/ LET: Parent Effectiveness } \\
\text { Training/ Teacher Effectiveness } \\
\text { Training/ Leaders Effectiveness } \\
\text { Training: available from Catholic } \\
\text { Education Office training personnel, } \\
\text { led by Leo Cahill -meant that my } \\
\text { communications were upgraded } \\
\text { further - Thomas Gordon was the } \\
\text { author and originator, and I completed } \\
\text { all the courses available through CEO } \\
\text { and applied them for better } \\
\text { communication at home, at school and } \\
\text { in study milieux. }\end{array}$ & $\begin{array}{l}\text { Effectiveness } \\
\text { Training courses } \\
\text { ensured quality in } \\
\text { communications }\end{array}$ & $\begin{array}{l}\text { Integration of } \\
\text { communication } \\
\text { s expertise into } \\
\text { existing } \\
\text { pedagogical } \\
\text { practice }\end{array}$ & \\
\hline N7 & $\begin{array}{l}\text { More integration of my thinking made } \\
\text { it possible to have a blended and more } \\
\text { advanced and hopefully more expert } \\
\text { professional approach to teaching- } \\
\text { synthesis in action. }\end{array}$ & $\begin{array}{l}\text { Integrated thinking } \\
\text { for advanced and } \\
\text { expert professional } \\
\text { approach to } \\
\text { teaching }\end{array}$ & $\begin{array}{l}\text { Professional } \\
\text { integrated } \\
\text { approach to } \\
\text { new initiatives } \\
\text { in pedagogy }\end{array}$ & \\
\hline
\end{tabular}




\begin{tabular}{|c|c|c|c|c|}
\hline REF & COMMENT & CONSTRUCT & CONCEPT & THEME \\
\hline N10 & $\begin{array}{l}\text { My innate understanding was } \\
\text { becoming more expert and detailed in } \\
\text { my approach and I shared these } \\
\text { matters with colleagues on different } \\
\text { faculty teams. Some understood, some } \\
\text { did not, some were drowning in their } \\
\text { own problems and were unable to } \\
\text { internalise the point of my } \\
\text { discussions. }\end{array}$ & $\begin{array}{l}\text { Understanding and } \\
\text { integration of } \\
\text { pedagogical } \\
\text { strategies and } \\
\text { techniques leading } \\
\text { to sharing with } \\
\text { colleagues }\end{array}$ & $\begin{array}{l}\text { Difficulties in } \\
\text { sharing with } \\
\text { colleagues } \\
\text { because of } \\
\text { their problems } \\
\text { as well as my } \\
\text { efforts to } \\
\text { upgrade my } \\
\text { approach }\end{array}$ & $\begin{array}{l}\text { Change } \\
\text { agentry }\end{array}$ \\
\hline
\end{tabular}

\section{Qualifications}

In this phase, I embarked on further studies in an attempt to understand the bigger picture, since I recognised the importance of expanding my knowledge of Education. I steadily integrated my formal and informal learning as I applied my various new knowledge of pedagogical finesse, of the incorporation and blending of new initiatives such as Lipman's Community of Inquiry, managing the communication of new knowledge to colleagues (sometimes unsuccessfully), and recognising what role Change Agentry would play in challenging me to become a better teacher.

\section{Experiential learning}

This was a significant stage of my professional development:

\footnotetext{
I joined the Education Union and each of the professional associations where I was expected to teach: English, History, Social Studies and Religious Education - either in the Catholic scenario or the wider Victorian milieux (N2).
}

These experiences enable me to integrate local and universal elements of a broad unfolding picture of education. At the same time, I was encouraged to develop advanced methodologies and to upgrade my pedagogical skills in order to encourage richer student learning. By being able to match teaching and learning styles in my pedagogical practice, I advanced my personal concept of praxis in the classroom. 
To accommodate the emerging need for systematic understanding of pedagogical change in my own work, I set about upgrading my teaching and leading skills by changing schools on a regular basis. In turn, this led to an improvement in my understanding of broader student needs, including meeting the stage of learning development through the use of scaffolding techniques.

\section{Pedagogical changes}

My participation in a number of effectiveness training courses enabled me to integrate communications expertise with my developing pedagogical practice; I was encouraged, as a result, to take a professionally integrated approach to new initiatives in pedagogy. I had a 'blended and more advanced and hopefully more expert professional approach to teaching' (N11).

\section{Change agentry}

At the same time, I took the first faltering, difficult, and not altogether successful steps into change agentry: I realised that change in organisations was more complex than I had originally recognised.

Within my process of norming, I discovered that I was assisting the norming of student behaviour, at first tentatively and then more confidently, as they participated in experiential learning, ultimately being able to evaluate their own learning outcomes. I had gained experience in practising Shulman's (1987) 'knowledge of learners and their characteristics'; I felt relief that I could successfully undertake both the art and science of teaching thinking skills. It would take me a full school year, each year, to facilitate student progress and to achieve this with all my classes: I was learning with the students - a true collaborative experience. I became more confident in this regard over a number of years with students in Years 8, 9 and 10, as did they; it was here that I learned, in a Community of Inquiry approach, to distinguish between a teacher-directed classroom and what I called a 
'student-directed classroom'- without recourse at that point to finding the complete research on this concept.

\section{Storming}

In the lengthy storming stage, I was intent on recognising and adapting the various strands of experiential and formal learning in pedagogical terms, by engaging in a synthesis of thinking about teaching skills for the changing world of the students and the consequences for teachers: the role of change agentry merged and blended with my experiential learning, so that leadership initiatives were emerging for me as I recognised and responded to the politics in Education. One consequence was further research and qualification into the teacher skills of Philosophy for Children, and the identification and maintenance of a student-centred learning atmosphere in the classroom, regardless of which subject area was being taught or in which Year Level I was teaching. These six themes are summarised in Table 5.3

\section{TABLE 5.3 STORMING}

\begin{tabular}{|c|c|c|c|c|}
\hline REF & COMMENT & CONSTRUCT & CONCEPT & THEME \\
\hline S1 & $\begin{array}{l}\text { In the 80s: there were many } \\
\text { Professional Development courses, } \\
\text { some one -day length, but many were } \\
\text { longer. I attended in particular } \\
\text { decision-making in classes, in } \\
\text { schools, in teacher delivery and } \\
\text { management of change. }\end{array}$ & $\begin{array}{l}\text { Attendance at many } \\
\text { and varied PD courses } \\
\text { for curriculum } \\
\text { content, for P4C } \\
\text { development, for RE } \\
\text { units, for teacher } \\
\text { development and } \\
\text { strategies for change }\end{array}$ & $\begin{array}{l}\text { Development of } \\
\text { understanding } \\
\text { and practice in } \\
\text { so many areas } \\
\text { where change } \\
\text { was promoted }\end{array}$ & \multirow[b]{2}{*}{$\begin{array}{l}\text { Change } \\
\text { agentry }\end{array}$} \\
\hline S2 & $\begin{array}{l}\text { Bullying/ Buddying and Peer Support } \\
\text { systems approach: I completed } \\
\text { seminars in Peer Support and with } \\
\text { several colleagues inserted the whole } \\
\text { system into the junior secondary } \\
\text { school -much time in negotiations to } \\
\text { make this a reality was invaluable in } \\
\text { showing me the detail of school } \\
\text { administration planning for the first } \\
\text { time. }\end{array}$ & $\begin{array}{l}\text { Peer Support and } \\
\text { Buddying systems: } \\
\text { PD and } \\
\text { implementation made } \\
\text { general practice more } \\
\text { understandable }\end{array}$ & $\begin{array}{c}\text { Clearer } \\
\text { classroom } \\
\text { administrative } \\
\text { measures were } \\
\text { linked with my } \\
\text { formal and } \\
\text { informal } \\
\text { change } \\
\text { measures in the } \\
\text { school. }\end{array}$ & \\
\hline
\end{tabular}




\begin{tabular}{|c|c|c|c|c|}
\hline REF & COMMENT & CONSTRUCT & CONCEPT & THEME \\
\hline S3 & $\begin{array}{l}\text { By now, I was finishing the } \\
\text { postgraduate B.Ed. St. at University } \\
\text { of Melbourne, so I had opportunity to } \\
\text { delve into further new knowledge and } \\
\text { studies during that course on } \\
\text { pedagogy, and the various teaching } \\
\text { styles: -Joyce and Weil; and attending } \\
\text { a conference where Basil Moore } \\
\text { presented the five most useful } \\
\text { teaching styles that he and Norm } \\
\text { Habel had published. This became my } \\
\text { central focus and constantly informed } \\
\text { my teaching practice for the whole of } \\
\text { my career. }\end{array}$ & $\begin{array}{l}\text { Central focus on } \\
\text { efficiency, } \\
\text { effectiveness based on } \\
\text { effort regarding new } \\
\text { pedagogy }\end{array}$ & $\begin{array}{l}\text { Management of } \\
\text { change }\end{array}$ & \\
\hline S11 & $\begin{array}{l}\text { This resulted in finding the } \\
\text { Philosophy for Children movement; } \\
\text { involvement with that over the next } \\
20 \text { years coloured my teaching } \\
\text { dramatically and influenced my } \\
\text { preparation for the MEd at University } \\
\text { of Melbourne. I embarked on a } \\
\text { preparation for this by identifying } \\
\text { John Wilson at Oxford Department of } \\
\text { Education Studies in Norham } \\
\text { Gardens, Oxford as a means of } \\
\text { understanding moral education, as } \\
\text { well as Matthew Lipman's Philosophy } \\
\text { for Children and especially his } \\
\text { pedagogical adjunct: the Community } \\
\text { of Inquiry in the classroom milieu. }\end{array}$ & $\begin{array}{l}\text { Finding the P4C } \\
\text { movement involved } \\
\text { further varied and full } \\
\text { time (one year) study } \\
\text { over a } 20 \text { year period }\end{array}$ & $\begin{array}{l}\text { Integration of } \\
\text { P4C, previous } \\
\text { learning, new } \\
\text { studies and } \\
\text { classroom } \\
\text { practice to } \\
\text { consolidate } \\
\text { elements into } \\
\text { firm } \\
\text { pedagogical } \\
\text { practice }\end{array}$ & \multirow{4}{*}{$\begin{array}{l}\text { Experi- } \\
\text { ential } \\
\text { learning }\end{array}$} \\
\hline S12 & $\begin{array}{l}\text { On returning after an academic year } \\
\text { with John Wilson, I began the } \\
\text { fashioning of what became the } \\
\text { substance of my MEd studies at } \\
\text { University of Melbourne, though I did } \\
\text { not complete this project till mid-90s. }\end{array}$ & $\begin{array}{l}\text { Return from Oxford, } \\
\text { reshaping content into } \\
\text { MEd substance }\end{array}$ & $\begin{array}{l}\text { Resettlement } \\
\text { into Victorian } \\
\text { education } \\
\text { scenario }\end{array}$ & \\
\hline S13 & $\begin{array}{l}\text { Gender balance in class activities } \\
\text { became a political issue, so I began to } \\
\text { marshal my understandings of my } \\
\text { experience of teaching in single } \\
\text { gender and coeducational classrooms- } \\
\text { this ongoing activity gave impressive } \\
\text { results in coeducational situations and } \\
\text { highlighted that single gender class } \\
\text { members and their teachers did not } \\
\text { have suitable opportunity to } \\
\text { understand this area fully. }\end{array}$ & $\begin{array}{l}\text { Gender balance issues } \\
\text { meant using my } \\
\text { accumulated } \\
\text { knowledge of single } \\
\text { gender and mixed } \\
\text { class information- } \\
\text { realisation that single } \\
\text { gender class } \\
\text { experience was not } \\
\text { enough }\end{array}$ & $\begin{array}{l}\text { Realisation of } \\
\text { broader } \\
\text { student } \\
\text { diversity and } \\
\text { the teacher } \\
\text { response } \\
\text { needed }\end{array}$ & \\
\hline S16 & $\begin{array}{l}\text { Following that, came incorporation } \\
\text { and managing students with disability } \\
\text {-legally blind, cerebral palsy, } \\
\text { profound deaf students were accepted } \\
\text { into the schools and teachers were } \\
\text { given in-house PD to help with these } \\
\text { students' needs. This was a separate } \\
\text { development from the language needs } \\
\text { of second and third phase students } \\
\text { from migrant families. }\end{array}$ & $\begin{array}{l}\text { Incorporation and } \\
\text { management of } \\
\text { students with } \\
\text { disability and local } \\
\text { PD to integrate } \\
\text { students as far as } \\
\text { possible }\end{array}$ & $\begin{array}{l}\text { Deeper teacher } \\
\text { learning for } \\
\text { individualised } \\
\text { student } \\
\text { progress }\end{array}$ & \\
\hline
\end{tabular}




\begin{tabular}{|c|c|c|c|c|}
\hline REF & COMMENT & CONSTRUCT & CONCEPT & THEME \\
\hline S17 & $\begin{array}{l}\text { Further Conferences o/seas in 1990s } \\
\text { and early 2000s, promoted by the } \\
\text { Association of Moral Education, } \\
\text { Philosophy for Children and British } \\
\text { Educational Research Association } \\
\text { (BERA), gave me the opportunity to } \\
\text { keep up with new initiatives in related } \\
\text { areas and allowed for personal PD at } \\
\text { the same time. }\end{array}$ & $\begin{array}{l}\text { O/seas conferences in } \\
\text { three related } \\
\text { knowledge areas used } \\
\text { for personal PD } \\
\text { purposes }\end{array}$ & $\begin{array}{c}\text { Curriculum } \\
\text { and } \\
\text { pedagogical } \\
\text { changes were } \\
\text { highlighted } \\
\text { through } \\
\text { attendance at } \\
\text { these } \\
\text { conferences, } \\
\text { leading to } \\
\text { eventual } \\
\text { decision for } \\
\text { PhD studies }\end{array}$ & \\
\hline S18 & $\begin{array}{l}\text { Language literacy and facility for } \\
\text { senior students - internal PD for } \\
\text { junior, middle and senior secondary } \\
\text { class work -made it obvious how } \\
\text { important creativity, age level and } \\
\text { background of each student aided } \\
\text { their progress. This needed constant } \\
\text { monitoring to make sure I was } \\
\text { including the separate skills to attend } \\
\text { to their many needs. }\end{array}$ & $\begin{array}{l}\text { PD for language } \\
\text { literacy for Junior, } \\
\text { Middle and Senior } \\
\text { students }\end{array}$ & $\begin{array}{l}\text { Targeted PD in } \\
\text { language } \\
\text { literacy and } \\
\text { array of skills } \\
\text { for expanded } \\
\text { student } \\
\text { progress in } \\
\text { learning and } \\
\text { expression }\end{array}$ & \\
\hline S19 & $\begin{array}{l}\text { Team teaching in classroom } \\
\text { situations: this needed expertise in a } \\
\text { number of ways because teachers } \\
\text { were loath to give away what they } \\
\text { saw as precious autonomy in a } \\
\text { classroom situation. This objective } \\
\text { would take place eventually and } \\
\text { teacher response began to appreciate } \\
\text { how each teacher brought new ideas } \\
\text { and expertise which could be } \\
\text { incorporated in one's own armoury of } \\
\text { skills. }\end{array}$ & $\begin{array}{l}\text { Introduction of team } \\
\text { teaching in variety of } \\
\text { strategies. Teacher } \\
\text { response varied but } \\
\text { eventually accepted } \\
\text { new initiatives }\end{array}$ & $\begin{array}{l}\text { Postmodern } \\
\text { approaches and } \\
\text { real change in } \\
\text { pedagogical } \\
\text { practice } \\
\text { introduction } \\
\text { beginning with } \\
\text { team teaching }\end{array}$ & \multirow{3}{*}{$\begin{array}{l}\text { Experi- } \\
\text { ential } \\
\text { learning }\end{array}$} \\
\hline S20 & $\begin{array}{l}\text { Comparative religions studies, } \\
\text { ecumenical initiatives and interfaith } \\
\text { connections: three annual summer } \\
\text { schools at Melbourne College of } \\
\text { Divinity meant deeper understandings } \\
\text { of diversity, democracy, religious and } \\
\text { secular perspectives and the need to } \\
\text { incorporate patterns into delivery style } \\
\text { to accommodate such matters. }\end{array}$ & $\begin{array}{l}\text { Through summer } \\
\text { school studies, deeper } \\
\text { understandings and } \\
\text { initiatives to } \\
\text { accommodate } \\
\text { diversity and related } \\
\text { perspectives into } \\
\text { teaching and delivery }\end{array}$ & $\begin{array}{l}\text { Specialisation } \\
\text { in interreligious } \\
\text { and secular } \\
\text { curriculum for } \\
\text { student } \\
\text { learning }\end{array}$ & \\
\hline S21 & $\begin{array}{l}\text { Integration of multi-intelligences and } \\
\text { their consequences into teaching } \\
\text { styles was a challenging task because } \\
\text { it needed to be blended into the } \\
\text { existing initiatives mentioned already. } \\
\text { Such new information changed much } \\
\text { about how I viewed assignments and } \\
\text { how assessments were approached. }\end{array}$ & $\begin{array}{l}\text { Changes to integrate } \\
\text { the consequences of } \\
\text { multi-intelligences } \\
\text { meant change to } \\
\text { curriculum content, } \\
\text { pedagogical practice, } \\
\text { assignment and } \\
\text { assessment tasks }\end{array}$ & $\begin{array}{l}\text { Updated } \\
\text { teacher learner } \\
\text { styles and } \\
\text { curriculum } \\
\text { content }\end{array}$ & \\
\hline
\end{tabular}




\begin{tabular}{|c|c|c|c|c|}
\hline REF & COMMENT & CONSTRUCT & CONCEPT & THEME \\
\hline S22 & $\begin{array}{l}\text { Multiculturalism and interculturalism: } \\
\text { development of last two points and } \\
\text { integration of new material -improved } \\
\text { my curriculum focus and development } \\
\text { of self-evaluation for students and } \\
\text { self. Personal impact was highlighted } \\
\text { by informal PD/study with Professor } \\
\text { Mews at Monash Centre of Religious } \\
\text { Studies and with the Parliament of } \\
\text { World Religions. }\end{array}$ & $\begin{array}{l}\text { PD for cultural and } \\
\text { religious perspectives } \\
\text { expanded to include } \\
\text { efficiency in } \\
\text { curriculum planning } \\
\text { and self-evaluation for } \\
\text { students and for self }\end{array}$ & $\begin{array}{l}\text { Upgraded } \\
\text { pedagogical } \\
\text { practice in } \\
\text { cultural, social } \\
\text { and religious } \\
\text { perspectives in } \\
\text { curriculum and } \\
\text { self evaluation }\end{array}$ & \\
\hline S4 & $\begin{array}{l}\text { Australian Catholic Principals } \\
\text { Association conferences, each year } \\
\text { (80s), were the channel in which I } \\
\text { consolidated my professional } \\
\text { knowledge and widened my network } \\
\text { of valued colleagues who could help } \\
\text { with my further teacher education. }\end{array}$ & $\begin{array}{l}\text { Conferences } \\
\text { consolidated present } \\
\text { knowledge and } \\
\text { broadened } \\
\text { professional network }\end{array}$ & $\begin{array}{l}\text { Taking my } \\
\text { place in } \\
\text { leadership and } \\
\text { experiential } \\
\text { knowledge in } \\
\text { wider } \\
\text { education }\end{array}$ & \multirow{5}{*}{$\begin{array}{l}\text { Leader- } \\
\text { ship } \\
\text { initiatives }\end{array}$} \\
\hline S5 & $\begin{array}{l}\text { I also was secretary and editor of the } \\
\text { Journal pre and post conference } \\
\text { journal magazine for several years. }\end{array}$ & $\begin{array}{l}\text { Secretarial/editorial } \\
\text { duties }\end{array}$ & $\begin{array}{l}\text { Broadened } \\
\text { educational } \\
\text { focus }\end{array}$ & \\
\hline S6 & $\begin{array}{l}\text { Australian Association of Religious } \\
\text { Education conferences were central to } \\
\text { my career improvement. RE based, } \\
\text { but ecumenically based, I found the } \\
\text { breadth of understanding and } \\
\text { academic flavour to my liking and } \\
\text { learned well -each year, I did some } \\
\text { summer courses at Catholic Education } \\
\text { Office and other venues to aid further } \\
\text { academic development and } \\
\text { curriculum expertise. }\end{array}$ & $\begin{array}{l}\text { AARE conferences } \\
\text { central to career } \\
\text { improvement, aided } \\
\text { by CEO courses }\end{array}$ & \multirow{3}{*}{$\begin{array}{l}\text { Insertion of } \\
\text { advanced RE } \\
\text { perspectives } \\
\text { into existing } \\
\text { personal } \\
\text { pedagogical } \\
\text { and curriculum } \\
\text { practices }\end{array}$} & \\
\hline S7 & $\begin{array}{l}\text { As a result I became involved in a } \\
\text { large enterprise with Dove } \\
\text { Communications in which curriculum } \\
\text { was a key: six magazines a year, } \\
\text { aimed at each of Years 7-9, piloted } \\
\text { first in about ten schools and we } \\
\text { developed patterns to incorporate } \\
\text { feedback from those teachers and } \\
\text { students, so that each magazine could } \\
\text { be used as class texts. }\end{array}$ & $\begin{array}{l}\text { Involvement in } \\
\text { preparation, piloting } \\
\text { and publishing of RE } \\
\text { curriculum content, } \\
\text { parallel to teaching } \\
\text { practice }\end{array}$ & & \\
\hline S8 & $\begin{array}{l}\text { A special team of teachers also } \\
\text { included the writing of year-long RE } \\
\text { textbooks again for each year for Yrs. } \\
7,8,9,10 \text { and a number of magazine } \\
\text { developments to be used for internal } \\
\text { Yrs. } 11 \text { and } 12 \text { units, which was } \\
\text { familiar territory in } 80 \text { s. Other } \\
\text { specific themed books to support the } \\
\text { textbooks were included over a } 7 \text {-year } \\
\text { period. }\end{array}$ & $\begin{array}{l}\text { Expansion of } \\
\text { preparation, piloting } \\
\text { and publishing of RE } \\
\text { curriculum content, } \\
\text { paralleling teaching } \\
\text { practice }\end{array}$ & & \\
\hline
\end{tabular}




\begin{tabular}{|c|c|c|c|c|}
\hline REF & COMMENT & CONSTRUCT & CONCEPT & THEME \\
\hline S9 & $\begin{array}{l}\text { This ongoing teacher learning and use } \\
\text { of increased knowledge and personal } \\
\text { expertise gave me a valid and realistic } \\
\text { understanding and knowledge of } \\
\text { curriculum initiatives in this subject } \\
\text { and an appreciation for how students } \\
\text { responded -I was learning what the } \\
\text { thinking skill of synthesis could teach. }\end{array}$ & $\begin{array}{l}\text { Increased knowledge } \\
\text { and expertise } \\
\text { connecting to student } \\
\text { response }\end{array}$ & $\begin{array}{l}\text { Expanded } \\
\text { leadership in } \\
\text { curriculum, } \\
\text { and } \\
\text { pedagogical } \\
\text { practice within } \\
\text { faculty of RE } \\
\text { connected to } \\
\text { student } \\
\text { learning }\end{array}$ & \\
\hline S10 & $\begin{array}{l}\text { I developed a Decision-making } \\
\text { instrument and a Discussion } \\
\text { instrument during this decade with the } \\
\text { help in Psychology of Professor } \\
\text { Holian at RMIT to provide students } \\
\text { with better understanding of how they } \\
\text { could honestly help themselves and } \\
\text { each other. }\end{array}$ & $\begin{array}{l}\text { Development of } \\
\text { instruments to provide } \\
\text { students with self-help } \\
\text { tools }\end{array}$ & $\begin{array}{c}\text { Expanded } \\
\text { confidence in } \\
\text { personal ability } \\
\text { to design } \\
\text { curriculum and } \\
\text { pedagogical } \\
\text { instrument for } \\
\text { student needs }\end{array}$ & \\
\hline S23 & $\begin{array}{l}\text { The role and function of teachers } \\
\text { became a changing scenario: further } \\
\text { to Professor Cherednichenko and her } \\
\text { work, delving into the in loco parentis } \\
\text { features, legal responsibilities as they } \\
\text { were changed in government action } \\
\text { added to curriculum development. }\end{array}$ & $\begin{array}{l}\text { Focus on role and } \\
\text { function of teacher } \\
\text { with political } \\
\text { initiatives for } \\
\text { upgraded legal } \\
\text { responsibilities for } \\
\text { teachers }\end{array}$ & $\begin{array}{l}\text { Changed } \\
\text { community } \\
\text { experience } \\
\text { pushed } \\
\text { teachers into } \\
\text { pastoral care } \\
\text { role beyond } \\
\text { that of earlier } \\
\text { eras }\end{array}$ & \multirow[b]{2}{*}{$\begin{array}{l}\text { Politics in } \\
\text { education }\end{array}$} \\
\hline S24 & $\begin{array}{l}\text { Subsequent addition of Civics and } \\
\text { Citizenship studies -beyond what was } \\
\text { available in government kits while } \\
\text { inserted for political purposes from } \\
\text { the Federal Government was } \\
\text { immediately integrated with } \\
\text { perspectives of multiculturalism and } \\
\text { interculturalism. }\end{array}$ & $\begin{array}{l}\text { Government kits on } \\
\text { Civics and Citizenship } \\
\text { imposed on the } \\
\text { curriculum }\end{array}$ & $\begin{array}{l}\text { Change agentry } \\
\text { and political } \\
\text { presence } \\
\text { impinging on } \\
\text { existing } \\
\text { curriculum for } \\
\text { multiculturalis } \\
\text { m, } \\
\text { interculturalis } \\
\text { m and cultural } \\
\text { studies }\end{array}$ & \\
\hline S15 & $\begin{array}{l}\text { Added to this mix was the exciting } \\
\text { new knowledge of how to develop the } \\
\text { Community of Inquiry from the } \\
\text { Philosophy for Children movement. I } \\
\text { made several journeys overseas and } \\
\text { interstate to learn enough to qualify in } \\
\text { different milieux both for Matthew } \\
\text { Lipman's purposes and for my own. }\end{array}$ & $\begin{array}{l}\text { Advanced pedagogical } \\
\text { practice and overseas } \\
\text { qualification in } \mathrm{P} 4 \mathrm{C}\end{array}$ & $\begin{array}{c}\text { Use of P4C } \\
\text { initiatives for } \\
\text { individualised } \\
\text { student } \\
\text { learning }\end{array}$ & $\begin{array}{l}\text { Research } \\
\text { and } \\
\text { qualificati } \\
\text { on in P4C }\end{array}$ \\
\hline S14 & $\begin{array}{l}\text { In the 90s much was made of the } \\
\text { integration of students with special } \\
\text { needs as far as possible into the } \\
\text { mainstream classroom. ESL in the } \\
\text { Classroom courses added to my } \\
\text { detailed knowledge of student needs } \\
\text { and were integrated carefully with my } \\
\text { knowledge of the five teaching } \\
\text { models that Moore (1986) had } \\
\text { recommended and much of what } \\
\text { Joyce and Weil (2000) had detailed in } \\
\text { their book. }\end{array}$ & $\begin{array}{l}\text { Special needs students } \\
\text { in class, ESL courses, } \\
\text { integration of new } \\
\text { pedagogy into the five } \\
\text { Moore models of } \\
\text { teaching I used. }\end{array}$ & $\begin{array}{c}\text { Differentiation } \\
\text { and Integration } \\
\text { of pedagogical } \\
\text { initiatives } \\
\text { recommended } \\
\text { for individual } \\
\text { student } \\
\text { learning }\end{array}$ & $\begin{array}{l}\text { Student } \\
\text { centred } \\
\text { learning }\end{array}$ \\
\hline
\end{tabular}




\section{Change agentry}

There were three concepts associated with the theme of change agentry. The first: development of understanding and practice in so many areas where change was promoted has resulted in a blending of epistemological sources and praxis that resulted in the development of new units of work. The second: clearer classroom administrative measures were linked with my formal and informal change measures in the school, and resulted in the third: the requirement for a better understanding of the management of change. As a result, I sensed that the emergence of a new paradigm, aimed at an alternative approach to the delivery of curriculum content, had begun.

\section{Experiential learning}

As a consequence of this emergent approach, there was an explosion of experiential learning in my classrooms at each Year Level: integration of elements of Philosophy for Children; previous learning; new studies and classroom practice to consolidate elements into firm pedagogical practice. The students were thrilled to bits; they saw it as an exciting innovation that they thought 'wasn't real school!'. This eased my resettlement into the Victorian Education scenario, after my 12 months' academic experience at Oxford University. This new experience led me to a deeper realisation of the reality of broader student diversity, as well as the need for a different teacher response: the conscious construction of experiential learning resulted in new classroom strategies and techniques - further enhancing the perception of the emergence of a new paradigm. This paradigm demanded deeper teacher learning for the purpose of individualised student progress; in turn, this led to urgency in making curriculum and pedagogical changes, the nature of which were highlighted through my attendance at national and international conferences, and which culminated in my eventual decision to undertake PhD studies. Overall, I had gained a keener interest in the following: 
- targeting Professional Development in language literacy and an array of skills for expanded student progress in learning and expression;

- introducing postmodern approaches and real change in pedagogical practice: beginning with team teaching;

- specialising in an interreligious and secular curriculum for student learning.

I updated my teacher-learner styles and curriculum content, and upgraded my pedagogical practice in cultural, social and religious perspectives in curriculum and resultant self-evaluation - first for their topics and eventually for their personal lives. At the same time, I focussed on the development and maintenance of a thoroughly democratic classroom approach.

\section{Leadership initiatives}

This was essentially the first time that I 'gave myself permission' to undertake leadership responsibilities beyond the classroom. This resulted in the following:

- Taking my place in leadership and experiential knowledge in wider education.

- Broadening my educational focus.

- Inserting advanced religious education perspectives into existing personal pedagogical and curriculum practices.

- Expanding teacher leadership in curriculum and pedagogical practice within the Faculty of Religious Education.

- Expanding confidence in my personal ability to design curriculum and my own pedagogical instruments to meet student needs.

As a consequence, my conscious efforts in the line of formal and informal leadership dramatically expanded and, for the first time, were acknowledged in the workplace. 


\section{Politics in education}

Two themes emerged: a changed community experience pushed teachers into a pastoral care role beyond that from earlier eras; change agentry and political presence impinged on existing curricula for multiculturalism, interculturalism and Cultural Studies. In Shulman's (1987) terms, pedagogical content knowledge, consisting of putting pedagogy into practice and developing classroom content that was appropriate, became one of the ideological features of the times. While this created some personal discomfort. I knew that I had to adapt to these external demands: Schwab’s (1983) 'commonplaces of the classroom' - what happened in the classroom in relation to students, teachers, the curriculum and the milieu itself - were under tighter political scrutiny than I had previously experienced. The times they 'were a-changing!'

\section{Research and qualification in Philosophy for Children}

After returning from further research and the gaining of qualifications in Philosophy for Children, in Horsham, UK, I returned to my post in Victoria, Australia, certain enough of my experiential learning in the use of Philosophy for Children initiatives for individualised student learning; I had taken in the full proffered professional learning about how to manage at Levels 1 and 2. My self-confidence in my application of a Community of Inquiry had grown sufficiently to provide the foundation for my teaching strategies for the rest of my career.

\section{Student centred learning}

I made a connection between the five teaching models of Moore (1986) and the expanded list of teaching models of Joyce and Weil (1986). This led to my differentiating and integrating the pedagogical initiatives recommended for individual student learning, in order to decide what I needed to construct a student-directed classroom: the school curriculum and the 'knowledge of learners and their characteristics' (Tuckman, 1965). 


\section{Performing}

In this final phase, which brings me to the present - performing - I put into practice a synthesis of the cognitive and attitudinal behaviours that I had acquired in the previous three stages.

Emerging understanding, aided by the ten years spent in developing this thesis, added to the discovery of new knowledge and the synthesis of existing knowledge, into a precise picture of what education for the new century might include: experiential learning that was pedagogically shaped; personal growth and change agentry that was subsumed into the overall pedagogical approach, in order to benefit students and teachers alike; further qualifications solidified the foundations. These five themes are detailed below in Table 5.4 .

\section{TABLE 5.4 PERFORMING}

\begin{tabular}{|c|c|c|c|c|}
\hline REF & COMMENT & CONSTRUCT & CONCEPT & THEME \\
\hline P7 & $\begin{array}{l}\text { The result of the 1990-91 academic } \\
\text { year in UK, I spent under the tutelage } \\
\text { of John Wilson at Department of } \\
\text { Educational Studies in Oxford was } \\
\text { ground-breaking. It was a private } \\
\text { arrangement under the auspices of the } \\
\text { Farmington Trust, and supported by } \\
\text { the Oxford Trust and the Worthington } \\
\text { Trust, whereby I attended and audited } \\
\text { Wilson's MEd classes, was included } \\
\text { in his D. Phil. tutorials, and had my } \\
\text { personal tutorial with him on a weekly } \\
\text { basis. }\end{array}$ & $\begin{array}{l}\text { Ground-breaking } \\
\text { academic studies } \\
\text { stretched and } \\
\text { expanded my } \\
\text { knowledge and } \\
\text { understanding } \\
\text { especially in } \\
\text { educational studies } \\
\text { and informed all } \\
\text { my subsequent } \\
\text { efforts to develop } \\
\text { expertise and } \\
\text { knowledge relevant } \\
\text { to curriculum, } \\
\text { student learning, } \\
\text { leadership and } \\
\text { philosophy } \\
\text { especially } \\
\text { analytical } \\
\text { philosophy } \\
\text { movement }\end{array}$ & $\begin{array}{l}\text { Academic } \\
\text { studies in } \\
\text { Oxford } \\
\text { provided } \\
\text { foundation } \\
\text { for all } \\
\text { subsequent } \\
\text { studies and } \\
\text { educational } \\
\text { practice }\end{array}$ & \multirow[t]{2}{*}{$\begin{array}{c}\text { Experiential } \\
\text { learning }\end{array}$} \\
\hline P10 & $\begin{array}{l}\text { This I found useful at junior } \\
\text { secondary levels as students were } \\
\text { keen to use computer research. Much } \\
\text { confusion about plagiarism, and the } \\
\text { use of printouts as evidence of their } \\
\text { facility with IT was obscured by the } \\
\text { fact that they had to read what they } \\
\text { downloaded, and this proved a } \\
\text { sticking point for several years. }\end{array}$ & $\begin{array}{l}\text { Computer research } \\
\text { was useful but } \\
\text { plagiarism and } \\
\text { printouts provided } \\
\text { hurdles as sticking } \\
\text { point in junior } \\
\text { secondary students }\end{array}$ & $\begin{array}{c}\text { Incorporation } \\
\text { of computer } \\
\text { research into } \\
\text { teaching } \\
\text { learning } \\
\text { process }\end{array}$ & \\
\hline
\end{tabular}




\begin{tabular}{|c|c|c|c|c|}
\hline REF & COMMENT & CONSTRUCT & CONCEPT & THEME \\
\hline P 13 & $\begin{array}{l}\text { Over a period of } 25 \text { years, I mentored } \\
\text { and monitored about } 30 \text { - } 36 \text { student } \\
\text { teachers, sometimes from two } \\
\text { Universities in one year, as a means of } \\
\text { understanding their new perspectives } \\
\text { and providing them with realistic } \\
\text { experience in classroom settings }\end{array}$ & $\begin{array}{l}\text { responsibility for } \\
\text { student teacher } \\
\text { experience }\end{array}$ & $\begin{array}{l}\text { Development } \\
\text { of mutual } \\
\text { understandin } \\
\text { g of classroom } \\
\text { strategies in } \\
\text { partnership } \\
\text { with student } \\
\text { teacher } \\
\text { program }\end{array}$ & \\
\hline P8 & $\begin{array}{l}\text { In } 2000 \text { and beyond: my leadership in } \\
\text { most schools were as subject head of } \\
\text { various faculties, and were mainly } \\
\text { curriculum based, but pastoral care } \\
\text { positions in } 70 \text { s and } 80 \text { s led to } \\
\text { classroom initiatives to do with } \\
\text { student self-evaluation, and led to } \\
\text { teaching at broader level of applied } \\
\text { thinking skills, especially at senior } \\
\text { secondary levels. }\end{array}$ & $\begin{array}{l}\text { Leadership of } \\
\text { different faculties } \\
\text { meant practice of } \\
\text { all threads of } \\
\text { personal studies } \\
\text { and keeping current } \\
\text { impetus for change } \\
\text { and internal } \\
\text { educational } \\
\text { delivery, } \\
\text { particularly at } \\
\text { senior secondary } \\
\text { classes }\end{array}$ & $\begin{array}{l}\text { Leadership, } \\
\text { current, and } \\
\text { edgy, in } \\
\text { curriculum, } \\
\text { in class } \\
\text { practice, and } \\
\text { for senior } \\
\text { students in } \\
\text { particular }\end{array}$ & \\
\hline P3 & $\begin{array}{l}\text { Subject integration and } \\
\text { compartmentalisation: an effort I } \\
\text { made to help students to recognise } \\
\text { how skills in one subject area could } \\
\text { be utilised in other subject areas. This } \\
\text { initiative was a personal one and } \\
\text { culminated in much student support } \\
\text { when subject choices were being } \\
\text { made in Yr 10, and when subject } \\
\text { choices were reviewed for VCE and } \\
\text { eventually when initial subjects were } \\
\text { chosen for career purposes and } \\
\text { tertiary study. }\end{array}$ & $\begin{array}{l}\text { Inclusion of subject } \\
\text { integration and } \\
\text { compart- } \\
\text { mentalisation led to } \\
\text { design and } \\
\text { implementation of } \\
\text { decision-making } \\
\text { and other initiatives } \\
\text { for student } \\
\text { decision-making }\end{array}$ & $\begin{array}{l}\text { Expansion of } \\
\text { Life Skills, } \\
\text { applied } \\
\text { thinking skills } \\
\text { and future } \\
\text { planning in } \\
\text { curriculum }\end{array}$ & \\
\hline P 14 & $\begin{array}{l}\text { From } 1992 \text { till 2000, I was a home } \\
\text { stay place for tertiary students from } \\
\text { country Victoria, interstate, especially } \\
\text { Queensland, and all South-East Asia } \\
\text { countries except Myanmar. About } 26 \\
\text { all told, stayed for varying lengths of } \\
\text { time from several weeks to five } \\
\text { years(PhD student) }\end{array}$ & $\begin{array}{l}\text { mutual } \\
\text { development of } \\
\text { cultural and } \\
\text { educational matters } \\
\text { for them and for me }\end{array}$ & $\begin{array}{c}\text { Cross- } \\
\text { cultural } \\
\text { fertilisation }\end{array}$ & \multirow{3}{*}{$\begin{array}{c}\text { Personal } \\
\text { growth }\end{array}$} \\
\hline P9 & $\begin{array}{l}\text { IT in the classroom meant that } \\
\text { professional development was needed } \\
\text { as changes to assignments, research } \\
\text { methods, and assessments could be } \\
\text { incorporated in my preparation and } \\
\text { delivery, and also to students own } \\
\text { armoury for progress. }\end{array}$ & $\begin{array}{l}\text { Regular IT use } \\
\text { needed PD to } \\
\text { accomplish } \\
\text { efficiently }\end{array}$ & $\begin{array}{l}\text { Upgrade to } \\
\text { include IT in } \\
\text { my work and } \\
\text { in planned } \\
\text { classroom } \\
\text { activities }\end{array}$ & \\
\hline P1 & $\begin{array}{l}\text { Leadership studies -partly as a result } \\
\text { of threads in B.ED Studs and MEd } \\
\text { studies were the next stage in my } \\
\text { studies. Formal and informal } \\
\text { leadership: how did it operate? - } \\
\text { especially in view of content like } \\
\text { Caldwell \& Spinks and the self } \\
\text { managing school concept? }\end{array}$ & $\begin{array}{l}\text { Leadership studies } \\
\text { incorporating new } \\
\text { initiatives from } \\
\text { educational } \\
\text { theorists } \\
\text { foundational to } \\
\text { early shaping of } \\
\text { what culminated in } \\
\text { PhD studies }\end{array}$ & $\begin{array}{l}\text { Threads of all } \\
\text { postgraduate } \\
\text { learning } \\
\text { culminating } \\
\text { in shaping of } \\
\text { leadership } \\
\text { studies }\end{array}$ & \\
\hline
\end{tabular}




\begin{tabular}{|c|c|c|c|c|}
\hline REF & COMMENT & CONSTRUCT & CONCEPT & THEME \\
\hline P2 & $\begin{array}{l}\text { At this stage, the beginnings of the } \\
\text { integration of John Wilson's Moral } \\
\text { Education scenario, with Lipman's } \\
\text { Philosophy for Children and the } \\
\text { Community of Inquiry were } \\
\text { completed and incorporated into the } \\
\text { M. Ed. substance. }\end{array}$ & $\begin{array}{l}\text { Integration of } \\
\text { Wilson's work and } \\
\text { Lipman's P4C } \\
\text { initiatives laced } \\
\text { into M Ed studies }\end{array}$ & $\begin{array}{l}\quad \text { P4C } \\
\text { integrated } \\
\text { with Lipman } \\
\text { and included } \\
\text { in a new } \\
\text { curriculum }\end{array}$ & \multirow[b]{2}{*}{$\begin{array}{l}\text { Change } \\
\text { agentry }\end{array}$} \\
\hline P6 & $\begin{array}{l}\text { Eventually, by a succession of various } \\
\text { small projects at junior levels, } \\
\text { students began to see the excitement } \\
\text { of the new and broad canvas that they } \\
\text { could use. Life became bearable for } \\
\text { them in the classroom, because self- } \\
\text { evaluation exercises at the end of each } \\
\text { topic within a subject brought } \\
\text { interesting and validating results for } \\
\text { them and for me. }\end{array}$ & $\begin{array}{l}\text { Self-evaluation } \\
\text { exercises following } \\
\text { use of applied } \\
\text { thinking skills in } \\
\text { class received } \\
\text { positively }\end{array}$ & $\begin{array}{l}\text { Applied } \\
\text { thinking skills } \\
\text { delivery } \\
\text { through } \\
\text { medium of } \\
\text { subject or } \\
\text { topic accepted } \\
\text { by receptive } \\
\text { students }\end{array}$ & \\
\hline P11 & $\begin{array}{l}\text { The Associate Diploma of Theology } \\
\text { in } 2007 \text { highlighted some of the facets } \\
\text { of teaching in RE that I consciously } \\
\text { needed to pursue about the balance of } \\
\text { knowledge of theological matters. It } \\
\text { was central to the development of my } \\
\text { reshaped theory in the thesis, that I } \\
\text { was sufficiently knowledgeable about } \\
\text { secular and religious perspectives. }\end{array}$ & $\begin{array}{l}\text { Ass. Dip. Theol. } \\
\text { Needed to balance } \\
\text { my knowledge base } \\
\text { to distinguish } \\
\text { between secular } \\
\text { and religious } \\
\text { paradigms }\end{array}$ & $\begin{array}{l}\text { Qualification } \\
\text { needed for } \\
\text { classroom, } \\
\text { curriculum } \\
\text { and for } \\
\text { preparation } \\
\text { for PhD thesis }\end{array}$ & \multirow[b]{2}{*}{ Qualification } \\
\hline P12 & $\begin{array}{l}\text { Having tried to shape this thesis } \\
\text { question while still at University of } \\
\text { Melbourne; I was unsuccessful at PhD } \\
\text { and Dr of Education levels, was } \\
\text { invited to try again at VU and now the } \\
\text { thesis is complete, because I resigned } \\
\text { from teaching to work in the last } 3 \\
\text { years to shape and reshape the detail } \\
\text { to accomplish the research. I was able } \\
\text { to have the administration of the } \\
\text { research instrument for this thesis } \\
\text { completed before I resigned. }\end{array}$ & $\begin{array}{l}\text { Shaping of thesis } \\
\text { question caused } \\
\text { difficulties till last } \\
\text { few years when } \\
\text { shaping and } \\
\text { development of } \\
\text { knowledge meant } \\
\text { completion could } \\
\text { take place }\end{array}$ & $\begin{array}{l}\text { Shaping and } \\
\text { implementati } \\
\text { on leading to } \\
\text { delivery and } \\
\text { completion of } \\
\text { thesis }\end{array}$ & \\
\hline P4 & $\begin{array}{l}\text { I infused much of the thinking skills } \\
\text { apparatus into the development of } \\
\text { options, the advisement needed from } \\
\text { experts, and the 'ME' diagram with } \\
\text { its consequences of a broad canvas } \\
\text { upon which to place their plans. }\end{array}$ & $\begin{array}{l}\text { Applied thinking } \\
\text { skills infused into } \\
\text { decision-making } \\
\text { and advisement for } \\
\text { successful student } \\
\text { completion of } \\
\text { choices for career } \\
\text { and beyond }\end{array}$ & $\begin{array}{l}\text { Implementati } \\
\text { on of applied } \\
\text { thinking skills } \\
\text { in teaching } \\
\text { learning } \\
\text { delivery }\end{array}$ & \multirow[b]{2}{*}{$\begin{array}{c}\text { Teaching } \\
\text { thinking skills }\end{array}$} \\
\hline P5 & $\begin{array}{l}\text { By now, the broadening of thinking } \\
\text { skills development: Splitter \& Sharp’s } \\
\text { list became part of my delivery at the } \\
\text { beginning of each class. I introduced } \\
\text { one skill at a time and at first, students } \\
\text { were not all impressed! }\end{array}$ & $\begin{array}{l}\text { Applied thinking } \\
\text { skills infused into } \\
\text { decision-making } \\
\text { and advisement for } \\
\text { successful student } \\
\text { completion of } \\
\text { choices for career } \\
\text { and beyond }\end{array}$ & $\begin{array}{l}\text { Regular use } \\
\text { of change in } \\
\text { teaching } \\
\text { relevant } \\
\text { applied } \\
\text { thinking skills } \\
\text { for } \\
\text { curriculum } \\
\text { purposes in } \\
\text { each class }\end{array}$ & \\
\hline
\end{tabular}




\section{Experiential learning}

At this time I was conscious of a change in my personal perspective that amounted to the recognition of a need, and the confidence to develop a new paradigm: one that put the student at the centre of the teaching-learning process. In this I was supported by

- My academic studies in Oxford that provided the foundation for all of my subsequent studies and educational practice.

- My incorporation of computer research into the teaching-learning process.

- My emerging development of mutual understanding of classroom strategies in partnership with the school's student-teacher programs;

- Leadership studies that were current, and sometimes at the cutting edge, in the following: curriculum, classroom practice and, in particular, for senior students;

- Expansion of three elements: the time devoted to the teaching of Life Skills; the teaching of applied thinking skills; and futures planning in curriculum.

These were integrated in my academic and personal growth, as discussed in the next sub-section.

\section{Personal growth}

Home, and school, and personal influences, resulted in a cross-cultural and cross-disciplinary fertilisation that contributed to my cultural and professional growth. This was complemented by my making a major personal effort to upgrade my ICT skills that led me to include IT in my work and in planned classroom activities. Finally, the threads of my various postgraduate learnings culminated in the shaping of my leadership studies; these also became a foundation for sections of this thesis. 


\section{Change agentry}

The Community of Inquiry integrated into Philosophy for Children by Lipman, became the starting point for all my teaching - a point to which I could return time and time again - and eventually emerged as the new curriculum and pedagogical practices. I determined that the new paradigm needed to begin by teaching students how to construct their own questions, with the specific academic aim of efficient research. The inclusion of this extra qualification broadened the knowledge foundation upon which I developed an insistence towards the development of each student through the medium of any given subject or topic. Together, these underscored the new process of change agentry that I applied.

\section{The qualifications}

The sum total of all my formal learning, my experiential learning and my learning from my students, resulted in my seeking admission to a $\mathrm{PhD}$ program; this filled an urgently-felt need for me to pursue the investigations leading to understanding of perceptions of teachers about the teaching of thinking skills, and allowing me to pursue the question of how to promote the teaching of the concepts of virtues, values and ethics for student rich learning. At the same time, the qualifications that I had gained to meet the needs of classroom pedagogy (pedagogical content knowledge), the curriculum commonplaces (students, teachers, subjects taught and milieu), and required for preparation for $\mathrm{PhD}$ thesis, shaped the delivery and completion of my thesis.

\section{Teaching thinking skills}

Applied thinking skills had to become the central factor in the teachinglearning process, as were the regular use of change in teaching relevant applied thinking skills for curriculum purposes in each class: Naming, Introducing, and Modelling each new thinking skill (Splitter \& Sharp, 1995) must become the central factor in the teaching-learning process. 


\section{Looking forward}

As a result of my self-analysis, the auto-ethnographical introspective began to settle fully as new knowledge; I commenced the application of my process on a continuous basis and in a collaborative manner in each school in which I taught. This led to the development of what has become my new theory. For reasons that will become apparent, I call this edu-tensegrity. I set out fully the theory and the indicators with which its application might begin, in Chapter 6. 


\section{CHAPTER 6}

\section{From Twelve Categories of Influence to the Concept of Edu-tensegrity}

\section{Introduction}

In Chapter 6, the construction of a broad theory of education begins to emerge. The Categories of Influence are listed, because they provide a foundation upon which the impulse to teach thinking skills formally would lead to efficient democratic decision-making skills by students. I had been trialling a number of tools that I had, over a number of years, built with the aid of secondary students. I found that promotion of trilectic thinking patterns, rather than dialectic or lineal thinking patterns, fitted the diversity that is central to learning today. Tensions that arise from complicated situations can be harnessed through the use of trilectic logic in a way that allows diversity, democracy and autonomous decision-making skills to become central to student learning.

The geodesic dome, the foundation of my theory of edu-tensegrity, allows for the inclusive concerns and considerations that swirl around the plurality of peoples in their sociological and communal experience in Australia today. The application of trilectic logic is tested at the level of thinking, and is an effort to show how to approach inclusive decisionmaking. It also addresses some of the recent educational ideas of Crowther, Hargreaves and Beare. 
Having reflected on my own experiences and learning and investigated other people's experiences and ideas I have identified what I call twelve 'Categories of Influence' that I believe could shape a new architecture for teaching virtues, values and ethics. This chapter uses the 12 Categories of Influence and the concept of 'Edu-tensegrity' to build a new architecture for teaching virtues, values an ethics that puts the student at the centre of the teaching and learning process.

1. Dimensions of daily life

2. Dimensions of thinking

3. Dimensions of education

4. Curriculum theorists

5. Constructs about knowledge and the thinking curriculum

6. Historical and philosophical theorists

7. Historical and philosophical constructs

8. Teachers and teaching

9. Instrumental contributions

10. Outcomes and issues of dependence and rich learning

11. Construction of a belief system, the good life and a career

12. Integration of student, teacher and curriculum.

But before looking at the detail of each category and the combined effect of the 12 categories it is important to consider the idea of tensegrity as I believe this gives a shape with which the findings can be understood.

\section{What is tensegrity?}

Donald E. Ingber (Ingber, 1997, p. 48-57) indicates that tensegrity is a concept under which medical and allied scientists have found a universal set of rules that seem to guide the design and shape of organic structures - from simple carbon compounds to complex cells and tissues (p. 48). He has outlined carefully that these entities depend on a structure that uses selfassembly hierarchically as tiers of systems within systems (p. 48). Ingber (p. 
49) realised that tissues are removed and replaced in a maintained pattern of architecture then 'tensegrity structures are mechanically stable not because of the strength of individual members but because of the way the entire structure distributes and balances mechanical stresses'. Ingber also tells of this phenomenon being taken up by architects who construct geodesic structures relying on the balance between tension and compression. By distributing and balancing weight the push-pull factors become counteracting forces, which equilibrate throughout the structure to enable it to stabilise itself (p. 49).

\section{The concept of bio-tensegrity}

According to Ingber (1997, p.48- 57) when the concept of tensegrity is applied to the study of the human body; it uses the concept of bio-tensegrity, initially constructed and applied by Stephen Levin. He argues that medicine and natural therapies need to be carefully balanced to achieve the ultimate health of each human body. Ingber relates these concepts by considering the tension and compression elements found in a geodesic dome, as represented in Figure 6.1. What is most noticeable in the picture is that the points at which the arms of the structure meet, are the tension and the compression element which is being controlled within the dome cover. It is

\section{FIGURE 6.1 PICTURE OF A GEODESIC DOME}



Source: Scientific American, January, 1998, p. 50 
also noticeable that the parallels in this structure are similar in each section and become the basis of Ingber's claimed illustration of form. It also highlights the size of the human person standing on the dome, in relation to the size of the dome itself! Ingber also demonstrates that all cells of microscopic, visible and invisible, small and large sizes have this structure. There is potentially a reason to use that parallel to highlight a universal pattern: human cells, water molecules, and he argues that even a giraffe's neck has the same geodesic structure incorporating tension and compression (see Figures 6.2 and 6.3).

FIGURE 6.2 PICTURE OF A GIRAFFE'S NECK

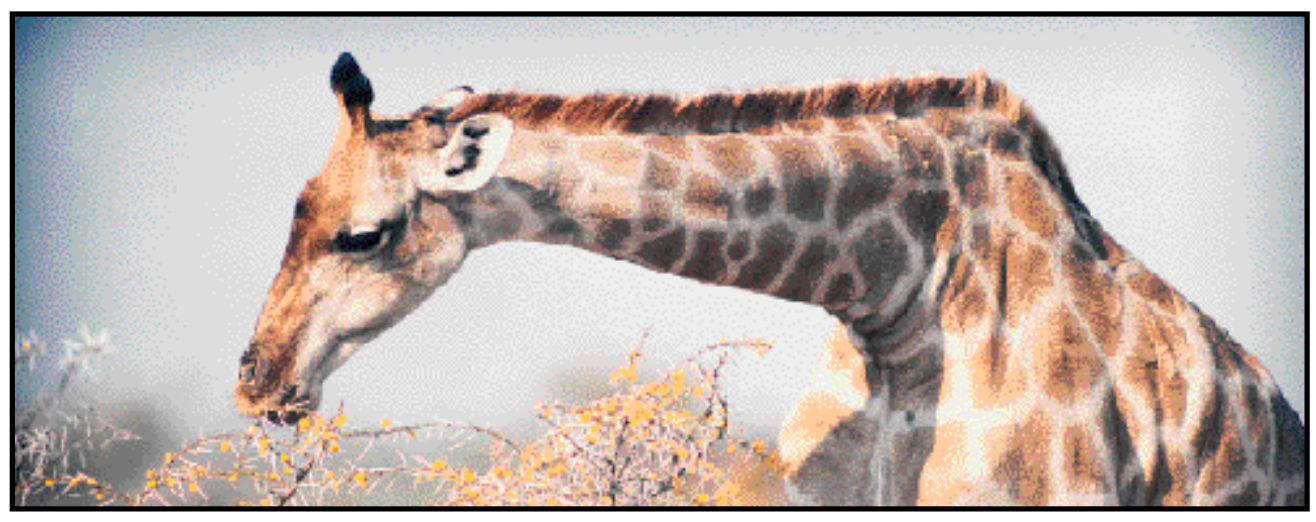

Source: Scientific American, January, 1998, p. 56

FIGURE 6.3 MODEL: GIRAFFE'S NECK OR CABLE-AND-BEAM SCULPTURE

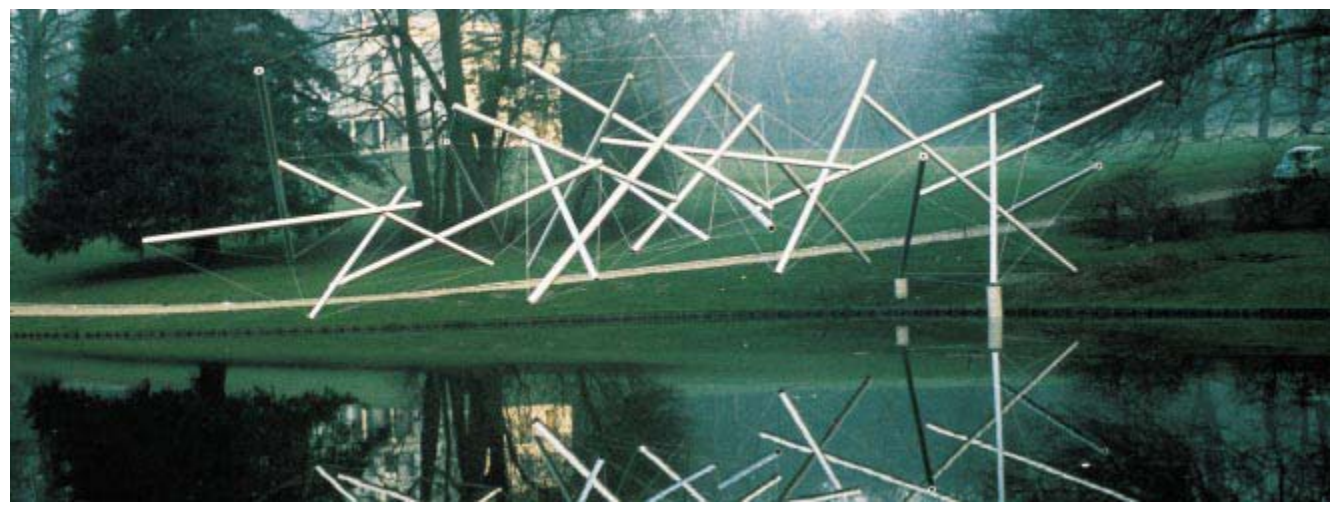

Source: Scientific American, January, 1998, p. 57 
What is noticeable about the Giraffe's bone structure (shown as a model in Figure 6.3 and 6.4) is that the bones are at various angles to each other so that when the giraffe sees what it wants; its reach is enabled by the movement of its neck. It is a study in tension and compression, distribution and balance of weight. The opposing forces allow the balance and form to operate in the required way and the giraffe can proceed. In the educational metaphor, students can see and reach what they want, by recognising the daily experience of tension and compression, of balance and the likely pushpull factors that may be apparent at times in their experience of education.

There are two further ideas that are important in biotensegrity. The first is to remember that cells thrive and evolve when stretched, and die if left stagnant. The second is that the structure actually strengthens under mechanical stress and is called 'prestress' by Ingber (1997, p.49).

So it is important that the concepts/sets which have been titled Categories of Influence are fully tested and proven true. For this theory to be successful, each of the Categories of Influence needs to be tested under rigorous scrutiny. Every effort has been made in my personal classroom experience over a long secondary teaching career to pilot much of the content that contributes to this reshaped theory. By the duplication of these conditions in many classrooms, as was done with Lipman's Community of Inquiry and Vardy's Five-Strand Religion and Values Education initiatives, it is possible to strengthen the understanding and practice that will support the principles that parallel the initial Tensegrity.

\section{Tensegrity as a concept for education}

The initial concept of Tensegrity is an architectural concept in which buildings can be successfully erected on a principle of tension and compression. In this concept, the exact weight and angular precision of uprights and horizontally connected beams rely on each other for balance, for accuracy of design and for longevity. If calculations, weighting and relevant 
texture are accurate, this building will remain intact. If for any reason, there is a break of one element or a weakening of one or more controls, then the structure loses balance, and the push-pull factors of all elements are affected. By degrees, the whole structure can be decimated if precise remedial action is not provided immediately.

Perhaps as a consequence, I reasoned, the title of my parallel in education might be called edu-tensegrity. It follows then that the twelve Categories of Influence that are listed above are equally important, and none must be diminished or weakened; and none must be strengthened unnecessarily. The result of either of these approaches would weaken and loosen the structure. It becomes obvious that in the last four decades, this over-emphasis on certain parts of the educational structure have been less successful than expected. Beare (2010), Hargreaves (2009), Hargreaves and Shirley (2009) Crowther et al. (2010) and Crowther (2010) seem to highlight in their different ways the effects of these push-pull phenomena.

As with Buckmaster Fuller's geodesic domes (Ingber 1997), we can build the reshaped philosophy of education using this construct of tension and compression, distribution and balance, incorporating the twelve Categories of Influence stated above - in what Ingber calls geodesic precision. The geodesic dome becomes a metaphor for overall education; a geodesic dome, on which I have imposed educational Categories of Influence as depicted in Figure 6.4. It is important to recognise that we can not afford to confuse the reality of the geodesic buildings in architecture with the concept of the geodesic dome, which is the underpinning influence in the new paradigm.

It follows, as a consequence, that the twelve coloured triangles in the centre provide an organisational framework around which the subjects we teach may be integrated. There are 12 separate areas in the centre of the dome pictured, all of which represent a particular 'category of influence' listed. All other triangles represented in the geodesic dome refer first to the myriad of topics, subject areas, specialisations and particular learning areas 
FIGURE 6.4 EDU-TENSEGRITY AND THE 12 CATEGORIES OF INFLUENCE

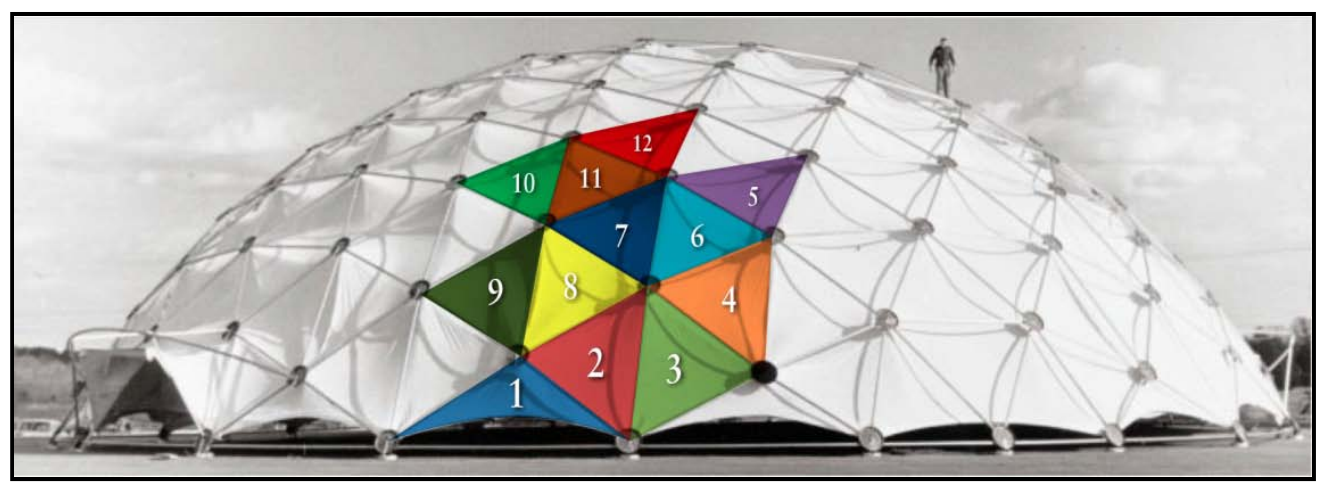

Source: Scientific American, January, 1998, p. 48; digitally altered by Scott Larritt-Evans

which exist in education today. Also included in the triangles are the metaconcepts of education: philosophy of education, sociology of education, administration, curriculum development, psychology of education, provision of myriads of necessary resources for both students and teachers, and most importantly the area of professional development for teachers, for specialists, for theorists, for administrators, in the service of the teaching- learning process.

\section{A new concept of education: Edu-tensegrity}

The concept of the Categories of Influence parallels the example of the geodesic dome in architecture. We see the same geodesic dome with the triangles coloured to represent the Categories of Influence. Each triangle has three sides, all of which need to function fully and connect with each other in order to produce a complete and balanced concept of how education needs to function in twenty-first century conditions. In fact, we cannot afford to leave out any of the Categories of Influence without finding that the whole structure weakens and crashes. We become aware that each category of influence is equal in significance and importance.

In a parallel with the giraffe's neck, depicted in Figures 6.2 and 6.3, the student coming in contact with all of these elements within the Categories of 
Influence, will eventually find rich and real learning, in the parallel way as the giraffe sees and finds his compass bearings and his food.

And so is born edu-tensegrity, in which all 12 Categories of Influence become the poles of strength that shape and reshape the student experience of education, by the emphases placed on alternative Categories of Influence over the period of the students' whole education, without dropping any of the list at any time! Emphasis on the political needs of an era is not more important than the Categories of Influence delineated here.

This reshaped theory takes account of the life work of Wilson, Lipman and Vardy and makes a crucial attempt to synthesise competently what each legacy leaves for us in education. Nothing I can say encompasses the aim and objective of this whole investigation than to refer to the obituary article written by Roger Sutcliffe of Matthew Lipman. The article appears as Appendix D as it details the direction of my learning.

One last pertinent point harks back to a remark about the importance of values made by Mulford (1994, p. 38) in the last decade of the twentieth century:

Education should be preparing tomorrow's adults to meet ethical as well as economic imperatives - preparing them not only for a life of work, but also a life of worth. We must not only educate to serve the purposes of others, but also develop their capacity to question the purposes of others. We must bolster students' will to seek wisdom. We must enable them to communicate, to think creatively about complex issues, to act responsibly, and - when necessary - to act selflessly. ....A skills crisis would indeed be bad enough but a values crisis would be devastating.

My concept of edu-tensegrity, shown in pictorial form in Figure 6.4, is an attempt to harness the various 'Categories of Influence' that drift through the education sector. These can be connected by the unifying theme of a geodesic dome, in which the architectural principles underlying structures like the London millennium dome or the Melbourne soccer stadium can be applied in parallel to biology and now to education. 
In the investigation that has taken place, there are a series of sets or triangles whereby it is noted that each of the three connected concepts interlink, as exploration of a reshaped theory of education emerges. On the geodesic dome above, the 12 triangles, that I have called Categories of Influence, are equal in significance and priority, and colour-coded to emphasise the points that follow:

Each 'category of influence',(shown in separate colours), highlights triangulation of interdependent elements of an educational concept, and together they form the structure that allows the push-pull factors of political, personal and professional elements of educational experience to be attended, without failure overtaking the enterprise we call education.

\section{Three dimensions for each Category of Influence}

The first step in describing edu-tensegrity is to outline three interconnected components for each category. I have aspired to reach similar knowledge and understanding in this study.

1. Dimensions of daily life incorporates virtues, values and ethics

2. Dimensions of thinking incorporates ontological, epistemological and pedagogical influences

3. Dimensions of education incorporates philosophy of education, sociology of education and psychology of education

4. Curriculum theorists I have included allow for my view about the centrality of three experts - Wilson, Lipman and Vardy

5. Constructs about knowledge and the thinking curriculum incorporate Wilson's Moral Components List, Lipman's Community of Inquiry and Vardy's study of Religious and Values Education

6. The three central historical and philosophical figures in education are Aristotle, Dewey and Schwab

7. The particular historical and philosophical constructs are Meaning, Reasoning and Choice 
8. Teachers and teaching are shaped by the philosophical, the eschatological, and the educational goals

9. Instrumental contributions to support thinking and decisionmaking for life skills beginning with personal understanding (using the ME diagram) extending this to decision making (using my Six Steps) and then exploring behavioural concepts and their consequences (using the HUG/BUG)

10. Outcomes and issues of dependence and rich learning include dependence (requiring distinct help with acquisition of new knowledge), independence (requiring the space to access and display new knowledge) and interdependence (requiring the scaffolding or support of collaborative experience for internalisation of new knowledge)

11. The need for students to construct a belief system, make decisions about the good life and a career - students knowingly to accept responsibility for connecting and constructing their personal belief system, their preferred future and their career of choice

12. Integration of student, teacher and curriculum requires that more emphasis is placed on the collaboration and interdependence of the student, the teacher and the curriculum expert, so that they can correspond more precisely with today's educational needs.

\section{Explaining the twelve Categories of Influence}

This section provides more detail about each of the 12 categories.

\section{Dimensions of daily life}

Virtues, values and ethics are the three components that go to make up the foundation of twenty-first century life based on quality of decisions for each student. In the geodesic dome, the relevant triangle representing this category of influence is blue. The central outcome from this thesis is to focus on how 
to provide key ideas that will contribute towards a specific architecture for the teaching of virtues, values and ethics. Some significant authors who contribute to my considerations are Wilson et al. (1967), Seligman (1990, 2002), Kristjannsson (1998, 2000), Golding (2006), and Taylor (2007). These sources give breadth and meaning to the inductive thinking that accompanies the reflections so central to the individual meaning of a person's life.

\section{Dimensions of thinking}

Ontological, epistemological and technical influences underpin this investigation. In the geodesic dome, the relevant colour representing this category of influence is red. The big ideas that are the foundation of the ontological influence lead us towards the epistemological content that represents the new knowledge emerging from creative and careful thinking. This of course gives rise to the decisions that will form the bases of pedagogical (techne) actions in life's journey for the individual. I am indebted to many of the authors and philosophers who feature in the extensive literature review of chapter 3 , but it is particularly significant to explore the relevant work of Wilson (1987), H. Gardner (1993a), and Fisher (2001, 2003).

\section{Dimensions of education}

Philosophy of education, sociology of education and psychology of education: each represents three interactive disciplines for effective delivery of curriculum. These three elements of the education scenario are represented in the geodesic dome by the citrus colour. Leading thinkers of the ilk of Dewey (1944), Ayer (1973), Boomer (1982), Shulman (1987), Constas (1998), Hargreaves (2009), Beare (2010), Crowther (2010), Seligman (2011) and others who feature in the literature review give substance to the paradigm shifts that accompany our accuracy in the fields of philosophy of education, sociology of education and psychology of education. 


\section{Curriculum theorists}

Three expert theorists: Wilson, Lipman, Vardy represent theoretical and practical thinking frameworks, whose influences can and are being integrated for better quality of educational delivery. In the geodesic dome, these are represented by the orange colour. These educational philosopherpractitioners are supported by the scaffolding of Aristotle, Dewey and Schwab, whose emphases are placed on the equal importance of the student, the teacher, the curriculum content and the classroom milieu.

\section{Constructs about knowledge and the thinking curriculum}

Wilson's Moral Components List, Lipman's Community of Inquiry and Vardy's RAVE (study of Religions and Values Education) become channels of constructs and practices within the thinking curriculum for curriculum integration. In the geodesic dome, this category of influence is represented by the colour purple. Their classroom content and focus on teaching the student, rather than just the subject knowledge, is central to the considerations of my newer paradigm. At the end of the first decade of the new century, Hawkes (2008, 2010), Cherednichenko, (2000), and Wellham (2010) accompany towering figures like Crowther (2010, 2011), Beare (2010), Brock (2010), Hargreaves (2003, 2009), and Walker (2011).

\section{Historical and philosophical theorists}

The influence of three significant historical and philosophical figures in education: Aristotle, Dewey and Schwab, are linked by their indications of the inductive and deductive thinking involved in delivery of holistic education. In the geodesic dome, this category of influence is represented by sea-green. In fact, this category of influence indicates much of the historical paradigm changes within the development of education for today. 


\section{Historical and philosophical constructs}

The historical and philosophical constructs from these towering figures offer the focus and function of reasoning: Aristotle, and the broad sweep of meaning in education; Dewey and the means of experiential education; and the four commonplaces that provide the vehicles for student choice: Schwab. In the geodesic dome, this category of influence is represented by a dark-blue colour. The work of these three figures is central to my considerations of the distinction between a teacher-directed classroom and a student-directed classroom. Each distinguishes between the skills needed to teach content of the curriculum and the skills needed to reach and communicate with each student: educational meaning; experiential education and the quadrilectic logic of Schwab’s commonplaces.

\section{Teachers and teaching}

Teachers provide a range of goals which students can explore: philosophical goals, eschatological goals and educational goals. In the geodesic dome, this category of influence is represented by the yellow colour. This outcome is already well in evidence but it can be swamped by the variety of short-term topics that modern politics wishes to include in education. This category of influence allows for the precondition of teaching the steps of thinking skills so that students can learn how this process operates for decision-making and life choices. There is a need for serious consideration of the safe environment for students. They then can survive some mistakes as they learn the skills of decision-making that will become life skills needed for career choice, for personal philosophy, for the eschatological speculations that accompany each individual's life journey. Philosophers and others who support the search for meaning are numerous in the literature review, but Singer (1979), McNamara (1983) and Brink (1989) give valuable insights to highlight the significance of this category of influence. 


\section{Instrumental contributions}

My contribution to the possibility of a reshaped theory of education includes the Heasly Behaviour Uncertainty Grid (HUG/BUG); the 'ME' diagram; and the Six Steps of Decision-making and in the geodesic dome, this category of influence is represented by the olive-green colour. I have relied on all of the authors within the literature review to identify, understand and apply the best of thinking skills in historical and current modes to the construction of a career, the good life and the meaning of life. Students have a right to be able to construct and build for themselves as much as possible from the foundation of thinking skills. Decision-making skills regarding their attention to virtues, values and ethics will then follow. The particular figures that accompany Wilson, Lipman, and Vardy for the length of this inductive modelling of the new paradigm include Schwab (1983), Shulman (1987), Splitter \& Sharp(1995), Zigler (1998, 1999), Constas (1998), Costa (2001), Greenfield (2004, 2007, 2008, 2011), Roth (2005), Beattie (2009), Hargreaves (2009), and Crowther (2010), Seligman (1990, 2002, 2011).

\section{Outcomes and issues of dependence and rich learning}

Consequences for students who achieve in different ways by constructing a mode of being: appropriately dependent, independent and interdependent as needed, and are therefore able to expand their skills and deepen their knowledge. One of the main consequences of the new paradigm has been to search by inductive means to construct a category of influence that provided for the three differing states of attitude that underpin the impetus for learning identified here. In the geodesic dome, this category of influence is represented by the green colour. A focussed reference to one such dialogue (between Coch and Tancredi,) shows in one themed issue of the Journal of Moral Education (2007). The stage noted in my Six Steps of Decisionmaking which calls for advisement, rather than advice or counselling, is central here, as the teacher needs the precise skills of advisement so that the student can use the three states mentioned: dependent, independent and 
interdependent modes -which are all part of learning skills. Costa (2001), Fogarty (1991, 1993, 2001, 2005), Lipman (1988, 1991, 1993, and his novelettes), Vardy (1998 and the DAN professional resources), Goleman (1996, 2007), and H Gardner (1991, 1993, 2009) lead the academic analysis that point the way for teachers and educationalists.

\section{Construction of a belief system, the good life and a career}

Education provides students then with channels of learning by taking account of the recent work in neuro-science of Susan Greenfield, (2004, 2007, 2008, 2011) focussing on research into how the brain works; Norman Doidge, (2007) focussing on research into rehabilitation in how the brain works; and Joe Dispenza, (2007) focussing on research into how to harness the potential new abilities of the brain for learning and for life. In the geodesic dome, this category of influence is shown by the dark-orange colour. These three figures provide us with consequent relevance that influences the precise measures that characterise the construction of a belief system, building of a preferred future and making career decisions, rather than just an amalgam of topic knowledge which may or may not be specifically useful in the future of the individual student.

\section{Integration of student, teacher and curriculum}

Promotion of an accurate channel for the student achievement of such educational excellence means that teachers at the coalface need to be heard. Their comments: hesitations, confusions, concerns, silences were central to the prosecution of this investigation about what their perceptions are about the teaching of thinking skills - in relation to virtues, values and ethics - in educational terms. In the geodesic dome, the category of influence representing this concept is a dark-red colour. Virtues, values and ethics are systems for organising our view of the world and, in Greenfield's terms, need to be taught as new knowledge. So the triangle here is the collaboration between the student, the teacher and the curriculum expert. I am indebted to 
Fisher (1990, 2003), Golding (2006), Vardy (1998), Kristjannsson (1998, 2000), Hargreaves (Hargreaves, 2009; Hargreaves \& Shirley, 2009), Leckey, (2009), and Crowther (2010a and 2010b).

Having identified these twelve Categories of Influence which appear to be unconnected, it then seemed important to think about the connections between each category and the sum of the combined categories. I started thinking about the concept of tensegrity and education as a geodesic dome in which the push-pull factors are central to the overall balance of an education experience for today's students. I have found that the Categories of Influence can be grouped according to their ontological, epistemological and technical characteristics.

In ontological terms there are links between the following Categories of Influence:

1. Dimensions of daily life

2. Dimensions of thinking

3. Dimensions of education

4. Curriculum theorists

5. Constructs about knowledge and the thinking curriculum.

In epistemological terms there are links between the following Categories of Influence:

6. Historical and philosophical theorists

7. Meaning, reasoning and choice

8. Teachers and teaching

9. Heasly instrumental contributions.

There are links in terms of human experience, emerging as pedagogical, technical strategies and techniques in the classroom between the following Categories of Influence:

10. Outcomes and issues of dependence and rich learning

11. Construction of a belief system, the good life and a career

12. Integration of student, teacher and curriculum. 


\section{Looking forward...}

Having identified 12 Categories of Influence and developed an argument for Edu-tensegrity as an over-arching concept, the following chapters apply these ideas to the teaching of virtues and values (Chapter 7) and ethics (Chapter 8). In each chapter, the 12 Categories of Influence are used as an organising structure to build this architecture for improved teaching practices. 


\section{CHAPTER 7}

\section{A new architecture for teaching virtues and values}

\section{Introduction}

In this chapter, I set out how Categories of Influence might allow a pedagogical and inclusive architecture to be built for the purposes of teaching, in the twenty-first century, about virtues and values first, prior to addressing decision-making about ethical behaviours. The sequence of this architecture needs to be built first around virtues and values because both are developed autonomously and privately through thinking skills prior to decisions being made about desired action. By testing whether each Category of Influence could be separately applied to the concept of education, I hoped to strengthen and deepen appreciation of existing understandings about where education could contribute further in an inclusive and transparent, but accountable process.

In this Chapter the focus is on the centrality of individual internal responses to understanding virtues and values. I will develop a further variant and significant way of applying the theory of edu-tensegrity: taking the Categories of Influence and aligning them with Schwab’s (1983, p. 240) four commonplaces of the curriculum, where

\footnotetext{
...curriculum is what is successfully conveyed to differing degrees to different students, by committed teachers using appropriate materials and actions, of legitimated bodies of knowledge, skill, taste, and propensity to act and react, which are chosen for instruction after serious reflection and
} 
communal decision by representatives of those involved in the teaching of a specified group of students who are known to the decision makers.

In a thesis associated with the positioning of curriculum coordinators in schools, Ling (1998, p. 233) points out that

\begin{abstract}
...Schwab identified the essential pinch of the problem for curriculum coordinators as maintaining the balance between means and ends that affect the four commonplaces of education-teacher, student, what is taught and milieux of teaching-learning—in determining the curriculum...
\end{abstract}

Furthermore, Ling (1998, p. 233) points out an essential link between Aristotle and Schwab:

\begin{abstract}
Schwab makes the essential point that the curriculum is not necessarily the same for all students or a given age and standing, that it does not differ necessarily in all respects for each and every student or school, and that it is not something about which decisions can be certified in advance of trial as the best decisions. Schwab (1983, p, 243) affirms the two sets of arts (derived from Aristotle and expounded upon in his essays on liberal education) required to meet the need for localism of curriculum: arts of the practical, i.e., prudence and deliberation, and arts of eclectic.
\end{abstract}

It makes sense, therefore, for me to group the Categories of Influence that I have identified previously within the framework of Schwab's four commonplaces, while taking account of the ontological, epistemological and pedagogical aspects of virtues, values and ethics - thus creating a further impact shown in Table 7.1 below.

TABLE 7.1 SCHWAB'S COMMONPLACES AND CATEGORIES OF INFLUENCE

\begin{tabular}{|c|l|l|}
\hline COMMONPLACE & $\begin{array}{c}\text { CATEGORY OF } \\
\text { INFLUENCE }\end{array}$ & \multicolumn{1}{c|}{ SUBJECT FOCUS } \\
\hline Student & $\begin{array}{l}\text { 7. Meaning } \\
\text { reasoning and } \\
\text { choice }\end{array}$ & $\begin{array}{l}\text { The historical and philosophical constructs from these } \\
\text { towering figures offer the focus and function of } \\
\text { reasoning: Aristotle, and the broad sweep of meaning in } \\
\text { education: Dewey and the means of experiential education, } \\
\text { and the four commonplaces that provide the vehicles for } \\
\text { student choice: Schwab. }\end{array}$ \\
\hline
\end{tabular}




\begin{tabular}{|c|c|c|}
\hline COMMONPLACE & $\begin{array}{l}\text { CATEGORY OF } \\
\text { INFLUENCE }\end{array}$ & SUBJECT FOCUS \\
\hline Student & $\begin{array}{l}\text { 9. Heasly } \\
\text { contributions }\end{array}$ & $\begin{array}{l}\text { Heasly contribution to the possibility of a reshaped } \\
\text { theory of education: Behaviour Uncertainty Grid } \\
\text { (HUG/BUG); ME diagram; Six Steps of Decision-making. }\end{array}$ \\
\hline Student & $\begin{array}{l}\text { 10. Outcomes and } \\
\text { issues of } \\
\text { dependence and } \\
\text { rich learning }\end{array}$ & $\begin{array}{l}\text { Consequences for students who achieve in different ways } \\
\text { by constructing a mode of being: appropriately dependent, } \\
\text { independent and interdependent as needed, and are } \\
\text { therefore able expand their skills and deepen their } \\
\text { knowledge. }\end{array}$ \\
\hline Student & $\begin{array}{l}\text { 11. Construction of } \\
\text { a belief system, } \\
\text { the good life } \\
\text { and a career }\end{array}$ & $\begin{array}{l}\text { Education provides students then with channels of } \\
\text { learning by taking account of the recent work in neuro- } \\
\text { science of Susan Greenfield, (research into how the brain } \\
\text { works) Norman Doidge, (research into rehabilitation in } \\
\text { how the brain works) and Joe Dispenza (research into how } \\
\text { to harness the potential new abilities of the brain for } \\
\text { learning and for life), with consequent relevance that } \\
\text { influences construction of a belief system, building of a } \\
\text { preferred future and making career decisions. }\end{array}$ \\
\hline Teacher & $\begin{array}{l}\text { 4. Curriculum } \\
\text { theorists }\end{array}$ & $\begin{array}{l}\text { Three expert theorists: Wilson, Lipman, Vardy, whose } \\
\text { influences can and are being integrated for better quality of } \\
\text { educational delivery. }\end{array}$ \\
\hline Teacher & $\begin{array}{l}\text { 5. Constructs } \\
\text { about } \\
\text { knowledge and } \\
\text { the thinking } \\
\text { curriculum }\end{array}$ & $\begin{array}{l}\text { Wilsons Moral Components List, Lipman's Community } \\
\text { of Inquiry and Vardy's RAVE (study of Religions and } \\
\text { Values Education) become channels of Constructs and } \\
\text { practices within the thinking curriculum for curriculum } \\
\text { integration. }\end{array}$ \\
\hline Teacher & $\begin{array}{l}\text { 6. Historical and } \\
\text { philosophical } \\
\text { theorists }\end{array}$ & $\begin{array}{l}\text { The influence of three significant historical and } \\
\text { philosophical figures in Education: Aristotle, Dewey and } \\
\text { Schwab - linked by their indication of the inductive and } \\
\text { deductive thinking involved in delivery of holistic } \\
\text { education. }\end{array}$ \\
\hline Teacher & $\begin{array}{l}\text { 7. Meaning } \\
\text { reasoning and } \\
\text { choice }\end{array}$ & $\begin{array}{l}\text { The historical and philosophical constructs from these } \\
\text { towering figures offer the focus and function of } \\
\text { reasoning: Aristotle, and the broad sweep of meaning in } \\
\text { education: Dewey and the means of experiential education, } \\
\text { and the four commonplaces that provide the vehicles for } \\
\text { student choice: Schwab. }\end{array}$ \\
\hline Teacher & $\begin{array}{l}\text { 8. Teachers and } \\
\text { teaching }\end{array}$ & $\begin{array}{l}\text { Teachers provide a range of goals which students can } \\
\text { explore: philosophical goals, eschatological goals and } \\
\text { educational goals - for decision-making and life choices. }\end{array}$ \\
\hline Teacher & $\begin{array}{l}\text { 12. Integration of } \\
\text { student, teacher } \\
\text { and curriculum }\end{array}$ & $\begin{array}{l}\text { Promotion of an accurate channel for the student } \\
\text { achievement of such educational excellence means that } \\
\text { teachers at the coalface needed to be heard. Their } \\
\text { comments, hesitations, confusions, concerns, silences were } \\
\text { central to the prosecution of this investigation about what } \\
\text { their perceptions are about the teaching of thinking skills in } \\
\text { relation to virtues, values and ethics in educational terms } \\
\text { So the triangle here is the collaboration between the } \\
\text { student, the teacher and the curriculum expert. }\end{array}$ \\
\hline Teacher & $9 \begin{array}{l}\text { Heasly } \\
\text { contributions }\end{array}$ & $\begin{array}{l}\text { Heasly contribution to the possibility of a reshaped } \\
\text { theory of education: Behaviour Uncertainty Grid } \\
\text { (HUG/BUG); ME diagram; Six Steps of Decision-making. }\end{array}$ \\
\hline Milieu & $\begin{array}{l}\text { 1. Dimensions of } \\
\text { daily life }\end{array}$ & $\begin{array}{l}\text { virtues, values and ethics as the foundation of twenty-first } \\
\text { century life based on quality of decisions for each student. }\end{array}$ \\
\hline Milieu & 2. Dimensions of & The ontological, epistemological and pedagogical \\
\hline
\end{tabular}




\begin{tabular}{|c|c|l|}
\hline COMMONPLACE & $\begin{array}{l}\text { CATEGORY OF } \\
\text { INFLUENCE }\end{array}$ & \multicolumn{1}{|c|}{ SUBJECT FOCUS } \\
\hline thinking & influences that underpin this investigation \\
\hline Milieu & $\begin{array}{c}\text { 3. Dimensions of } \\
\text { education }\end{array}$ & $\begin{array}{l}\text { Philosophy of Education, Sociology of Education and } \\
\text { Psychology of Education that each discipline interacts } \\
\text { with each other for effective delivery of curriculum. }\end{array}$ \\
\hline Milieu & $\begin{array}{l}\text { 11. Construction of } \\
\text { a belief system, } \\
\text { the good life } \\
\text { and a career }\end{array}$ & $\begin{array}{l}\text { Education provides students then with channels of } \\
\text { learning by taking account of the recent work in neuro- } \\
\text { science of Susan Greenfield, (research into how the brain } \\
\text { works) Norman Doidge, (research into rehabilitation in } \\
\text { how the brain works) and Joe Dispenza (research into how } \\
\text { to harness the potential new abilities of the brain for } \\
\text { learning and for life), with consequent relevance that } \\
\text { influences construction of a belief system, building of } a \\
\text { preferred future and making career decisions. }\end{array}$ \\
\hline
\end{tabular}

This table emphasises the continuity between the emergent Categories of Influence and the four commonplaces of Schwab - with the subject focus as an independent variable and student, teacher and milieu as dependent variables. The dependent variables are listed in the order different students, committed teachers and those involved (the milieu) in serious reflection, and communal decision by representatives of those involved in the teaching of a specified group of students, who are known to the decision makers. The subject focus comes out of a consideration of the twelve Categories of Influence that have been identified in this study, resulting in an entity that is consistent with Schwab’s definition of curriculum, above.

In the next chapter, each category of influence will be applied to ethics within the curriculum. In the succeeding sections of this chapter, each category of influence will be applied to virtues and values within the curriculum. Prior to this, however, an emergent paradigm involving, in the first instance, virtues, values and education; in the second instance, the placement of virtues and values in its central place in the curriculum, will be discussed.

\section{Virtues and values and education}

In the new paradigm, virtues and values are part of the emerging new knowledge that affirms students in their understanding of their world. 
Given the conceptual lead in my teaching instrument - the Heasly Uncertainty Grid or alternatively named Behaviour Uncertainty Grid (HUG/BUG) content, discussed in Chapter 4 - students explore and discuss meanings of concepts and behaviours listed, follow this activity with comparison of patterns of behaviours, and begin to recognise how they can make personal choices.

Experts and educationalists, philosophers and planners have provided a wealth of behavioural concepts that can be included later in curriculum designed for studies within this area of new knowledge for students and teachers alike; their work is discussed more fully in Chapter 3; this broad spectrum includes:

- Human flourishing (Wilson 1987; (Allix, 2000);

- Compassion (McNamara 1983, and Ruiz and Vallejo 1999);

- Freedom, responsibility, necessity, moral luck, and free will (Watson, 1982 and Wilson et al 1967);

- Truth, falsification, rigor (Gardner, S. 1998);

- Pride, envy, anger, covetousness, gluttony, sloth, lust (Schwartz 2000);

- Respect, honesty, sensitivity, reasonableness and strengthening of judgment (Splitter \& Sharp, 1995) ;

- Moral argument and two forms of moral thinking (Hare, 1981, 1992);

- Religious perspectives and secularism (Hick 1973, Grayling 2011);

- Obama (2006, 2008) and his autobiographical construction of a religious paradigm;

- Love and hate, with rage, despair and death (O’Connor 2001);

- The connection between thinking processes with emotional processes (Lipman 1988, Wilson 1971);

- Wisdom (Macdonald 1996); 
- Anxiety and conflict and moral critique (De Botton 2004, Rowland 1993, Seligman 2002, Goldhagen 2002);

- Good work, excellence and ethics (H. Gardner 1993a, Gardner et. al. 2001);

- Reinventing knowledge and recognising guilt, shame and forgiveness and their consequences (Mackay, 1997, 1999, 2004; McNeely \& Wolverton, 2009).

Addressing elements of moral meaning and values provide a level of content to be added to the above list:

- Connection between neuroscience, biology and psychology (Hauser 2006);

- Moral instinct (Greenfield 2008, Lipton 2008, Doidge 2007);

- Authenticity (Macqueen 2009, Obama 2006);

- Ecological integrity and respect (Theobald, 1997);

- Interlinking of human learning with cognitive variation, personality and socio-cultural diversity in language acquisition (Brown 1980);

- Responsibilities of administration (Evers \& Lakomski 1991, 1996);

- Self-management (Caldwell \& Spinks 1986, 1992: Smyth 1993);

- Educational coherentism: Keeves \& Lakomski (1999) on the substance in values that is foundational to the concept of coherentism;

- Schooling the emotions (Goleman 1996, 1998b, 2007) and educating the emotions (Wilson, 1971);

- Emotional intelligence, emotional habits and emotional literacy (Wellham 2010, and Goleman 1996, 1998b, Nussbaum 2001, Zigler (1998);

- Power and authority, liberty and obligation (Raphael 1976, Giddens 1998, Rich 1972). 
Added to this set of inter-related concepts is the work of Strawson (1963, 1985) that highlights meaning in terms such as inconsistency, patterns of reasoning like deductive and inductive reasoning, assumptions of cultural meaning and attitudes of scepticism and resistance to the acceptance of reasonable conclusions. These were explored by Winch in his writings about Weil (Winch, 1989) and by Kenny (1973) as he battled through the writings of Wittgenstein. Wittgenstein (1972) wrote about the depth of meaning in life. Discovering Seligman’s (2011) profile of Wittgenstein brought me relief and understanding about Wittgenstein's reversal of thinking and philosophical content, allowing for deeper appreciation of the breadth of concepts underpinning teaching of thinking skills.

Particular attention can be given to the understanding of eschatological, theological and spiritual perspectives within the confines of these deliberations: Isaac Singer (1983) and Sacks (1997, 2000b; 2003) on a Jewish perspective; and the long list of religious thought displayed by Pelikan (1990), Tarnas (1991), Carroll (2004), Taylor (2007), Russell (2004) and the relevance identified by Pratt (2003) of teaching about before teaching in religious matters. Mews (2009) explores the respect needed for interreligious dialogue with reference to multiculturalism and its distinctive partnering concept -interculturalism: to highlight the implied reasoning in these eschatological, theological and spiritual perspectives. Also implied is the same respect for dialogues in secularism. Watt, (1976), Roth (2005b, 2005a) and Beattie (2009) deliver valuable directions and imperatives to inform such dialogues, so that reshaping of curriculum by linking applied thinking skills, neuroscience findings and the discovered insights, and the dawning understanding of behaviour patterns commonly within virtues and values, can be achieved.

Underpinning all these concepts is the existence of H. Gardner's (1993a) multi-intelligences, Edward de Bono’s (1996) thinking practices, Robert J. Marzano (2001, p.379-383) and the language of thinking, Fogarty (1993, 2001) and the brain-compatible classroom, and the emerging issues for 
educational pedagogy emerging from a neuro-scientific perspective (Greenfield, 2007). Dialectic and dialogical thinking (Paul, 2001) together with the globalism connotations (Saul, 2009) help the understanding of broad perspectives outlined by Splitter \& Sharp (1995), Fukuyama (1999), Klein (2007), Giddens (1998), Howe (2007) and Mackay (1993). The final element of consideration here is Kristjannsson (1998) and the concept of megalopsychia (the 'crown of virtues' according to Aristotle): self-respect as defined by him. Combined, these concepts and perspectives assist in preparing a program about values, virtues and ethics that is suitable for student discussion and exploration.

Virtues can be taught, so that students have knowledge of the internal concepts of possible life experience based on intention, reasons, connections and emotional response. It must not be forgotten that Virtues are first an internal response and a sifting and sorting process in which decisions about how to express self in various ways can be chosen (Wilson, 1987). Virtues as a concept emanates from a range of cultural distinctions, religious underpinnings, identifiable goods, political impulses, and philosophical frameworks through which an individual can express the best of human behaviours on a daily basis.

Anih (1992, 1995) placed emphasis on ecumenical tolerance in religious and political matters, including the element included by Lipman about the need for self-correction. The term used by Anih, 'fallibilistic flexibility', refers to the need for individual people to strengthen their motivation and adjustment for mutual co-operation, collaboration and plurality: the community aspect of living the good life, vital as a foundation for recognising the relevance of virtues and values.

As a means of distinguishing the fundamentalist thinking that focused on warlike behaviours, Sacks had previously written with a great welling-up of feeling in the virtue of Hope (Sacks, 2000a) which he based not only on covenant but also on contract. He called this the politics of responsibility. 
FIGURE 7.1 TWELVE CATEGORIES OF INFLUENCE

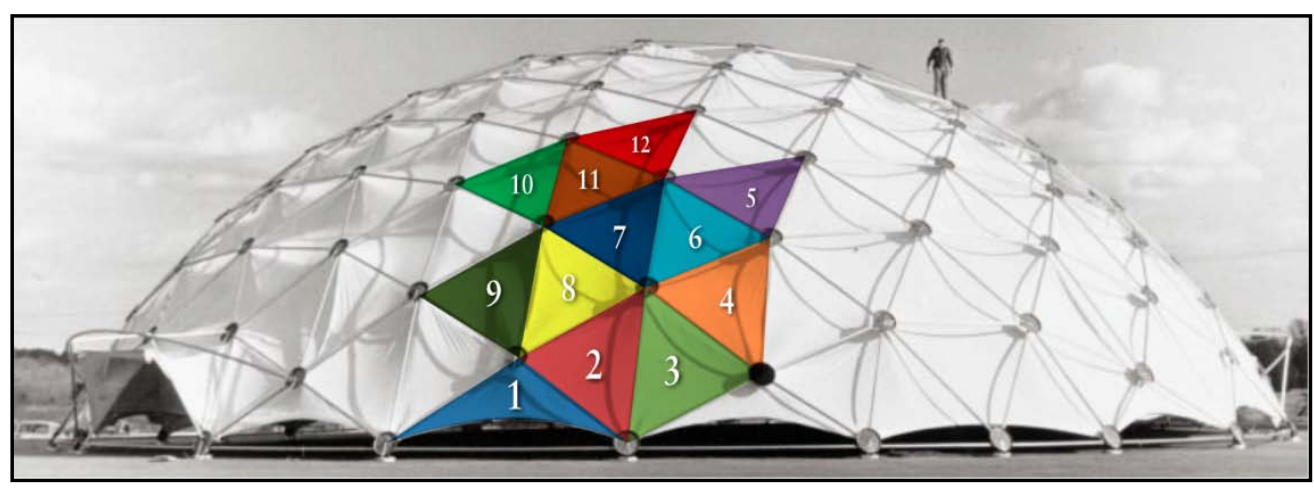

There was accurate theology behind the values and virtues that an individual can monitor and then contribute in neighbourhoods, communities, voluntary groups as well as religious communities. Sacks shows the interconnection between the internalisation that breeds virtues and the communal connections that promote values. There is a parallel here to Obama (2006, 2008). Kristjannsson (1998, p. 5) posits that Aristotle's indication is that self-respect was central to the balance in moral education. This consideration draws our attention again to Aristotle's crown of virtues called megalopsychia: self- respect.

The centrality of individual virtues and their relevant values provide a foundation that helps students in making decisions. The wealth of material represented above, now needs to be shaped and re-shaped in order to produce a manageable architecture for a virtues, values and ethics curriculum. It is my assertion that edu-tensegrity, through the Categories of Influence, will assist in this shaping and re-shaping. Others will find ways to test whether practice will mirror my theory and whether philosophy of education concepts have been adequately and accurately integrated into a refreshed focus on one area of twenty-first century education which seems to have been sometimes unaddressed.

Notice that, in Figure 7.1, there are coloured triangles to represent the twelve Categories of Influence. The other triangles represent all the available subjects taught within Schwab’s (1983) concept of the 'commonplaces' of 
the curriculum: inter-connection of the student as central, the teacher as role model and facilitator and director of learning, the topics and applied thinking skills of the subject area, and the milieu which takes firmly into account the physical, emotional, social, intellectual and spiritual elements of the student and the milieu in which learning takes place. In this consideration, we see the connection to the central column of the Heasly HUG/BUG shown in Chapter 4.

\section{Where do virtues and values fit in education?}

Virtues and Values can be identified, in research and by query, so that students are able to recognise, understand, evaluate for themselves the broad evidence base needed to decide on which of the community values that they will express from the underpinning cultures in which they live (see Wilson, Appendix C; Hawkes, 2010).

In this chapter, I apply the concept of edu-tensegrity to a consideration of virtues, values and education. This will involve the Categories of Influence list, as shown in Table 7.1. Virtues and values will only 'fit' in education, if they are applied - considering, analysing and inspecting the meanings accompanying the push and pull factors - using the tension and compression elements inherent in edu-tensegrity.

\section{Category of Influence 1: Dimensions of daily life}

The dimension of daily life that I consider under this category of influence is concerned with ontological approaches, which I have indicated addresses the internal dimension of daily life, the world where virtues are internalised; where intention, preference, and response-ability are found, prior to integrating and translating these impulses into Values and the epistemological dimension (Wilson, Vardy). 


\section{The ontological approach}

To make the most effective use of ontological perspectives, I define ontology, which is a branch of philosophy that deals with the nature of existence (University of Birmingham \& COBUILD, 2001) as encompassing personal perspectives, personal philosophies and daily experiences of any individual who will integrate these considerations with previous understandings, constructing a philosophical framework for daily life concerns, in particular following both Aristotle and Wilson. In different ways, both Aristotle and Wilson have explored the connections mentioned. The variety of thinking skills that build what is to count as a concept of virtues is displayed in Splitter \& Sharp (1995).

The ability to develop and teach thinking skills (Wilson, 1963; Lipman, 1993b), has become essential for effective and efficient teaching (Cherednichenko, 2000). Yet to teach the art of decision-making and the broad variety of reasoning and thinking, reason and logic support the need for deeper knowledge and understanding for all learning areas and for social competence (Dewey, 1944, p. 80).

This research, in its central purpose, investigates understandings and practices that explicitly focus on teaching thinking, particularly selfcorrection (Lipman, 1993b), exploring relationships between current learning, and personal decision-making, behaviours, attitudes, and preferences through ... 'the construct of continuous reconstruction of experience' (Dewey, 1944, p. 80; and Wilson, 1963).

Teachers' perceptions, opinions and attitudes have been critical in trying to understand how curriculum decisions are made. The research provides a foundation for exploring where connections to morality: virtues, values and human flourishing are found (Wilson, 1987; Skorupski, 1993; Costa, 2000; Costa \& Garmston, 2001). 


\section{Virtues as dimensions of daily life}

Various elements of the concepts of virtues are linked through the development of the broad arc of thinking skills that are taught systematically throughout secondary curriculum to students via various disciplines and classroom practices (Splitter \& Sharp, 1995). Virtues according to John Wilson’s (1987) approach are concepts needing teaching and understanding at a deep level, so that individual choices and decisions can be made accurately for appropriate use.

Careful definition of the nature of emotional intelligence highlighted empathy and how emotional intelligence may be applied. Goleman (1996) detailed relearning emotional literacy and understanding of one's own temperament. So integration of new knowledge from Splitter \& Sharp, Wilson and Goleman is needed at the level of the class or grade to be taught about virtues as dimensions of daily life.

\section{Values as dimensions of daily life}

It is important in the construction of this enterprise to appreciate the ordinariness of virtues and values which are foundational to life and to remember that both shape the quality of decisions that students make (Wilson (1987), Garmston (1999), M. Lewis (2010; 2011), and Riso (1992). Consideration of the concepts of individual values like fair play or respect, whether secular or religious, and community values like equal opportunity, tolerance and understanding of cultural diversity, which underpin daily cultural behaviour, are inspected, analysed and connected to thinking skills. The aim is to recognise that where virtues begin internally as concepts for living; values become the result of new knowledge, personally and in the community: based on thinking skills, applied systematically and coherently to the epistemological extension gained from the Heasly HUG/BUG concept.

In terms of the commonplaces of the curriculum (Schwab), this category provides the foundation of twenty-first century daily life; it impinges on the milieu in which the curriculum is delivered. 


\section{Category of Influence 2: Dimensions of thinking}

The underpinning influences that emerge from the various dimensions of thinking are widespread. They include the teachers' efforts ontologically to provide opportunities for deep learning and internal reflection. This is scaffolded by epistemological impulses that provide the student with the avenues to collect, integrate and internalise knowledge and understanding of various conceptual content, e.g., individual virtues and communal values. This will lead to the technical phronesis that means the knowledge and understanding is deep enough to provide evidence of learning by applied thinking skills; so that the student advances towards a personal body of knowledge and learning. Liberal education needs further re-shaping for effective and successful deep learning; edu-tensegrity addresses this consideration.

As the thesis map in Chapter 1 indicates, each category of influence contributes in its turn to the eventual outcome of deep learning for students, aided by accurate teacher delivery of the thinking framework and the systemic curriculum framework. The concept of edu-tensegrity requires that all Categories of Influence are maintained within the tension and compression identified in the geodesic dome (of education) to provide monitoring of balance and accuracy of aim and outcome.

The evolution of liberal education has resulted in some conflicting outcomes (Wilson, 1987) and educating children for good citizenship has resulted in contradictions in some communities between rights and responsibilities. Values-free, religious-free and neutral teaching strategies have served Victorian education by concentrating on the content of curriculum alone, but have left constraints upon teachers and students (Snook, 1972); Giddens, (Giddens, 1998; Hargreaves, 2009). While this kind of education brings knowledge of individual rights, it has begun the enterprise evolving into the beginnings of the stakeholder society (Giddens, 1998; Howe, 2007). 


\section{A culture of reflection and a culture of resilience}

Before beginning the exciting project of embedding a culture of reflection and a culture of resilience in the classroom (both are indeed separate, but opposite sides of the one coin rather than adversaries), it is a significant consideration to find ways for teachers to be clear about how and what their own preparation needs to be. Kincheloe (2005, p. 155) states succinctly: 'Teacher education provides little insight into the forces that shape identity and consciousness. Becoming educated, becoming a critical practitioner necessitates insight into the construction of selfhood and personal transformation'. My experience has taught me that the construction of selfhood is connected to a culture of reflection and that personal transformation is connected to a culture of resilience. That same experience has allowed me to bridge the gap, the divide, between the old paradigm and the new paradigm: devoting attention in Kincheloe's (2005, p. 158) terms, with post-formalism, which grapples with purpose, devoting attention to issues of human dignity, freedom, authority, and social responsibility.

Kincheloe when examining the scholar-teacher as auto/biographical researcher in post-formal inquiry continues:

\footnotetext{
In more constructivist and critical forms of inquiry researchers who do not understand themselves tend to misconstrue the pronouncements and feelings of others. ... In a sense, the objectivist tradition provides a shelter in which the self can hide from the deeply personal issues that permeate all socio-educational phenomena.
}

It becomes obvious that the subtle overlap of these two issues, the culture of reflection and the culture of resilience, is foundational to the development of teacher expertise, careful curriculum planning and accurate pedagogical strategies. Roger Sutcliffe in his Questionnaire responses and in his Personal and Social Philosophy (2009), described on the p4c website, has made clear that wisdom is part of the thinking skills practice. 
In Latin, SAPERE means to be wise. SAPERE also is an acronym for the Society for Advancing Philosophical Inquiry and Reflection in Education. Philosophy for Children (P4C) can enhance the teaching and learning in any curriculum area and can contribute to many school initiatives, from targeting under-achievement, improving literacy and providing for gifted and talented students - to developing thinking skills, emotional literacy, and independent learning (SAPERE website).

Another claim that Sutcliffe (2010) made in his personal correspondence, paralleling my considerations, is that Philosophy for Children has

been linked to higher achievement in National Curriculum tests at Key Stages 2 and 3 and GCSE, (UK), and has been shown to have positive effects on children's social and emotional development, particularly ... in faith and multi-faith groups, prisoners and young offenders, organisations addressing global citizenship, and local community groups.(SAPERE Information promotion-2010).

Tishman et al. (1995) state clearly and immediately that this is so! And they continue: 'The purpose of teaching thinking is to prepare students for a future of effective problem solving, thoughtful decision-making, and lifelong learning'. To establish with clarity the purpose and aim of a culture within each classroom, they highlight a significant concept of how we understand the establishment of a culture of thinking.

Their claim is precise; they list six dimensions of a culture of thinking:

1. A language of thinking

2. Thinking dispositions

3. Mental management

4. The strategic spirit

5. Higher order knowledge

6. Transfer

Enculturation includes that the teacher will be able to provide personal examples of modelling, explanation, interaction, and feedback 
Tishman et al. (1995, pp. 7-8) provide examples of making meaning through the development of the thinking culture which highlights the subtle differences between:

Laugh, giggle, chortle, chuckle, cackle, guffaw; guess, suppose, surmise, presume, assume, speculate.

In the building of usable language for the thinking classroom, terms made familiar for students include:

\footnotetext{
Alternative, think, believe, guess, conjecture, hypothesis, evidence, reasons, estimate, calculate, suspect, doubt, theorise, conclusion, opinion, point of view, interpret, dialogue.
}

A longer list of 124 verbs by Tishman et al. (1995, pp. 8-13) gives impetus to my emphasis on the need to teach students how to construct their own questions. Only then, can the process of establishing meaning by examining these examples of the language of thinking for active meaning- making through mental management be established for accurate transfer.

\section{Highlighting the culture of reflection}

If the education revolution, which currently is emerging in political spheres, is to have any grounding in reality given stark world-wide conditions, exploration of what a culture of reflection would include, needs to be introduced, grounded and further facilitated through today's best practice. Such an aim would lead to development of an emerging consciously focussed culture of resilience. What this framework will become in practical professional terms may not mean serious jettisoning of valued pedagogical features already in use, but may benefit from an insertion of different emphases as outlined in this investigation (Costa and Garmston, 2001 p 1822).

The emphasis is on the imperative to register what Australia and its education might actively need. This is necessary to recognise social changes 
since early twentieth century, and gives the higher profiles that seem to be signalling implications of good and evil (Lewis, 1940, 1946). Howe (2007) constructs a very compelling argument when he details sociological changes in the lives of individual Australians placing emphasis on meaning-making (which he derives from Plato's ideas) and the imperative for life-long learning and what that means. He outlines the risks of this new phase in social development of Australia. He believes in investing in people and building Australia's skills. This then gives an extra impetus to the needs of our students. Howe (2007, p. 17) states:

The aim of this book is to set out an approach to framing a values-driven social policy that responds to the fundamental changes both in our economy and our society. It is an urgent challenge. It is now widely accepted that the dynamism and fluidity of economic and personal relationships today require a different response from the wage-earners welfare state (Castles, 2001) which, many would argue, underpinned Australian social policy in the twentieth century.

These detailed changes in society command our attention and consideration. It is in responding to what Howe says that a particular imperative for teachers and mentors within the educational system as it stands, to jettison unwieldy ideologies and concentrate on the delivery of basic but not simplistic strategies, maintaining development of the highest integrity in education. Looking across the bodies of work produced by Wilson, Lipman, Vardy, Hargreaves, Beare and Crowther it is clear that they all testify to the connections between morality and virtues - we need to be accomplished and teach these concepts more universally (Wilson, 1987).

\section{Linking the culture of reflection and the culture of resilience}

The culture of reflection provides the basis of systematic observation needed. Howe is reflecting social policy as it operated in twentieth century Australia - it is just as imperative that care and consideration be given to what Richard Leonard calls the mystical gaze of the cinema as he reflects on and examines 
the films of Peter Weir (Leonard, 2009). Leonard leads us through careful research with elements of insights from Jung, film theory and theology, and provides a perspective that will inform teaching of concepts like justice, guilt, shame, moral virtue, punishment, moral rules (Skorupski, 1993).

Australians try to distinguish between religion and spirituality (Tacey 1995, 2003) as they delineate the apparent boundaries between theology and philosophy. At both ends of the spectrum, there are indications that a culture of reflection is a positive insertion (Seiger-Ehrenberg, 2001; Golding, 2006). In Tacey's (1995, p. 1-12) explorations, an examination and analysis of Australia at the end of the second millennium addresses the ecological crises, racism, violence, macho-cultural mores, alcohol and other abuses. The connection between Howe (2007), Leonard (2009) and Tacey (1995, 2003) does point towards a spiritual renewal in Tacey's opinion. He has allowed for reflection in a secular and a religious sense.

The implied channel for development of understanding spurs realisation that thinking skills of specific range and focus will be needed to successfully negotiate the scholarly study of film, novel, study of other media, and the fashioning of creative writing particularly at Year 12 and university level. This is so that simplistic responses are limited to facts and surface response as a first step in framing an individual response. Considering conceptual understandings (Kramer \& Alstad, 2009) and social change means that this response to the considerations of virtue, values, ethics, decision-making, and personal philosophy is defining the individual, and where it exists, theological considerations and eschatological speculations can address concepts like fear, freedom, evolution, toleration (Burwood \& Wyeth, 1998) by systematic reflection.

\section{Highlighting the culture of resilience}

The culture of reflection and connection to the culture of resilience become central for today's students as Howe (2007, p. 144) says that there is a strong connection between skills, economic security and individual opportunities: 
The importance of education as an investment in Australia's future no longer seems to be contestable. .... There are already strong signs of Australia's labour market dividing into two groups. One is those who are clearly part of the knowledge economy, who are rewarded with good pay and conditions, who are confident about planning their lives because they have a sense of security. However, another Australia is emerging. It is made up of those Barry Jones might call the information poor, who if they are able to work at all are increasingly working in part-time or casual jobs, who never really escape some dependence on social security benefits.

Such skills contribute to a just society and minimise discrimination and inequality, starting in secondary schooling.

Ethical thinking, based on the broad spectrum being delineated, (Fitzgerald 1993) needs to be taught specifically. Decision-making at the level of school leadership will be required so that students are surrounded by the modelling of strategic decisions that make sense for democratic purposes and for educational aims and objectives. Chittenden (2004) has given careful consideration to these requirements. So also Caldwell \& Spinks (1992) but their principles are challenged by Smyth and his colleagues(Smyth, 1993) and this means that teachers and administrators need to know and understand each side of these considerations. Joyce \& Weil (1986, p. 25, p. 317, p. 383) identify that concept development is important as underpinning for decisionmaking for students, but it follows that school leadership needs to demonstrate support and cohesion in the quality of decisions (p. 337). This applies when behaviour theory and mastery learning is central to learning about virtues and by extension, the internalising of values. We could call this experience the gaining of cultural intelligence.

\section{Connecting through and significance of the process of maieutic thinking}

Attention has been paid to the efforts of Socrates (de Bono 1996) to birth an effective process allowing a philosopher to reflect and plan how to make random thoughts more substantial and to find order in the thinking process. 
Socrates did this, by means of marshalling the thoughts around a given concept, and discussing all the elements of those thoughts in an ongoing and continuous communication for shared thinking, experience and conclusions. This he saw as learning. Paul (2001) gives a clear analysis and meaning to dialogical and dialectical thinking underpinning the Socratic Method with diagrams and descriptions. The Socratic Method has been described as that which induces a thinker to formulate concepts through dialectical or logical sequence of questions as outlined by Fisher $(1990,2003)$ later in this Chapter. The aim is to assist a person to become fully conscious of Constructs previously latent in the mind (Oxford English Dictionary).

Constant Mews in a personal communication (2008) advised that in 1885, W. H. Payne defined maieutics as the art of giving birth to ideas and that in 1981, J. Simon conceptualised a writer as being the midwife between the reader and the dictionary - a parallel to a person, using questions within a dialogue to assist in becoming fully conscious of significant constructs. Mews said:

Socrates employs the method, but never wrote it down. His pupil Plato uses it in his dialogues, making Socrates the central character, but remains fascinated by the concept of eternal aesthetic and moral truths. Plato's pupil, Aristotle, uses the method but puts forward his own resolution of questions relating to every sphere of knowledge, including systematising the principles of dialectic. In the renaissance, there was a rediscovery of the Platonic dialogues, a rediscovery of that mode of maieutics, as a pedagogical technique - although the tendency was to promote the concepts of Plato, rather than the method of Socrates - widely viewed as transforming philosophical tradition by his consistent application of the questioning technique not just to cosmological questions, but to ethical questions of how to live.

\section{The consequences of a reshaped philosophy}

The ontological element of this enterprise calls for careful, recognised, comprehensive philosophy and related concepts with specific teaching 
method in sequential processed steps, for the whole student cohort (preschool, primary, secondary, tertiary both academic and TAFE). My theory is a reshaping of much data and substance that already exists, but different emphases and uses for technical strategies and techniques will cause divergent outcomes in learning. Applied thinking skills strongly underpin this theory, hence the emerging new paradigm where teachers and students together pursue questions and quests with freedom from constraints.

The underlying epistemological matters rely on teachers' breadth of knowledge, the variety of professional skills, and the effectiveness of their decision-making processes. It is possible to merge my Findings, my Theory, and the consequent Practices into a new and reshaped theory of teaching and learning. Reference to the various models of teaching outlined by Joyce \& Weil (1986) highlights the broad spectrum of knowledge used by teachers already, so the reshaped practice emerges. My theory has fitted within Crowther's (2010) COSMIC C-B model of school capacity-building. My whole investigation led directly to the new and reshaped pedagogy and comes under the fourth of Crowther's six dynamics: micro-pedagogical deepening (Crowther et al., 2010). There are strong links and connections with Parallel Leadership (Crowther, 2010) which emerged out of the CONSTRUCTS project in Victoria, Australia. Some participants mentioned the CONSTRUCTS approach in their responses to the Questionnaire, though it was not shown by all teachers as part of their personal pedagogies. In developing the CONSTRUCTS process (Crowther, 2010), there were numbers of questions for teachers, administrative personnel and curriculum leaders (pp. 22 \& 23) of a school that highlight the role of questions in a similar format to that which has been emerging from the considerations of my Questionnaire.

Perceptions of teachers, who teach thinking skills, seem to be focused on such matters within their area of expertise, but it is possible to provide deeper emphases, effective and efficient strategies and techniques in curriculum planning, ontological streamlining, and epistemological connections. 


\section{Separating moral matters from religious belief}

An initial definition of thinking skills includes the use of the list of open and closed questions: How? When? Where? Why? What? Which? Who? What if? Part of separating moral matters from religious belief, as well as sifting and sorting through the various learned thinking skills mean personal plans and new knowledge can emerge, depending on understanding the connections and links between all facets of thinking and how such skills translate into life skills, as shown in Figure 7.2.

All questions accumulate a conglomerate picture used to test whether a proposed action is moral or not, good or evil, appropriate or not, significant or not, right or wrong with an individual explanation. Following these considerations, we develop elements of thinking skills about moral matters that broaden understanding of the whole spectrum of moral education - or if I use today’s terminology: Values Education.

\section{FIGURE 7.2 THINKING SKILLS AND PERSONAL GOALS}

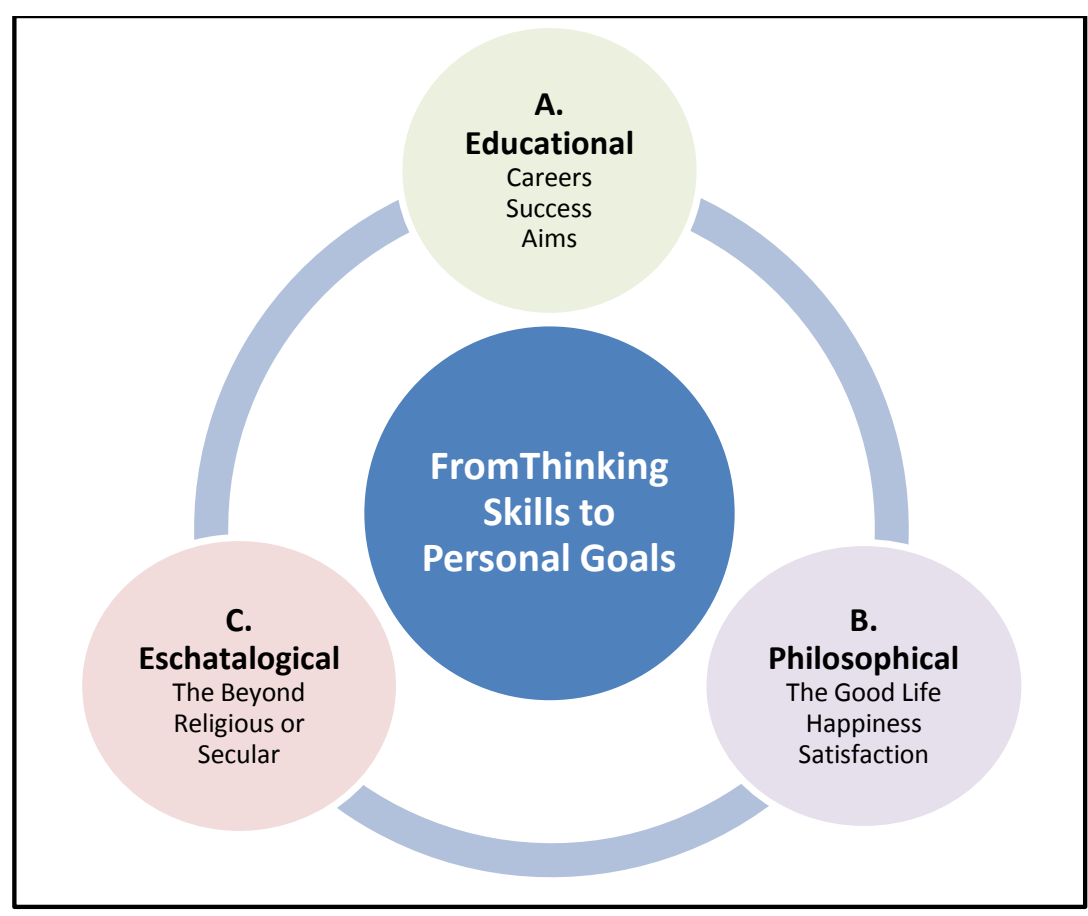


The circumstances surrounding the deliberately neutral starting point of Wilson (1967, 1990) has illuminated the subsequent development of curriculum, and the separation of moral education from the religious paradigms (Hopkins, 1978), (Singer, 1983), (Stockton, 1995), (Taylor, 2007) so visible in the 1940-60 era. Questions about whether thinking skills are also moral education when applied to content like that addressed by Freakley \& Burgh (2000) will provide some clarity.

\section{Levels of moralisation: A reshaped concept of moral sensitivity}

Moralisation as a process is being morally sensitive. New knowledge that allows for different understandings and differing emphases that seem to turn on a personality type provide for careful logic. Moral conviction is the strength of response to contentious phenomena. Moral attitudes connect to suffering, reciprocity and personality type. Each has an associated primary emotion (Riso 1992).

The Shared Praxis model of teaching that Moore (1986, see the most distinctive features in Appendix E) promoted, allows teachers to provide such a process. This process is also set out in detailed steps by Joyce \& Weil (1986) who focus on the quality of decision-making. Based on the principles of Habermas, Joyce \& Weil make links to: their Inductive Thinking model of teaching (p. 55); their Non-directive Teaching model (p. 141); their Model 10: Synectics, in which deliberate enhancement of creative thought is promoted (p. 159); and in their Model 11: Increasing Awareness, by expanding personal and social horizons (p. 184). There are echoes and connections here to Roger Sutcliffe's (2009) Personal and Social Perspectives. So the teaching about the existence of moralisation is broadened and deepened for accuracy in decision-making.

The concept is based on the degree to which a person cares about his or her own preferences about moral sensitivity. Differences in moral issues across cultures and political ideologies become fraught with danger. Concepts of compassion, powerlessness and meaninglessness (Singer, 1979; 
McNamara, 1983; Nouwen, 1983; Seligman, 1990; Sacks, 2000b, 2000a; de Botton, 2004) are crucial to opportunities in which the individual chooses a better level of moralisation after understanding all the indicators rather than a top-down set of precepts, to which reward and punishment is attached (Snook 1972).

The use of narrative helps to provide positive influence for the students without coercive action, engendering an experience of emotions that have a moral component like sympathy and empathy. If Greenfield's theorising about the way the brain functioning for learning is universally accepted, then there is a problem if sympathy and empathy is not taught explicitly. This is one indication that shows the need to name and teach concepts of virtues, values and ethics.

Open discussion about immorality and amorality, identifying heroes and villains, is needed to give substance to these concepts. Modelling of moral judgment and empathy; highlighting what is to count as unethical behaviour and evil motives, is involved in the teaching for democracy. Self correction, in Lipman's terms (and Anih's fallibilism), must be freely chosen rather the student being pressured. Deep learning invites a choice to abandon amorality in many decisions.

\section{Establishing open-mindedness, close-mindedness, bias, prejudice, personal opinion and point of view}

Moral sensitivity can be manifest in either narrow or broad personal perspectives. To allow thinking skills to produce a balanced approach to choices and decisions, it is significant that we examine the two ends of the spectrum: positive open-mindedness or negative close-mindedness.

It is therefore important to heed Fisher (1990, p. vii):

That the ... foundation for thinking skills needs to be laid early in life, for open-mindedness begins in the formative years when a child's identity as a thinking person is being established. As children become adults there is an increasing tendency to close-mindedness where beliefs are ego-centred 
(where what $I$ believe becomes more essential that what I believe) where those who disagree are regarded as biased and as not having the capacity to enter into a reasoned and open-ended discussion.

Until recently, the research in Australia often found that students struggle to think critically and creatively within the typically confined secondary school structures (Leckey, 2009), (Wilks, 1995). It is a significant consideration to include research about the various desires and needs that are part of the Western cultural patterns (Mackay, 2010) to develop an accurate overview of moral sensitivity whether from religious or secular origins. I have tried to address this concern in the Heasly HUG/BUG (Chapter 4).

\section{Teachers and moralisation}

It is important to use thinking skills and research sources to recognise and develop the elements of moral education. These levels of moralisation use deductive and inductive thinking patterns in accurate understanding of moral matters -that which I have called values education. There is a need for response to the following complicated points:

- Discussion opportunities in which the individual chooses, as distinct from imposition, a better level of moralisation after understanding all the indicators -rather than a top-down only set of precepts, to which reward and punishment is attached (Community of Inquiry -Lipman format).

- The use of narrative that helps positive influence on the students without coercive action but engendering moral emotions like sympathy and empathy enough to improve the lot of a victim. If Greenfield's theory is accepted, then there is a problem if sympathy and empathy is not taught explicitly - (Lipman's novelettes and the morality tale of Saxby and Gross (1999).

- Open discussion about amorality and immorality, identifying heroes and villains, is needed to give deeper substance to these concepts - (Vardy's RAVE curriculum and format). 
- Teacher modelling of moral judgment or empathy highlighting what counts as unethical behaviour or evil motives needs attention- (Wilson's Moral Components List).

- Self correction, in Lipman's terms, must be freely chosen rather than students being pressured, even though there may be minority positions in a class response. This is especially important in conflict resolution processes. Sharing feelings and perceptions also helps to reach the final understanding and the students' eventual choices - (see Moore's Praxis model of teaching in Appendix E).

In terms of Schwab's commonplaces of the curriculum, this category provides the foundation of twenty-first century dimensions of thinking; it impinges on the milieu in which the curriculum is delivered.

\section{Category of Influence 3: Dimensions of education}

Dimensions of education as a category of influence refer to philosophy, psychology and sociology of education. Each discipline is intimately connected to the understanding of virtues and values. Fogarty (2001, p. 145) refers to 'Architects of the Intellect' who 'can sustain pedagogical diversity'. In education, these 'architects', reasonably referred to as master architects, have provided effective channels promoting integration of thinking skills and intellectual progress; the theories that each has constructed are listed in Figure 7.3.

It is significant that teachers endeavour to connect seamlessly with my final conclusions, in which the architecture of this whole investigation leads towards a reshaped theory of education. Respondents to the Questionnaire gave specific details of their personal pedagogy, shown in Chapter 4. 
FIGURE 7.3 ARCHITECTS AND THEIR THEORIES

\begin{tabular}{|l|l|}
\hline MASTER ARCHITECT & \multicolumn{1}{c|}{ THEORY } \\
\hline Dewey & Experiential Learning \\
\hline Montessori & Discovery Learning \\
\hline Piaget & Constructed Learning \\
\hline Vygotsky & Social Interactions \\
\hline Feuerstein & Cognitive Modifiability \\
\hline Costa & Habits of Mind \\
\hline Gardner & Multiple Intelligences \\
\hline Sternberg & $\begin{array}{l}\text { Successful Intelligence } \\
\text { Learnable Intelligence }\end{array}$ \\
\hline Perkins & Enriched Environments \\
\hline Diamond & Computational Theory of Mind \\
\hline Pinker & Emotional Intelligence \\
\hline Goleman & Moral Intelligence \\
\hline Coles &
\end{tabular}

Current historical emphases, I discover, have solidified into a quest for educational, ecumenical and inter-faith communities to have tools for higherorder thinking in three specific areas for the students:

- Decision-making and consequent action for construction of a career path;

- Decision-making and consequent actions for construction of a philosophy of life-quality of life;

- Decision-making and consequent actions for meta-questions regarding the meaning in life; the 'beyond' and relevant questions -in religious and /or in secular terms.

\section{Uncovering detail in dimensions of education}

My Questionnaire was designed to explore the broad arc of dimensions of education and to discover what teachers' perceptions of teaching thinking skills contained. Questions were constructed from a problem-centred starting-point (Flick, 2002). Eight of the nine specific themes picked up in 
the Findings Chapter (4) refer in detail to technical strategies and techniques already in classroom practice. The last section about hesitations, confusions, concerns, and silences comes out of this evidence and implies the presence of ontological and epistemological practices. The Questionnaire title points directly to existing pedagogical practice. Ontological and epistemological matters are gleaned later as the analysis of responses deepened.

Questions in the Questionnaire were placed in an order which was designed to flow like a verbal interview in order to help facilitate the participants' thinking for efficient recall in a minimum of time (Flick, 2002, p. 102-103). Research was planned methodically, was based on educational principles and reflection, and followed the criteria for successful research shown by Flick (2002, p. 275). Given the breadth of pressures on teachers in the classroom, in collegial interactions for planning, and in curricular delivery and professional development, the critical opportunity for teachers to have a voice, which was authentic to their experience, was chosen as the starting point.

In terms of the commonplaces of the curriculum, this category too provides the foundation of twenty-first century dimensions of education; it impinges on the milieu in which the curriculum is delivered.

\section{Category of Influence 4: Curriculum theorists and shaping the curriculum}

The three curriculum theorists that have impact within this thesis are John Wilson, Matthew Lipman and Peter Vardy. Shaping the curriculum through their contributions promotes deep learning and aims to teach the student rather than just delivering the curriculum content itself.

\section{Wilson}

Wilson explored the philosophical theory, unusual for that time, that moral education was not necessarily a religious matter. His body of published work 
began with introducing the analytical theory approach to the understanding and teaching of concepts. He saw the links between concepts and virtues. He recognised the signs of the times that change may include dismissal or avoidance of familiar religious and cultural mores. By publishing careful explorations of what was to count as being moral, or in today's language: understanding values and acting on them, he taught and modelled his philosophy of education in his writing. The links and connections between cultural or sociological behaviours, psychological responses and analytical development of an inner sense that constituted morality and moral education in a broader sense he said just followed the precepts of English Christianity. One outcome was the construction of a list of virtues to which he gave nonsensical names. His aim was to ask us to inspect, analyse, exemplify and deeply understand these virtues and choose whether to practise such virtues personally. By reason and logical sequence, he led the reader through subtle and detailed considerations of what constituted a virtue. His own teaching experience led him to use the instructional mode in the classroom and the lecture theatre, with the result that he did not extend these theories into complete curriculum units, familiar to today’s specialists.

His list of moral components rested on a focus on attitude, with regard to respect for others and their wellbeing as well as a respect for self (a kind of philosophy); with a focus on ability and awareness of the presence of emotions (a kind of empathy); with a focus on attainment in many respects: of relevant knowledge (knowing that), of understanding (knowing how and when, where, why ...), attainment as application in order to develop insight and accuracy, of taking responsibility where necessary, of accurate translation of all this into action (decisions).

\section{Lipman}

Lipman, however, chose to produce a format which began at the point where children could ask many questions about any contentious matter, and this would be followed by strategies that taught the students many thinking skills 
as they experienced the deep learning about the same questions that led Wilson to publish his own considerations.

If teachers are familiar with the role and function of questions, it will lead to a deeper appreciation of the achievement of mastering and using the broad spectrum of thinking skills (Cherednichenko \& Wilks, 1997; Cherednichenko, 2000). The invaluable work done within the Philosophy for Children movement of Lipman and his colleagues over half a century extends this exploration.

Two of the three practitioner-experts who responded to the Questionnaire did recommend the acquisition of the expertise practised in the community of inquiry which is the essential central kernel of the Philosophy for Children pedagogy.

\section{Vardy}

My experience of the work of Vardy for the studies in Philosophy and Religious Units at years 11 and 12, lead me to recognise the connections I see from Wilson, to Lipman, to Vardy. Vardy (from 1998 to the present) has produced the content and pedagogical breadth that was indicated for eventual development of thinking about moral education by Wilson, and the classroom experience of the Community of Inquiry that led Lipman to engage students as thinking learners who proceeded at times in dependency, but increasingly were independent thinkers, always allowing for the times when interdependence was addressed by team work. The final outcome here is the confident use of interdependency as the third channel for deep and rich learning, implied by Vardy's writing for students, and his professional development materials for teachers.

We can include in the curriculum the teaching about concepts leading to a compilation of a Moral Components List equal or parallel to that of Wilson: through historical research, through relevant knowledge, and through Lipman's Community of Inquiry processes -for successful decision-making expertise for all students. 
We can integrate ontological concerns, epistemological matters and technical pedagogical strategies and procedures set out in the Vardy's Religious and Values Education (RAVE) programs through the agency of DialogueAustraliaNetwork (DAN) for further democratic human flourishing and thriving.

Interestingly, each category of influence can be linked with emerging new knowledge from neuro-science sources showing how the brain works. Each can be linked with the concepts of virtues and values, because the definitions given previously refer to internalisation of understanding for virtues, and application in personal behavioural terms in community to understand the concept of values - that which is important and beneficial and seen to be so by a large number of those within the educational and social community.

In terms of the commonplaces of the curriculum, this category provides the foundation of twenty-first century thinking provided by curriculum theorists; it impinges on the teachers who are delivering the curriculum.

\section{Category of Influence 5: Constructs about knowledge and the thinking curriculum}

The influence that these three theorists, Wilson, Lipman and Vardy, have provided is due to the particular constructs that they have used to shape the practical application of reasoning, in order to find meaning, and contributing to the meaningfulness of the decisions that will be made as students travel through the education process.

As a result of the detail given by respondents, we have accurate themes listed, that show teachers' perceptions about the teaching of thinking skills. The conclusions from the participants of the Questionnaire provide important issues and varied responses that need inspection, analysis, acceptance and action. In theory, the embedded-ness of such thinking skills would pervade all manner of daily experience in subject areas, life skills, in pastoral care 
initiatives without dismissing the balance that humour and entertainment provide. Thinking skills impact on:

- Decision-making and choices.

- Relationships

- Construction of a career

- Development of quality of life - the good life

- Possibility of choices made for either a religious or secular approach to the construction of meaning in life.

\section{The cycle of inquiry}

From the evidence of Questionnaire respondents, I found that it is clear that there is a cycle of inquiry with separate elements contributing to the role, function and usefulness of questions in discussion. There is a vital central relevance to the quest for understanding the efficacy of questions and questioning.

I have represented in Figure 7.4 a process of the cycle of inquiry. At each stage in this process, it is important to recognise that no specifically lineal thinking is expected. Each stage has a variety of applications and lateral thinking is used so that wide ranges of relevant issues can be examined and integrated. Teachers know the accepted channels stimulating students' ability to process content through kinaesthetic, auditory and visual channels of learning. The brain is wired so that two of the three channels are used more than the third one. The first assumptions of respondents include that it is necessary to use different stimuli and activities to facilitate an effective, efficient and ethical atmosphere in which the students feel safe to explore all possibilities and ask questions as are needed. 
FIGURE 7.4 HEASLY CYCLE OF INQUIRY

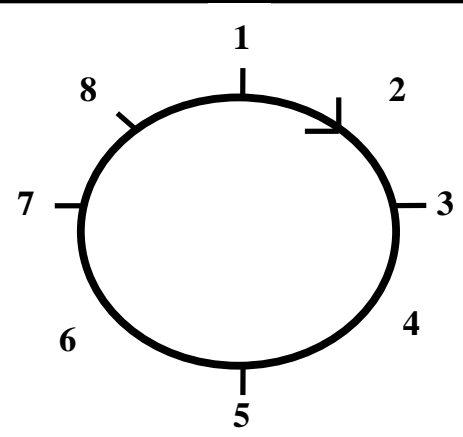

KEY:

1. Myriad styles of Stimuli

2. Connections between stimulus questions and emerging topic questions

3. Emerging questions

4. Connections between the emerging questions and students thinking

5. Students thinking

6. Connections between students' thinking and actual class topic discussion

7. Class topic discussion.

8. Discussion feedback emanating from all sources within the class topic discussion leading to further stimulus.

\section{Things that get in the way of teaching thinking}

It is important to be realistic so that we listen to teachers' voices accurately. Change happens in disrupted periods and in cyclical patterns. The disruption and patterns of interruption that I experienced contain such spasmodic experiences that include the following:

- time constraints;

- $\quad$ special one-off programs;

- intrusion of special attendance for all-school assemblies: speakers, fire drill ;

- pilot programs;

- changes in daily time-tables to accommodate extra-curricular school projects like a mini-school concert, or special effort for social justice appeals; 
- lack of details seen to be forthcoming regarding the disruptions that cause difficulties in engaging students in ongoing education about how to use thinking skills, which are always based on the effective use of questioning.

- remembering that this question was not asked in the Questionnaire and no relevant comment seems to have surfaced.

FIGURE 7.5 WASSERMANN'S FIVE ES MODEL

\begin{tabular}{|c|l|}
\hline THE 5Es & \multicolumn{1}{c|}{ THEIR IMPORT } \\
\hline Engage & Create interest, stimulate curiosity \\
\hline Explore & Inquire into student questions, test their Constructs \\
\hline Explain & Interpret the evidence, construct possible answers \\
\hline Elaborate & Test knowledge in a new situation, check accuracy of new knowledge \\
\hline Evaluate & $\begin{array}{l}\text { Review and reflect, apply new conclusions to existing knowledge, beliefs, and } \\
\text { skills. }\end{array}$ \\
\hline
\end{tabular}

\section{Useful approaches: the role and function of questions}

Wassermann's grid, shown in Figure 7.5, identifying the kinds of questions and their intent, has become a vital skill for directing students' interest and for enhancing rich learning. It is an opportunity to introduce and teach about inductive thinking, as it is possible to start at any of the five and build a deeper meaning and understanding, as the answers and indications emerge from the considerations and study engaging the students. According to Wassermann (1993) there are unproductive questions and productive questions that have been used by teachers. Though she detailed ten of each category, it is the distinction that matters, and the productive questions that warrant inspection. Some questions are unproductive and require dismissal from the armoury of questions that teachers used to stimulate students' thinking. However, productive questions are focused on the development of student thinking and reasoning and appear in the list shown in Figure 7.6. 
FIGURE 7.6 A PICTURE OF TEACHER - SCAFFOLDING OF QUESTIONS

\begin{tabular}{|c|c|}
\hline TYPE & DETAIL: Occurs when \\
\hline More generatively based questions & $\begin{array}{l}\text { - the teacher prompts the students to compare, observe, } \\
\text { classify, hypothesise, interpret, interpret data, } \\
\text { evaluate, decide, create, apply principles to new data }\end{array}$ \\
\hline Clearly stated question & $\begin{array}{l}\text { - the teacher clarifies through precise language exactly } \\
\text { what is being asked }\end{array}$ \\
\hline Accurately focused questions & $\begin{array}{l}\text { - the teacher distinguishes between Constructs, issues } \\
\text { and concepts, making sure exactly which is being } \\
\text { examined }\end{array}$ \\
\hline $\begin{array}{l}\text { Inviting questions, rather than } \\
\text { intimidating questions }\end{array}$ & $\begin{array}{l}\text { - the teacher moderates tone of voice, expression and } \\
\text { focus so that students are engaged in exploring their } \\
\text { response }\end{array}$ \\
\hline $\begin{array}{l}\text { Questions that are positive but } \\
\text { demanding in tone and expectation }\end{array}$ & $\begin{array}{l}\text { - students are able to explore, investigate, identify } \\
\text { ambiguity and uncertainty, making exploratory } \\
\text { choices prior to finalising the learning episode }\end{array}$ \\
\hline Respectful questions & $\begin{array}{l}\text { - the teacher uses a respectful tone to underline the } \\
\text { value of engendering trust between teacher and } \\
\text { student in a partnership of inquiry }\end{array}$ \\
\hline $\begin{array}{l}\text { Questions that go beyond recall and } \\
\text { recapitulation into areas in which } \\
\text { they can demonstrate thinking skills }\end{array}$ & $\begin{array}{l}\text { - the teacher shows that it is expected that students can } \\
\text { show new meanings and understandings about the } \\
\text { topic }\end{array}$ \\
\hline $\begin{array}{l}\text { The challenge to make why questions } \\
\text { invitational rather than inquisitorial }\end{array}$ & $\begin{array}{l}\text { - the teacher can rephrase a question that allows } \\
\text { students to go beyond being defensive about their } \\
\text { views into exploratory or self-correcting domains }\end{array}$ \\
\hline Diplomatically phrased questions & $\begin{array}{l}\text { - a student is helped to recognise the need for self- } \\
\text { correction or clarification of a seeming contradiction }\end{array}$ \\
\hline Creative questions & $\begin{array}{l}\text { - the teacher feels free to invite the student to articulate } \\
\text { all options even what at first seems like wild } \\
\text { Constructs }\end{array}$ \\
\hline
\end{tabular}

\section{Role and function of questions: teachers' perceptions}

The perceptions of respondents in the Questionnaire seemed to indicate that questions had a simple role only in the teaching-learning process. Perhaps they were useful as convenient ways of bringing a student back on task, or advancing the understanding of information at a given point in the lesson. It was useful also to distinguish between the role and the function of questions.

According to Fisher (1990) the questions have multiple functions. They need to provide:

- A direct opening for student stimulation to recall previous content before proceeding with new information. 
- An opportunity for both teacher recall and student reflection on content in the process of learning to that point.

- A valuable chance for careful clarification to avoid confusion and contradiction.

- The requirement for students to learn what is involved in overview and review of material when involved in personal or group work.

\section{Teachers' responses}

Structured strategies underpin the curriculum content, embedded in the language of questions and questioning, following the process of teacherlearning. Particularly in the UK teaching milieu, there exists a recognised teaching strategy to have a series of introductory 'starters' activities followed by main activities as Frank stated: he had a distinct framework for the teaching of philosophy in education. He described how thinking skills were taught in starters and main activities and he introduced these thinking skills during the starters. Stimulus strategies included use of video; anecdotes; questions in all their forms; written exploratory work.

Paul expected students to evaluate, to make abstractions, to use generalisations and to associate. It appeared to imply that he wished students to associate in as many ways as possible from the stimulus and from discussions which were also used. Susan expected her students to 'investigate/ design/ produce/ evaluate their work' as they progressed in Technology Design. George expected students to keep an open mind, use different perspectives and allow for 'shades of grey'.

These were opportunities to measure the outcomes of learning which were tested in various ways to help to further embed and integrate the new content, so teachers' assumptions were based on this complex scenario as a channel to maximise the efficacy and effectiveness of the teaching-learning process. This embedded-ness was an underlying stimulus organised by the teacher, leading to activities which gave opportunities for the students to 
explore, investigate, question, develop hypotheses, evaluate and eventually internalise the emerging knowledge. This was shown in teachers' responses:

- Dennis indicated that it was a clear and recognised experience for the students that material was introduced, familiarising them with the implications, and this was done by extensive discussion.

- Connie described her process as a cyclical or spherical set of steps: the question is put on the board, pupils contribute. There was opportunity for teacher prompting; there were also chances for pupils' questions to what she called 'trigger' other pupils to respond. Connie made it clear that she used question and answer; discussion and brain gym.

- Rose showed an extensive list of stimulus for introductory and then main activities: Venn diagrams/concept mapping; debates; fish bowl group work; argumentative essays; POE (predict/observe/explain); open-ended questions; continuums; word posters; deep thinking challenges.

- Lavenda gave an interesting step-by-step process: Open questions; processing information; co-operative learning (groups and pairs); reviewing and applied reality testing.

In terms of the commonplaces of the curriculum, this category provides the foundation of twenty-first century thinking that affects constructs about knowledge and the thinking curriculum; it impinges on the teachers who are delivering the curriculum.

\section{Category of Influence 6: Historical and philosophical theorists}

Three significant historical and philosophical theorists have contributed a particular focus to the construction of new knowledge that leads us towards the theory of edu-tensegrity. They are Aristotle, Dewey and Schwab. 
Ostwald (1962), the translator of Aristotle's tome Nichomachean Ethics has given us a careful overview of lecture notes believed to be from the sage himself. His ethical system as Aristotle called it has three categories of scientific knowledge: metaphysics; physics; mathematics. Aristotle does not use the twenty-first century term: values.

Aristotle saw:

a) Metaphysics as being what today might be called theology or being or reality; he meant by

b) Physics the notion of motion, growth and decay; he included in

c) Mathematics such concepts as number, shape, size, angles, connections.

Using these three categories of distinction, Aristotle developed a system whereby he could promote pursuit of reason for the study of truth as truth, and thereby improve the understanding of the workings of reality. He was able to recognise the relevance of virtue, moral imperatives and consequent ethical behaviour. According to Wilson, Aristotle's philosophy, or point of view, was a consistent picture based on consequences for humankind of the power of speech and reasoning:

\footnotetext{
Aristotle and Plato before him - believed that acting like a human being is tantamount to acting like a rational human being, and acting like a rational human being is the same thing as acting like a good human being.
}

Aristotle proceeded to examine human problems with the aim of finding lucid principles, and the consequent careful rigour was that which Wilson applied. Aristotle explored choice, what made something moral, what was included in the good life, what was ethical behaviour and how all this could make a person happy. Wilson applied these Aristotelian principles in order to develop the secular approach to today's efforts to explore reality; to achieve the good life. Wilson explored the notions of virtue or the philosophy of choosing to be the best person possible. This was not far removed from today's thinking about virtues, values and ethics and can be linked with the beginning of the system of secular thinking today. 
Dewey (1944) was concerned to make sure that education was a progressive experience that promoted the knowledge leading to the gathering of information in which concepts like virtues and today's term: values, could be safely and accurately understood. He emphasised the quality of education as the seat of the process to achieve this aim.

While Schwab did not talk in terms of virtues or the related values, he has outlined the equivalent of tensegrity or push-pull factors in which students, teachers, the curriculum content and the classroom must be sometimes equal and sometimes not. Schwab (1983, p. 48) points out that:

There are times and places when the welfare of the state is of paramount importance (during threat of war for example) and what contributes most to the future happiness of individual students, or justice to a subjectmatter, or the bents of teachers, must take subordinated positions.

Schwab's careful emphasis on the reasoned balance between the four commonplaces alerts us to the continued moral imperative of reflection, monitoring, creative planning and realistic outcomes. They are in themselves a form of values and knowledge from which the various strategies and techniques considered significant can be produced in the classroom itself.

In terms of the commonplaces of the curriculum, this category provides practical applications obtained from historical and philosophical theorists; it impinges on the teachers who are delivering the curriculum.

\section{Category of Influence 7: Meaning, reasoning and choice - blending and synthesising reflection and resilience}

Much time for reflection is part of the underpinning that we need to develop our constructs about meaning, to recognise and become efficient in reasoning, and to find the patterns of logic that allow us to make systematic choices efficiently and appropriately. To reflect, as I define it, means to register something new and exciting, (or its opposite), outside of current 
personal experience, that seemingly invites participation from oneself. To reflect means to recognise the inner impetus to think accurately about meaning; after explanation; after learning; after thinking about the content and its consequences; after testing for authenticity; after evaluating validity; after identifying possible spin or unbalanced presentation of that something new.

The model of teaching that is mirrored here is that of Shared Praxis (see Moore in Appendix E). This process parallels the process which I identified; it became the stepping-off point in the Heasly Six Stages of Decisionmaking. Teachers give evidence of understanding this impetus in all educational scenarios, but their perceptions of how they teach their students the art of efficient thinking skills within a democratic context is diverse. Tishman et al. (1995), in discussing the contributions of Joyce \& Weil (1986) Splitter \& Sharp (1995), Schwab (1983), Crowther (1996; 2010; 2010), and Hargreaves (2003, 2009), helped me to recognise the depth of enculturation needed from teachers to develop the idea of a defined culture of thinking.

Therefore, to engender a culture of reflection on a consistent basis, requires that a balance must be struck between history of yesteryear and today; between the experience of another and that of oneself; inserting a recognition of the part played by consultation, expertise, advice and advisement. To produce the best practice of a culture of reflection, emphasis is placed on the value of research and on applied thinking skills, and to the unending use of computer-based information available. This is best exemplified by Joyce \& Weil (1986, p. 463). They place models of teaching into four separate categories which have direct influence on the engendering of a culture of reflection within the classroom.

It is important to realise that students will have certain difficulties becoming familiar with the requirements they are expected to understand but they emerge with understandings ontologically that benefit their thinking both inductively and deductively and which merge into epistemological channels of knowledge gained and understandings substantiated. 
Time spent in classroom teaching-learning processes in which the culture of reflection is encouraged and consistently practised, is to allow the valuable asset of time to be available for the introduction of all the thinking skills, whether by discrete subject frameworks or by the inclusion of such thinking skills within the subject. The list that appears in Splitter \& Sharp (1995) is a good starting point because the Philosophy for Children movement has given half a century to the development of broad thinking skills as a contribution to best practice in education.

\section{Linking perceptions of theorists and experts in curricula terms}

The perceptions of teachers will be ready for change towards best practice in education (Splitter \& Sharp, 1995). Schwab’s (1983) contributing reflections on this process reveals a likely pitfall that teachers will appreciate, because he believes balance is achieved when curricular specialists are grounded in close contact with classroom conditions, with teachers, and with the existing curriculum.

Using a number of historical approaches available (Beare et al., 1989; Hargreaves, 2003; Hargreaves, 2009; Beare, 2010) has translated into a paradigm allowing for the sustainable change and capacity-building detailed in Crowther's (2010; 2010) terms by inspection of constructs about meaning, reasoning and choice. Important sign posts have become available enabling students to identify depth and richness in learning, to recognise the experience of democracy in the classroom, and to choose in each moment, whether and when dependence, independence or interdependence is the most significant and useful method of proceeding with their education.

Hargreaves (2009) has an overview of three waves of educational history beginning in 1970s showing the weaknesses of each framework. He constructs a fourth way, a Hargreaves' framework that accounted for all features, negative and positive. His conclusion is succinct.

His Fourth Way reform includes: 
- Less bureaucracy and more democracy;

- More collaboration and less competition;

- More innovation and inspiration, less data-driven intervention;

- Less fear factor and more peer factor as the instigator of school reform;

- Includes the individual innovation and inspiration of the 70s;

- Adds also the quest for community and common cause of the 80s;

- Builds on the greater sense of urgency in the 90s;

- Does not exclude the increased reliance but not dependence on data and evidence of this century.

And Hargreaves (2009, p. 32) adds:

\begin{abstract}
Our better future lies in a hopeful vision of a more just world that we can create together, rather than a swift and superficial one that will distract us, or a greedy and fearful one that will control and eventually destroy us. This is our fragile world. This can be its future. Now is our moment of choice.
\end{abstract}

Our understandings about meaning, reasoning and choice, rest precisely on the discovery of new knowledge, and the application of such knowledge. Built on the ontological exploration and questions that precede this point, the new knowledge is also interrogated to uncover the consequences of such matters, consequently the applied thinking itself leads to new skills. The discovery of links and connections takes the place of assumptions and unproved possibilities. Category of Influence 7, meaning, reasoning and choice, connects these Crowther Constructs, and shapes the thinking curriculum for twenty-first century thinking.

\title{
Some historical links between values-based schools and meaning, reasoning and choice
}

I have known that there are values-based schools in Australia, dotted throughout all the States and Territories. Attention to the sweep of Australian educational history will support how teachers delivered content for student 
discussion, for recognition of contentious issues and for eventual understanding and rich learning. Making this practice universal in Australian education is one facet of this reshaping exercise and would be achievable in time because of the paradigm change being indicated.

In Victoria, Australia, education in my experience was encouraged to have values-based mission statements despite an Educational Act of 19th century Australia which legislated against school use of religious studies and beliefs of any given Christian denomination or other religion. We began from this 19th century policy to avoid the basing of Education on any particular religious schema, and to distinguish between Church and State. I grew up trying to make sense of the confusing overlaps which seemed to be a heritage of the nations from which Australia sprang. The role of teacher was to transmit basic information and knowledge, usually in instructional mode even of religious matters.

Then in the mid-twentieth century, the ethos of Australian schools was directed towards an inclusive academic standard. Curriculum planning fed the system of schooling in Australia which encompassed government schools, independent schools and a separate, systemic Catholic schools network within the independent sector. This situation was further influenced by a government policy linking the construct of a values-free or valuesneutral educational classroom. The result, among other considerations, was a broadening of opportunity for all students. The role of the teacher was to open ways of viewing the world to students, but without the intrusion of religious or secular values.

Now, there is a recognition that religious and secular values are present, whether overt or covert, in classroom work. Students can learn through the investigation and experience of such values being expressed in educational content. To accomplish this aim, we can find various curriculum frameworks that teach thinking skills, as one vital channel for students to holistically experience and choose how they shape their lives. Consequently, teachers and students could, more specifically, learn about the diversity of values and 
the reasoning underpinning such values, regardless of all personal commitments.

There is value to be gained in having conscious classroom experience in the systematic construction of questions, and how to then begin the process of finding options, of the prioritisation of those options, the checking of each option for possible use, and resolution upon the most likely and best final choices for action.

\section{Extending the links between thinking skills and decision-making}

My project has been aimed at engendering firm, reasoned, culturally authentic thinking skills in physical, emotional, social, intellectual and spiritual (religious or secular) matters, for individual students in a democratic classroom milieu. Pertinent emphases in development of consciously understood emotional intelligence rests on how teachers and students construct (that is: think about, understand and synthesise) their content for virtues, values and ethical behaviours. One example of such construction has been undertaken in a Brisbane boys' school with remarkable results. Wellham (2010) mentions four emotional intelligence categories in his research:

- Emotional Recognition and Expression (of oneself).

- Understanding Others' Emotions.

- Emotions Direct Cognition.

- Emotional Management and Control.

Teachers will find these elements of the research valuable to their own professional development, especially if they have been immersed in the process of teaching the curriculum content only.

In terms of the commonplaces of the curriculum, this category provides the foundation of meaning, reasoning and choice; it impinges on the students 
who are engaged in the learning and the teachers who are delivering the curriculum.

\section{Category of Influence 8: Teachers and teaching}

Central to this Category of Influence is how teachers view their own delivery and progress as they pursue a range of goals. Philosophical goals and eschatological goals within the curriculum content shape the practice of pedagogical excellence by informing the technical strategies and techniques necessary to achieve educational goals.

Teachers' perceptions of how they teach thinking skills need continuous consistent inspection and support in order to promote confidence and expertise, through the acquisition of a consciousness of their abilities to monitor and develop the breadth of thinking skills. It is not necessary to promote a framework where teachers may have personal experience of only a particular theological, ethical, philosophical or teaching focus. Teachers mostly teach successfully about political and religious beliefs without compromising their personal stances.

By reference to the tables in my Chapter 4, I find that the perceptions of teachers, in responses to the Questionnaire, span all points of view on a given spectrum in contrasting directions:

- some who are not including such matters in their teaching portfolio favour concentration on curriculum content exclusively, and

- some who are highlighting the need for such matters as teaching the student through the medium of the topics and subject studies, try to use teaching thinking skills as part of the educational curriculum.

My constructed grid integrating these five elements: physical, emotional, social, intellectual and spiritual elements of the individual (Heasly HUG/BUG), shown in Chapter 4, provides reasons for considering the: 
- Inclusion of fully-nuanced pedagogical steps into teachers' skills range (drawing on Lipman, Wilson, Vardy, Sutcliffe, Golding, as well as Howard Gardner and Susan Gardner).

- Profiling of reshaped teaching strategies to take account of emerging teaching-learning conditions (drawing on Crowther, Hargreaves, Boxall and Lucas).

- Construction of technical strategies that take account of the familiar approaches to teaching the topic, teaching to the test, teaching life skills and teaching applied thinking skills (drawing on Costa, Fogarty).

- Provision of a safe and focussed opportunity for students to develop their own questions and answers to the issues and concerns that affect their personal lives, independent of unnecessary influences, but congruent with the apparent role of the school to provide, parental-like responsibility for maturational opportunities, including fruitful experience in academic matters (drawing on Costa, Garmston, Splitter \& Sharp, Skorupski, Hawkes, Riso).

- Taking into account of the latest research about the brain's response in learning, since education is a powerful contributor to brain maturation (drawing on Burns, Greenfield, Lipton, Dispenza, Doidge and Lucas).

Significant present conditions needing integration by teachers, one with the other, include:

- The existence of multiple forms of Information and Communications Technology, increasingly used in daily life, even by the youngest of children.

- The deliberate insertion of electronic technology into the teaching-learning process. 
- The emerging brain research that brings accurate knowledge about how the brain works and learns, with direct consequences for the reshaping of the teaching-learning process.

- Other emerging research indicating apparent changes in the human brain as a direct result of proximity of various technologies to the brain. Research mentioned in Greenfield's (2007) presentation in Norrkoping indicates neuronal patterns (dendrites) in the frontal lobe of the brain in today's learners are adjusting to accommodate the passing spectrum of instant and fleeting external stimuli of today's environment (see also Greenfield, 2008 p.30, 47, 48, 71 on dendrites).

- The continual contemporary emphases on attending almost exclusively to, and experiencing, even in risky situations, the myriad of external stimuli. This happens at the expense of personal attention to interior reflection, relaxation, maturing resilience and the quality of reconciliation necessary for quality of life and for resilience in human relationships to thrive.

Splitter \& Sharp (1995, p. 58) identify four kinds of questions:

- Closed procedural questions.

- Open procedural questions.

- Closed substantive questions.

- Open substantive questions.

Consider the answer given by a teacher responding to my Questionnaire. Giovanni states: 'I raise an issue/theme/topic and we discuss it [emphasis in original]. I ask questions/they ask questions throughout the discussion'. The relevant consideration is the identification of what kind of questions and how many questions are procedural or substantive in nature, how many questions that were philosophical, ethical or factual were not used.

Questions outside the norm were exciting and led to re-assessment of meaning offering an opportunity of using the strategies of Splitter \& Sharp 
(1995, p. 9) and Bena Kallick (2001). Beyer, Costa and Presseisen (2001) have included a glossary which contributes broader daily language for teachers. Consistent with this broadening, participant teacher, Jane states: 'I always say you are welcome to disagree but you need to be able to articulate your reasons and be prepared for your comments to be responded to'.

Reasoning and inquiry alone require 24 separate strategies if we follow the delineation by Splitter \& Sharp (1995). There is also the question of whether, in the perceptions of secondary colleagues, it becomes obvious that they do not see significance in the underlying objective of this study: that deeper learning is promoted when virtues, values and ethics are included in the deliberations and discussion of the classroom, broadening concepts of knowledge. There is also the matter that secondary students themselves seldom recognise: that they limit themselves to questions within the topic because of assessment considerations, rather than deepening their knowledge and understanding.

\section{The unique contribution of Fisher}

Fisher (in Heasly, 1995) delineates the differences between the Socratic Method and Plato's Academic tradition as he highlights the finer points of teaching children to think; these differences are listed in Figure 7.7. The professional teacher can recognise the differences between the two concepts and to use each or both as needed. 
FIGURE 7.7 SOCRATIC V. ACADEMIC APPROACHES

\begin{tabular}{|c|l|l|}
\hline $\mathbf{1}$ & \multicolumn{1}{|c|}{ THE SOCRATIC METHOD } & THE ACADEMIC TRADITION \\
\hline $\mathbf{2}$ & Philosophy is an active process & $\begin{array}{l}\text { Philosophy is a learned body of } \\
\text { knowledge }\end{array}$ \\
\hline $\mathbf{3}$ & Philosophy is inductive & Philosophy is dogmatic \\
\hline $\mathbf{4}$ & Philosophy is linguistic & Philosophy is deductive \\
\hline $\mathbf{5}$ & Philosophy is open to all & Philosophy is conceptual \\
\hline $\mathbf{6}$ & Philosophy is applicable to life & Philosophy is for the few \\
\hline $\mathbf{7}$ & Philosophy is dialogue (oral) & Philosophy is written \\
\hline
\end{tabular}

There is a difference also between Philosophy for Children, with its Community of Inquiry conventions, and Socratic Dialogue. Fisher delineates these as separate and useful means of adding to the teacher's praxis as shown in Figure 7.8. Teachers would become familiar with Philosophy for Children and with Socratic Dialogue and the separate conventions which surround the successful use of these strategies. The role of questioning in each set of strategies is subtly different and it is my experience that those subtle differences add to the potential for learning and knowledge.

FIGURE 7.8 CONTRASTS

\begin{tabular}{|l|l|}
\hline \multicolumn{1}{|c|}{ PHILOSOPHY FOR CHILDREN } & \multicolumn{1}{c|}{ SOCRATIC DIALOGUE } \\
\hline Philosophical story as starting point & Philosophical question as starting point \\
\hline Free ranging discussion & Focus on one question or problem \\
\hline Expression of alternative viewpoints & Aim for consensus of opinion \\
\hline Inquiry through dialogue & Dialogue includes a meta-discourse \\
\hline Questions written before discussion & Questions/ statements written during discussion \\
\hline Oral review of discussion & Written review of discussion \\
\hline
\end{tabular}


FIGURE 7.9 RESPONSES TO SOCRATIC QUESTIONING

\begin{tabular}{|l|l|}
\hline \multicolumn{1}{|c|}{ QUESTIONS } & \multicolumn{1}{c|}{ RESPONSES } \\
\hline Questions that seek clarification: & $\begin{array}{l}\text { These include explaining, defining, giving } \\
\text { examples, supporting, enquiring. }\end{array}$ \\
\hline Questions that probe reasons and evidence: & $\begin{array}{l}\text { These include forming an argument, assumptions, } \\
\text { reasons, evidence and counter examples. }\end{array}$ \\
\hline Questions that explore alternative views: & $\begin{array}{l}\text { These include re-stating a view, speculation, } \\
\text { alternative views, counter argument, distinctions }\end{array}$ \\
\hline Questions that test implication and consequences & $\begin{array}{l}\text { What follows includes implications, consistency, } \\
\text { consequences, generalising rules, testing for truth }\end{array}$ \\
\hline Questions about the question or the discussion: & \\
\hline
\end{tabular}

In addition, Fisher provides a list, shown in Figure 7.9, that pinpoints how Socratic questioning might be processed. Fisher wrote the paper, 'Socratic Education - a new paradigm for philosophical inquiry?' for the International Philosophy for Children in July, 1995 in Melbourne; this is discussed in Heasly (1995).

If we promote a culture of reflection, it is to provide opportunities to delve into paradoxes and moral dilemmas. This becomes a first step in the process of resilience-building for the teacher. The process of continuous and consistent reflection, built on thinking skills, (Splitter \& Sharp, 1995) has provided a channel of increasing expertise, efficiency and effectiveness, underpinning and engendering my concept of a culture of resilience. No question in the Questionnaire explicitly asked for detailed kinds of thinking skills and no respondent listed the kinds of thinking- though some teachers did mention options and the allowing for different answers, especially in Art, Design and Technology. The reshaped philosophy of education being constructed here keeps discovering the emerging ways that teachers use the curriculum content of a given topic to engender rich, deep learning through the teaching of thinking skills.

Using the content of the curriculum plan leads to the immediate inclusion of a particular thinking skill. If the teacher ensures that this skill is used regularly, and consistently, the student will internalise this skill and be comfortable using its proper name in addition to the curriculum content. This 
practice enhances the ability of the student to prepare for examination, project work and research (Foot, 1967; Mackay, 1993; Wilks, 1996; Mackay, 1997, 1999, 2004; Revell \& Arthur, 2007).

Of course, it is necessary to fire the enthusiasm of teachers and their delivery of content, and to heed the warning of Seiger-Ehrenberg (2001) regarding how hackneyed a term can become if it is not treated delicately and accurately. Her example is the effort to make distinctions between a concept, a fact, a principle, an attitude, a skill and even a disposition, like Burwood and Wyeth (1998) who together pinpoint distinguishing between the concepts of tolerance and toleration! Fitzgerald (1993) explores what he called the Eleven Deadly Sins, giving detail to each concept and also highlighting the requirement for linguistic accuracy that can be a minefield for the teacher, given the changes in meanings of many terms that the last few generations use.

\section{Evidence of teacher-oriented languages used}

The teachers' responses show evidence which I detail below, that they used six differing kinds of language to ask questions about topics and issues. I have given them titles to help to identify the subtle links and meanings that can be part of the teacher practice in classroom:

- Instructional transmission pedagogy, in which teachers planned to confine themselves in an academic way to the educational jargon inherent to their subject area by instruction exclusively for the content of their curriculum topic. Questions were designed and timed to elicit existing knowledge of the relevant topic under consideration at the beginning, during and at the end of a given lesson.

- Dennis indicated one example of this style of pedagogy as he used question and answer, group discussion, guided reading to achieve the stated learning process. 
- Michelle stated: use curriculum topics/design process to engender discussion; and teaching strategies - written instruction, one-onone discussion, direction, and questions.

- Student-centred persuasive pedagogy, in which teachers offered questions about content for student decisions, based on levels of interest within the class. Questions were couched in persuasive, engaging formats to entice the students enough to become deeply engaged in the process of inherent learning.

- Terence described this style as he used questions, flexibility and causes a positive environment for thinking in his classroom strategies.

- George stated: 'I can go either way depending on the level of interest'.

- Collaborative and creative pedagogy, in which teachers and students were in partnership in one or more of the pedagogical formats available, so that investigation, research and discussion formed the basis of a successful learning outcome.

- Jane made this quite plain in her responses: “... encourage students to use strategies such as brainstorming, PMI activities, debates, personal reflections, examination of current affairs and opinions relevant to the topic ...'

- The Community of Inquiry was one format that incorporated the development of relevant questions, the grouping and categorising of parallel types of questions and the process of learning by constructing the bones of the thinking skills that underpin the format.

- Roger, Clint and Matthew, the three practising experts, gave descriptions of this model and its processes and outcomes.

- The process of Socratic questions is my overarching title for discussion and the separate but similar process of Aristotelian questions in discussion were two other formats. These two processes have different outcomes: one asks questions only about the topic itself from every angle, 
while the other asks questions from any angle and queries how the topic may impinge on other related topics.

- Vaughan used this approach as he looked at the use of lateral thinking, embedding thinking skills within any unit for deeper meaning.

- Roger, the UK practising expert underscored the 'breadth and range of the philosophical nature of thinking and the value of questions, creating radical thinking, urging students to strive for better quality in their learning'.

- Clint, the NZ practising expert, highlighted that the teacher needed to be focussed on student thinking processes, not on the correct answer.

Students seemed to learn the specific jargon attached to a given teacher or attached to a subject area. Some students, in my experience, concluded that the thinking skills that pertained to one subject area or teacher did not get carried over to other subject areas. This I have called 'compartmentalisation':

- This may be a reason why Bernard said that his students have freedom to fail; freedom to create; and put things into perspective.

- It also inclined George in his challenge to keep an open mind; use different perspectives; allow for shades of grey.

- It perhaps induced Henry when describing the process he used to stimulate discussion to state: I challenge individual perceptions (emphasis in original), and is why he also stated at the end of the Questionnaire: 'Education must be about more than knowledge!'

\section{Analysis of the concept of virtue}

For the purposes of this investigation, a virtue, as a concept, I define as the assembled broad thinking, reflecting, sifting and discernment, seen to be foundational to the best expression that an individual can be. Virtue emerges from a range of cultural distinctions, religious underpinnings, identifiable 
goods, political impulses and philosophical frameworks through which an individual can express the best of human behaviour on a daily basis. There are certain virtues that have from time immemorial been accepted as useful, helpful and seamless stepping-stones towards understanding, and they have a revered place in society. They include tolerance, fairness, justice, prudence. Emphasis on some virtues, at the expense of other chosen virtues, changes as the generations pass. The outcome in each situation is the discovery of new knowledge in learning how and when to use these learned responses as a basis of human behaviour (Wilson, 1971; Watt, 1976).

While Wilson was essentially concerned with the business of philosophical and analytical inquiry into what constituted moral education, he began from a logically apt stepping-off point. He considered that moral education itself was a concept. It is acknowledged in his collaborative work (Wilson et al., 1967) that there are a great many muddles to be cleared up and prejudices to be removed, before we can get a clear view of the subject at all.

It was relevant also to the above considerations to recognise the significance of what is to count as moral values that reside in secular patterns of thought. Rowland (1993) and Seligman (1990, 2002) have given a complete scenario in which the logic of exploring what was to count as the good life and what was to count as the right or wrong thing to do is clearly outlined. Over almost 20 years (1993-2010) Mackay takes up a similar vein of exploration of how some Australian people, who prefer a secular world view, have chosen their standards of moral rectitude. While there were noticeable parallels between the outcomes of what was to count as right and wrong in both the religious and secular scenarios, the reasons differed considerably. Religions other than Christianity also had parallels in the search for what was to count as right, and what were to count as wrong behaviours.

I have found it useful to make the distinction between two approaches: an opinion and a point of view. The former, which for our purposes is seen as being the chosen position of an individual, based on some logic and 
sometimes based also on high levels of emotional response; the latter is a chosen position taken after considerable reflection and exploration. It provides the thinker with a more placid and accurate response in which emotional reaction has subsided or was not really present during the analysis of the problem, the dilemma, the difficulty or the paradox being addressed. This is clarified in the stages of decision-making that I have recommended.

The diverse nature of what was to count for all people as rigorous thinking and careful logical process was part of the experience of learning that accompanied the content of the topic in the classroom in any discipline. Maturation of thought and internalisation of the consequences of such diversity is part of the educational enterprise, even if conclusions lead to diverse opinions.

\section{Teachers' perceptions, assumptions and expectations}

It was expected that, by exploring and analysing the material in the Questionnaire carefully, applying the theories of educational excellence, including all that these three initial sources (Wilson, Lipman, Vardy) provided, and also applying the various new theories offered by Greenfield and others, some conclusions, responses, recommendations and cautions would be possible.

The teaching-learning process is essentially, I believe, a seven-phase process, comprising input, realisation, understanding, assimilation, implementation, retention and the relevant/appropriate use of content (Heasly 1995, p. 17).

Naomi Klein's (2007) The Shock Doctrine tells us a story that could be a significant contribution to the understanding the patterns of society and the future. It could be said that democracy would be better served if students were able, through the teachers' contribution of successful thinking skills, to address the decisions they must make for their future wellbeing: personally, politically and in problem-solving. 
Many teachers already structure diversity into their strategies showing what the channels for learning involve: hearing, listening, internalising, recognising, understanding, learning. Kerri said she did so when stating that she used the construct of the multiple intelligences to underpin her teaching strategies.

The process can be promoted through repetition, recapitulation, recognition, reorganising and relevance to the new material, along with the previously accepted and understood content on the topic. This is demonstrated clearly in the responses to the Questionnaire by Samantha who used thematic approaches, linear development of the topic and inquiry-based learning, to enhance the opportunities for learning in her classroom.

Importance of change in student experience was enhanced by the existence and then promotion of information and communications technology (ICT) in Australian culture and its consequences for the teaching-learning experience. I remember the consequences for student teachers coming from a climate of values-neutral classrooms: often it was the unseen influence which permeated the classroom experience and, with its subtle overt and covert changes, had an unknown effect on the culture of the classroom.

There are consequences for teachers, whose classroom experience has had to negotiate the divide between their own experience of the valuesneutral classroom and the existence of gender-based classes, or classes of fifty or more students, or the opposite: when classes were for a variety of reasons only ten to twelve students.

\section{Emerging relevance of brain research for teaching thinking}

The significance of research about how the brain works; about how the brain learns; about the evolution of brain-dendrite accommodation of technology; and about the physical impact of ICT experiences especially in early childhood on the structure of the brain, are all central questions for my considerations. Connecting this to the conclusions to her pharmacological projects over fifteen years, Greenfield (2004, 2007, 2008, 2011) has 
highlighted about how technology was changing the patterns of dendrites in the brains of the young. Kagan's (1994) efforts were directed towards the construction of techniques and strategies for effective brain-compatible learning. There is a need to incorporate the significant new knowledge about the emerging data of how the brain processes learning, coming from the study of the mind. Investigations by Greenfield appear to be supported by an array of neuro-plasticity specialists (Jensen, 2006; Dispenza, 2007; Doidge, 2007; Lipton, 2008). Knowledge about how environmental factors can change the brain and enhance learning is central to aligning learning outcomes to classroom pedagogy.

It should be noted that Fogarty (in her work between 1990 - 2003) and Costa and Kallick (2001) have made concerted efforts to fashion what they have called brain-compatible classrooms (Fogarty's term), in the service of the teaching fraternity, so that pedagogical processes incorporate such considerations with coherence and harmony.

Teachers already signal a deep appreciation of the teaching of thinking skills when they help secondary students with the preparation for career choices; in Years 9 and 10, especially. The impact of using the full range of thinking skills has become apparent on:

- Decision-making and choices - a democratic issue.

- Relationships - an emotional issue.

- The construction of a career - an educational and social issue.

- The development of the 'good life' - a psychological and philosophical issue.

- The possibility of choices made for either a religious or secular approach to the construction of meaning in life - an intellectual, spiritual and theological or secular issue.

These impacts can be seen to connect directly with the central column of the Heasly Behaviour Uncertainty Grid (HUG/BUG). If they are alerted to the full enhancement of rich learning that can result from its application, teachers can identify the various streams of thinking outlined, and incorporate 
elements into topics and discussions. The aim is to engender a new habit of mind according to the indications of Costa (2001b) in which reflecting on daily interactions with teachers, other students and the content of the curriculum is central. The consequence of this aim is the teaching of the growing student how to handle perceived outcomes of daily experience and learning challenges in an effective and resilient way.

As a result, there appears to be the opportunity to fashion the widest diversity of questions that may be pertinent to a given topic. It was obvious when I began the practice, which students would benefit from the actual naming of each new thinking skill as it was introduced. Teachers' perceptions of the link between cultures of reflection, resilience and the promotion of thinking skills for decision-making, would become integral to their pedagogical effectiveness.

Splitter \& Sharp (1995) have demonstrated clearly how philosophy can underpin all areas of the academic curriculum. Their diagram appears as Appendix B and I found ways to find out that this was possible. Curriculum experts would become responsible for inclusion of this content into curriculum. Schwab (1983) details his preferences for effective curriculum expertise and the improved suggestions for curriculum experts. Assessment tasks would vary from the appraisal towards a process whereby the completion of tasks would rely on: demonstration of reasoning; the highlighting of conclusions; integration of the task into reality; and the provision of the student with the knowledge that the tasks are worth their time and are effective for the development of new knowledge, new perspectives and new horizons. The need to include content which addresses the maturation of students in emotional intelligence was another difficulty which was initially addressed in my reshaped pedagogy and later when I discovered Crowther's design of sustainable capacity building for reform (Crowther et al., 2010):

- Senge's two principles that professional learning is integral to organisational development and that school is 
involved with creation and sustainability of significant new knowledge (Senge et al., 2000, p. 6)

- Hargreaves \& Fink's five practical core components for educational improvement (Hargreaves \& Fink, 2006, p. 7)

- Fullan's eight elements of school sustainability which speak of, among other things, moral purpose and deep learning (Fullan, 2005 , p. 8).

In terms of the commonplaces of the curriculum, this category provides the foundation for teachers and teaching; it impinges on the teachers who are delivering the curriculum.

\section{Category of Influence 9: My contributions}

I have undertaken extensive professional development during my long career as a teacher as a means of providing me with the best collegial practice for myself and my colleagues, and the best pedagogical practice for my students. As a means of further development within the classroom, I have constructed a number of instruments which have been used in a variety of classrooms: for students of mixed abilities, for inclusive classrooms, for specialist classes, and for Homeroom practice. These are detailed in Chapter 4.

The Cycle of Inquiry appears earlier in this chapter and there is an emphasis on Questioning throughout. The Cycle has been an instrument that students have especially appreciated. It surprised them to think that failure could recede into oblivion if they followed this pattern. The element of inquiry in its different guises is central to the business of Questions and questioning.

Students regularly found the Heasly HUG/BUG instrument helpful because they could explore in their own time and at their own rate just what perspectives were already parallel to their own beliefs and experiences; but it also allowed for personal and sometimes private exploration of what might be ahead of them, as they explored the positive side (Heasly Uncertainty Grid 
- HUG and the negative Behaviour Uncertainty Grid - BUG) side of the concepts they encountered in the Grid- as opposite sides of one coin rather than contrasts that needed to be compared with one choice.

The Heasly ME diagram was of considerable satisfaction to me as a professional engaged in providing for real and deep learning for my students in that they considered it useful. The potential it held for future planning caused them to follow up many possibilities before they made final choices for career purposes, and indications were that other more private considerations were being explored also.

In the Heasly approach to the Six Steps in Decision-making, junior secondary students found parts of this process a bit difficult, because they were unsure of the steps in providing themselves with a wide variety of possible choices, and were a bit shy when faced with finding three or more experts who could provide advisement (chosen experts who provide knowledge for prioritising) rather than advice (the communication of any personal experience alone). Older students were more confident in using the instrument for their own purposes, but were a bit impatient when it seemed necessary to allow the time needed when a mistake was made, and evaluation needed to take place in order to identify what had already been learned. Many students tended to jump straight to the next possible action on their list, without doing the evaluation, and so missed significant new knowledge by dismissing this part of the process.

The Leadership grid, appearing in chapter 4 showing informal and formal leadership behaviours: careful use of much evidence and study could provide me with the content to compile the list of 48 characteristics of both an informal leader and a formal leader, then I had what I judged to be a comprehensive instrument to aid in my development of professional expertise. This grid needs, more than other Heasly contributions, to be tested for validity and accuracy for possible universalizability, as I have tailored it to the rest of this investigation over the years, but other eyes and voices need to give evidence as to its further usefulness or otherwise. 
There is significance in noting the differences between practices of indoctrination and practices promoting higher-order thinking skills. Like the Leadership grid, this set of contrasts needs other eyes to provide approval of the content for further pedagogical purposes. The construction of this instrument was undertaken for personal purposes pertaining to professional development which began in the 1970s.

As a result of these considerations, it became apparent that the concepts of accountability and transparency were part of the professional development of twenty-first century teaching skills. To strengthen accountability, there is an implied inspectorial role; however, I am not advocating the political concept of failing schools - quite the opposite. Transparency also needs to be clearly defined: About what? To whom? About whom? At what stages? For exactly what purpose: political expediency or educational outcomes? Most particularly: where is remedial teaching in all of these considerations? Where and what, then, constitutes failure?

And so we address the concept of edu-tensegrity: where, like the giraffe with its long neck's bone structure - that is consonant with the geodesic dome. Those Categories of Influence that form part of the tension and compression forces that strengthen and give support to the architectural concept itself, and the parallel in biotensegrity, now parallel my theory, acknowledging that the same underlying theory exists in contemporary education.

In terms of the Schwab's commonplaces of the curriculum, this category provides my practical contributions to teaching and learning; it impinges on the students who are engaged in the learning and the teachers who are delivering the curriculum.

\section{Category of Influence 10: Outcomes, issues of dependency, rich and deep learning}

Students achieve different ways of being by choosing, by decision-making, by new knowledge, by personal research and by experience within the 
education scenario. The outcomes contribute, through decision-making, to their choices of career, study patterns, successful outcomes in acquiring new knowledge and new skills.

Students learn through an amalgam of the various processes and systems alluded to in this chapter, processes and systems that it is necessary to recognise and to use successfully, when students are dependent and need help and advisement in various ways; when they can with confidence proceed independently with various projects and enterprises, in relationships, and in community, making their mark in their community if it pleases them to do so, and earning their place as persons of experience and importance in their environment. Allied to this, are the knowledge and skills to operate successfully in an interdependent manner, acquiring skills of team-work, of research, of interaction with people around them. They are able to proceed to construct a relevant, reasoned and reliable belief system, build a preferred future and career, and make such decisions that promote wellbeing and human flourishing (Seligman, 2011).

Rich and deep learning is acquired through the auspices of the dedicated teaching fraternity who take up the challenges of the concept of edutensegrity and find consistency, expertise and steadiness of mind to steer when necessary, to support where necessary, and to provide advisement when requested.

In terms of the commonplaces of the curriculum, this category provides outcomes for issues of dependence and rich learning; it impinges on the students who are engaged in the learning.

\section{Category of Influence 11: The construction of a personal belief system, a good life and a career}

Embedded in this investigation is the process of decision-making that lies at the heart of how the individual student can develop the life skills needed to accomplish their preferred goals and achieve outcomes influencing the whole 
of their life experience: in effect to provide the new knowledge of how to understand and apply virtues, values and ethics.

A personal belief system is one which is built one step at a time, taking account of the many influences around us, communal and individual, that give us the confidence that makes sense of what are termed here eschatological speculations. A belief system refers either to a religious or a philosophical approach to how the world is. If religious, it will perhaps rely on a particular faith, a particular formulation of a god or gods that will allow us to make decisions to be made about the outer elements of the universe that the religious person inhabits; however, it is equally possible, in taking account of the many influences around us, to develop a belief system which does not include the presence of a god or gods in those eschatological speculations. Eventually, each person needs to find a personal answer.

The 'good life' is what Aristotle (translated by Ostwald in 1962) chose to investigate when he tried to find a system in which the conditions and the attitudes, the preferences and the intentions, are constructed for ourselves and for those around us. His system was to make the best of whatever conditions we find around us, and to establish the best conditions for our life that we can. In doing so, there is the logic and reasoning that is necessary to find holistic ways that account for integration of one's own needs with the needs of those with whom one lives.

Educationally, teachers need to incorporate the development of the skills and knowledge necessary for the construction of the many aims and objectives that accompany the building of a career.

In terms of the commonplaces of the curriculum, this category provides the foundation for construction of a belief system, the good life, and a career; it impinges on the students who are engaged in the learning and on the milieu in which the curriculum is delivered. 


\section{Category of Influence 12: The integration of student, teacher and the curriculum expert to form the nexus of pedagogical delivery}

The almost umbilical connection between the student, the teacher and the curriculum expert for the effective, efficient and accurate delivery of integrated learning, is central to the understanding of what the concept of education actually contains.

The role of the student is sometimes that of a dependent, who must rely on the authority and knowledge of teachers and other significant figures for direction. The student must also incorporate into their understanding of educational experience those times when independence is the better approach - especially where rich learning is concerned. Also equal in significance for the student design: is the choice of whether or not to incorporate interdependence - when ICT, other students, teachers and others, can collaborate to achieve more complete understandings of curriculum content.

The role of the teacher is crucial in allowing for efficient and effective delivery and understanding of the curriculum content. It is also to provide the necessary insights to promote the rich learning needed for the aim of education to take place. Just teaching curriculum content is not enough to ensure the full aim of education. This thesis posits that the emphasis is in the teaching of the student, through the medium of the curriculum content.

The role of the curriculum expert is to find ways to embed knowledge, skills, and interaction into the curriculum balance that interconnects where needed to all areas of the curriculum. Compartmentalisation, a term I used when faced with students who did not realise that they could carry skills from subject discipline to another, results if the curriculum does not include real efforts to make necessary connections between knowledge and skills among the various disciplines and subjects as the student progresses through the education system.

In terms of the commonplaces of the curriculum, this category provides rich learning by the integration of teacher inputs, their impact on students, 
and the curriculum elements addressed; it impinges on the teachers who are delivering those curriculum elements.

\section{Looking forward...}

The consequences of applying the Categories of Influence and the knowledge emerging from this new theory of edu-tensegrity are a reshaping of curriculum to include a deeper emphasis on applied thinking skills pointing towards the understanding of virtues, values and ethics. In this chapter the focus has been on giving students opportunities to learn about various levels of virtues and values education. The students are able to understand that both virtues and values are methods by which they can pattern their own behaviours with a view to taking responsibility for their futures.

In the next chapter the focus turns to a direct examination of a new architecture for teaching ethics. Linking into the curriculum initiatives indicated here will be the careful reshaping of further detail to include applied thinking skills in relation to school leadership and ethics in action. 


\section{CHAPTER 8}

\section{Reshaping Curriculum: Linking Applied Thinking Skills to Ethics}

\section{Introduction}

Chapter 8 further tests the application of thinking skills to ethics by attempting to reshape where curriculum might be linked, and the possible choices available for ethical behaviours chosen autonomously by both students and teachers. It is important to recognise that I see ethics and ethical behaviour as the response of an individual within a community; on the other hand, virtues and values are more personal and might be more private. No attempt has been made to include specific curriculum content, as I have limited the thesis to building an emerging concept of education, taking account of the three sources that I believe are necessary for education about virtues, values and ethics.

This investigation identifies the development of an understanding of the links and connections that I have been constructing; contradictions and gaps as well as hesitations and concerns occurring in classroom strategies and techniques coming from the teachers' perceptions; discussions and learning about the education process from various theorists, philosophers, teachers and experts. Under the auspices of this process, students have opportunities to inspect, analyse and evaluate fully what is presented to them in the way of learning by their teachers; teachers have opportunities to benefit professionally and personally from newly indicated links to ontological matters, to epistemological endeavours and to the development of 
pedagogical strategies and techniques that enable them to interrogate, understand and choose whether and where to integrate this content into their own practice. The subjects taught facilitate an integration of a virtues, values and ethics curriculum; the education whole is embedded in the particular milieu in which the curriculum is operating. In practice, the concept of EduTensegrity accommodates the changing nature of the students, the teachers and the subjects taught, into the current milieu and also into the developing needs that will be encountered in later twenty-first century education.

To reshape the curriculum to integrate the ontological and epistemological elements of knowledge regarding concepts of individual virtues and values with ethics and ethical behaviours, it is important to recognise the place of three of my instrumental tools, contributed in Chapter 4: the HUG/BUG, the ME Diagram, and the Heasly approach to the Six Steps in Successful Decision-making. The application of thinking skills to the reshaping of the curriculum requires the provision of the ontological constructs and the epistemological concepts of new knowledge that allow for individual decision-making in a democratic manner. Students learn to make decisions based on the best available options, according to the best knowledge and experience available, for the best possible outcomes in recognising and solving a problem, a difficulty, a dilemma or a paradox. The place of these three tools, which focus on students being able to upgrade their skills, relies on the prior recognition of big ideas (ontological considerations). These big ideas translate as a Philosophy of Education for each teacher, who may then shape and reshape the content into new knowledge (epistemological concerns and consequences). With help of other grids I have provided, this new knowledge includes an expanded understanding of the role and functions of teacher, and addresses the discomfort that may be experienced in this field of teaching over matters of possible indoctrination. The new knowledge emerges as a blended and synthesised response by the teacher, and emerges as an approach to the relationship between student, teacher, the subject taught and the milieu (Schwab, 1983). The translation 
into classroom strategies and interpretation of what is to count as a deeper teacher-learner process allows Aristotle's techne to emerge as efficient and effective pedagogy in its newer and more complete profile as a concept, and as the Art and Science of Teaching.

In this chapter, ethics, ethical leadership and edu-tensegrity and present educational practice will be shaped and integrated into a new educational paradigm.

\section{Ethics, ethical leadership, edu-tensegrity and education}

I will apply edu-tensegrity to a consideration of ethics and of education.

This will place ethics in the Categories of Influence considered in the previous Chapter, as follows:

- Dimensions of daily life, in which ethics is seen as a foundational shape for quality decisions that students will make and act upon;

- Dimensions of thinking, in which learning about and understanding each concept of the broad arc of possible ethical behaviours, for ethical leadership and practice, is central to their possible use in choices; reasons for action are central;

- Dimensions of education, in which ethics are acted upon, tested and discussed within many subject areas for students to evaluate for purposes of their integration of resilience into ethical decisionmaking; resilience is central to effective decision-making;

- Curriculum theorists - Wilson, Lipman and Vardy - whose subject materials progressively develop an increasingly practical virtues, values and ethics curriculum showing the connections between ontological considerations, epistemological concerns and their consequences, and the technical strategies and techniques that must be included in what is to count as the complete concept of pedagogy; 
- Constructs about knowledge of the thinking curriculum, apply as their developmental constructs need to shape emerging thinking about ethics curriculum, when teachers may lead and facilitate discussions in which students test and argue the merits of various options for ethical action;

- Historical and philosophical theorists such as Aristotle, Dewey and Schwab provide the particular translations by Costa, Garmston, Kagan and Fogarty, who model inductive and deductive thinking from medical science and philosophical sources for what Fogarty calls brain-compatible pedagogy, i.e. matching classroom learning experiences to her research on how the brain processes new information and engages in rich learning. So a cohesive, caring, constructive, and creative approach emerges from traditional sources of educational excellence into the new conditions of twenty-first century Education;

- Meaning, reasoning and choice, takes the concepts and constructs first enunciated in the works of Aristotle, Dewey and Schwab in conjunction with the crucial developments recounted by Greenfield and others, into how the brain works. The resultant educational developments initiated by Costa and others shape the practical application of reasoning, the finding of meaning and making of choices about learning, about applied thinking skills and about ethical behaviours. They become the new building materials highlighted in the geodesic dome approach, new and continuing teachers to emerge with a deeper emphasis on meaning, reasoning and choice for themselves and their students.

- Teachers and teaching, gleaned from the teachers' voices heard in their responses to the Questionnaire items: these teachers, within their range of educational goals, provided valuable information from the coalface of the classroom. They provided a realistic commentary on the possibility of teaching about ethics and ethical 
behaviours. It is vital therefore that the teachers' voices are continually heard as new phenomena emerges. It is equally vital that the imposition of single-issue phenomena is not pushed by single-issue lobby groups. Each generation faces new cultural experience and teachers address this phenomenon on a continuous basis, with varying success.

- My own additions (Heasly contributions) at the practitioner level, contribute to the teaching about ethics for student understanding and evaluation: by the use of curriculum instruments; by the development of thinking skills linked to regular reflection and resilience; by the identification of emerging neuro-scientific concepts in brain research; and by extension, in the inspection of the problematic responsibilities of ethical educational leadership. Inevitably, changes will occur and the education ethos may follow suit, but positive approaches will provide for the emergence of excellence, efficiency and effectiveness.

- Outcomes and issues of dependence and rich learning from the work of four neuroscientists - Greenfield, Doidge, Lipton, and Dispenza - are central to the careful development of understanding a curriculum concerned with ethics, ethical leadership and daily experience. If the educational goal is to equip students with authentic and valid new learning, from which they make their decisions for their future, then it is not a large step from today's education to reach towards the teaching of a virtues, values and ethics curriculum. Students are obviously dependent on the teachers' abilities to provide educational material and methods that introduce new ideas, new knowledge and new ways of accomplishing the acquisition of their applied thinking skills.

- Construction of a belief system, the good life and a career to encompass students' own expanding knowledge: achieving differing ways of efficiently handling dependence, independence 
and interdependence as the knowledge of ethics and ethical decisions expands into deep learning, and into an emergent conscious practice of individually chosen and wise, culturally appropriate behaviours. It is crucial that students are able to recognise the stages which accompany the respective states of dependence, independence and interdependence - enough to take personal advantage of each stage to maximise their learning and balance their ethical behaviours. This provides the basis from which they may emerge as confident and educated people, able to clarify their intentions and preferences regarding a belief system, their own construction of the 'good life' and the establishment of a career; all of which will exist in the future, as they exist already, in very differing circumstances and conditions to those of the twentieth century.

- Integration of student, teacher and curriculum - emphasising the almost umbilical connection between the student, the teacher and the curriculum expert for effective, efficient and accurate delivery of integrated learning - is crucial to the experience of education for rich and lifelong learning for each student. The close proximity of teacher, student and the curriculum is familiar territory to today's teachers, but the existence of the various channels of learning (i.e., visual, aural, kinaesthetic, holistic, experiential and communal) which can be triggered by, for example, ICT facilities need to be acknowledged to achieve best practice for teachers and best learning results for students.

It is important to find ways to relate all of these Categories of Influence making links and distinctions that will inform the facilitation of the teaching and learning process. Where the previous chapter focuses on connections in knowledge of virtues and values, this chapter extends these considerations to include ethics in the process. 


\section{Category of Influence 1: Dimensions of daily life}

Having given due thinking and reflection time to the exploration of ontological constructs - those big ideas that help students (and teachers) to make sense of the world around them - I construct a cyclical channel of inquiry. This approach leads to possible identification of learning contradictions, gaps, assumptions and concerns experienced in daily life, and helps students (and teachers) to gather evidence into a coherent conglomeration of knowledge and skills. As John Wilson's Moral Component List (Appendix C) indicates, the variety of thinking skills required extends into every subject area, and the very long list of those thinking skills can be found when needed in Splitter \& Sharp (1995).

If I posit that virtues are the internal dimension of education, and values are the external and communal dimension of education, then ethics becomes the construction of a personal set of standards of behaviour of the individual to enhance a career, to construct the 'good life' and to allow for eschatological speculation of a spiritual, religious or secular nature. Those standards have been present in every generation in contrasting societies and represent the best of what humankind can become - for themselves and for each other.

Application of the knowledge gained towards the exploration of the meaning and consequences of ethical behaviours, expands under this new paradigm: by mastering the use of the Heasly HUG/BUG; by the realisation of the potential within the ME diagram; by the recognition of what is to count as informal and formal leadership, especially as regards a deeper understanding and knowledge of what constitutes democracy. This is what I have gleaned from my observations within my own experience, studies and teaching.

In terms of the commonplaces of the curriculum, this Category of Influence provides the practical basis for twenty-first century daily life; it impinges on the milieu in which the curriculum is delivered. 


\section{Categories of Influence 2: Dimensions of thinking}

The influences that form the foundation of the ontological, epistemological and technical dimensions that teachers employ become absorbed into the technical and strategic classroom pedagogies that focus students on the learning process (Lipman, 1993; Heasly, 1995; Vardy, 1998; Costa, 2000; Fogarty, 2003). The Dialogue Australasia Network journals and seminars, from the Vardy network, have sought to support teachers' work.

It is not necessary to promote a framework where teachers may have only a particular theological, ethical, philosophical or teaching focus, as seems historically to have happened in some traditional or confessional schools. Teachers teach successfully about political, secular and religious beliefs without compromising their personal stances. It is entirely acceptable to teach well about such matters, without divulging personal and private stances. Dimensions of thinking necessarily emerge as students internalise the learning that a topic provides, and the potential that opens up as they investigate and research. I have found, however, that it makes discussion and reflection about contentious matters easier to manage, if a certain transparency is possible for the individual teacher.

A culture of reflection is promoted here in order to provide opportunities to delve into paradoxes and moral dilemmas (Watt 1976; Wilson, 1987; Haring, 1978; Snook, 1972; Fukuyama, 2007; Singer, 2002). This becomes a first step in the process of resilience. No question in the Questionnaire explicitly asked for detailed kinds of thinking skills and no respondent listed the kinds of thinking - though some teachers did mention a construct of options and of allowing for different answers, especially in Art, Design and Technology. The application of the Heasly Six Steps of Decision-making would add a realistic dimension to these reflections, grounding the process in foundations for action.

Decisions made by individuals in classroom settings are part of the concept of edu-tensegrity, where the teaching of the student is paramount and is done through the overt curricular content and its extension to the level of 
teaching the student, especially through the use of my proposed instruments. Integration of the contributions of Wilson, Lipman and Vardy also ground pedagogical strategies and techniques in searching democratic thinking, using many of the various thinking skills set out in the list of Splitter \& Sharp (1995).

A new school of ethical thought emerges from these deliberations for emphasis and practice. After the jettisoning of certain familiar educational beliefs, one discovers the changed and upgraded role of questioning and the need to show students how to construct their own list of questions; the more central role of discussion, including the use of the Community of Inquiry; the changed pedagogical rhythm teachers would use in the new paradigm ; the centrality of the teaching of the broad arc of named thinking skills; the specifics of the proposed new sequential processes in the teaching and learning process for rich learning; the deliberate ensuring of the implementation of democratic principles and the careful use of consensus in decision-making. Reference to Lipman's Community of Inquiry strategies is one way of achieving this, and Splitter \& Sharp (1995) concur; Sutcliffe (UK expert) and Wills (Australian expert) in their responses to the Questionnaire, are complemented by Fogarty (2001b).

In terms of the commonplaces of the curriculum, this category provides a practical basis for twenty-first century dimensions of thinking; it impinges on the milieu in which the curriculum is delivered.

\section{Categories of Influence 3: Dimensions of education}

In all areas of Education, there is opportunity to refine, develop and promote the improved and accurately-focussed implementation: of teaching skills; of specialisations in ethically controversial educational questions; with researched developments in principles for leadership and for the teachinglearning process. 
When preparing new topics, the inclusion of broad arc of thinking skills needed is crucial. Using the renewed content of the curriculum plan requires immediate integration of the particular and ethical thinking skill(s) congruent with the part of the subject for the lesson. If the given skill is used regularly, without default, and consistently, the student will internalise that skill comfortably with its proper name, with the curriculum content and with the impetus that provides a basis for successful writing of essays, presentations, playwriting, and mathematical design as Sutcliffe alludes to, in his Questionnaire response. This provides conscious and rich learning rather than thin learning (Davis,1995), and does not rely on the unpredictability of memory (Heasly, 1995). This process changes how technology is used to achieve independent learning strategies. The extension into the matter of thinking skills and decision-making as a firm foundation for the successful choice of ethical behaviour emerges from this patterning (Foot, 1967; Mackay, 1993; Wilks, 1996; Mackay, 1997, 1999, 2004; Revell \& Arthur, 2007). The process of patterning is analysed and described throughout the works of these experts.

Of course, it is necessary to fire the enthusiasm of teachers for their accurate delivery of content, as Seiger-Ehrenberg (2001) warns. The example applied is the effort to make distinctions between a concept, a fact, a principle, an attitude, a skill and a disposition - like Burwood and Wyeth (1998), who together exemplify the teaching of the difference between the concepts of 'tolerance' and ‘toleration'. Fitzgerald (1993) explores what he called the Eleven Deadly Sins, giving the changes in cultural meanings that accompany the last two generations at least. These changing meanings alert us to the need for ethical vigilance, accuracy and communication skills.

The next step I posit leads to the reshaping of a systematic approach to effective technical (techné) strategies and techniques for pedagogical authenticity. We can heed Hodgkinson (1991) and his construction of the links between education and leadership, as he provides us with careful delineation of what he calls the moral art, outlining connections between the 
theory and the practice of morality, moral complexity and leadership; and the accompanying legal, formal and moral responsibility- all of which feed into an understanding of the practice of ethics.. We can heed Hospers (1982) who analyses the whole concept of ethics, taking account of our need to explore: the meaning surrounding relativism; what is to count as good; the links between these two concepts and the various theories that inspect the connection between obligation, duty, ethics, human rights, justice and moral responsibility. It will include finding whatever we mean by the term 'conscience' and how we construct our responses to such matters. The classroom Community of Inquiry provides the opportunity for democratic exploration of such matters. In fact, the concept of democracy will, in turn, become the subject of extensive inquiry as students and teachers, together, explore, discuss and finally determine the best understandings to emerge about what democracy means. This becomes an indicator for the understanding of the standards and actions which ensure ethical behaviours.

Consider the stress likely to accompany the participant teacher, Kerri, whose four years of teaching are based on a Bachelor of Multicultural Studies. Compare the likely difference in the development of thinking skills for the respondent Hyacinth whose 32 years of teaching are based on a Bachelor of Arts, Diploma of Education, Graduate Diploma of English as a Second Language, Graduate Diploma in Administration, Graduate Diploma in Student Welfare and Master of Social Work.

The consequence is that support for teachers rests on the sustainability concept that Crowther $(2010 ; 2010)$ identifies. The preferred list of virtues, values and ethics - which can be endless and diverse - relies at least in part on the work of Clark (2004), Gardner (1993a), Mackay (1993, 2004), Postman (1986, 1996), Watson (1982), Wittgenstein (1972) and Strawson (1963, 1985) whose lengthy explorations alert teachers to the complexity of the exposed curriculum content as discussions about ethics emerge from studies based on materials across the range of different subjects. 
By engendering a culture of reflection through the extensive use of thinking skills, management of risk becomes part of the processes when students are introduced to every learning domain that becomes part of the art and skill of resilience. Knowledge of the work and reflections of Wilson, Lipman and Vardy leads to the construction, teaching and development of the kinds of techné (technical strategies and techniques) that allow ethically for the overlaps between each of these concepts.

The enhancement of resilience allows for learning experience accompanying

- Crowther's COSMIC C-B fourth of his six dynamics (2010b);

- the acquisition of the long list of thinking skills of Splitter \& Sharp (1995);

- the learning from Neil Hawkes and the Values-based Schools (2010);

- the integration of information from Sutcliffe and the Inquirybased learning principles (shown in particular responses to the Questionnaire);

- Fisher $(1990 ; 2003)$ with the precision of his pedagogy for teaching children to think.

So the student needs to register and to integrate the overview that there is knowledge, there is research, there is evaluation, there is a skills base and there is an assessment task that proves successful maintenance of learning. This is all-inclusive in the teaching learning process. Splitter (1995 lists) shows how. The Heasly Six Steps of Decision-making shows compare-andcontrast procedures that perform this function, too. Students are then choosing experts, whose role is clearly that of advisement (providing access to specific options to solve a problem) and mentoring (walking with the student or other person as problem-solving takes place), for relevant material which becomes central to the teaching-learning process and consequent, ethical classroom strategies. 
Resilience, I posit, as an amalgam of determination, knowledge, accurate aim and the reasoning to recognise various mistakes and misunderstandings as stepping stones towards the eventual understanding and successful completion of educational goals.

Splitter \& Sharp (1995) demonstrate what teachers of Philosophy for Children see regularly: the emerging of a more mature and reflective student self; the ability to engage with others fairly; the valuing of good thinking; ability to learning from one another and together; and the development of the ability to recognise one's own faulty thinking and to decide on relevant change. The process has been called self-correction in the Philosophy for Children movement. This, then, becomes ethical inquiry and ethical education. Anih's (1992) term becomes understandable: fallibilistic flexibility. My students in Ethics Studies in year-2000 classes were fascinated by the term, and it proved an invaluable teaching tool!

Part of the value of engendering a culture of resilience is to give due attention to the crucial role played by questions which serve to stimulate the culture of reflection as a means of reaching forward towards resilience whether because of dependence, independence or interdependence in democratic learning and responsible decision-making. There is a direct link to the emergence of brain-compatible teaching strategies and techniques for teachers (Costa, 2000; Fogarty, 2001b; Fogarty \& Pete, 2003; Fogarty, 2005) that is bolstered by the research and investigations of Greenfield (2007, 2008), Doidge (2007) and many others. The impact on how our understanding of what is to count as ethical decision-making, in Wilson's terms, is beyond the scope of this investigation. It is enough, here, to point to a crucial existing link.

In terms of the commonplaces of the curriculum, this category provides the practical basis for twenty-first century dimensions of education; it impinges on the milieu in which the curriculum is delivered. 


\section{Categories of Influence 4: Input from research theorists and the shaping of the curriculum}

Greenfield (2004, 2007, 2008, 2011) has been involved in investigations of research into the way the brain operates. Her keynote announcements at the Thinking Conference in Norrkoping, Sweden in 2009, alerted the many listening educators to a need to collaborate and compare existing educational principles and mores with her twenty-first century findings. In particular, she indicated a need to accommodate the changes in modern living conditions, which appear to change physical components of the brains of children because of their daily use of computer technology. The subject matter she raised in her address to the Australian National Press Club in 2011 adds to the complexity of educational responsibilities.

It is imperative that we include emerging new knowledge affecting how the brain learns best: Lipton (2008) has pursued how the brain is a link between mind and matter in a definitive way, whereas Dispenza (2007) especially in his Introduction to that work) has emphasised the development of how the brain itself is capable of evolving from one generation to the next, and in response to regular conditions of living. Doidge (2007) has made substantial progress in research in the broad spectrum of how the brain responds and expands as needed for relevant personal trauma and conditions like mood management, learning difficulties, how the brain learns, memory recall and brain retention abilities.

There are ethical elements, emerging from my research, that can integrate and operate within the reshaping of the curriculum; these considerations are conditional on how teachers:

- Teach nuanced and age-specific thinking skills to all students. These are already operative in the program of Philosophy for Children, originated by Matthew Lipman. Scope and sequence steps are already in existence.

- Identify the practical application of thinking skills as a mark of democratic practice. This is the kernel of the Community of 
Inquiry in Philosophy for Children. The format is followed as a means of expanding the strategies of Inquiry-based teaching and learning to cover the exploration of virtues, values, and ethics. Elements of Thinking Styles are explored in detail by Lozano (2001) including the forms: monarchic, hierarchic, oligarchic and anarchic. This is a theory which opposes and contrasts with de Bono’s idea about Thinking Hats (1987, 1992, 1993, 1994, 1996), with long-familiar See/Think/Act systems; my six stages of decision-making process (see Chapter 4); and Stop/Reason/Try experiments that feed into Hargreaves $(2003,2009)$ and Crowther (2010; 2010; 2011).

- Encourage and actively teach students to develop and construct relevant questions. Relevant questioning can begin with the questions of how, when, where, why, what, which, who and what if? I have found it to be a good exercise to introduce this activity on a regular basis early in the new school year so that students recognise and expect to be provided with new thinking skills. Questions can be either open or closed, but each question will provide steps to further the inquiry relevant to understanding of concepts, knowledge bases, and ethical behaviours (Wassermann, 1997). Along with the naming of a particular skill from Splitter \& Sharp (1995), it is possible to incorporate into each topic the modelling and practice of the construction of questions.

- Make the necessary connections between the intentions and preferences of the individual, and the virtues and values that inhabit that person's lifestyle, without intrusions into privacy issues. This will allow students to recognise the links to ethical behaviours, and the need to know the reasons for action or inaction. Questions then take on a particularly interesting and central role and function as part of the thinking skills and decision-making process applicable to stages in career or aspects 
of lifestyle (Dewey, 1944; Wilson, 1971; Singer, 1979; Laurence, 1991; Riso, 1992; Singer, 1993; Rohr \& Ebert, 2009; Singer, 2009) and provide an opportunity to address twenty-first century ethical concepts such as globalisation (Singer, 2002); preventive war (Fukuyama, 2007); human rights and children's understanding of justice (Hicks, 1997); and the recognition of the overlaps between the concepts of evangelisation and indoctrination (Hodgkinson,1991; Stead, 1996).

- Use these skills in practical application in all subject areas and daily primary or secondary home-room administration time, so that students can grasp, understand and share meaning in a tolerant, respectful and intelligent manner (Fogarty, 1990, 1991, 1993; Costa, 2000; Costa, A., 2001b; Fogarty, 2001a; Greenfield, 2004; Fogarty, 2005; Doidge, 2007; Greenfield, 2007, 2008; Lipton, 2008).

- Teach what efficient reasoning is and is not; how illogical responses limit creativity; how indoctrination defies human rights; how decision-making leads to personal action, resulting in personal responsibilities, personal response-ability, and personal respect (Snook 1972, 1975; Hirst \& Peters 1970; Singer, I.B, 1983; Sacks, 2000a, 2000b, 2003; Mackay, 2004; Mackie, 1977).

- Develop further curriculum content, pedagogical procedures and assessment strategies because they become vital. Crowther's (2010) fourth dynamic makes such an ethical aim manageable. Crowther's fourth dynamic refers to deepening meaning using pedagogical strategies and techniques.

- Include the teaching of concepts leading to a compilation of a Moral Components List equal or parallel to that of Wilson (1971): through historical research, through relevant knowledge, and through Community of Inquiry processes for successful decisionmaking expertise (Wilson, 1965; Lipman, 1967; Wilson, 1973; 
Wilson \& Straughan, 1983; Wilson, 1986, 1990; Vardy, 1998; Wilson, 1998; Garmston \& Wellman, 1999; Vardy, 1999; Costa, 2000; Wilson, 2000; Costa, A., 2001b; Lipman, 2003).

- Integrate the ontological concerns, epistemological matters and technical pedagogical strategies and procedures set out in the Vardy's Religious and Values Education (RAVE) programs through the agency of the accompanying journal called DialogueAustraliaNetwork (DAN). This will enhance teacher and student evaluations that can then be developed so as to provide relevance and expertise in the application of thinking skills and the achievement of learning outcomes, especially with regard to expanding the identification and understanding of ethical behaviours (Lipman \& Sharp, 1978, 1980a, 1980b; Lipman, Sharp \& Oscanyan, 1980; Hoy \& Miskel, 1982; Lipman \& Sharp, 1985, 1986; Moore, 1986; Lipman \& Gazzard, 1988; Splitter \& Sharp, 1995; Sharp, Reed \& Lipman, 1996; Costa, A., 2001b, 2001a).

Greenfield (2007), in particular, poses the problem whereby the current generation of students has very little response on an empathetic level to what they see either in the real world or the virtual world. The philosophy and the psychology of education, even the pedagogy of all teachers, are affected if her claims are capable of universalisation. If not, there may then be a need to analyse and understand how current teaching efforts may need to be radically different to suit such altered student needs. The teaching of social justice, democracy, respect, resilience, and ethics would all need to be structured differently, allowing for the actual teaching of each virtue, value, ethic to be delivered first as a concept, and then developed as a possibility for personal examination and internalisation, before being allowed to germinate in the students' minds as possibilities for action.

If democracy becomes a reality in our schools, we will need to stimulate and foster decision-making for students. Study of English novels would need 
such underpinning of what is possible and what can be internalised. The teaching of philosophy, psychology, art and design, even politics and economics, would require adjustment to incorporate this phenomenon. Religious or secular culture would contribute to deep learning. Home- room discussions and Life Skills classes would also be part of the larger effort for harmonious learning about what is to count as ethical behaviour.

When a positive climate reigns, everyone learns, skills in consultation and prediction are promoted, and problems, paradoxes and puzzles are managed or solved. The efficiency, effectiveness and effort needed for this framework to be inserted will begin with teachers who are confident in their ability to teach all of the thinking skills.

\section{Specific linking of brain research, neuro-plasticity and decision- making}

Attention can also be paid to the research by Doidge, Lipton, Dispenza, and Burns (2010), regarding the plasticity of the brain. The connections between listening and learning, experimenting and learning, memorising and learning, all need further expert analysis by educational authorities able to develop the links between the present evidence and the consequences for teaching and pedagogical expectations. While conferences and learned papers have already paved the way, there is a need for further concrete research. The perceptions of teachers revealed in the responses to my Questionnaire do not include such content.

Wilson attempted to shape the preliminary thinking consonant with the virtues, values and ethics of the cultural milieu he inhabited, with his Moral Components List which he meant for students to analyse. Lipman, with his Community of Inquiry, provides a similar channel but also includes the quest for community in his democratic approach; and an insistence of developing the art and skill of questioning. Vardy and his Five Strand Approach to the Studies of Religion and Values Education, with teacher development resources and construction of curriculum content for senior secondary 
religion and philosophical studies, is proceeding with the same educational aim of deep understanding of virtues, values and subsequent ethical behaviour, according to both religious and secular principles. Connecting the emerging new knowledge on how the brain works and learns, with the need to learn, not only subject content, but also Life Skills programs, leads to reflection on how decision-making is performed in an individual's life.

De Bono (1987) provides a set of simple effective patterns often used at primary and early secondary levels. Underlying this approach - which Roger Sutcliffe, in his response to my Questionnaire, deems to be somewhat mechanical - is a dependent attitude. It is therefore a feature of this analysis that the Heasly Six Steps of Decision-making give space for reflection, as well as interdependent and independent exploration for the maturing senior secondary and tertiary student, to enable them to find the subtle, sometimes contradictory elements that intrude into their life decisions. These attitudes rest on the growing understanding of concepts of individual virtues, communal values and subsequent ethical behaviour (Heasly, 1995).

There is an expectation that clear democratic thinking, using the whole array of thinking skills that are outlined especially by Splitter \& Sharp (1995), and by Costa and his colleagues (Costa, A., 2001a) contribute to the highest effectiveness and efficiency. Peter Singer (1979, 1993, 2002, 2009), Mackay (2004), and Shaun Carney (2009), comment on the lack of empathy in a pertinent indicator of their concern. Carney objected to a skit on a TV program that was supposed to be humorous when it ridiculed dying children. The lack of empathy expressed in this program underlined the point Susan Greenfield made at Norrkoping.

In terms of the commonplaces of the curriculum, this category provides a practical basis for the foundation of twenty-first century thinking that affects constructs about knowledge and the thinking curriculum; it impinges on the teachers who are delivering the curriculum. 


\section{Categories of Influence 5: Constructs and knowledge about the thinking curriculum}

Wilson provided the Moral Components List, but did not proceed as far as providing a precise curriculum in scope and sequence proportionate to the levels and grades of the education system. Having completed the theory and used the content in his own classes at both secondary and tertiary levels, he left it to others to develop the actual curriculum aspect.

Lipman provided the Community of Inquiry and the accompanying expansion of the understanding of the role and function of questions in the process of Inquiry. He followed this up with novelettes that were designed for particular year levels exploring certain questions about life, goodness and how to choose to live well.

Vardy began where Lipman and Wilson left off. Wilson had provided the concept development directions; Lipman had provided the pedagogy of the necessary thinking skills and the new knowledge; Vardy chose to focus particularly on the development of Constructs about the thinking curriculum and about learning at the upper level of secondary education.

In the work of each of these theorists, the general focus is distilled and extended for young and older student study levels. Greenfield (2007) talked of changed dendrite patterns in the frontal lobe of children who were exposed to strong visual and aural stimuli beyond their very young brains' capacity. The result, Greenfield indicates, is an apparent inability to develop empathy, because the immature brain capacity can not process the feelings that usually accompany the fast-moving images, and a blurring of the real and the virtual takes place. So education must teach this consequence and link it to virtues, values and ethics. There is no catch-up capacity in Greenfield's terms. 


\section{Categories of Influence 6: Medical or biological and philosophical theorists lead us to Edu-Tensegrity}

It is important to recognise the epistemological connections between the neuro-scientific research of Kagan (1994) and educational research of Costa and his colleagues (Costa, A., 2001a). The consequence of brain research investigations has led to this team providing valuable new knowledge and ongoing development of pedagogical strategies and techniques that, for teachers, link and build on Greenfield (2007), Doidge (2007), Dispenza (2007) and Lipton (2008). They develop a deep and clinical approach by research towards a better understanding how the brain operates. There are significant ramifications for teaching, far beyond the framework of this investigation.

The edu-tensegrity concept that underpins this enterprise, explained in Chapter 6, gains its momentum from the premise of Aristotle: that logic and reasoning are needed to fashion precise and descriptive definitions of what is to count as a virtue; what is to count as morality (or in today's language values); and what is to count as Ethics. It also gains immeasurably from the integration being attempted with the work of Greenfield and others, noted above; from Wilson, Lipman and Vardy; and from Costa and others, also noted above. The edu-tensegrity concept also gains its plausibility from the work of Dewey (1944), whose ability to tease out the meaning of education in the last century, was so central to teachers' understanding then and now.

Finally, the edu-tensegrity concept benefits from Schwab’s (1983) insistence that the internal balance of education rests on the equality of the student, the teacher, the curriculum and the classroom milieu: what he calls the four commonplaces. To reiterate, Schwab (1983, p. 240) gives us a dramatic definition of what is to count as curriculum:

Curriculum is what is successfully conveyed to differing degrees to different students, by committed teachers using appropriate materials and actions, of legitimated bodies of knowledge, skill, taste, and propensity to act and react, which are chosen for instruction after serious reflection and 
communal decision by representatives of those involved in the teaching of a specified group of students who are known to the decision-makers.

The commonplaces that he insists need to be equal are teacher, student, what is taught and the milieu of teaching-learning (Schwab, 1983, p. 241) but I point out that my concept of edu-tensegrity applies as the push-pull factors of differing needs at differing times by the state, the students, or the intended value of education for all.

Without, however, the integration of momentous changes being flagged by indications outlined in this Chapter, there is still a gap between the expected teaching-learning process of mid-twentieth century educational processes and the need for accuracy and precision for changed twenty-first century circumstances. The connection between education and ethical behaviours is enhanced by the integration of these considerations. They are an urgent wakeup call and are integrated firmly in the concept of edutensegrity.

In terms of the commonplaces of the curriculum, this category provides practical applications obtained from historical and philosophical theorists; it impinges on the teachers who are delivering the curriculum.

\section{Categories of Influence 7: Constructs about meaning, reasoning and choice}

Constructs of depth and importance about meaning, reasoning and choice, are central to the experience of students. Wilson, Lipman and Vardy build on Aristotle, Dewey and Schwab. They become the foundations upon which Greenfield and others directly work in neuro-science, as well as Costa and others, whose creativity is pointed sharply towards constructing a braincompatible learning ethos in the classroom. These threads emphasise the concept of architectural tensegrity, where tension and compression keep the building from collapse. The parallel in medico-biological considerations, in growth of cells, is in Ingber's (1997) terms, called biotensegrity, and this 
provides the foundation for my concept of edu-tensegrity. These threads are all crucial to my twenty-first century concept of what is to count as Education. The concept today is already embedded within our daily experience, almost unconsciously. Reshaping the focus for best practice will change the conditions of teaching, but will not obscure the modern relevant subject-based curriculum; it will point directly towards meaning, reasoning and choice.

The perceptions of teachers indicated in their responses to my Questionnaire, seem to indicate that questions have a simple role in the teaching-learning process: perhaps questions were useful merely as convenient ways of bringing a student back on task, or of advancing the understanding of information at a given point in the lesson. Wasserman's (1997) work indicates to me in the following ways:

- The teacher prompt [question] can be applied by the teacher at any time during the lesson.

- The provision of links, distinctions and connections are present within a curriculum study.

- The opportunity to recall previous or existing knowledge in pretesting or post-testing activities is paramount.

- The opportunity for the teacher to demonstrate and model how broadly questions can expand any investigation or analysis or review.

- The chance to allow the teacher to provide scaffolding for student use, that is, for the teacher to insert an activity to compose a list of maximum relevant questions before student research commences: How? When? Where? Why? What? Which? Who? Even What if?

- Once the students have composed or constructed the questions starting with the relevant word in this list, they are equipped to investigate the main titular question.

It is useful also to distinguish between the role and the function of questions.

The eight functions or aims of questions, to me, will include: 
- A direct opening for student stimulation to recall previous content before proceeding with new information.

- An opportunity for both teacher recall and student reflection on the content in the process of learning to that point.

- A valuable chance for careful clarification to avoid confusion and contradiction.

- The requirement for students to learn what is involved in overview and to review material when involved in personal or group work.

- Provision of the opportunity to promote innovative student thinking.

- The identification of links with other topics and considerations in the manner of Plato.

- The identification of logical progression and consequences pertaining to new content and how it is integrated, in the manner of what Plato related of Socrates.

- The efficient and effective use of questions provided by the teacher (Fisher, 2001, 2003) as well as the questions arising from the list mentioned above to prepare for personal research.

The teacher participants whose answers to my Questionnaire are given earlier in the study have structured diversity into their strategies by providing students with the channels for learning: hearing, listening, internalising, recognising, understanding, learning. Kerri states this in her use of the construct of the multiple intelligences to underpin her classroom strategies. Her ethical process is promoted through repetition, recapitulation, recognition, reorganisation and relevance to the new material and with the previously accepted and understood content. This is demonstrated clearly by Samantha, who uses thematic approaches, linear development of the topic and inquiry-based learning, to enhance the opportunities for learning in her classroom. 
The four perfections of Maimonides (Singer, 2009, p. 68) highlight this construct:

- perfection in the acquisition of worldly goods

- $\quad$ perfection of the body, its conformation and skills

- moral perfection - the highest degree of excellence in man's character

- true perfection (and highest) - the possession of the highest intellectual faculties.

The teacher will facilitate and teach about the connections between attitudes and dispositions in a democratic classroom. Lipman's Community of Inquiry is a precise process, beginning with the listing of all questions generated by all students, followed by a process of grouping the questions. And all of this prior to the beginning of the actual research directed at formulating the knowledge sought and taught. Adjustments relevant to cultural mores in different parts of the world would, of course, apply.

Given Greenfield's indications, Costa's habits of mind, Aristotle’s insistence on logic and reasoning, ethical decisions based on vexed questions such as racial and sexual discrimination and the hurt and distress they deliver, are at least exposed. By use of applied thinking skills, it is even possible that voting patterns could be based on such concepts of virtues, values and become ethical decisions in their own right. We could have a series of resolves within education that would deepen knowledge about what constitutes a virtue, what constitutes a value, what constitutes ethics. It is also the foundation of democracy as practised by those who expect the realisation of the most good for the most people and the generosity of all to support those who need a helping hand. It is Virtues and Values in action. Virtues and Values in action are, according to my earlier definitions called Ethics. Dewey (1906) wrote extensively about the links and connections between these concepts, referring to the imperative of experiential education. Bruner (1960) also distinguished the forms of intuitive and analytic thinking as necessary for deep learning. 
This aim fits harmoniously into the Crowther six dynamics especially under Dynamic No. 4 of COSMIC C-B. School Wide Pedagogy is his aim. It is also the aim of the reshaped theory of education being constructed in this enterprise. Further development of Crowther's thesis is set out in ACEL 2011 four editions by Lewis and associates. Dynamic no. 4 is Micro-pedagogical deepening. The new pedagogical reshaping emerging in my theory of edutensegrity, which has been explored in this and the previous chapter, I claim, shows promise of attending to those challenges that Crowther says, seem unsurmountable.

It is worth examining Crowther and his colleagues’ (2010) assessment of this phenomenon:

\footnotetext{
Schools that are seeking to enhance their outcomes frequently get to the point of making a commitment - and then stall. They stall because of a lack of capacity to establish a sense of coherence. Some get to the point of establishing coherence and then stall. They stall because of the challenges involved in generating a vision and a SWP [School Wide Pedagogy]. Some others manage to create a vision and SWP - and then stall. They stall because the challenges involved in extending SWP into classroom practice seem insurmountable.
}

Armon \& Dawson (1997) provide a glimpse of the process of what happens when the teacher within Crowther's CONSTRUCTS project has become a teacher leader. The sustainability of and capacity for inserting meaningful change for students and for teacher-colleagues is a vital component of the professional development required within twenty-first century education; it also underpins the challenge of the whole theory and concept of edutensegrity. Taking account of Senge's (1992) Fifth Discipline and the accompanying resource (Senge et al., 2000) allows for the development and integration of thinking skills and systems thinking for the classroom, through the use of leadership skills and with consequences for teaching. A valuable conversation between Howard Gardner and Senge provides for a firm understanding of the complexity faced in the teaching and learning process. 
Edu-tensegrity feeds into Senge's concept of change and sustainability in school development, especially through the practice of stated steps supporting each school as organisational change is developed.

Various efforts are made by teachers to enhance flexibility in thinking; towards applying past knowledge to new situations. But success rests squarely on the ontological concerns, the epistemological matters and the technical consequences highlighted in the major schema titled edu-tensegrity - where all the Categories of Influence contribute to the geodesic dome of education that represents best practice, by reshaping constructs and practices regarding meaning, reasoning and choice. The push-pull factors we see in the history of the last five decades of Australian Education need to be in balanced tension to accomplish deep and rich learning (Chapter 6).

In terms of the commonplaces of the curriculum, this category provides the practical basis for meaning, reasoning and choice; it impinges on the students who are engaged in the learning and the teachers who are delivering the curriculum.

\section{Categories of Influence 8: Teachers and teaching}

Teachers find deeper appreciation of the achievement of a process when they master the broad spectrum of thinking skills in a focused ethical way. The Philosophy for Children movement of Matthew Lipman and his colleagues will ultimately be streamlined by these informed and practised teachers. Two of the three experts who responded to the Questionnaire did in fact recommend the acquisition of the specific expertise practised in the Community of Inquiry which is the essential central kernel of the Philosophy for Children pedagogy. I remember that Lipman confessed in a phone-call twenty years ago that he began his extraordinary journey in thinking skills when his grand-daughter asked so many questions as a three-year-old child. The process of questioning can once again be seen as the catalyst for knowledge acquisition. 
Questioning is used as introductory strategy so often that analysis of this matter is warranted. Wassermann (1993) gives responses to six mental traps for teachers to locate in their students' responses:

- Look for assumptions. Learn how to find them and teach students the same approach.

- Be cautious about generalisations (don't exaggerate from the data). The teacher models and shows students how assumptions and generalisations work.

- Avoid seeing things in extremes; as dichotomies (look for shades of grey in data). Again, teacher facilitates how students can do the same, recognising the effort to avoid dualistic thinking.

- Be alert for dogmatism and close-mindedness. (Watch for overcertainty, lack of caution, being unyielding, and allow for ambiguity). Vigilance must be exercised to teach students. Similarly - use questions to highlight where dogmatism and close-mindedness appears.

- Discern personal bias: - opinion versus evidence; value judgements may be unfounded; look for stated reasons. Questions can highlight and discern students' weaknesses in recognising cause and effect or false reasoning. Take time to think: look deeply into facts and evidence, take time to come to authentic conclusions, evaluate thinking processes and enhance meaningmaking.

Teachers need to be sure that education does not become part of the problem of teaching the curriculum content rather than the student, rather than part of the solution. Teachers' perceptions of the value of the topics that they teach provide them with the opportunity to evaluate thinking skills for the existing curriculum. Flexibility of mind, flexibility of thinking, flexibility of action: all these are education; the marriage of the culture of reflection and the culture of resilience. In the engendering of a culture of reflection and a culture of resilience the distinctions between competition and co-operation, 
co-ordination and individual responsibility, relevance and resilience, must all be made clear.

The first step of a Cycle of Inquiry, I recommend therefore, is to choose the various stimuli that will aid introductory impulses for a new topic or stage in the learning process. The reason is important because the question starter can provide a broad palette for enquiry. These stimuli can be grouped into several categories that will support the teacher-administrators' teaching experience; the outcome from using the responses to the Questionnaire are shown in Figure 8.1.

FIGURE 8.1 TEACHERS USE OF STIMULI IN CLASSROOM

\begin{tabular}{|c|c|}
\hline CATEGORIES & STIMULI USED IN CLASSROOM \\
\hline \multirow{9}{*}{ Graphic Organiser } & Brain-storming \\
\hline & Concept maps \\
\hline & Continuums \\
\hline & Corners/ Jigsaw activities \\
\hline & Mind mapping \\
\hline & Research task \\
\hline & Student interest introductory activities \\
\hline & Think Pair Share activities \\
\hline & Thinking Keys \\
\hline $\begin{array}{l}\text { Question or interrogation e.g., } \\
\text { hypothesising, testing, clarifying }\end{array}$ & Specific clarifying questions: teacher- or student-based \\
\hline \multirow{7}{*}{ Story } & A fact \\
\hline & A quote \\
\hline & Anecdotes \\
\hline & Case studies \\
\hline & Chalk and talk presentations \\
\hline & POE (predict, observe, explain) activities \\
\hline & Presenting problem or problematic situation \\
\hline \multirow{2}{*}{ Text } & Text \\
\hline & Written source material \\
\hline \multirow{8}{*}{ Visual } & An experiment \\
\hline & An object \\
\hline & Artwork \\
\hline & Comprehension activity often a newspaper handout \\
\hline & Venn diagrams \\
\hline & Video \\
\hline & Visuals \\
\hline & Word posters \\
\hline
\end{tabular}


Many teachers will be familiar with the seven points of the cycle of pedagogical delivery in the classroom. These are central to the technical strategies and techniques presently included in the thematic sections concluded and collated in Chapter 4. Of these introductory or randomly placed activities, teacher and students first pursue the aim of analysis for understanding, either of a skill or of an experiment, or of recognition of meaning, reason and value - Roger Sutcliffe makes this clear in his response to Section C of the Questionnaire, when he asks of students whether a workin-progress could be better:

My basic approach to teaching questioning is to open students' minds to the nature and importance of philosophical questioning, that is, questioning of meaning, reasoning, and value. There also needs to be an element of creative/radical alternatives, best captured in the overall strategy of asking: Could this thinking/ acting/ product be better?

Figure 8.1 (above) is the next step in developing the Cycle of Inquiry (which began with Figures 7.2 on pg. 268 and 7.3 on pg. 273). Taken together, ethical and courageousconnections of all kinds can be made:

- between presented questions;

- between contradictory content;

- between early and later thinking about the stimuli;

- between what students may have understood before and what the stimuli is indicating; and

- between different thinking skills that operate to uncover the next step in the cycle, depending on whether the recognition of meaning, or of reason, or of value is being pursued.

This has been thoroughly explained by Splitter \& Sharp (1995), who state that a

willingness to entertain and confront divergent views, paradigms or even whole cultures is a crucial aspect of different forms of inquiry (historical and scientific, for example). 
It is not surprising that Philosophy for Children should give a special place to dialogue - that is, the art and craft of speaking with one's peers about topics on which there may be substantive disagreement.

We can teach children to look for unstated assumptions and inferences when they read, and to explore conceptual boundaries and possibilities when they write, as long as reading and writing, like talking and listening, are regarded by them as genuine forms of inquiry, and not simply as mechanical activities by which they acquire, record and report information.

Both teacher participants Hyacinth and Samantha stand out with their lists of the stimuli used to support this particular strand in the Cycle of Inquiry. Franco indicates that he is clearly looking for children's responses and a flow of ideas. As Matthew also responded in the questionnaire, the model that we as practitioners of the Community of Inquiry use allows students to engage in higher-order thinking.

The third step in the Cycle of Inquiry is the emergence of questions based on any links and contradictions or gaps in the stimulus material. George indicates that he could go either way in a discussion depending on the level of interest. Teachers recognise this phase by using these efficient strategies and they expect many questions: to clarify, to test for authenticity, to draw distinctions and connections; to pinpoint relationships such as part-or-whole, means-or-end, cause-or-effect, classifying and categorising: unavoidably dualistic as a starting point but necessary for broadening and for correcting one's own misconceptions as the emerging questions provide recognition of new knowledge (Splitter, 1995). Pietr, in Physical Education, describes his process of questioning: 'Post a question relating to the topic, and ask students to stand in a certain place depending on their point of view; to the new and then discuss and break up, then re-discuss'.

The fourth step in the Cycle of Inquiry is one in which the teacher begins to help students to make stronger connections and to classify the central and relevant concepts, sub-topics and apparent contradictions (Splitter \& Sharp, 1995). The teacher will be 'fishing' for more information, as indicated by teacher-respondent Terence, with his use of a series of comments and 
contributions designed to highlight the meaning, the reasoning and the value of the new knowledge. Kerri uses a general approach, employing brain storming techniques and mind-mapping on a white board, or group tasks.

The fifth step is used exclusively by teachers to develop a climate of discussion, by challenging students to decide for themselves the most important elements of the emerging questions (Splitter \& Sharp, 1995). Teachers then begin helping the students by posing carefully-worded questions to distinguish the lesser problems that can be addressed after the main problems have been dealt with. In this way, both teacher and students are using the kinds of questioning that lead to the prioritising, commenting, responding, categorising, analysing, synthesising and contributing of answers to ethically advance the investigation.

The dynamics of this step can be supported by the teachers' expertise in generating an easy investigatory style; according to Giovanni: I ask questions, they ask questions and we discuss it’.

The sixth step in the Cycle of Inquiry operates after clarifications and exploratory questions have enabled a grasping of the content by class members, remembering that the Community of Inquiry includes the teacher as teacher, facilitator and learner. It is mainly an oral/aural set of activities. Discussion (Splitter \& Sharp, 1995) now becomes more focussed around an exchange of information about parts or about the whole of the topic.

In a seventh step, Connie and Georgia both use questions, posed randomly throughout the class discussion, for retention and recall so that students can construct their own knowledge during and from the discussions. There is an interchange of thinking about deeper questions (Splitter \& Sharp, 1995). There is an emerging integration of Constructs. There is discussion and debate, either formal or informal and on the spot. Teachers can organise a seminar to further student internalisation of meaning, of elements of the content material, and of the likely major questions that appear to need further research. 
In the UK teaching milieu, particularly, there is a recognised teaching strategy which employs a series of introductory 'starters' activities followed by main activities. Frank states that he has a distinct framework for the teaching of Philosophy in education. He describes how thinking skills are taught in starters and main activities and introduces these thinking skills during the starters. Stimulus strategies include use of video, anecdotes, questions in all their forms, and written exploratory work.

- Paul expects students to evaluate, make abstractions, and use generalisations and to associate. He appears to imply that he wishes students to associate in as many ways as possible from the stimulus and from discussions which are also used.

- Susan expects her students to 'investigate/design/produce/ evaluate' their work as they progress in Technology Design.

- George expects students to 'keep an open mind, use different perspectives and allow for shades of grey’.

There are, therefore, embedded opportunities to measure the outcomes of learning. This embedded-ness is an underlying purpose for the use of stimulus organised by the teacher, leading to activities for the students to explore, investigate, question, develop a hypothesis, evaluate and eventually internalise the emerging knowledge. The familiar teaching-learning process is showing below the various strategies used:

- Dennis makes it clear that it is a recognised experience for the students that material is introduced, familiarising them with the implications; and that this is done by extensive discussion.

- Connie describes her process as a cyclical or spherical set of steps: the question is put on the board, pupils contribute. There is opportunity for teacher prompting and there are also chances for pupils' questions to 'trigger' other pupils to respond. Connie makes it clear that she uses question and answer, discussion and brain gym. 
- Rose provides an extensive list of stimuli for introductory and then main activities: Venn diagrams/concept mapping; debates; fish bowl group work; argumentative essays; POE (predict/observe/explain); open-ended questions; continuums; word posters; deep thinking challenges.

- Lavenda gives an interesting step-by-step process: Open questions; processing information; co-operative learning (groups and pairs); reviewing and applied reality testing.

Relevant consequences for student teachers whose own experience of education and schooling was in the climate of values-neutral classroom are the unseen influence which often permeates the classroom and has an unknown effect on the culture of classroom. Emerging elements of a multicultural classroom depend in my experience on the presence of migrant populations from a variety of regions in the world, and the possibility of refugee students whose reasons for being present are very different.

The significance of research in the field of understanding of how the brain works; how the brain learns; evolution of the brain-dendrite accommodation of technology; the physical impact of ICT experiences especially in early childhood on the structure of the brain, are all central to these considerations. To connect this content to Greenfield's statements and Kagan's research requires a taking into account of very new knowledge that impinges on present educational concepts from the perspectives of ethical behaviours, school leadership as well as applied thinking skills.

The possibility that Australia, or any other country, could begin to fashion a culture of giving (Singer, 2009) would not be feasible without the generalised enculturation of the reflection and resilience being proposed here. Today's scenario of individual acquisitionism, says Peter Singer, is directly contradicted by the teachings of Jesus of Christianity, and by Maimonides of Judaism. Singer (2009, p. 68) presents details of a study into how ethical Australians actually are. In today's Australia, other religious pundits would 
be included as well as Jesus and Maimonides. This would be a useful stepping-off point for teachers in discussing the wisdom of the ancients:

For millennia, wise people have said that doing good brings fulfilment. Buddha advised his followers: Set your heart on doing good. Do it over and over again, and you will be filled with joy. Socrates and Plato taught that the just man was happy. Epicurus did, too.... The wisdom of the ancients still holds.

Considerations such as these seem closer to the concept of holistic education than the present Australian trend to insert testing at every level and the intrusion of league tables, which are so easily manipulated towards unsound conclusions.

In terms of the commonplaces of the curriculum, this category provides a practical foundation for teachers and teaching; it impinges on the teachers who are delivering the curriculum.

\section{Categories of Influence 9: My curriculum}

The insertion of a number of relevant technical strategies and techniques, as tools for the teaching of applied thinking skills, to the matters surrounding learning about virtues, values and ethics, provides a contrast and a link to the responses identified in the Questionnaire.

It is significant that we all make choices based on either an impulse - a feels-right response in a situation of emergency - or that we take further time to mull over possibilities when something of real import is faced in life. It is also significant that there are a variety of ways that individuals arrive at those decisions. In Chapter 4, the detail of the processes of thinking skills is shown by my classroom instruments and grids. These are designed to highlight and improve the likelihood of successful and ethical decision-making by highlighting the various points of view along any spectrum and their consequences. 
The process of the Six Steps of Decision-making has been explained in Chapter 4. Teaching the practice of thoughtful decision-making for action describes the foundations of what Ethics is. I had success by using the cycle pattern (Figure 8.2 below) to insist that there is a distinction to be made between making a mistake or error of judgment, and falling into failure. It had the benefit as far as junior secondary students were concerned of being the same diagram of cyclical patterning that is used in the previous chapter for questions and questioning in virtues and values. Here it is used to follow the steps of an exploration, and where a mistake might be made by a student, the steps to avoid failure are embedded in order to produce a stronger or more appropriate decision or conclusion.

Students responded to both by discovering that there were two ways they could look at their own experience: one was in tiny time slots limited to one incident at a time. The other was to recognise that behaviour is often patterned because of our distinctive unique personality types. Their discovery was deepened when taking account of the Heasly HUG/BUG and its arc of possible consequences. The shift in understanding led to more reflection on students' own behaviours and, by degrees the students could see a much bigger picture of the world around them.

The 'ME' diagram explained in Chapter 4, reinforced this, and led the students to formulate consciously-developed reasons for their chosen behaviours - which is what decision-making is. Their action following this decision-making was as a consequence of stronger import for them and ethical behaviour became a live consideration as they realised how responseability was the starting point, and responsibility was the end point. Knowledge and skills, they concluded, were needed to address the ability to respond, before assuming the commitments of responsibility.

These six steps, shown in Figure 8.2, follow a cycle which is shown diagrammatically to illustrate that decision-making is ongoing, refining as we proceed; and inaccurate outcomes are maybe mistaken; but failure does not apply. It is important, I estimate, to recognise that failure is not an outcome. 
Failure happens when the individual gives up, collapses into inaction and bears uncomfortable consequences as a result. One or more action-for-

\section{FIGURE 8.2 CYCLICAL THINKING- THE SIX STEPS}

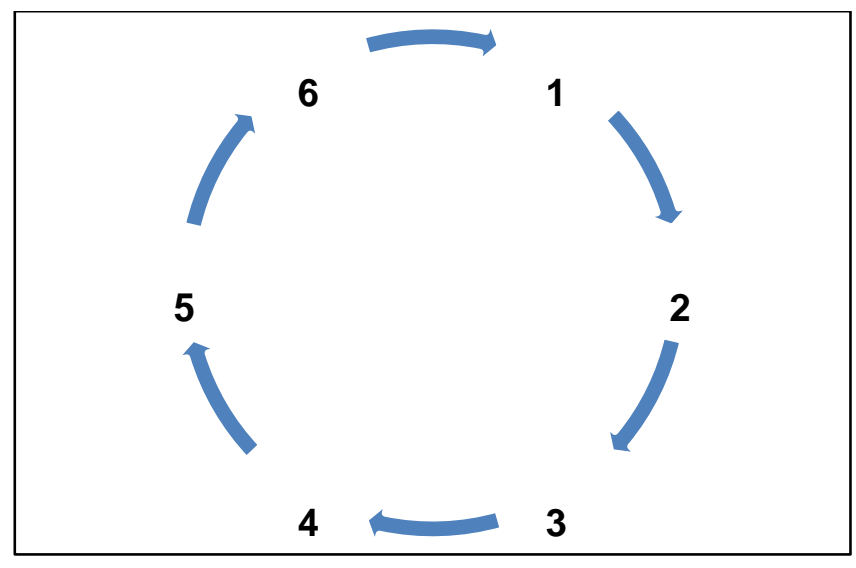

resolution steps can be a sign of patience, further understanding and resilience. A mistake is not a failure. Connections to the work of de Bono (1996) are important for broadening thoughts into concepts, emphasising skills in thinking (phronesis).

Of course, time is a constraint, but students respond in a remarkably positive way as they explore the insights that this instrument offers. It is not necessary to concur completely with all parts of the Heasly Uncertainty Grid (HUG/BUG). What is of value, though, is an understanding of all of the terminology sufficiently well to explore creatively all parts of the instruments with students. The student cohort needs to negotiate what is meant by Values, Virtues and Ethics in a changing world in which personal, national and international decisions will be central to their lives. This is not a measurement tool. It (the HUG/BUG) is an exploratory instrument designed to identify some patterns of human behaviour and the possible consequences of such behaviours. It highlights the potential for new knowledge for students and the potential for directing them towards an exciting future. 
The Grid (see Chapter 4, Figure 4.3) begins with the elements of personality in its centre column. A HUG is potentially a positive experience and a BUG is potentially a negative experience. This approach fits the Australian student vernacular and probably would resonate with New Zealanders and English students.

By dint of self-exploration of potential behaviour patterns, it is possible for students to check their responses in daily living for negative or positive results. Discussion and students' experiences combine to provide choices, stimulating students to recognise behaviours they may not yet understand fully, and allowing for their choice to be positive, i.e., perseverance and courage, rather that collapse and aggression; honesty rather than deceit. The concept of edu-tensegrity requires that these matters are as much part of a vital balanced curriculum as Mathematics, Music and Mapping!

In terms of the commonplaces of the curriculum, this category provides practical steps to new perspectives surrounding issues of dependence and rich learning; it impinges on the students who are engaged in the learning.

\section{Categories of Influence 10: Students new knowledge, understanding and expanded skills for learning}

In the previous chapter, attention was paid to the development of the Categories of Influence as they pertained to the matters of teaching about virtues and values. Ethics has been separated out because it is action as a result of the application of decision-making and the processes used to attain efficient and effective results through the integration of new knowledge, deeper understanding of the processes proposed and the resultant expansion of skills.

To reshape the curriculum towards the integration of the educational outcome of teaching the student as well as delivering the content of the topic or subject to be taught, teachers contribute each day to ethics in action. Students can be given the opportunities to analyse, inspect, interrogate, choose and conclude, just what ethics are to be acted upon. 
Wilson attempted to provide a channel, consonant with the virtues, values and ethics of the cultural milieu he inhabited, with his Moral Components List, which is meant for students to use for analysis and the construction of logical conclusions; Lipman's Community of Inquiry provides a similar channel, but includes the quest for community in a democratic and ethical approach to classroom discussion, and an insistence on developing the art and skill of questioning. Vardy, with his Five Strand Approach to the Studies of Religion and Values Education, offers the ongoing provision of teacher development resources and his wide-ranging construction of curriculum proceeds with the same educational aim.

In terms of the commonplaces of the curriculum, this category provides processes for resolving issues of dependence and independence; it impinges on the students who are engaged in the learning.

\section{Categories of Influence 11: Emerging connections of neuroscience to learning}

Greenfield (2007, 2011) alerts us to new considerations as we expand our education scenario for modern student cohorts. Previously, exploring the idea of changing perceptions on thinking, Fogarty and others (in Costa, A., 2001a) seem to have taken a lead by exploring the brain-compatible classroom. Curriculum must provide students with skills in thinking, as well as the subject knowledge, as relevant channels of learning. Greenfield, Doidge and Dispenza, separately, point the way, so that teachers can promote new abilities for learning and lifelong decision-making. In such a manner is the student equipped to develop a considered religious or secular belief system; the student is provided with life learning about what are to count as virtues, values and ethics, in order to build their future - what Wilson took from Aristotle, still called the 'good life'; the student is accompanied on the journey of planning and accomplishing an accurate choice of career and of personal future. 
For the purposes of this investigation, I define the concept of an ethic as the considered result or outcome in action (as well as a recognised system) by the individual in a daily contribution to one's owned culture, society, nation, or tribe. (There are deep and vexed issues in this question when migrant transitional behaviours, and refugees' problems of domicile, are taken into account; issues that lie well beyond the scope of this project). The outcomes of recognition and development of personal ethics can be employed to solve many of the dilemmas, questions, paradoxical situations and vexed problems of life, based on whatever the individual teacher or student considers and chooses as the best way to act. They include honesty, fair rather than foul play, social inclusion, social justice and active care for all forms of disablement. Regardless of ignorance about some relevant matters, these decisions are likely to be based on reason, impulse, previous experience, risk taking, needed outcomes, possibilities or obedience to previously internalised concepts; be they virtues-based or values-based; or religious, political or philosophical in texture. The outcome is new knowledge for the individual teacher or student.

To influence students in their deep learning, teachers know that it is critical to deliver content, including the possibilities opened up by neuroplasticity, in an accessible form, so that students can internalise the new knowledge, process and integrate the new curriculum content into existing knowledge, concentrating on expanding skills in both applied thinking and relevant strategies and techniques. Inspection of Barack Obama's presidential addresses is a good example for this aim.

The teaching of ethics across any subject areas requires significant consideration. Careful analysis of course, will be based on serious and authentic evidence towards the identification of personal and community values. Admission of evidence and the analysis level for Year 7 students will necessarily differ from that of Years 10, 11 and 12 and beyond, especially in tertiary levels. Both secular and religious mores are involved. The conceptual foundations used by Glasser (1969), Goleman (by himself 1996; 1998b, 
1998a; and with the Dalai Lama 2004, 2007) and Gribble (1969), have been analysed, developed and expanded by Snook (1972), Wilson (1967), Hargreaves (2003), and Crowther (1996; 2010; 2010; 2011) to provide new foundations for sustainability.

I propose therefore:

- The construction of a curriculum for Years 7 to 12 in each subject area, based on the various thinking skills of Splitter \& Sharp (1995), which can be carried out by means of the Community of Inquiry format (Lipman et al). By way of expansion, there are connections and links for curriculum content from Goleman (2007), Pritchard (1985), Nussbaum (2001) and Rich (1972).

- The connection focussed on the new format of decision-making about careers, the Good Life, and religious or secular eschatological speculations for clarity (Kaldor et al., 2010).

- Construction of and research into links to people in the poorest countries on any continent, including Australia, through channels like those that Singer has provided e.g.: Fair Share and Fair Trade. Singer (1979), Wilson (1987), De Botton (2004), Seligman (1990) and Sacks (2000, 2003) all highlight the ethical dimensions of what also accompanies moral mindfulness, moral instinct, morality, values, distributive justice in the Giddens (1998) approach, and parallel leadership in Crowther's (2010a) format of responsibilities and response-abilities. These considerations are present too in studies in Language acquisition, English, Social Studies and more.

- Compilation of a balanced mix of concepts, moral obligations and ethical arguments about a list of virtues, both personal and communal, e.g.: compassion, clear thinking, empathy; being moral, as well as physical: courage, strength and determination, honesty, generosity (Wilson, Vardy, and Hawkes) can be constructed. The use of the Community of Inquiry (Lipman, 
Fisher, Sutcliffe, Cherednichenko, Wilks and Susan Gardner) will allow ethical dimensions of subtle and diverse concepts to emerge as discussions and the teachers' consequent role become more useful, more exhaustive and more skilful (Crowther, 1996), but also transparently democratic, rather than indoctrinating in content.

- Development and use of realistic assessment tasks that reward processes of research, community action, leadership, innovation and consistency, will support classroom strategies. These imperatives appear to answer comprehensively the six dynamics of Crowther's COSMIC C-B (Crowther, 2010b). An underpinning philosophy of education that takes account of Howard Gardner's (2008) scenario of the five minds enhances Crowther's model which is needed for the future.

Discussion may take the form of small group interchanges in what has been called 'corners' or the 'jigsaw' process -Hyacinth suggested using corners and Samantha emphasised student-based inquiry and inquiry-based research in the Questionnaire responses. The small groups learn deeply about their particular allocated area of inquiry, but need to rely on the abilities of other teams for deeper understanding of the rest of the topic. The reliable teacher always has a list of questions to re-engage those whose interest may have waned: Rose employed her open-ended questions; Henry clearly challenges individual perspectives; George expects students to keep an open mind, use different perspectives and allow for shades of grey; and Magda always has a list of questions to re-start the discussion, or divert pupils when they have digressed. All manner of interesting elements to engage students' attention emerge in class discussions.

Discussion may also take the form of the Community of Inquiry, on which Matthew Lipman's Philosophy in the Classroom is formulated. Here, the discussion, using applied thinking skills to generate as many questions as 
are needed by the students themselves, is based on the expectation that every member of the class will provide a question, which is then recorded on the white board. The whole class helps to categorise, sort and group sets of similar questions. Selection of the main questions that are deemed to need immediate action happens quickly, and students organise themselves into groups responsible for extra research and information-gathering. The cyclical pattern that operates can delve deeper still, as students pursue the questions which they consider are not fully completed. As Hyacinth says:

\footnotetext{
Philosophy for Children the overall title of the Community of Inquiry as per Lipman provides a framework for teaching any topic, but is especially helpful in teaching a literary text. Again, this framework takes students out of their traditional roles and develops their thinking in a different environment and a different way. They take control of their thinking and generate their learning.
}

The blueprint of the six dynamics used by Crowther and his associates (2010) gives succinct direction to the value of focussed, sharply alert participants who keep the business approach and thereby achieve the best understanding out of any discussion.

Discussion also can be in the form of the 'fish-bowl', in which 10 to 12 students sit in a circle and conduct a discussion about a topic using applied thinking skills, identifying the questions and connections needed to provide for all the class members to have enough notes to further their own investigations. The rest of the class sit in an outer circle and take as many notes as possible. Lavenda describes a version of the fish-bowl, thus:

Give a scenario (true-to-life or actual incident) which has a variety of characters or stakeholders with different points of view/interests. Students are given roles in small groups, or a half-half class split, or 2 whole class situation (e.g., mock court case) and have a period to prepare their case or perspective. It may be that a mock jury makes a decision, or a mock council, voters or politicians. It may be a community group. At the end, 
students de-role and write up what and how they would decide (and whether their initial stance has changed or not).

It is worth taking account of Crowther's (2010 p. 39) 'Rules for Skilful Discussion' as they encompass some central pitfalls: the direct language helps to highlight each possible pitfall:

- Talk only to improve the silence. Occasionally stop talking, not only to others but to yourself.

- Balance advocating or sharing your position with inquiry into someone else's.

- $\quad$ Contribute to shared meaning - seek to clarify what others are saying.

- $\quad$ No ping ponging back and forth between two people.

- Talk into the space in the middle of the group.

- Do not interrupt others. Slow down the discussion and be patient.

- $\quad$ Speak only positively - don't be critical or disparaging.

- Be happy if the group reaches an impasse, because it is on the verge of a breakthrough - don't stop - keep the process moving.

- Let silence happen and see what occurs out of that process ... every now and again take a vow of silence.

This list provides a neat circular set of links that help teachers to construct the content they need. In all types of discussions, applying each of these rules focuses on the skills needed.

In terms of the commonplaces of the curriculum, this category provides the pedagogical processes that lead to constructing: an ethical belief system, the ethically good life, and an ethical, logical career; it impinges on the students who are engaged in the learning and on the milieu in which the curriculum is delivered.

\section{Categories of Influence 12: Collaboration between teachers, student and curriculum experts}

The outcome of education at all times must be predicated on the accuracy of channels of teaching and learning which openly and efficiently promote student achievement. The links between the roles and functions of teachers and curriculum experts provide for the teaching prowess needed. Immediate questions for the student to link into the learning scenario best suited for their success in education emerge. Collaboration, leadership and pastoral care feed 
into the links between student, teacher and curriculum expertise at every level of education. This is the single most important aim and is crucial for promotion of efficient, effective and rich learning in today's education scenario, though there will always be the student who does not accommodate this teaching-learning process.

Democracy and authority are part of daily life, yet seem dimly understood. Every effort is needed to facilitate students and teachers in acquiring the understanding and applied knowledge necessary to make both concepts manifest in educational terms, thereby strengthening bonds between teacher, student and curriculum expert. The boundaries between the two concepts need to be extrapolated clearly, so that their meanings and the reasons for possibility of both can be addressed to facilitate deep learning in matters of virtues, values and ethics. Diversity in responses to the Questionnaire showed that democracy and authority were seen by the teacher respondents as the opposite sides of one coin, but they are not the opposite of each other. This needs discussion to enable students' new knowledge to emerge. Students will be changed by the experience and understanding of the concepts of authority and democracy. It is necessary for them to recognise and clarify each element in relation to the other.

As a means of exemplifying those elements of rich learning that need to be delivered to students by direct work at appropriate times, and also by the modelling of such matters, I define democracy as a concept that can be constructed:

- An assumption of equal rights that permeates all aspects of daily life.

- An expectation of equal treatment between all members of society.

- A provision for the help and support of those with less, by those with more; and by everyone of everyone else.

- A regular analysis and free vote on matters of importance: justice, social regulation and possible conflict. 
- A free and respectful interaction in matters of physical, emotional, social, intellectual and spiritual concern for all members of society.

- A set of social institutions that is responsible for the setting up of such organisations as can be given authority to manage these concerns.

Similarly, the example of analytical processes that underpin the teaching of authority as a concept, I recommend that several precise points can to be made about authority:

- Authority in the classroom must by and large be earned, not demanded.

- Respect is the natural due of people in positions of authority, as distinct from the earning of authority through the recognition of learners and colleagues.

- Authority can be hierarchical, autonomous, shared, distributed, and team-based, but always according to the tasks required.

- Authority in one area of expertise does not automatically flow over to other areas of responsibility - individuals can be multigifted, and multi-skilled, but always the recognition of authority must be earned.

This set of requirements for democracy and the notions of authority in classrooms seem simple and achievable. Inserting knowledge of the boundaries, the links and the connections between the old paradigm (which existed prior to the contemporary learning conditions of ICT and brain research), and the new paradigm (in which new knowledge augurs new pedagogical imperatives) is central to this set of requirements.

I have found that teachers and students need to construct classroom strategies that will facilitate real educational aims and avoid unnecessary pitfalls:

- Clashes of expectations between teacher, students and parents may intrude. 
- Pressures of a full curriculum may exhaust both teacher and the student cohort as they strive to authentically finish the content in time for tests and examinations.

- Teachers may expand or contract curriculum content to prepare students for testing, without having time for reflection and questioning from the students. This is not to be judged as unethical, but it is unfinished.

- Some students may not be ready for the content of the given class, and cause difficulties for others already understanding the work.

- Extra-curricular, but central learning experiences may intrude unavoidably but necessarily into the daily timetable, with sometimes disastrous disruptions in student learning.

- Learning styles of students and teaching styles of teachers may not always be congenial, or cohere systematically, and may result in some students being further confused.

- Allocation of time for ICT research and the completion of tasks can erode the time available for general arrangements of assessment routines and timetables.

- Over-use of guidance by some teachers in the required steps to complete the delivery of learning can stifle the creativity of some students, who can become dependent on teacher time and teacher prompts for completion of tasks. That dependency needs to be balanced by experiences of independence and interdependence. Confusion between the roles of facilitation, counselling, proffering of advice, and advisement techniques can become blurred in both teachers' understanding and students' experience.

- Time for extensive questioning in either Socratic Method or the Aristotelian Method can be curtailed by a teacher giving answers that do not allow for internalisation and depth of understanding in applying of such knowledge to further problems in learning. The time constraints are consequently stressful. Socrates' style of 
analysis allows for any number of questions around a central problem or concept as students analyse and deepen their recognition of the underlying meaning as it emerges. Aristotle's style allows for the questions to flow into further allied areas of concern as students find ways of making distinctions, detecting links, uncovering contradictions and identifying flawed reasoning surrounding the content to be learned and applied (Fisher, 2001, 2003). Students and teachers need to choose together which strategy is to be used.

- The stress on teachers, including misconceptions from those outside the classroom who misunderstand the time needed for student resilience to become established. Students need periods of reflection as they internalise more deeply their understanding of concepts prior to applying new knowledge to more advanced requirements of the next stages of schooling (Sergiovanni \& Corbally, 1984; Sergiovanni, 1992).

In reshaping the curriculum, teachers will lead the impetus towards school leadership in efficient and effective student learning, by the inclusion of the instruments presented in this theory of edu-tensegrity and the considerations surrounding this thesis. Democratic and cultural practices will follow as the student cohort takes up the challenge to include the philosophical and ontological constructs of the new paradigm, the epistemological basis of new knowledge and the integration of these matters into an personal ethical perspective which makes logic and wisdom part of their considerations of the 'good life' (Aristotle, 1962). When supported by administrative and curriculum specialists, teachers' perceptions of their use of applied thinking skills will expand and students' appreciation of deep learning will be further manifested. Reference to Crowther's sustainability is central to the development of a holistic picture of what is involved in the challenge of inserting edu-tensegrity into today's classroom. So, 
consequently, are the considerations of Hodgkinson (1991) and Hospers (1982).

The teaching and learning process is the channel through which students are eased into the culture around them and are exposed to their future (Burns, 2010). It begins within the home, expands through educational channels both formally and informally as brain maturation proceeds (Heasly ME diagram). Costa and Garmston's Passions of the Mind (2001) and Costa's Habits of Mind (2001b) are balanced settings for learning outcomes. This knowledge base must include learning in formal and informal settings, as well as blanket acceptance of ICT and other electronic communications devices and technologies. This is the link between the old paradigm and the new paradigm.

By analysing Barack Obama’s books (2006, 2008), examples of a leader with clarity of thought is enhanced. We can delve into the principles proposed by Caldwell \& Spinks (1992, pp. 1-64), taking account of the reservations assembled by Smyth (1993) and identifying the perfections listed by the philosopher Maimonides (Zigler, 1999). We find the specialised themes about the importance of structure, readiness for learning, intuitive and analytic thinking and motives for learning. These are, of course, outlined by Bruner (1960), and support exploration for meaning.

Much about Virtues, Values and Ethics is already embedded within the curricula in myriad ways. Some careful tweaking could provide threads that would emerge for middle school students as a broad-based preparation for Year 11 and 12 studies of English, English Literature, Texts and Traditions, Philosophy, Business Studies, Commerce, Economics and Religion and Society. Ethical behaviour is so often built on the bedrock of embedded virtues, beliefs, and values bequeathed to us from earlier generations (Mackay, 1997).

Of equal importance and significance, the teacher actually introduces particular and relevant thinking skills from Splitter \& Sharp, and uses some of the activities within the planned curriculum content. The naming and 
modelling of the particular thinking skill takes but a few minutes, yet requires of the teacher a meticulous planning of what is and is not to be learned as a relevant thinking skill. If done efficiently, ethically and effectively, little extra time is taken at the beginning of class, especially as the process becomes an expected adjunct by the students. It also enhances the ability of the students to prepare for examinations, for project work and for research, as well as providing a basis for the successful composition of essays, presentations, dramas/plays and in mathematical design. This approach provides for conscious and rich learning rather than thin learning (Davis, 1995), and does not rely on the unpredictability of memory for its success (Heasly, 1995). The process can change how information is used by the students for achieving independent learning strategies applicable to lifelong learning pursuits.

The function of the teacher needs some defining to allow for the making of distinctions between the activities of facilitator, monitor, modeller, supervisor, mentor and instructor, especially in the face of multiple uses of published subject content, and also because of the new knowledge available regarding how the brain learns. It is possible to glean so much from the writings of Dispenza, Lipton, Doidge, Costa, Fogarty and others that merges and emerges by degrees, as the theory of edu-tensegrity is understood and applied.

\section{Connections of the various Categories of Influence to each other and the classroom}

Imprints of ontological, epistemological and technical considerations are now present as a result of the various influences that pervade this thesis. Those influences include the reshaped, underpinning Philosophy of Education, Sociology of Education and Psychology of Education, in order to fulfil the social contract of the teaching-learning process where each fulfils the teaching and learning. There is, I contend, reason to promote in this investigation the Categories of Influence which result from work of the three 
experts: Wilson, Lipman, and Vardy. If students are taught not just topics or units, then concepts of Virtues, Values and Ethics will be included, so that students are enabled to make independent decisions. This is the linking of applied thinking skills to students' progress by school leadership and the expanding practice of ethics and ethical behaviour. Historically, the various Categories of Influence have been addressed, applied and advanced since Aristotle, Dewey and Schwab, but these three theorists have significant content to offer for the accurate delivery of deep learning to students. Finally, as has been delineated earlier, I have, as practitioner, provided three instruments: The Heasly /Behaviour Uncertainty Grid (HUG/BUG), the Six Steps of Decision-making, and the ME diagram - all of which aid in the implementation of the various Categories of Influence. The links and connections between all the proposed Categories of Influence help to construct the architecture of the theory I call edu-tensegrity. This theory then is an attempt to formulate an educational basis for the new paradigm.

Perhaps Australian society expects that education will provide experiences of success in learning in the domains of virtues, values, ethics, community, democracy and, by implication the arts and disciplines of resilience, courage, foresight, maturity, empathy, independence and relevant learning - all of which rely on accurate, balanced decision-making. The links and assumptions that can be connected to the development of Emotional and Social Intelligence (Goleman, 1996) are central to student learning.

Unless these concepts and the accompanying skills are blended and delivered in an authentic manner, it is not certain that students will learn the skills of resilience and courage until life trauma challenges them - and that is often too late to help them negotiate the difficulties that surround them and is not the business of classroom education. There are already some impulses towards addressing this part of education (Senge, 1992; Hargreaves, 2009); (Wilson, 1963; Gardner, 1993a; Lipman, 1993b; Vardy, 1998; Lipman, 2003; Crowther, 2010; Crowther et al., 2010). The teaching of thinking skills that already forms part of the pedagogical practice of some teachers appears to be 
the logical starting point for today's instant generation and ethics education can explicitly hone their abilities and their understanding in these matters.

Education in philosophy and the history of ideas seems to be a valuable consideration. 'Educere' (going back to the roots to evaluate and learn) which is connecting with epistemological concerns and considerations, and 'Educare' (training in skills in all relevant ethical learning areas) which is connecting with technical (techné) strategies emerging, will be needed. This amplifies and influences professional development work by continuously stimulating the agency of curriculum corporations, internal school leadership programs, current research and especially the work of such specialists as the ACEL and their academic journal.

If we consider the implications for teachers set out by Costa (2001a), Kagan (1994), Fogarty (2003), Gardner (1993a), Ekman (2001, 2008) and Ayer (1973) the expectations are enormous. Who is to approach, analyse and deliver enough knowledge, learning, research and insight for each student? Concepts such as freedom, responsibility, necessity, moral luck, tolerance, moral mindfulness and perhaps others, connect with the Moral Components List of Wilson, and show how the teaching of concepts needs to permeate all content in the classroom. There is a need to investigate the role and function of teacher - as facilitator, mentor, supervisor, examiner, counsellor - in the lives of the student cohort. Crowther (2010; 2010) makes a significant contribution with their work on Parallel Leadership and the IDEAS project.

Teachers already display great knowledge, balanced approach and commitment to students, despite the sometimes overwhelming stresses they face. The demand to include more of the social and cultural capital that was initially experienced in the homes of two or three generations ago is used by Mackay (1993) and Singer (1979) highlighting the changes in educational scenarios that teachers may adapt to their classroom strategies. This adaptation is essential before even recognition of the implications regarding the changes in dendrite patterns described by Greenfield (2004), Doidge (2007), Lipton (2008) and Dispenza (2007) can be addressed. 
Decision-making steps can be learned. There are many available strategies which begin with the de Bono Six Hats system (de Bono, 1987, 1993) and progress through a number of other systems and gradually introduce the student to complex, abstract as well as concrete thinking skills. This ensures that the Gardner (1993) multi-intelligences are incorporated in the teaching-learning process. Care must be taken so that students do not assume that mechanical thinking patterns are promoted at the expense of the Gardner style of emotional intelligence, particularly noted by Goleman (1996). Roger Sutcliffe (in Chapter 4) alerts teachers to a different approach that can include reflection, emotional intelligence and values. There is also the need to investigate further the relevance of the work of Stephen Kagan (1994) of Chicago University where researchers have tried to identify how the brain learns. Using material to track progress in brain response from newborn onwards Kagan is discovering how the dendrites (electrical impulses within the brain) form and what stimulates the best conditions for rich learning. Costa's work (2000; 2001a) in this area is allied to Kagan's and needs to be taken into account when restructuring pedagogy and curriculum, which is though significant ethically outside the parameters of this study.

Costa and colleagues (2001), Lipman (1993b) and Vardy (1998) identify for us that seminars, teacher resources and ICT curriculum support are invaluable for expanding the teachers' repertoire. The democratic classroom relies on discussion, collaborative research, sharing of information, strategizing towards sound pedagogically accurate outcomes by using inquiry-based techniques and Community of Inquiry formats to attend to this urgent need (Fisher, 1990; Splitter \& Sharp, 1995; Vardy, 1998; Lipman, 2003; Golding, 2006; Hargreaves, 2009; Leckey, 2009; Crowther, 2010; Sutcliffe, 2010). As Davies (2005), succinctly says: 'we need to actively rethink, respond, reshape, reconceptualise, reconstruct and recreate'.

As detailed earlier, one particular skill needed by teachers in the classroom is the understanding of what constitutes indoctrination and how it differs in concept, understanding and teaching strategy from the delivery of 
higher order thinking skills. Both Snook and Wilson do their best to delineate the distinctions between the two practices (Snook, 1972; Wilson, 1972).

It is necessary to spend time analysing the actual concepts themselves (ontological approaches) so that the further steps of distinction are clarified. Teachers and students will benefit from exploring what indoctrination is. My experience over 36 years indicates that it is important ethically to distinguish indoctrination about religious belief from offering teaching about religious belief; to recognise the differing roles of lineal and lateral thinking; to understand at a deep level what the difference is between fundamentalist thinking patterns and democratically constructed personal and communal options (epistemological development). It is also vital that students actually recognise that while some of these thinking patterns can be dangerous, it is not logical to ignore them. They have existed from time immemorial, and have had their (questionable) uses in (hopefully only previous) generations of learners. Stimulating discussions have occurred in my classes when senior students become adept at abstract thinking and can concretise at will, in order to develop the best and most balanced personal understanding (the emergence of pedagogical-techné): ethics in action.

Although aimed at the needs of teachers who are responsible for teaching deaf or hard-of-hearing students, the work of Easterbrooks and Scheetz (2004, p. 255-263) provides a worthy summary of the links and connections between three interlinking concepts: character education; values clarification; critical thinking.

Students who are deaf or hard of hearing must learn to think critically. Character education (CE) refers to the effort to teach basic values and moral reasoning (Doyle \& Ponder, 1977). Values clarification (VC) is the process of examining one's basic values and moral reasoning (Rokeach, 1973). Character education and values clarification as subject matter foster the development of critical thinking (CT), a tool used both to develop and to modify moral reasoning. 
These three areas mutually support one another. The development of sets of values and their underlying moral reasoning is the foundation for thinking critically and ethically about values (Vardy, 1998). Easterbrooks \& Scheetz (2004) examine the components (connecting, I believe, to Wilson's earlier approach), of critical thinking, character education, and values clarification, summarise the literature, and provide a template for appropriate lesson plans. They also describe strategies that promote the development (connecting, I believe, to Lipman's Community of Inquiry and Vardy's RAVE approach) of critical thinking, character education and values clarification. The links are definitively established with appropriate sources for the concepts from their earlier research. The terms used are central to the thinking skills being taught for the understanding and insights needed for deeper learning by Wilson and his teaching of concepts, by Lipman and his Community of Inquiry, Vardy and RAVE, and by Hawkes and his all-school values education. All of these sources provide striking examples of the considerations required for this new emphasis in edu-tensegrity. Teachers use formal background studies and professional development strategies for continuity and deployment in all subject areas. Busy teachers often have conflicting calls on their time. Specialist teachers who choose to develop their skills and expertise being explored in this thesis and which are often called Life Skills programs, can find important content in this exploration. As Easterbrooks \& Scheetz (p. 255-263) contend that critical thinking

\footnotetext{
skills must ultimately become the agent by which individuals self-monitor their thoughts, feelings and actions. However, until one has developed the ability to self-monitor, he or she needs to have these experiences mediated by competent, supportive individuals, in this case, teachers.
}

\section{Teachers' recognition of inquiry-based learning in classes}

Teachers are aware of these various patterns of inquiry and use them to develop thinking skills in their teaching-learning process. Below are variations on the inquiry-based learning strategies used in class today. 
Sometimes, students are given a task, in which each will need to independently follow the instructions for completion. That all students complete the same task is not necessary, because this strategy is used to help students learn a step-by-step skill that they are going to use repeatedly. Often their task is research-based, and all students need to complete the work to understand the learning strategy embedded within it. Hyacinth stated: 'We overtly plan curriculum using Thinking Hats ...to introduce de Bono to Year 8, I put the Thinking Hats up on the wall and explained each colour and the questions that related to that colour'.

Some teachers consciously taught thinking skills through the use of the questioning procedure. Others blended without highlighting the thinking skills but allowed for persistent and consistent questions and research to help students to reach understanding of new knowledge. George stated: 'I ask questions/give information/tell stories/give counter arguments. I monitor interest then modify my approach'. By comparison, Giovanni stated: 'Always respect others' right to have an opinion; always support your opinion with a rational argument'.

It is worth delineating the various forms that inquiry in the new paradigm can take:

- Classroom-based inquiry

This strategy is commonly called the Community of Inquiry and is a central part of the strategies put together by Matthew Lipman to ensure that all children experienced a democratic classroom. This classroom is one where there is room to interrogate the source material, question the teacher, question and discuss among themselves, and to self-correct when they discovered mistaken assumptions in their own thinking and learning patterns. Lipman's approach relies on the class members coming together in a particular way making sure that individual students recognise the experience of consensus, inquiry and puzzlement as they control the rate of questions, and which questions are best investigated within the given class or within a set time frame. 
Hyacinth said: 'Philosophy for Children (the overall title for the Community of Inquiry as per Lipman) provides a framework for teaching any topic, but is especially helpful in teaching a literary text. The framework takes students out of their traditional roles and develops their thinking in a different environment and different way. They take control of their thinking and generate their learning. It removes dependence on teacher as a fountain of knowledge'.

- Problem based inquiry

This automatically means to both teacher and students that questions and questioning are the crux of the learning procedure so that their expectations and their experiences cohere fully.

Sandy taught the full International Baccalaureate program and made it clear that lots of questioning - open-ended and problem-solving - were central to the way he expected students to manage their learning. He expected thinking skills and relevant questions and questioning to be used at all stages. He would provide analytical strategies to this end, stimulating discussion with a lot of group work and with focus questions.

- Subject-specific inquiry:

This can take a variety of forms. Often the use of content and understanding of a topic to extract values and concepts is central to humanities subjects like Social Studies, Business studies, Religious Studies, Philosophical history of ideas, English Literature and English itself. The questions and questioning have the effect of highlighting thinking skills, which result in an understanding of topic-embedded concepts.

In Music Studies, Olag's priority was on the skills that become central to playing in a band. He lists Leadership skills, Predicting skills, Synthesising skills, Analytical skills, Cooperation skills.

- There is, in the new paradigm, a sequence:

The teacher will predict, co-opt, and affirm. It is then that students are influenced, taught and become efficient learners. On the other hand, 
teachers of subjects like Physical Education recognise the display of progress in thinking and reasoning for their learning from the beginning of the year to the end of the year and at different year levels. Pietr noted: 'From $\mathrm{K}$ to $\mathrm{Yr}$ 7, self-knowledge is used to identify cognition development, Inquiry: like why is it so, etc., creativity: from $\mathrm{Yr} 8$ to $\mathrm{Yr}$ 10 - analytic, creativity, evaluation application, critical thinking, assessment strategies in all levels, problem solving and guided discovery strategies'.

So, we have to separate the four styles and recognise that the technical strategies and techniques applied in each case may overlap, but also that they induce different learning experiences, one from the other, and assumptions about learning outcomes will vary consequently.

Teacher-respondents to the Questionnaire indicated that when they were teaching issues, probing for deeper thinking and higher-order thinking, there were emerging areas concerning the meaning of life, and the quest for happiness and the good life.

As George stated (when exploring reasons for responses in the classroom), on an archaeological topic called Origins, which was incorporated in the Year 9 curriculum: 'RVE (Religion, Values, Ethics education): “Origins”- why do they believe in any theory? Where do they get it from?' Later, he added: 'Ethics is the application of Values - scenario situations work really well’.

Bernard stated: 'Philosophy - not always one way of doing things ... Also, basic religious values are shared across most religions'. Giovanni indicated a deeply-held teaching framework: 'Ethics seems to mean Virtues and Values. I DON'T (his emphasis) believe we should teach any particular ritual to be ethical, e.g., going to church is good OR dying for your country is admirable’.

Michael indicated that in his classroom thinking strategies for the teaching of Virtues, he would promote that it is virtuous to move forward in a way that contributes to the common good: 'I always ask the students if their 
beliefs or actions work in a way that is good for the wider group and not just themselves'.

Michael also stated: 'My understanding is that ethics takes the idea of virtue further to the principle of agreed rules. This notion was central to the pursuit of scientific thinking (my subject area)'. He further stated that teaching an understanding and literacy in the area of rules and regulations is what he means by Ethics Education.

While teachers indicated similar thinking patterns that appeared in their teaching about virtues, values and ethics, there was a wide pattern of thinking skills for rich learning. The connection to formal and informal educational leadership is pertinent here, because teachers identify deeper thinking issues. They are not always in the position to find personal resources to expand into consistent formal and informal leadership behaviours as outlined in Chapter 4; even when they are aware of these distinct issues as they are encountered at the coalface.

In terms of the commonplaces of the curriculum, this category allows the integration and collaboration of teacher inputs, teacher impact on students, and the cohesion of the subjects taught; it impinges on the teachers who are delivering those curriculum elements in the particular ethical milieu.

\section{Looking forward...}

After such a wide-ranging exploration and investigation, it is important to recognise that what we can do to integrate some of these initiatives into already existing theory, practice and praxis in current education in Australia, perhaps in New Zealand and United Kingdom too, is pertinent, possible, probing and thoroughly exciting. Our responses, our response-abilities and our responsibilities are all part of this project. These recommendations and conclusions are not unfamiliar in themselves, but the particular inductive way in which I have linked and promoted these various concepts provide channels 
for consideration. Therefore, the answers that have emerged from all the themes and sub-themes, all the questions and consequential considerations, all the Categories of Influence that underpin my thesis are contained in the last chapter. I endeavour to answer the questions that I posed in Chapter 1, logically, ethically and coherently. 


\section{CHAPTER 9}

\section{Reshaping the Values, Virtues, and Ethics (V.V.E.) Future: Responses, response-abilities and responsibilities}

\section{Introduction}

Chapter 9 separates inductively the indications that would point to the future if this theory were to be examined and tested by educational colleagues in the classroom. The trilectic approach is useful here to identify how reshaping the inclusion of a democratic and secular approach to curriculum content resulting from this theory would operate. The geodesic dome highlights the tension between many of the three overlapping concepts within each Category of Influence discussed in Chapter 6. The Responses are to assemble the assessment and judgement of educational leaders today. The Responseabilities are an effort to register and examine the gap that may occur when there is insufficient knowledge or skills or understanding to proceed. The Responsibilities are the new knowledge and new concerns that emerge by inductive thinking, building for teachers from an examination of the content of this thesis. I finish with a number of personal reflections about enhanced learning as I have travelled through the construction of this thesis: I answer each question raised in the first chapter.

As a means of contributing content to the general debate on moral education - sometimes called values education - I offer my reflections as a teacher of nearly four decades. These have led me to a reshaping process for 
teaching in Australian and other classrooms, to a new paradigm designed to address the changed and new emerging conditions for education for twentyfirst century conditions. I need to state here that when I hear discussion of values, I find that it almost invariably includes an implication of the addition of virtues and ethics, even when these terms are not explicitly used. I reason that this situation is a result of several generations of emphasis on secular forms of education, and as such needs to be given its own worth, rather than indulging in the deficit form of assessing knowledge.

I set out to seek knowledge to enliven teacher practice and to identify and construct a specific architecture for the teaching of virtues, values and ethics. In striving to accommodate topics and subject matter into an already crowded curriculum, at the behest of particular sections of society, we can lose sight of the meaning and implications of the concept of rich and deeper learning through applied thinking skills (Burdett, 1999); Cherednichenko, 1997; (Brenda Cherednichenko, 2000); (Splitter, 1999) (Mackay, 1999).

Howard Gardner (2008) offers a project that he has admitted has overwhelmed him at times, in which he provides a glimpse of how emerging brain research required of him a corresponding examination of how learning and the good life would be accomplished according to his concept of how the mind works. He set a challenge that echoes throughout contemporary education; a challenge that I have attempted to address in this investigation. He has made his earlier research the pivot between the old paradigm of education towards conforming and apparently suitable educational qualifications, and the new paradigm moves towards collaborative and interactive richness in both formal and experiential learning. He adamantly describes how twenty-first century living conditions will require the cultivation of what he calls five minds: disciplined, synthesising, creating, respectful, and ethical minds - and shows how these will come to form the whole mind of the individual. He (2008, p. 153) suggests that cultivating those elements within each person falls under the auspices and responsibility of education: 
Now the time has come to take stock to review the major claims and clear up some lingering questions [...] I recapitulate the principal features of each kind of mind [...] I review some of the obstacles to the formation of these minds; speculate about the order in which these minds might be developed, and then offer suggestions about how the ensemble of minds might best be cultivated.

This provides an exciting point in my efforts towards qualitative research that this thesis represents; a stepping stone on the path that moves from the old paradigm to a new paradigm of and by the teaching about virtues, values and ethics.

According to the old paradigm, the Australian Government began a zealous political push to deliver Civics and Citizenship education into the 1990s' school systems. The consequences, always unforseen by myopic politicians with short term and short-sighted ideological imperatives, have included the demands and requirements of educators that they teach and use understanding of all underlying concepts (Mackay, 1993), including the dictate that the promotion of such behaviours as foresight, prudence and commitment become part of the curriculum (Cam, 2002a). Government comment on these requirements has implied nothing but an expectation of passivity and docile acceptance of the economic-rationalist framework of today’s political landscape (Gardner, Susan, 1999); (Splitter \& Sharp, 1995). The contrast between this view and Mackay’s, Gardner's and Cam’s (2002,) collective view to the government initiative is stark.

In shaping and developing the writing of this thesis, care has been taken to identify and highlight so many links and connections between experts and educationalists, philosophers and teachers; and between my responses in undertaking experiential learning, formal studies, informal and formal leadership roles, and the praxis of publishing and practising of curriculum development. These intricate linkages between theoretical considerations and the actualities of practice have been crucial to the modelling of my theory of edu-tensegrity. I believe the theory of edu-tensegrity travels part of the initial 
journey that Gardner (2008) is asking educationalists, teachers and society to undertake, to promote and to accomplish.

To accommodate his request, I provide another triangle, in the tradition of trilectic logic, to aid in this aim of reshaping: Responses, Responseabilities and Responsibilities. This triangle is patterned on my initial integrative schema, shown in Figure 3.1 (Chapter 3), and begins by addressing ontological considerations, then epistemological concerns and consequences before indicating the emergence of technical or pedagogical strategies from the process. The application of this integrative schema closes my investigation by answering specifically the questions posed in Chapters 1 and 2.

To provide a specific architecture for the teaching about virtues, values and ethics that will address their overt or covert unfolding across the expanse of twenty-first century culture, we must reshape the future through these Responses, Response-abilities and Responsibilities The ontological considerations are present in the Responses of the teachers who provided their ideas, opinions, and points of view in the aid of this study; the practitioner experts (and I include my own contributions); and the many voices from the literature, who all illustrate the emerging requirements for accuracy, diversity and reality in contemporary education.

Epistemological notions are present in the Response-abilities - the ability to respond by the use of appropriate knowledge, accurate skills and relevant planning; all knowledge-based, as they emerge from: my investigation; from beginning and continuing teachers in informal and formal leadership (curriculum, administration and support networks); teacher-education course content collaborating for best student outcomes rather than as a response to local, short-sighted political impetus. The aim is a richer quality of student learning.

The concept of Responsibilities contains the pedagogical elements of this process of reconstruction of the edifice of Education that emerges inductively from my investigation: all educationalists across all branches of primary, 
secondary and tertiary areas of education, without exception, can and should contribute in democratic, collaborative and knowledgeable ways, to the reshaping and reemphasising of the needs of contemporary education according to the processes contained in the Categories of Influence in edutensegrity.

\section{Responses: Reshaping the future}

Competition is the key tenet in the harsh world of economic-rationalism; students prefer to engage with daily tussles only in order to complete that which is to be assessed. They do not want to give credence to any special topic unless it follows the predictable formula of teacher input, consequent investigation and a mark at the end. That is where the old paradigm of education should end, too.

The pervasive influence of media and advertising on contemporary culture exerts a very real narrowing upon perspectives; a narrowing that is subconsciously experienced as a disallowing of real choice; Klein (2007) highlights this dramatically and gives cause to for concern about an insidious form of subliminal indoctrination that may be underway, thus giving a sound reason for entering this new paradigm of education.

In their efforts to construct their future, students see that they are dependent, until about the age of 25 years (Mackay, 1993). It can lead so often to serious personal risk-taking that can be breathtakingly lifethreatening. Students appear to be aware of a new world focus, but they experience much of the old societal scenario.

In today's highly technological world, the phenomenon of blurring the edges is a legacy students accept from a previous generation, who dignified it with philosophical titles like deconstructionism. We see the emergence of cynicism, nihilism and the consequentialism/anti-consequentialism bind as we move from class to class (Connell, 1982). It seems the content and format of teaching needs refurbishment at least (Gardner, 1993a) and claims that 
Philosophy for Children does address these phenomena are therefore pertinent (Splitter \& Sharp, 1995) to this discussion. Wilson's Moral Components List (in addition to his other relevant works), Lipman's Community of Inquiry, and Vardy's RAVE curriculum all provide pivot points for the introduction of the new paradigm of education that must be undertaken prior to the full implementation and achievement that Gardner's (2008) propositions outline for all educators and administrators.

\section{Response-abilities: Improving teaching about V.V.E.}

The ability to respond to the demands for change, for sustainability, for connections with traditional teaching practice, for cognisance of emerging brain science and the consequences for teaching; all these demands require recognition, articulation and resolution of the contradictions, the connections, the links, the assumptions in the conceptual foundations of education as we know it. This is still the preserve of the old paradigm.

As a result of my investigation - an overview of the analytical spectrum covered by Aristotle, through the developments of Dewey and Schwab and incorporating the extensions implicit in Wilson and Lipman - we can trace the expansion and history of what have become various forms of education today. It has been important, in a curricular sense, to recognise that the teaching of thinking skills is already happening in the classroom: the different emphases that have been discovered have been useful to constructing a schema that addresses the concerns generated by conditions of learning and reasons for learning that have been changing and adapting to a new intensely physical and technological era. This has resulted in my proposed virtues, values and ethics framework for coherent curriculum to meet students' needs - especially as brain research has uncovered significant thrusts towards a reshaped approach and the emergence of a new paradigm.

My study has uncovered and solidified into recognition, student need and teacher support,- so that ecumenical, inter-faith and educational communities 
can have access to tools for higher-order thinking. These tools are necessary for these students' future decision-making abilities as the attempt to:

- construct of a career path;

- construct of a personal philosophy of life and quality of life; and even to

- develop meta-questions regarding the meaning of life and a concept of eschatological matters, by being able to formulate relevant questions and questioning techniques in either religious or secular terms.

The data within the Questionnaire give teachers a voice about these matters and indicates in various ways that teachers attempt to the best of their ability in prevailing circumstances, to fulfil their obligations. Their responses to my Questionnaire led me to significant conclusions: that teachers may well be unwittingly already reaching out towards the new paradigm of education.

These are the first tentative steps along the road that Gardner has indicated. To achieve these purposes within the new paradigm of education, I propose that scholar-teachers now allow themselves, as post-formal auto/ethno/biographers, to perpetually reshape themselves through their own relationships to knowledge and the resulting patterns of perception and behaviour (Kincheloe, 2005, p. 172). Looking back over my ethnographical retrospective, I can see that the continual blending, learning and application over thirty-six years extended my own learning by instilling an understanding of what it means to be a 'bricoleur' (Ling, 2012). That learning was an exciting venture into the realm of inductive data reduction, and even though this learning occurred late in my life it can still fire further application of my knowledge - an example of all that I have provokingly proposed here.

\section{Integration of all Categories of Influence into the dome of education}

It is vitally important to link the ontological concerns, those big-picture underlying philosophical constructs into this reshaping exercise. A first 
attempt at this linking strategy is undertaken consistently throughout Chapters 6, 7 and 8 of this study. But this is a strategy that needs building upon if it is to actively translate such speculative thinking and exploratory constructs into a reshaping exercise. This is a step that requires the recognition, naming, and integration of relevant epistemological matters: those elements of knowledge and relevant skills that lead to the final practical, pragmatic objective.

The technical (techné) consequences of these two steps appear here because Curriculum does matter. Explorations, conclusions and recommendations will serve to further the planning for the content of various relevant professional development initiatives, so that refinement of educational aim, insertion of accurate knowledge and skills and the accompanying classroom strategies and techniques will emerge for all educational practitioners.

The parallels revealed by an overlaying of Lipman's Philosophy for Children and Vardy's Religion and Values Education can form the bedrock of these considerations. Certainly Wilson's Moral Components List will require revision, because the nonsense titles that Wilson assigned to each of the concepts are now largely redundant. The conditions of schooling, especially the imparting of cultural and covert agendas, are far more generic, and rely less on such archaic distinctions as those revealed in earlier elitist concepts such as the teaching of Classics, as if there were the possibility of a level of education that was somehow quantitatively different and in some way superior to the more mundane education content offered by government school curricula. Wilson's List is central to the teaching of Virtues, Values and Ethics as concepts in accordance with this revised and reshaped theory.

Thinking skills regularly feed into an individual's decision-making forays as they explore their career, for their construction of the good life and how they face eschatological questions about the possibilities of an after-life, and about meaning in this life (Kaldor et al., 2010). My research indicates the need to have a particular re-shaping of existing knowledge for effective 
teaching of thinking skills for rich deep learning, especially about virtues, values and ethics (V.V.E.) to fully enable these explorations

\section{Practical implications for the role and function of teacher}

It is important to emphasise an understanding of what constitutes decisionmaking; it is a process that appears in education; in planning of life goals; in the development of one's life philosophy; in the choices made to include religious or secular mores. The need to make decisions is ubiquitous in everyday life; it is only logical that the skills necessary for effective and ethical decision-making are available to all, students and their teachers alike, through schooling.

It is important to recognise that today's students, who respond most naturally and receptively to contemporary notions of individualism, need to have channels available to them for the exploration of their own decisions, for the development of their own philosophies and moral attitudes, and to deal with the consequences of decisions emanating from such channels of experience. This can be enhanced by the inclusion, if such is the individual's decision, of religious mores and, in some cases, the living of a religious life to some degree. Education in Philosophy or the History of Ideas seems to be a valuable arena for consideration. Educere, (going back to the roots of existing concepts and familiar knowledge to evaluate and learn), and Educare (training in skills in all relevant learning areas) are two basic concepts that will be needed to achieve a holistic education (Heasly, 1995).

Teachers appear to be today's societal instrument of choice in the approach, analysis and delivery of enough knowledge, learning, research and insight for students to learn how to include such goals in their lives. Upon such knowledge rests the opportunities of each individual (and today's students are tomorrow's individuals and society) to decide, independently or with altruistic support, how their life decisions might unfold and might be capable of being unfolded. This will allow student knowledge to develop in the cause of personal resilience, compassion, interactivity to be the 
foundation beneath the more businesslike characteristics of effort, effectiveness and efficiency.

The structure of an individual classroom, indeed of an individual school lie well within the scope of investigations into what is to constitute education in Australia (Mackay, 2010); (Crowther et al., 2010); (Hargreaves, 2003; Hargreaves, 2009) in the twenty-first century. It becomes the ongoing responsibility of the today's social and political leaders, alongside teachers, to examine the interlocking concepts that feed this new educational paradigm with such promise with the deliberate and stated aim of improving the studies and experiences of these teachers. As Crowther et al. (2010) stress the idea of sustainable change needs careful planning.

\section{Responsibilities: Creating a future for V.V.E.}

Any reshaping of the future of education rests on attention to detail and on the development of all of the Categories of Influence listed in this theory of edu-tensegrity. No single component is more important than any other. The teaching of decision-making as part of the teaching applied thinking skills is a result that is crucial to the curriculum.

The research indicates that it is appropriate to examine how decisionmaking is taught, so that a new generation of diverse and independent students may employ the processes:

- Education: how they make choices regarding all personal aspects of career often called Life Skills;

- Preferred planned life style: how to make choices that depend on developing and maturing, ethically substantial, personal philosophy;

- Choice between a preferred secular or religious belief systemhow to make choices in accordance with the consciously chosen religious or secular belief system and how to live those choices in a logically coherent and independent life-style. 
So, yes, despite the human tendency to prefer conformity, there is a need to include the likelihood that some people may opt for a religiously belief-based personal framework; some will opt for a non-religious belief-based personal framework; and some will choose an agnostic-based personal framework; some will choose never to actively consider any of these options. Thinking skills are a necessary adjunct to the decision-making that is central to all four areas.

Emerging brain research into the place of creativity (Hosseinee, 2008) and thinking is further supported and enhanced by McAdam (2011, p. 7- 8) in highlighting the need for what she calls modern folk art. This aids people with memory loss to reflect on her concept of an inner script to find meaning from their own lives. I contend that the parallel of the inner script to find meaning in life is vital for students. They can otherwise be swamped by ICT, by other-centred pursuits and by extreme risk-taking practices. I contend also that our concept of creative arts in education can include the arts of applied thinking skills, with specific benefits for career choice, for recognition of the precepts of Aristotle's 'good life', and for successful approaches to personal eschatological speculations.

\section{Teachers and planning}

Central to the planning of a thematic curriculum that links and connects to other learning frameworks, and that will include the thinking skills approach of Lipman, the Moral Components of Wilson, the Five Strand Approach to Religion and Values Education of Vardy (as well as the reshaped pedagogical approach being developed as a result of this investigation), is the forging and shaping a harmonious meta-curriculum format.

Crowther (2010) outlines the fourth Dynamic of his COSMIC C-B theory: micro- pedagogical deepening. The initiatives that appear in Chapters 4, 5, 6 and 7 begin such a fundamental change:

Micro-pedagogical deepening incorporates deep conceptual understandings, intense personal analysis and rigorous strategies. Our 
research affirmed these conclusions from the authoritative international literature. Relatedly, our research affirmed the attractiveness to teachers of the 3- dimensional view of $21^{\text {st }}$ century teacher professionalism that underpins Micro-pedagogical deepening ... (Crowther, 2010, p.16)

Micro- pedagogical deepening involves teachers engaging in one or more of three forms of pedagogical practice: intensive reflection on personal gifts and talents; conceptual expansion and exploration of the schools pedagogical principles (SWP = schoolwide principles); and refinement and implementation of strategies relating to the SWP. (Crowther, 2010, p. 23)

\section{Categories of Influence as responsibilities for education}

The question of which content the teacher prepares for the classroom now becomes central to establishing the new paradigm. Apart from the subject and skills areas that are already the responsibility of the teacher, there now comes expansion and integration of and into four additional areas:

- Content to convert into knowledge - ontological concerns.

- Decision-making for applied thinking about content epistemological matters.

- Virtues, values, ethical and philosophical thinking for a personally preferred secular life-style and consequences of an eschatological nature - ontological and epistemological considerations.

- Virtues, values, religious mores, theology, ecumenism or interfaith studies for a religion-based lifestyle; alternative technical consequences of an eschatological framework - ontological and epistemological considerations.

Teachers will be able to expand their practical techniques to the teaching of a wide array of thinking skills. Splitter and Sharp's (1995), diagram shows this very clearly (appendix B). The set of abilities to improve the pedagogical frameworks emerging from the Categories of Influence that teachers are responsible for applying is varied.

I consider that there is a need for teacher education program to rest deeply on the following structures: 
- Knowledge of relevant curriculum design. Construction and implementation of a set of personal classroom pedagogies. Deliberately and carefully chosen, named, taught and planned and prepared thinking skills that are embedded in the existing curriculum throughout primary, secondary, tertiary learning

- Recognition of precisely how different the students' classroom experience is - recognition of the operation of a teacher-directed classroom pedagogy or a student-directed pedagogy; knowledge of the differing thinking practices operating within each discipline, naming them accurately and identifying when they carry over from one discipline to another.

The naming and modelling of particular thinking skill takes a few minutes only but still requires of the teacher a meticulous planning of what is and is not to be learned as a thinking skill. Although not much extra time is taken up, care must be taken to heed the warning of Leckey (2009), whose research into the difficulties experienced in the setting up of the format of the Community of Inquiry reveals some unexpected considerations about time, external conditions and the uncertainty regarding usefulness within the classroom. The value of introducing students to the Community of Inquiry at an early age, as Lipman did, to promote the efficient use of time and enhance effective learning is displayed in her work.

\section{Categories of influence in action}

Teachers seem stressed by the requirements of formatted reports, league tables, and emerging new requirements in various national curriculum perspectives. The time requirements to implement the Community of Inquiry may then appear, to the uninitiated, as a possible misuse of teaching time; hence the apparent contemporary preference for individualised learning. 
In the new paradigm advocated in this thesis, we can, as Hodgkinson (1991, p. 42) suggests, go back to Aristotle and recognise that we are addressing a trichotomy:

Not two things: theory and practice, but three things: theory, practice and praxis. Administration is not art or science; nor is it art and science, it is art, science and philosophy.

The art, science and philosophy of the new paradigm for education in the twenty-first century are encapsulated in the twelve Categories of Influence:

- Category of Influence 1 - dimensions of daily life: teachers are familiarised with pastoral care initiatives that can be developed and shared in each milieu.

- Category of Influence 2 - dimensions of thinking: teachers develop further the knowledge and pedagogical skills to teach the student through the medium of the subjects taught.

- Category of Influence 3 - dimensions of education: teachers refining and focussing their various informal and formal leadership roles and functions both inside and outside the classroom for deeper educational learning.

- Category of Influence 4 - input from research theorists and the shaping of the curriculum: teachers focussing on the refinement of understanding of ontological, epistemological and pedagogical knowledge from a wide variety of sources, from their student teacher days and continuing throughout their professional development and formal qualifications to promote rich student learning.

- Category of Influence 5 - constructs and knowledge about the curriculum: teachers reshaping and incorporation of the thinking curriculum as a means of enhancing the progress of student understanding and academic progress. 
- Category of Influence 6 - medical or biological and philosophical theorists leading to the theory of Edu-Tensegrity: teachers incorporation, from primary, through secondary and tertiary levels of education, the significant emerging knowledge from neuroscience, and from extant secular and spiritual belief systems for their integration into the developing the virtues, values and ethics of their respective cultures by study and reflection, thereby enhancing their levels of resilience.

- Category of Influence 7 - constructs about meaning, reasoning and choice: teachers development of pedagogical and pastoral stratagems to allow students to recognise when they are dependent, interdependent or independent in their efforts to democratically build their own personal meanings, their own reasoning and their own various choices regarding careers, 'the good life' and exploration of eschatological speculations in maturity.

- Category of Influence 8 - teachers and teaching: teachers focussing on the benefits of collaboration, of democratic leadership in all its forms, and how these may lead to harmonious and positive experiences of learning; professional development in the classroom for the enhancement of the teaching about virtues, values and ethics.

- Category of Influence 9 - my contributions: my long term application of the principles outlined; testing and validating the accuracy in this investigation; the inclusion of pedagogical tools which when used with discernment, provide exciting systematised progress for students and teachers alike.

- Category of Influence 10 - students' new knowledge, understanding and expanded skills: teachers facilitation, teaching and monitoring in the expanded role and function of informal and formal leadership, both in and out of the classroom, the whole 
gamut of creativity: of applied thinking skills, of decision-making, of reflection and of resilience, for rich student learning and progress.

- Category of Influence 11 - emerging connections of neuroscience to learning: teachers' incorporation of the remarkable work that emerges from the enhanced understanding of medical and biological elements of brain function and learning, for the provision of rich and sustained student learning.

- Category of Influence 12 - collaboration between teachers, students and curriculum experts: teachers' development of a focussed Philosophy of Education based on the principles displayed by the analogy of the geodesic dome, which accompanies them into the classroom, balancing the stresses and tensions, and supporting all that happens in the subjects taught, so that the basic principle of edu-tensegrity can fully support the reshaped concept of twenty-first century education.

The re-shaped pedagogy strives to find effective means for the provision of rich learning through and about thinking skills, through and about decision-making and through and about the various channels in an individual's life that rely on that learning and thinking and on those decisions. It also entails a radical return to the roots of historical development of logic and reason developed across cultures and eras of Western civilisation: Aristotle, Plato and Socrates are equally as relevant as Wilson, Lipman and Vardy. In order to respond to and cohere with the conditions of Australian needs this investigation advocates reshaping of education and points to the new paradigm that I have been exploring. Using the reasoning process of the ancient Greek philosophers provides clear, valued and validated processes.

A change of paradigm that involves re-shaping of classroom pedagogy also involves promoting urgent change in the emphases that politicians and teachers place on pedagogical details. This change begins with: 
- De-emphasising the aims and objectives of teaching of units, topics and subjects in favour of using those same units, topics, subjects for dual purposes.

- Inculcation of deep and detailed knowledge and skills of virtues, values and ethics necessary for management of personal career choices; for constructing personally fulfilling quality of life; for opportunities that arise to consciously decide, through eschatological speculations, on a secular or religious life focus.

To achieve these aims and educational objectives, teachers and educational leaders need to become more expert in curriculum planning; they need to be capable of teaching coherent conceptual development techniques in matters of virtues, values and ethics; they must be skilled in advice and advisement techniques in order to promote decision-making skills in a democratic teaching learning process; and, of course, they must be ontologically and epistemologically advanced (dare I say wise and knowledgeable) enough to teach both the content of the curriculum and the life skills explored therein.

\section{Consequences}

There is need for reflection on any positive and negative consequences of any change: and where this suggested content would best fit. First, the notion of constant change can easily build into any government framework an attitude of change- for- changes' sake - it is important to allow for pilot studies to prevent any too-hasty jumping to conclusions that may take place. The reshaping requires real time, settling and the giving of space to bring out an effective and efficient set of evaluations prior to the more 'official' changes that must inevitable take place.

Second, it needs to be understood that the goal is not the outcome-on-theground results of the newer emphases and strategies being inserted through the Melbourne Declaration (Curriculum Corporation, 2008). Agreed national goals are just that - a set of end-points which teachers and students strive to reach. Careful attention to all the variables is needed before conclusions can be confirmed and accepted. Learning does not become effective because a 
policy exists, nor because an agreement exists, nor because we wish to use a construct or set of standards as a benchmark. A negative approach could produce conditions of close to harassment for both students and teachers, and the end result would be totally counter-productive.

Third, stronger partnerships need careful monitoring in order to produce positive psychological conditions, even when strictly applied, so that real, rich learning can be experienced by the team of parent, student and teacher.

Fourth, quality teaching is a term that has somewhat simplistically been attached to the type of political manoeuvring, that reaches for some short term results, such as the use of league tables, over-testing and attention to administrative and organisational minutiae. In such a milieu, quality in teaching may be impossible.

Fifth, the level of learning in any classroom stops and starts according to seasons, bouts of illness, special programs, extra-curricular activities, emotional stress, teacher or student absence and more. Time is also a factor for those students whose patterns of learning are not lightning fast. The timeconstraints that teachers face every year is a central part of this framework.

To enhance rich learning, I believe the whole picture of a given topic, as shown in Figure 8.1 on pg.340, needs to be supported by a consistent model for teaching students how to question. Obviously, this kind of learning, based on critical and creative thinking (Cherednichenko \& Wilks, 1997) and couched in very democratic frameworks, needs to be infused into the strategies used in the classroom in the construction of what is to count for students as rich learning. A move in this direction is outlined in Figure 9.1.

\section{FIGURE 9.1 ENHANCING RICH LEARNING}

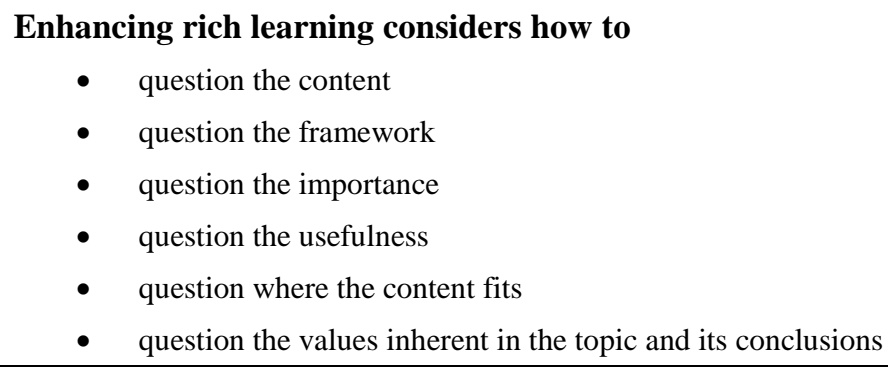


- develop a fearless approach to reason and logic

- $\quad$ recognise and make distinctions pertaining to physical, emotional, social, intellectual, sexual, political, philosophical, ethical, religious matters within the topic

- $\quad$ identify and eventually internalise at their own pace the consequences of these questions

World-class curriculum and assessment is a very woolly concept. If these things are divorced from the local culture, the appellation 'world-class' assumes connotations of a political, religious or secular ideological agenda of a covert nature. Democracy demands any concept as important as a form of curriculum be as transparent as possible and that it be monitored for such corrupting influences. Otherwise, the new paradigm too is immediately corrupted.

If critical and creative planning is employed rather than implementing policies addressing any and every disadvantaged student group in any and every school, then real and rich learning can take place. Monitoring of such programs needs to be exactly the same as it is for the myriad other students. Refugees are not mentioned in the disadvantaged groups, and they are an increasing educational consideration today.

We require an adequate definition of transparency. How? Where? When? What? Which? Who? Why? - These are good questions from which to start. They provide transparency about: What? To whom? About whom? At what stages? Where is remedial teaching in all this? Where is Failure in all this? How can we accomplish what is required? It is possible that critical and creative thinking, no matter how ethical, will lead to angst and anger rather than the desired and implied outcomes. It is necessary to promote a very careful and respectful approach to this area of action.

\section{Dependence, independence and interdependence}

In the new paradigm, the role of teacher is that of channeller, connecter and manager of the interaction between the various students and the content of the curriculum through curriculum planning; via topic management, student experiential learning and student analysis and questioning (Lipman \& 
Montclair State College. Institute for the Advancement of Philosophy for Children., 1980, 1981, 1985b, 1986, 1987b, 1988b; Splitter \& Sharp, 1995; Cherednichenko \& Wilks, 1997; Crowther, 2010). The function of teacher is to know in advance how to use ontological questions and Constructs; how to translate through experience, understanding and commitment, the processing of the big Constructs into epistemological detail (phronesis); and how to use the various technical (techne) strategies and techniques that produce rich and deep learning (Snook, 1972; Vardy, 1998; Swartz, 2001; Fisher, 2003; Hargreaves, 2009).

In the democratic inquiry classroom, the teachers' role is multi-faceted, challenging and complex: facilitator, provoker, modulator, monitor and supporter (Cherednichenko \& Wilks, 1997) and simultaneously for each student; this list can be varied to accommodate the contingencies of the new paradigm, and expanded in the manner shown in Figure 9.2:

FIGURE 9.2 TEACHERS' ROLE IN THE DEMOCRATIC INQUIRY CLASSROOM

\begin{tabular}{|c|l|}
\hline ROLE & \multicolumn{1}{|c|}{ FUNCTION } \\
\hline Teacher & providing epistemological content and skills (phronesis) \\
\hline Facilitator & $\begin{array}{l}\text { communicating, explaining, and helping to find answers to } \\
\text { problems, dilemmas, paradox and mystery }\end{array}$ \\
\hline Mentor & $\begin{array}{l}\text { leading students through the learning process, providing } \\
\text { opportunities for analysis }\end{array}$ \\
\hline Supervisor & organising, arranging regularly and completely \\
\hline Counsellor & giving directions, listening to problems \\
\hline Assessor & measuring evidence of learning (rich or thin) from student work \\
\hline Transactor & providing channels to ease students in unfamiliar study \\
\hline Transformer & $\begin{array}{l}\text { providing democratic vehicles in classroom pedagogy promoting } \\
\text { the widest sweep of thinking skills for applied knowledge for } \\
\text { further learning. }\end{array}$ \\
\hline
\end{tabular}

The function of the teacher and the influences of the new paradigm on teaching, I conclude, are broad:

- To understand assigned curriculum at a deep level.

- To extract relevant and important themes, issues, considerations from relevant curriculum documents. 
- To introduce, explain, trial, provide information for students on content; to lead and facilitate thinking patterns, problems pertaining to a given topic/theme/skill/problem.

- To provide whatever relevant activities are required to ensure student success and achievement.

- To ensure that effective, efficient teaching-learning strategies are used until student success in learning takes place.

- To provide a variety of measurement activities so that student confidence is established firmly.

- To design and use assessment instruments that will provide valid, focussed and authentic results of the student attainment.

Practical connections regarding the deepening role and function of a teacher can be developed further by integrating the sustainable school capacity building of Crowther and his colleagues (2010). His concept of Parallel Leadership, or distributed leadership (Crowther, 2010) broadens the teacher role and function yet again and eventually needs separate yet integrated action.

In all, the Categories of Influence lead us to the metaphor of the geodesic dome and provide a fresh impetus towards rich and deep learning. The pushpull factors observable in the dome, similar in form to those tensions and stresses familiar to teachers, will resonate in a new paradigm for the reshaped theory of education. Much will rest on teachers' perceptions of the teaching of thinking skills. The voices of the teachers heard in the Questionnaire responses give the impetus needed to develop this reshaped theory further.

\section{Questions raised by this investigation: Results and answers}

By examining the responses, response-abilities and responsibilities that I have identified throughout this investigation, it is possible for me here to 
posit a final personal response to the questions raised in the early chapters. As this research has been a qualitative initiative, proposed meanings were examined through a number of lenses by expert practitioners, theorists and educational leaders, to appraise the applicability of the links and connections that have been postulated.

\section{Research questions raised in Chapter 1}

1. How do secondary teachers perceive their teaching of thinking skills?

The Questionnaire contains questions designed to allow teachers' perceptions to come to the surface and the Findings Chapter gives extensive detail both quantitatively and qualitatively of the perceptions of those teachers; their voice is the first source of the whole project.

2. What is the relationship between secondary teachers' perceptions, opinions and attitudes of their teaching skills and the three different curriculum perspectives discussed: Lipman's Philosophy for Children; Wilson's Moral Components List; Vardy’s Five-Strand RAVE?

Many teachers have shown how they use pedagogical strategies and techniques to facilitate their particular subject areas. However, some tea3chers are not familiar with the detail of how questions can be used as a comprehensive classroom tool for rich learning. The resulting construction of edu-tensegrity as a reshaped emphasis on the Categories of Influence is intended as an aid in the provision of rich learning for twenty-first century educational conditions.

\section{Research questions raised in Chapter 2}

1. Ontological Question: What significance can be assigned to development of personal perspectives surrounding teachers' 
attitudes to the teaching-learning process; how do teachers maintain emphases needed to teach students -rather than teach just the subject content - for rich learning?

Using the huge body of work surrounding Wilson's Moral Components List, it is possible for the teaching about virtues, values and ethics to be communicated in pedagogical terms. Equally, by using the huge body of work surrounding Lipman's Philosophy for Children - with particular reference to the accompanying Community of Inquiry - it is possible to provide experiential learning regarding the central role of questions in constructing rich learning for students. By accessing the ever-increasing body of work surrounding Vardy's Religions and Values Education and its Five Strands, the manner of constructing a rigorous and wide-ranging inquiry into philosophical and other ethical concerns for senior secondary students, especially in the realm of recognising and understanding the dichotomy of religious belief and philosophical belief systems.

\section{Epistemological Question: How do teachers ensure that students} learn all the thinking skills relevant to educational knowledge and skills, so that applied thinking skills are recognised, identified, modelled, assessed and demonstrated in all learning outcomes in all subject areas?

In my Questionnaire, the deliberate aim of giving teachers at the coalface an opportunity to voice their particular insights also provided an opportunity for each teacher to review their own teaching practice. Indications are that most of the teachers were satisfied with the comprehensive teaching that they accomplished within their respective subject areas. Several respondents stood out, however by their detailed strategies for teaching beyond the confines of their subject area. Use of a number of strategies and processes, in which specific attention was given to the broad arc of applied thinking skills were recognised. Because no 
specific question asked for serious details, most teachers did not follow through with the steps indicated in the question: recognising, identifying, modelling, assessing and demonstrating individual thinking skills. And those detailed by the Philosophy for Children theorists were also not in evidence. Certain skills and certain learning outcomes were used although some applied thinking skills were not always given their operative name.

\section{Pedagogical (techné) Question: How do teachers understand and} acquire a comprehensive list of thinking skills to model, teach and require students to use in every educational sense: in career choices, in quality of life decisions, and in both secular and religious dimensions and approaches to life's eschatological speculations?

Given that teachers were noting their current practice, this question is a prediction based on current practices, but indications of what more could be included where advisement by teachers facilitates students' career choices was given by a number of the responders. Teachers use many applied thinking skills, but it would be providing an even higher level of expertise if teachers were able to broaden the list of thinking skills with their given titles for rich learning. Teachers can provide useful research and inquiry about virtues, about values and about ethics, preparatory to students proceeding to choose among life decisions about secular and philosophical stances or religious perspectives without the need to resort to any form of indoctrination or coercion.

Finally, as was shown in Chapter 3, the impetus towards the teaching of thinking in education needs a refocussing upon pedagogy. Hill (2011) and Thornton, Phelps and Graham (2011) emphasise this need: a feature of which is shown by Kristjannsson (1998) and his reference to Aristotle's crown of the virtues, 'greatness of soul' (megalopsychia): 


\begin{abstract}
I recommend, in line with Lipman's Constructs, that for the purpose of raising or consolidating students self-respect, the classroom be converted into a community of inquiry in which students learn to listen to one another with respect, build on one another's constructs, challenge one another to supply reasons for otherwise unsupported opinions, assist each other in drawing inferences from what has been said, and seek to identify one another's assumptions.
\end{abstract}

It could be said that the practice and praxis of teaching is unfinished until pedagogy actively includes these matters in the service of knowledge acquisition of, rather than in, all these concepts about Virtues, Values and Ethics, to address twenty-first century expectations in education. These, I realised at the Adelaide Conference, 2011 feed into the professional standards currently being developed by the Australian Institute for Teaching and School Leadership for both teachers and principals. The implication seems to be that there are other educational leaders who are also addressing the new paradigm for twenty-first century conditions. This augurs well for the teaching profession and for the student cohort.

In keeping with the effort to concentrate on inductive thinking, I have used applied thinking skills to model my approach in the reshaped theory of edu-tensegrity. I have concentrated on finding links and connections, filling gaps, identifying contradictions, in order to build up the details of research, analyses, discovery, experiential learning and epistemological knowledge to be gleaned from teachers.

\title{
Emerging elements for twenty-first century learning conditions
}

The emerging elements of twenty-first century learning conditions are becoming increasingly apparent and the precision of application of the elements of my theory points to the need for a critical review of teacher education. As was announced at its Adelaide Conference in 2011, the Australian Institute for Teaching and School Leadership (AITSL) is already 
deeply involved on such a project and has also provided a map of crucial resources.

When speaking of his scenario of 'five minds for the future', Gardner (2008, p. 165) makes a connection that implies a specific architecture for the teaching of virtues, values and ethics when he states:

\footnotetext{
It is up to the educational system as a whole - the educational system in the broadest sense - to ensure that the ensemble of minds is cultivated. In one sense, this is a job of synthesis - making sure that all five kinds of minds are developed. But equally, it is an ethical obligation: in the years ahead, societies will not survive - let alone thrive - unless as citizens we respect and cultivate the quintet of minds valorised here.
}

Future areas for research and consideration include the:

- Inclusion in the present curriculum for teacher education of a substantial curriculum of what counts as the various virtues, values and ethics for individual, communal and everyday life. This would be the first step towards the construction of the architecture for a virtues, values and ethics curriculum.

- Expansion of our understanding of crucial curriculum theorists as they lie along a historical, ontological, epistemological and pedagogical progression, and their synthesis into a curriculum, designed to aid teacher recognition and internalisation of links, connections, gaps, duplications, contradictions and assumptions in the understanding of a virtues, values and ethics curriculum. This would constitute a second step in the construction of the architecture of a virtues, values and ethics curriculum.

- Exploration of the consequences for curriculum content in primary, secondary and tertiary level in the face of the inclusion of such a reshaped curriculum: from the perspectives of thinking and logic; from psychological perspectives; from the familiarity of new, beginning and continuing teachers with the broad arc of the many virtues, the variety of values and the choices implied in 
ethics. This would develop into the third step in the construction of a specific architecture for a virtues, values and ethics curriculum.

Each of these three steps would need to be explicitly stated and understood at a deep level to facilitate the achievement of rich learning for students within the architecture of a virtues, values and ethics curriculum.

I have indicated the primacy of the new paradigm. I have noted that it dovetails with the work of other twenty-first century educational leaders such as Crowther and Hargreaves. I have situated the whole into a historic inductive concept which identifies at each crucial step how new knowledge emerges from the evaluation and deep analysis of matters uncovered by my three authoritative sources of research.

\section{My Final Reflections on the experience of being a researcher}

\section{Reflection 1}

I have endeavoured to recognise the levels of continuity in the work of those who have gone before me, and to whose work I hope to have made a useful contribution and extension for twenty-first century education. Consistently therefore I have followed Newton's idea of standing on the shoulders of those who have preceded me.

\section{Reflection 2}

I have learned so much about the expertise of my colleagues within the teaching profession and within my academic community throughout the long and tortuous journey of this investigation. I hope that the use of their responses has provided for an accurate and logical construction of a philosophy of education, based on relevant ontological matters, epistemological knowledge and accurate pedagogical conclusions.

\section{Reflection 3}

I have discovered that a deep understanding of the many likely virtues, values and ethics, that can be part of education as a preparation for and part of daily life, is a conduit by which a harmonious structure of reflection, 
resilience and rich learning for teachers and students alike can be achieved. It has been a privilege to complete this investigation and submit the results for examination by my academic colleagues.

\section{Reflection 4}

The thread of continuity that informs edu-tensegrity begins with teachers who teach their students - through the medium of subjects taught - how to apply the broad arc of thinking skills, including knowledge about virtues, values and ethics, for their own understanding and rich learning. This allows teachers to promote the practice of quality decision-making for their students which may then apply to students' careers, to quality in their life decisions, and to their belief systems - whether they be secular or religious in texture, so that all may take part in an education system that is characterised by discernment, democratic cohesion and determined use of academic rigor for rich and sustained learning throughout life.

\section{Reflection 5}

It is to be understood that I offer this thesis as being based within a pedagogical reality that allows for intercultural diversity, international understanding and internal individual choice. It is important to me that the goal is not confused with a utopian dream that would negate the significance and usefulness of its content for $21^{\text {st }}$ century education. 


\section{REFERENCES}

Allix, N. M. (2000). Transformational leadership: Democratic or despotic. BEMAS, 28 (1). 7-20.

Andrews, D. (2010). Editorial. Leading and Managing, 16 (1). ii-iii.

Anih, S. C. (1992). Religious ecumenism and education for tolerance in nigeria. Enugu, Nigeria: Institute of Ecumenical Education Thinkers Corner.

Anih, S. C. (1995). Third millenium god-talk. Enugu, Nigeria: Institute of Ecumenical Education Thinkers Corner.

Aristotle. (1962). Nichomachean ethics. Indianapolis, USA: Bobbs-Merrill.

Armon, C. \& Dawson, T. L (1997). Developmental trajectories in moral reasoning across the life span, Journal of Moral Education, 26 (4), pp. 433-453.

Aspin, D. (2002). An ontology of values and the humanisation of education. In Values in education. Pascoe, S. (Ed.). Deakin West, Australian Capital Territory: Australian College of Educators. 12-24.

Aspin, D. (2003). Actions speak louder. Melbourne, Victoria: Curriculum Corporation.

Ayer, A. J. (1973). The central questions of philosophy. London, UK: Penguin Books.

Barrett, C., (Ed.). (1966). Ludwig wittgenstein: Lectures and conversations on aesthetics, psychology and religious belief. Oxford, UK: Basil Blackwell.

Barrett, W. (1964). What is existentialism? New York, USA: Grove Press, Inc.

Beare, H. (2010). Six decades of continuous school restructuring: Swimming through the waves of reform without being drowned. Penrith, N.S.W.: Australian Council of Educational Leaders.

Beare, H., Caldwell, B. J. \& Millikan, R. H. (1989). Creating an excellent school. London and New York: Routledge.

Beattie, M., (Ed.). (2009). The quest for meaning: Narratives of teaching, learning and the arts. Rotterdam, Nederlands: Sense Publishers.

Beckett, D. (1994). Doing things for the right reasons: Professional knowledge for adult educators. Australian Journal of Adult and Community Education, 34 (2). 104-111. 
Bellanca, J. A. \& Fogarty, R. (1991). Blueprints for thinking in the cooperative classroom. Palatine, Ill: Skylight Publishing.

Beyer, B., Costa, A. \& Presseisen, B. (2001). Glossary of thinking terms. In Developing minds: A resource book for teaching thinking. Costa, A. (Ed.). Moorabbin, Victoria: Association for Supervision and Curriculum Development.

Bezzina, M. (2009). Leading learning: Eight perspectives. Independent Education, 39 (2). 24-26.

Biggs, J. B. \& Telfer, R. (1981). The process of learning. Sydney: PrenticeHall Australia Pty Ltd.

Blackmore, S., (Ed.). (2005). Conversations on consciousness. Oxford, UK: Oxford University Press.

Bloom, B. S., (Ed.). (1956). Taxonomy of educational objectives, the classification of educational goals - handbook i: Cognitive domain. New York: McKay.

Bohm, D. (1998). On dialogue. Thinking: a Journal for Philosophy for Children, 14 (1). 2-7.

Boomer, G., (Ed.). (1982). Negotiating the curriculum. Gosford, NSW, Australia: Ashton Scholastic.

Boostrom, R. (1998). The student as moral agent. Journal of Moral Education, 27 (2). 179-190.

Brenda Cherednichenko, B. (2000). Critical and Creative Thinking, 8 (2).

Brink, D. O. (1989). Moral realism and the foundations of ethics. Cambridge, UK \& New York, USA: Press Syndicate of the University of Cambridge.

Brown, H. D. (1980). Principles of language learning and teaching. Englewood Cliffs, N.J., USA: Prentice-Hall, Inc.

Bruner, J. S. (1960). The process of education. Cambridge, Mass., USA: Harvard University Press.

Burdett, J. (1999). The community of inquiry as means of reducing the incidence of youth suicide. Critical and Creative Thinking, 7 (1).

Burns, M. (2010). Neuroscience applications for building teacher capacity. Australian Educational Leader, 32 (3). 7-10.

Burwood, L. \& Wyeth, R. (1998). Should schools promote toleration? Journal of Moral Education, 27 (4). 465-473.

Caldwell, B. J. \& Spinks, J. M. (1986). Policy-making and planning for school effectiveness. Hobart, Tasmania. Australia:

Caldwell, B. J. \& Spinks, J. M. (1992). Leading the self-managing school. London, UK: The Falmer Press. 
Cam, P. (2002a). Critical and Creative Thinking, 10 (1).

Cam, P. (2002b). Fact, value and philosophy education. Critical and Creative Thinking, 10 (1). 21-28.

Carney, S. (2009). Empathy goes missing. The Age. 10 June 2009. Melbourne.

Carroll, J. (2004). The wreck of western culture. Humanism revisited. Carlton, Victoria, Australia: Scribe Publications Pty Ltd.

Cave, D., (Ed.). (1976). Problems in education. A philosophical approach. Stanmore, NSW, Australia: Cassell Australia Limited.

Cherednichenko, B. (2000). Teaching thinking: Reform for educational equity. Critical and Creative Thinking, 8 (2).

Cherednichenko, B. \& Kruger, T. (2002). Those who can, do! Australian Association of Research in Education, Brisbane.

Cherednichenko, B. \& Wilks, S. (1997). Philosophical inquiry in the classroom: Teaching strategies for improved outcomes. Curriculum and Teaching, 18 (1).

Chittenden, A. H. R. (2004). Ethical leadership in the context of structural and organisational change in pastoral care. Leading and Managing, 10 (1).

Clandinin, D. J. \& Connelly, F. M. (1994). Personal experience methods. In Handbook of qualitative research. Lincoln, N. K. D. Y. S. (Ed.). London: Sage. pp. 413-427.

Clark, L. (2004). Preparing learners for their future not our past. Ideasys Seminar, Taylors Lakes, Melbourne. Australia.

Coch, D. (2007). Neuroimaging research with children: Ethical issues and case scenarios. Journal of Moral Education, 36 (1).

Connell, A., Kessler and Dowsett. (1982).

Connell, R. A., Ashenden, D., Kessler, S. \& Dowsett, G. W. (1982). Making the difference. School, families and social division. Sydney, Australia: George Allen \& Unwin Australia.

Constas, M. A. (1998). Deciphering postmodern educational research. Educational Researcher, 27 (9). 36- 42.

Costa, A. (1992). The school as a home for the mind. A collection of articles. Melbourne, Victoria, Australia: Hawker Brownlow Education.

Costa, A., (Ed.). (2000). Teaching for intelligence Il. A collection of articles. Frenchs Forest, NSW, Australia: Skylight-Hawker-Brownlow.

Costa, A., (Ed.). (2001a). Developing minds: A resource book for teaching thinking. Moorabbin, Victoria: Hawker Brownlow Education. 
Costa, A. (2001b). Habits of mind. In Developing minds. Costa, A. (Ed.). Moorabbin, Victoria: Association for Supervision and Curriculum Development.

Costa, A. \& Garmston, R. J. (2001). Five human passions: The origins of effective thinking. In Developing minds. Costa, A. (Ed.). Moorabbin, Victoria: Association for Supervision and Curriculum Development. 18- 22.

Costa, A. \& Kallick, B. (2001). Building a system for assessing thinking. In Developing minds. Costa, A. (Ed.). Moorabbin, Victoria: Association for Supervision and Curriculum Development.

Costa, A. L., (Ed.). (2001). Developing minds. A resource book for teaching thinking. Moorabbin, Victoria, Australia: Hawker Brownlow Education.

Crowther, F. (1996). Unsung heroes: The leaders in our classrooms (sixth annual william walker oration). Australian Council for Educational Administration Annual Conference, Perth. WA

Crowther, F. (2010). Parallel leadership: The key to successful capacitybuilding. Leading and Managing, 16 (1).

Crowther, F. (2011). From school improvement to sustained capacity: The parallel leadership pathway. Thousand Oaks, California: Corwin Press Inc.

Crowther, F., Abawi, L., Andrews, D., Conway, J. M., Dawson, M., Lewis, M., Morgan, A. R. \& Petersen, S. (2010) Building and sustaining capacity in your school - the cosmic c-b model. Crowther, F., Abawi, L., Andrews, D., Conway, J. M., Dawson, M., Lewis, M., Morgan, A. R. \& Petersen, S.

Curriculum Corporation. (2008). Melbourne declaration on educational goals for young australians. Melbourne: Ministerial Council on Education, Employment, Training and Youth Affairs.

Davies, A. C. (2005). A new democracy for professional development and research: Learning to find the future. Unpublished thesis: Victoria University.

Davis, A. (1995). Criterion-referenced assessment and the development of knowledge and understanding'. Journal of Philosophy of Education, 29 (1).

de Bono, E. (1987). Cort thinking program. Workcards and teachers' notes. Chicago USA: Science Research Associates.

de Bono, E. (1992). Serious creativity. London, UK: HarperCollinsPublishing.

de Bono, E. (1993). Teach your child how to think. New York, USA: Penguin. 
de Bono, E. (1994). Parallel thinking. London, UK: Viking.

de Bono, E. (1996). Teach yourself to think. London, UK: Penguin Books.

de Botton, A. (2004). Status anxiety. Camberwell, Victoria Australia: Hamish Hamilton.

de Spinoza, B. (1955). On the improvement of the understanding. The ethics. Correspondence. New York. USA: Dover Publications. Inc.

Dewey, J. (1906). The experimental theory of knowledge. Mind: A Quarterly Review of Psychology and Philosophy, 59 (July). 293-307.

Dewey, J. (1944). Democracy and education: An introduction to the philosphy of education. New York: The Free Press.

Dialogue Australasia Network. Dialogue journal. Retrieved from http://www.dialogueaustralasia.org/?page_id=77. 12 March 2012.

Dispenza, J. (2007). Evolve your brain. Deerfield Beach, Florida, USA: Health Communications, Inc

Doidge, N. (2007). The brain that changes itself. Carlton, Victoria, Australia: Scribe Publications Pty Ltd.

Doidge, N. (2010). Changing your mind. Toronto, Canada: 90th Parallel Productions Inc.

Dwyer, B. \& English, G. (1988a). Catholics in australia: Our story (student book). Blackburn: Collins Dove.

Dwyer, B. \& English, G. (1988b). Catholics in australia: Our story (teachers' book). Blackburn: Collins Dove.

Easterbrooks, S. R. \& Scheetz, N. A. (2004). Applying critical thinking skills to character education and values clarification with students who are deaf or hard of hearing. American Annals of the Deaf, 149 (3). 255263.

Edman, P. (2009). Being fully human: Values education. Australian Catholics, (Winter 2009).

Ekman, P. (2001). Telling lies: Clues to deceit in the marketplace, politics and marriage. New York, USA: W. W. Norton \& Company, Inc.

Ekman, P., (Ed.). (2008). Emotional awareness. A conversation between the dalai lama and paul ekman. New York, USA: Holt Paperbacks.

Eliot, T. S. (1962). Notes towards the definition of culture. London, UK: Faber and Faber Limited.

Elliott, R. H. (1986). Exploring religions and faith at school. Bellevue Heights, South Australia 5050: The Australian Association for Religious Education.

Elliott, R. H. (1988). In quest for integrity for protestant church-related schools. Religious Education Journal of Australia, 4 (1). p. 15- 21. 
Evers, C. W. \& Lakomski, G. (1991). Knowing educational administration Oxford. UK: Pergamon Press.

Evers, C. W. \& Lakomski, G. (1996). Exploring educational administration. Coherentist applications and critical debates. Oxford, UK: Pergamon.

Fairbairn, G. (1999). Empathy, intuition and the development of expertise in teaching. Analytic Teaching, 19 (2).

Fisher, R. (1990). Teaching children to think. Cheltenham, UK: Nelson Thornes.

Fisher, R. (2001). Teaching children to think. Cheltenham, UK: Nelson Thornes.

Fisher, R. (2003). Teaching thinking London, UK: Continuum.

Fitzgerald, R., (Ed.). (1993). The eleven deadly sins. Melbourne, Victoria, Australia: William Heinemann Australia.

Flew, A. (1972a). Indoctrination and doctrines. In Concepts of indoctrination. Snook, I. A. (Ed.). London: Routledge \& Kegan Paul.

Flew, A. (1972b). Indoctrination and religion. In Concepts of indoctrination. Snook, I. A. (Ed.). London: Routledge \& Kegan Paul.

Flick, U. (2002). An introduction to qualitative research. London, UK: Sage Publications.

Fogarty, R. (1990). Designs for cooperative interactions. Palatine, Ill.: Skylight Publishing.

Fogarty, R. (1991). The mindful school: How to integrate the curricula. Palatine, Ill.: IRI Skylight Pub.

Fogarty, R. (1993). Integrating the curricula: A collection. Palatine, Ill.: IRI/Skylight Pub.

Fogarty, R. (2001a). Differentiated learning: Different strokes for different folks. Chicago: Fogarty \& Associates.

Fogarty, R. (2001b). Our changing perspective of intelligence: Master architects of the intellect. In Developing minds. Costa, A. (Ed.). Moorabbin, Victoria: Association for Supervision and Curriculum Development. 144- 149.

Fogarty, R. (2005). Ten things new teachers need to succeed. Moorabbin, Victoria, Australia: Hawker Brownlow Education.

Fogarty, R., Perkins, D. N. \& Barell, J. (1992). The mindful school: How to teach for transfer. Palatine, Ill.: Skylight Pub.

Fogarty, R. \& Pete, B. (2003). Twelve brain principles that make the difference. Hawker Brownlow Education.

Foot, P., (Ed.). (1967). Theories of ethics. Oxford, UK: Oxford University Press. 
Freakley, M. \& Burgh, G. (2000). Engaging with ethics. Ethical enquiry for teachers. Katoomba, NSW, Australia: Social Science Press.

Freeman, E. \& Appel, D. (1956). The wisdom and ideas of plato. New York, USA: Premier Books/ Fawcett World Library.

Freire, P. (1972). Pedagogy of the oppressed. London UK: Penguin Books.

Fukuyama, F. (1999). The great disruption. London. UK: Profile Books.

Fukuyama, F. (2007). After the neocons. London, UK: Profile Books Ltd.

Fullan, M. (2005 ). Leadership and sustainability: System thinkers in action. Thousand Oaks, CA: Corwin Press.

Gagné, R. M. \& Briggs, L. J. (1974). Principles of instructional design. New York: Holt, Rinehardt and Winston.

Gardiner, P. (1988). Kierkegaard. Oxford, UK: Oxford University Press.

Gardner, H. (1991). The unschooled mind. How children think, and how schools should teach. London: Fontana Press.

Gardner, H. (1993a). Frames of mind: The theory of multiple intelligences. London, UK: Fontana Press.

Gardner, H. (1993b). The unschooled mind. How children think, and how schools should teach. London: Fontana Press.

Gardner, H. (2008). Five minds for the future. Boston, USA: Harvard Business Press.

Gardner, H., Csikszentmihalyi, M. \& Damon, W. (2001). Good work. When excellence and ethics meet. New York, USA: Basics Books.

Gardner, H. \& Senge, P. (2000). The context of reframing learning. In Schools that learn: A fifth discipline fieldbook for educators, parents and everyone who cares about education. Senge, P., CambronMcCabe, N., Lucas, T.et al (Ed.). London: Nicholas Brealey Publishing.

Gardner, S. (1996). Inquiry is no mere conversation (or discussion or dialogue). Facilitation of inquiry is hard work. Analytic Teaching, 16 (2).

Gardner, S. (1997). Philosophy: A potential gender blender. Analytic Teaching, 17 (2).

Gardner, S. (1998). Truth: In ethics and elsewhere. Analytic Teaching, 19 (1).

Gardner, S. (1999). Interactive reasoning: The road to freedom. Critical and Creative Thinking, (7).

Gardner, S. (1999). Interactive reasoning: The road to freedom. Critical and Creative Thinking, 7 
Garmston, R. \& Wellman, B. (1999). Adaptive schools: A sourcebook for developing collaborative groups. Norwood, MA: Christopher Gordon.

Giberson, K. W. \& Collins, F. S. (2011). The language of science and faith. Downers Grove, Ill. USA: InterVarsity Press.

Giddens, A. (1998). The third way. The renewal of social democracy. Cambridge, UK: Polity Press.

Glaser, B. J., (Ed.). (2002). Relevance, history, features. Process and theories. London, UK: Sage Publications.

Glasser, W. (1969). Schools without failure. New York, USA: Harper \& Row.

Goldhagen, D. J. (2002). A moral reckoning. London, UK: Little, Brown/ Time Warner Books UK.

Golding, C. (2006). Thinking with rich concepts. Heatherton, Victoria, Australia: Hawker Brownlow Education.

Goleman, D. (1996). Emotional intelligence. Why it can matter more than iq. London, UK: Bloomsbury Publishing Plc.

Goleman, D. (1998a). Vital lies, simple truths. London, UK: Bloomsbury Publishing Plc.

Goleman, D. (1998b). Working with emotional intelligence. London, UK: Bloomsbury Publishing Plc.

Goleman, D. (2007). Social intelligence. London UK: Arrow Books.

Goleman, D. \& Lama, D. (2004). Destructive emotions. London UK: Bloomsbury Publishing.

Grayling, A. C. (2011). The good book: A secular bible. London: Bloomsbury.

Greenfield, S. (2004). Tomorrow's people. How 21st-century technology is changing the way we think and feel. London. UK: Penguin Books.

Greenfield, S. (2007). Keynote address: Wednesday morning. 'Education today'. Thinking Conference, Norrkoping, Sweden.

Greenfield, S. (2008). I.D.: The quest for identity in the 21st century. London, UK: Sceptre, an imprint of Hodder \& Stoughton.

Greenfield, S. (2011). A dementia free future: Fantasy or reality? National Press Club Address, Canberra, Australia.

Gribble, J. (1969). Introduction to philosophy of education. Boston, USA: Allyn and Bacon, Inc.

Grierson, D. (2009). How secular are we? Mulgrave, Victoria, Australia: johngarrattpublishing. 2: 
Griffiths, A. P., (Ed.). (1993). Ethics. London: Cambridge University Press.

Gula, R. M. (1989). Reason informed by faith. Foundations of catholic morality. Mahwah, NJ., USA: Paulist Press.

Habermas, J. (1987). Knowledge \& human interests. Oxford, UK: Polity Press.

Handy, C. (2001). The elephant and the flea. London, UK: Hutchinson.

Hare, R. M. (1981). Moral thinking. Its levels, method and point. Oxford, UK: Clarendon Press/ Oxford University Press.

Hare, R. M. (1992). Essays on religion and education. Oxford. UK: Clarendon Press.

Hargreaves, A. (2003). Teaching in the knowledge society. Education in the age of insecurity. New York, USA: Teachers College Press.

Hargreaves, A. (2009). The fourth way of educational reform. Melbourne: ACEL.

Hargreaves, A. \& Fink, D. (2006). Sustainable leadership. San Francisco CA: Jossey-Bass.

Hargreaves, A. \& Shirley, D. (2009). The fourth way: The inspiring future for educational change. Thousand Oaks, California: Corwin Press Inc.

Haring, B. (1978). Free and faithful in christ. Homebush, NSW. Australia: Society of St Paul Publications.

Harrington, D. J. (2005). How do catholics read the bible? Lanham, Maryland, USA: Rowman \& Littlefield Publishers, Inc.

Hauser, M. D. (2006). Moral minds: How nature designed our universal sense of right and wrong. London, UK: Abacus.

Hawkes, N. (2010). Does teaching values improve the quality of education in primary schools? A study about the impact of values education in a primary school. Saarbrücken, Germany: VDM Verlag Dr. Müller Aktiengesellschaft \&Co. KG.

Heasly, B. T. (1995). Moral bases for professional teachers' planning and practice. Unpublished thesis: University of Melbourne.

Hick, J. H. (1973). Philosophy of religion. Englewood Cliffs, New Jersey. USA: Prentice-Hall, Inc.

Hicks, A. J. (1997). Children's concepts of procedural justice. Unpublished thesis: University of Melbourne.

Hill, J. (2011). How does acel develop leading learners for effective change? The Australian Educational Leader, 33 (2). 4- 6.

Hirst, P. H. \& Peters, R. S. (1970). The logic of education. London, UK: Routledge and Kegan Paul Ltd. 
Hodgkinson, C. (1991). Educational leadership: The moral art Albany,NY., USA: SUNY.

Hopkins, A. (1978). The school debate. Middlesex, UK: Penguin Books Ltd. Horgan, J. (1999). The undiscovered mind. New York, USA: The Free Press.

Hospers, J. (1982). Human conduct -problems of ethics. New York, USA: Harcourt Brace Jovanovich, Inc.

Hosseinee, A. A. (2008). Investigating the impact of the creativity teaching program on teachers' knowledge, attitude, and skills. Journal of Educational Innovations, 22 (Winter). 67-72.

Howe, B. (2007). Weighing up australian values. Sydney, Australia: University of New South Wales Press Ltd.

Hoy, W. K. \& Miskel, C. G. (1982). Educational administration New York. USA: Random House.

Ingber, D. (1997). The architecture of life. Scientific American, January, 1998 p. 48- 57.

Jackson, Y. (2001). Reversing underachievement in urban students: Pedagogy of confidence. In Developing minds. Costa, A. (Ed.). Moorabbin, Victoria Australia: Association for Supervision and Curriculum Development. 222- 228.

Jensen, E. (2006). Enriching the brain: How to maximize every learner's potential. San Francisco: Jossey-Bass a Wiley imprint.

JohnPaul 11. (1994). Catechism of the catholic church. St Pauls, Homebush, NSW, Australia.

JohnPaul. (1994). Catechism of the catholic church. St Pauls, Homebush, NSW, Australia.

Joyce, B. \& Weil, M. (1986). Models of teaching. Englewood Cliffs, NJ., USA: Prentice-Hall, Inc.

Kagan, S. (1994). Cooperative learning. San Clemente, CA: Kagan Coperative Learning.

Kaldor, P., Hughes, P. \& Black, A. (2010). Spirit matters -how making sense of life affects well-being. Preston, Victoria, Australia: Mosaic Press.

Kallick, B. (2001). Teaching thinking through technology; assessing growth in thinking abilities; building a system for assessing thinking. Moorabbin, Victoria: Association for Supervision and Curriculum Development. p. 472, 496, 517- 524 p. 472, 496, 517- 524.

Kay, W. (1975). Moral education. A sociological study of the influence of society, home and school. London.UK: George Allen \& Unwin Ltd.

Keeves, J. P. \& Lakomski, G., (Ed.). (1999). Issues in educational research. Oxford,UK: Pergamon. An Imprint of Elsevier Science. 
Kenny, A. (1973). Wittgenstein. Middlesex, UK: Pelican/ Penguin Books Ltd.

Kincheloe, J. L., (Ed.). (2005). Auto/biography and critical ontology: Being a teacher, developing a reflective persona. Rotterdam, Ned: Sense Publishers.

Kincheloe, J. L., McLaren, P. \& Steinberg, S. R. (2011). Critical pedagogy and qualitative research: Moving to the bricolage. In The sage book of qualitative research: 4. Denzin, N. K.\& Lincoln, Y. S. (Ed.). Thousand Oaks, CA: Sage Publishers, Inc.

Klapwijk, R. \& Rommes, E. (2009). Career orientation of secondary school students $(\mathrm{m} / \mathrm{f})$ in the netherlands. International Journal of Technology and Design Education, 19 (4). 403- 418.

Klein, N. (2007). The shock doctrine. Camberwell, Australia: Allen Lane (Penguin).

Knowles, M. S. (1984). Andragogy in action. San Francisco: Jossey-Bass.

Kramer, J. \& Alstad, D. (2009). The passionate mind revisited. Berkeley, California, USA: North Atlantic Books.

Krishnan, S. (2009). Student experiences of problem-based learning in engineering: Learning cultures of pbl teams. Unpublished thesis: Victoria University.

Kristjannsson, K. (2000). The didactics of emotion education. Analytic Teaching, 21 (1).

Kristjansson, K. (1998). Self-respect, megalopsychia, and moral education. Journal of Moral Education, 27 (1).

Laurence, G. (1991). People types and tiger stripes. Florida, USA: CAPT.

Leckey, M., (Ed.). (2009). Turning points: Evaluation of a philosophy for children program with adolescents. Peter Lang. Internationaler Verlag der Wissenschaften.

Leonard, R. (2009). The mystical gaze of the cinema. The films of peter weir. Carlton. Victoria. Australia: Melbourne University Press.

Levin, S. (2002). The tensegrity-truss as a model for spine mechanics: Biotensegrity. Journal of Mechanics in Medicine and Biology, 2 (3 \& 4). 375-388.

Lewis, C. S. (1940). The problem of pain. Glasgow, Scotland, UK: Fount Paperbacks/ Collins.

Lewis, C. S. (1946). The great divorce. Glasgow, Scotland, UK: Fount Paperbacks/ Collins.

Lewis, M. (2010). Editorial Leading and Managing, 16 (2). p.ii- iv. 
Lewis, M. (2011). The hitchhiker's guide to the leadership cosmos. The Australian Educational Leader, 33 (1).

Lim, T. K. (1998). How to evaluate a philosophy for children program. Critical and Creative Thinking, 6 (1).

Lincoln, Y. S., Lynham, S. A. \& Guba, E. G. (2011). Paradigmatic controversies, contradictions, and emerging confluences, revisited. In The sage handbook of qualitative research 4 . Denzin, N. K.\& Lincoln, Y. S. (Ed.). Thousand Oaks, CA: Sage Publishers, Inc.

Ling, I. M. (1998). The role of the curriculum coordinator: An exploration through discursive practice (Unpublished DEd thesis). The University of Melbourne, Victoria, Australia.

Ling, I. M. (2012). The bricolage and the multiple spaces of tesl research. In Critical qualitative research reader. Steinberg, S. R.\& Cannella, G. (Ed.). New York: (In press) Peter Lang.

Lipman, M. (1967). What happens in art. New York: Appleton-CenturyCrofts.

Lipman, M. (1969). Discovering philosophy. New York,: Appleton-CenturyCrofts.

Lipman, M. (1988). Philosophy goes to school. Philadelphia: Temple University Press.

Lipman, M. (1991). Thinking in education. Cambridge ; New York: Cambridge University Press.

Lipman, M. (1993a). Thinking children and education. Dubuque, Iowa: Kendall/Hunt Pub. Co.

Lipman, M., (Ed.). (1993b). Thinking children and education. Dubuque, Iowa, USA: Kendall/ Hunt Publishing Company.

Lipman, M. (2003). Thinking in education. New York: Cambridge University Press. xii xii.

Lipman, M. \& Gazzard, A. (1988). Getting our thoughts together : Instructional manual to accompany elfie. Upper Montclair, N.J.: Institute for the Advancement of Philosophy for Children, Montclair State College.

Lipman, M. \& Montclair State College. Institute for the Advancement of Philosophy for Children. (1980). Mark. Upper Montclair, N.J.: Institute for the Advancement of Philosophy for Children, Montclair State College.

Lipman, M. \& Montclair State College. Institute for the Advancement of Philosophy for Children. (1981). Pixie. Upper Montclair, NJ: Institute for the Advancement of Philosophy for Children, Montclair State College. 
Lipman, M. \& Montclair State College. Institute for the Advancement of Philosophy for Children. (1985b). Lisa. Upper Montclair, N.J.: [First Mountain Foundation].

Lipman, M. \& Montclair State College. Institute for the Advancement of Philosophy for Children. (1986). Kio \& gus. Upper Montclair, N.J.: Institute for the Advancement of Philosophy for Children, Montclair State College.

Lipman, M. \& Montclair State College. Institute for the Advancement of Philosophy for Children. (1987b). Suki. Upper Montclair, N.J.: Institute for the Advancement of Philosophy for Children, Montclair State College.

Lipman, M. \& Montclair State College. Institute for the Advancement of Philosophy for Children. (1988b). Elfie. Upper Monclair, N.J.: Institute for the Advancement of Philosophy for Children.

Lipman, M. \& Sharp, A. M. (1978). Growing up with philosophy. Philadelphia: Temple University Press.

Lipman, M. \& Sharp, A. M. (1980a). Social inquiry: Instructional manual to accompany mark. Upper Montclair, N.J: Institute for the Advancement of Philosophy for Children, Montclair State College.

Lipman, M. \& Sharp, A. M. (1980b). Writing: How and why: An intructional manual to accompany suki. Upper Montclair, N.J: Institute for the Advancement of Philosophy for Children, Montclair State College.

Lipman, M. \& Sharp, A. M. (1985). Ethical inquiry: Instructional manual to accompany lisa. Lanham, Md.: University Press of America.

Lipman, M. \& Sharp, A. M. (1986). Wondering at the world: Instructional manual to accompany kio \& gus. Lanham, Md. Upper Montclair, NJ: University Press of America ; Institute for the Advancement of Philosophy for Children.

Lipman, M., Sharp, A. M. \& Oscanyan, F. (1977). Philosophy in the classroom. Upper Montclair, N.J.: Institute for the Advancement of Philosophy for Children, Montclair State College.

Lipman, M., Sharp, A. M. \& Oscanyan, F. S. (1980). Philosophy in the classroom. Philadelphia: Temple University Press.

Lipman, M., Sharp, A. M. \& Oscanyan, F. S. (1984). Philosophical inquiry: An instructional manual to accompany harry stottlemeier's discovery. Lanham, MD. [Montclair, N.J.]: University Press of America, Institute for the Advancement of Philosophy for Children.

Lipton, B. H. (2008). The biology of belief. Carlsbad, CA., USA: Hay House, Inc.

Lovat, T. J. (1989). What is this thing called religious education? Wentworth Falls, NSW, Australia: Social Science Press. 
Lozano, A. (2001). A survey of thinking and learning styles. In Developing minds. Costa, A. (Ed.). Moorabbin: Association for Supervision and Curriculum Development. 192- 196.

Lucas, S. (2010). Nurture groups in schools London.UK: SAGE Publications Ltd.

Lushyn, P. \& Kennedy, D. (2000). The psychodynamics of community of inquiry and educational reform: A cross-cultural perspective. Thinking: a Journal for Philosophy for Children, 15 (3).

Macdonald, C. (1996). Towards wisdom. Willowdale, Ontario, Canada: Hounslow Press.

Mackay, H. (1993). Reinventing australia. Sydney, Australia: Angus\&Robertson.

Mackay, H. (1997). Generations. Sydney, Australia: Pan Macmillan Australia.

Mackay, H. (1999). Turning point. Australians choosing their future. Sydney, NSW. Australia: Pan Macmillan Australia Pty Ltd.

Mackay, H. (2004). Right and wrong. Sydney, Australia: Hodder Headline Australia P/L.

Mackay, H. (2010).

Mackie, J. L. (1977). Ethics. Inventing right and wrong. London, UK: Penguin Books.

Macqueen, S. (2009). Preservice teaching and values education: Achieving authenticity. The Social Educator, 27 (1). 16- 22.

Markova, D. (1992). How your child is smart. Berkeley. USA: Conari Press.

Martin, D. (2011). The future of christianity. Farnham, Surrey, UK: Ashgate Publishing Limited.

Marzano, R. J. (2001). A new taxonomy of educational objectives. In Developing minds. Costa, A. (Ed.). Moorabbin: Association for Supervision and Curriculum Development. 181- 188.

Mathews, R. (1999). Jobs of our own. Sydney, Australia and London, UK: Pluto Press/ Comerford and Miller.

McAdam, J. G. (2011). The magic of colour, the role of art and the future of dementia care. Society for the Arts in Healthcare -22nd Annual International Conference:Advancing Patient-Centred Arts, Burlingame, CA, USA.

McNamara, J. (1983). The power of compassion. New York. USA: Paulist Press.

McNeely, I. F. \& Wolverton, L. (2009). Reinventing knowledge. New York, USA: W.W.Norton and Company. 
Menec, V. H. (2003). The relation between everyday activities and successful aging: A 6-year longitudinal study. Journal of Gerontology, 58B (2).

Mews, C. J. (1/12/2008). to Heasly, B. Email.

Mews, C. J. (2009). Catholicism, nostra aetate, and interreligious dialogue: From multiculturalism to interculturalism. Champagnat, 11 (1).

Michelli, N. M. (1999). Teacher education in a democracy: Towards an apprenticeship of liberty. Journal for Philosophy for Children, 14 (3).

Mills, C. W. (1978). The sociological imagination. Middlesex, UK: A Pelican Book. Penguin Books Ltd.

Moore, B. (1986). Five strategies for teaching. Australian Association for Religious Education, Brisbane, Australia.

Mulford, W. R. (1977). Structured experiences for use in the classroom: Primary, secondary, tertiary, inservice. Canberra: Centre for Continuing Education, Australian National University.

Mulford, W. R. (1994). Shaping tomorrow's schools Melbourne: Australian Council for Educational Administration.

Murris, K. (2000). The role of the facilitator in philosophical inquiry. Thinking: a Journal for Philosophy for Children., 15 (2).

Newton, I. (15 February 1676). to Hooke, R. Letter.

Norman, D. A. (1969). Memory and attention. An introduction to human information processing. New York, USA: John Wiley \& Sons, Inc.

Nouwen, H. J. M. (1983). I gracias! A latin american journal. San Francisco, USA: Harper \& Row.

Nussbaum, M. C. (2001). Upheavals of thought. The intelligence of emotions. Cambridge, UK: Cambridge University Press.

O'Connor, P. (2001). Beyond the mist. Crows Nest, NSW. Australia: Allen \& Unwin.

O'Neill, O. (1993). Duties and virtues. In Ethics. Griffiths, A. P. (Ed.). Cambridge UK: Cambridge University Press.

Obama, B. (2006). The audacity of hope. New York and Melbourne: Random House and The Text Publishing Company.

Obama, B. (2008). Dreams from my father. Melbourne, Australia: The Text Publishing Company.

Pascoe, S. (1996). Broadening our horizons. Hawthorn, Australia: Australian Council for Educational Administration.

Pascoe, S., (Ed.). (2002). Values in education. Deakin West ACT: Australian College of Educators. 
Paul, R. W. (2001). Dialogical and dialectical thinking. In Developing minds. Costa, A. (Ed.). Moorabbin, Australia: Association for Supervision and Curriculum Development. 427- 436.

Pears, D. \& McGuinness, B. F. (1971). Wittgenstein. Glasgow, Scotland, UK: Fontana/Collins.

Pelikan, J., (Ed.). (1990). The world treasury of modern religious thought. Boston, USA: Little, Brown and Company.

Postman, N. (1986). Amusing ourselves to death. London. UK: Heinemann.

Postman, N. (1996). The end of education. Redefining the value of school. New York, USA: Knopf.

Pratt, D. (2003). Rethinking religion. Exploratory investigations. Adelaide, Australia: Australian Theological Forum Press.

Pritchard, M. S. (1985). Philosophical adventures with children. Lanham, MD, USA: University Press of America, Inc.

Raphael, D. D. (1976). Problems of political philosophy. London, UK.: The Macmillan Press Ltd.

Redshaw, S. (1995). Philosophical inquiry: Promoting thinking in tertiary education. Thinking: The Journal of Philosophy for Children, 12 (2).

Reed, R. F., (Ed.). (1992). When we talk: Essays on classroom conversation. Fort Worth, Texas, USA: Analytic Teaching Press.

Reich, K. H. (1995). From either/or to both/and through cognitive development. Thinking: Journal for Philosophy for Children., 12 (2).

Revell, L. \& Arthur, J. (2007). Character education in schools and the education of teachers. Journal of Moral Education, 36 (1).

Rich, J. M., (Ed.). (1972). Readings in the philosophy of education. Belmont, CA. USA: Wadsworth Publishing Company, Inc.

Richardson, B. (1997). Teaching for presence in the democratic classroom. Thinking: Journal for Philosophy for Children, 13 (1).

Riso, D. R. (1992). Discovering your personality type. New York, USA: Houghton Mifflin Company.

Rohr, R. \& Ebert, A. (2009). The enneagram. A christian perspective. New York, USA: The Crossroad Publishing Company.

Roth, W.-M., (Ed.). (2005a). Auto/biography and auto/ethnography: Finding the generalized other in the self. Rotterdam, Ned: Sense Publishers.

Roth, W.-M., (Ed.). (2005b). The write of passage: Reflections on writing a dissertation in narrative methodology

Rotterdam, Ned.: Sense Publishers.

Rousseau, J.-J. (1974). Emile. London, UK: J. M. Dent \& Sons Ltd. 
Rowland, M. D. (1993). Absolute happiness. Blackheath, NSW, Australia: Self Communications $\mathrm{P} / \mathrm{L}$.

Ruiz, P. O. \& Vallejos, R. M. (1999). The role of compassion in moral education. Journal of Moral Education, 28 (1).

Russell, B. (2004). History of western philosophy. London, UK Routledge Classics.

Sacks, J. (1997). The politics of hope. London UK: Vintage.

Sacks, J. (2000a). The politics of hope. London UK: Vintage.

Sacks, J. (2000b). Radical then, radical now. On being jewish. New York: Continuum.

Sacks, J. (2003). The dignity of difference. Kondon UK: Continuum.

Saul, J. R. (2009). The collapse of globalism. Melbourne, Australia: Penguin Group (Australia).

Saxby, M. \& Gross, J. (1999). The devil's trousers - a folktale from bologna. Milsons Point, NSW: Random House Pty Ltd, Sydney, Australia.

Schon, D. (1983). The reflective practitioner: How professionals think in action. London: Temple Smith.

Schon, D. (1987). Educating the reflective practitioner. San Francisco: Jossey-Bass.

Schwab, J. J. (1983). The practical 4: Something for curriculum professors to do. Curriculum Inquiry, 13 (3).

Schwartz, S., (Ed.). (2000). The seven deadly sins. New York, USA: Gramercy Books.

Schweigert, F. J. (1999). Learning the common good: Principles of community-based moral education in restorative justice. Journal of Moral Education, 28 (2).

Seiger-Ehrenberg, S. (2001). Concept development. In Developing minds. Costa, A. (Ed.). Moorabbin, Victoria: Association for Supervision and Curriculum Development. 437- 441.

Seligman, M. E. P. (1990). Learned optimism. Milsons Point, NSW, Australia: Random House Australia Pty Ltd.

Seligman, M. E. P. (2002). Authentic happiness. Sydney, Australia: Random House Australia.

Seligman, M. E. P. (2011). Flourish. North Sydney, Australia: William Heinemann.

Senge, P., Cambron-McCabe, N., Lucas, T., Smith, B., Dutton, J. \& Kleiner, A. (2000). Schools that learn: A fifth discipline resource. London, UK: Nicholas Brealey Publishing. 
Senge, P. M. (1992). The fifth discipline. The art \& practice of the learning organisation. Milsons Point, NSW, Australia: Random House Australia Pty Ltd.

Senge, P. M. (2004). The fifth discipline: The art \& practice of the learning organization. Milsons Point, NSW, Australia: Random House Australia Pty Ltd.

Senge, P. M. (2011). Keynote address: Education today. Australian Council of Educational Leadership, Adelaide.

Sergiovanni, T. J. (1992). Moral leadership: Getting to the heart of school improvement. San Francisco, USA: Jossey-Bass.

Sergiovanni, T. J. \& Corbally, J., (Ed.). (1984). Leadership and organisational culture. Urbana and Chicago, USA: University of Illinois Press.

Sharansky, N. \& Dermer, R. (2004). The case for democracy. The power of freedom to overcome tyranny and terror. New York, USA: Public Affairs.tm.

Sharp, A. M., Reed, R. F. \& Lipman, M. (1996). Studies in philosophy for children: Pixie. Madrid: Ediciones de la Torre.

Sharp, A. M. \& Splitter, L. J. (1995). Teaching for better thinking. The classroom community of inquiry.

Shulman, L. (1987). Knowledge and teaching: Foundations of the new reform. Harvard Educational Review, 57 (1). 1-22.

Singer, I. B. (1983). The penitent. London, UK: Penguin Books.

Singer, P. (1979). Practical ethics. Cambridge, UK: Cambridge University Press.

Singer, P. (1993). How are we to live? Ethics in an age of self-interest. Melbourne: The Text Publishing Company Pty Ltd.

Singer, P. (2002). One world -the ethics of globalisation. Melbourne, Australia: The Text Publishing Company.

Singer, P. (2009). The life you can save. Acting now to end world poverty. Melbourne, Victoria, Australia: Text Publishing Company.

Skorupski, J. (1993). The definition of morality. In Ethics. Griffiths, A. P. (Ed.). Cambridge: Cambridge University Press.

Smyth, J., (Ed.). (1993). A socially critical view of the self-managing school. London UK: Falmer Press.

Snook, I. A., (Ed.). (1972). Concepts of indoctrination: Philosophical essays. London, UK: Routledge \& Kegan Paul.

Snook, I. A., (Ed.). (1975). Indoctrination and education. London, UK: Routledge and Kegan Paul. 
Solomon, R. C., (Ed.). (1974). Existentialism. New York, USA: Random House, Inc.

Splitter, L. (1999). Thinking: a Journal for Philosophy for Children, 14 (3).

Splitter, L. J. (1997). Philosophy and democracy in asia and the pacific: Philosopphy and civic education. Thinking: a Journal for Philosophy for Children, 13 (3).

Splitter, L. J. \& Sharp, A. M. (1995). Teaching for better thinking: The classroom community of inquiry. Melbourne, Victoria, Australia: Australian Council for Educational Research.

Stead, B. (1996). The influence of critical biblical study on the teaching and use of scripture in catholic primary schools in victoria. Unpublished thesis: Latrobe University.

Stockton, E. (1995). The aboriginal gift. Spirituality for a nation. Alexandria, NSW. Australia: Millenium Books.

Storer, D., (Ed.). (1985). Ethnic family values in australia. Sydney, Australia: Prentice-Hall of Australia Pty. Ltd.

Strauss, A. \& Corbin, J. (1990). Basics of qualitative research. London: Sage.

Strawson, P. F. (1963). Introduction to logical theory. London UK University Paperbacks, Methuen \& Co. Ltd.

Strawson, P. F. (1985). Skepticism and naturalism. Some varieties. London, UK: Methuen \& Co. Ltd.

Sutcliffe, R. (2009). Personal and social philosophy. p4c.com. Retrieved from http://p4c.com/articles/newsletter-october-2009. 29 November, 2011.

Sutcliffe, R. (2010). to Heasly, B. Letter.

Swartz, R. (2001). Thinking about decisions. In Developing minds. Costa, A. (Ed.). Moorabbin, Victoria: Association for Supervision and Curriculum Development. 144- 149.

Swartz, R. J., Costa, A. L., Beyer, B. K., Reagan, R. \& Kallick, B. (2008). Thinking-based learning. Activating students' potential. Norwood, MA, USA: Christopher-Gordon Publishers, Inc.

Tacey, D. J. (1995). Edge of the sacred. Transformation in australia. Blackburn, Victoria, Australia: HarperCollinsPublishers.

Tacey, D. J. (2003). The spirituality revolution. Sydney, NSW: HarperCollinsPublishers.

Tancredi, L. (2007). Hard-wired behaviour: What neuroscience reveals about morality. Journal of Moral Education, 36 (1). 
Tarnas, R. (1991). The passion of the western mind. New York: Ballantine Books.

Taylor, C. (2007). A secular age. Cambridge, MASS. USA: The Belknap Press of Harvard University Press.

Taylor, M., (Ed.). (1975). Progress and problems in moral education. NFER Publishing Company Ltd, UK.

Theobald, R. (1997). Reworking success. New communities at the millenium. Gabriola Island, BC., Canada: New Society Publishers.

Thomas, R. M. (2003). Blending qualitative and quantitative research methods in theses and dissertations. Thousand Oaks, California: Corwin Press, Inc.

Thornton, P., Phelps, R. \& Graham, A. (2011). Teacher leaders driving change: Inquiry process. The Australian Educational Leader, 33 (2). 8- 12.

Tishman, S., Perkins, D. N. \& Jay, E. (1995). The thinking classroom. Needham Heights. MA, USA: Allyn \& Bacon.

Tuckman, B. (1965). Developmental sequence in small groups. Psychological Bulletin, 63 (6). 384-399.

University of Birmingham \& COBUILD. (2001). Collins cobuild english dictionary for advanced learners. London \& Glasgow: HarperCollins.

Van Hooft, S. (2012). Religious classes in state schools must be about teaching, not preaching, The Age, p. 13, 2 March.

Vardy, P. (1998). The five strand approach to religious and values education in australia. Melbourne, Australia:

Vardy, P. (1999). What is truth? Sydney: University of New South Wales Press.

Verharen, C. C. (1997). A philosophical approach to teaching reasoning. Thinking: a Journal for Philosophy for Children, 13 (2).

Victorian Board of Studies. (2000). Curriculum and standards framework ii (csf). Carlton, Victoria: Board of Studies.

Walker, A. (2011). School leadership as connective activity. Monograph 48ACEL,

Warnock, M. (1970). Existentialism. Oxford: Oxford Univeristy Press.

Wassermann, S. (1993). Getting down to cases. Learning to teach with case studies. New York USA: Teachers College Press, Columbia University.

Wassermann, S. (1997). The secrets of asking the right questions. SCORE, 4 (3). 13-16.

Watson, G., (Ed.). (1982). Free will. Oxford UK: Oxford University Press. 
Watt, A. J. (1976). Rational moral education. Carlton, Victoria, Australia: Melbourne University Press.

Wellham, D. (2010). Emotional intelligence development for churchie boys in 'making of men'. The Australian Educational Leader, 32 (3).

Wilks, S. (1995). Critical and creative thinking -strategies for classroom enquiry. Eleanor Curtain Publishing.

Wilks, S. (1996). Talking it over beats the bullies. Analytic Teaching, 17 (1).

Wilks, S., (Ed.). (2005). Designing a thinking curriculum. Camberwell, Vic: ACER Press.

Williams, B. (1972). Morality. An introduction to ethics. Cambridge, UK: Cambridge University Press.

Wilson, J. (1963). Thinking with concepts. London, UK: Cambridge University Press.

Wilson, J. (1965). Logic and sexual morality. Middlesex, UK: Penguin Books Ltd.

Wilson, J. (1971). Education in religion and the emotions. London,UK: Heinemann Educational Books Ltd.

Wilson, J. (1972). Indoctrination and rationality. In Concepts of indoctrination: Philosophical essays. Snook, I. A. (Ed.). London, UK: Routledge \& Kegan Paul.

Wilson, J. (1973). The assessment of morality. London, UK: NFER, UK.

Wilson, J. (1986). What philosophy can do. London, UK: Macmillan.

Wilson, J. (1987). A preface to morality. Hampshire,UK: The Macmillan Press Ltd.

Wilson, J. (1990). A new introduction to moral education. London, UK: Cassell Education.

Wilson, J. (1998). Two aspects of morality. Journal of Moral Education, 27 (1).

Wilson, J. (2000). Learning to love. London UK: Macmillan Press Ltd.

Wilson, J. \& Straughan, R. (1983). Philosophising about education. London, UK: Pelican Original.

Wilson, J., Williams, N. \& Sugarman, B. (1967). Introduction to moral education. Middlesex, UK: Pelican Books.

Winch, P., (Ed.). (1989). Simone weil. "The just balance". Cambridge, UK: University of Cambridge.

Wittgenstein, L. (1972). Tractatus logico-philosophicus

London, UK: Routledge \& Kegan Paul. 
References

Zigler, R. L. (1998). The four domains of moral education: The contributions of dewey, alexander and goleman. Journal of Moral Education, 27 (1).

Zigler, R. L. (1999). The formation and transformation of moral impulse. Journal of Moral Education, 28 (4). 


\section{Appendix A: Questionnaire}

Investigating Teachers' Perceptions of their Teaching of Thinking Skills Questionnaire.

Your careful and clear responses to each of the following questions will help to identify many aspects of how teachers today approach their planning, and how they make authentic and legitimate curriculum decisions for the teaching of thinking skills to secondary students.

Please write clearly.

A. This section seeks to provide an accurate profile of teachers who teach thinking skills.

PERSONAL DETAILS

1. Name:

2. Gender: $\quad M$

F

3. Address.

Postcode. 
Appendix A

4. School

5. Personal qualifications

6. Years of Service/ Teaching history

7. Please list the subject areas and year levels in which you teach thinking skills:

B. This section seeks to focus on your professional knowledge and practice.

CURRENT TEACHER PRACTICE:

8) Are there particular curriculum frameworks that you use for the teaching of thinking skills? If so, please indicate what these are. 
Appendix A

9) Please describe how thinking skills are taught in your classes.

10) At what stage of the teaching process do you prefer to introduce thinking skills?

11) How do you organise your curriculum topics and teaching strategies to introduce teaching skills? Please provide an example of this organisation if possible.

C. This section seeks to provide an opportunity to understand your classroom strategies and management of student learning process.

CLASSROOM PEDAGOGY: 
Appendix A

11) How frequently do you as teacher raise questions?

Often $\square \quad$ Regularly $\quad \square \quad$ Sometimes $\quad \square \quad$ Rarely

12) How frequently do you as teacher ask for questions?

Often $\quad \square \quad$ Regularly $\quad \square \quad$ Sometimes $\quad \square \quad$ Rarely

13) When do you raise the questions?

At the beginning of topic

At the end of topic

Randomly during the topic

14) When does discussion take place?

As a result of student questions

As a result of teacher questions

As a result of the text content

15) Describe the process you use to stimulate discussion, including whether it is teacher- or student- directed?

D. This section seeks to find out about the process you use to teach specific tasks and student response to this.

PROCESS FOR TEACHING: 
Appendix A

16) Which students do you stimulate towards thinking skills?

Leaders

Quiet one's

Noisy one’s

Boys

Girls

Alternating boy and girl $\square$

Random choice

Student choice

All students

17) What sequential processes do you use in the teaching of thinking skills? e.g.,: pre- post testing; question starters such as - how, when, where, ; de Bono's hats

18) Please specify and explain, especially if there are other processes: 
Appendix A

19) What particular thinking skills do you teach and at which particular levels?

Please list and given some explanation.

20)Please explain if you concentrate on some thinking skills and not on others?

Yes $\square$

21)Please comment on the thinking skills activities you use most often.

22) Please comment on the reasons for promoting these thinking skills and/or knowledge.

23) How do you reward (not assess ) the display of thinking skills? (e.g., Teacher approval? Inclusion in teaching framework? Do more thinking skills?)

E. This section seeks to discover your knowledge and possible use of three particular curriculum frameworks that pertain to thinking skills. 
Appendix A

SIGNIFICANT CURRICULUM FRAMEWORKS:

24) Have you used Matthew Lipman's Philosophy for Children to teach thinking skills? Yes

No

If so, please say how this is done.

25) Have you used John Wilson's Moral Components List to teach thinking skills?

Yes $\square \quad$ No

If so, please say how this is done

26) Have you used Peter Vardy's Five-Strands for teaching World Religions and Values?

Yes $\square \quad$ No

If so, please say how this is done. 
Appendix A

27) Have you used other curriculum frameworks designed to teach thinking skills? Yes $\square \quad$ No

If yes, please specify and say how you use it.

28) Have you attended professional development or faculty in-service on the following?

Philosophy for Children (Matthew Lipman) (strongly Ethics based)

Yes $\square \quad$ No

Moral Components List (John Wilson) (strongly Virtues based)

Yes $\square \quad$ No

Five-Strands for World Religions/Values (Peter Vardy) (strongly Values based)

Yes $\square \quad$ No 
Appendix A

29) If you do use any of these curricula, please discuss your reasons for including them and their strategies in the planning and implementing of teaching of thinking skills.

30) Please describe your approach/ planning for the teaching of thinking skills. Include common strategies that you use.

F. This section seeks to determine the process for teaching thinking skills that you use

TEACHING PRACTICES:

31) Do you teach decision-making?

Yes $\quad \square \quad$ No 
Appendix A

32) If so, please describe how the classroom process works, e.g., a typical incident:.

33) How do you respond to students who disagree with other students' answers, or responses, in discussion? Please describe a typical incident.

34) What is your approach to resolving possible disagreements? Is there a recognised process or does it depend on certain localised factors? Please describe a typical example. 
Appendix A

35) Do you ask students to explore reasons for their responses? Please describe a typical incident in which students clarify their responses.

36) What do you do if students disagree with your own reasons? Please describe a typical incident showing how such a matter is normally resolved if it happens in your classroom.

37) Do you teach the meaning of 'consensus' as part of thinking skills?

Yes $\square \quad$ No

38) If so, do you teach the process of consensus as part of thinking skills?

Yes $\square \quad$ No

39) Do you teach the meaning of 'democracy' as part of thinking skills?

Yes $\square \quad$ No

40) If so, do you teach the process of democracy as part of thinking skills?

Yes $\square \quad$ No 
41) What strategies do you use to teach the above skills of consensus and democracy? Please describe by using a couple of typical incidents about the teaching of consensus and democracy.

42) Please describe your views and practices, if any, on the teaching of Virtues within a thinking curriculum for your class.

43) Do you think it is important to teach about Virtues Education as part of the teaching of thinking skills in your classroom?

Yes $\square \quad$ No

44) If yes, can you describe what you mean by Virtues Education? 
45) If no, please give an indication of what you believe Virtues Education to be, and why it may not be important in your classroom.

46) Do you think it is important to teach the skills inherent within Virtues Education in your classroom?

Yes $\square \quad$ No

47) If you do, can you describe what you mean by Virtues Education:

48) Please describe what you see as the distinction between Virtues Education and Values Education, if any.

49) Please describe your views and practices, if any, of the teaching of Values within a thinking skills curriculum in your classroom. 
Appendix A

50) If you do, can you describe what you mean by Values Education.

51) If not, please give an indication of what you believe Values Education to be, and why it may not be important in your classroom.

52) Please describe your views and practices, if any, of the teaching of Ethics within a thinking skills curriculum in your classroom.

53) If you do, can you describe what you mean by Ethics Education. 
Appendix A

54) If not, please give an indication of what you believe Ethics Education to be, and why it may not be important in your classroom.

G. This section concerns itself with where the teaching of thinking skills 'fits' in the daily work of involvement in your teachinglearning process.

55) Do you teach thinking skills in subject areas such as

* Study of Society and Environment (SOSE) $\quad$ Yes $\quad \square \quad$ No

* Religious studies Yes $\quad \square$ No

* Bible studies Yes $\quad \square$ No

* Values education

Yes

No

* Ethics education

Yes

No

* History of Ideas

Yes

No

* Philosophy

Yes

No

* Philosophy for Children

Yes

No

* English

Yes

No

* Literature studies

Yes

No 
Appendix A

* Life Skills education

* Personal Development and Physical education studies

Yes

No

* Mathematics

Yes

No

* Languages other than English

Yes

No

* Science and Technology

Yes

No

* The Arts

Yes

No

* Other (please specify)

56) Please describe a typical incident of the teaching of thinking skills in at least one subject area above.

57) In the teaching of Virtues Education, Values Education and Ethics Education, please explain with reasons, which, if any, of the three curriculum areas is more important in your teaching of thinking skills to secondary students. 
58) Are there different teaching approaches that you use in different subject areas? Please describe using a typical incident to clarify your statements.

59) Please tell us about the way in which you teach thinking skills in the subject areas indicated.

60) When students respond inappropriately, how do you encourage engagement with thinking skills?

61) Are there are any other significant matters or comments you would like to add about the teaching of thinking skills to secondary students which is about 
Appendix A

investigating teachers' perceptions of their teaching of thinking skills to secondary students.

62) Please describe clearly and give an example to help highlight the classroom process.

Thank you for your efforts.

Your responses are appreciated. 
Appendix B: Splitter \& Sharp

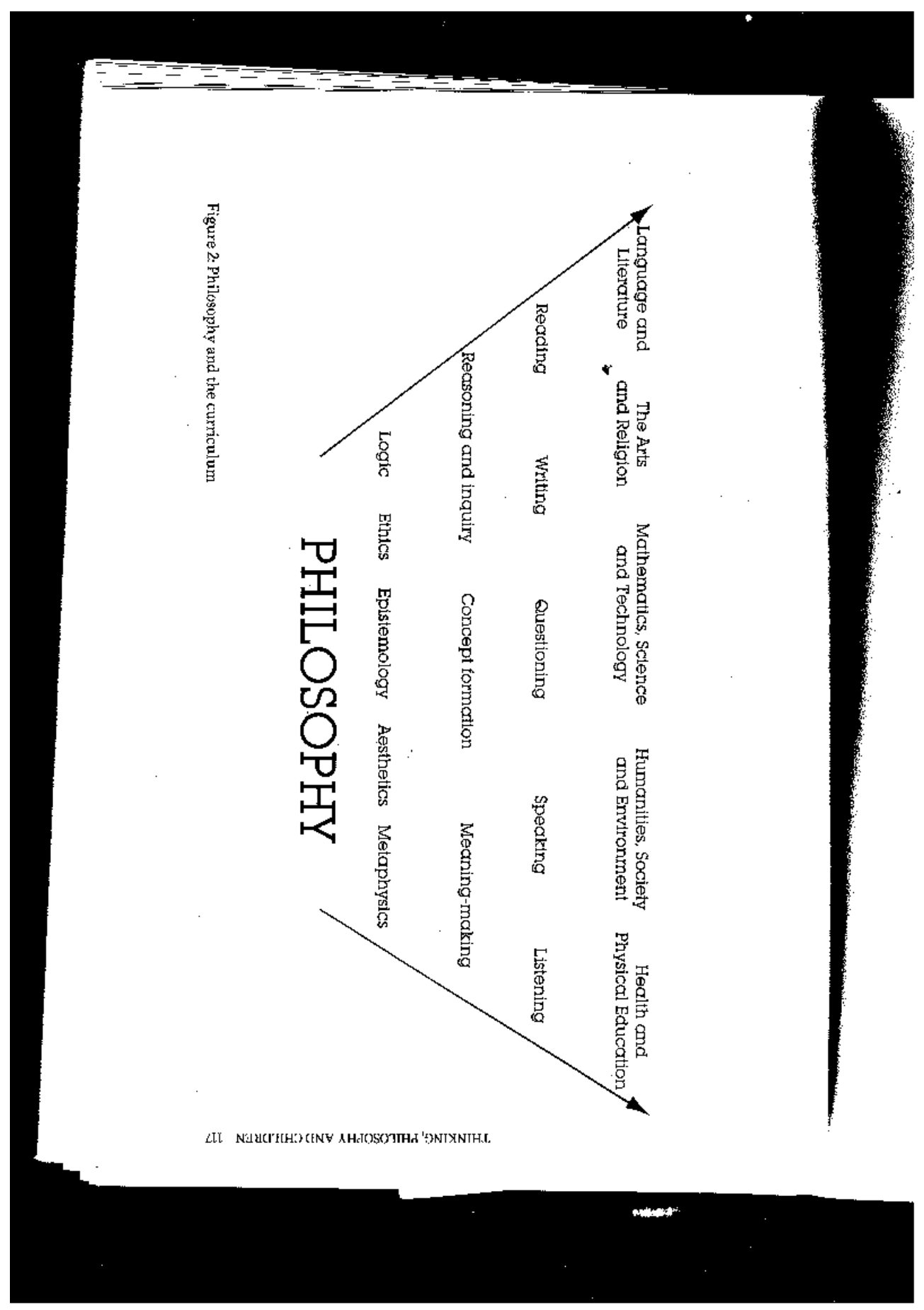




\title{
Appendix C: John Wilson
}

\section{Wilsons Moral Components List}

\section{PHIL attitude respect/concern for others}

PHIL (1) Respect for equals, justice, giving others their rights

PHIL (2) Benevolence, love, altruism

Separable into
(a) Belief that others count
(b) Feeling of respect/ concern
(c) Appropriate action taken as a result of (a) and (b), at least under normal circumstances.

\section{EMP ability awareness of emotions}

EMP (1) (AUTEMP) awareness of one's own emotions

EMP (2) (ALLEMP) awareness of others emotions

\section{Separable into}
(a) Awareness of conscious emotions
(b) Awareness of unconscious emotions (insight)

\section{GIG attainment mastery of relevant knowledge}

GIG (1) knowledge of facts, knowing that

GIG (2) knowhow, social and other relevant skills

\section{KRAT (1) attainment bringing to bear of above components}

\author{
KRAT (1a) noticing \\ KRAT (1b) thinking fully \\ KRAT (1c) taking responsibility
}

The above, taken together, will lead to a decision about what to do or to feel. We mark this stage by the term DIK (when other peoples' interests are involved) or PHRON (when they are not).

\section{KRAT (2) attainment translation of the decision into action}

In the equation form: 


\title{
PHIL EMP GIG (plus KRAT (!) > DIK/ PHRON: plus KRAT (2) > right action and emotion.
}

For purposes of assessment, i.e. of assigning some kind of score to individuals or groups for each component, it is necessary to consider various dimensions and life areas for each component in which scores may differ. For instance, subjects may vary in the range of people for who PHIL (high PHIL for gang members, low for adults), or the consistency within that range: or they may have high KRAT (1) for sex but low for drug taking: high GIG (1) for facts concerned with safe driving, low for facts concerned with laws and social norms: and so forth. These and some other assessment problems are discussed in Problems of Research in Moral Education and Testing and Assessment in Moral Education (Conceptual Notes).

From John Wilson Education in Religion and the Emotions (p. 261 262).

\begin{abstract}
It is important, I submit, to note that the attitude, ability and attainment that Wilson has italicised is the point at which the effort to synthesise the elements of logic and reasoning with the existence of the emotional landscape of the individual. Wilson was at the cutting edge of the thinking that was emerging at that time for the integration of psychology, philosophy, logic and sociology into what was to count as right and wrong in intention, in behaviour and in consequences for the community.
\end{abstract}




\title{
Appendix D: Lipman Obituary by Roger Sutcliffe
}

\author{
Matthew Lipman (1923 - 2010)
}

The name of Matthew Lipman is still not as widely known, let alone honoured, as that of John Dewey, who Lipman himself suggested in his autobiography was the greatest philosopher of education of all time.

Yet the legacy that Lipman left on his death, just after Christmas 2010, may yet prove to be of greater impact as the twenty-first century continues its desultory advance towards a new paradigm of education - one fit for globalising, fast changing societies, whose citizens increasingly struggle to make sense of the world and of their lives.

Lipman's autobiography was titled, A Life Teaching Thinking (IAPC, 2008, ISBN 0916834417), and in it he expressed the modest hope that he had some part of that process of locking ourselves in to the new educational approach, the toward thinking orientation.

In fact, the practice of Philosophy for Children, that he conceived in the late 1960s and that has developed in the 40 years since, was and remains in the vanguard of the movement towards more thoughtful curricula.

There is no room for complacency in this regard, though. So long as national curricula are evaluated largely through objective examinations, which emphasise the so-called hard skills of information recycling, rather than the smart skills of critical interpretation and evaluation, and creative conceptualisation and application - so long will there be a need for educational radicals such as Lipman.

Not that Lipman was always a radical. As one of seven sons of Russian Jewish parents who immigrated to the USA in the early 1900s, he did not rebel against the comparatively conventional lifestyle that they pursued in order to establish themselves in their new country.

Probably the most unconventional trait that his machinist father passed on to him was that of valuing practical invention. One longer range consequence of my father's inhabiting a world of tools, machines and motors was that, without being conscious of it, I came to prefer a world of principled practicality, while considering theory empty and sterile.

Later Lipman undoubtedly saw himself not only as the radical inventor of the principles of Philosophy for Children, but also as the determined pioneer (along with Ann Margaret Sharp) who turned its principles into practice.

But, to emphasise the point that this radicalism came comparatively late, he said himself: Up until the university riots of 1968, I had always been a gradualist as far as educational reconstruction was concerned... But the university riots suggested that both educational theorists and educational practitioners had been much too sluggish. A whole new plan, a whole new practice, a whole new theory - all of these had to be drawn up and set in place virtually instantly.

What emerged in the years either side of these riots in Columbia University (where Lipman was a tenured professor teaching Contemporary Civilisation) were, firstly, a book that became the foundation of the Philosophy for Children initiative - Harry Stottlemeier's Discovery (1967); and, secondly, the development of the practice more widely known as the community of inquiry - which is best exemplified in P4C but which is quietly becoming the touchstone for good pedagogy throughout the curriculum.

Here is how Lipman describes the origins of this pedagogy: The new educational dispensation I was starting to envision involved putting the spotlight on thinking in the classroom - not especially on the memorisation of facts, but on the greater, grander concept of thinking itself. This could be done by exposing the class to a specially prepared text that would exhibit a new way of thinking, encouraging the members of the class to interpret the passage as they saw fit, and having them discuss their interpretations together. The contention and deliberation that this process would stir up in the classroom would lead students to have opinions they would turn into judgements, and discussions they 
would turn into dialogues... About ten years later, borrowing a phrase invented by Charles Peirce, I would call such a group a community of inquiry.

Now is not the time to elaborate or refine this account. Suffice it to say (a) that Lipman himself elaborated it in his scholarly chef d'oeuvre, Thinking in Education (2003), especially explicating the essential concepts of Critical, Creative and Caring thinking; and (b) that others have enriched the theory by emphasizing, also, the concept of Collaborative thinking, and have enriched the practice by extending specially prepared text to include specially selected stimuli, such as picture books and artefacts.

It has to be acknowledged that Lipman himself did adhere strongly to the belief that his own texts/stories were the best materials for introducing children (and teachers) to philosophical inquiry; and they do, indeed, provide a resource of unparalleled richness. They have been used successfully, moreover, in the 40 or more countries where P4C has been taken up.

But Lipman had learned from Dewey's book (Intelligence in the Modern World) that philosophy had a lot to do with the relationship between theory and practice. Dewey was very emphatic on this point. What is more, he called his position experimentalism. I liked the term because it seemed to encourage the creative side of education and the imaginative side of life.

So it would probably not have upset him too much to see others experiment, successfully, with other materials, not least one's with imaginative and aesthetic dimensions. (Lipman's doctorate was in fact in the field of aesthetics, and he had a deep interest in both children's art and nonverbal thinking.)

What one could and should, indeed, say is that Lipman's breadth of education and interest was probably well beyond that of most educators or even professors.

Perhaps this was partly a result of reflection on his experience as a naïve American in his early 20s caught up in a war on another continent: I was more responsive to the aesthetic aspect of my wartime experience than to its ethical aspect... I didn't really understand the depth of tragedy that had taken place in the concentration camps, nor did the other Americans I was with.

At any rate, within a year of that experience he had studied for two semesters at the American University in Shrivenham, UK, where he was introduced to formal philosophy, and had introduced himself to Spinoza: It was my first experience of philosophy as sheer intellectual power - in this case, the power of understanding. Spinoza's Ethics wasn't just contemplative: it was visionary philosophy at its most exciting.

Then, in 1946, he enrolled in the School of General Studies at Columbia University, where he attended courses in fine arts and psychology as well as philosophy. Again, he read beyond the given syllabus, and must have been one of the first Western undergraduates (or even graduates) to read and appreciate Vygotsky: In the Psychology Library I was able to peruse the back issues of Psychiatry, and it was there that I discovered Vygotsky. I found Vygotsky's work intriguing because it brought together Philosophy, Psychology, Language, Social Studies and many other areas of learning.

There can be little doubt that the development of the theory and practice of the community of inquiry, some 25 years later, was informed by this reading; and the same might be said in regard to another great thinker in the field of language and learning, as this quote indicates: There had never been any question for me of the originality and power of George Herbert Mead... Thus the fifties ended for me with an avid interest in striking out on my own, but finding that the way to do so involved committing myself to those of the previous generation or two who had already done so successfully.

Another mark of Lipman's growing independence can be found in his personal life. In 1950, shortly after he had graduated, he had gone to study at the Sorbonne in Paris, as a Fulbright scholar. In the same cohort of scholars was Wynona Moore, a black student from the French department at Columbia. In France they shared many acquaintances and opportunities to develop their ideas, and they also became close friends, to the point that in January 1952 they married in the Paris City Hall. The following is a revealing comment on their decision to get married.

Given that we were in love, we thought it appropriate for the times. We were susceptible enough to the existential emphasis on the exemplary act ... that we were inclined to see such an act as being the kind of powerful example one should set for others... We had too much respect for one another to engage in a marriage solely for political or philosophical reasons, but that incentive was there nevertheless.

The couple had two children, and the marriage lasted for 20 years. It probably would have lasted longer but for the fact that Wynona had struck out on a political career, eventually becoming a Senator for New Jersey. Their two life paths were diverging, and although Lipman said divorce was not an action he could have taken for himself, his relationship with Wynona remained friendly and affectionate, and it is clear that he continued to take pride in her achievements as a black politician.

His own politics, like his personal relationships, were founded upon a deep respect and empathy for others. The politics are captured simply in the following statement: The reduction of social 
discrimination through insistence on equality in matters of employment, housing and education is bound to be, as we have seen, an approach so slow and tedious as to make one wonder if it represents progress at all. Nevertheless, we cannot afford not to go this route.

The ethics closer to his own growing professional interests - are expressed in the following: I was deeply moved by how children suffer, and how little they can do about it. And I think it was at that time (the 1960s) that I was beginning to see the importance of freedom of inquiry, not simply for teachers, but for children as well... I was aware of the possibility that what I was planning to develop was something that could be useful for the purpose of moral education and not just for the improvement of thinking skills.

Once that plan had been put into effect, there was early success, not only in hard evidence that thinking skills were indeed advanced by $\mathrm{P} 4 \mathrm{C}$, but also in recognition of its positive social and emotional effects - even its value in deliberate moral education (as distinct from indoctrination). The mass media, intrigued by our quixoticism, called dramatic attention to our activities. We were not just tinkerers; we were visionaries and activists who came from so far outside of the field of education that we might just possibly be right.

Even the BBC became interested in the late 1980s and produced Socrates for 6 year olds, a remarkable documentary which launched P4C on its path in the UK and of which Lipman himself asked the question: What kind of society is it that can turn its back on such evidence of the kind of difference Philosophy for Children can make in the classroom?

In fact, $\mathrm{P} 4 \mathrm{C}$ is far from being eschewed, either in the UK or elsewhere, and continues to make quiet but solid progress towards changing the culture of education and indeed of public discourse. A recent conference at the LSE indicated strong support from a varied range of speakers for the restoration of philosophy at the heart of liberal education.

Lipman advanced one possible (and powerful) argument for this in a globalised society: The teaching of religion in the schools is attacked by the secularists, and the standoff that results seems capable of being resolved by replacing the time given to religion in the elementary school curriculum with time given to the teaching of philosophy.

Perhaps that is still too radical a thought even for an advanced liberal society. More moderate might be the argument that Personal and Social Education can best be conceived as a philosophical enterprise - indeed, that it cannot be conceived as education proper unless it does have a philosophical, and especially an ethical, dimension.

This was expressed by Lipman as follows: The approach that I have created in Philosophy for Children is about process, not content. It is not about prescribing any one philosophy to children but about encouraging them to develop their own philosophy, their own way of thinking about the world. It is about giving the youngest of minds the opportunity to express ideas with confidence and in an environment where they feel safe to do so.

One might just add that the process encourages interdependent, critical thinking as well as independent, creative thinking, and that this is why P4C remains, at its best, the most holistic and wholesome thinking skills approach.

That at any rate, is the view of the thousands of educators throughout the world who continue to share Lipman's own hope that Philosophy for Children will become accepted across the world, not just as another strand in the overcrowded curriculum but as a pedagogy that will build a better and more reasonable world for our children and their children to inhabit: a world that looks as beautiful from across the street as it does from the distance of space.

That hope, expressed by Lipman in 2008, demonstrates not only his humanity but also his courage, for by this time he was suffering considerably from the Parkinson's disease with which he had been diagnosed nearly ten years earlier. As if that was not hard enough to bear, in 2006 his second wife, Teri, who was 20 years younger than he, died unexpectedly.

A man who could lose so much, and yet write, looking back, both: For me, wherever Teri was, there was love and spirituality; there was divinity, and I love the world that has produced something as beautiful as philosophy is a rare man. Matthew Lipman will certainly be remembered for Philosophy for Children, but he should also be remembered as a remarkable philosopher for adults.

Roger Sutcliffe, December,2011 


\section{Appendix E: Strategies for teaching (Moore)}

(Basil Moore, 1986 Conference AARE, Brisbane; one day was assigned to each model for the conference)

- Concept Attainment Model

- Inquiry Learning Model

- Communications Model

- Negotiated Model

- Shared Praxis Model

To make it easier both to read each model in its entirety and to compare and contrast the various models, I have used the following format to reconstruct each model:

\begin{tabular}{|c|c|}
\hline ELEMENTS & DETAILS \\
\hline Its developers & $\begin{array}{l}\text { An introduction to some of the key people who have developed the model } \\
\text { and their writing }\end{array}$ \\
\hline $\begin{array}{l}\text { Its distinctive and key } \\
\text { goals }\end{array}$ & $\begin{array}{l}\text { No model of teaching has an entire set of educational goals which are } \\
\text { unique to it. Models are, however, in part characterised and distinguished by } \\
\text { their central and focussing goals }\end{array}$ \\
\hline $\begin{array}{l}\text { Its critique of traditional } \\
\text { education }\end{array}$ & $\begin{array}{l}\text { Each model of teaching is clarified and distinguished to some extent by the } \\
\text { specific way in which it characterises traditional education and criticises it } \\
\text { for its shortcomings. It is helpful to identify this criticism as it sharpens } \\
\text { insights into what the model proposes as a corrective. }\end{array}$ \\
\hline Its rationale & $\begin{array}{l}\text { Each model believes that the style of learning it proposes is important, if not } \\
\text { essential, for effective human functioning. As teachers we will usually } \\
\text { adopt (or adapt) any particular model if we are persuaded about its } \\
\text { importance and value for our students. }\end{array}$ \\
\hline $\begin{array}{l}\text { Its key theoretical } \\
\text { assumptions }\end{array}$ & $\begin{array}{l}\text { Models of teaching which are genuinely worth considering by thoughtful } \\
\text { teachers have carefully thought through and researched theoretical } \\
\text { foundations especially concerning the nature of knowledge (Epistemology) } \\
\text { and the process of human learning (learning theory). Important distinctions } \\
\text { between teaching models are to be found in the particular theories of } \\
\text { knowledge and of learning which inform them. }\end{array}$ \\
\hline $\begin{array}{l}\text { Its basic } \\
\text { teaching/learning } \\
\text { structure }\end{array}$ & $\begin{array}{l}\text { In the end the value of a model of teaching for practising teachers is its } \\
\text { ability to provide a basic plan or structure for the teaching/learning } \\
\text { interaction in the classroom (or other settings). No model of teaching tries to } \\
\text { impose on teachers an inviolable set of steps which must be followed } \\
\text { slavishly. But each does attempt to provide teachers with a basic guide } \\
\text { which they can adapt. A major distinguishing characteristic of each model is } \\
\text { the distinctive sequencing it provides the teaching/learning process. This } \\
\text { sequencing of the teaching/learning process is, perhaps, what best enables } \\
\text { us to understand a model of teaching and to apply it to our own teaching. }\end{array}$ \\
\hline A hypothetical example & $\begin{array}{l}\text { I conclude the exposition of each model with an attempt to illustrate how } \\
\text { each particular model might be developed in the classroom to teach/ learn } \\
\text { something of the theme associated with it in the Conference structure. But I } \\
\text { do hope that the practical example will go some way towards clarifying the } \\
\text { methodology. }\end{array}$ \\
\hline
\end{tabular}

Basil Moore's reference is Joyce and Weil. 'Models of Teaching'. Englewood Cliffs, Prentice Hall, $2^{\text {nd }}$ edition 1980.

I used the $3^{\text {rd }}$ edition of Joyce and Weil 1986. 